\title{
Modulation of monocyte responses by progressive multiple sclerosis therapy.
}

Вy

Carl Zimmermann Beyers

A thesis submitted to Victoria University of Wellington in fulfilment of the requirements for the degree of Doctor of Philosophy.

Victoria University of Wellington

2020 



\section{Table of Contents}

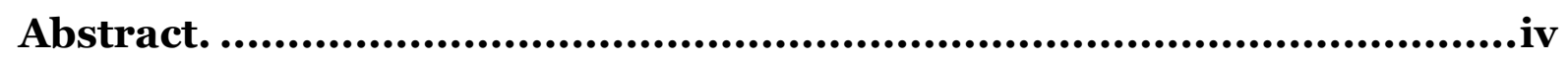

Acknowledgements. ..............................................................................vi

Figures......................................................................................vii

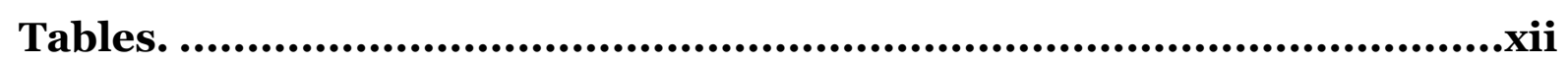

Abbreviations. ............................................................................... xiii

Chapter 1. General Introduction. ................................................. 1

1.1 The immune system. ………………………................................................ 1

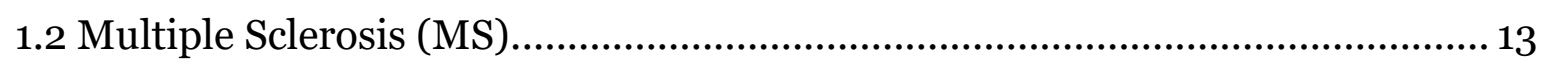

1.3 Immunophenotyping in MS..........................................................................24

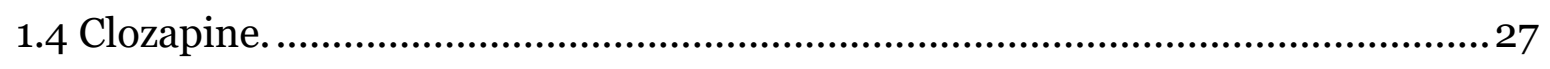

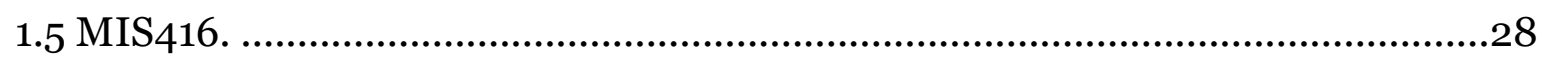

1.6 Overall aims and objectives. ........................................................................... 30

Chapter 2. Immune phenotyping of whole peripheral blood in progressive multiple sclerosis............................................................................... 31

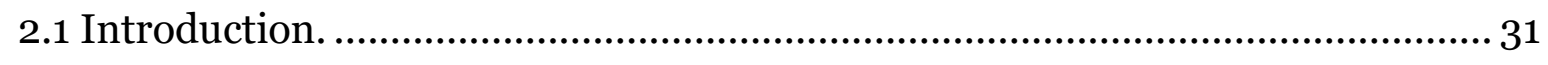

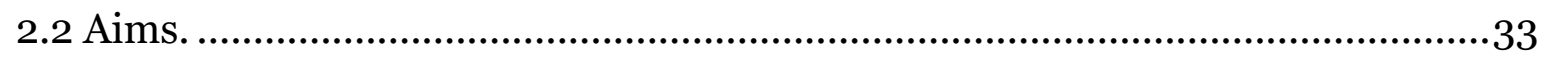

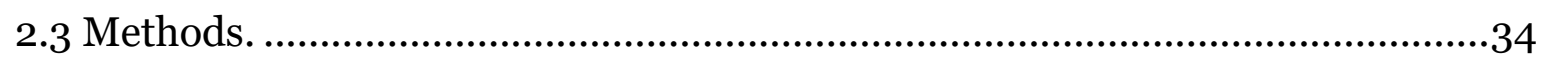

2.4 Results. .....................................................................................................

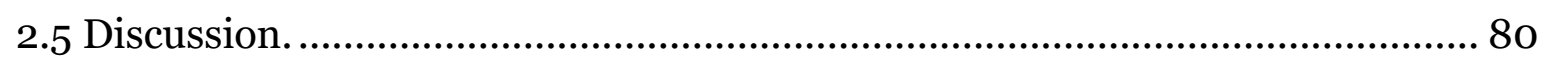

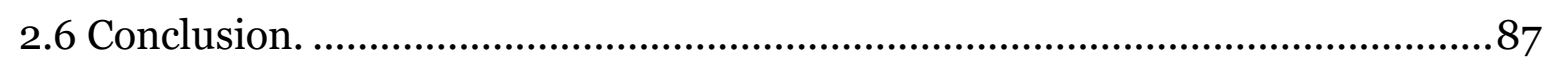

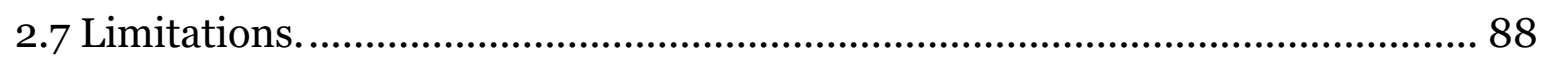

Chapter 3. Materials and Methods for in vitro culture. ....................... 89

3.1 Sample collection and storage. …….................................................................. 90

3.2 PBMC processing, staining and data acquisition................................................ 91

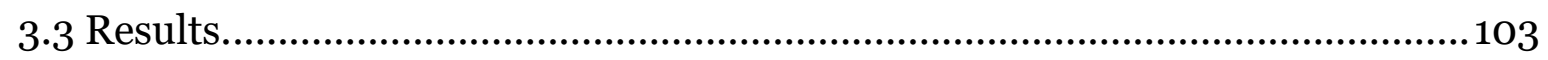

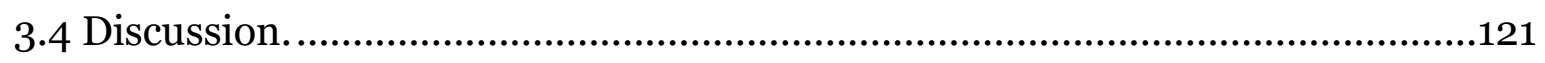

Chapter 4. The effects of in vitro clozapine on dopamine receptor and cytokine expression in progressive MS...............................................124 
4.1 Introduction.

4.2 Aims. 127

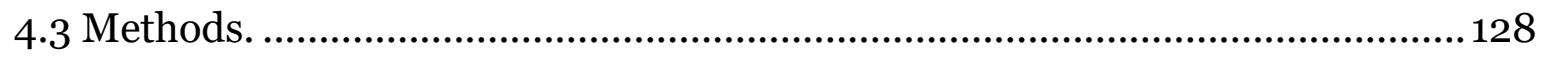

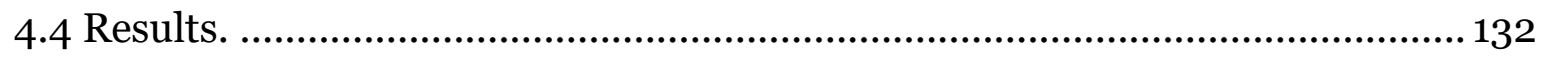

4.5 DR expression patterns of in vitro cultured monocytes.............................. 135

4.6 In vitro clozapine and its effects on intracellular cytokine expression............151

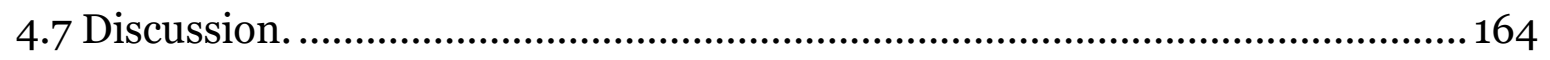

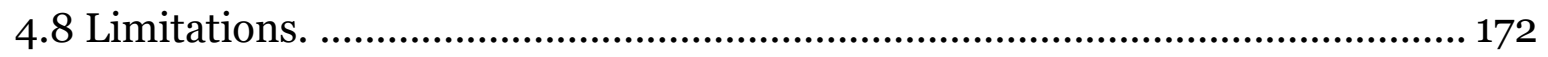

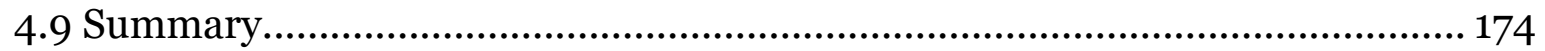

Chapter 5. Transcription factor and cytokine responses following MIS416 treatment in vitro......................................................... 175

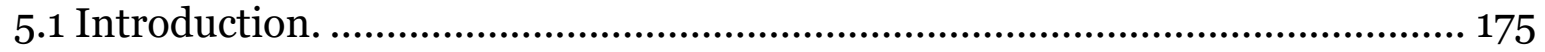

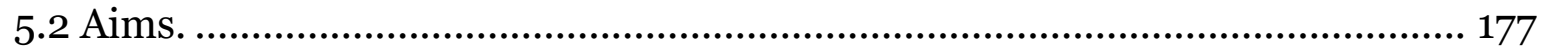

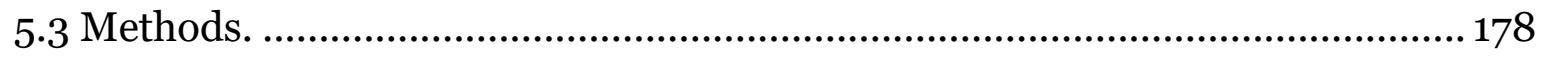

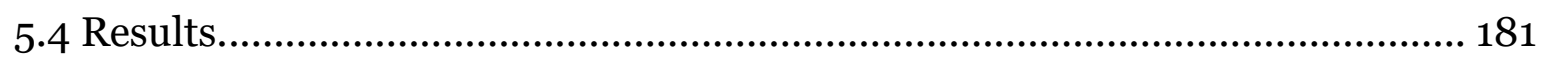

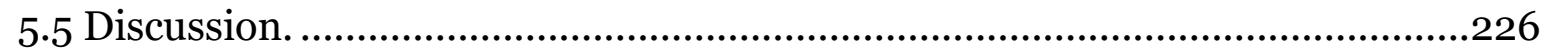

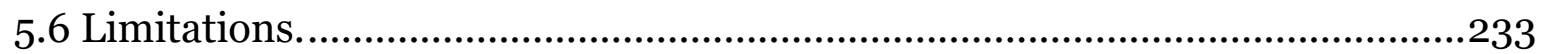

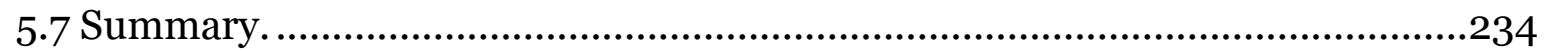

Chapter 6. t-SNE \& SPADE of multicolour cytometry data.................235

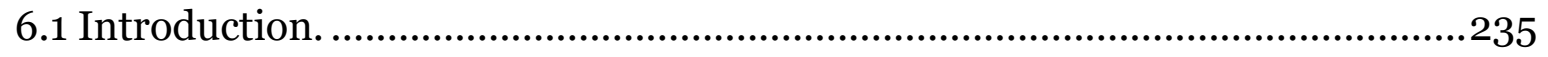

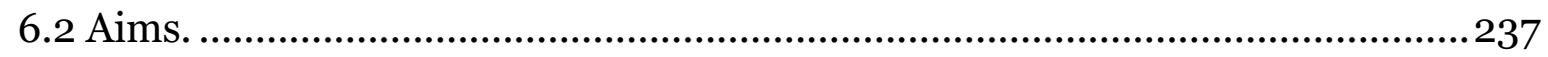

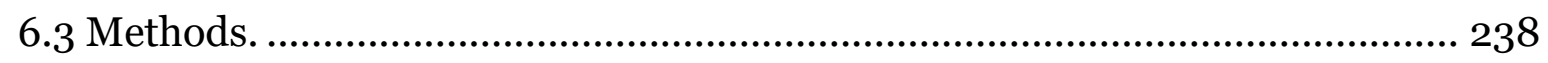

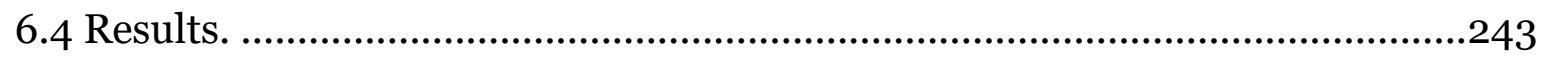

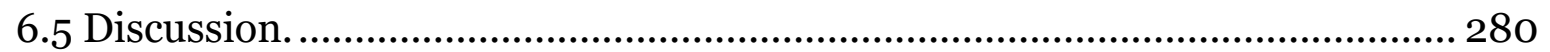

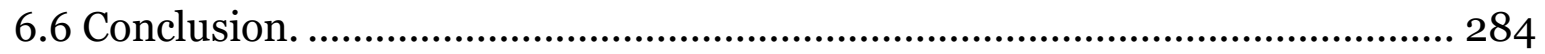

Chapter 7. Summary and future directions ..................................285

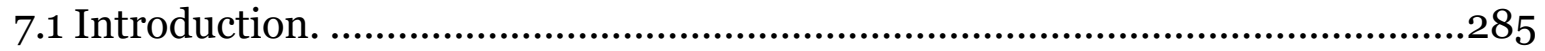

7.2 Chapter 2. Immune phenotyping in progressive MS............................... 286

7.3 Chapter 3. Materials and Methods for in vitro culture................................287 
7.4 Chapter 4. The effects of in vitro clozapine on dopamine receptor and cytokine expression in progressive MS..........................................................................28

7.5 Chapter 5. Transcription factor and cytokine responses following MIS416 treatment in vitro ........................................................................................... 289

7.6 Chapter 6. t-SNE \& SPADE of multicolour cytometry data.............................. 291

7.7 Future directions................................................................................................293

Bibliography. ....................................................................................295 


\section{Abstract.}

Multiple sclerosis (MS) is an immune-mediated neurodegenerative disorder that is distinguished by neuroinflammation and demyelination. MS is severely debilitating and remains the most common cause of disability arising from non-traumatic brain and CNS damage in adults. In its progressive phase there are no effective treatments, so new therapy options are an urgent research priority. Extensive work has been done on the role of the adaptive immune system in contributing to the disease pathology and on the effects of therapies targeting lymphocytes in relapsing-remitting MS. Fewer studies have examined innate immune cells in people with progressive MS. This thesis addresses that gap by profiling monocyte phenotype and function in response to new and repurposed drugs that may provide benefit in progressive MS. This was achieved by modelling the drugs' effects in vitro using peripheral blood cells from people with progressive MS and healthy subjects.

Clozapine is an atypical antipsychotic with broad receptor affinity that is primarily used to treat refractory schizophrenia. In addition to is antipsychotic action through dopamine receptor (DR) D2, its broad neuro-immune receptor affinity is thought to dampen inflammatory responses in the CNS. This thesis highlights clozapine's antiinflammatory effect by demonstrating a reduction in the expression of proinflammatory cytokines that are associated with MS pathology in treated monocytes. Clozapine also induced a significant increase in the expression of D1. We observed that D1 expression changes happened alongside alterations to immune cell activity and that MS participant monocytes were much more susceptible to DR expression changes compared to healthy people. Together this data substantiates clozapine as a potential treatment for progressive disease.

MIS416 is a large, non-soluble microparticle suspension that induces nuclear factor kappa B $(\mathrm{NF \kappa B})$ dependent cytokine induction. We show here that monocytes are key cytokine responder cells to MIS416 and explore the molecular mechanism by demonstrating its effects on transcription factor activity. Our data showing increased production of cytokines by MIS416 suggests a route of treatment efficacy through tolerisation mechanisms, and by reducing inflammation through upregulation of antiinflammatory cytokines and negative feedback from pro-inflammatory cytokine 
release. Furthermore, we demonstrate how disease heterogeneity, phenotype, and genotype could significantly affect drug response outcomes in patients who received the drug as part of a phase 2 clinical trial.

Much of this work was done using new spectral cytometer technology. Its use allowed for the novel approach that enabled the subtraction of autofluorescent noise from out data, and we demonstrate its efficient functioning, ease of use, and utility in acquiring high dimensional datasets. The resulting large dataset allowed us the opportunity to interrogate it using bioinformatics tools, and we show their utility as adjunct tools to conventional methods of gating and statistical analysis. These analyses help demonstrate that monocytes are a heterogenous immune cell subset that is functionally distinct in people with progressive MS when compared to monocytes from healthy individuals. 


\section{Acknowledgements.}

Foremost thanks go to Prof Anne La Flamme. Her direction, help, and friendship were instrumental in seeing me through the last four years. This thesis would be substantially less without her input, and her mentorship inspired new ideas and help drive this research.

To Dr Gill Webster who very generously donated all the MIS416 clinical trial material, helped supervise this thesis, and offered amazing insights and direction, thank you.

To my loving, supportive, and patient partner David who deserves an immeasurable amount of gratitude. Your eloquent diplomacy helped me keep my head up. Without you I would never have been able to take up the opportunity to pursue this work. I love you very much.

To my colleagues and friends, particularly those in the LAF lab, I also very much appreciate the comradery and confidence when at times things did not go as planned. Special thanks to the people who helped with the experiments presented here: $\mathrm{Dr}$ Vimal Patel who performed the MTT assay work in Section 4.4.2; Dr Jun Yan at the University of Queensland who performed the DNA sequencing analysis for NFKBIA in section 5.4.5; and Cynthia Morgan who performed very many ELISA runs to collect the data in section $5 \cdot 4 \cdot 4$.

To my loving father Tertius, who gave me every opportunity to succeed, I am very grateful. Finally, I dedicate this work to my mother Carin. Her perseverance and optimism will always be an inspiration. 


\section{Figures.}

Figure 1-1 The evolution of MS. ................................................................. 17

Figure 2-1 T-cell (Panel 1) gating strategy........................................................ 45

Figure 2-2 B-cell (Panel 2) gating strategy.....................................................46

Figure 2-3 DC/NK/Neutrophil (Panel 3) gating strategy.......................................47

Figure 2-4 Myeloid (Panel 4) gating strategy. .................................................... 48

Figure 2-5 Representative titration data from PB antibody panel titrations............. 51

Figure 2-6 CD40 expression controls. …......................................................53

Figure 2-7 Expression of CD85k................................................................54

Figure 2-8 Gating control for HLA-DR............................................................54

Figure 2-9 Simple regression analysis of central pathology (path), and flow cytometry (flow) absolute count data sets. .......................................................................... 57

Figure 2-10 Absolute number $(\mathrm{A}-\mathrm{C})$ and proportion $(\mathrm{D}-\mathrm{F})$ of major T-cell subsets

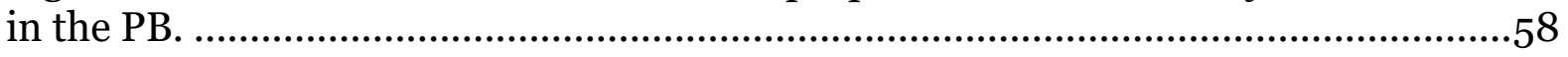

Figure 2-11 Absolute number (A\&B) and proportion (C\&D) of minor T cell subsets in

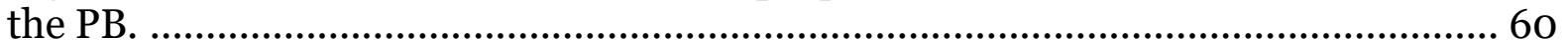

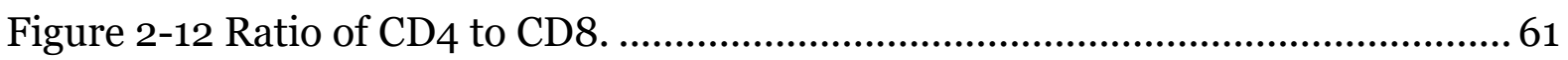

Figure 2-13 Absolute number of B cells, T cells and leukocytes in the PB from B-cell

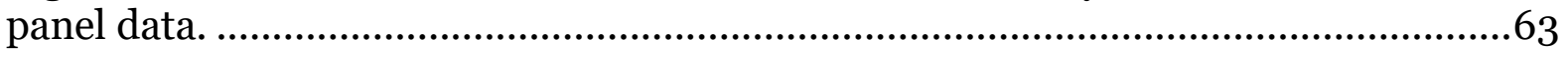

Figure 2-14 Absolute number of B-cell subsets in the PB. ....................................63

Figure 2-15 The proportion of B-cell subsets relative to all CD19+ events................64

Figure 2-16 Proportional composition of the B-cell compartment in HC and MS....64

Figure 2-17 Total number of myeloid cells by immune subset...............................66

Figure 2-18 Expression (gMFI) of activation markers of all monocytes..................67

Figure 2-19 Expression (gMFI) of HLA-DR, CD40 and CD85k on monocytes by subset. 68

Figure 2-20 Proportional makeup of the monocyte compartment relative to all PBMC.

Figure 2-21 Total proportion of immune subsets relative to all CD45+ cells. ........... 71 
Figure 2-22 Representative plots of immune cells subset variation in the HC. 73

Figure 2-23 Heatmap of marker expression of SPADE tree plots for all $\mathrm{T}$ cells (CD4\&CD8).

Figure 2-24 SPADE tree heatmap of marker expression of for all $\mathrm{T}$ cells (CD45RA\&CCR7).

Figure 2-25 SPADE tree heatmap of marker expression for all B-cells

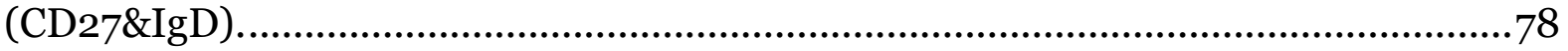

Figure 2-26 SPADE tree heatmap of marker expression for all B-cells

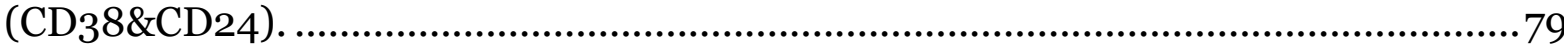

Figure 3-1 Sankey diagram of PBMC immune subsets by lineage marker phenotyping and the expected average proportional composition in a sample............................97

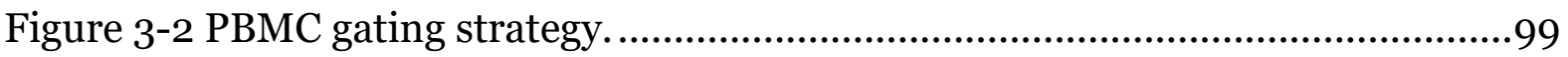

Figure 3-3 FMO gating for cytokine expression from stimulated monocytes. .........102

Figure 3-4 Inter-cohort comparison of the average DR expression for D1 - D5 .......106

Figure 3-4 Comparison of the average intensity of cytokine expression between CRISP and MIS416 trial participants.

Figure 3-5 Inter-cohort comparison of active transcription factor expression for CRISP and MIS416 participants. 108

Figure 3-7 PBMC lineage cocktail antibody titration. 111

Figure 3-8 Autofluorescence signal of unstained PBMC. 113

Figure 3-9 Monocyte autofluorescence by channel.

Figure 3-10 Average medium bead emission as fluorescence (gMFI) for each detector of the Aurora platform.

Figure 3-11 Representative donor comparison of STAT protein phosphorylation detection using methanol and saponin buffers. 118

Figure 3-12 The effect of saponin and methanol containing permeabilisation buffers on monocyte phosphoprotein responses in a representative donor. 119

Figure 3-13 Aurora cytometer spectral emission over all channels for APC-H7 and viability dye.

Figure 4-1 Effect of in vitro clozapine on PBMC viability. 134

Figure 4-2 The expression of DR in monocytes for all in vitro conditions. 136

Figure 4-3 Baseline (To) proportion of D5 positive monocytes. 137 
Figure 4-4 Baseline (To) DR expression of D1-5 in HC and MS participants. 138

Figure 4-5 D1 expression on monocytes of all FMO positive gated cells. .141

Figure 4-6 D5 expression on monocytes of all FMO positive gated cells. 144

Figure 4-7 D2 expression on monocytes of all FMO positive gated cells. 146

Figure 4-8 D3 expression on monocytes of all FMO positive gated cells. .148

Figure 4-9 D4 expression on monocytes of all FMO positive gated cells. .150

Figure 4-10 IL-6 expression in monocytes over all in vitro conditions. 152

Figure 4-11 IL-1 $\beta$ expression in monocytes over all in vitro conditions. 154

Figure 4-12 TNFa expression in monocytes over all in vitro conditions. 156

Figure 4-13 INFy expression in monocytes over all in vitro conditions. 158

Figure 4-14 IL-10 expression in monocytes over all in vitro conditions. 160

Figure 4-15 Linear regression of cytokine and D1 (dependent variable) expression for monocytes from the MS cohort. 163

Figure 5-1 Time series of fluorescent MIS416 uptake by monocytes from 3 healthy donors. 183

Figure 5-2 Phosphorylation of STAT1, STAT3, CREB and p65 in monocytes stimulated with MIS416 over a time series. 184

Figure 5-3 The mean proportion (A \& D) and level of activated NFאB p65 in monocytes. 187

Figure 5-4 The mean proportion (A \& D) and level of activated STAT1 in monocytes. 189

Figure 5-5 The mean proportion (A \& D) and level of activated STAT3 in monocytes. 191

Figure 5-6 The mean proportion (A \& D) and level of activated CREB in monocytes. 193

Figure 5-7 MIS416 response curve of cytokine expression. 196

Figure 5-8 Effect of MIS416 on in vitro cytokine production in cells stimulated overnight. 197

Figure 5-9 Representative, single donor LPS stimulation series. 199

Figure 5-10 The mean difference in the proportion (A, E \& I) and relative expression of IL-1 $\beta$ in monocytes following overnight in vitro stimulation. 201 
Figure 5-11 The mean difference in the proportion (A, E \& I) and relative expression of IL-6 in monocytes following overnight in vitro stimulation. 203

Figure 5-12 The mean difference in the proportion (A, E \& I) and relative expression of IL-10 in monocytes following overnight in vitro stimulation. 205

Figure 5-13 The mean difference in the proportion (A, E \& I) and relative expression of INF $\gamma$ in monocytes following overnight in vitro stimulation. 207

Figure 5-14 The mean difference in the proportion (A, E \& I) and relative expression of TNFa in monocytes following overnight in vitro stimulation. 209

Figure 5-15 The mean total cytokine detected by ELISA in tissue culture supernatants from concurrent in vitro experiments. 211

Figure 5-16 Pearson's Correlation of cytokine expression. 213

Figure 5-17 Linear regression of total cytokine expression. 214

Figure 5-18 The mean difference in total marker expression (gMFI) for NFкB p65, CREB, IL-6 and IL-1 $\beta$ in monocytes. 218

Figure 5-19 Mean serum immune factor response for MS participants stratified by NFKBIA promotor mutation. 219

Figure 5-20 The mean proportion, and level of activated NFkB p65 in monocytes. 221 Figure 5-21 The mean proportion, and level of activated CREB in monocytes. .......222 Figure 5-22 The mean proportion, and level of activated STAT1 in monocytes.......224 Figure 5-23 The mean proportion, and level of activated STAT3 in monocytes. .....225 Figure 6-1 SPADE analysis data-flow for a simulated dataset. ................................. 240 Figure 6-2 t-SNE plot representative of all in vitro monocytes (Row A)...................245 Figure 6-3 SPADE analysis plot using t-SNE parameters of all concatenated in vitro monocyte data. ......................................................................................................246

Figure 6-4 t-SNE plot overlay of outlier cluster monocyte data. ..............................249

Figure 6-5 t-SNE plot overlay of central clustered monocyte data........................... 250

Figure 6-6 SPADE analysis plot using lineage markers of all concatenated in vitro monocyte data. .252

Figure 6-7 TNFa expression comparison between HC and MS cohorts for LPS, MIS416 and untreated cells. 254

Figure 6-8 IL-1 $\beta$ expression comparison between HC and MS cohorts for LPS, MIS416 and untreated cells. 
Figure 6-9 IL-6 expression comparison between HC and MS cohorts for LPS, MIS416 and untreated cells.

Figure 6-10 INF $\gamma$ expression comparison between HC and MS cohorts for LPS, MIS416 and untreated cells.

Figure 6-11 IL-10 expression comparison between HC and MS cohorts for LPS, MIS416 and untreated cells.

Figure 6-12 t-SNE plot of TF expression by monocytes for all participants and all conditions.

Figure 6-13 t-SNE analysis of TF expression in monocytes from all participants and all conditions.

Figure 6-14 Heatmap overlay of Monocyte D1 expression in MS participants. .262

Figure 6-15 Clustering analysis of all in vitro monocytes and expression (gMFI) of DR1, DR3 and DR5..... 264

Figure 6-16 SAPDE analysis of monocyte diversity. .265

Figure 6-17 SPADE analysis depicting normalised DR expression in monocytes for MS participants. 267

Figure 6-18 Inter-cohort comparison of DR expression for DR3 and DR5 at To... 268 Figure 6-19 Density distribution SPADE analysis of t-SNE parameters for monocytes. .269

Figure 6-20 t-SNE of all PBMC data depicted as a density overlay of all PBMC (A) and displaying manually gated subsets (B). 271

Figure 6-21 t-SNE plot of PBMC from MS participants and the effects on DR1 expression in untreated and LPS cultures respectively. 272

Figure 6-22 t-SNE plots of DR expression by the PBMC. .274

Figure 6-23 t-SNE analysis of cytokine expression by the PBMC .276

Figure 6-24 Heatmap of TNFa expression in the PBMC of MS participants by t-SNE analysis. .278

Figure 6-25 t-SNE plot of TNFa expression in untreated and MIS416 treated PBMC. .279 


\section{Tables.}

Table 2-1 Whole blood cytometry antibody cocktails...........................................36

Table 2-2 FACS Canto II whole blood cytometry panel design...............................37

Table 2-3 Major T-cell fractions enumerated in Panel 1. ..........................................38

Table 2-4 Major B-cell fractions enumerated in Panel 2.......................................39

Table 2-5 Innate cell subsets enumerated in Panel 3 ............................................39

Table 2-6 Monocytes cell subsets enumerated in Panel 4...................................... 40

Table 2-7 Whole blood analysis participant demographics. ..................................50

Table 3-1 Antibody cocktail mixture for in vitro PBMC staining..............................94

Table 3-2 PBMC panel for all in vitro assays...................................................... 98

Table 3-3 Demographic distribution of all in vitro study participants....................103

Table 3-4 Demographic distribution of MS study participants. ..............................104

Table 3-5 Average per channel CV of the combined data series for the medium CS\&T bead collected on the Aurora platform over 6 months....................................... 116

Table 4-1 Dopamine receptor antibodies.........................................................130

Table 4-2 Demographic distribution of study participants................................... 132

Table 4-3 Summarised results of the effects of in vitro culture conditions on

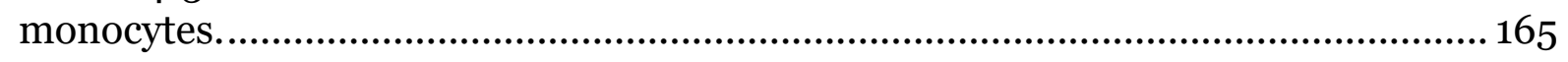

Table 5-1 Demographic distribution of study participants.................................. 181

Table 5-2 Single donor stimulation of PBMC over an LPS dose range. ...................198

Table 5-3 Cell proportions following overnight LPS stimulation............................198

Table 6-1 Proportional composition of central cluster events by treatment condition. 251

Table 6-2 t-SNE overlay of the proportional distribution of the PBMC. 271

Table 6-3 Expression (gMFI) of DR for all PBMC by treatment condition. .272

Table 6-4 Heatmap of average expression (gMFI) of cytokines for gated immune

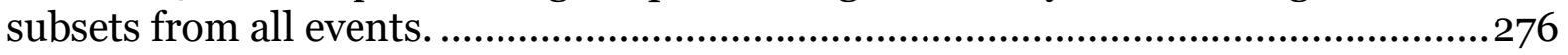

Table 6-5 Effects of clozapine on TNFa expression by subset. ..............................278 


\section{Abbreviations.}

ATP

$\mathrm{AC}$

ANOVA

APC

AHSCT

$\mathrm{Bcl}$

BCR

BBB

BM

$\mathrm{Ca}$

CREB

CNS

CSF

CIS

Cloz

CRISP

CD

$\mathrm{R}^{2}$

$\mathrm{CV}$

CI

cAMP

CS\&T

CTL

DC

DNA

DMF

DMT

DR

D1
Adenosine Triphosphate

Adenyl Cyclase

Analysis of variance test

Antigen Presenting Cell

Autologous Haematopoietic Stem Cell

Transplant/Therapy

B-cell lymphoma

B-cell Receptor

Blood brain Barrier

Bone Marrow

Calcium

cAMP response element-binding

Central Nervous System

Cerebrospinal Fluid

Clinically Isolated Syndrome

Clozapine

Clozapine and Risperidone for the Treatment of Progressive Multiple Sclerosis

Cluster of Differentiation

Coefficient of Determination

Coefficient of Variation

Confidence Interval

Cyclic Adenosine Monophosphate

Cytometer Setup and Tracking

Cytotoxic T cells

Dendritic Cells

Deoxyribonucleic Acid

Dimethyl Fumarate

Disease Modifying Therapies

Dopamine Receptor

Dopamine Receptor D1 
D2

D3

D4

D5

DMEM

EM

EMRA

EGF

EBV

EAE

$\mathrm{FTY}_{720}$

FACS

FMO

FoxP3

FSC

gMFI

GA

$\mathrm{HC}$

HLA

Ig

INF

IL

ICC

LPS

MRI

MHC

MIS

MAPK

MAIT

MS

MDP

mDC

NTZ

NK cells
Dopamine Receptor D2

Dopamine Receptor D3

Dopamine Receptor D4

Dopamine Receptor D5

Dulbecco's Modified Eagle Medium

Effector Memory

EM re-expressing CD45RA

Epidermal Growth Factor

Epstein Barr Virus

Experimental Autoimmune

Encephalomyelitis

Fingolimod

Flow Cytometry

Fluorescence Minus Control

Forkhead Box $\mathrm{P}_{3}$

Forward Scatter

Geometric Mean Fluorescence

Intensity

Glatiramer Acetate

Healthy Control

Human Leukocyte Antigen

Immunoglobulin

Interferon

Interleukin

Intracellular Cytokine

Lipopolysaccharide

Magnetic Resonance Imaging

Major Histocompatibility Complex

MIS416

Mitogen-Activated Protein Kinases

Mucosal Associated Invariant T cells

Multiple Sclerosis

Muramyl Dipeptide

Myeloid DC

Natalizumab

Natural Killer cells 
NKT

NLR

$\mathrm{NF \kappa B}$

NOD

PAMP

PRR

PDG

PBMC

PMT

pDC

PPMS

PMS

PML

QC

Treg

RRMS

ROR

SPMS

STAT

$\mathrm{Na}$

SPADE

S1P

SD

TfH

Th

TCR

t-SNE

TLR

$\mathrm{TF}$

TNF
Natural Killer T-cells

NOD-Like Receptors

Nuclear Factor Kappa B

Nucleotide-binding Oligomerization

Domain-containing protein

Pathogen Associated Molecular

Patterns

Pattern Recognition Receptor

Peptidoglycan

Peripheral Blood Mononuclear Cell

Photon Multiplier Tube

Plasmacytoid DC

Primary Progressive MS

Progressive MS

Progressive Multifocal

Leukoencephalopathy

Quality Control

Regulatory T cell

Relapsing-Remitting MS

Retinoid-related Orphan Receptor

Secondary Progressive MS

Signal Transducer and Activator of Transcription

Sodium

Spanning-tree Progression Analysis of

Density-normalized Events

Sphingosine-1-phosphatase

Standard Deviation

$\mathrm{T}$ follicular Helper

T Helper

T-cell Receptor

T-distributed Stochastic Neighbour

Embedding

Toll-Like Receptors

Transcription Factor

Tumour Necrosis Factor 
VCAM-1

VLA-4
Vascular Endothelium Cell Adhesion

Molecule-1

Very Late Antigen-4 


\section{Chapter 1. General Introduction.}

\subsection{The immune system.}

The mammalian immune system functions to distinguish self from non-self, aiming to remove infections caused by pathogenic, parasitic, and opportunistic organisms that occupy and harm the body. This function includes the removal of autologous cells in which a loss of homeostatic control has occurred (e.g. cancer) and the clearing up of debris from senescent or dying cells. Overall, the system is highly complex, comprising many distinct structures, cells, and molecules that mount and execute the immune response. In classifying the system, we commonly refer to two complementary arms, namely, the adaptive and the innate immune systems.

\subsubsection{The adaptive immune system.}

Adaptive processes are targeted responses that are also engaged with building and executing immune memory. These processes facilitate what is initially a slow but specific response to non-self antigen, with a more rapid response on subsequent repeat antigen exposure. Specificity is developed during immune cell maturation, where bone marrow (BM) derived lymphoid precursor cells mature through a process that generates vast numbers of potential antigen receptors that can bind an estimated $10^{16}$ different molecules (Robins et al., 2009). The two major adaptive immune cell types are B cells, which have B-cell receptors (BCR), and T cells, which have T-cell receptors (TCR).

\subsubsection{Adaptive receptors.}

Receptor diversity is generated through a process where gene segments are rearranged to produce a combination of variable (V), diversity (D), and joining (J) segments called VDJ recombination (Tonegawa, 1983). VDJ recombination, together with a combination of subsequent diversity generating processes, form the antigen receptor 
sequences that comprise the adaptive immune repertoire. Each daughter cell that survives selection is unique and has the capacity to expand clonally upon antigenic stimulation. The rapidity of response is established by memory lymphocyte subsets that have had previous antigen exposure. Antigen binding stimulates lymphocytes to proliferate, generating effector, and longer-lived memory subsets that persist long after the antigen has been eliminated. Memory cells, upon a second exposure to antigen, begin clonal expansion that results in a rapid and specific response to infection.

\subsubsection{B cells.}

B cells function to produce cytokine and antibodies. Antibodies, also known as immunoglobulin (Ig), are secreted BCR. Their functions include roles such as complement activators, acting as toxin and pathogen neutralising agents, or as opsonisation agents to direct phagocytosis (Casadevall and Pirofski, 2004). B cells mature in the BM, and usually undergo clonal selection in the secondary lymphoid organs (e.g. spleen or lymph nodes). Most B cells develop into antibody secreting cells by first differentiating into plasma blasts and then into long lived Ig-secreting plasma cells (Zinkernagel et al., 1996). Plasma cells are established primarily in the BM, or at sites of inflammation, while a small proportion also develop into memory B cells (Murphy, 2012). B cells can also have a significant impact on inflammation by producing pro-inflammatory (e.g. IL-6) as well as anti-inflammatory (e.g. IL-10) cytokines and by acting as regulatory cells (Barr et al., 2012, Iwata et al., 2010, Fillatreau et al., 2002).

\subsubsection{T cells.}

T cells also arise in the BM but their maturation occurs in the thymus. The three main sub-populations of $\mathrm{T}$ cells are cytotoxic $(\mathrm{CD} 8+)$, helper $(\mathrm{CD} 4+)$, and regulatory $\left(\mathrm{FOXP}_{3}+\right)_{\mathrm{T}} \mathrm{T}$ cells. These subsets have distinct roles in directing, regulating, and enacting immune responses, although not exclusively so (Murphy, 2012). Unlike in B cells, TCR recognition of antigen only occurs when an antigen has been pre-processed 
by antigen presenting cells (APC) and presented via major histocompatibility complex (MHC) molecules.

\subsubsection{CD4+ T helper (Th).}

As the name suggests this subset functions primarily to support the actions of other immune subsets. Th cells were initially subcategorised into only two polarising subsets, namely Th1 \& Th2, as based on distinct cytokine production (Mosmann et al., 1986), but due to the range of functions and plasticity of this subset we now appreciate this was insightful, but overly simplistic.

Th1 were one of the original subsets described by Mosmann et al. They arise following infection by intracellular bacteria and viruses. Th1 produce INF $\gamma$ and are identified by the transcription factor Tbet (Szabo et al., 2000). Th1 cells are involved in cellmediated immunity and the elimination of bacterial infected cells. Th2 were the second subcategory and they function to support the induction of B cell activation, antibody production, and class switching, so are often described as central to the elimination of extracellular pathogens. Th2 are identified by the transcription factor GATA3, and produce IL-4, IL-5 and IL-13 (Zhu et al., 2006).

Th17 are distinct from Th1 and Th2 (Harrington et al., 2005). Th17 act as mucosal surface and -barrier defenders, and recruit and induce neutrophil production. Their differentiation requires expression of the retinoid-related orphan receptor gamma $t$ (ROR-yt) (Manel et al., 2008), that is also used to identify this subset. IL-17 is the main effector cytokine for this subset and the subset functions to protect against extracellular bacteria and fungi (Tesmer et al., 2008).

\subsubsection{2 $\mathrm{T}$ follicular helper (Tfh).}

Tfh cells are a specialised subset of helper cells aiding B cell function. They are characterised by the expression of the transcription factor B-cell lymphoma 6 (Bcl6). Tfh cells home to B-cell follicular structures where they play an essential role in the establishment and formation of germinal centres. As germinal centres are where plasma and memory B cells develop, Tfh cells play an essential role in effective B cell function. 


\subsubsection{Regulatory T cells (Treg).}

Processes during T-cell maturation aim to eliminate most self-reactive cells (see section 1.1.6 Tolerance). Some TCR with low self-reactivity escape deletion and must be regulated. Treg act to maintain and regulate these autoreactive cells, and to temper immune responses. Treg are distinguished from other Th cells by their expression of the transcription factor Forkhead Box P3 (FoxP3) (Sakaguchi, 2005; Sakaguchi et al., 2008). Their regulatory capacity is centred around their ability to supress the immune function of other T cells through the production of cytokines such as IL-10 (Annacker et al., 2003). They also function to prevent autoimmunity through processes like the impeding of co-stimulation of self-recognising T-cells (Walker and Sansom, 2011).

\subsubsection{CD8+ Cytotoxic T cells (CTL).}

CTL are cytokine producing cells that act to kill other cells bearing their antigenic target. CD8 is a glycoprotein expressed on the CTL surface that is essential for the interaction of a CTL with MHC class I on the target cell (Milstein et al., 2010). CTL are central to the body's defence against intracellular pathogens, cancer, and viral infections. Upon activation, CTL release cytotoxins that induce apoptosis of the target cell. One way this is achieved is via the presence of vesicular perforin stored and released by CTL. Perforin makes the target cells permeable to other CTL cytotoxins (e.g. granzyme) that have serine protease activity (Liu et al., 1995). Serine protease can trigger a caspase cascade, resulting in protein cleavage and cell disassembly, and ultimately in cell death (Nagata and Suda, 1995). Alternatively, CTL can also act on their targets through Fas-Fas interactions which are direct contact processes also mediated by caspase action (Rouvier et al., 1993).

The adaptive immune system is not an isolated system. Clonal activation of naïve adaptive immune cells requires signals in addition to activation through the BCR or TCR. These additional signals, in the case of $\mathrm{T}$ cells, often come from dendritic cells, while for naïve B cells these signals often come from helper T cells. Such signals, termed co-stimulation and antigen presentation (see section 1.1.5 APC), are regulated by the innate immune system (Murphy, 2012). Co-stimulation encompasses those secondary signals that are essential for the activation of immune cells. Generally, costimulation occurs in the presence of antigen presenting cells (APC), that then together with an antigen specific signal, results in immune cell activation. For T cells, these two 
signals come from the TCR ligating to its target antigen that is presented by and APC, and from activation of a co-stimulation receptor expressed on the $\mathrm{T}$ cell ligated to a ligand that is present on the APC surface. T-cell proliferation, differentiation and survival are highly dependent on these co-stimulation signals. Furthermore, innate system factors such as cytokines can prime the response of adaptive cells and act as attractive agents that induce the migration and transportation of immune cells to the sites of inflammation and damage.

\subsubsection{Innate immune system.}

The innate immune system can be described as the initial reflexive response to immunological challenge that usually precedes an adaptive response. Innate reactions recruit, activate and mediate cellular responses, and prevent entry of pathogens through barrier mechanisms and structures. Barrier structures like the skin form the first line of defence and include mucosal tissues and the action of cilia in the gut and respiratory tracts that prevent and remove infectious agents. Innate responses also include the complement pathway, and the functions of secreted immune factors such as cytokines and chemokines.

\subsubsection{Innate receptors.}

In contrast to the generation of novel lymphocyte receptors described for the adaptive system, innate cells possess germline encoded, evolutionarily conserved pattern recognition receptors (PRR). PRR survey for highly conserved pathogen antigens termed pathogen associated molecular patterns (PAMP). These include molecules such as bacterial DNA, lipopolysaccharide (LPS), and bacterial cell wall constituents including peptidoglycan (PDG) (Meylan et al., 2006). PRR are both intra and extracellular receptors that span a broad range including a collective of inflammasome-activating proteins, toll-like receptors (TLR), and NOD-like receptors (NLR). Of these, a range of TLR and NOD1 \& NOD2 have best been described. Each TLR functions in the detection of different PAMP antigens (Akira and Hemmi, 2003), while NOD1 and NOD2 are best described as crucial to the detection of bacterial cell wall constituent muramyl dipeptide (MDP), a subunit of peptidoglycan (Franchi et al., 2009). The activation of PPR induces the activation of transcription factors that lead 
to the production of cytokines, chemokines, adhesion molecules and enzymes that act as part of the host defence. These factors are produced following receptor activation and downstream signalling though the nuclear factor kappa B (NFkB), mitogenactivated protein kinases (MAPK), and type I interferon pathways that result in inflammation, immune factor production, and cell death responses (Meylan et al., 2006).

The cells of the innate system include those subsets that present antigen or produce mediators like cytokines, as well as cells that have phagocytic functions (e.g. monocytes) and regulatory roles (e.g. NK cells). They also include macrophages, dendritic cells, and large granulocytes (neutrophils, eosinophils, basophils).

\subsubsection{Monocytes.}

Monocytes are the largest of the leukocytes and are short-lived granulocytic responder cells. They comprise roughly $10 \%$ of the peripheral leukocyte population, with this proportion increasing during infection. Their common precursors are long term multipotent haematopoietic stem cells (HSC) that are resident in the BM. HSC give rise to common a myeloid progenitor, the myeloblast, that precedes all peripheral granulocytes, including monocytes (Monie, 2017). The BM is therefore the primary source for peripheral monocytes, ensuring continual replenishment of this short-lived subset. The BM and spleen further act as a cellular reservoir, facilitating the rapid release of monocytes during infection and tissue damage (Rees, 2010). Once released from the BM monocytes tend to stay in circulation for up to three days, although most have a short lifespan $(<24 \mathrm{~h})$ and are removed by apoptosis. Some monocytes may migrate into tissues or sites of damage and differentiate into macrophages and DC (Peters et al., 1991).

Peripheral monocytes are highly heterogeneous but can be broadly characterised into three subsets (classical, intermediate, nonclassical) by their expression of CD14 and CD16 (Table 2-6). Recently, this phenotypic distinction has been substantiated using extensive gene expression studies (Cormican and Griffin, 2020). While there is some overlap, the three subsets are functionally distinct: classical cells comprise the majority (>80\%) being highly effective phagocytic scavenger cells; intermediate 
monocytes are effective antigen presenting cells that produce the most reactive oxygen species (ROS); and non-classical cells may be anti-inflammatory and engage in T-cell proliferation and stimulation (Sampath et al., 2018). These subsets have also been demonstrated to show differential outcomes in their responses to stimulation, gene expression, and activation (Portevin et al., 2015; Smedman et al., 2012).

The role of monocytes during the immune response relate to their aforementioned functions. All monocytes express PRR that enable pathogen identification. Following activation, monocytes can also mediate inflammation through the release of cell signalling molecules and cytokines, and are known to affect a broad range of interactions in response to stimulation (Smedman et al., 2012). Furthermore, monocytes are also highly effective at T-cell co-stimulation, for example in presenting peptide in association with MHC to induce T cell mediated cytotoxic killing (Abeles et al., 2012; Ziegler-Heitbrock, 2006).

Monocytes well known for their pathogenic role as inflammatory mediators in autoimmune diseases (Cormican and Griffin, 2020). Conversely, they have an important functional role as immune regulators and in tissue repair, making these cells essential in resolving inflammation (Ma et al., 2019). Monocytes have been shown to infiltrate the CNS (Burdo et al., 2010), and are known to cause nerve damage upon activation and differentiation (Pulliam et al., 1997). In progressive MS, widespread activation of CNS mononuclear phagocytic cells, including monocytes, macrophages, and microglia is characteristic (Mallucci et al., 2015). In MS, these activated cells show strong associations with tissue damage, atrophy, impaired neuronal function, and decreased regenerative capacity (Ma et al., 2019). From this, it has been suggested that monocytes are key to the development of autoimmune disorders, specifically MS (Chuluundorj et al., 2014).

\subsubsection{Macrophages}

Furth et al. were the first group to describe the interrelationship between peripheral circulating monocytes and their tissue resident counterparts called macrophages (Furth et al., 1972). Together with other phagocytic cells, monocytes and macrophages form a group called mononuclear phagocytic cells with common morphology, function, and origin. Macrophages tend to be tissue resident, having been established 
before birth or can differentiate from monocytes at the sites of inflammation (Ovchinnikov, 2008). They have the same biology and functional capacity as monocytes and are often referred to interchangeably. Furthermore, macrophages have additional functions and names based on their anatomical location (e.g. microglia in the brain, osteoclast in bone etc.), for example in maintaining tissue homeostasis (Davies et al., 2013; Rees, 2010).

\subsubsection{Dendritic cells (DC).}

DC are very potent APC (see section 1.1.5 APC). DC in the periphery are as yet not fully categorised, but from their expression and function we can divide DC into three subclasses. These subclasses include a plasmacytoid ( $\left.\mathrm{CD}_{3} 3^{+}\right)$and two myeloid subsets (CD1c+ and CD141+ respectively) (Ziegler-Heitbrock et al., 2010). DC development occurs in the BM, from both myeloid and lymphoid precursors, or they may differentiate from monocytes (Peters et al., 1991). DC themselves may also differentiate into macrophages (Robinson et al., 1999). Despite this high degree of inter-relation DC are as distinct a cellular population as macrophages with respect to their tissue resident specificity (Chen et al., 2016). Furthermore, DC are distinct from monocytes/macrophages in their surface protein and gene expression which, together, supports their position as a unique terminal lineage subset (Robbins et al., 2008).

Monocytes, macrophages and DC are present both at the sites of inflammation and in peripheral circulation. As discussed, all three subsets have overlapping innate responder functions that include phagocytosis of targets by direct binding of their PRR, cytokine production of both a pro- and anti-inflammatory nature, and as APC. Together, these cells represent typical innate system architypes that span the gap between innate and adaptive processes.

\subsubsection{Natural Killer cells (NK cells).}

NK cells are cytotoxic cells that target and kill virally infected and cancer cells through overlapped mechanisms as seen in $\mathrm{B}$ and $\mathrm{T}$ lymphocytes, but without previous sensitisation. These include direct lysis using exocytosis of perforin and granzyme, as well as antibody-dependent cytotoxicity against IgG coated targets (Moretta et al., 2007). NK cells differentiate from the same lymphoid progenitor as $B$ and $T$ lymphocytes (Murphy, 2012). Unlike T cells, most NK cells do not express CD3, so 
recognition of target molecules (i.e. PAMP) is through innate PRR. Furthermore, NK cell activity is in turn regulated through activator and inhibitory receptors that recognise MHCI like molecules or by cytokines (Murphy, 2012). NK cells also mediated cytokine production and have a central role in shaping the innate immune milieu by facilitating DC maturation as well as in the reciprocal activation of DC, mast cells, monocytes and large granulocytes (Parolini et al., 2007).

\subsubsection{Large granulocytic cells.}

As with monocytes - neutrophils, basophils, and eosinophils arise in the BM and originate from the myeloblast. Of these, neutrophils form the most abundant leukocyte in the blood, while basophils are the largest. Neutrophils are short-lived $(\sim 8 \mathrm{~h})$ phagocytic cells that secrete a range of anti-microbial factors (Witko-Sarsat et al., 2000). These factors include cytokines that act as effector molecules for other immune cells (Ear and McDonald, 2008), and include the contents of cellular granules (Koenig et al., 2017). Basophils are the least common granulocyte and behave similarly to mast cells, a tissue resident granulocyte, in that they release histamine, serotonin, and heparin when stimulated. These agents increase vascular permeability and recruit other immune cells (Stone et al., 2010). Basophils, alongside eosinophils, are key mediators in responding to parasitic infection, as well as in the allergic response (Voehringer, 2009).

Common to all granulocytic cells are the presence of cellular granules. Granules contain myeloperoxidase, hydrolases, defensins \& permeability increasing protein and complement activators. Together, these agents target pathogens engulfed by phagocytosis using a membrane permeabilisation and oxidative killing strategy (Faurschou and Borregaard, 2003). Some granules also contain plasma proteins and membrane receptors that, when fused with the granulocyte surface, rapidly increase their density to facilitate transmigration and phagocytosis (Koenig et al., 2017).

\subsubsection{Antigen presenting cells (APC).}

APC are present in both adaptive (e.g. B cells) and innate (e.g. dendritic cells) immune systems. APC are so named because they process and present antigen to T cells. T 
cells require pre-processing of their antigenic targets and expression of these processed epitopes to facilitate recognition (Murphy, 2012). APC process fragments of antigens and display them to T cells using the two classes of human MHC I \& II. Both classes of MHC proteins are encoded by the highly polymorphic human leukocyte antigen (HLA) genes. The first class, MHCI, is ubiquitously expressed by nucleated cells and present protein fragments of internal origin that have been degraded by the proteasome. MHCI associated antigens are therefore of intracellular bacterial, or viral origin but can also originate from the host cell itself. Processing includes catalytic fragmentation and transportation to the APC surface where it is presented to CD8+ T cells in association with MHCI (Vyas et al., 2008). The second class, MHCII, present antigen that is exogenous in origin primarily to $\mathrm{CD} 4 \mathrm{~T}$ cells. MHCII associated antigen is therefore present on phagocytic cells such as dendritic cells (DC), B cells, and macrophages (Jensen, 2007). DC divert somewhat from the two methods described above due to their ability to cross present exogenous antigen to CD8 T cells using the MHCI. Conventionally only endogenous antigen is presented to CD8+ T cells, but DC are phagocytic cells that can engulf infected cells and fragments, that is then delivered to the MHCI pathway for presentation (Bevan, 1976; Jensen, 2007). Each individual person expresses at least three and up to six different MHCI, and at least three to four MHCII molecules on the cell surface. Different types of MHC molecules are better suited to presenting different antigens (Murphy, 2012). The MHC system and the HLA genes that encode it are central to the immune system's response capacity and diversity. This diversity also generates the unique genetic fingerprint that distinguishes individuals. Together, defects or subversion of these systems may result in autoimmune disease, transplantation rejection, and cancer.

\subsubsection{Tolerance.}

Massive immune diversity is required to recognise a broad range of antigenic targets but must also limit a self-directed response. The random nature of genetic rearrangement in the VDJ process of lymphocyte receptors invariably give rise to receptors that will recognise self-antigen. Cells expressing highly reactive self-directed receptors must be eliminated or, for less reactive types, restricted as some pathogenic antigens may have a high degree of similarity to self-epitopes. The process by which this is achieved is collectively termed immune tolerance. Tolerance functions to 
restrict the identification of self-antigen (Palmer, 2003), and to remove nonfunctional cells (Starr et al., 2003). There are two main processes, termed central and peripheral tolerance. Central processes are engaged in the primary lymphoid organs (thymus and BM), where lymphocyte maturation occurs. Central tolerance arises from the negative selection of self-recognising receptors expressed by lymphocytes in these lymphoid organs. High and sustained concentrations of antigen also induce tolerogenic processes in lymphocytes (Iezzi et al., 1998), thereby avoiding damage to many tissues and cells of the body. Peripheral tolerance restricts immune activity outside primary lymphoid tissues, as well as limiting reactions to commensal organisms and other non-pathogenic molecule such as food, gut bacteria, pollens and dust.

\subsubsection{Autoimmunity.}

Distinguishing self from non-self is not always executed successfully. Such failure can result in a breakdown where the immune system targets host tissues for immune mediated removal (i.e. autoimmunity). Autoimmune reactions usually target selfantigens, or the antigens of commensal microbiota (Tlaskalová-Hogenová et al., 2004), and characteristically, results in tissue damage. The main method of action is through autoantibodies, but auto-reactive T cells are also involved (Firestein et al., 2014). The pathology of autoimmune reactions is diverse, and it is in the combination of the method and target epitopes that the mechanism of an autoimmune condition derives. It is from this that can we define autoimmunity as an adaptive-immune system mediated disease.

Somewhat distinct from autoimmune diseases are the autoinflammatory disorders. These are commonly monogenic disorders classically observed as a pathology of the innate immune response. However, the close interface between adaptive and innate arm functions mean that autoimmunity spans a range of disorders of both an autoimmune and -inflammatory nature.

Several factors contribute to the likelihood of autoimmune disease. These risk factors are collated into genetic and environmental factors. Genetic predisposition and phenotype describe the contribution of the genetic background to autoimmune 
disease. This is commonly studied in large association studies that identify genetic regions or risk loci associated with a disease phenotype (Inshaw et al., 2018). Commonly, risk loci fall in the non-coding region of the genome, thought to be regulatory in function, which limits our understanding of their mechanistic contribution to autoimmune disease (Dendrou et al., 2018). Large cohort studies can also identify environmental risk factors for autoimmunity, such as lifestyle, behavioural, and even geo-locational, as exemplified by multiple sclerosis - the central topic of this thesis. 


\subsection{Multiple Sclerosis (MS).}

MS is an immune mediated neurodegenerative disorder of the brain and CNS. Other neurodegenerative disorders such as Huntington's, Alzheimer's and Parkinson's diseases, where neuroinflammation is a key component, contrast distinctly with MS (Bar-Or, 2008). In MS neuroinflammation is also present, though focal plaques of demyelination form the primary feature of MS pathology. This trifecta of inflammation, neurodegeneration, and demyelination, combined with an underlying autoimmune disorder, distinguishes MS from other neurodegenerative diseases (Fischer et al., 2013). MS is the most common cause of disability arising from nontraumatic brain and CNS damage in adults (Oh et al., 2019). Furthermore, nearly all genetic regions identified in genome wide association studies of MS are involved with immune mechanisms, underpinning the disease as primarily an immune mediated disorder (Lassmann, 2019).

The autoimmune component of MS is thought to be driven by autoreactive T cells that target epitopes present on the myelin sheath that surrounds axons of the brain and CNS. The T-cell attack of myelin causes the destruction/disruption of the myelin sheath, and by extension, nerve conduction. The symptoms of MS, while highly polymorphic, collectively deduce from the disruption of nerve conduction, from nerve damage, and from plaque formation. Symptoms may include: a loss of vision, fatigue, altered tactile and pain sensations, muscle pain and spasm, loss of motor-, bladder-, and bowel control, and cognitive impairment.

\subsubsection{MS aetiology and epidemiology.}

The cause of MS is unknown. Several risk factors for the disease have been identified that include both environmental and genetic components. These risk factors are believed, in combination, to cause MS (Olsson et al., 2016).

\subsubsection{MS Risk factors.}

Four key observations support the premise that environmental risk factors contribute significantly to MS aetiology: a latitude gradient of increased MS risk (Alla et al., 2016; 
Simpson et al., 2011), migration affecting MS risk (BMJ, 2019; Gale and Martyn, 1995; Kurtzke et al., 1985), an observed increase in the prevalence of MS likely attributable to lifestyle factors (Alla et al., 2014; Olsson et al., 2016), and the premise of the environment being a major driver of the adaptive immune repertoire. Recent evidence supporting environmental risk factors comes from data by Belbasis et al. They analysed 44 unique meta analyses and found three environmental risk factors that showed convincing evidence of an association with MS: Epstein Barr virus (EBV) nuclear IgG seropositivity, history of infectious mononucleosis (caused by EBV infection), and smoking (Belbasis et al., 2015). Further risk factors such as adolescent obesity, vitamin D deficiency, and organic solvent exposure also show evidence of an association (Olsson et al., 2016). Furthermore, clinical data underpins these association factors, with incident case controls demonstrating that both smoking and low sun exposure significantly increased the risk of onset of CNS demyelination (van der Mei et al., 2015).

\subsubsection{Genetic risk factor for MS.}

The strongest genetic risk factors for MS identified so far occur within the HLA gene region. The first of these was identified using linkage analysis by researchers in California in 1972 (Naito et al., 1972). The identification of other, non-random associations within the HLA region eventually revealed the DRB1*1501 locus as having a primary role in MS susceptibility (Oksenberg et al., 2004). DRB1*1501 is an HLA class II variant and the most common of this subtype found in Europeans with white ancestry (Sawcer, 2008). Genetic loci may also offer protection from the disease, an example of which comes from a class I variant HLA-A*02. Furthermore, disease risk can also be modified through the presence, lack of, or from a combination effect for some MHC haplotypes (Olsson et al., 2016).

\subsubsection{MS disease distribution and prevention.}

The prevalence of MS is unequally distributed around the globe and holds to a pattern whereby rates are significantly higher in low latitudes such as in North America and Europe. In 2013 there were an estimated 2.3 million people living with MS and the global median prevalence stood at 33 persons per 100,000, up from 30 in 2008 (MSIF, 2013). 
The relatively low incidence of MS in the population as a whole (1:1000) severely limits the epidemiological study of the disease. Study tools require very large population cohorts (i.e. statistical power) to be effective, and confounding bias factors contribute and hamper accurate population frequency determinations and obscure the extent to which environmental factors contribute to disease (Sawcer, 2008). There is currently not enough evidence of the causes of MS to instigate an effective program of prevention (Ascherio et al., 2012).

\subsubsection{MS pathophysiology.}

MS pathophysiology is a highly heterogeneous process. Disrupted physiology in MS and the resulting symptoms are primarily caused by disrupted nerve condition (Smith and Macdonald, 1999). This disruption stems from inflammation, demyelination, plaque formation, and CNS damage that are all mediated by the breakdown of the blood brain barrier. Recovery from relapse occurs due to remyelination, the expression of ion channels on denuded axon, and the resolution of inflammation.

\subsubsection{Demyelination.}

Removal of the myelin sheath from an axon results in the exposure of the underlying axonal plasma membrane (i.e. axolemma), which has far fewer ion channels with which to conduct an action potential, and results in the loss of signal conduction for which the extent of the loss is dependent on the site and the extent of demyelination (Smith and Macdonald, 1999). Remyelination may occur on remission that restores conduction (Chari, 2007), or it may be restored due to axonal expression of voltagegated ion channels in the exposed plasma membrane. Axons also degenerate during MS, primarily thought to be due to persisted $\mathrm{Na}^{+}$influx following demyelination that reverses $\mathrm{Na}^{+}-\mathrm{Ca}^{2+}$ exchanger channels that then pumps damaging levels of $\mathrm{Ca}^{2+}$ into the axon (Stys et al., 1992). This channel activity results in non-remitting pathological damage (Chari, 2007).

\subsubsection{The MS plaque.}

In the brain and CNS, focal plaques of demyelination caused by inflammation are identifiable as a key feature of MS. These sclerotic plaques are the sites of disease 
activity, and based on the area affected, may relate to disease symptoms (Confavreux et al., 2000). Within the MS plaque a diverse array of processes contributes to the collective effects that are demyelination, glial scarring, and axonal loss, while the cellular inflammatory contribution come from infiltrating reactive lymphocytes and macrophages (Bobermin et al., 2018). These plaques are not stationary lesions but an evolving process that may show deposits of IgG (i.e. complement), oligodendrocyte/neuronal/astrocyte loss, and may present with or without remyelination (Wu and Alvarez, 2011). Plaques may appear as both white and grey matter lesions. The localisation in either grey or white matter may be due to distinct disease processes, as grey matter lesions commonly present with lower levels of leukocyte infiltration (Peterson et al., 2001), are more common in progressive disease (Kutzelnigg et al., 2005), and correlate with the presence of cognitive disability better than white matter lesions (Amato et al., 2007). Another distinctive feature of MS pathology is the destruction of myelin and oligodendrocytes without immediate axon loss, that has been suggested as being primarily driven by a demyelinating agent (Lassmann, 2014).

\subsubsection{The blood brain barrier (BBB).}

Infiltration of peripheral leukocytes into the CNS is a critical component of MS pathology that is facilitated by a breakdown of the blood brain barrier (BBB) (Correale and Villa, 2007). The BBB comprises an endothelia cell layer between the brain parenchyma and vascular system that limits the entry of cells and proteins into the CNS, unless it is disrupted, or where distinct transport mechanisms exists (i.e. extravasation) (Zlokovic, 2008). Transport mechanism that facilitate leukocyte entry in the CNS regulate immune response mechanisms that are a critical part of CNS immune surveillance. BBB breakdown is also a common feature in neurodegenerative disorders. This breakdown can be a disruption that occurs at selective diffusion barrier proteins, known as tight junctions in the epithelia. Breakdown may also occur from a subversion of the immune privilege that usually excludes pathogens. In MS both forms of BBB breakdown are thought to contribute, and extravasation of selfrecognising T cells into the CNS may be a crucial initiating factor in MS (Minagar and Alexander, 2003; Ortiz et al., 2014; Ransohoff et al., 2003). Once in the CNS immune cells trigger inflammation factors that are known to mediate MS, and further exacerbate BBB breakdown (Minagar and Alexander, 2003). 


\subsubsection{CNS damage.}

In addition to demyelination and plaque formation, profound neurological damage occurs through neuronal, axonal and synapse degeneration (Dutta and Trapp, 2010). This degeneration is one of the hallmarks of progressive disease (Brück, 2005). What drives this tissue damage in MS is yet unknown, but oxidative force from mitochondrial damage may be a contributing cause (Lassmann, 2014). As discussed, the demyelinated axon detects an increase in $\mathrm{Na}+$ influx that requires active extrusion. Active extrusion eventually can result in ATP (i.e energy) insufficiency and oxidative damage (Brown and Bal-Price, 2003; Kapoor et al., 2003). This damage theory is substantiated by gene expression data that indicates decreased ATP production in chronically demyelinated neurons (Dutta et al., 2006), and from immunohistochemistry studies that showed functional defects in respiratory chain proteins in MS lesions (Mahad et al., 2008). Lastly, there are significantly more oxidatively damaged neurons present in MS lesions over other neurodegenerative disease controls (Fischer et al., 2013).

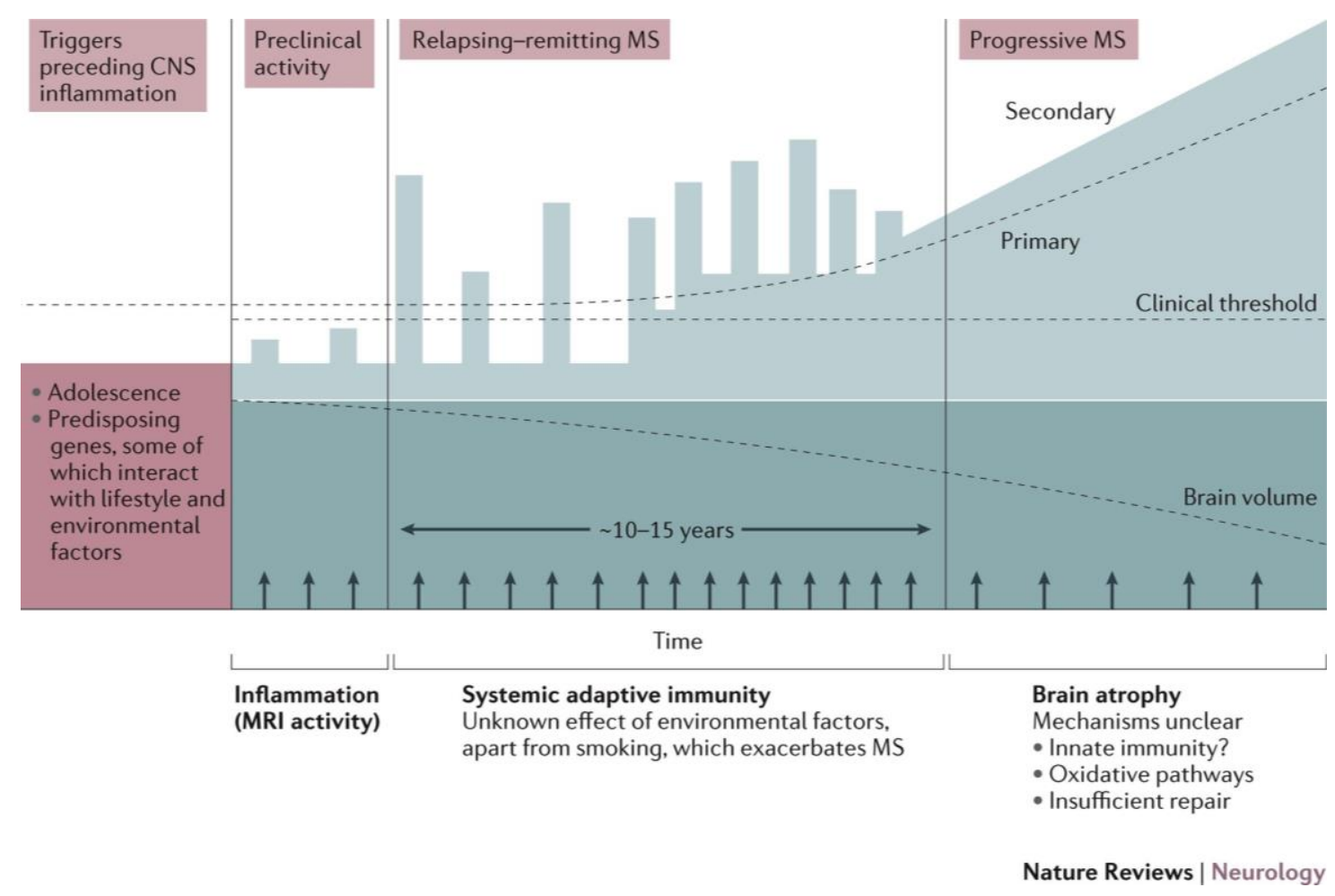

Figure 1-1 The evolution of MS.

The light blue area indicates progression, with bars representing relapses. Arrows indicate detection of inflammation by MRI. Adapted from Olsson et al., 2016 Reused with permission. 


\subsubsection{MS presentation and phenotype.}

Initial symptoms of MS are highly varied but clearly reflect CNS involvement, with optical neuritis, ataxia, motor weakness and general heat intolerance being common. MS is generally a long-term chronic disease that is not immediately life threatening but mortality is significantly higher than the general population due to compounding factors that result from long term disability (Scalfari et al., 2013). MS disease phenotype is either relapsing or progressive depending on the current stage and disease process. Three general phenotypes are used to distinguish disease phases: initial presentation, early stage intermittent disease that may span decades, and an end stage progressive course.

1.2.3.1 Clinically isolated syndrome (CIS).

CIS is defined as an initial presentation of symptoms of inflammatory demyelination that could be MS (Lublin et al., 2014). CIS presentation may also be classified directly as MS based on MRI scan criteria (Polman et al., 2011). Treatment with MS disease modifying agents following CIS presentation significantly reduces the risk of a second exacerbation that would then define a definite diagnosis of MS (Comi et al., 2001).

\subsubsection{Relapsing-Remitting MS (RRMS).}

Most patients present with a relapsing remitting disease course (Figure 1-1) that over time sees neurological disabilities accumulate. During this early phase, disease processes fluctuate with relapse and remitting periods. These may resolve completely, or such periods may lead to the accumulation of relapse induced damage and neurological disability from incomplete recovery that compounds to worsening disability. This early phase is often distinguished by the appearance of new, or the reactivation of old lesions in the brain and spinal cord.

\subsubsection{Progressive MS (PMS).}

Compounding damage and disability is unfortunately the outcome for the majority of RRMS patients. It may be that the functional capacity to resolve relapsing attacks diminishes, or the accumulating damage reaches a tipping point. Irrespective, eventually a progressive phase of the disease, termed secondary progressive MS 
(SPMS) starts. People with MS may also present with a progressive course although this is uncommon and occurs in only 10-15\% of MS presentations (Lublin et al., 1996, 2014). A progressive presentation is termed primary progressive MS (PPMS) and is defined as a presentation of MS in the absence of prior disease exacerbations (unremitting). The basic pathology of relapsing and progressive MS is, however, the same but occurs at a higher frequency in the progressive phase.

\subsubsection{Treatment with disease modifying therapies (DMT).}

While symptomatic treatment can alleviate some the disease effects, no cure for MS is available. Significant advances in reducing relapse rates have been made in the use of disease modifying therapies for RRMS However, once the disease advances to the progressive stage it becomes refractive to therapy, limiting options to symptomatic treatment (Lassmann et al., 2012).

Sequestration in the periphery, to prevent immune cells from moving into the CNS, is the premise for many DMT used in MS. Some of the currently licensed therapies available in RRMS often target the migratory capacity of peripheral lymphocytic cell populations (fingolimod and natalizumab) or eliminate specific subsets such as CD2O expressing cells (rituximab and ocrelizumab). The complex interplay of immune cell subsets and their interactions, abrogated through therapy, can together be considered broadly immunomodulatory with both treatment benefit and risk. Here we outline these drug induced changes where known, with a focus on the most common and broadly prescribed agents and their impact in the peripheral immune compartment, followed by the most promising treatment option - haematopoietic stem cell transplant therapy (see 1.2.4.7).

\subsubsection{Interferon Beta (INF $\beta)$.}

INF $\beta$ was one of the first DMT for MS and remains a current therapeutic option. It is a recombinant protein product with longstanding evidence of moderately reducing disease activity in RRMS. While the therapy aims to emulate the endogenous product, there are some unresolved questions on the impact and potential risks of disease exacerbation from therapy (Sellebjerg et al., 2012). The impact of INF $\beta$ on PB T-cells 
has been well defined (Chiarini et al., 2011; Praksova et al., 2012), yet few authors report the impact on other immune subsets, specifically innate immune cells. INF $\beta$ mechanistic action is diverse and impacts on myeloid cell co-stimulatory molecule expression, with treatment non-responders profiled with higher expression of activation markers like CD86 on myeloid cells prior to induction of therapy (Comabella et al., 2009). Compared to other DMT, INF $\beta$ may be a cost-effective option that limits the risk of annual relapse (Hamidi et al., 2018), but it has not been shown to limit the rate of progression in MS (Mantia et al., 2012).

\subsubsection{Glatiramer acetate (GA).}

GA was another early DMT that remains a treatment option. GA is a random polymer of four amino acids found in myelin basic protein that can effect a more antiinflammatory T-cell repertoire and inhibit T-cell activation in MS (Dhib-Jalbut, 2002). Furthermore, treatment results in a shift of the PB T-cell compartment towards an antigen inexperienced population (i.e. naïve) with a concomitant decrease in the memory T-cell compartment (Praksova et al., 2012). This change offers insight into the efficacy of the drug in MS in that it reduces antigen-experienced T cells where the bulk of autoreactive cells may reside (Praksova et al., 2012). The drug also induces an alteration of monocyte subsets and activation markers that include the expansion of transitional $\mathrm{CD} 14^{+} \mathrm{CD} 16^{+}$monocytes and sees the normalisation of innate activation markers on monocytes from MS patients compared to those seen in healthy controls (Chuluundorj et al., 2016). Specifically, the proportion of the co-stimulatory marker CD40 expression on DC is noted to be lower in GA treated MS patients (Sellebjerg et al., 2012a), while homing and activation markers CCR5 and CD86 expression is also reduced in GA treatment on DC and monocytes (Sellebjerg et al., 2012b). CD40 expression on DC also showed a relationship with the rate of relapse in treatment cohorts on GA and INF $\beta$, where lower CD40 expression levels correlated with longer relapse free days (Sellebjerg et al., 2012b, 2012a). As with INF $\beta$, GA has no beneficial effects on progression and only offers a slight reduction of the annual rate of relapse (Mantia et al., 2010).

\subsubsection{Fingolimod.}

Fingolimod (a.k.a FTY720) is an oral immunosuppressive therapy for RRMS and structural analog of sphingosine. It acts as an agonist to bind and internalise the 
sphingosine-1-phosphatase $(\mathrm{S} 1 \mathrm{P})$ receptor that modulates trafficking of lymphocytes (Brinkmann et al., 2002). Reduced S1P expression impairs lymphocyte exit from the lymph nodes (Teniente-Serra et al., 2016). Fingolimod therefore acts to directly impact immune phenotype and has a disease modifying effect through reducing the CCR7+ population of central memory T-cells, including Th17 cells, those putative drivers of autoimmune inflammation in MS (Hla and Brinkmann, 2011; TenienteSerra et al., 2016). T-cell subsets have been well defined in the context of this therapy (Mehling et al., 2010). Specifically, treatment effects an early 60\% reduction in total lymphocyte count after induction, which was maintained during a four week follow up, mostly in B cells, while Th1 and Th2 subsets showed an increase, while overall the Th17 compartment and NK cell populations remained unaffected (Sato et al., 2014). Treatment also impacts innate cells, including a down-regulation of activation markers in myeloid DC (CD1c $\left.\mathrm{c}^{+}\right)$and monocytes, and an increase in the proportions of monocytes, DC, and subset specific cytokine positive T-cells (Thomas et al., 2017). While treatment may increase the likelihood of patients being relapse free at 24 months, and efficacy is improved when compared to GA and INF $\beta$, there is no effect on preventing the worsening of disability (Mantia et al., 2016).

\subsubsection{Dimethyl fumarate (DMF).}

The methyl ester of fumaric acid, DMF, is as a first line therapy that results in broad lymphocyte depletion that is effective in MS due to collaterally depleting CD8+ autoreactive T-cells present in the PB (Longbrake et al.; Spencer et al., 2015). Drug induced leukopenia was noted in initial trials of DMF, and it also effects B-cell subset composition by specifically depleting mature B-cells through apoptosis while also decreasing pro-inflammatory activity ( $\mathrm{Li}$ et al., 2016). The depletion effect from DMF therapy is akin to that seen in fingolimod, which may explain why the drug's effects in MS are similar and attributable to a reduction in the autoreactive T-cell burden (Longbrake et al., 2015). Treatment reduces the number of relapse events after two years, and there is minimal evidence that treatment can reduce disability worsening (Xu et al., 2015).

\subsubsection{Natalizumab (NTZ).}

NTZ is a monoclonal antibody (anti-CD49d) targeting the alpha-4 subunit of the very late antigen-4 (VLA-4), negating the binding of VLA-4 with vascular endothelium cell 
adhesion molecule-1 (VCAM-1) on the surface of the vascular endothelium (Sellebjerg et al., 2012a; Wipfler et al., 2010). Treatment prevents peripheral leukocytes from crossing the BBB into the CNS and results in their accumulation in the PB (Polman et al., 2006). Due to this effect NTZ is one of the first anti-inflammatory treatments for MS. Treatment further compounds the PB leukocyte burden due to the mobilisation of CD34+ progenitor cells, also known as haemopoietic stem cells (Mattoscio et al., 2015; Zohren et al., 2008). The reduction of extravasation of inflammatory leukocytes into the CNS and modulation in peripheral immune homeostasis from an altered subset composition is thought to jointly result in a reduction of pathological MS brain lesions and relapses (O’Connor et al., 2005; Skarica et al., 2011). Treatment with NTZ can reduce relapse and disability progression (Filippini et al., 2013), and NTZ remains one of the best treatments for the prevention of relapse (Tramacere et al., 2015).

\subsubsection{Anti-CD20 therapy.}

B-cell depletion through ant-CD20 monoclonal antibodies result in a decrease in RRMS relapse rate and offers a compelling insight into the role of B-cells in MS relapse (Montalban et al., 2017). It has been suggested that the efficacy of B-cell depleting agents is due to the presence of a soluble disease-causing demyelinating agent that is produced by B cells (Lassmann, 2014). Two such therapies, ocrelizumab and rituximab, selectively activate complement mediated NK cell targeted depletion of CD20 expressing cell subsets (Palanichamy et al., 2014). Many of these deleted cells are B-cells, but the drug also targets a minor yet diverse T-cell population. Rituximab decreases disease in RRMS by reducing brain inflammatory lesions and moderately reduces the risk of relapse as compared to placebo (Hauser et al., 2008). While rituximab does not decrease the time to confirmed disease progression in PPMS, stratification into younger disease cohorts and those with more inflammatory lesions may moderate disease progression (Hawker et al., 2009). Ocrelizumab therapy showed very good results in a comparative analysis phase III trials of RRMS: lower rate of relapse, disease activity, and disability progression over INF $\beta$ (Hauser et al., 2017). Results in PPMS was however only very modest, showing reduced MRI and clinically measured progression rates (Montalban et al., 2017). The risk of infection while on these agents is acute and is further compounded as infection is also a late stage MS co-morbidity. This means full immune monitoring is needed as part of the dosing regimen for anti-CD20 therapies. 
1.2.4.7 Autologous haematopoietic stem cell transplant.

Unfortunately, despite the significant advances and benefit of DMT for some patients, others continue to experience relapse and disability progression while on treatment. Autologous haematopoietic stem cell transplant/therapy (AHSCT) is a one-off treatment procedure that aims to reset the haematopoietic cell compartment. It is mainly used in haematological malignancy but has seen adoption as therapy for highly aggressive and treatment resistant autoimmune conditions such as MS (Passweg et al., 2017; Snowden et al., 2018). The treatment regimen generally consists of four stages: stem cell mobilisation and harvest, a BM ablation and conditioning regimen, an infusion of autologous haematopoietic cells, and long-term follow-up care to monitor for transplant efficacy and prevent infection or malignant cancers. AHSCT aims to eradicate pathogenic autoimmune cells and repopulate the $\mathrm{BM}$ with a more tolerogenic immune compartment thereby resolving underlying autoimmune driven neuroinflammation (Ismail et al., 2019).

Several studies have been undertaken that show a high degree of clinical efficacy and treatment outcome success (Bose et al., 2018; Burt et al., 2019; Das et al., 2019). While safe and effective delivery of this treatment in MS is possible, it requires a multidisciplinary team approach, significant follow up care, and education for neurological communities to identify and support suitable patients (Ismail et al., 2019). Due to its success in some centres, MS is currently the biggest clinical indicator for BM transplant therapy in Europe (Sharrack et al., 2019; Snowden et al., 2017).

\subsubsection{Immune monitoring for DMT in MS.}

Long term monitoring of leukocyte subsets in MS patients on some DMT, especially NTZ, is considered required practice as these patients are at severe risk of developing progressive multifocal leukoencephalopathy (PML) due to reduced CNS immune surveillance. PML is caused by the JC virus, which under reduced immune surveillance reactivates and causes damage and inflammation of the white matter of the brain. This then makes studies of NTZ therapy a good reference for experimental designs aiming to monitor immunomodulation in the MS therapy milieu (Mattoscio et al., 2015; Mellergard et al.). PML has also been reported in fingolimod and DMF treatment regimens, further substantiating the need for routine leukocyte monitoring 
in MS patients on DMT (Doshi and Chataway, 2016). Such data collection, facilitated by cytometry tools, has the added potential to reveal additional features of MS immune pathology.

\subsection{Immunophenotyping in MS.}

Immunophenotyping is the technique of profiling cellular markers and characteristics by flow cytometry. It is particularly useful in diseases where the cells of the peripheral blood are involved as these are readily accessible and their investigation may offer insight into disease pathology. It has great utility at rapid singe cell discrimination and enables rare subset monitoring. In MS, the disease processes, progression and presentation are highly diverse. This results in a large dynamic range for leukocyte population numbers in the blood, and when coupled with the lack of a known diseasecausing cell, restricts the utility of $\mathrm{PB}$ phenotyping as a diagnostic tool. However, there is some longstanding evidence of $\mathrm{PB}$ phenotypic alterations in MS, such as an increase in $\mathrm{CD} 4 / \mathrm{CD} 8$ ratio for $\mathrm{T}$ cells (Bach et al., 1980) likely due to a decrease in $\mathrm{CD}^{+} \mathrm{T}$ cells (Pender et al., 2014). This starts to suggest a profile of alterations may exist and, as advances in technology facilitate more detailed subset monitoring, further changes have come to light (Posová et al., 2017). Even with highly specialised phenotyping techniques the heterogeneity inherent to MS can confound immunophenotyping data, yet accurate diagnosis and MS subtyping can help resolve differences in group comparisons. This type of monitoring is therefore essential when assessing risk and benefit for patients before and on DMT (Mattoscio et al., 2015; Ontaneda et al., 2014).

\subsubsection{The phenotype of T cells in MS.}

The T-cell compartment in the PB is the best-defined immune cell subset in MS. As mentioned, a general theme in MS pathology is the persistent reduction of CD8+ T cells, independent of disease process, that is present at onset through to the progressive phase, and is seen as a primary defect rather than as being secondary to the disease (Pender et al., 2014). The ratio of $\mathrm{CD}_{4} / \mathrm{CD}^{+} \mathrm{T}$ cells is therefore often skewed (Bach et al., 1980), and is suggestive of aberrant immune homeostasis. Flow cytometry has been used to show that this ratio change results from the decrease in $\mathrm{CD}^{+}{ }^{+}$effector memory (EM) and EM re-expressing CD45RA (EMRA) CD8+ $\mathrm{T}$ cells 
(Ajami et al., 2011). Advances in cytometry technology have also allowed for a clarification of the T-cell changes in MS pathology. Examples from the literature demonstrate T-cell subsets and their activation state in MS, and showed Tfh cells, which are often implicated in autoimmune disease development, as being activated in MS (Fan et al., 2015; Simpson et al., 2010). Cytometry analysis has also been effective at identifying Th17 cells and how different treatment regimens affect specific T-cell subsets (Negrotto et al., 2015).

\subsubsection{The phenotype of B cells in MS.}

As with T-cell work, phenotypic profiling of minor B-cells has offered insights into disease processes in MS, such as changes in the proportion and number of cells in the periphery correlating with disease progression and duration (Christensen et al., 2013; Seidi et al., 2002). Historically, the total number of B cells was not known to vary significantly in MS patients when compared to HC (Jones et al., 2016; Seidi et al., 2002), although no longer conclusively so as some authors show regulatory B cells subset in MS to be altered (Niino et al., 2012). Such work has only recently been possible due to the application of detailed multicolour phenotyping. Profiling B cells in MS pathology has only in the last decade become commonplace - an unfortunate oversight. We now know that in active disease, the total frequency of B cells in the periphery decrease due to the loss of memory and class switched subsets from circulation (Haas et al., 2011). Periods of remission and treatment with fingolimod for example can redress this shift (Haas et al., 2011; Schwarz et al., 2016). Immunophenotyping has also been used to show that MS disease shifts the relative frequency of B-cell subsets to increase naïve and reduce the switched memory subset during active disease (Schwarz et al., 2016). This relationship and the proven efficacy of leukopenia inducing therapy (e.g. NTZ, see 1.2.4), adds substantial weight to the use of B-cell depleting monoclonal therapies (anti-CD2O) in MS.

Unfortunately, a large gap still exists in the analysis of the impact of progressive disease on B-cell subsets. This information gap is exasperated by a lack of full spectrum B-cell phenotyping from most experimental designs. 


\subsubsection{The phenotype of monocytes in MS.}

Monocytes are often overlooked in MS phenotyping studies, but they are key players in the disease pathogenesis as they act to promote inflammation (Bar-Or et al., 2003). By showing the up-regulation of markers such as HLA-DR, CD40, CD86, CD64, detailed phenotyping of monocytes has revealed that these cells in MS are classically activated (Chuluundorj et al., 2014, 2016). Monocytes in MS also show altered cytokine expression (Chuluundorj et al., 2016; Waschbisch et al., 2016) which in turn effects Th1 and Th17 cells, which are known to promote disease process in MS (Rostami and Ciric, 2013).

Data from phenotyping studies on the monocyte compartment has shown that CD16 ${ }^{+}$ monocyte subsets together comprise about $10 \%$ of total peripheral compartment, but that they are expanded in autoimmune diseases including MS (Waschbisch et al., 2016). Data from our own lab has shown that in MS, CD16+ monocytes are proinflammatory and secrete higher levels of IL-6 with significantly less IL-10 compared to the $\mathrm{CD} 14^{+}$subset (Chuluundorj et al., 2014). Coupled with an increased infiltrating capacity through preferential recruitment to endothelial lined vessel walls, CD16+ monocytes drive inflammatory processes (Ancuta et al., 2003). Cytometry studies have facilitated the identification of the altered phenotype and function of these cells in MS and implicates this subset as an early infiltrating cell in MS development, with some treatments normalising the activation profile to that seen in HC populations (Chuluundorj et al., 2016; Waschbisch et al., 2016).

Unfortunately, there is limited information on monocyte subsets in MS disease and in studies of therapy response. Closing this gap offers the potential to dramatically expand the scope of our understanding of these innate immune cells.

\subsubsection{Dendritic cell profiles in MS.}

Given their low abundance in the PB, DC studies are limited by the need for more specialised equipment, such as high flow rate cytometers. The lack of literature citing treatment impacts on DC subset composition in MS is acute, likely due to technical limitations. What data is available shows that DC numbers are not known to vary to a significant degree in MS compared to HC (Han et al., 2014; Sellebjerg et al., 2012a), but DC do show an increased proportion of co-stimulatory marker expression (e.g. 
CD86 and CD80) (Comabella et al., 2009; Thewissen et al., 2013). The future work on this subset will likely highlight their complex interaction of T-cells and their role in driving inflammation in MS.

\subsubsection{NK cells.}

Insufficient data is available to justify what, if any, alterations there are in the NK cell proportional makeup of the PB in MS. NK cells are usually omitted from whole blood analysis in MS and are only described when there is an anticipated therapeutic impact on the subset. What limited data there are show no know variation to any degree of significance in NK cell numbers in MS when compared to control populations that comprise other inflammatory and non-inflammatory disease cohorts (RodríguezMartín et al., 2015). A single study by Jager et al. has however described a substantive decrease in MS patients' total NK cell population compared to HC (Jager et al., 2008).

\subsubsection{NKT cells}

Natural killer T-cells (NKT) are a further NK like subset where data indicate their numbers are reduced and their cytokine production is impaired in MS (Berzins et al., 2011). Innate-like NKT are a further specialised subset of innate-like lymphocytes that have been found in some SPMS disease course patients to have higher IL-17 production compared to control and PPMS, suggesting NK cell have a more inflammatory phenotype in MS disease (Biasi et al., 2016).

Using the background described in the preceding sections it becomes clear that progressive MS patients have very limited treatment options. Furthermore, innate immune cell profiles and function are an overlooked research topic in MS. From this then stems the practical work for this thesis in profiling the effects on innate immune cell phenotype and function of two potential drugs in progressive MS.

\subsection{Clozapine.}

Clozapine is an atypical antipsychotic primarily used for the treatment of refractory schizophrenia. It works by suppressing psychosis through a transient and comparative antagonism of dopamine at the dopamine receptor D2 (D2) (Schrader et 
al., 2019). Clozapine also binds and antagonises a wide range of other neuroreceptors including serotonin and other dopamine receptors. Furthermore, clozapine is an effective immunomodulatory agent capable of altering T-cell differentiation and cytokine expression (Chen et al., 2012), specifically following whole blood in vitro stimulation (Himmerich et al., 2011). Because of its immunomodulatory action and the low affinity to a broad range of receptors, it was posited that clozapine treatment may result in the dampening of the pro-inflammatory immune responses present during neuroinflammation (Ribeiro et al., 2013).

The parallels between CNS inflammation in schizophrenias and MS, and the efficacy of clozapine in redressing and modulating the immune system, led our group to investigate the impact of clozapine in an animal model of MS. We found that clozapine could ameliorate disease severity in a dose-dependent manner and detected lower $\mathrm{T}$ cell and myeloid cell CNS infiltration (Green et al., 2017; O'Sullivan et al., 2014). This work also characterized clozapine's immunomodulatory effects by showing its ability to modify bone marrow-derived macrophage responses in vitro and determined that the activity was independent of type 2 dopaminergic antagonism (O'Sullivan et al., 2014). In a second study, our group went on to assess the impact of clozapine treatment on remyelination and functional outcomes in a cuprizone model (Templeton et al., 2019). This showed that clozapine administration improved functional recovery following cuprizone withdrawal, with recovery outcomes correlating with decreased microglial and astrocyte activation and increased myelination in the corpus callosum. From this success in animal models, a small clinical trial (i.e. CRISP study) was undertaken to assess whether the administration of clozapine during progressive MS would be suitable and appropriate.

\subsection{MIS416.}

MIS416 is a large, non-soluble microparticle that induces a characteristic cytokine response in human peripheral blood mononuclear cell (PBMC) cultures in vitro (Girvan et al., 2010). The molecular structure of MIS416 comprises two PAMP, namely $\mathrm{CpG}$ and MDP, targeting TLR9 and NOD2 respectively. TLR9 agonism from $\mathrm{CpG}$ results in TNFa and IL-6 induction via nuclear factor kappa B (NF- $\mathrm{B})$ activation 
and associated transcriptional activity (Tsujimura et al., 2004). NOD2 agonism induces IL-10, also in response to NF- $\kappa \mathrm{B}$ activation (Macho Fernandez et al., 2011). It has been suggested that MIS416 as a therapeutic agent would induce an altered immune state through the activation of the NF- $\kappa B$ mediated signalling responses. It has been observed that MIS416 administration results in elevated serum INFY in humans (White et al., 2014), and in animal models that it affects T-cell responses and alters immune cell subpopulations though cytokine production and phenotypic changes (White et al., 2018). MIS416 can also result in T-cell differentiation and DC maturation in vitro (Girvan et al., 2010).

It was therefore suggested that if used in chronic progressive MS, that the drug would upregulate innate driven anti-inflammatory responses with a potential therapeutic benefit. In line with this postulation our lab showed the efficacy of MIS416 in suppressing CNS disease in animal models of MS through suppressing Th1, Th2, and Th17 cell responses and by inducing serum INFY (White, 2015; White et al., 2014).

Based on this data, MIS416 was used as a therapeutic intervention in humans under a compassionate use program (section 25 and 29 of New Zealand (NZ) medicines Act 1981). Eight progressive MS participants received drug intravenously weekly for twelve weeks. The drug was well tolerated, and participants self-reported an improvement in symptoms with the majority requesting continued use after the initial program concluded (private communication, Dr. Gill Webster). MIS416 was then formally evaluated for dosing safety and tolerability in humans through a phase 2 dose escalation trial (Luckey et al., 2015). The drug was deemed safe and well tolerated in an MS cohort. Modest adverse events following treatment were anticipated due to NF$\kappa \mathrm{B}$ mediated signal transduction that resulted in flu like symptoms (pyrexia, headache, fatigue etc.), while no disease exacerbation was observed. The drug then proceeded through to phase 2 testing but failed to show "clinically meaningful or statistically significant differences in measures of neuromuscular function or patient reported outcomes" (Quinn, 2019). 


\subsection{Overall aims and objectives.}

The work is based on the broad hypothesis that peripheral blood immune cells, specifically monocytes, in untreated patients with progressive multiple sclerosis will differ significantly from those of a healthy comparison group, and that treatment with immunomodulatory agents in vitro will have a modest but demonstrable effect in changing phenotype and other immune factors such as cytokines and phosphoproteins.

Our specific aims with which to test our hypothesis are as follows:

1.6.1.1 Compare whole blood phenotyping data from people with progressive MS to a healthy control (HC) cohort and establish whether MS specific features are present.

1.6.1.2 To study the effect of clozapine on the innate immune response by demonstrating its effects on phenotype, cytokine, and dopamine receptor expression of monocytes in vitro.

1.6.1.3 To determine the specific effects of MIS416 treatment on PBMC transcription factor and cytokine responses in vitro with a particular focus on the effects on monocytes, and observe if there are inherent differences in the innate response to MIS416 during MS.

To achieve our aims for this thesis, we set out by profiling the peripheral immune cell compartment of untreated patients with progressive MS using multicolour flow cytometry. 


\section{Chapter 2. Immune phenotyping of whole peripheral blood in progressive multiple sclerosis.}

\subsection{Introduction.}

MS disease processes are diverse, being highly varied in clinical presentation and progression rates (Doshi and Chataway, 2016). This disease heterogeneity result in significant variation of leukocyte populations in the peripheral blood (PB) and central nervous system (CNS), and restricts the utility of immune phenotyping as a diagnostic tool for MS (Jones et al., 2017). As such, no characteristic peripheral immune cell phenotype for MS has been identified. Some features are however present, with an increase in the CD4/CD8 ratio for $\mathrm{T}$ cells being common (Bach et al., 1980). This change in ratio is primarily caused by a decrease in $\mathrm{CD}^{+} \mathrm{T}$ cells and is noted in many patients (Polman et al., 2011). Some patients also have decreased proportions of B cells and NK cells (Posová et al., 2017). Analysing PB immune cells with flow cytometry is an excellent way for compiling in-depth phenotyping profiles. Its utility at single cell discrimination helps with rare subset identification, enables the monitoring of the peripheral response during trials of immunomodulatory therapies in MS (Ghadiri et al., 2017; Li et al., 2017; Lieberman et al.; Plavina et al., 2017; Teniente-Serra et al.), and aids in the assessment of risk for patients on treatment (Ontaneda et al., 2014). Furthermore, the populations of circulating PB immune cells often reflect those present in the CNS where proportional alterations can suggest a change in inflammatory state, or where the functional defects of regulatory cells can suggest a loss of control over self-targeting T-cells (Jones et al., 2017).

The CRISP trial (CRISP; Clozapine and Risperidone for the Treatment of Progressive Multiple Sclerosis; ACTRN12616000178448) (La Flamme et al., 2020), was a blinded, randomized, placebo-controlled clinical trial conducted at Wellington Hospital (Wellington, NZ). The trial aimed to assess the safety and suitability of two atypical antipsychotics, clozapine and risperidone, as possible treatments for people with progressive MS. The trial was based on pre-clinical results from two animal model studies done by our group, the first of which showed that clozapine and risperidone 
effected a dose dependent reduction in disease severity, while clozapine treatment was also able to resolve disease (O'Sullivan et al., 2014). In the second study, our group went on to assess the impact of clozapine treatment on remyelination and functional outcomes in a cuprizone model (Templeton et al., 2019) and showed that clozapine administration improved functional recovery following cuprizone withdrawal, with recovery outcomes correlating with decreased microglial and astrocyte activation and increased myelination in the corpus callosum. The CRISP trial was a natural next step in moving our work from animal models into patients. The trial set out to repurpose clozapine and risperidone in MS, with a primary endpoint aimed to assess their safety and acceptability. The trial reached this primary endpoint early as it was observed, from an increase in adverse events and participant drop-out, that PMS patients showed heightened sensitivity to clozapine. The trial therefore recruited only nine of a planned thirty-six participants. Concurrently, we recruited a cohort of healthy participants (HC), also from the Wellington region, to act as a control cohort for our laboratory-based work.

Our work in this chapter focusses on PB immune phenotyping of participants enrolled in the CRISP trial. This chapter outlines a freestanding but also introductory project for the rest of this thesis. It contains all the relevant technical, method, and optimisation steps as they were not repeated for any other project. We initially aimed to compile a longitudinal profile over six months of participant in vivo treatment, but the early termination of the CRISP trial limited our dataset to baseline (untreated) samples. With this smaller than anticipated cohort, we focussed our efforts to conducting an exploratory study of immune phenotyping in MS. We set out to determine whether baseline phenotyping could discriminate MS participants from $\mathrm{HC}$, and what value longitudinal blood sample analysis, using only HC, could yield. We further wanted to compile a dataset with which to test alternative cytometry data analysis methods. Unconventionally, we profiled adaptive and innate immune cells as targets for our analysis, as phenotyping of innate cells has often been overlooked in studies of MS pathology. 


\subsection{Aims.}

Our aim for this work was to profile the peripheral immune cell compartment of untreated patients with progressive MS using multicolour flow cytometry. To achieve this aim we compared peripheral blood immune cell numbers, proportion, and phenotype of MS patients with healthy subjects. The work is based on the hypothesis that the PB phenotype in untreated patients with MS will differ significantly from those of a HC comparison group. These differences should be observable as changes in the total number and the proportion of some immune cell subsets. We further posit that while the immune system is flexible allowing it to respond, and three sequentially sampled blood draws over a consecutive six-month period should yield highly similar numbers and proportions for each of the immune cell populations monitored in healthy people.

\subsubsection{Specific aims.}

2.2.1.1 Compare whole blood phenotyping data of absolute count and proportion of immune cells from people with MS to a HC cohort and establish whether MS specific features are present.

2.2.1.2 Assess the stability of the healthy immune system by profiling PB immune subsets at three time-point intervals and assess what if any variation can be detected.

2.2.1.3 Explore the use of spanning-tree progression analysis of density-normalized events (SPADE) plots to analyse high dimensional single cell data. 


\subsection{Methods.}

\subsubsection{Study participants.}

The study comprised two cohorts: a progressive MS group $(\mathrm{n}=9)$ and a healthy control group of age- and sex-matched individuals $(n=15)$. All MS participants were screened and enrolled as previously described (La Flamme et al., 2020; Luckey et al., 2015), and had either primary or secondary progressive disease with no significant confounding comorbidities and were not receiving any disease-modifying therapies.

\subsubsection{Ethical approval.}

Full ethical approval was in place before we commenced any experiments, with the CRISP trial being approved by the Central Health and Disability Ethics Committee (15/CEN/216) and the Standing Committee on Therapeutic Trials (15/SCOTT/177).

\subsubsection{Experimental procedures.}

We compiled standardised whole blood processing and immunophenotyping protocols for use during the study. These are detailed in the subsequent sections. Briefly summarised: individual participant blood samples were collected and stored in a Cyto-chex BCT (Streck, US) tube. For each panel stain, $100 \mu \mathrm{l}$ of blood was aliquoted into a $1.5 \mathrm{ml}$ polystyrene FACS tube (Falcon, US) and stained with a prepared combination of antibodies for 15 minutes in the dark at room temperature. Next, $2 \mathrm{ml}$ FACS lyse/fix (BD, US) was added, the sample mixed and allowed to lyse in the dark at room temperature for 15 minutes. The tubes were then centrifuged to pellet the cells, and $1.5 \mathrm{ml}$ supernatant was aspirated and discarded. The cells were then resuspended in the remaining supernatant and stored at $4^{\circ} \mathrm{C}$ protected from light until flow cytometric acquisition the next day.

\subsubsection{Sample collection.}

Peripheral blood was collected from consented participants by a phlebotomist using standard aseptic techniques from the median cubital, cephalic or basilic vein. On 
average, participants donated $10 \mathrm{ml}$ of whole blood of which $2 \mathrm{ml}$ was collected into a Cyto-chex BCT tube (Streck, 2019) for whole blood immunophenotyping and $8 \mathrm{ml}$ for isolation and storage of peripheral blood mononuclear cells (PBMC).

\subsubsection{Cyto-chex BCT tube.}

Samples for immunophenotyping analysis on whole blood was collected and stored for up to one week after isolation in a Cyto-chex tube. The Cyto-chex BCT tube is a CE marked, US FDA cleared product for clinical immunophenotyping on human peripheral white blood cells subsets (Streck, 2019). Samples are considered stable, retaining morphology and surface antigens thereby enabling consistent recovery of immune cells subsets for up to two weeks post collection.

\subsubsection{Whole blood staining.}

Antibody cocktails were prepared fresh each day in a $1.5 \mathrm{ml}$ polystyrene tube (Table 21). One test volume $(100 \mu \mathrm{l})$ of peripheral blood from a Cyto-chex tube was aliquoted into a separate polystyrene flow cytometry tube (FACS) tube for each panel stain and a test volume of each respective antibody cocktail added. Prepared antibody cocktails included $50 \mu$ Brilliant Stain buffer (BD, US) to avoid staining artifacts that may arise from the use of multiple BD Horizon Brilliant dyes in the cocktails. In total, each participant was stained for 4 panels. Each tube was then briefly vortexed and incubated in the dark at room temperature for 15 minutes. $2000 \mu \mathrm{L}$ of $1 \mathrm{x}$ FACS Lysis (BD, US) buffer was then added to each tube, mixed, and incubated at room temperature for 15 minutes. The tubes were then centrifuged at $300 \mathrm{~g}$ for 5 minutes and $1500 \mu \mathrm{l}$ of the supernatant discarded. $50 \mu \mathrm{l}$ of CountBright Absolute Counting Beads (Thermofisher, US) was then added to the cells and mixed. 
Table 2-1 Whole blood cytometry antibody cocktails.

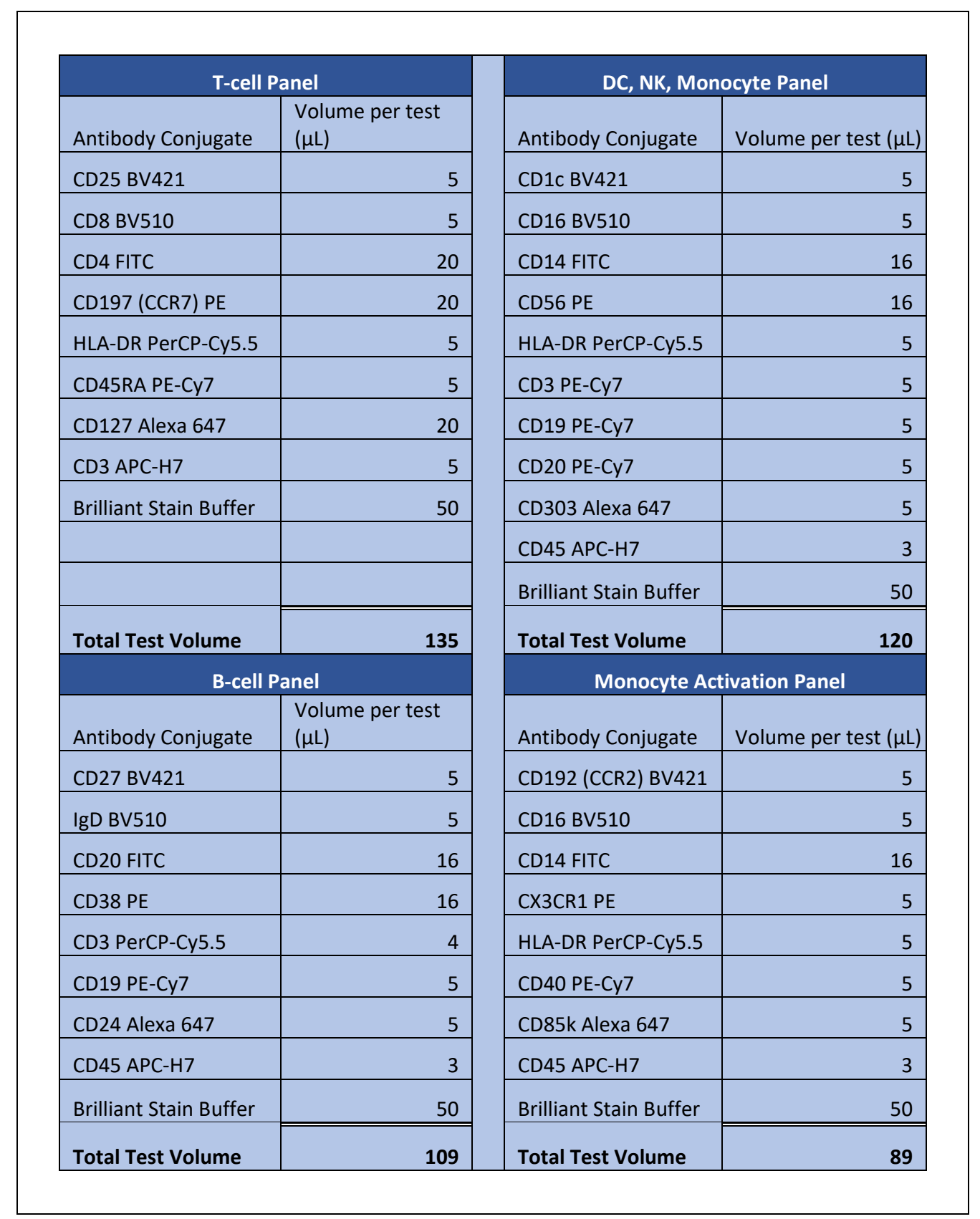

The Total Test Volume of each panel was for a single $100 \mu \mathrm{l}$ whole blood test, with this volume scaled to accommodate staining of multiple tubes. 


\subsubsection{Conventional multicolour cytometry.}

All whole blood analyses were performed with conventional cytometry methods on a FACS Canto II (BD, US). The Canto II setup comprised a three-laser system (4-2-2 configuration), facilitating eight colour cytometry runs. All whole PB samples were processed and acquired within a twelve-month period.

\subsubsection{Panel design.}

Optimal panel design was based on the sample source: red cell lysed peripheral blood (PB). Our aim for participants from the CRISP study was to get an overview of all the major immune cell subsets present in $\mathrm{PB}$, and to enumerate each subset accurately. Significant efforts have been made to standardise reagents and markers in clinical cytometry, but research data often lack standardisation (Hasan et al., 2015; Streitz et al., 2013). To ensure reproducibility over the course of our clinical trial sampling period we based our PB panel on optimised and validated markers published by the Human Immunology Project (Maecker et al., 2012).

Table 2-2 FACS Canto II whole blood cytometry panel design.

\begin{tabular}{|c|c|c|c|c|c|c|c|c|}
\hline \multirow{3}{*}{$\begin{array}{l}\text { Excitation Source } \\
\text { Emission/Detector } \\
\text { BD Fluorochromes }\end{array}$} & \multicolumn{2}{|c|}{$\begin{array}{l}405 \mathrm{~nm} \text { Violet } \\
\text { Laser }\end{array}$} & \multicolumn{4}{|c|}{488 nm Blue Laser } & \multicolumn{2}{|c|}{$633 \mathrm{~nm}$ Red Laser } \\
\hline & $450 / 50$ & $510 / 50$ & $530 / 30$ & $585 / 42$ & $670 \mathrm{LP}$ & $780 / 60$ & $660 / 20$ & $780 / 60$ \\
\hline & BV421 & BV510 & FITC & PE & $\begin{array}{l}\text { PerCP- } \\
\text { Cy5.5 }\end{array}$ & PE-Cy7 & AF647 & APC-H7 \\
\hline Panel 1: T Cell & $\mathrm{CD} 25$ & CD8 & CD4 FITC & $\mathrm{CCR}_{7}$ & HLA-DR & CD45RA & CD127 & CD3 \\
\hline Panel 2: B-cell & CD27 & $\operatorname{IgD}$ & $\begin{array}{l}\text { CD20 } \\
\text { FITC }\end{array}$ & CD38 & CD3 & CD19 & CD24 & CD45 \\
\hline $\begin{array}{c}\text { Panel 3: } \\
\text { DC/NK/Mono }\end{array}$ & CD1c & CD16 & $\begin{array}{l}\text { CD14 } \\
\text { FITC }\end{array}$ & $\mathrm{CD}_{56}$ & HLA-DR & $\begin{array}{c}\text { CD3/CD19/ } \\
\text { CD20 }\end{array}$ & CD303 & CD45 \\
\hline Panel 4: Monocyte & $\mathrm{CCR} 2$ & CD16 & $\begin{array}{l}\text { CD14 } \\
\text { FITC }\end{array}$ & $\mathrm{CX}_{3} \mathrm{CR}_{1}$ & HLA-DR & $\mathrm{CD} 40$ & CD85k & CD45 \\
\hline
\end{tabular}

\subsubsection{Marker selection.}

The total number of immune subsets that can be delineated in a single tube is limited by the number of makers included in the panel stain. With our instrument restricted to 8-colour panels, we required several tubes (panels) to capture data on a range of specialised cells subsets (Table 2-2). We selected a dim channel (APC-H7) in the far- 
red spectrum for CD45 as a common and overlapping feature. This antigen is reliably detected on most PB subsets and facilitated total cell count enumeration. CD45 was however not incorporated into the T-cell panel, as CD3 was a reliable T-cell discriminator. With three tubes we were able to characterise the majority of PB subsets, so we included an additional fourth tube focussing solely on monocyte activation.

Panel 1: T cells.

For this panel we implemented $\mathrm{CD}_{3}$ only acquisition using an APC-H7 channel threshold that ignored most non-T cells but allowed a minimal number of negative events through to discriminate a positive and negative population. Mostly, CD3 positive events were recorded into the raw cytometry files, with any non-T-cell excluded with subsequent gating. The major T-cells subsets delineated are outlined in Table 2-3.

Table 2-3 Major T-cell fractions enumerated in Panel 1.

\begin{tabular}{|c|c|c|c|c|c|c|c|c|}
\hline & CD3 & CD4 & CD8 & CCR7 & CD45RA & CD127 & CD25 & HLA-DR \\
\hline Naïve & + & $+/-$ & $+/-$ & + & + & - & - & $\S$ \\
\hline TCM & + & $+/-$ & $+/-$ & + & - & - & - & $\S$ \\
\hline Teff & + & $+/-$ & $+/-$ & - & + & - & - & $\S$ \\
\hline TEM & + & $+/-$ & $+/-$ & - & - & - & - & $\S$ \\
\hline Activ & + & $+/-$ & $+/-$ & $\S$ & $\S$ & $\S$ & $\S$ & + \\
\hline Treg & + & + & - & $+/-$ & $+/-$ & + & + & $+/-$ \\
\hline
\end{tabular}

$\mathrm{TCM}=$ central memory, Teff = effector, $\mathrm{TEM}=$ effector memory, Activ = activated, Treg $=$ regulatory $\mathrm{T}$ cells. $\S$ not used to delineate the subset.

Panel 2: B cells.

B cells were discriminated by scatter, CD45 and CD19 positivity while being negative for CD3.

We typed all the major subsets commonly found in the PB as outlined in Table 2-4. 
Table 2-4 Major B-cell fractions enumerated in Panel 2

\begin{tabular}{|l|c|c|c|c|c|c|c|c|}
\hline & CD45 & CD3 & CD19 & CD20 & CD38 & CD27 & IgD & CD24 \\
\hline Tran & + & - & + & + & + & $\S$ & $\S$ & + \\
\hline Plasb & + & - & + & - & + & + & $\S$ & - \\
\hline Mem & + & - & + & + & - & + & $+/-$ & $\S$ \\
\hline Naive & + & - & + & + & - & - & + & $\S$ \\
\hline
\end{tabular}

Tran $=$ transitional, plasb $=$ plasma blast mem $=$ memory $\mathrm{B}$ cells. $\S$ not used to delineate the subset.

Panel 3: DC/NK/Granulocytes.

This more complex panel required the use of a combined 'dump' channel containing three antibodies to which all cells of interest would be negative (Lin-: CD3-CD19CD2O-). This effectively excluded all lymphocyte subsets, limiting the cells of interest in this panel to those cells of the innate immune system (Table 2-5).

Table 2-5 Innate cell subsets enumerated in Panel 3.

\begin{tabular}{|l|c|c|c|c|c|c|c|c|c|}
\hline & CD45 & Lin & CD14 & CD16 & $\begin{array}{l}\text { HLA- } \\
\text { DR }\end{array}$ & CD303 & CD1c & CD56 & SSC-H \\
\hline pDC & + & - & - & $\S$ & ++ & + & - & $\S$ & $\S$ \\
\hline mDC & + & - & - & $\S$ & ++ & - & + & $\S$ & $\S$ \\
\hline Mono & + & - & + & $+/-$ & + & $\S$ & $\S$ & $+/++$ & $\S$ \\
\hline NK & + & - & - & + & - & $\S$ & $\S$ & $+/++$ & $\S$ \\
\hline Neut & + & - & $\S$ & ++ & \pm & $\S$ & $\S$ & ++ & ++ \\
\hline Eosin & + & - & $\S$ & $\S$ & $\S$ & $\S$ & $\S$ & $=$ & ++ \\
\hline
\end{tabular}

$\mathrm{pDC}=$ plasmacytoid dendritic cell $(\mathrm{DC}), \mathrm{mDC}=$ myeloid $\mathrm{DC}$, mono $=$ monocyte, neut $=$ neutrophil, eosi $=$ eosinophil. $\S$ marker not used to delineate the subset. $\mathrm{SSC}-\mathrm{H}=$ side scatter height. $\mathrm{Lin}=\left(\mathrm{CD}_{3} / \mathrm{CD} 19 / \mathrm{CD} 20 \mathrm{mix}\right)$

Panel 4: Monocytes.

We were interested in profiling PB monocytes in more detail. Commonly, three subsets of monocyte are present in the PB as outlined in Table 2-6. 
Table 2-6 Monocytes cell subsets enumerated in Panel 4.

\begin{tabular}{|l|l|l|l|l|l|l|}
\hline & CD45 & HLA-DR & CD14 & CD16 & CCR2 & CXCR3 \\
\hline Class & + & + & ++ & - & ++ & + \\
\hline Inter & + & + & ++ & + & $\S$ & $\S$ \\
\hline Non-c & + & + & + & ++ & + & ++ \\
\hline
\end{tabular}

Class $=$ classical, inter $=$ intermediate, non-c $=$ non-classical monocytes. § not used to delineate the subset.

We also aimed to establish the potential impact of our drugs in monocytes, so included two activation markers (CD40 \& CD85k) commonly expressed by monocyte subsets. Unfortunately, early trial termination limited the utility of these markers.

\section{ILT3/CD85k}

The expression of ILT3 on antigen presenting cells (APC) is linked to graft acceptance in transplantation. CD8+ tolerogenic T cells (from transplant accepting donors) can cause up regulation of ILT3 expression on APC. In MS, CD8+ CD28- T-cell function, especially during relapse, is significantly compromised yet reverts to normal during remission (Jensen et al., 2009). Numerous approved RRMS therapies increase ITL3 expression. In vitro interferon $\beta$ stimulation of cultured monocytes, DC and macrophages has been shown to increase ILT3 expression, while those isolated from untreated RRMS patients show decreased levels of expression when compared to treated patients in remission and healthy controls (Jensen et al., 2009).

$\mathrm{CD} 40$

To activate T cells through the TCR, a second signal is needed (co-stimulation). Costimulatory molecules are either positive or negative in their effect on T-cell signalling, and CD40 is a positive co-signalling molecule. It is primarily expressed by B-cells and APC. In animal models of MS, the positive/negative co-signalling of T cells have been shown to have an important role in the pathophysiology of the disease, and in vivo upregulation of $\mathrm{CD} 40$ is associated with treatment response in RRMS (Wiesemann et al., 2007). 
We included CD85k and CD40 in our monocyte panel to allow us to assess if either of the drugs from the CRISP study could alter their expression while also keeping a view to future work to assess the impact of CRISP study protocols.

\subsubsection{Gating.}

Our PB gating strategy are based on the lineage phenotyping methods published by the Human Immunology Project (Finak et al., 2016; Maecker et al., 2012). We used hierarchical and Boolean gating to ensure that terminal lineage subsets would only include events that also met the full phenotypic designation. Full hierarchical gating strategies for each tube using representative data is depicted in Figures 2-1 through 24 .

\subsubsection{Assay settings and instrument setup.}

Correct instrument setup verifies the optimal running capacity of a cytometry platform and calibrates the instrument detectors to ensure reproducible data across a longitudinal acquisition period (Maecker and Trotter, 2006). Optimal gain settings for detectors enable the operator to distinguish low dim staining populations from background noise (Maecker and Trotter, 2006). To ensure reproducibility we used FACSDiva v8.o (BD, US) application settings. During setup, channel voltages were adjusted so that negative populations were within or above the 97th percentile of the dim cytometer setup and tracking (CS\&T) bead set (lot\# 910858_77840). This allowed for maximum low-end resolution sensitivity (Mukherjee et al., 2015) and was achieved by using a sample as an unstained control and a fully stained panel set from an individual donor to determine the optimal PMT voltages for each respective panel stain. These settings were then saved to use in future applications. Saving and reusing experiment settings is possible as the software links experiment settings to the daily CS\&T calibration thereby maintaining consistent values irrespective of daily changes to the instrument.

\subsubsection{Thresholding.}

Each signal detected by the cytometer is scrutinised by the various detectors to determine whether, based on intensity, the signal is recorded. User-assigned thresholds were applied to all acquisitions to limit events to those that would be relevant. Often this is set as a base level of forward scatter (FSC), correlating to just 
below the size of the cell subset of interest. While suitable for several applications, FSC thresholding should be avoided when absolute counting beads are used. These particles are much smaller than most cell subsets and setting low FSC thresholds will effectively cause those events to be ignored by the system.

It was therefore appropriate to include a second parameter, namely fluorescence thresholding. This type of threshold limits the instrument to recording events that are above a minimum level of fluorescence intensity. Commonly, CD45 was used for this limit, with the exception of Panel 1 where we used CD3 for T-cells.

2.3.2.10 Instrument maintenance and longitudinal quality control.

The FACS Canto was maintained according to manufacturer's recommendations and covered under a continual service contract. The instrument's longitudinal controls were maintained using single lot of CS\&T bead (BD, US) over the duration of the experimental use. This ensured that the set PMT voltages for our acquisitions remained near constant.

\subsubsection{Cytometry Controls.}

The use of isotype controls is common in research data. Their use is however only appropriate to determine the amount of background staining from an antibody to a target epitope and should only be used where there is a suspicion of non-specific binding (Andersen et al., 2016; Hulspas et al., 2009). There are many limitations in their use, mostly due to the inability to find a perfect matched isotype. The need for isotypes can also be completely negated by effective blocking of Fc receptors (Andersen et al., 2016). We did not include isotype controls for any lineage marker in our panels as our samples contained autologous serum, containing IgG. To confirm that any changes were not due to non-specific binding we included isotype controls for CD40 and CD85k.

Essential controls for multicolour panels are those that help limit errors from channel spill-over, namely compensation controls. Fluorophores emit light over a range of wavelengths, with an optimal peak. Detectors are designed to detect the emission for their respective fluorophore at that optimal peak as it is where the signal intensity is best. However, fluorophore emission is not just detectable at their peak, but tails off, 
extending the range of emission over a spectrum. Spill-over occurs when that tail end of signal is detected in an adjacent detector, giving false signal. Compensation controls are single stain reference emission controls that allows for a mathematical subtraction of such false signal (Maecker and Trotter, 2006). We prepared a weekly set of single stain compensation controls in FACS buffer using Ultracomp eBeads (Thermofisher, US) according to manufacturer's instruction. Compensation was performed and subtracted automatically in FACS Diva.

Correctly identifying positive and negative populations in our panels was always clear, owing to the confidence inherent in the bimodal expression of most PB phenotyping markers (Hulspas et al., 2009). This was due to the presence of a biological nonstaining subset for each epitope that could be used as a negative control. These 'internal negative' controls were used to accurately gate lineage subsets.

2.3.2.12 Data analysis.

All raw cytometry data were recorded, stored and compensated in FACSDiva (BD, US). Compensated cytometry data was analysed in Flowjo v10 (BD, US), with output data stored in Excel (Microsoft, US) tables. 
2.3.2.13 Absolute counting.

The addition of Countbright beads (ThermoFisher, US) facilitated absolute enumeration of immune subsets. This was done by relating cytometry events back to the absolute counts of cells present in the original blood sample using the formula below. All beads were from the same lot at 51000 beads per $50 \mu$ (from stock), and all samples were stained using $100 \mu \mathrm{l}$ of blood. Therefore $\mathrm{B}=510$.

To simplify absolute counts, we divided the constant B by 1000 for result readouts in $10^{3} \mathrm{cell} / \mu \mathrm{l}$. This may also be expressed as $10^{9} \mathrm{cell} / \mathrm{L}$ or $10^{6} \mathrm{cell} / \mathrm{ml}$ or $10^{3} \mathrm{cells} / \mu \mathrm{l}$

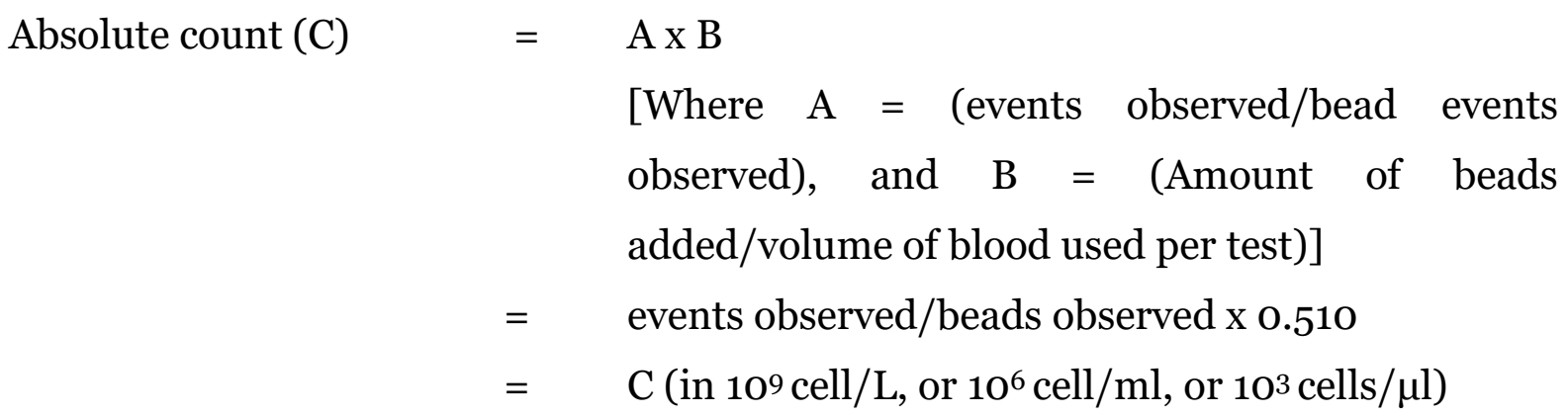

The volume of antibody and lysis buffer did not impact the count and was not considered a dilution factor as the beads and cells were diluted equally by these volumes. 


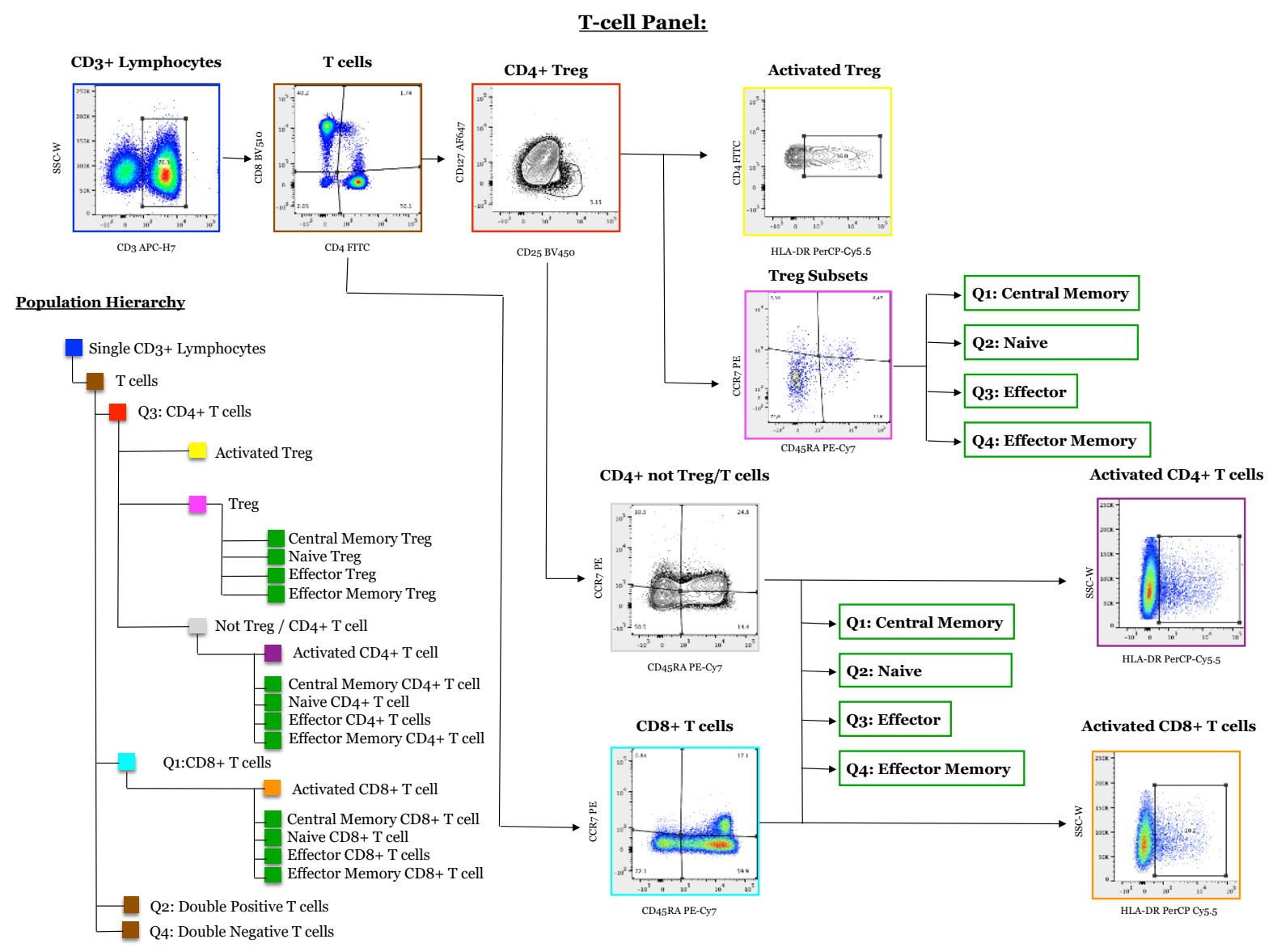

Figure 2-1 T-cell (Panel 1) gating strategy.

All, single, $\mathrm{CD}_{3}{ }^{+}$lymphocytes were sequentially gated with Panel 1 markers to terminal T-cell subsets. Quadrant gates for $\mathrm{CD}_{4}^{+}$and $\mathrm{CD} 8^{+}$are listed but not depicted. Arrows indicate all subsequent events were from the parent plot. Plots are labelled for the terminally gated subset they depict. The legend lists the population hierarchy and subset names corresponding to the matching plot outline colour. 


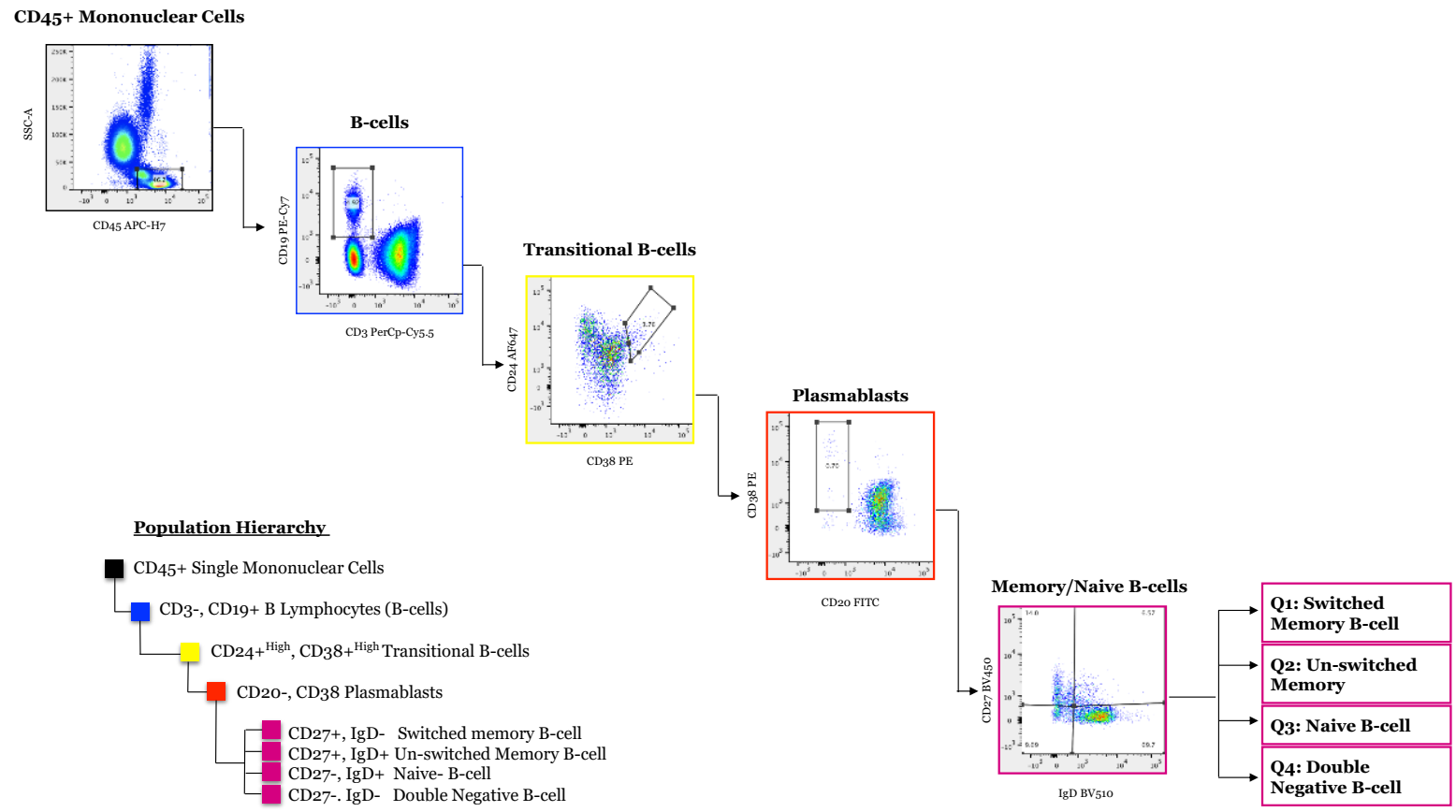

Figure 2-2 B-cell (Panel 2) gating strategy.

All, single, $\mathrm{CD} 45^{+}$events were hierarchically gated with Panel 2 markers to terminal Bcell subsets. Arrows indicate all subsequent events were from the parent plot. Plots are labelled for the terminally gated subset they depict. Memory/Naïve plots were gated using Boolean gating for events not gated as plasmablasts. The legend lists the population hierarchy and subset names corresponding to the matching plot outline colour. 


\section{Monocyte Panel:}

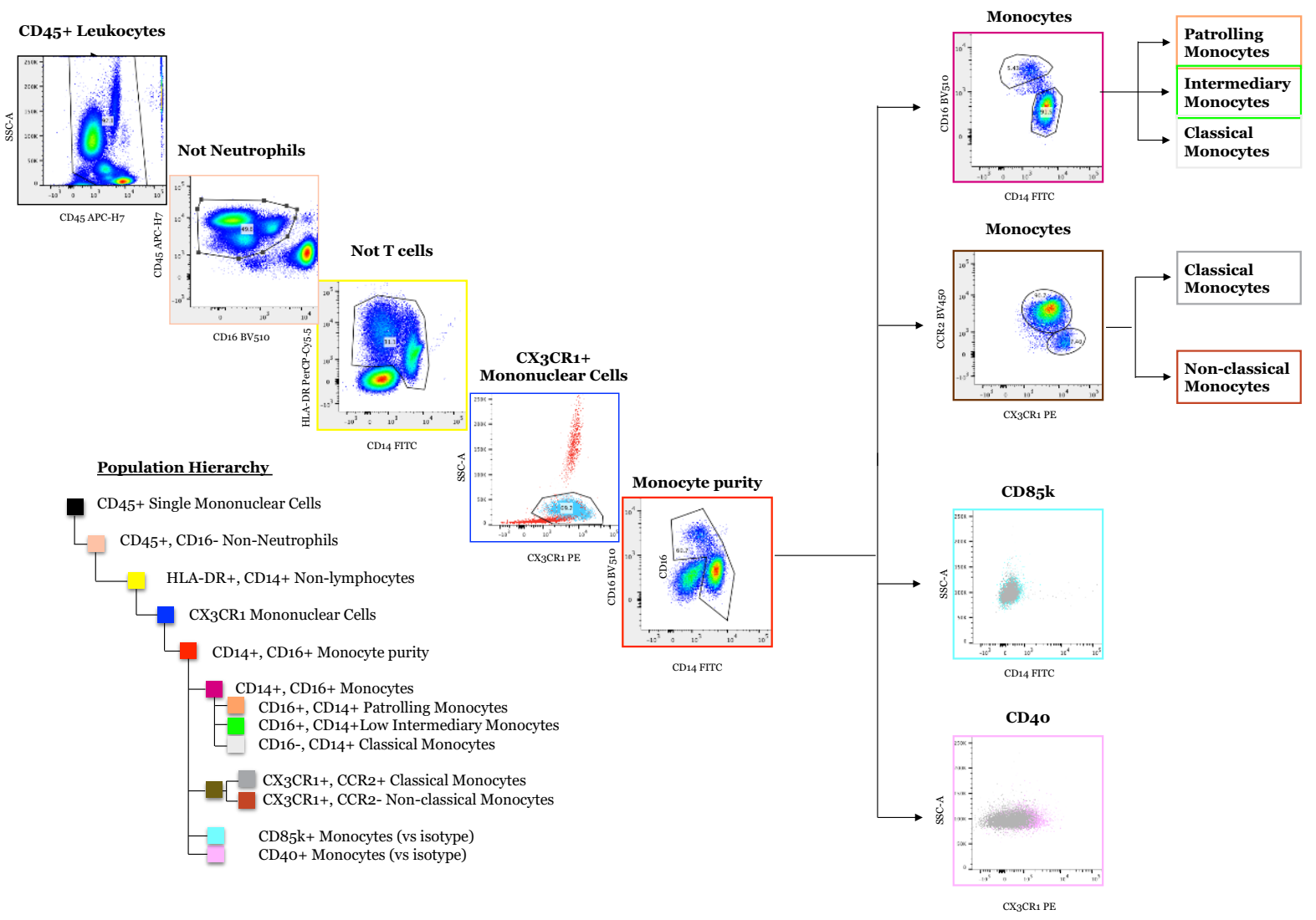

Figure 2-3 DC/NK/Neutrophil (Panel 3) gating strategy.

All, single, CD $45^{+}$events were hierarchically gated with Panel 4 markers to terminal subsets. Arrows indicate all subsequent events were from the parent plot. Plots are labelled for the terminally gated subset they depict. CD85k and CD40 are depicted as overlays with the respective isotype control. The legend lists the population hierarchy and subset names corresponding to the matching plot outline colour. 


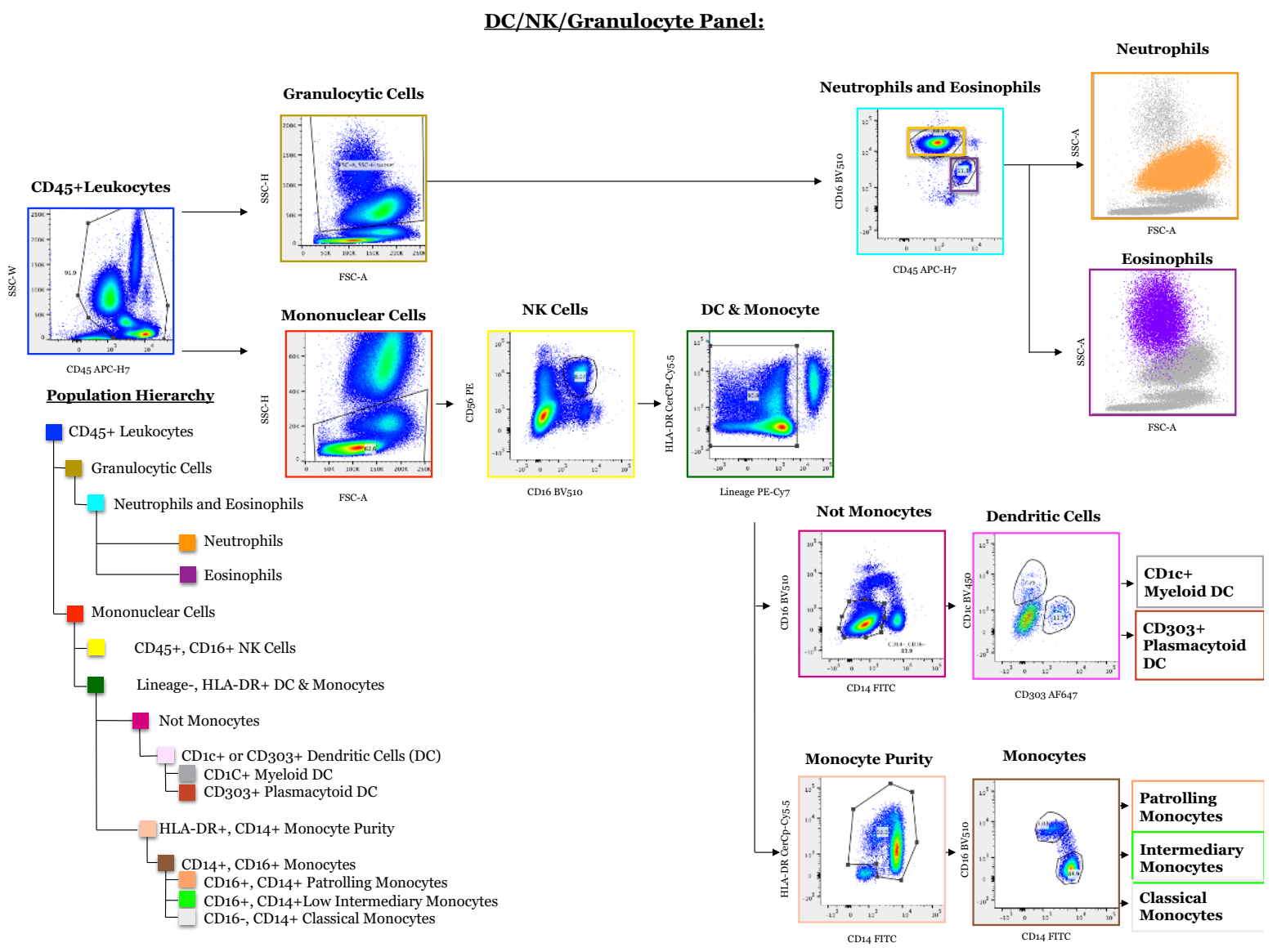

Figure 2-4 Myeloid (Panel 4) gating strategy.

All, single, $\mathrm{CD} 45^{+}$events were hierarchically gated with Panel 4 markers to identify monocytes. Stepwise plots and arrows indicate all subsequent events were from the parent plot. Plots are labelled for the terminally gated subset they depict. The legend lists the population hierarchy and subset names corresponding to the matching plot outline colour. 


\subsubsection{Statistics.}

Statistical analyses were performed in Prism 8 (Graphpad, US). We performed Spanning-tree Progression Analysis of Density-normalised Events (SPADE) using SPADE v3.o (Qiu et al., 2011). All datasets were subject to the D'Agostino-Pearson test with some data normalised prior. We preferentially analysed normally distributed subsets using Welch's T-test. Alternatively, Mann-Whitney was used. Given the exploratory nature of this type of investigation, we did not perform a correction for multiple comparisons except when we assessed an individual's immune variation at different time-points using the Friedman's test, and then corrected for multiple comparisons using the Holm-Sidak formula. For all instances $\mathrm{p}$ values at $\leq 0.05$ were taken as statistically significant while results close to the threshold were interpreted as indicative of a 'trend' so as not to exclude potentially meaningful results. All graphically depicted data show group averages plotted as mean values while error bars represent standard deviation (SD). 


\subsection{Results.}

\subsubsection{Participant demographics.}

The CRISP MS cohort comprised 9 participants, including secondary and primary progressive participants. 15 healthy persons were also recruited to act as a control cohort.

Table 2-7 Whole blood analysis participant demographics.

\begin{tabular}{|l|l|l|l|}
\hline & & $\begin{array}{l}\text { Healthy } \\
\text { Controls } \\
(\mathbf{n = 1 5})\end{array}$ & $\begin{array}{l}\text { Multiple } \\
\text { Sclerosis } \\
(\mathbf{n = 9})\end{array}$ \\
\hline Sex & Female (\%) & $8(54 \%)$ & $7(77 \%)$ \\
\hline $\begin{array}{l}\text { Age range } \\
\text { (mean) }\end{array}$ & & $34-64(48)$ & $45-69(57)$ \\
\hline
\end{tabular}

\subsubsection{Optimisation}

Our panel optimisation aimed to limit the requirement for titration by using reagents from a supplier where the products were already optimised for ex vivo use. Titration experiments served only to establish the validity of the manufacturer's claims and single antibody titrations showed no staining quality improvement over the manufacturer's recommendations (Figure 2-5, representative). Therefore, by limiting our antibody use to single batch numbers, we used the manufacturer's recommended amounts for each antibody in our panels. Furthermore, we used established combinations of lineage markers and fluorochrome conjugates limiting the need for further optimisation. 
CD38 PE

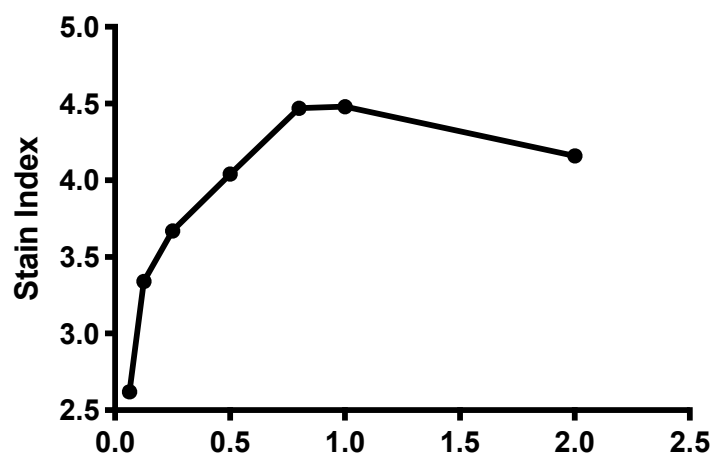

CD8 BV510

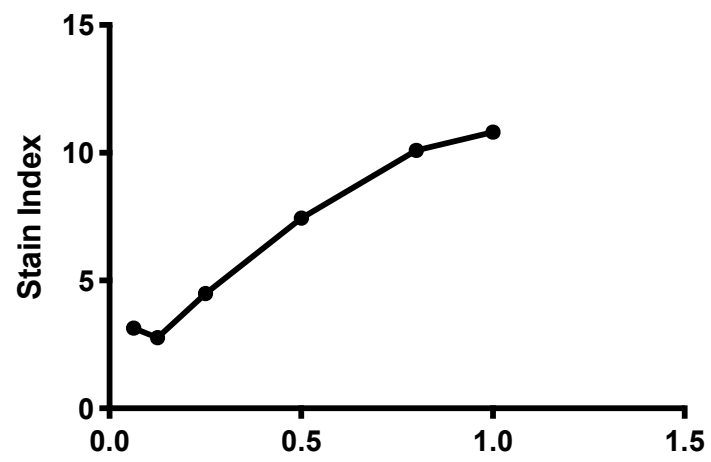

CD1c

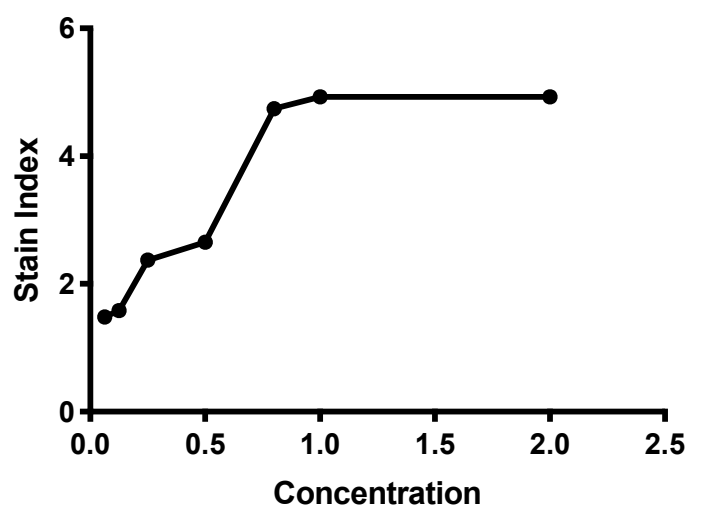

CD19 PE-Cy7

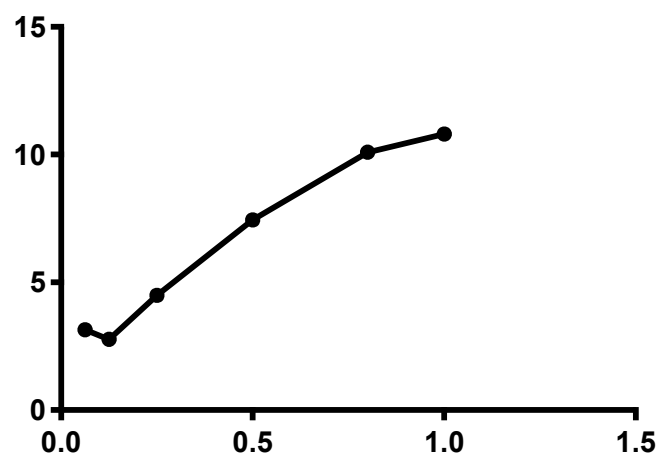

CD45RA PE-Cy7

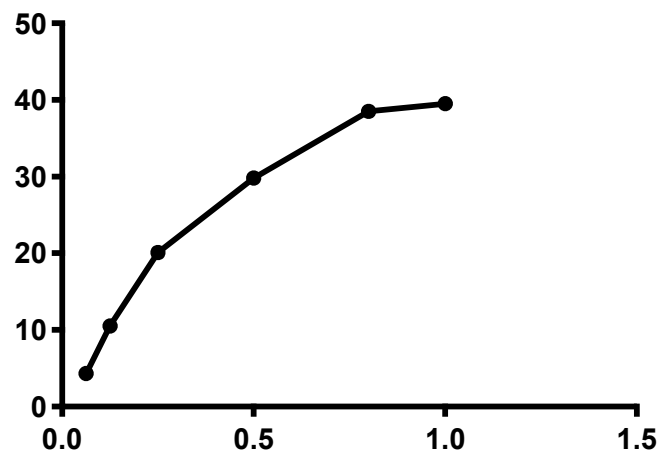

CD20 FITC

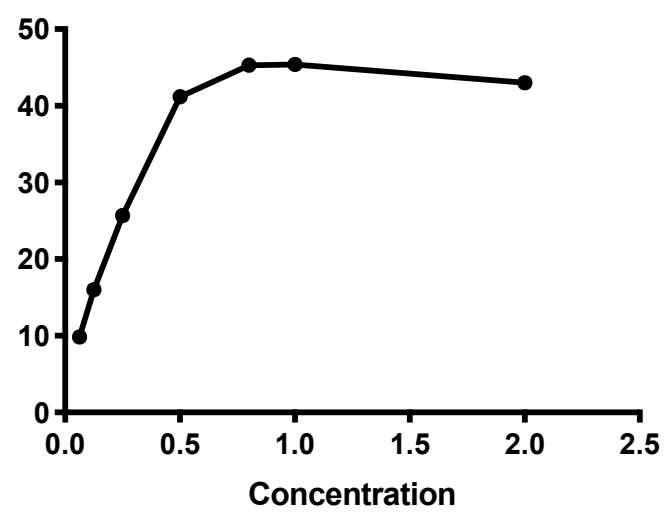

Figure 2-5 Representative titration data from $P B$ antibody panel titrations. Concentration at 1.0 times the manufacturer's recommended volume of reagent per million cells. Stain index $=\left(\mathrm{MFI}_{\text {positive }}-\mathrm{MFI}_{\text {negative }}\right) /\left(2 \mathrm{x} \mathrm{SD}_{\text {negative }}\right)$. 


\subsubsection{Activation marker validation.}

The level of activation marker expression was reported as the geometric mean fluorescence intensity (gMFI). We investigated expression of HLA-DR, CD40 and CD85k using internal negative staining and isotype controls. Ideally, a fluorescence minus (FMO) control would have had better utility as a gating control, but without it we calculated the expression of whole immune subsets (e.g. monocytes) to interrogate expression.

We collected isotype control data for CD40 and CD85k that were recorded in a separate panel with the same lineage markers but substituting CD4O and CD85k with their respective isotypes. CD40 expression is demonstrated in Figure 2-6. We calculated a significant difference in the gMFI of expression for the isotype control stain compared to fully stained monocytes $(\mathrm{p}=0.0001$ ) (Figure 2-6A) suggesting nonspecific staining was not an issue. We also observed high levels of expression on Bcells compared to the negative expression expected on T-cells (Figure 2-6C).

Unfortunately, we could not validate the staining of the CD85k isotype control owing to very high levels of background interaction seen from this clone. CD85k is however expressed by a range of other immune subsets including neutrophils (Bankey et al., 2010), but generally not by the majority of healthy B-cells (Kang et al., 2015). The differential expression of this epitope allowed us to confirm its use for our monocyte data (Figure 2-7).

For HLA-DR expression we used expression from neutrophils as a gating control. These cells have a high degree of autofluorescence and showed very dim staining. This allowed us to use neutrophil expression of HLA-DR as a gating control (Figure 2-8). Unfortunately, the absence of a comparable subset for CD40 and CD85k, or an FMO, prohibited subset enumeration of CD40 and CD85k markers. 

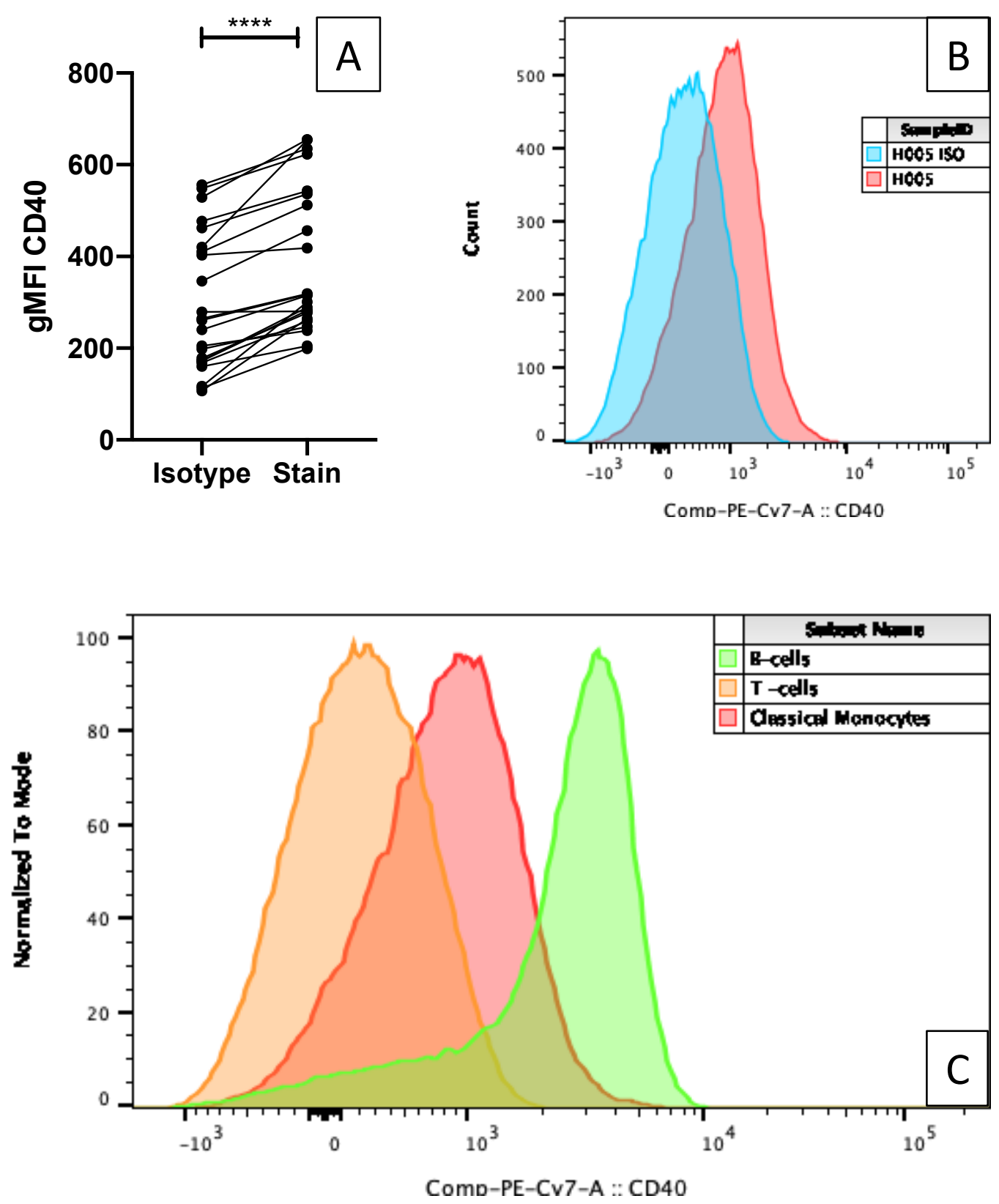

Figure 2-6 CD40 expression controls.

(A) Significant difference in the gMFI of isotype stained, compared to fully, stained monocytes. (B) representative histogram of isotope (blue) vs fully stained monocytes (red) of CD40. (C) Differential expression of CD40 in terminal immune subsets with B cells (green), T cells (orange), and monocytes (red). 

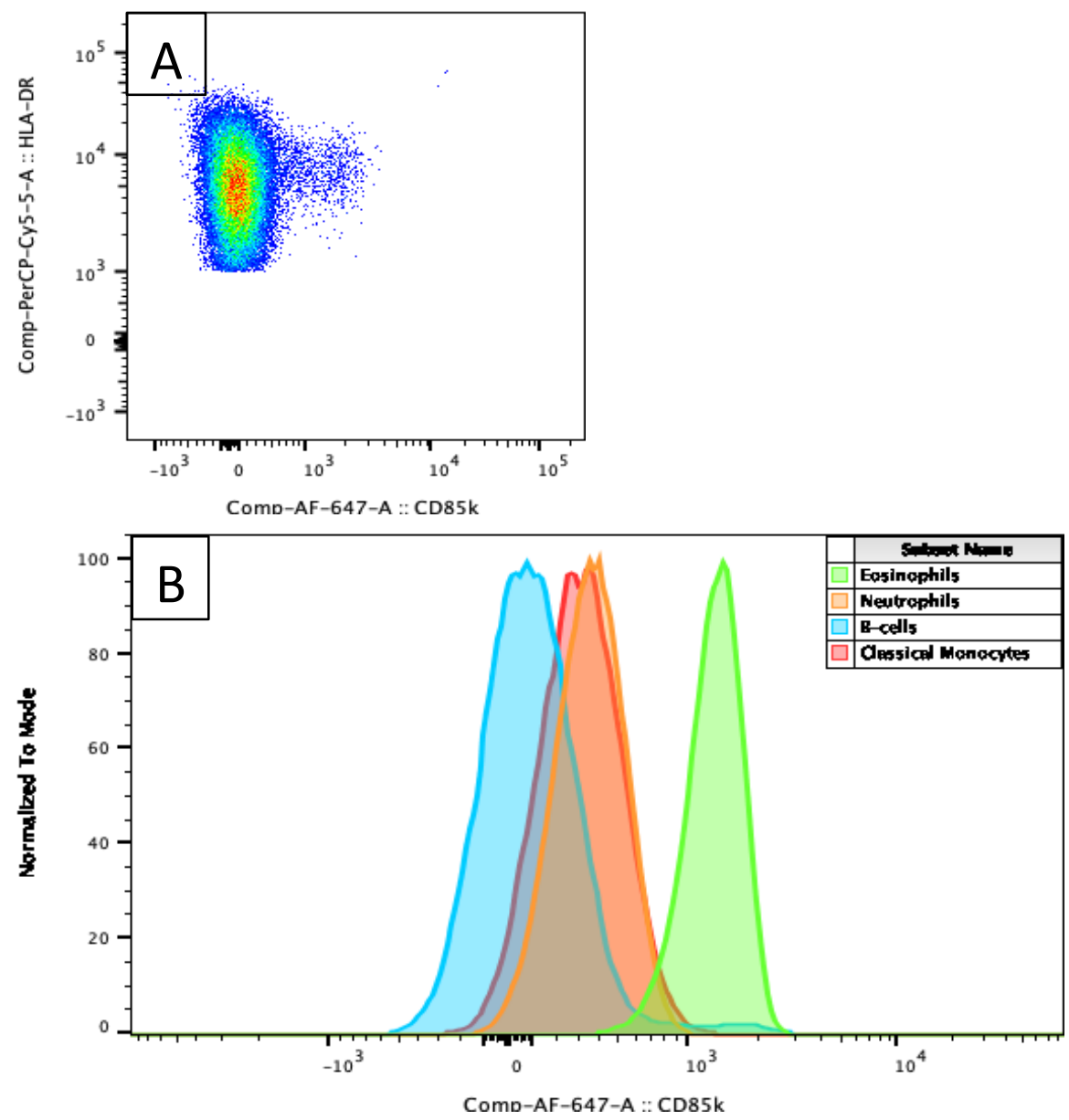

Figure 2-7 Expression of CD85k.

(A) Expression in a minor population of positive staining B cells. (B) Differential expression in the PBMC (blue = B cells; red = monocytes; orange $=$ neutrophils; green $=$ eosinophils).

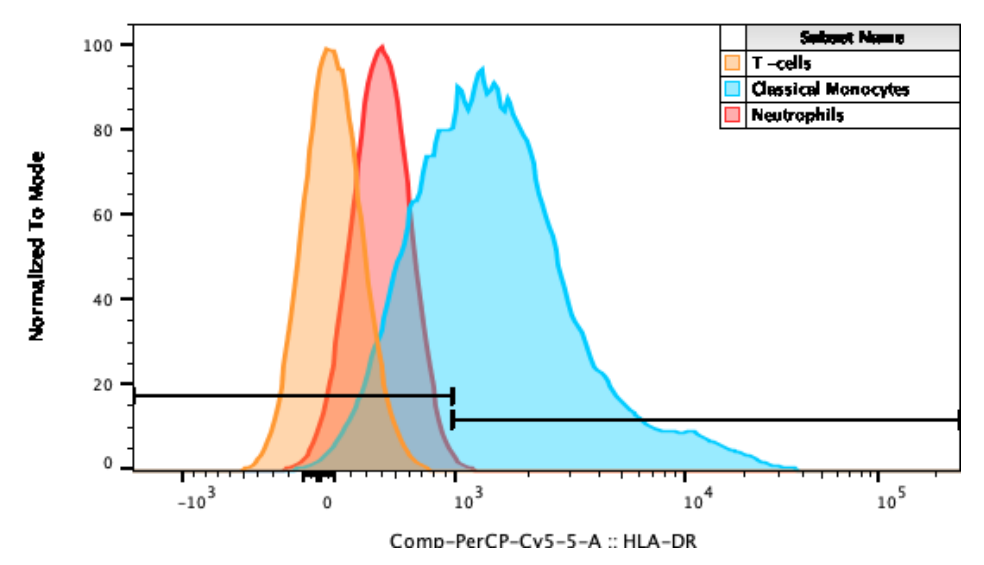

Figure 2-8 Gating control for HLA-DR.

Expression by monocytes (blue), using neutrophils (red) to account for autofluorescence that is absent in T cells (orange). 


\subsubsection{Absolute count comparison.}

All CRISP trial participants had a baseline full blood count sample processed by the central hospital pathology laboratory and we performed absolute counting of whole blood by flow cytometry on our samples from the same timepoint. We performed a simple linear regression for five blood count output measures between our baseline absolute count data and the pathology data. We observed a good correlation between the two measures with neutrophils $\left(\mathrm{R}^{2}=0.49 ; \mathrm{p}=0.034\right.$; Figure 2-9B), eosinophils $\left(\mathrm{R}^{2}=0.722 ; \mathrm{p}=0.0037 ;\right.$ Figure $\left.2-9 \mathrm{C}\right)$ and total lymphocytes $\left(\mathrm{R}^{2}=0.626 ; \mathrm{p}=0.012\right.$; Figure 2-9E) indicating a good line of fit. For monocytes $\left(\mathrm{R}^{2}=0.454 ; \mathrm{p}=0.080\right.$; Figure 2-9D) and total leukocytes $\left(\mathrm{R}^{2}=0.523\right.$; $\mathrm{p}=0.087$; Figure 2-9A), the trend was just short of statistically significant.

\subsubsection{Peripheral T cells in progressive MS.}

We calculated both the absolute number of cells in the original samples, as well as the relative frequency of $\mathrm{T}$ cells, by subset. The frequency was calculated as the proportional makeup as a fraction of all $\mathrm{CD}_{3}+$ cells. Data are presented here from high (major) abundance to lowest (minor), based on absolute counts.

\subsubsection{Major T-cell subsets differ in progressive MS.}

We found that while the total number of $\mathrm{CD}_{3}+$ cells did not vary significantly, there was a strong trend toward fewer CD8+ T cells in MS individuals (0.518 x $10^{3}$ cells $\left./ \mu \mathrm{l}\right)$ compared to $\mathrm{HC}\left(0.899 \times 10^{3}\right.$ cells $/ \mu \mathrm{l} ; \mathrm{p}=0.083$; Figure 2-10A). Similar to the enumerated CD8 cells, a trend indicated lower CD8+ cell proportions in people with progressive MS (22.22\%) compared to the HC (31.08\%), although this also was just short of statistical significance ( $\mathrm{p}=0.070$; Figure 2-10D). While enumerated CD4+ cells did not show a strong inter-cohort difference (Figure 2-10A), we observed a significant increase in the proportion of $\mathrm{CD}_{4}+$ cells in the MS cohort (MS 68.25\% vs. HC 58.17\%; $p=0.0457$; Figure 2-10D)

Within the CD4 compartment naïve, memory and effector cell numbers showed a high degree of interpatient variability and no significant difference between cohorts (Figure 
2-10B). This variation was also reflected in the proportional data with the exception of the central memory subset for MS participants, which was significantly larger (HC: 5.86\% vs. MS: $11.30 \%$; $=0.0177$; Figure $2-10 E$.

Within the CD8 compartment the naïve cells appeared to be reduced in the MS cohort ( $0.127 \times 10^{3}$ cells $\left./ \mu \mathrm{l}\right)$ when compared the $\mathrm{HC}\left(0.21 \times 10^{3}\right.$ cells $/ \mu \mathrm{l} ; \mathrm{p}=0.052$; Figure 2$10 \mathrm{C}$ ), with the same shift again appearing over the effector cells but also failing to reach significance. Similarly, the proportion of CD8 naïve cells (5.06\%) in MS appeared to be lower to the HC (8.59\%; $p=0.052$; Figure 2-10F), which was in line with the enumerated data. 
A

Leukocytes

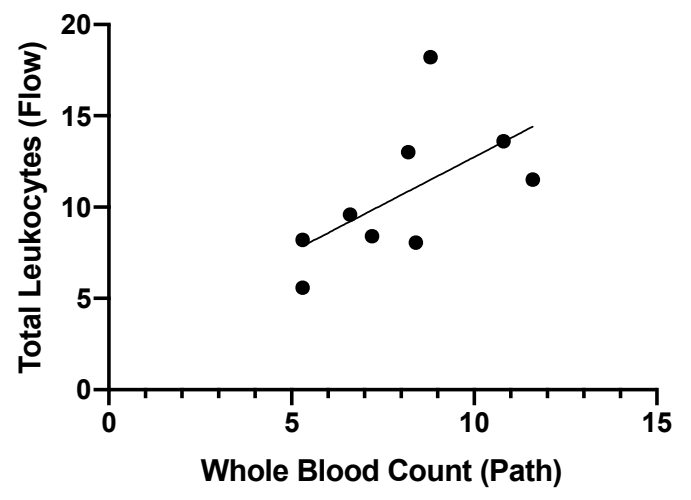

C

\section{Eosinophils}

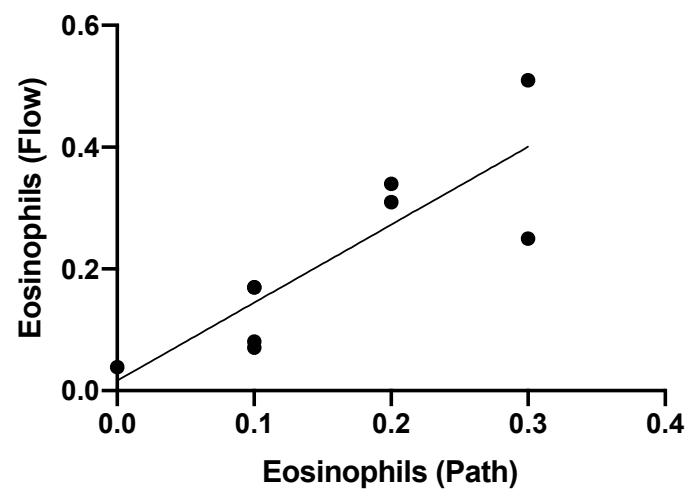

B

Neutrophils

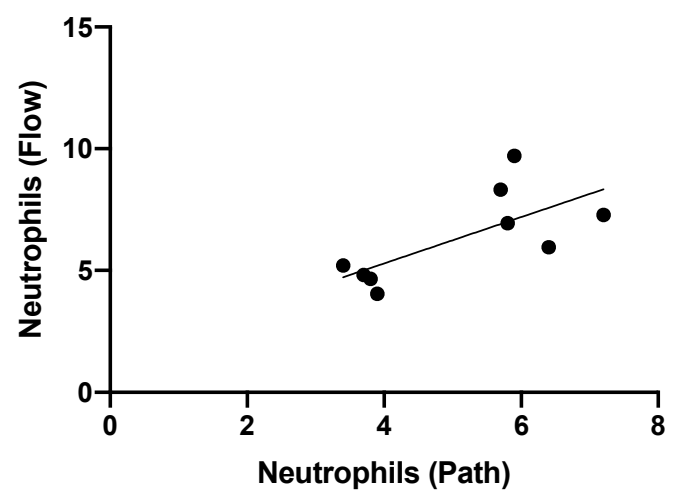

D

Monocytes

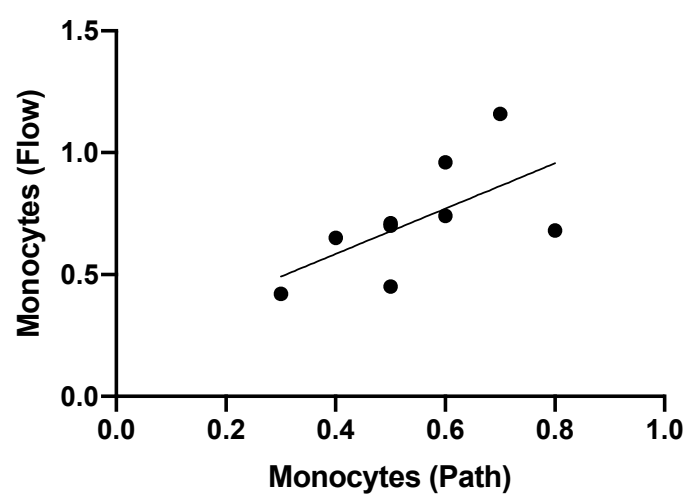

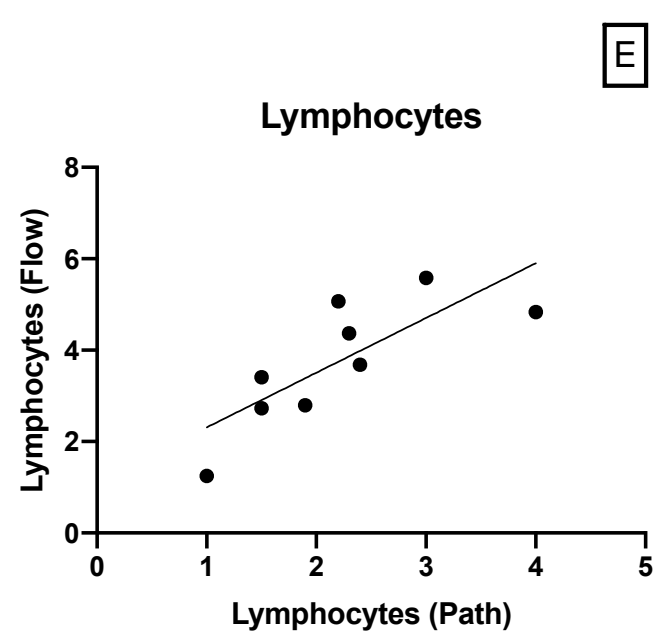

Figure 2-9 Simple regression analysis of central pathology (path), and flow cytometry (flow) absolute count data sets.

All values are in $10^{9}$ cell $/ \mathrm{L}$. 

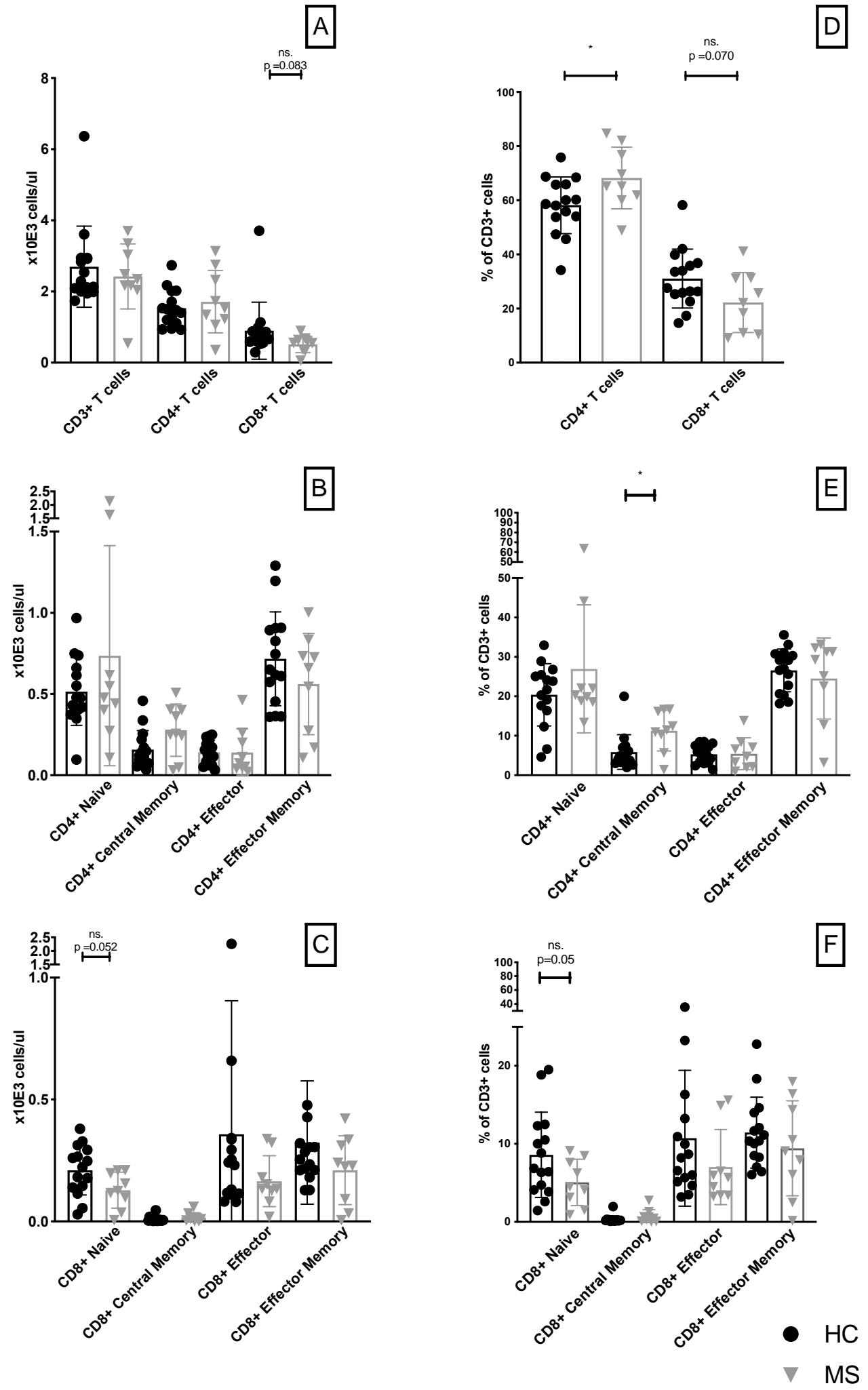

Figure 2-10 Absolute number $(A-C)$ and proportion $(D-F)$ of major $T$ cell subsets in the $\mathbf{P B}$.

Each data point represents an individual participant sample. Bars indicate mean and SD. ${ }^{*}(\mathrm{p} \leq 0.05),{ }^{* *}(\mathrm{p} \leq 0.01),{ }^{* * *}(\mathrm{p} \leq 0.001),{ }^{* * *}(\mathrm{p} \leq 0.0001)$. ns. $=$ not significant. 
2.4.4.2 The minor T-cell compartment in MS was significantly altered.

The data show that activated $\mathrm{CD}_{3}{ }^{+}$cells and $\mathrm{CD} 4{ }^{+} \mathrm{CD} 8^{+}$double positive cell numbers did not vary to any degree of significance (Figure 2-11A), but MS participants had significantly fewer double negative T cells ( $0.087 \mathrm{x103}$ cells/ $\mu \mathrm{l}$ ) compared to their HC counterparts (0.157 X103 cells $/ \mu \mathrm{l} ; \mathrm{p}=0.015$; Figure 2-11A). In contrast to the enumerated data, the difference in the proportion for double negative $\mathrm{T}$ cells did not reach the significance threshold, while the proportion of activated $\mathrm{T}$ cells $(\mathrm{p}=0.031)$ were found to be significantly higher in the MS cohort (Figure 2-10C).

Within the Treg compartment we observed that the subset group average numbers between the cohorts were broadly similar, but that activated Treg (HLA-DR+) were significantly increased in the MS cohort ( 0.0283 x $10^{3}$ cells $\left./ \mu \mathrm{l}\right)$ over the HC (0.0161 x $10^{3}$ cells $/ \mu \mathrm{l} ; \mathrm{p}=0.0251$; Figure $2-11 \mathrm{~B}$ ). In the proportional distribution, the difference was also significant, with MS participants showing an increased proportion of activated Treg (MS 1.21\% vs. HC 0.61\%; $\mathrm{p}=0.0017$; Figure 2-11D).

\subsubsection{The ratio of $\mathrm{CD} 4$ to $\mathrm{CD} 8$ in progressive MS.}

Lastly, we calculated the ratio of $\mathrm{CD} 4+$ to $\mathrm{CD} 8+\mathrm{T}$ cells and observed that this ratio was significantly altered in the MS cohort, with some MS participants showing values of up to 9.5:1 in favour of the CD4 compartment. This ratio was also significantly different in comparison to the $\mathrm{HC}(\mathrm{p}=0.0184$; Figure 2-12). 

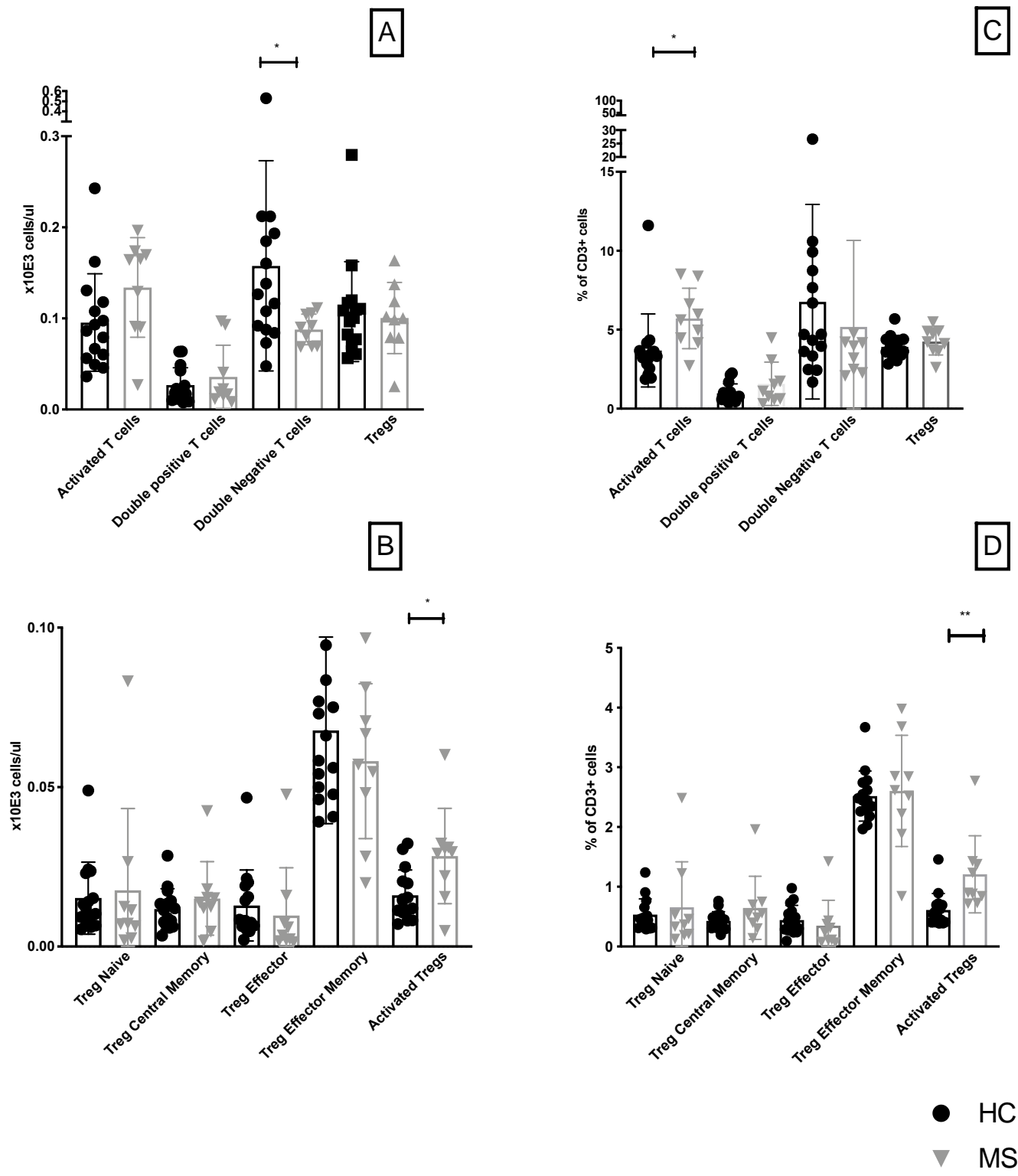

Figure 2-11 Absolute number (A\&B) and proportion (C\&D) of minor $T$ cell subsets in the $\mathbf{P B}$.

Each data point represents an individual participant sample. Bars indicate mean and SD. ${ }^{*}(\mathrm{p} \leq 0.05),{ }^{* *}(\mathrm{p} \leq 0.01),{ }^{* * *}(\mathrm{p} \leq 0.001),{ }^{* * * *}(\mathrm{p} \leq 0.0001)$ 


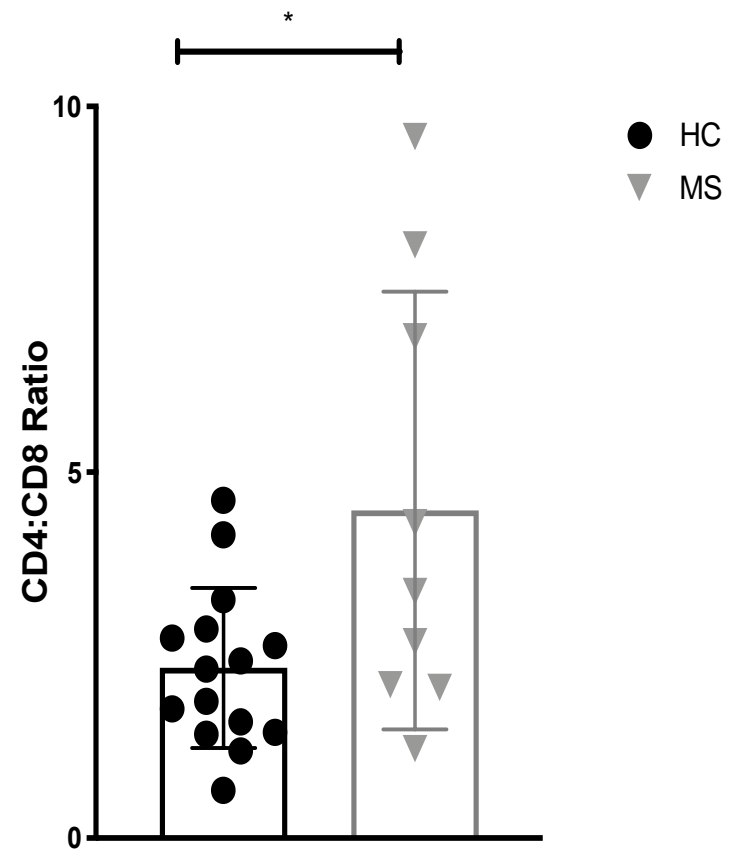

Figure 2-12 Ratio of CD4 to CD8.

Data calculated from the enumerated cell values. Each data point represents an individual participant sample. Bars indicate mean and SD. ${ }^{*}(\mathrm{p} \leq 0.05),{ }^{* *}(\mathrm{p} \leq 0.01),{ }^{* * *}(\mathrm{p} \leq$ $0.001),{ }^{* * * *}(\mathrm{p} \leq 0.0001)$ 


\subsubsection{Peripheral B cells in progressive MS.}

From our B-cell panel data, we calculated the total B-cell, T-cell, and leukocyte (CD45+) cell numbers. We observed no significant difference in the total number of these cells between the cohorts. B cells showed very little difference between the means, which may be due to the wide range in the total numbers from individual participants (MS 0.371 X103 cells/ $\mu \mathrm{l}$ vs HC 0.391 x103 cells/ $\mu \mathrm{l}$; Figure 2-13).

A detailed phenotypic breakdown of the B cell compartment and enumeration of the subsets did not reveal any difference in the average count per group, but we observed a trend where double negative B cells appeared less numerous ( $\mathrm{p}=0.0517$ ), while transitional B cells appeared more common $(\mathrm{p}=0.0848)$ in the PB of MS participants in contrast to HC (Figure 2-14). We then calculated the relative proportion of B-cell subsets as a fraction of all CD19+ events, allowing us to determine if the composition of the B-cell compartment was altered in MS. We observed differences in the switched memory subset, where MS participants (8.50\%) had a significantly smaller proportion compared to the HC (15.75\%; p = 0.0104). Similarly, double negative B-cell proportions were lower in MS participants (HC 20\% vs. MS 10\%; p = 0.0114) while the proportion of transitional B cells were significantly greater (HC 3.47 vs. MS 6.53; p = 0.0067; Figure 2-15). Together, these observations show that in progressive MS, the B-cell compartment is distinctly altered, with what is a larger transitional B-cell compartment, and fewer class-switched mature and double negative B cells (Figure 216). 


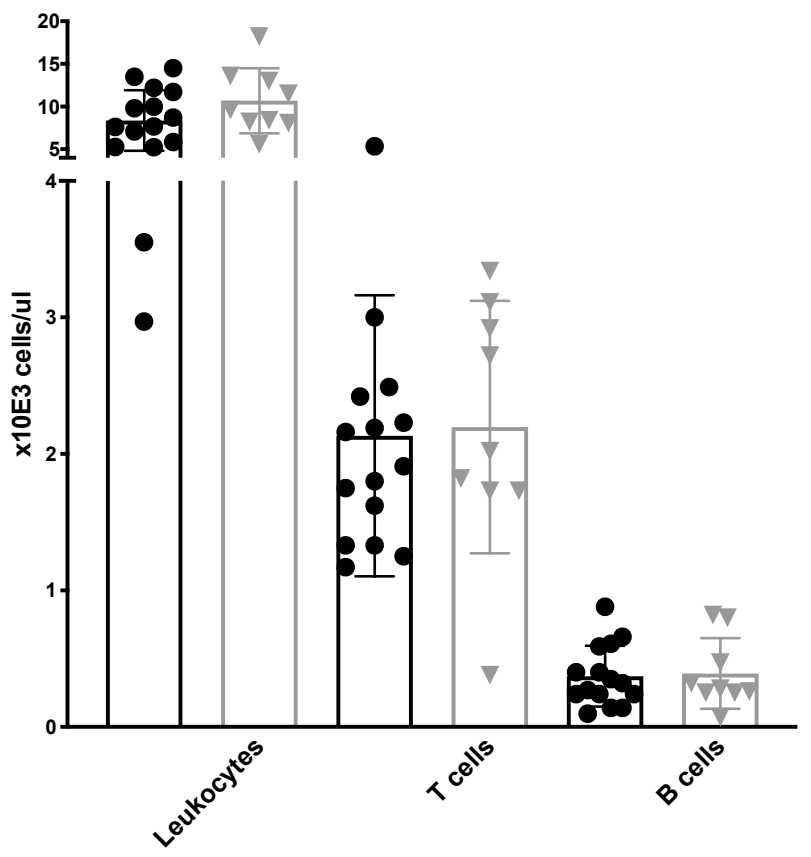

Figure 2-13 Absolute number of B cells, T cells and leukocytes in the PB from B-cell panel data.

Each data point represents an individual participant sample. Bars indicate mean and SD. ${ }^{*}(\mathrm{p} \leq 0.05),{ }^{* *}(\mathrm{p} \leq 0.01),{ }^{* * *}(\mathrm{p} \leq 0.001),{ }^{* * * *}(\mathrm{p} \leq 0.0001)$

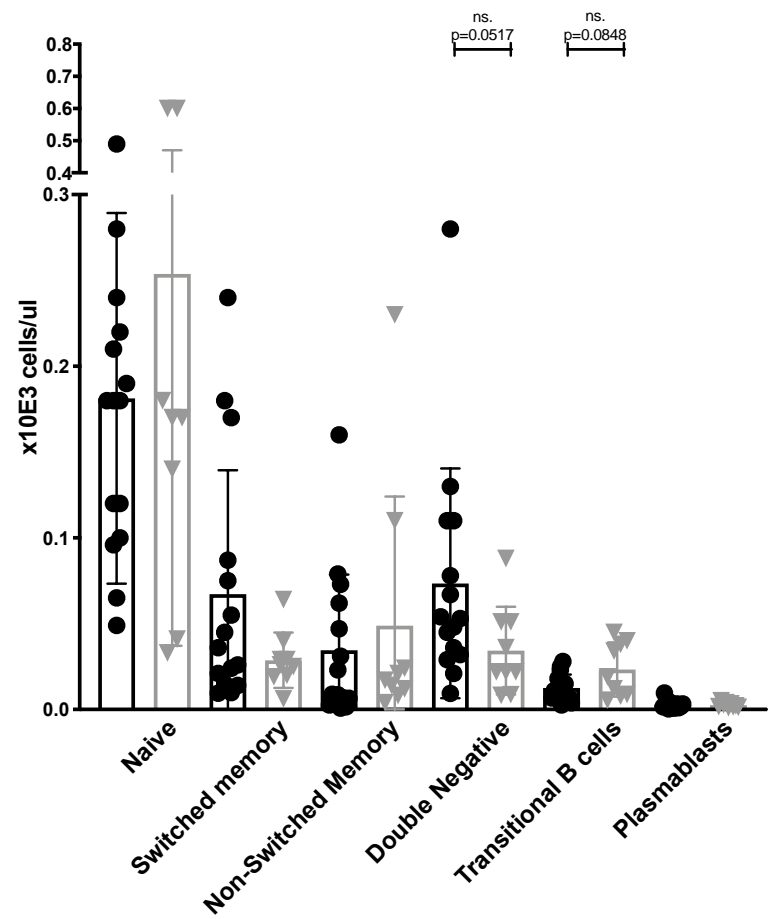

Figure 2-14 Absolute number of B-cell subsets in the PB.

Each data point represents an individual participant sample. Bars indicate mean and SD. ns. = not significant ${ }^{*}(\mathrm{p} \leq 0.05),{ }^{* *}(\mathrm{p} \leq 0.01),{ }^{* *}(\mathrm{p} \leq 0.001),{ }^{* * * *}(\mathrm{p} \leq 0.0001)$ 


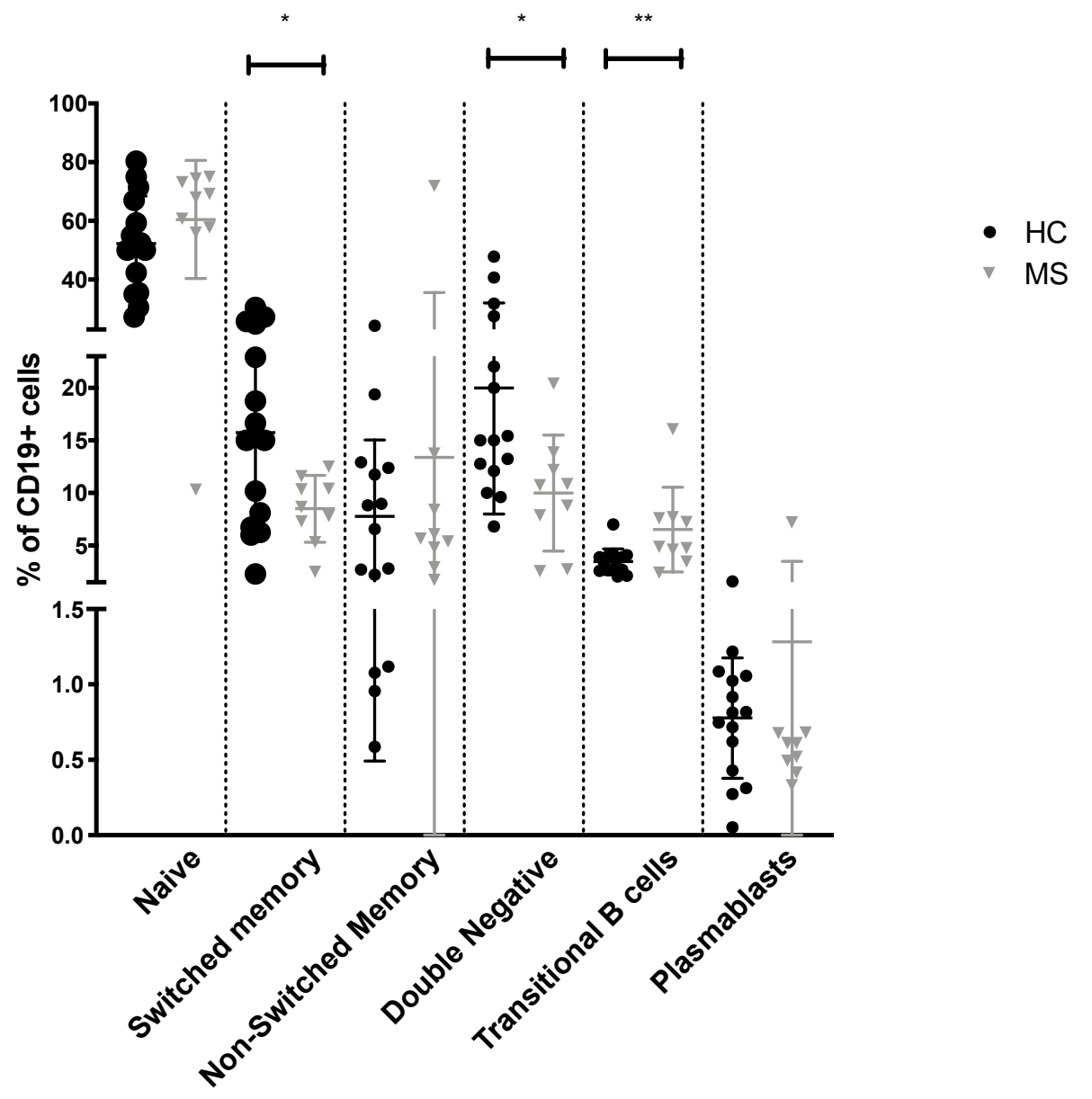

Figure 2-15 The proportion of B-cell subsets relative to all CD19+ events. Each data point represents an individual participant sample. Bars indicate mean and SD. ${ }^{*}(\mathrm{p} \leq 0.05),{ }^{* *}(\mathrm{p} \leq 0.01),{ }^{* * *}(\mathrm{p} \leq 0.001),{ }^{* * * *}(\mathrm{p} \leq 0.0001)$.

\section{Healthy Controls}

\section{Multiple Sclerosis}
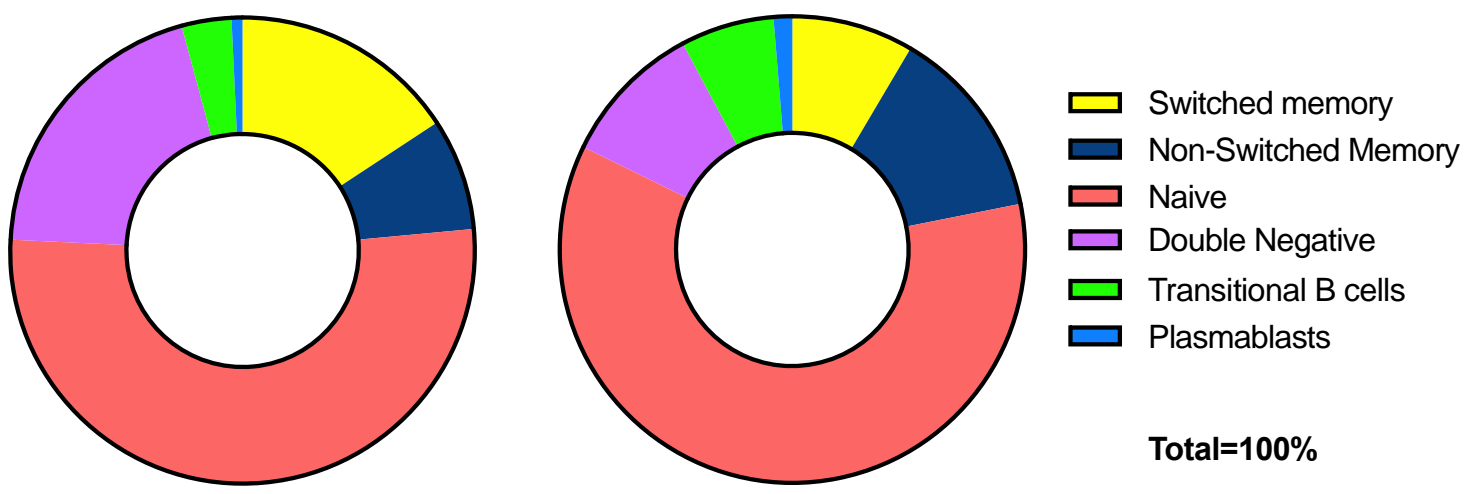

Figure 2-16 Proportional composition of the B-cell compartment in HC and MS.

Each section is representative of the group mean proportion for the relevant subset. 


\subsubsection{Peripheral myeloid cells in untreated MS.}

We enumerated myeloid lineage cell subsets with our NK/DC/granulocyte panel to determine if any changes in their absolute numbers were present in the MS cohort. Neither of the lineage cell group averages varied to a degree that met the definition of statistically significance (Figure 2-17A-D). We did, however, observe that neutrophil numbers appeared elevated in the MS cohort when compared to the $\mathrm{HC}$ although this trend fell short of the significance threshold ( $\mathrm{p}=0.071$; Figure 2-17A).

To determine the activation state of monocytes in our participants, we assessed expression of HLA-DR, CD40, and CD85k in our monocyte panel (Figure 2-18A-D). No significant differences in the expression of activation markers (Figure 2-18A-C) nor for the proportion of monocytes staining positive for HLA-DR (Figure 2-18D) were observed when the MS and HC cohorts were compared. Furthermore, no significant differences in expression of these markers was noted when we subdivided monocytes using CD14 and CD16 phenotypes, into classical, non-classical, and intermediate subsets (Figure 2-19).

We also calculated myeloid cell proportions in relation to the total cells commonly present in the peripheral blood mononuclear (PBMC) fraction thus excluding all large granulocytic cells. PBMC as a sample source is commonly used in immune research owning to the reliable long-term storage of such samples. We aimed to establish what the proportional makeup of the PBMC alone would show, and this would allow us to relate our data to previously published work. From this calculation we observed no trends and no significant inter-cohort variation (Figure 2-20). 
A

B
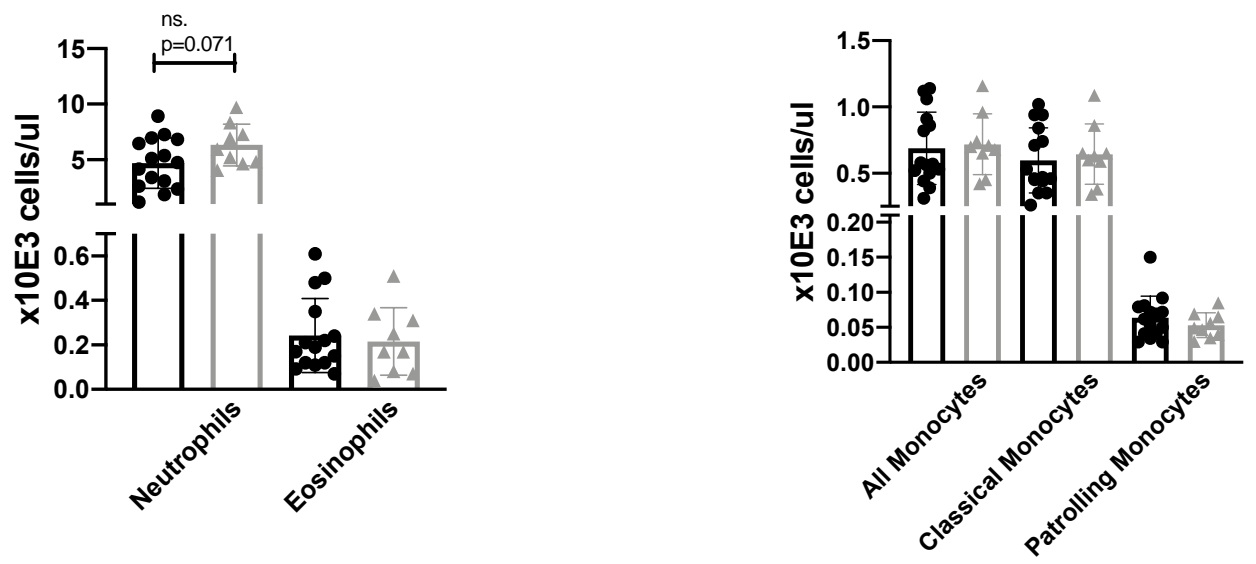

C
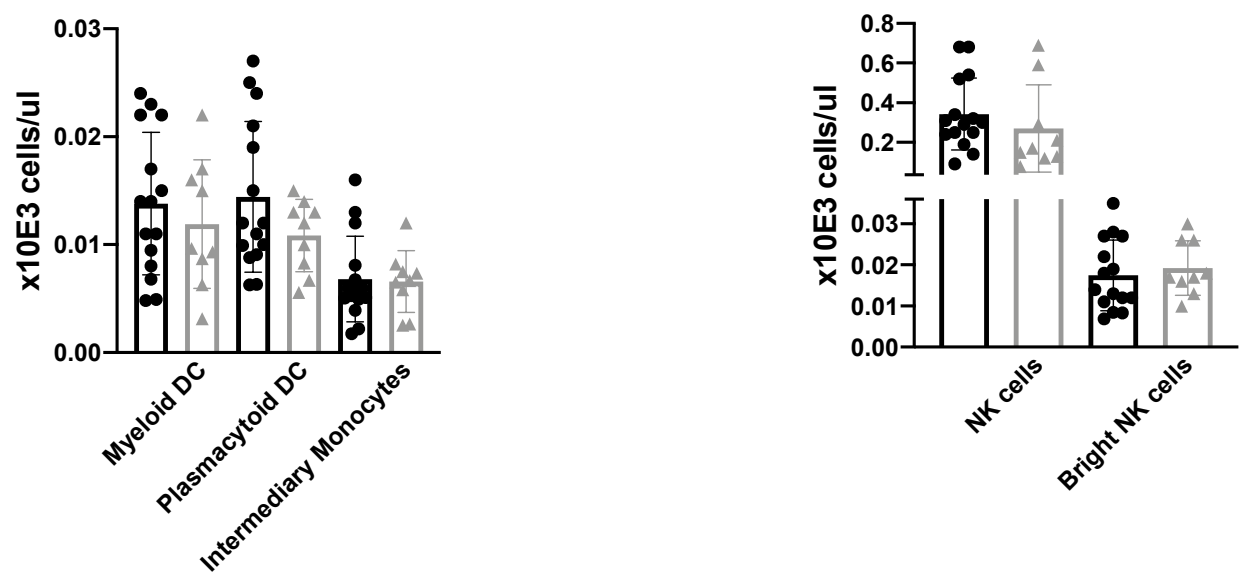

Figure 2-17 Total number of myeloid cells by immune subset.

A: large granulocytes, B: monocytes, C: dendritic cells (DC) and transitional monocytes, D: NK cells. Each data point represents an individual participant sample. Bars indicate mean and SD. ns. = not significant. ${ }^{*}(\mathrm{p} \leq 0.05),{ }^{* *}(\mathrm{p} \leq 0.01),{ }^{* * *}(\mathrm{p} \leq$ 0.001), ${ }^{* * * *}(\mathrm{p} \leq 0.0001)$ 

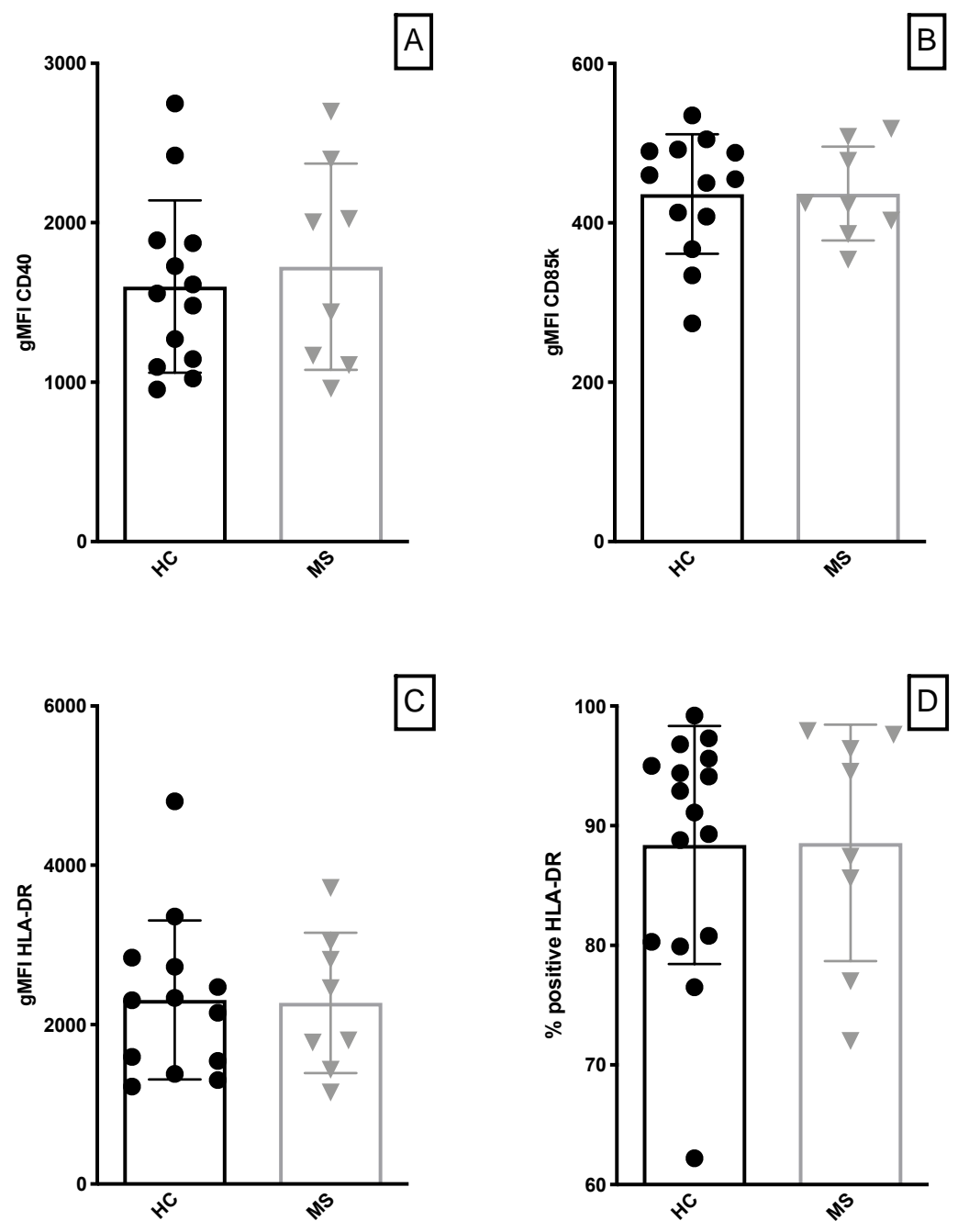

Figure 2-18 Expression (gMFI) of activation markers of all monocytes.

(A) CD40, (B) CD85k, (C) HLA-DR and \% of positive HLA-DR staining (D). Each data point represents an individual participant sample. Bars indicate mean and SD. 
HLA-DR
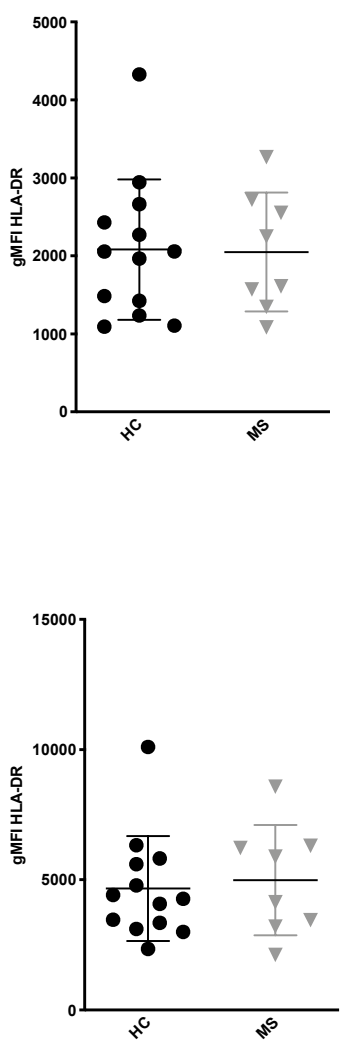

Intermediate

Non-classical
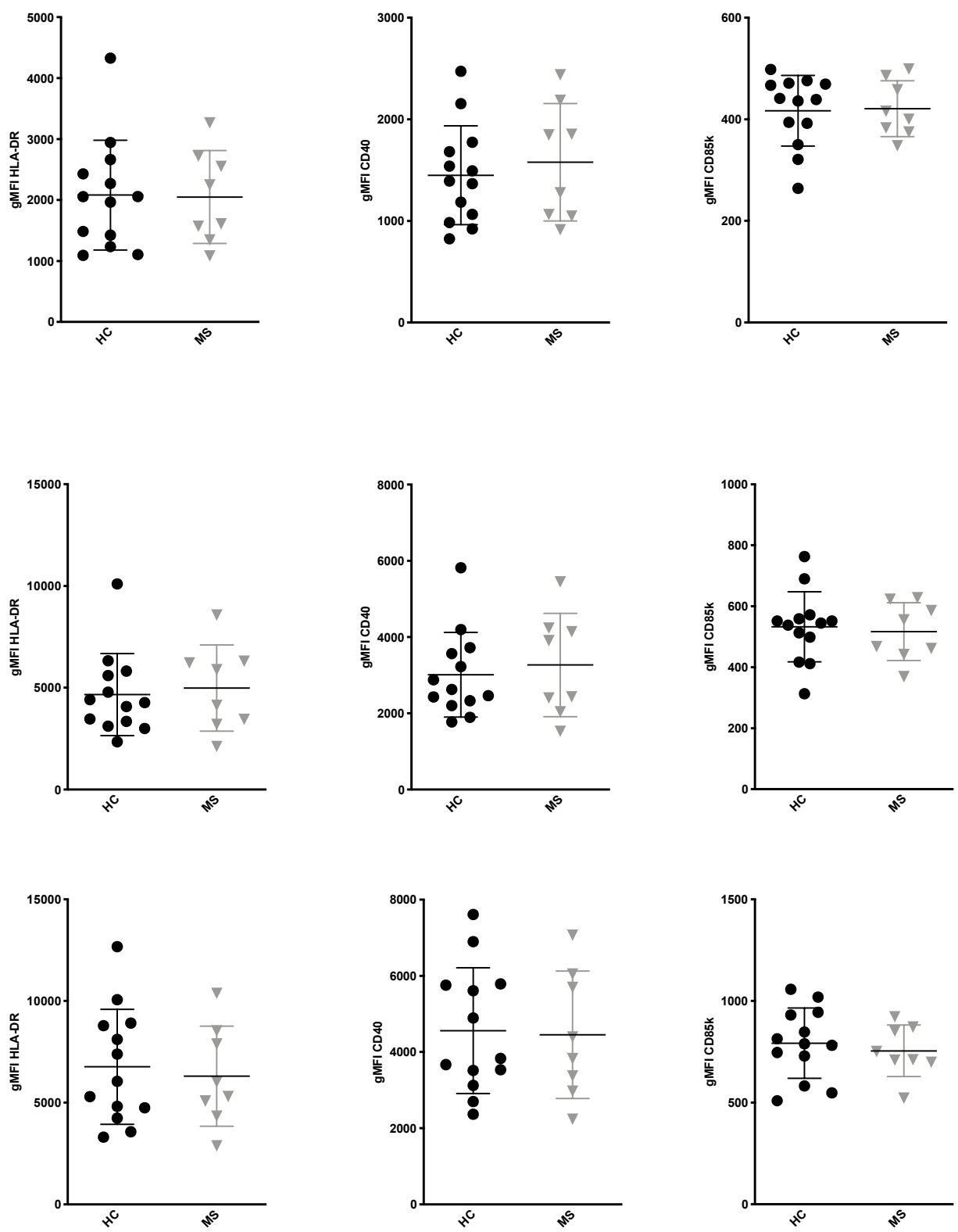

Figure 2-19 Expression (gMFI) of HLA-DR, CD40 and CD85k on monocytes by subset.

Each data point represents an individual participant sample. Bars indicate mean and SD. 
Total Monocytes

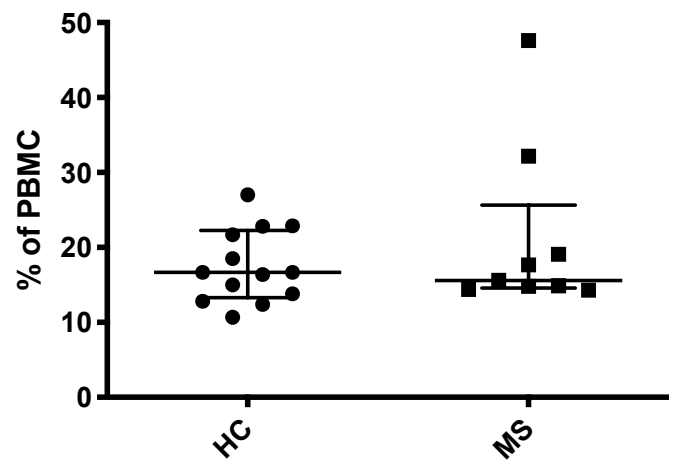

Non-classical Monocytes

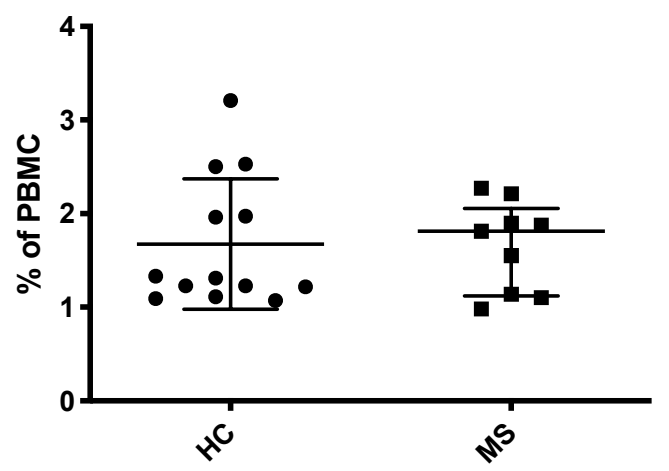

CCR2+CX3CR1+ Monocytes

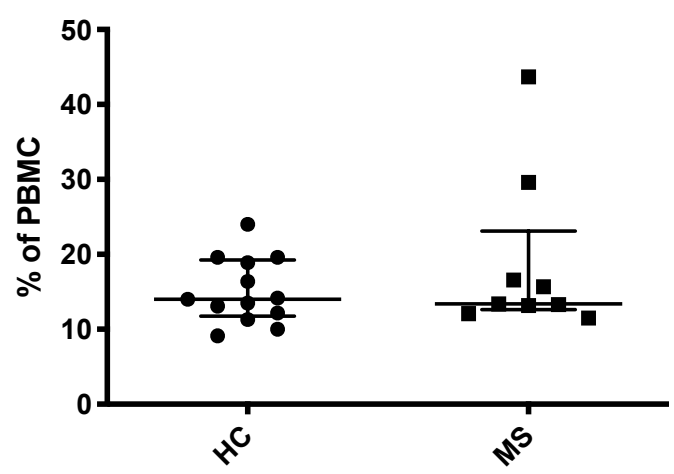

Classical Monocytes

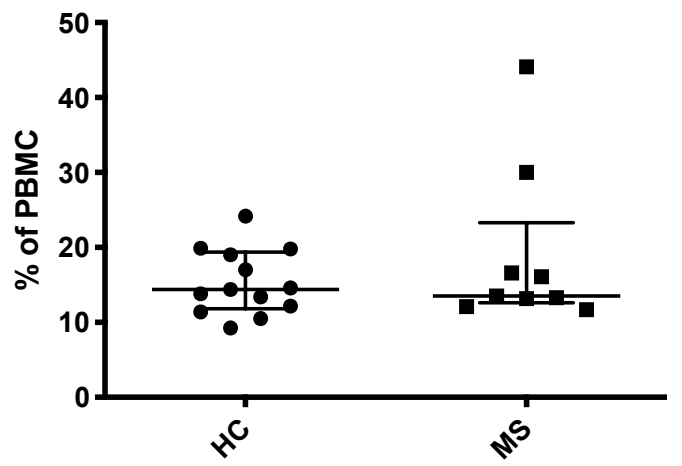

Intermediate Monocytes

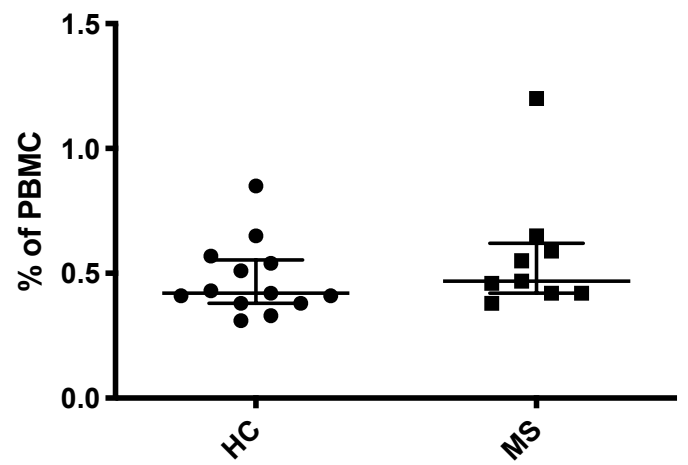

CCR2-CX3CR1+ Monocytes

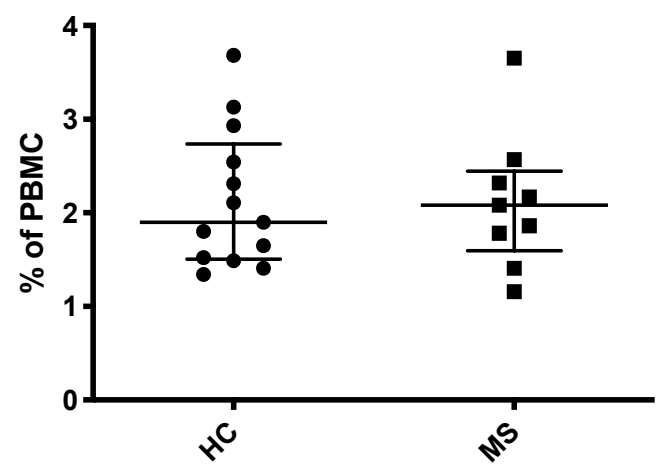

Figure 2-2o Proportional makeup of the monocyte compartment relative to all PBMC.

Each data point represents an individual participant sample. Bars indicate mean and SD. 


\subsubsection{The proportional composition of the immune system in MS.}

We pooled all T-, B- and myeloid cell panel data and calculated the relative proportion of each lineage subset as a percentage of the total leukocyte fraction (CD45+). The group average of each subset for MS and HC participants were then compared. We aimed to assess if there were any significant changes to the proportional makeup of the peripheral immune cell compartment as a whole, and to confirm alterations within the enumerated data. We depict in Figure 2-21 only those subsets that reached the definition of statistically significant or where a very strong trend presented.

Predominantly, T-cell subsets were affected, and we observed two trends whereby the proportion of CD3 T cells ( $\mathrm{p}=0.085$; Figure 2-21A), as well CD8 effector cells $(\mathrm{p}=$ 0.083; Figure 2-21C) were lower in the MS cohort. For some subsets the fraction present in the MS cohort were significantly lower than the HC, and these include: CD8 T cells ( $\mathrm{p}=0.0104$; Figure 2-21B), CD4 effector memory ( $\mathrm{p}=0.0491$; Figure 2-21D), double negative T cells $(\mathrm{p}=0.0212$; Figure 2-21F), double negative $\mathrm{B}$ cells $(\mathrm{p}=0.0113$; Figure 2-21G), and plasmacytoid DC ( $\mathrm{p}=0.0212$; Figure 2-21H). Conversely, we noted an increase in the proportion of activated Treg in untreated MS compared to $\mathrm{HC}$ ( $\mathrm{p}=$ 0.0177; Figure 2-21E). 

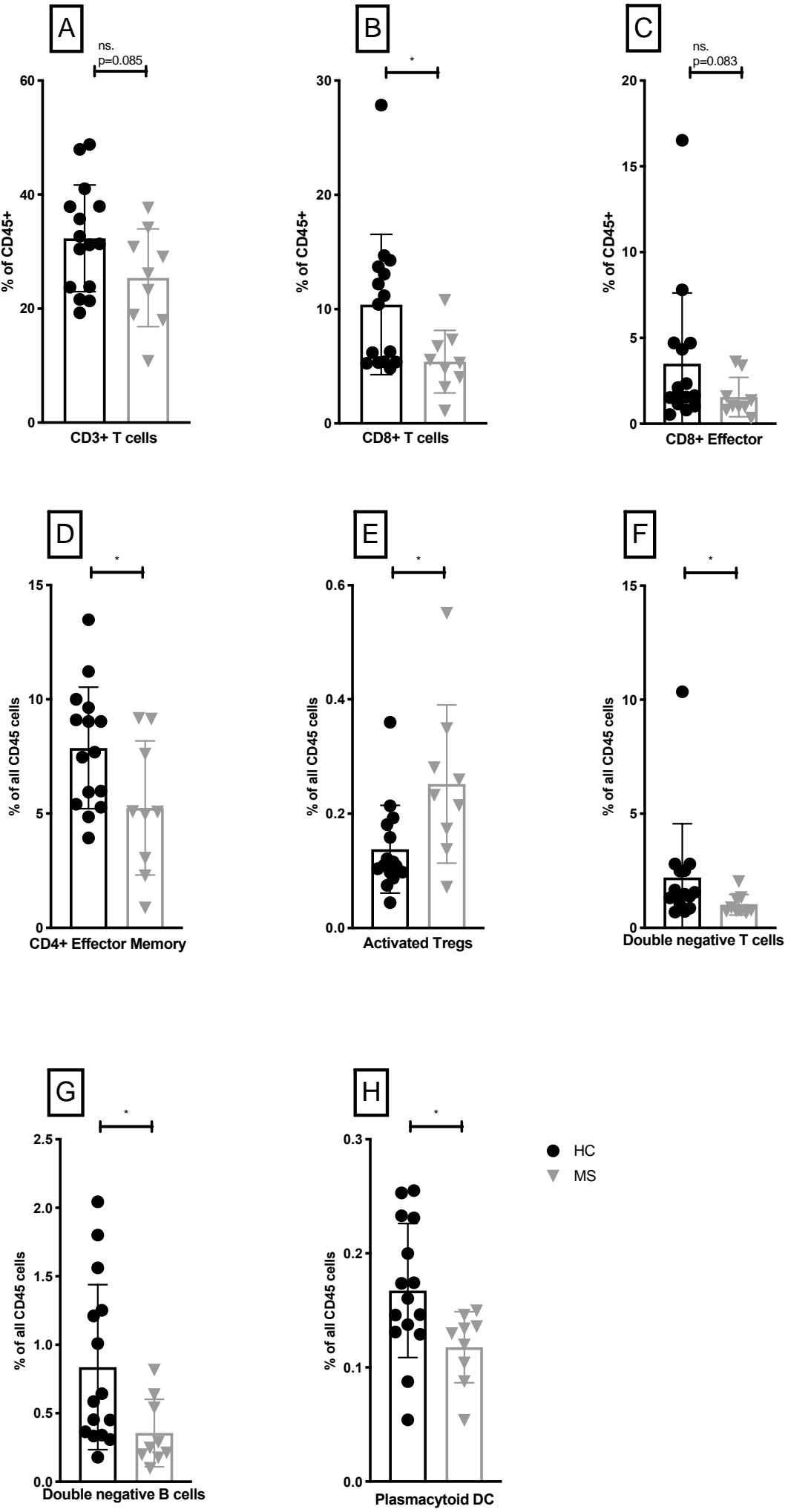

Figure 2-21 Total proportion of immune subsets relative to all CD45+ cells. Each data point represents an individual participant sample. Bars indicate mean and SD. ns. = not significant. ${ }^{*}(\mathrm{p} \leq 0.05),{ }^{* *}(\mathrm{p} \leq 0.01),{ }^{* * *}(\mathrm{p} \leq 0.001),{ }^{* * * *}(\mathrm{p} \leq 0.0001)$ 


\subsubsection{Immune system stability over time.}

To better relate our findings within the context of a dynamic system, we collected repeat samples at three-monthly intervals on 13 the 15 participants of our HC. For each immune subset, we calculated the absolute number of cells present at the three timepoints (To, T3 and T6). We collated this data into group averages to establish what variations occur naturally between the timepoints and to identify those immune subsets that may be prone to change. In total, thirty-nine terminally gated immune subsets were assessed. Given the large number of repeat tests, we corrected for multiple comparisons.

Overwhelmingly, we observed highly consistent numbers for the total number of cells within each subset (Figure 2-22A-E). We were not able to limit sample collection to a set time of day, which may have increased sampling variation. Only non-switched memory B cells numbers varied to the degree where a significant change between the time points could be described ( $\mathrm{p}=0.0078$, corrected; Figure 2-22F). We observed an increase in total numbers in this subset between To and T6, with substantially more of this cell type present in the periphery at T6. No other subset showed changes that suggested a significant change in the number of cells over the course of the study. 
A T cells (CD3+)

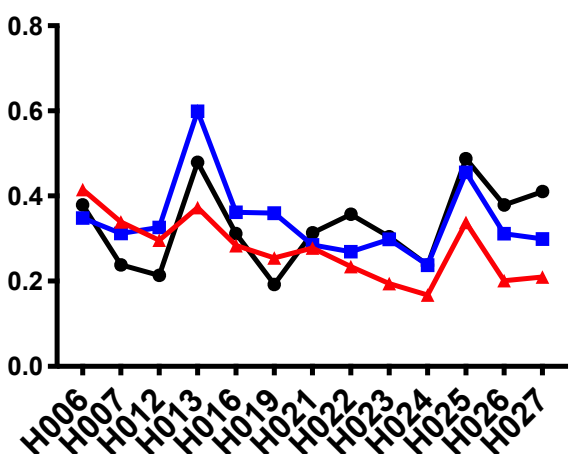

C Neutrophils

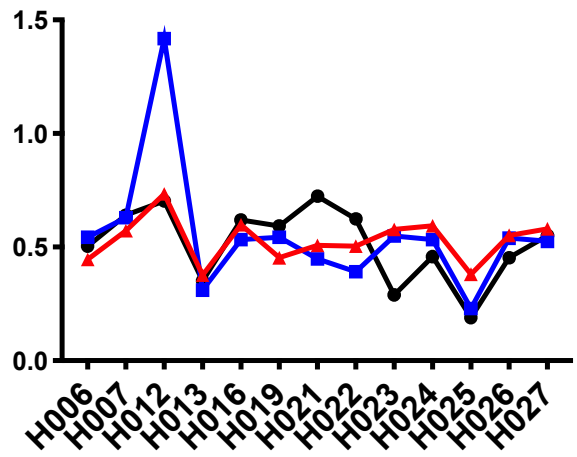

E Plasmacytoid Dendritc Cell

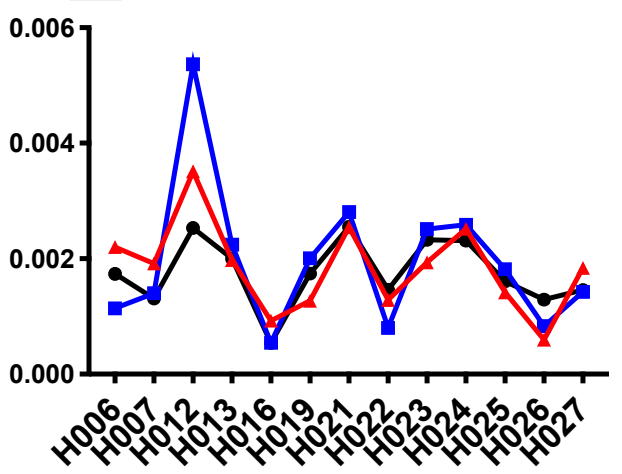

B B cells (CD19+)

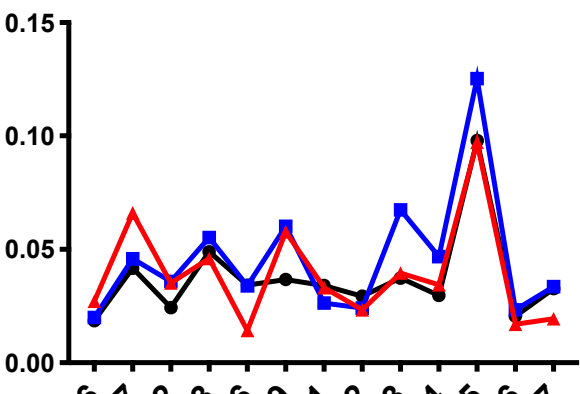

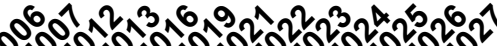
rok.
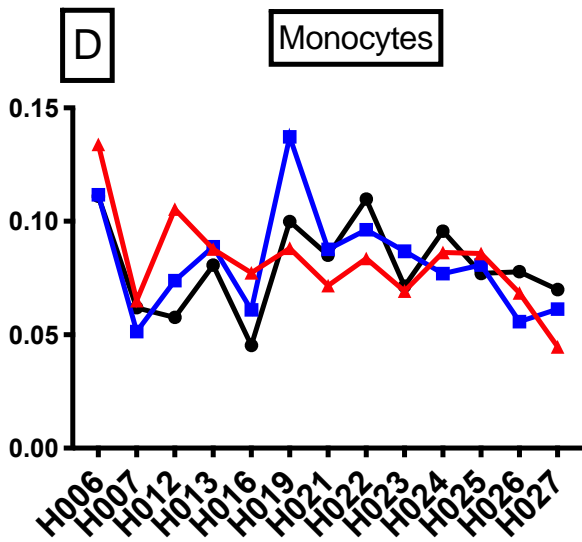

F Non-switched Memory B cells

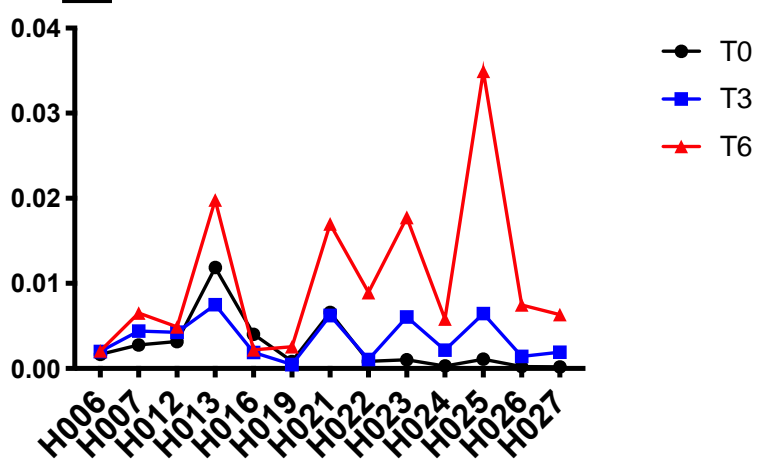

Figure 2-22 Representative plots of immune cells subset variation in the HC.

Each graph depicts the absolute number of cells from a separate immune subset for individual participants. 


\subsubsection{SPADE analysis.}

We performed an exploratory analysis on our dataset to establish the utility of high dimensional cytometry data analysis tools. While our dataset remained small, we aimed to establish what larger scale dataset handling would involve and understand the feasibility of alternative analytical methods. We further aimed to explore the combined use of classical and new techniques for data analysis to look for trends and verify the newer tools' ability to display classically-derived observations.

We sought out tools that contrasted strongly with standard cytometry gating. Classical gating and analysis can be very subjective as it allows for only two parameter visualisations at a time, whereas the entire dataset is far more complex and exists in multidimensional space. Due to its subjective focus, standard cytometry analysis can result in information being lost, which contrasts to high dimensional cytometry tools that identify trends by visualising the dataset in its entirety (Lugli et al., 2010). The use of these tools however remains exploratory, with conventional data analysis forming the basis of good reporting.

We selected SPADE as it has been reported in the literature to be effective at discriminating different cell populations from flow cytometry data (Bendall et al., 2011). SPADE is a form of cluster analysis where subpopulations are generated based on the available expression of antigens on the surface of the cell and how well each cell relates to other cells within the same analysis (Qiu et al., 2011). It helps identify known subtypes while not excluding rare or unknow ones. Cells are structured in a branch like plot detailing cluster relationships using all measured protein markers from the different cell types across the data.

We focussed on $\mathrm{T}$ cell and B cell data as these subset changes were most prominent for the MS participants. All MS and all HC participant raw data files were collated after which a separate SPADE analysis run was performed on each collated group. The SPADE trees for each group was then depicted as heatmap overlays for each antibody marker. We sequentially exported selections of nodes based on the marker expression back into FlowJo to confirm positivity using conventional gating. We then compared 
the proportion of nodes between $\mathrm{HC}$ and MS plots to illustrate the differences between the cohorts.

Together, the key subset alterations observed from our conventional analysis approach were also reflected in SPADE trees. We observed a smaller proportion of double negative T-cell nodes, a loss of CD8 cells (Figure 2-23), and an increase in CD4 memory cells (Figure 2-24) in the MS cohort. For B cells, the naïve compartment in MS was expanded and with a reduction in memory subsets observed (Figure 2-25), and transitional B cells in the MS participants were also expanded (Figure 2-26). 

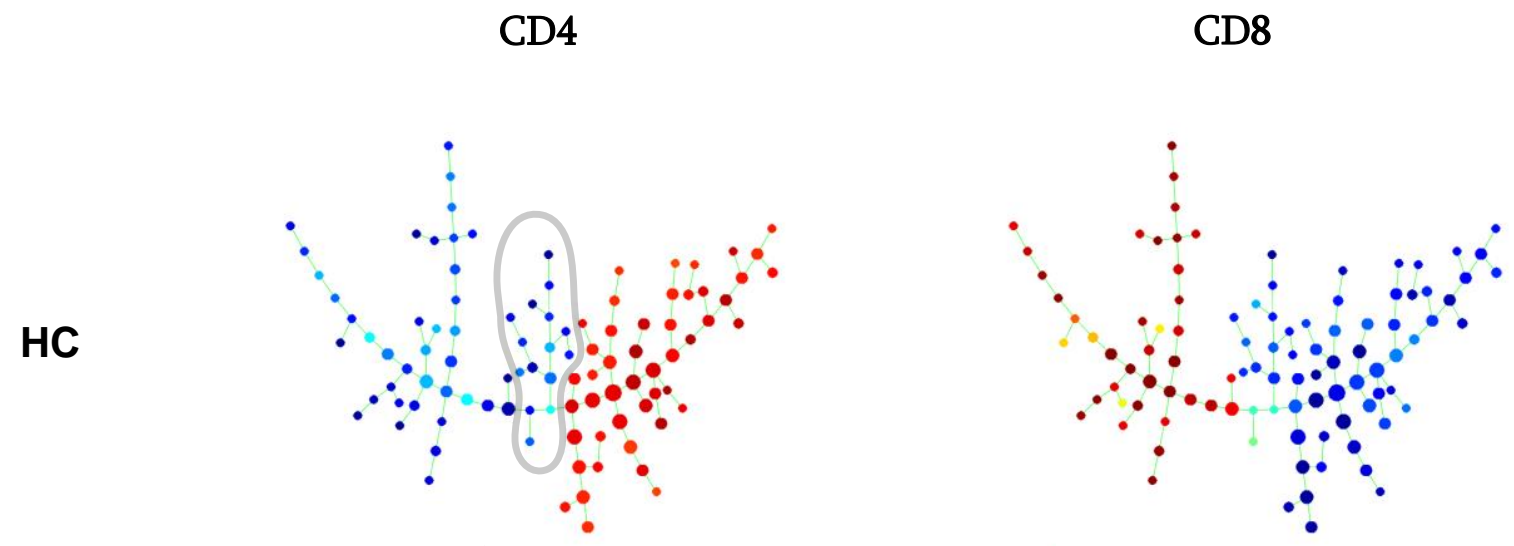

\section{MS}

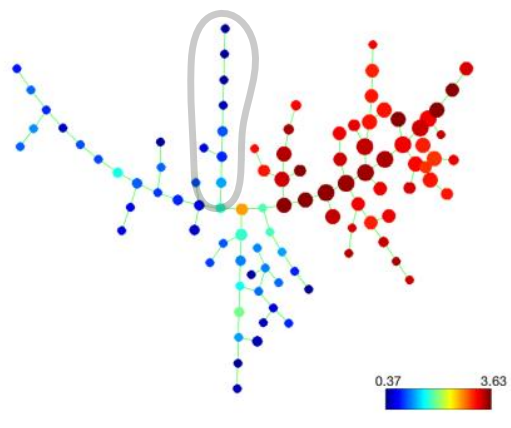

CD4-CD8- T cells

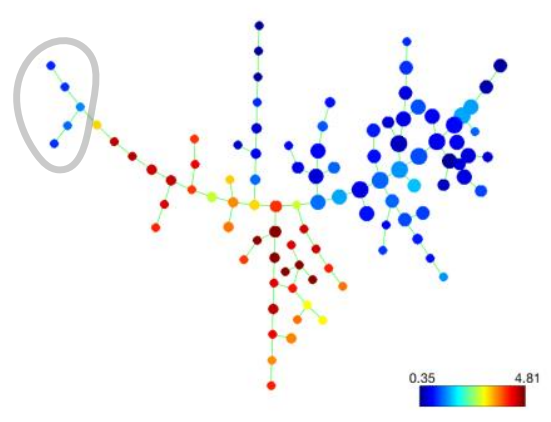

CD8+ T cells

Figure 2-23 Heatmap of marker expression of SPADE tree plots for all T cells (CD4\&CD8).

HC (top) vs. MS (bottom). Grey encircled events depict CD4-CD8- T cells (left) or the loss of CD8 events (right). 
CD45RA

HC

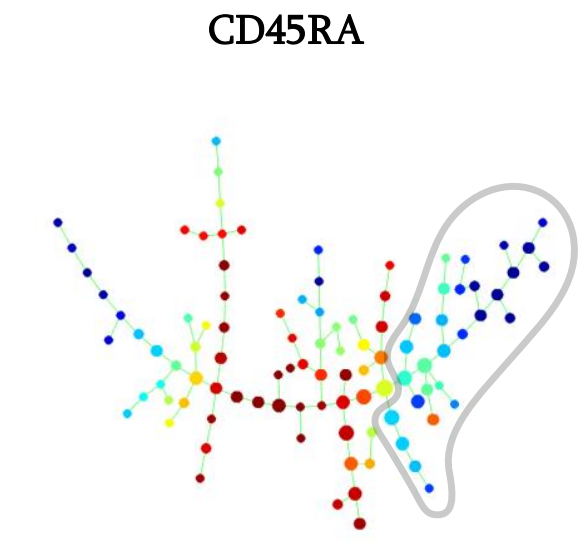

MS

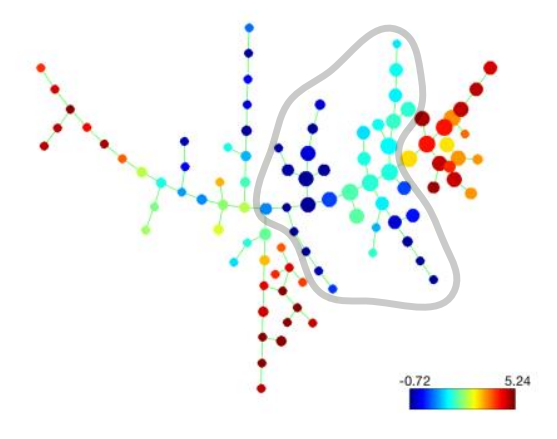

CD4+ Memory
CCR7
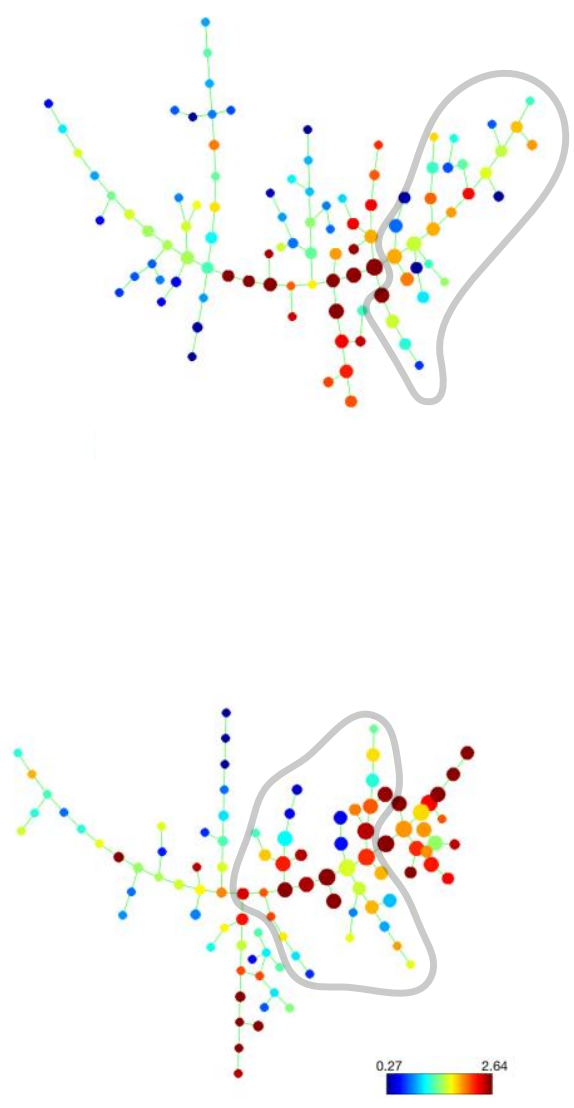

CD4+ Central Memory

Figure 2-24 SPADE tree heatmap of marker expression of for all $T$ cells (CD45RA\&CCR7).

HC (top) vs. MS (bottom). Grey encircled events depict memory (left) or central memory $\mathrm{T}$ cell events (right). 
HC
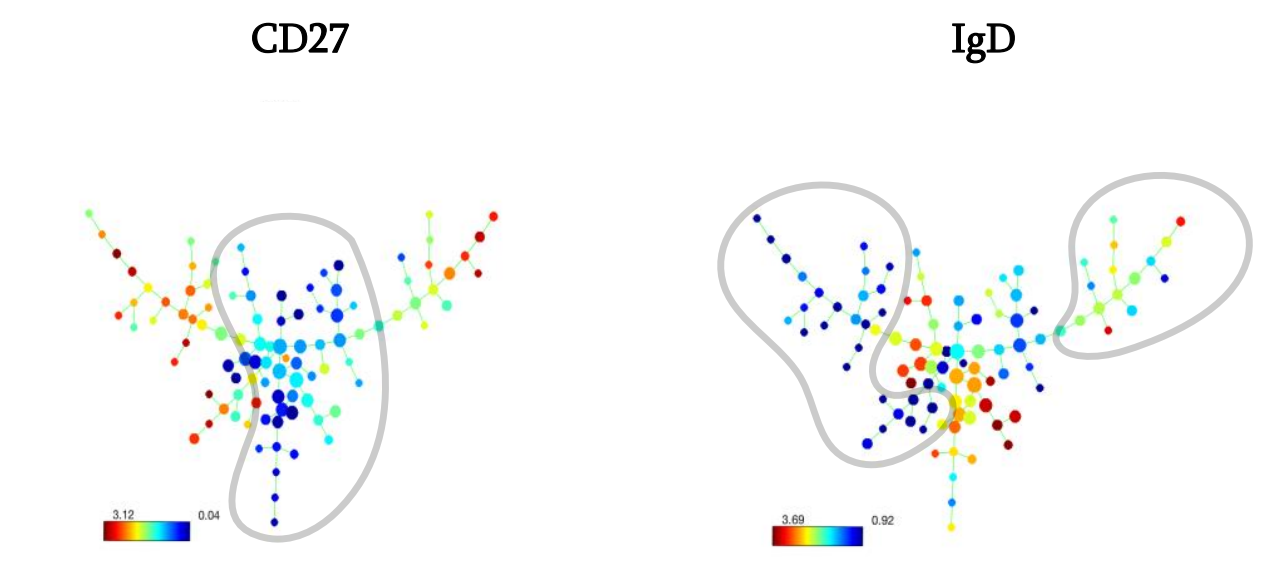

MS
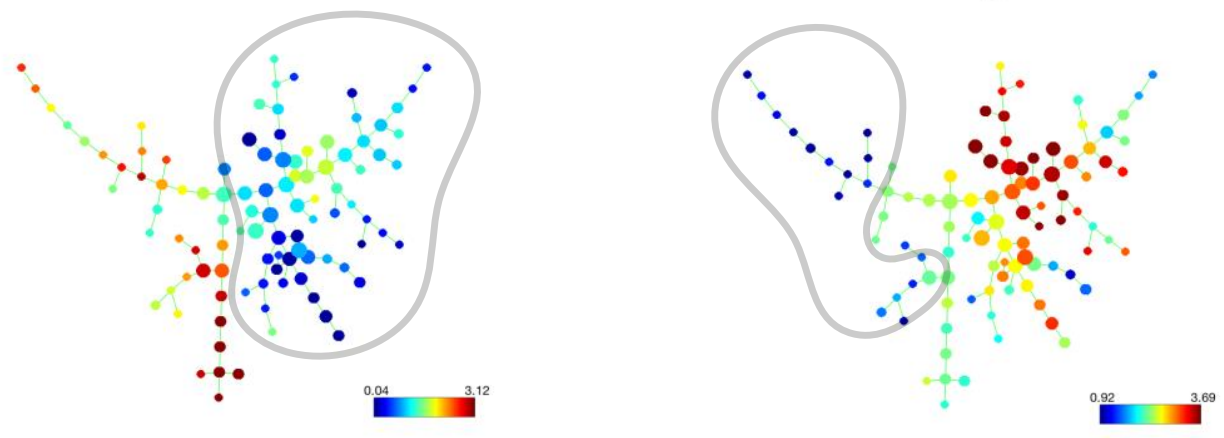

Naive B cells

Memory B cells

(CD27-)

(CD27+, IgD+/-)

Figure 2-25 SPADE tree heatmap of marker expression for all B-cells (CD27\&IgD).

HC (top) vs. MS (bottom). Grey encircled events depict naïve (left) or memory B cell events (right). 
CD38

HC

MS
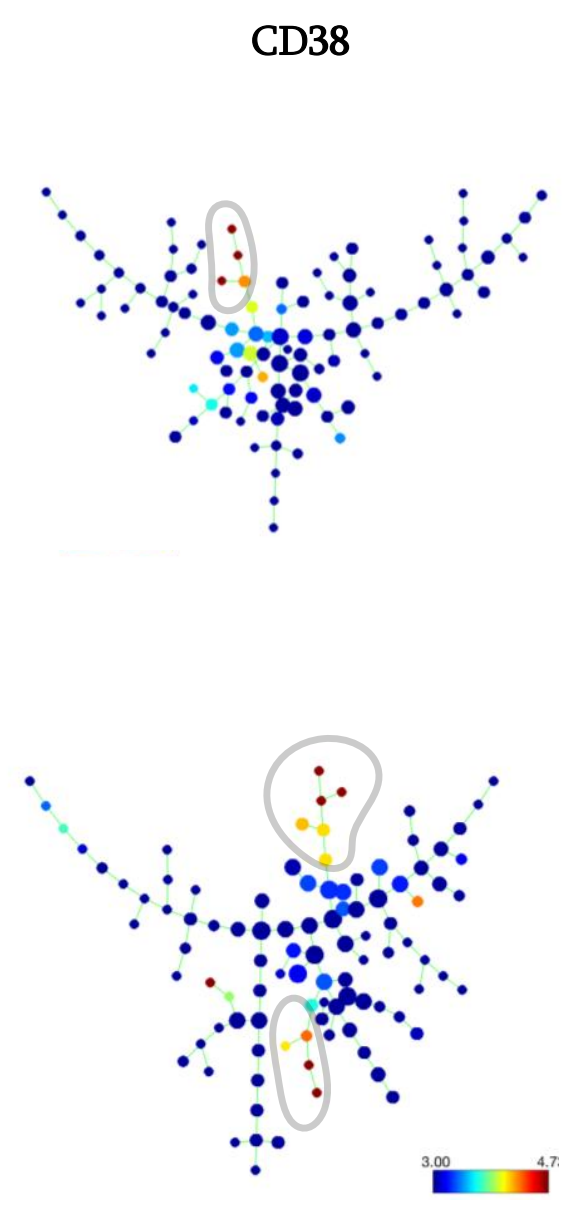

CD24
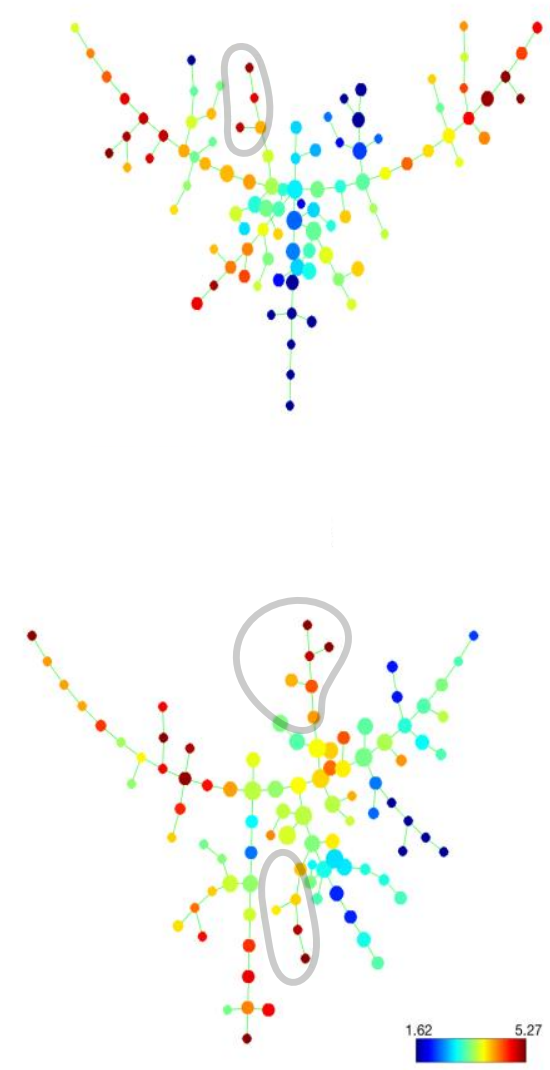

Transitional B cells

\section{(CD38++,CD24++)}

Figure 2-26 SPADE tree heatmap of marker expression for all B-cells (CD38\&CD24).

HC (top) vs. MS (bottom). Grey encircled events depict Transitional B cells by CD38 (left) or CD24 (right) expression. 


\subsection{Discussion.}

Overall, our data showed changes to the peripheral immune compartment in people with progressive MS. Our work was focussed on ex vivo peripheral immune cells from people with progressive MS, who were not on treatment, yet despite the broad scope of our phenotyping investigations, we found that the most substantive changes appeared in the T-cell compartment. T cells are a well-researched immune subset in MS, and this research has been aided by the available models for the disease in which the most commonly used, experimental autoimmune encephalomyelitis (EAE), is caused by autoreactive $\mathrm{T}$ cells. These models have been shown to be effective in drug discovery and determining treatment efficacy, although no single model can encompass all aspects of MS pathogenesis (Robinson et al., 2014). Furthermore, there is currently no model for progressive disease.

In expanding our investigation to all commonly present PB subsets, we aimed to form a complete picture of the peripheral immune compartment in MS. While we observed striking changes in the T-cell compartment, other subsets were also noteworthy. As such, these results warrant further discussion while some of our findings need to be contextualized in relation to previous literature.

\subsection{1 $\mathrm{T}$ cells in the periphery.}

Evidence suggests that the composition of T-, B- and regulatory cells are crucial in modulating the quality and magnitude of the immune response (Sakaguchi et al., 2008). Given the underlying pathophysiology of the untreated progressive MS cohort in this study, we hypothesised that we would observe significant changes to their immune subset composition. Indeed, we found substantial differences in cell numbers and percentages, particularly in the T-cell compartment.

We noted that in the CD8 compartment there were significantly fewer CD8+ cells in proportion to all CD45+ cells as well a trend toward fewer CD8+ cell numbers and a reduction in their proportion relative to all $\mathrm{T}$ cells. Within this subset, we further showed fewer CD8+ naïve cell numbers, underpinned by a reduction in their 
proportion relative to all $\mathrm{T}$ cells, although this data failed to reach significance. Overall, we can conclude that the CD8+ T-cell compartment in MS appears contracted.

Within the CD4 compartment, we noted a significant increase in CD4+ cells in proportion to all T cells for MS. Looking more closely at whether one population contributed to this elevation, we found the proportion of CD4+ effector memory cells relative to $\mathrm{CD} 45+$ cells was lower while the proportion of $\mathrm{CD} 4+$ central memory relative to $\mathrm{CD}_{3}$ was increased. Effector memory cells are those that migrate to sites of inflammation and can have immediate effector function while central memory requires stimulation input to differentiate into effector cells (Sallusto et al., 2004). The imbalance within the CD4+ compartment speaks to a loss of effector cells from the periphery, which have likely migrated to sites of active inflammation.

Our study identified a significant imbalance between the CD4 and CD8 compartments as evidenced by a significant increase in the CD4:CD8 ratio for our MS cohort. Our $\mathrm{HC}$ participants had an average ratio of 2.32, contrasting to 4.47 in the MS cohort. This ratio can be described by the general loss of cells from the CD8 compartment, particularly naïve CD8+ cells, and an increase in the CD4+ cells, particularly the CD4+ CM subset. Our findings are supported by a report, which also measured the T-cell compartment in MS (Pender et al., 2014). Similar to our data, Pender et al. show an increased CD4:CD8 ratio due to a loss of CD8 cells from a decrease in naïve CD8+ cells, specifically for their progressive cohort. They also report an imbalance in the CD8+ EM and CM compartment, which we did not observe. They do, however, note that phenotyping with CCR7 as used in our panel, over their use of CD62L, resulted in less significant differences, which may explain the differences in our results.

Treg are $\mathrm{T}$ cells that are enriched in suppressor activity and thought to play a dominant role in the regulation of autoreactive $\mathrm{T}$ cells (Rudensky, 2005). Between 20-30 \% of Treg are activated, expressing HLA-DR, and these cells are known to inhibit $\mathrm{T}$ cell proliferation and cytokine production (Baecher-Allan et al., 2006). Our data showed an increase in activated Treg in absolute count, and in the proportion relative to total CD3, and the proportion relative to CD45+ cells in the MS cohort. A report by Venken et al. found no significant difference in the proportion of Treg as they relate to total CD4 cells between HC, RRMS and SPMS patients (Venken et al., 2006). A direct 
comparison of our whole blood data to theirs is not possible owing to their use of a $\mathrm{CD} 4$ bead separation, but we suggest that our absolute enumeration data are a better representation of the Treg population in the periphery.

Another T-cell subset we observed to be altered were double negative (DN) T cells: those expressing a TCR but lacking either CD4 or CD8. We could, however, not exclude the presence of other low frequency subsets, such as NK T cells, or mucosal associated invariant $\mathrm{T}$ cells (MAIT) from this population. In autoimmune lupus, DN $\mathrm{T}$ cells are expanded, and produced significant amount of IL-17 and INF $\gamma$ and are considered to be contributing to disease pathogenesis (Crispín et al., 2008). In another report, for a cohort of individuals who had experienced a stroke, DN T cells were also found increased in the periphery, and observed to be associated with stroke lesions, enhancing neuroinflammation and contributing to brain injury (Meng et al., 2019). Our data showed a significant decrease in their numbers in the periphery and in the proportion to all cells in MS patients, which is in contrast to the changes reported during another autoimmune condition (i.e. lupus) and stroke. However, we could not find a report of their presence in MS in the literature but suggest that these cells may be involved in the disease or repair process and their contribution merits further exploration.

\subsubsection{B cells in the periphery.}

B cells (specifically plasma cells) produce oligoclonal immunoglobulin which are detected as bands in the cerebrospinal fluid (CSF) and are one of the most distinctive features of MS. B cell-targeting therapies are some of the more successful treatment options in MS, owing to their ability to drastically reduce the numbers of B cells in the periphery and by extension the CNS. However, because these therapies do not target plasma cells, oligoclonal bands persist in spite of the dramatic improvement in disease. B cells are very effective antigen presenting cells and are thought to contribute to MS by driving T-cell auto-reactive antigen responses (Jakimovski et al., 2017). B cells can also express a range of cytokines thus contributing to another key pathogenic process in MS and contributing to inflammation and innate immune activity (StaunRam and Miller, 2017). 
To better understand whether cells of the B-cell compartment might contribute to MS pathogenesis, we determined total cell numbers of the most common subsets present in the peripheral blood. We observed no significant change in the number of $B$ cells in our progressive cohort. This lack of change in enumerated B cells in MS compared to $\mathrm{HC}$ has previously been observed (Jones et al., 2017; Seidi et al., 2002), which is in line with our observations here.

B cell functions can be readily deduced by their expression of key markers of the subset's maturation phases. Naïve B cells are antigen inexperienced and differentiate to memory cells or plasma cells on stimulation with antigen. Memory cells are the reactive cells of the $\mathrm{B}$-cell lineage and can also mature to antibody secreting plasma cells on second stimulation. Class switching of memory cells is dependent on T-cell mediated activation, while unswitched memory B cells are $\mathrm{T}$ cell independent early responders (Haas et al., 2011).

The changes we observed were mostly in the proportional composition of the B-cell compartment and these changes suggest an altered functional capacity (Claes et al., 2015) in our MS cohort. Our data showed a contraction in the periphery of memory B cells, particularly the DN B cells and switched memory subsets with a corresponding expansion in transitional cells. Similarly, results by Haas et al. have shown an overall contraction in class switched and unswitched subsets, with a corresponding increase in naïve cells (Haas et al., 2011). It has been suggested that switched memory B cells, alongside plasmablasts and plasma cells have increased ability to cross the blood brain barrier (BBB) (von Büdingen et al., 2011; Niino et al., 2009). Even though the Haas et al. data was from untreated RRMS patients, the overlap in subset changes are worth noting particularly as others have also noted the increase in naïve cells, especially during active relapse (Niino et al., 2009). The increase in naïve cells for our cohort was not significant, possibly due to a lower power from a small sample number, as well as a single outlier participant with very low naïve cells numbers.

We further reported a contraction in DN B cells, and find this result also present in the literature but in an RRMS cohort (Haas et al., 2011). Lastly, we observed an increase in the absolute number and proportional compositional of transitional B cells in our 
cohort. A report in the literature showed this subset to be unaltered in a cohort of mixed relapse and progressive subjects (Michel et al., 2014), while others report that therapeutic efficacy in MS can go hand in hand with an increase in this subset (Blumenfeld et al., 2016; Schubert et al., 2015). Because our understanding of this subset is still developing, it is unclear what the collective impact of the changes in the B-cell compartment will have at this time. Overall, we note substantive change to the composition of the B-cell compartment in MS with key changes reflecting those from other authors.

\subsubsection{DC, NK cells and monocytes.}

Dendritic cells (DC) are very low abundance ( $\sim 13$ cells $/ \mu \mathrm{l})$ in the peripheral blood and are innate immune antigen presenting effector cells. Their role in MS pathogenesis is thought to be to activate and steer autoimmune-mediated inflammatory responses (Karni et al., 2006). Their total numbers have not been reported to vary significantly in MS compared to HC (Han et al., 2014; Sellebjerg et al., 2012), and we observed similar results. DC have been shown to have an increased proportion of co-stimulatory marker expression such as CD86 and CD80 in MS (Comabella et al., 2009; Thewissen et al., 2014) as well as increased cytokine production in response to LPS stimulation (Karni et al., 2006). Although these markers were not part of our assay for this study, they speak to a change in DC function and activity that may be crucial in MS. In this study, the only significant change we observed was a reduction in the plasmacytoid DC (pDC) subset in relation to all CD45 cells. Such a reduction in pDC cell proportions in the periphery, particularly in progressive patients, has previously been observed (Thewissen et al., 2014), with others also noting a significant change in expression of activation markers, cytokine responses and an ability to induce T-cell proliferation (Gandhi et al., 2010).

NK cells act as cytotoxic cytokine-producing cells targeting virally-infected or malignant cells (Cooper et al., 2001). We investigated two distinct NK cell subsets based on the level of CD56 expression. Dim cells are primarily engaged with cytotoxic activity, while the bright CD56 expressing cells are believed to effect a more regulatory function through cytokine production (Cooper et al., 2001). Data on NK cells change in terms of number and composition in the periphery in MS yield conflicting reports. 
We note a comment by Kastrukoff et al. suggesting disease heterogeneity, different disease course sampling, and technical limitations may limit accurate description of these cells in MS (Kastrukoff et al., 1998). Our data found no significant change in NK cells subset or proportions when compared to a HC. Furthermore, we note that some reports suggest substantive changes in the amount of bright NK cells subsets occurring alongside response to treatment, suggesting their enumeration during investigations for new immunomodulatory therapies as useful (Gandhi et al., 2010).

Monocytes are immune effector cells with three different subsets present in the periphery. Using a combination of overlapping markers to detect these subsets we observed no significant changes to either the absolute number or proportional composition in our MS participants when compared to HC. Our group had previously shown that the proportion of CD16+ monocytes as a proportion of PBMC increased in a cohort of untreated MS participants comprising primarily relapsing-remitting patients (Chuluundorj et al., 2014). Others have similarly shown that the composition of subsets of monocytes within the monocyte compartment may be altered in MS, noting that an increase in non-classical cells occurs with an associated reduction in classical cells (Gjelstrup et al., 2018). In contrast, some reports show an opposing reduction in CD16+ cells in the periphery of patients with MS (Waschbisch et al., 2016). As our data here focussed on a smaller cohort of only progressive phase patients, using absolute enumeration, our findings cannot be easily compared to these earlier works.

\subsubsection{Immune system stability and SPADE analysis of whole blood.}

We posited that our HC would show a high degree of stability in terms of the numbers and proportional composition of their peripheral immune cells over time. This was found to be the case, in that overwhelmingly, of the 38 terminal subsets analysed at three time points, only a single subset varied to the degree where the values differed enough to reach the definition of statistically significant. We would comment that for this analysis we corrected the data for multiple comparisons, ensuring a very high threshold for detectable differences. A well powered study $(n=638)$ has suggested that the overwhelming majority of variation that contributes to immune sampling readouts comes from interparticipant samples, whereas intraindividual (repeat) 
sampling has a very low level of variation, indicating that the longitudinal composition of the healthy immune system is highly stable (Carr et al., 2016). Collectively, this data supports high degree of stability in immune subset number and composition in the $\mathrm{HC}$ over time.

From our SPADE analysis we observed a high degree of overlap in the proportional makeup of the SPADE plots with those seen from conventional cytometry data. As our analysis was limited to only eight markers in each panel, we could not use this tool to identify new or unknown subsets. We conclude that the use here aids in whole subset visualisation, but that to get full use out of these tools, many more lineage markers, cytokines, and phosphorylation targets should be included in future work to aid in subset identification. 


\subsection{Conclusion.}

Our detailed subset phenotyping highlighted phenotypic alterations in progressive MS. These observations raise the question of whether phenotypic changes are primarily due to MS or secondary to progressive disease processes. Certainly, our lymphocyte data, which was in agreement with previous reports by others, suggests these changes are stable and characteristic of progressive MS. In contrast, we found only limited changes in innate immune cell numbers and composition, observing only that the proportion of plasmacytoid DC was significantly reduced. One possibility for this difference is that lymphocytes have been more thoroughly classified phenotypically, allowing us to infer their function from surface marker expression while for the less well characterised innate cells, we are more limited.

The observations from our progressive cohort are also often noted in patients with a relapsing-remitting disease course. These immune subset changes therefore appear to have persisted from early relapsing disease, as most relapsing patients transition into a progressive disease course. Progressive patients are also refractory to therapy, suggesting that in progressive pathology, phenotypic alterations become secondary to progressive disease processes. Lastly, we observed that SPADE plots are an informative tool to represent the phenotypic hierarchy and marker expression in immune subsets and we advocate for the use of such tools to complement cytometry data analysis. Together, this work aligns with the many reports of an activated phenotypic composition in MS, and forms the basis from which to focus our work into the functional characteristics of innate cells in progressive MS. 


\subsection{Limitations.}

Our control cohort was prospectively recruited with the aim of acting as a matched cohort to 36 MS CRISP participants. When the CRISP trial finished early, the age range and sex demographics of that cohort were not aligned well to the recruited HC. As such, these two factors may limit and add confounding variables to our dataset given that the heterogeneity of participants in terms of age and gender distribution, irrespective of disease phenotype, has been shown to contribute significantly to the compositional makeup of immune cells in a population (Aguirre-Gamboa et al., 2016; Carr et al., 2016).

Experimental based limitations that are worth noting are that the CD85k isotype control in our whole blood monocyte panel showed no difference in staining compared to its matched antibody. Therefore, non-specific binding of CD85k to its target epitope could not be excluded, although this was not anticipated. Also, our phenotyping of DN T-cells was limited by the lack of markers to correctly identify MAIT and NK T-cells (Juvet and Zhang, 2012) and speaks to a broader issue where very minor subsets of cells may be present in what are terminally gated populations. This issue is primarily due to a limitation inherent in phenotyping where restrictive numbers of lineage markers are used. Finally, SPADE trees were generated using separate data files for HC and MS participants. This limits the ability to interpret differences in nodes between the trees.

Lastly, instrument longitudinal controls used to maintain a cytometer over time are not always fully effective. While we tried to limit changes to our instrument over the duration of our study, we cannot determine what contribution variation in the instrument's settings may have had on our data. Including a biological control with repeat analysis over the duration of future longitudinal acquisitions would be the way to control for variation and allow for manual adjustments to be made. At worse, a variation of up to the limit of acceptable detector fluctuation could be introduced into our data. We noted no significant change in instrument variation that could not be corrected with an engineering calibration. This puts the quality of our data on par with those from other publications. 


\section{Chapter 3. Materials and Methods for in vitro culture.}

This chapter lists the methods we used to obtain a dataset that is analysed and discussed in Chapters 4 and 5. The methods are presented together as they formed a reproducible and consistent approach to sample processing for all our in vitro cytokine, transcription factor, and dopamine receptor expression data. The use of these methods resulted in a dataset that comprised 36 samples with near identical processing, treatment, and staining conditions. To produce this final dataset, each revived participant sample was split into separate culture tubes so that each sample could be processed for all in vitro conditions and be stained for all output measures. The analysis of each sample spanned a three-day process, starting with PBMC revival on day 1, stimulation and staining over days 1 and 2, and cytometric data acquisition on day 3. Furthermore, we processed two samples per week and interspersed healthy and MS participant samples over the course of the experimental period. We acquired all 36 samples consecutively over a period of eight months. The collated data generated from the 36 participant samples was then divided into treatment response groups: clozapine treatment responses are reported in Chapter 4 and MIS416 treatment responses in Chapter 5. In presenting clozapine and MIS416 data separately, we aim to show the respective drugs' immunomodulatory effects in progressive MS.

We further include in this chapter a section of results that validates the combination of participants from two separately recruited clinical trials into a single cohort. This validation includes cytokine and surface marker expression data. The 36 PBMC samples used in the in vitro analysis in this thesis were from the CRISP and MIS416 trials. The validation results are presented here to highlight any limitations that may arise from using participants from separately recruited cohorts, together.

Lastly, we introduce a new spectral cytometry platform used during the acquisition of our data, and detail its implementation, optimisation, and quality control. Autofluorescent noise has been a consistent issue in cytometry. With this new platform we demonstrate how it was effectively removed to improve the resolution of our cytometry data. 


\subsection{Sample collection and storage.}

All participant samples were collected and extracted using two sets of standardised methods. All CRISP and healthy control participant samples were collected, stored and processed by the author in Wellington. The MIS416 participant samples were collected from Auckland and Wellington, processed to frozen PBMC centrally by the team at Innate Immunotheraputics in Auckland, and then shipped to Wellington for in vitro culture and analysis by the author.

\subsubsection{CRISP trial sample processing.}

All CRISP samples were processed by the author. Blood was collected by venepuncture directly into a CPT tube with heparin (BD, US). This tube allowed for the closed separation of mononuclear cells using density gradient centrifugation (BD, 2019). These samples were collected and processed within two hours and PBMC isolation was performed per manufacturers' instruction.

Upon processing the tube was centrifuged at $1800 \mathrm{~g}$ at room temperature in a benchtop laboratory centrifuge. Following centrifugation, the plasma component was pipetted off and stored at $-80^{\circ} \mathrm{C}$. The PBMC fraction was pipetted off into $40 \mathrm{ml}$ cold Dulbecco's Modified Eagle medium (DMEM) (ThermoFisher, US) and a $50 \mu$ aliquot was taken for microscopic cell count. The remainder was inverted to mix and centrifuged at $350 \mathrm{~g}$ for 7 minutes. The supernatant was then discarded by decanting and cells gently

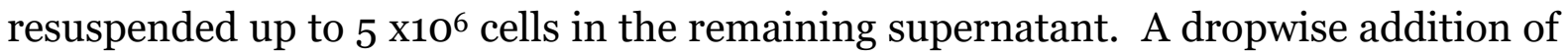
freezing media ( $80 \%$ FCS \& $20 \%$ DMSO) at 1:1 ratio was added, and the total suspension then transferred into a labelled cryovial. The cryovial vial was placed into a foam slow freezing container and frozen at $-80^{\circ} \mathrm{C}$ overnight. The following day, the vials of PBMC were transferred into liquid nitrogen for storage until use.

\subsubsection{MIS416 trial sample processing.}

All MIS416 trial participant samples were processed by technical staff from Innate Immunotheraputics (Auckalnd, NZ) with blood samples collected at trial sites. Briefly, blood was collected by venepuncture into standard BD heparinised sample tubes. In 
further contrast to CRISP samples, PBMC were separated by Ficoll (Sigma, US) density gradient separation. For this, 1:1 blood and PBS were combined, mixed by inverting and layered onto $3 \mathrm{ml}$ Ficoll media in a conical tube. The sample was then centrifuged at $400 \mathrm{~g}$ for 30 minutes at room temperature with no break. Next, the buffy coat was washed with cold PBS and pelleted as above. Pelleted cells were resuspended in 1:1 Freezing medium and frozen overnight using a slow freezing container. The next day, cells were transferred into liquid nitrogen for long term storage.

\subsection{PBMC processing, staining and data acquisition.}

All PBMC were processed consecutively over an eight-month period using the standardised methods listed below. All in vitro culture, data acquisition and analysis were performed by the author.

\subsubsection{PBMC culture and staining.}

\subsubsection{Culture Media.}

All PBMC were cultured in complete media. Media was prepared using the volumes described below from stock reagents. Stock concentration indicated in brackets unless stated as final.

$50 \mathrm{~mL}$ complete media - Stored at $4^{\circ} \mathrm{C}$ - contains:

43 mL DMEM (Gibco, US)

$5 \mathrm{ml}$ intact foetal calf serum (FCS) (heat inactivated and sterile filtered)

$1 \mathrm{ml}$ HEPES (1M) (Gibco, US)

$0.5 \mathrm{ml}$ Pen/Strep (5000 $\mu \mathrm{g} / \mathrm{ml})($ Gibco, US)

$0.5 \mathrm{ml}$ non-essential amino acids (NEAA) (100x) (Gibco, US)

$0.5 \mathrm{ml} \mathrm{L}$-glutamine (200 mM) (Gibco, US)

$18 \mu \mathrm{l}$ B-mercaptoethanol (50 $\mu \mathrm{M})$ (Gibco, US)

\subsubsection{PBMC Thawing.}

All participant PBMC were revived for in vitro culture by thawing cells rapidly in a water bath at $37^{\circ} \mathrm{C}$. Thawed cells were transferred into $50 \mathrm{ml}$ warmed complete media 
containing DNAse 1 (Invitrogen, US) $(27.5 \mathrm{u} / \mu \mathrm{l}$ final). An aliquot was taken for microscopic cell count and viability check by trypan blue exclusion. All samples were recovered with greater than $85 \%$ of cells viable. Cells were then immediately centrifuged at $350 \mathrm{~g}$ for 7 minutes, the supernatant decanted, and $50 \mathrm{ml}$ fresh warmed complete media added. Cells were again pelleted as above and resuspended in $50 \mathrm{ml}$ warm complete media and rested for 90 minutes at standard culture conditions $\left(37^{\circ} \mathrm{C}\right.$; $5 \% \mathrm{CO}^{2}$ ). Finally, cells were pelleted and resuspended at $10^{7}$ cells $/ \mathrm{ml}$.

\subsubsection{PBMC culture conditions.}

$450 \mu \mathrm{l}$ warmed $\left(37^{\circ} \mathrm{C}\right)$ complete media was aliquoted into polypropylene FACS tubes and stimulating and treatment agents added for final concentrations as follows: lipopolysaccharide (LPS; $0.1 \mathrm{ng} / \mathrm{ml}$ )(see 5.4.3.2), MIS416 (5 $\mu \mathrm{g} / \mathrm{ml})$ (see 5.4.3), and clozapine (40 $\mathrm{nM})($ see $4 \cdot 4 \cdot 2)$.

Into each stimulation tube we then added $0.5 \times 10^{6}$ cells. Baseline (To) cells were immediately viability stained and fixed (see 3.2.1.4), and then held at room temperature. All other tubes were incubated at standard in vitro conditions $\left(37^{\circ} \mathrm{C}, 5 \%\right.$ $\mathrm{CO}_{2}$ ). After three hours ( $\mathrm{T} 3$ ), tubes for phosphoprotein analysis were pelleted (450 $\mathrm{g}$ for 4 minutes), viability stained and fixed (see 3.2.1.4), and held at room temperature. We simultaneously added o.25 $\mu$ l GolgiPlug (BD, US) and o.13 $\mu$ l GolgiStop (BD, US) into tubes for cytokine analysis, followed by continuing overnight culture. To terminate the overnight cultures (T24) we added $50 \mu \mathrm{l}$ of a $20 \mathrm{mM}$ EDTA solution and pipetted the cultures to detach adherent cells. The tubes were then pelleted, viability stained, fixed and held at room temperature.

\subsubsection{PBMC viability stain and fixation.}

All PBMC samples for in vitro analysis were stained with a viability dye and fixed prior to any permeabilisation and antibody staining. With thawed PBMC as sample source, we though it pertinent to assess cellular viability on all our assays to limit the inclusion of dead or dying cells in the analysis. To this end, we included a viability dye in our cytometry panel. Live/Dead Fixable Yellow stain (Thermofisher, US) was user in all PBMC cytometry acquisitions follows. 
Immediately following the separate incubations from our in vitro culture (see culture conditions 3.2.1.3), cells were washed with $500 \mu$ l cold phosphate buffered saline (PBS) (Gibco, US), pelleted and stained with Live/Dead Fixable Yellow (Thermofisher, US) using $0.4 \mu \mathrm{l}$ of the reconstituted dye per $0.5 \times 10^{6}$ cells in cold PBS for 15 minutes protected from light. The stained cells were then pelleted, washed with $500 \mathrm{ul}$ cold PBS, pelleted again, and fixed with $500 \mu \mathrm{l}$ pre warmed $\left(37^{\circ} \mathrm{C}\right) 1 \mathrm{x}$ Lyse/Fix Buffer (BD, US) for 15 minutes at room temperature. All dead/dying cells were excluded from cytometry data using Live/Dead Fixable Yellow exclusion gating (Figure 3-2).

\subsubsection{PBMC permeabilisation, blocking and antibody staining.}

All cells for intracellular cytokine and transcription factor staining were permeabilised prior to staining. PBMC for dopamine receptor staining were not permeabilised prior to staining. Cells for permeabilisation were pelleted and washed cells with $1 \mathrm{ml} \mathrm{FACS}$ buffer (PBS with 2\% FCS and 0.1\% Sodium Azide (1M)) and then added $1 \mathrm{ml}$ of cold Perm/Wash buffer (BD, US) for 15 minutes on ice. Following incubation, the permeabilised cells were pelleted, the supernatant decanted, and then held on ice protected from light.

As monocytes/macrophages contain high numbers of Fc receptors that my interact and bind non-specifically to monoclonal antibodies, all cells for all conditions/stains were subject to a blocking step to negate this non-specific interaction. Blocking also eliminated the need for isotype controls during our acquisition (Andersen et al., 2016). All cells were blocked with $2.5 \mu \mathrm{l} \mathrm{Fc} \mathrm{Block} \mathrm{(BD,} \mathrm{US)} \mathrm{in} 100 \mu \mathrm{l}$ FACS buffer for 15 minutes on ice protected from light prior to any lineage, dopamine, transcription factor or cytokine staining.

All blocked cells were then stained with a cocktail of linage markers and interest markers (Table 3-1) by adding the relevant test volume (for $0.5 \times 10^{6}$ cells) of freshly made antibody cocktail, and then mix and hold the cells on ice protected from light for 15 minutes. Brilliant Stain buffer (BD, US) was added to all antibody cocktail tubes to prevent staining artefacts that can arise from the use of multiple BD Horizon dyes. All PBMC were stained with the PBMC lineage stain. In addition to the PBMC lineage stain, separate aliquots from the respective treatment conditions were stained with either tube 1, 2, 3 or 4 respectively. This resulted in a consistent lineage marker stain 
with only the reported dyes varying between the treatment conditions. All stained tubes were washed with $500 \mu \mathrm{l}$ FACS buffer, the supernatant decanted, and cells stored overnight at $4^{\circ} \mathrm{C}$ protected from light. Cytometry was then performed the next day.

Table 3-1 Antibody cocktail mixture for in vitro PBMC staining.

\begin{tabular}{|c|c|c|c|c|c|}
\hline \multicolumn{2}{|l|}{ Tube 1} & \multicolumn{2}{|c|}{ Tube 3} & \multicolumn{2}{|c|}{ PBMC Lineage Stain } \\
\hline Antibody Conjugate & Test $(\mu \mathrm{l})$ & Antibody Conjugate & Test $(\mu \mathrm{l})$ & Antibody Conjugate & Test $(\mu \mathrm{l})$ \\
\hline DR1 PE & 1,5 & TNF BV421 & 2 & CD45 APC-H7 & 1 \\
\hline DR2 AF488 & 1,5 & IL-6 PE-CF594 & 2,5 & CD3 AF700 & 1,5 \\
\hline KO AF647 & 1,5 & IL-10 PE & 2,5 & CD4 BV510 & 1,5 \\
\hline \multirow[t]{2}{*}{ DR5 AF405 } & 2,5 & IL-1b AF647 & 1,5 & CD8 PE-Cy5 & 4 \\
\hline & & INFg AF488 & 2,5 & CD14 BV480 & 2 \\
\hline Test Volume & 7 & Test Volume & 11 & CD16 BV786 & 2,5 \\
\hline \multicolumn{2}{|l|}{ Tube 2} & \multicolumn{2}{|c|}{ Tube 4} & CD19 BV605 & 1 \\
\hline Antibody Conjugate & Test $(\mu \mathrm{l})$ & Antibody Conjugate & Test $(\mu \mathrm{l})$ & CD56 BV711 & 1,5 \\
\hline DR3 AF488 & 1,5 & CREB AF488 & 10 & HLA-DR BV650 & 1 \\
\hline \multirow[t]{3}{*}{ DR4 AF647 } & 1,5 & STAT1 AF647 & 10 & CD123 PE-Cy7 & 1,5 \\
\hline & & STAT3 PE & 10 & Brilliant stain buffer & 25 \\
\hline & & P65 PE-CF594 & 2,5 & & \\
\hline Test Volume & 3 & Test Volume & 23,5 & Test Volume & 42,5 \\
\hline
\end{tabular}

For antibody titrations optimisation see 3.3.3. All PBMC were staining with the PBMC lineage stain. Separate aliquots from the respective treatment conditions 


\subsubsection{Spectral cytometry.}

Spectral cytometry platforms are based on the same principles as those used in conventional instruments, whereby fluorescence emission at single cell level is detected. Standard instruments utilise a single photon multiplier tube (PMT) channel to detect each fluorophore, limiting the total amount of conjugates in a standard cytometry panel to the number of PMT in the platform. In spectral cytometers, emission from each fluorophore is detected across all PMT (equivalent) channels and there are commonly many more channels in a spectral cytometer. The advances in the newer instruments allow for many more combinations of fluorophores in a single panel. For our PBMC assays we used the Aurora (Cytek, US) platform that could detect a combination of 28 fluorophores.

\subsubsection{Panel design.}

Our panel design strategy was based primarily on the sample source (e.g. PBMC) (Figure 3-1), and experimental conditions. Our first priority was to accurately detect and classify as many different immune subsets as reasonably practical. We used the same reference sources to select optimal lineage marker combinations as was used in our whole blood assays, settling on ten lineage markers to reliably discriminate all the major PBMC subsets (Table 3-2). As discussed, the use of revived PBMC as sample source required the inclusion of a viability marker to discriminate live from dead cells.

We further needed to detect phosphoproteins, cytokines, and dopamine receptors in all subsets in combination with the range of lineage markers. We determined that a consistent lineage marker approach would ensure inter-panel comparability, so settled on five channels for reporter marker expression, a single channel for viability staining, and ten lineage markers. This yielded four panel combinations to detect all output measures (Table 3-2). DR receptors were only available in a very limited set of conjugates, while phosphoprotein epitopes were selected based on their suitability for detection using Cytoperm buffer and the available conjugates (Cytobank, 2019). For cytokines a wider range was available, so we selected those based on the remaining panel slots. 
Spectral cytometers are not immune to fluorescence noise (i.e. spread), wherein emission from highly similar fluorophores is detected non-specifically within another channel, reducing the ability to discriminate noise from signal. As with conventional cytometers, this was the major consideration when fluorophore conjugates were selected. With a spectral platform this issue is however less problematic. We utilised the manufacturer's spill-over reference guide (Cytek, 2019) to select a range of conjugates to minimise spread issues.

\subsubsection{Gating.}

PBMC analysis was performed in immune cells subsets identified by lineage phenotyping. All phenotyping was performed in Flowjo (BD, US). We based our gating strategy on the phenotypic gating hierarchy of Han and colleagues (Han et al., 2014). The standardised hierarchical lineage phenotyping used is depicted in Figure 3-2. Given the bi-modal nature of the majority of our lineage markers, we were able to accurately phenotype the PBMC subset with the use of internal negative controls and Boolean gating (Hulspas et al., 2009). For monocytes, we were not able to resolve the classical, intermediary or non-classical subsets commonly found in freshly isolated blood samples. The data here was on stored and thawed PBMC after substantial in vitro manipulation. We therefore gated monocytes as a single subset. 


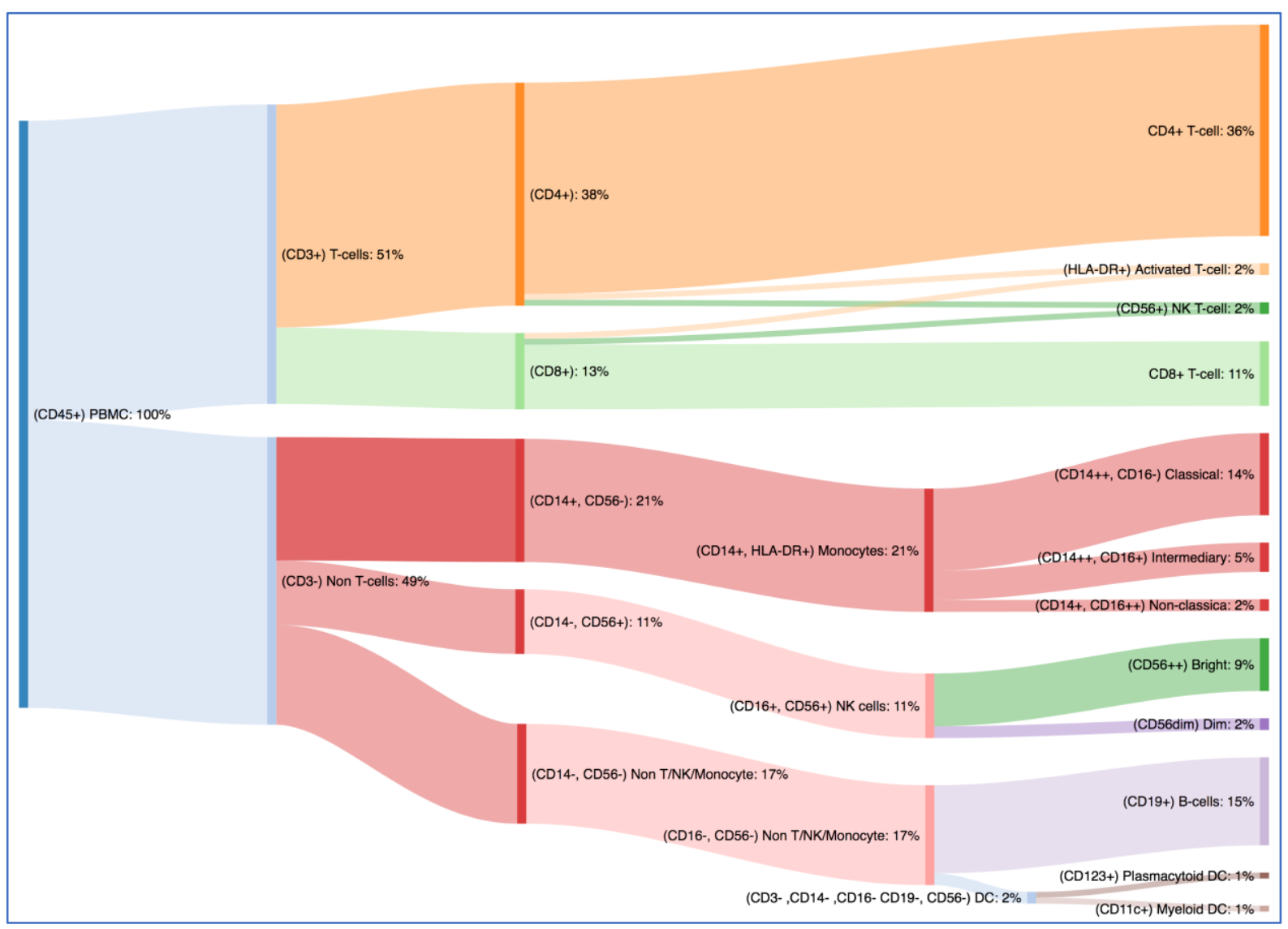

Figure 3-1 Sankey diagram of PBMC immune subsets by lineage marker phenotyping and the expected average proportional composition in a sample.

Terminal immune subsets are proportional to the total sample and are depicted as branched bars on the far right with parent subsets preceding. The top $50 \%$ comprise $\mathrm{T}$ cells, with myeloid and B cells the remainder. CD11c was omitted from the final staining panel due to manufacturer supply restrictions. 
Table 3-2 PBMC panel for all in vitro assays.

\begin{tabular}{|c|c|c|c|c|c|c|c|c|}
\hline \multirow{2}{*}{$\begin{array}{l}\text { Excitation Source } \\
\text { Virtual channel }\end{array}$} & \multicolumn{8}{|c|}{$405 \mathrm{~nm}$ Violet Laser } \\
\hline & V1 & V4 & V5 & V8 & V10 & V11 & V13 & V16 \\
\hline $\begin{array}{l}\text { Suggested } \\
\text { Fluorochromes }\end{array}$ & $\begin{array}{l}\text { BV421/ } \\
\text { AF405 }\end{array}$ & BV480 & BV500 & $\begin{array}{l}\text { Pacific } \\
\text { Orange }\end{array}$ & BV605 & BV650 & BV711 & BV786 \\
\hline Tube 1: DR & D5 & \multirow{5}{*}{ CD14 } & \multirow{5}{*}{ CD4 } & \multirow{5}{*}{ Viability } & \multirow{5}{*}{ CD19 } & \multirow{5}{*}{ HLA-DR } & \multirow{5}{*}{ CD56 } & \multirow{5}{*}{ CD16 } \\
\hline Tube 2: DR & $x x x x$ & & & & & & & \\
\hline Tube 3: Cytokines & TNF-a & & & & & & & \\
\hline $\begin{array}{l}\text { Tube 4: } \\
\text { Phosphoflow }\end{array}$ & $x x x x$ & & & & & & & \\
\hline FMO & $x x x x$ & & & & & & & \\
\hline Excitation Source & \multicolumn{5}{|c|}{$488 \mathrm{~nm}$ Blue Laser } & \multicolumn{3}{|c|}{$640 \mathrm{~nm}$ Red Laser } \\
\hline Virtual channel & B2 & B5 & B6 & B8 & B14 & R2 & R5 & R8 \\
\hline $\begin{array}{l}\text { Suggested } \\
\text { Fluorochromes }\end{array}$ & AF488 & PE & $\begin{array}{l}\text { PE- } \\
\text { CF594 }\end{array}$ & PE-Cy5 & $\begin{array}{l}\text { PE- } \\
\text { Cy7 }\end{array}$ & AF647 & AF700 & APC-H7 \\
\hline Tube 1: DR & DR2 & DR1 & $x x x x$ & \multirow{5}{*}{ CD8 } & \multirow{5}{*}{ CD123 } & ко & \multirow{5}{*}{ CD3 } & \multirow{5}{*}{ CD45 } \\
\hline Tube 2: DR & D4 & $x x x x$ & $x X X X$ & & & DR3 & & \\
\hline Tube 3: Cytokines & INF-g & IL-10 & IL-6 & & & IL-1b & & \\
\hline $\begin{array}{l}\text { Tube 4: } \\
\text { Phosphoflow }\end{array}$ & CREB & STAT1 & P65 & & & STAT3 & & \\
\hline FMO & $x x x x$ & $x x x x$ & $x x x x$ & & & $x x x x$ & & \\
\hline
\end{tabular}




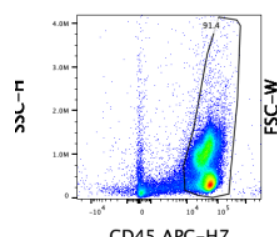

CD45 APC-H7

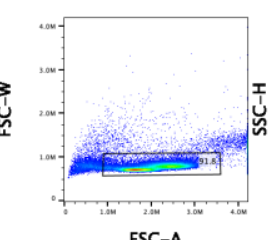

FSC-A
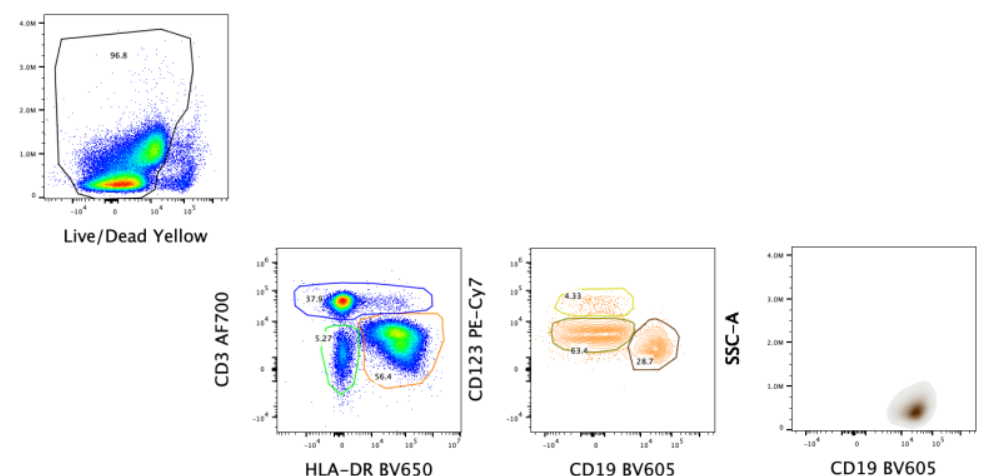

CD45+

Singles

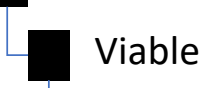

Live mononuclear cells

HLA-DR +

B-cells

Plasmacytoid DC

Myeloid cells

Monocytes

Myeloid DC

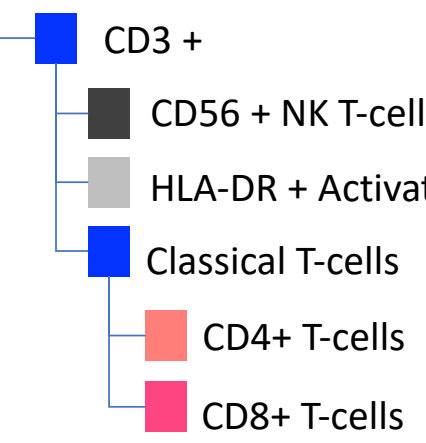

NK-cells

Bright NK cells
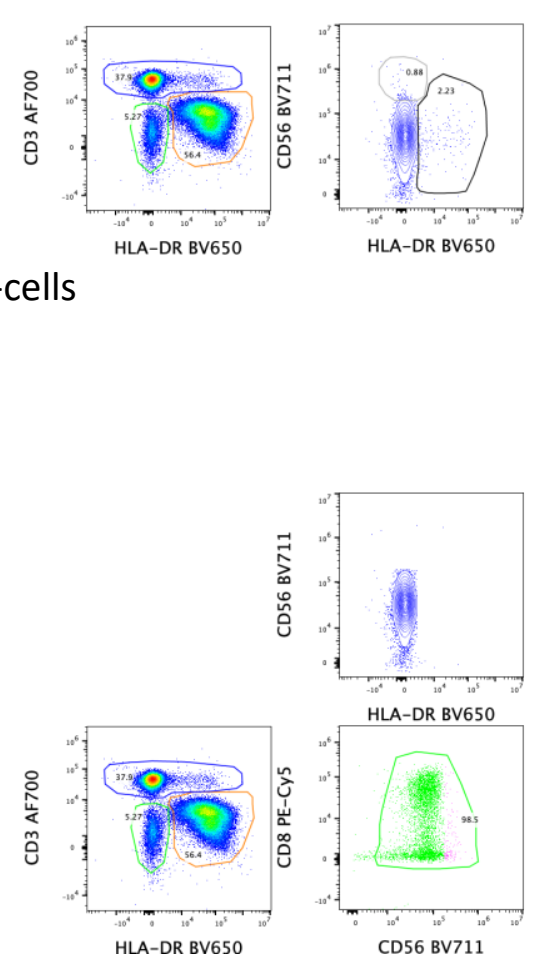
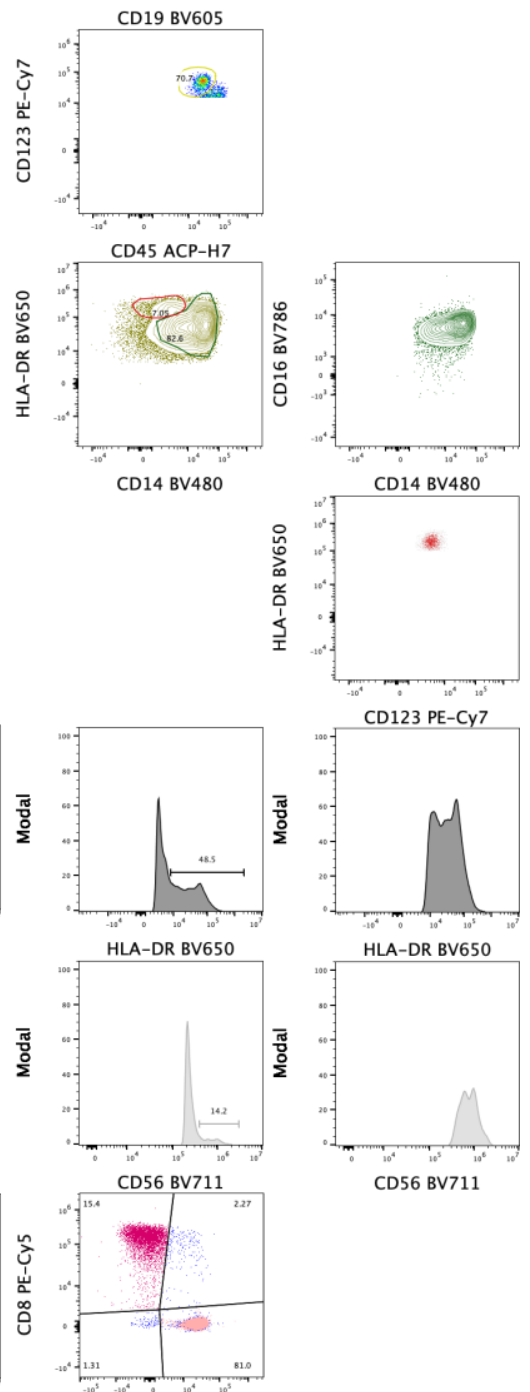

CD123 PE-Cy7
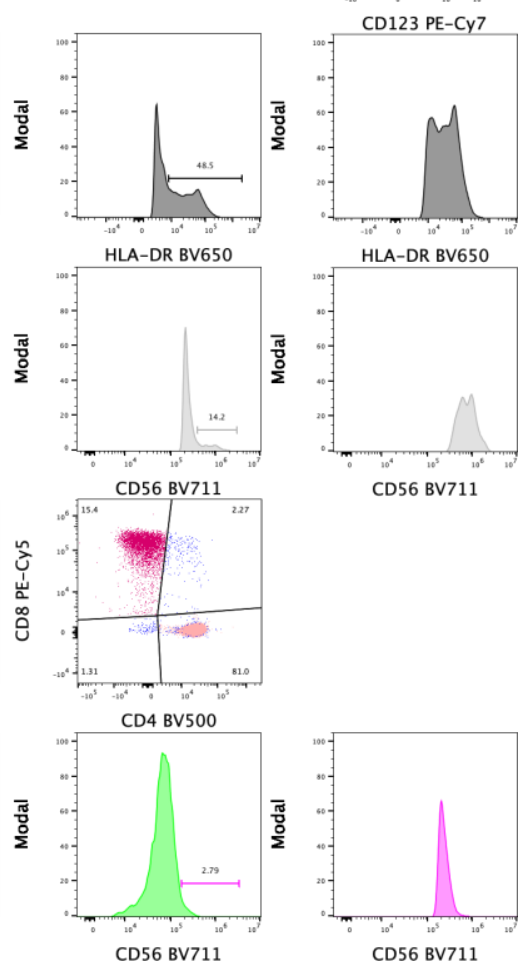

HLA-DR BV650

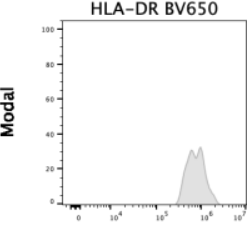

CD56 BV711

Figure 3-2 PBMC gating strategy.

All events were gated to be $\mathrm{CD}_{45}{ }^{+}$, single events and, viable. Using CD3 and HLA-DR yielded three populations containing 1: monocytes, DC and B cells; 2: T cells; and 3: NK cells. Legend indicates terminal immune subsets with colours corresponding to the subset population or gate colour. 


\subsubsection{Assay settings.}

As with conventional cytometry, spectral instruments require an adjustment of detector voltages, or gains, to ensure optimal detection within each detector. There where 38 detectors in our spectral system that needed to be individually adjusted. As part of the Aurora setup the manufacturer provides a set of gains optimised for multicolour blood analysis. We based our gain settings on these 'Instrument Settings' as a starting point. Running sequentially an unstained and then a fully stained sample from a single participant with these settings required only minimal adjustments to ensure all events were on scale for each detector. There was no need for optimal peak positioning on the scale, as the detector range was entirely linear. We then saved the new settings as a set of 'Application Settings'. Spectroflow v1.o software (Cytek, US) allows for saved gain settings to be linked to daily quality control (QC) calibration. We could therefore use these parameters during each acquisition, thus ensuring near identical gains were used for each acquisition. While many factors may influence the intensity of the signal detected, the use of essentially identical gains between different acquisitions runs ensured that any changes in the detected emission for a sample where much more likely to be biological than from experimental variation.

\subsubsection{Controls.}

Spectral cytometers do not require compensation controls. Where a fluorophore with a broad emission range over multiple detectors is a disadvantage in conventional cytometry, for spectral instruments this feature of fluorophores offers an advantage to discriminate fluorescent signals. Each fluorophore is detected over the entire range of detectors on the instrument, and given their different excitation and emission properties, allows for each dye to be profiled to create a fingerprint of emission over the detectors. When a sample is run the full spectrum of emission is recorded, and the software can unmix the separate fingerprints of the dyes from the full sample emission. The unmixed data output is to a virtual channel, identical in the data format to that obtained from conventional cytometers. To enable spectral unmixing, we ran a daily set of individual reference controls for each unique fluorophore present in our full PBMC panel stains, and an unstained sample from each participant. These reference controls were then used by the software to unmix the raw sample data. The unstained sample further acted as a reference baseline of auto-fluorescent emission and acted as a reference for the positioning of unstained events for each detector. 
The use of overlapping lineage markers between our four PBMC panels left 5 channels free to allow for the detection of reporter markers for cytokines, phosphoproteins and dopamine receptors (Table 3-2). As these reporters were central to our hypothesis, we included a fluorescence minus control (FMO) containing only lineage markers for each participant, to facilitate accurate reporting of expression in these channels. Panel stains with fewer than 5 reporter markers were also used as FMO. We used the FMO control during our gating of reporter channel expression to set a bisecting gate to delineate negative and positive signals for each reporter channel (Figure 3-3).

\subsubsection{Maintenance.}

The Aurora was maintained according to manufacturer's recommendations and covered under a continual service contract. The instrument's longitudinal settings were maintained using Spectroflow quality control beads (Cytek, US) from a single lot, over the duration of the experimental use. This maintained the Application Settings for our acquisitions at a constant, while comparable gains were being maintained and adjusted to a constant baseline, ensuring values remained near identical to those first recorded.

\subsubsection{Data analysis.}

All raw cytometry data were recorded and stored in Spectroflow (Cytek, US) in the .fcs file format. We also preformed all spectral unmixing and autofluorescence subtraction in the Spectroflow package. Cytometry data was then analysed in Flowjo v10 (BD, US), with output tables stored in Excel (Microsoft, US) tables. 


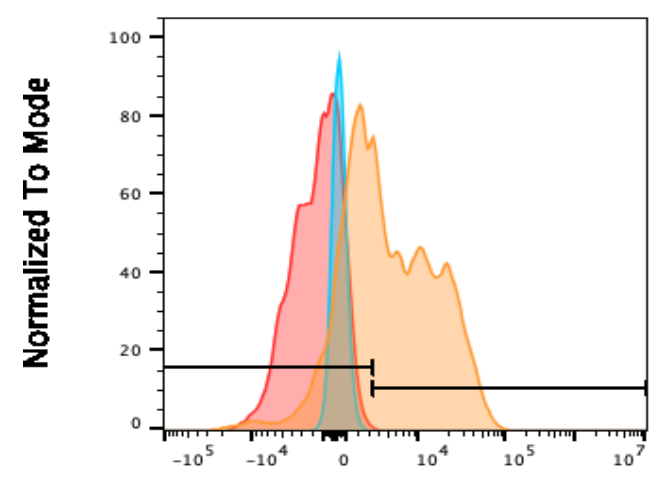

INFg AF488
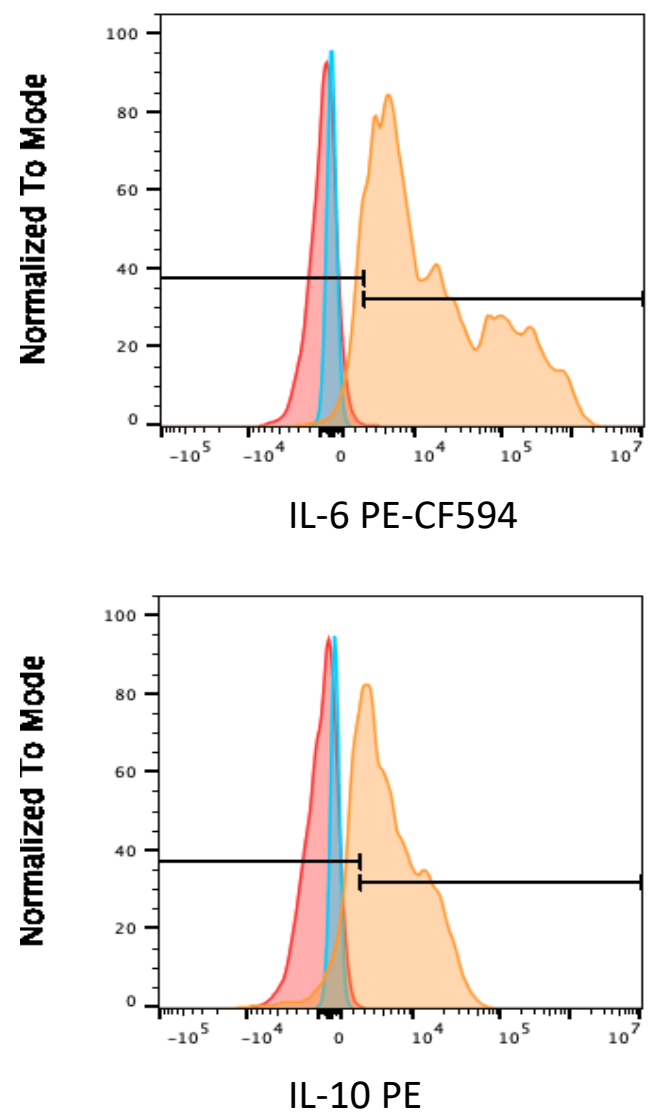
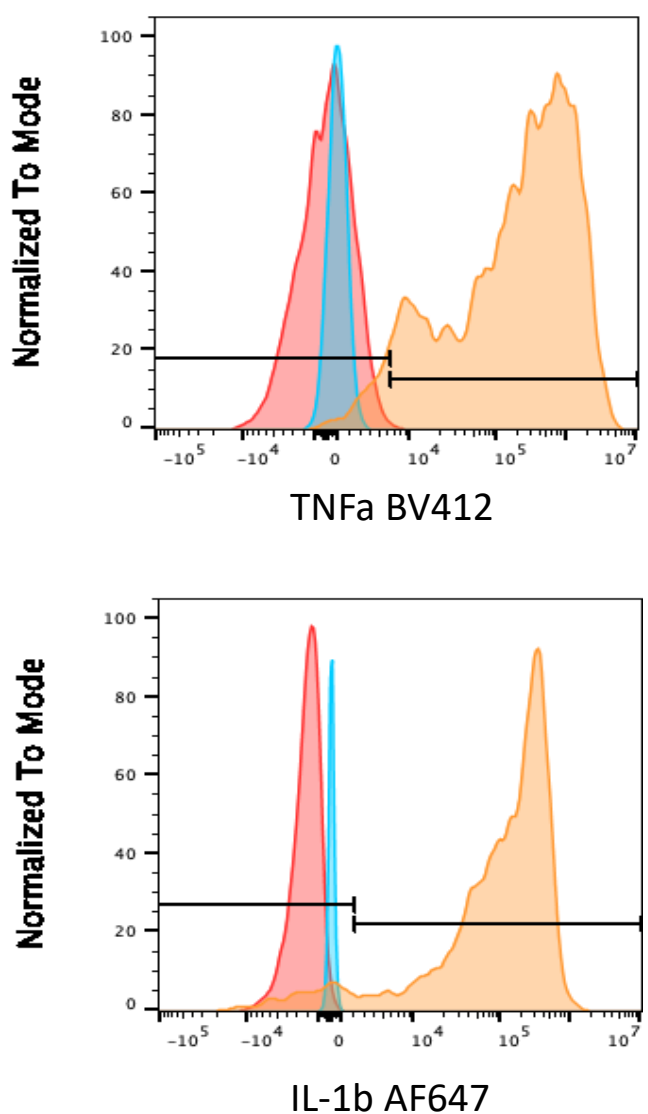

T24 MIS416 Treated

FMO

Unstained

Figure 3-3 FMO gating for cytokine expression from stimulated monocytes.

Representative data from a single individual. Red peak represents the FMO control, blue the unstained, and orange a sample of stimulated monocytes. The bisecting gate is representative of positive (right) and negative (left) data using the FMO as control. 


\subsection{Results.}

\subsubsection{Demographic data.}

We assessed in vitro-stimulated monocytes in PBMC cultures from two clinical trial cohorts comprising untreated progressive MS participants. Concurrently, we compared responses in PBMC cultures from an age- and sex-matched group of healthy control participants recruited locally from the Wellington region (Table 3-3).

Table 3-3 Demographic distribution of all in vitro study participants.

\begin{tabular}{|c|c|c|c|}
\hline \multicolumn{4}{|c|}{ Study Population } \\
\hline Healthy & & & \\
\hline & $\mathrm{N}$ & & 9 \\
\hline & Age & & \\
\hline & & Mean & 54.33 \\
\hline & & Range & $46-66$ \\
\hline & Gender & $\mathrm{F}: \mathrm{M}(\% \mathrm{~F})$ & $7: 2(77.8)$ \\
\hline MS & & & \\
\hline & $\mathrm{N}$ & & 27 \\
\hline & & CRISP & 9 \\
\hline & & MIS416 & 18 \\
\hline & Age & & \\
\hline & & Mean & 56.07 \\
\hline & & Range & $35-70$ \\
\hline & Gender & $\mathrm{F}: \mathrm{M}(\% \mathrm{~F})$ & $\begin{array}{r}21: 6 \\
(77.8)\end{array}$ \\
\hline
\end{tabular}




\subsubsection{Combined cohort validation.}

All the data for our in vitro experiments was obtained using the collated samples from two independently recruited cohorts $(n=27)$. To establish whether the participants from the cohorts were inherently different, we performed an inter-cohort comparison of our cytometry output measures to demonstrate the suitability of this strategy and to identify which output measures could be limited by the collation. To obtain this dataset, we collated data from all the MS participants also present in all our in vitro work. We divided their data based on the respective trial where the sample originated. We then compared the common cytometry output measures of the grouped participants (CRIPS vs. MIS416) to determine if there were any data bias introduced due to the differences in trial recruitment and sampling.

We observed no difference in the age or gender distribution between the MIS416 and CRISP participants (Table 3-4).

Table 3-4 Demographic distribution of MS study participants.

\begin{tabular}{|l|l|l|l|}
\hline & & CRISP & MIS416 \\
\hline $\mathrm{N}$ & & 9 & 18 \\
\hline Age & & & \\
\hline & Mean & 57.2 & 55.5 \\
\hline & Range & $45-69$ & $35-70$ \\
\hline Gender & F:M (\% F) & $7: 2(71.4)$ & $14: 4(71.4)$ \\
\hline
\end{tabular}

3.3.2.1 Dopamine Receptor (DR) expression.

We observed no significant differences in the expression of $\mathrm{D} 1-5$ between the CRISP and MIS416 cohorts (Figure 3-4), indicating the combination of the participants into a single group would not place any limitations on the data interpretation.

\subsubsection{Cytokine expression.}

In comparing the cytokine expression between the cohorts, we observed that the MIS416 cohort showed significantly increased levels of cytokine induction in comparison to the CRISP cohort. Specifically, there was a greater induction of pro- 
inflammatory cytokine at baseline, with IL-1 $\beta$ ( $\mathrm{p}=0.0485)$, IL-6 ( $\mathrm{p}=0.0338)$ and $\mathrm{TNFa}(\mathrm{p}=0.016)$ comparisons being significantly higher (Figure $3-5 \mathrm{~A}-\mathrm{C})$. This theme persisted for the LPS\&cloz and LPS stimulation data, although IL-1 $\beta$ LPS stimulation fell short of the significance threshold $(\mathrm{p}=0.0699)$. TNFa alone maintained this theme for the clozapine data also. Furthermore, we observed no difference between the cohorts for expression of IL-10 and INF $\gamma$ for any in vitro condition (Figure 3-5). 
A

$B$

D1

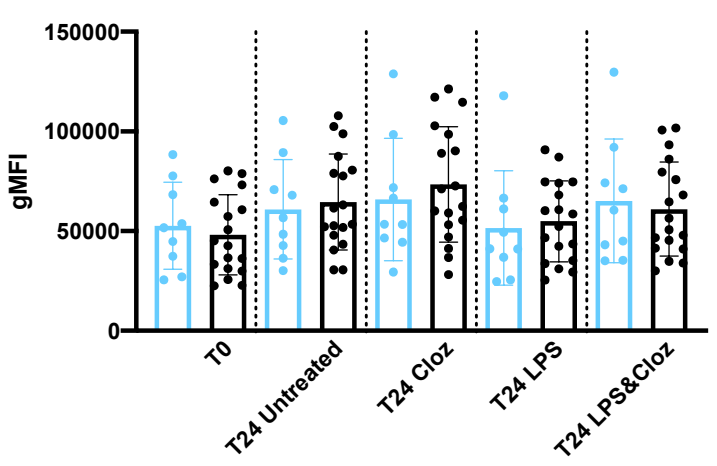

C

D3

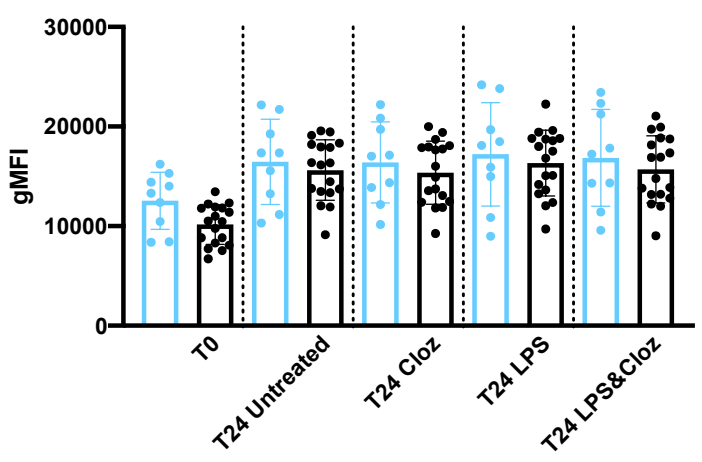

D5

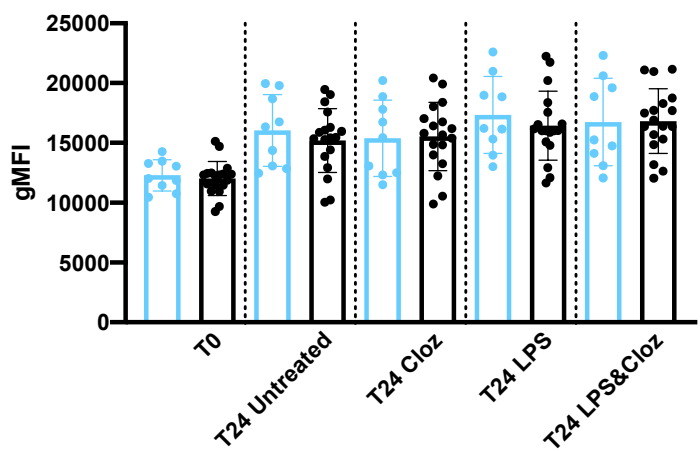

D2

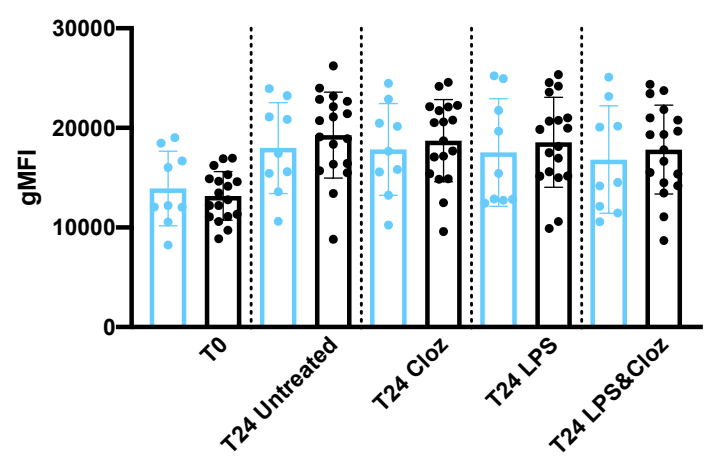

D

D4

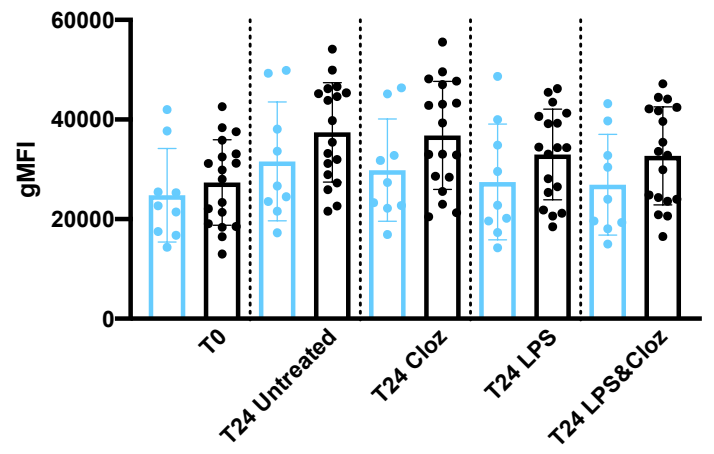

E

- CRISP

- MIS416

Figure 3-4 Inter-cohort comparison of the average DR expression for D1 D5.

Bars indicate mean, with error bars standard deviation. gMFI $=$ geometric mean fluorescent intensity. 

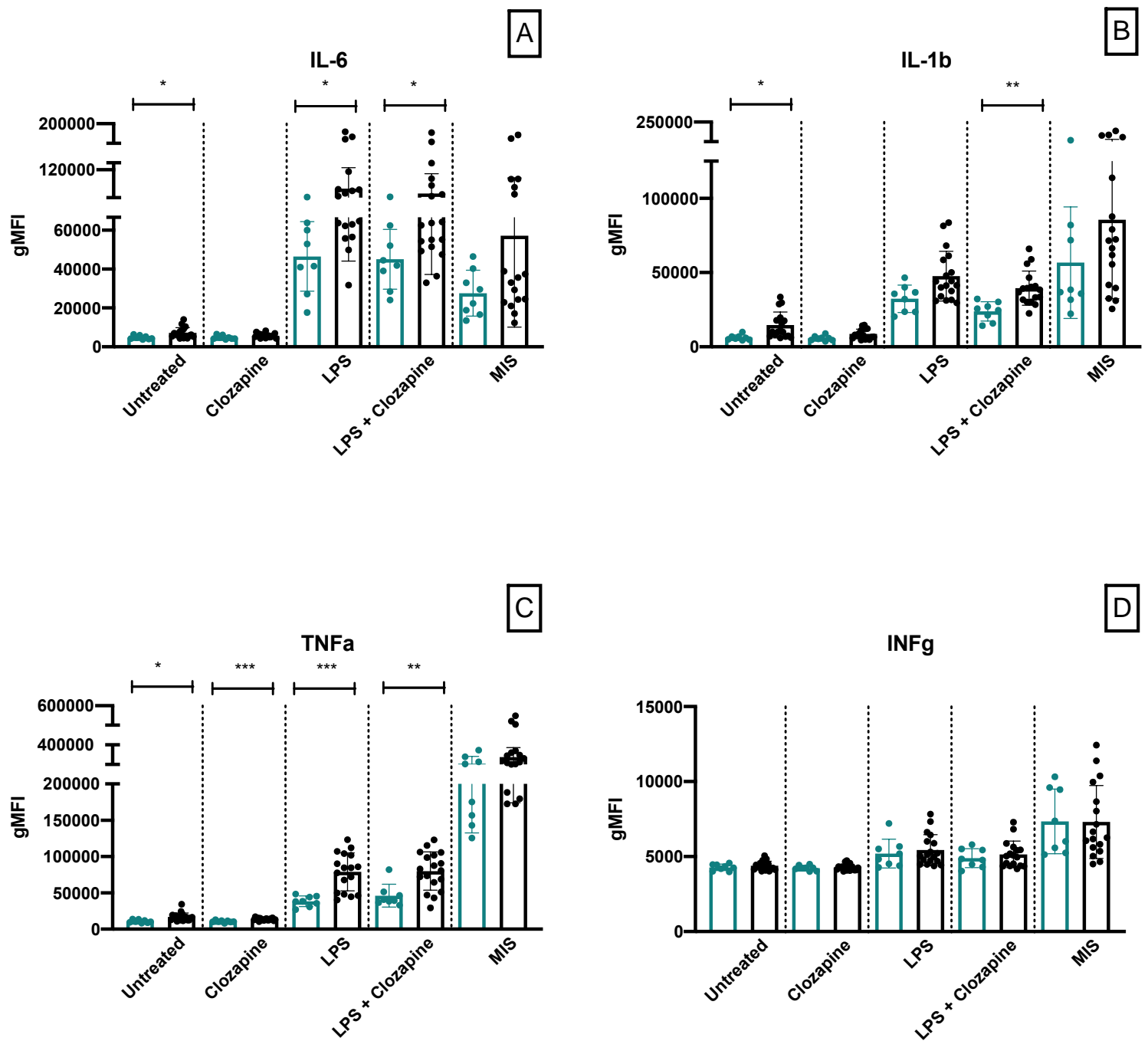

IL-10
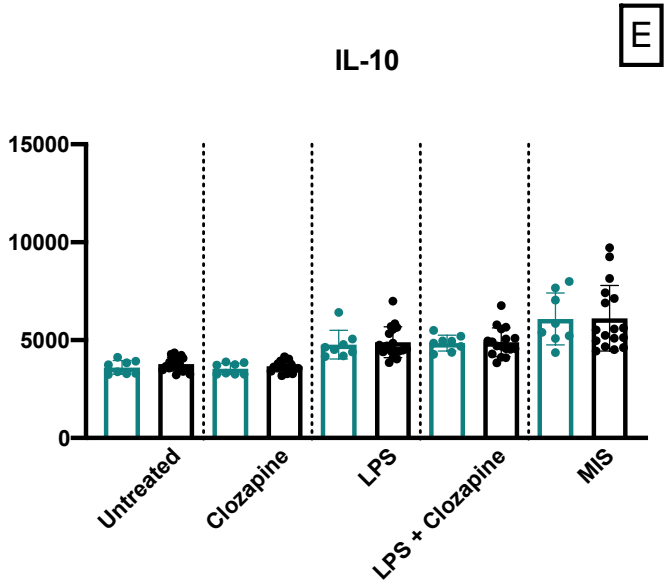

- CRISP

- MIS416

Figure 3-5 Comparison of the average intensity of cytokine expression between CRISP and MIS416 trial participants.

Bars indicate mean and SD. ${ }^{*}(\mathrm{p} \leq 0.05),{ }^{* *}(\mathrm{p} \leq 0.01),{ }^{* * *}(\mathrm{p} \leq 0.001),{ }^{* * *}(\mathrm{p} \leq$ 0.0001). 
3.3.2.3 Phosphoprotein expression.

The data from our phosphoprotein staining showed that MIS416 cohort had lower levels of active P65, and STAT1 compared to the CRISP cohort at the three sampling timepoints we assessed (Figure 3-6A-C). However, the amount of active STAT3 and CREB between the cohorts did not vary to any significant degree (Figure 3-6D).

A

CREB

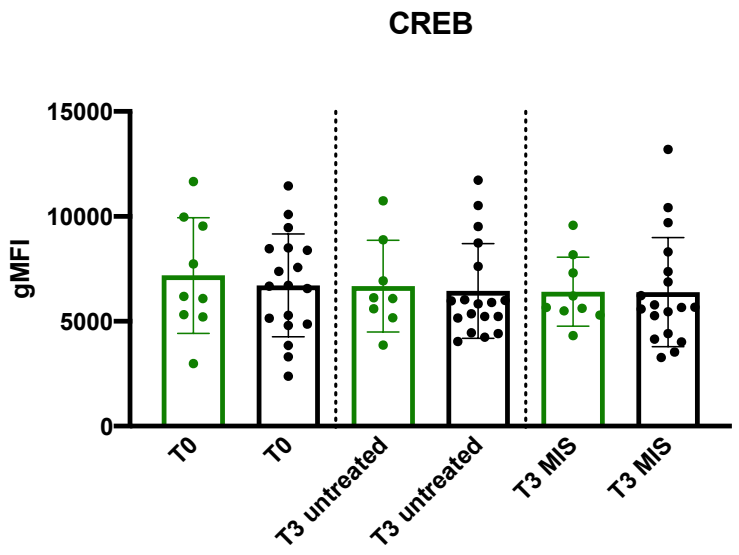

C

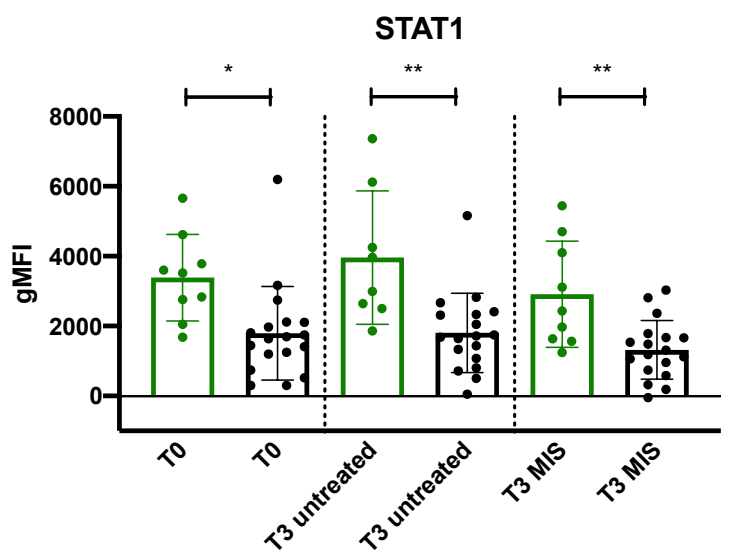

B

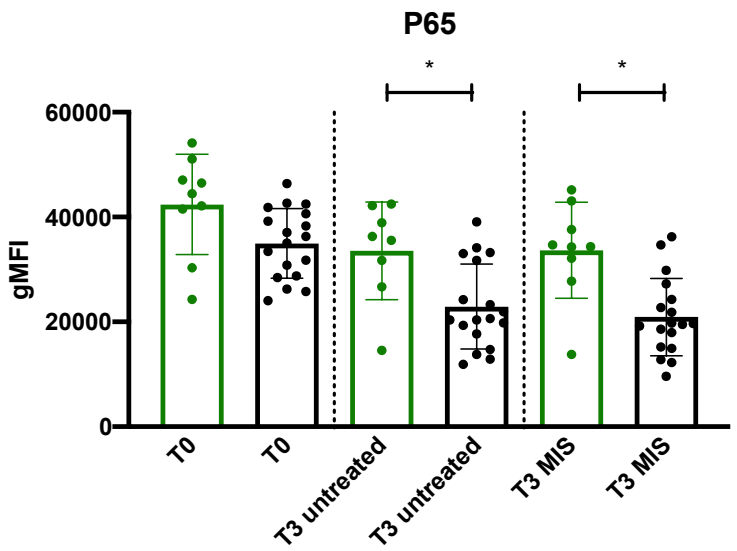

$\mathrm{D}$

STAT3

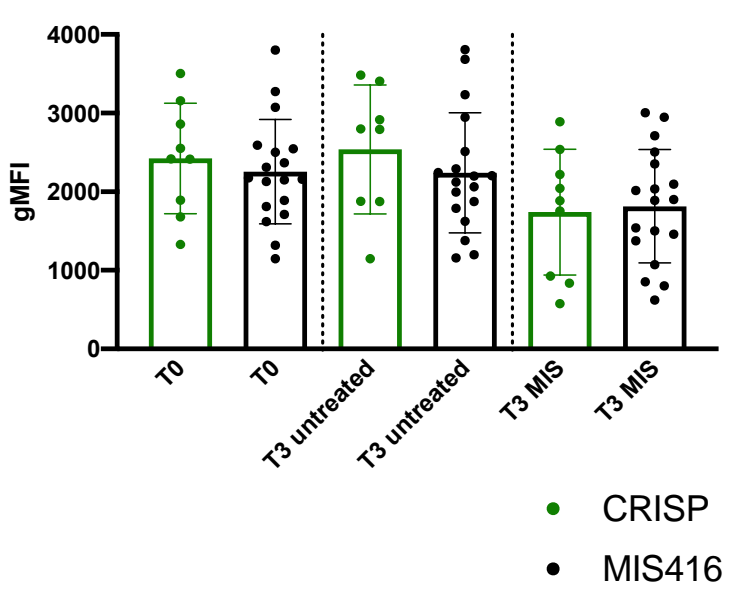

Figure 3-6 Inter-cohort comparison of active transcription factor expression for CRISP and MIS416 participants.

Bars indicate mean and SD. ${ }^{*}(\mathrm{p} \leq 0.05),{ }^{* *}(\mathrm{p} \leq 0.01),{ }^{* * *}(\mathrm{p} \leq 0.001),{ }^{* * * *}(\mathrm{p} \leq$ 0.0001). 


\subsubsection{PBMC Assay Validation.}

\subsubsection{Assay Optimisation and Titration.}

Our panel optimisation aim was to ensure that reproducible staining resulted over the duration of our in vitro assay period. We decided to limit the number of titrations by using the same antibody supplier as our PB work. These products were supplied ready diluted for use and allowed for staining with a manufacturer's recommended volume relative to the number of cells in each stain. The PBMC panel optimisation therefore comprised a verification of subset populations and resolution following a full panel stain. This was achieved by performing a cocktail titration approach, rather than individual antibody titrations. We performed the titration to model the effects of inter donor variation with respect to the proportional composition of immune subsets. We performed a serial dilution using a single donor and stained with a cocktail mixture of all lineage antibodies to manufacturers final concentration. The titration was based on the amount of PBMC in each stain, keeping the amount of antibody constant and varying the total PBMC concentration only, starting at $10^{6}$ cells (recommended)

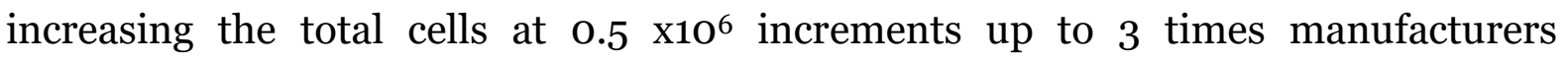
recommendation. Adding fewer cells was unnecessary, given that in our experimental staining protocol excess antibody was washed away before acquisition and the amount of cocktail added was adjustable based on the number of cells available for staining.

Data from the titration was analysed by gating on the stained PBMC to yield terminal immune subsets. For each subset we plotted the geometric mean fluorescence intensity (gMFI) using their main lineage discriminator antibody (e.g. CD19 for Bcells) (Figure 3-7). We observed high variability in staining intensity over the cell dilution series, with a general trend resulting in a drop in MFI when more cells were present. Several markers showed improved staining intensities at $2 \times 10^{6}$ cells. As the PBMC composition and subset proportion would be highly donor dependent, we concluded that further titration would be cost prohibitive. We also calculated the average coefficient of variation (CV) for the proportional makeup from the terminal immune subsets gated over the titration series. This showed that the average variation was $3.3 \%$. This modest variation over a dilution of up to three times the starting concentration further substantiated our decision to use the published antibody concentrations. We concluded that the recommended amounts offered an adequate 
staining result, and that shifts in subset composition anticipated from different donors would still yield acceptable results. Furthermore, we settled on using $0.8 \mathrm{x}$ the recommended amount of antibody to facilitate a modest cost saving. 

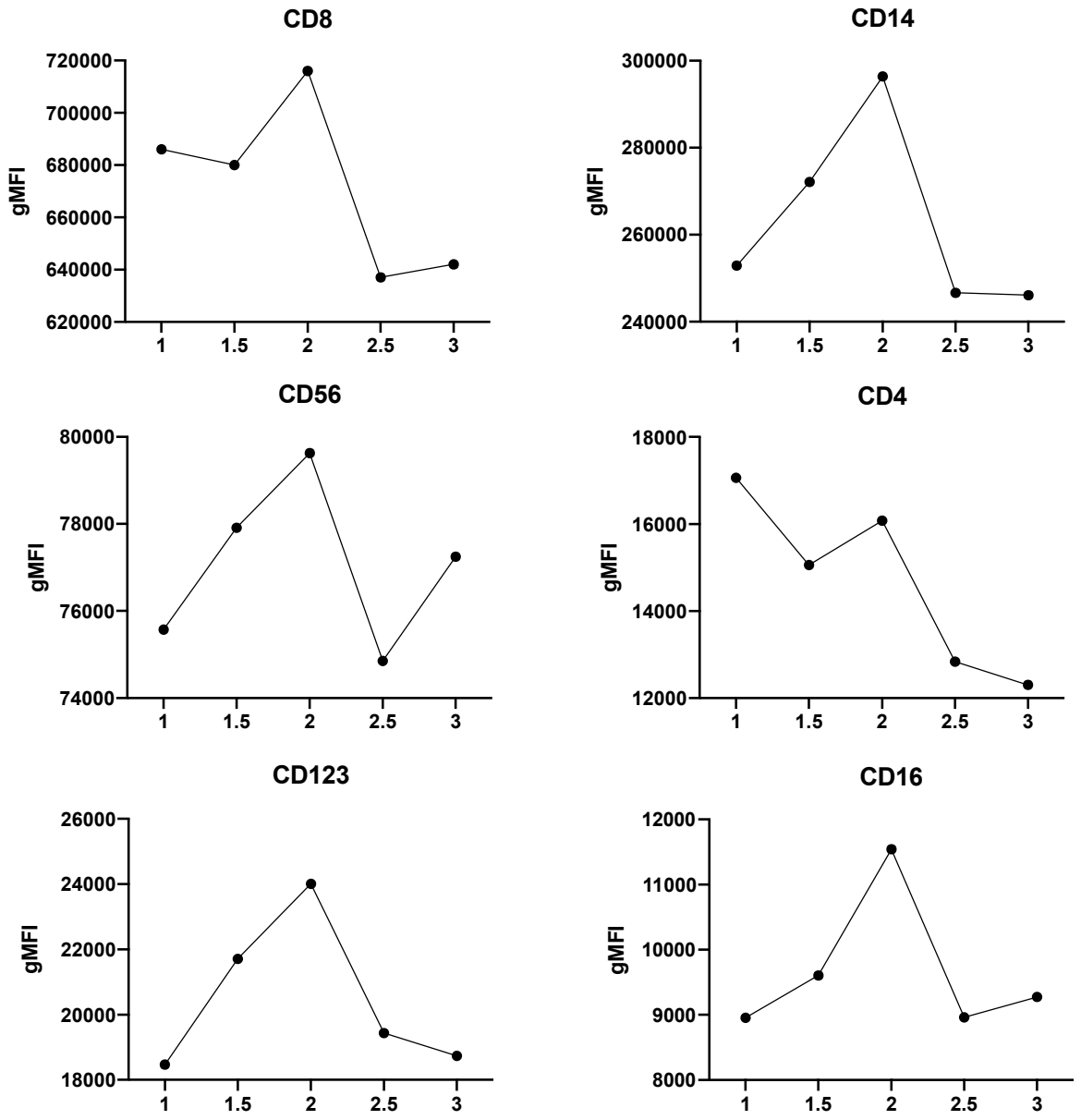

HLA-DR

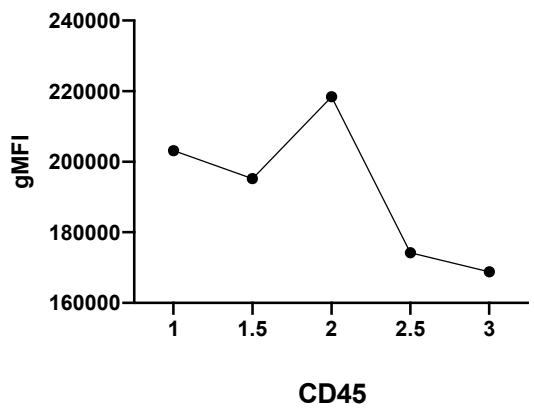

CD3
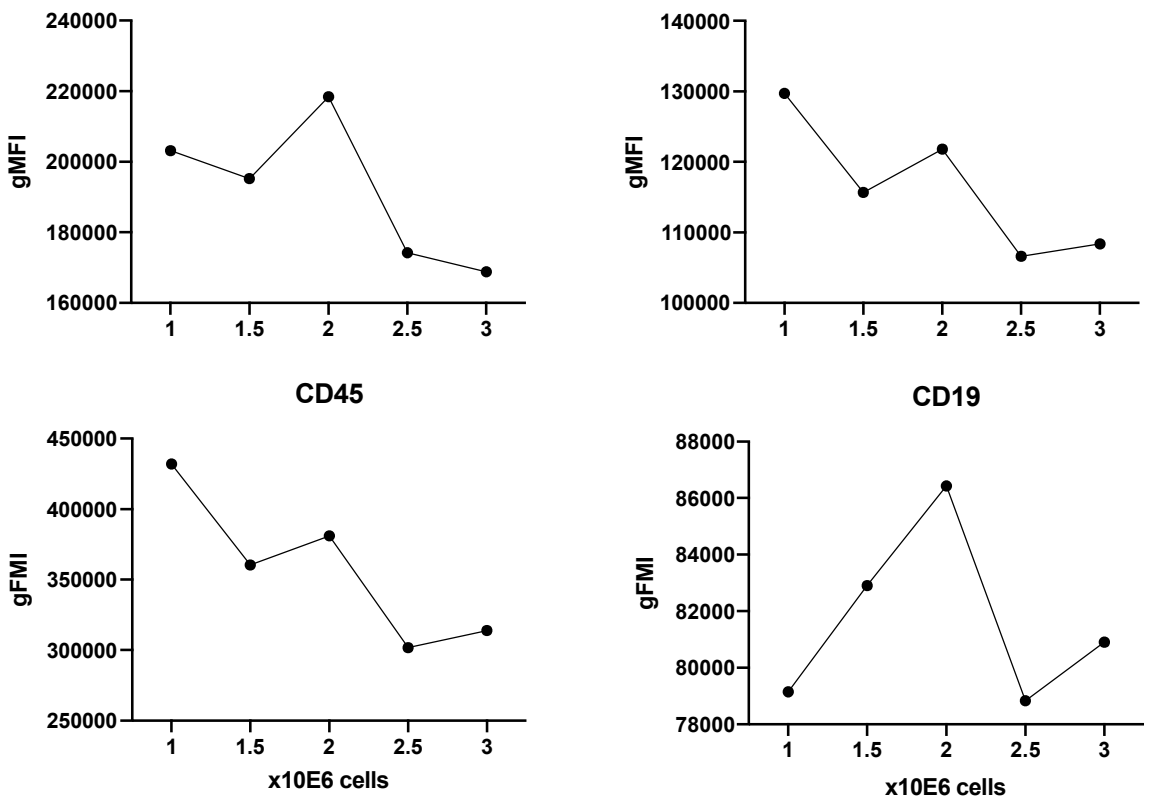

Figure 3-7 PBMC lineage cocktail antibody titration.

The gMFI of the subset expression for each marker is indicated over a dilution series. 


\subsubsection{Autofluorescence.}

Endogenous fluorescence, or autofluorescence, is the natural fluorescence inherent in cells due to the presence of fluorescent metabolites and structures (Monici, 2005). Autofluorescence is detectable over a broad wavelength, most prominently in the 350$500 n m$ range, and has long been known to affect the detection of reported dyes that emit in this spectral range (Billinton and Knight, 2001). Mostly, autofluorescence has been grudgingly accepted in cytometry, with workarounds such as using very bright reporter dyes, or by using only reporters that emit outside the known interference range. These workarounds are of limited use when large multicolour panels are required, as many dye conjugates are optimally detected in the 350-500 $\mathrm{nm}$ range, and may be dim.

Spectral cytometers are equipped to detect very dim signatures. Given the sensitivity of the detectors, autofluorescence was an anticipated source of background noise in our assays. We were able to profile autofluorescence by running unstained cells and observed that the signal was present over all the detectors. Furthermore, we observed an increase in the autofluorescence signal from cells when they were stimulated in vitro (Figure 3-8).

The Aurora software allows for autofluorescence to be profiled similar to the profiles constructed for reporter dyes. The autofluorescence signal can then be subtracted from the overall signal during the spectral unmixing process. This results in data where the detector signals contain only true reporter dye emission.

To confirm the validity of the manufacturer's claims that autofluorescence had a distinct profile, we recorded emission spectra from unstained monocyte, T-cells, whole PBMC and lymphocytes. We normalised expression over the detectors to the brightest emission channel and plotted the data (Figure 3-9). This showed that the emission profile for autofluorescence was highly similar for all samples. We performed a comparable acquisition on cells from different donors with similar results.

From this we concluded that subtracting autofluorescence noise from all our PBMC data during the unmixing step in the Spectroflow software package would substantially decrease background noise. 

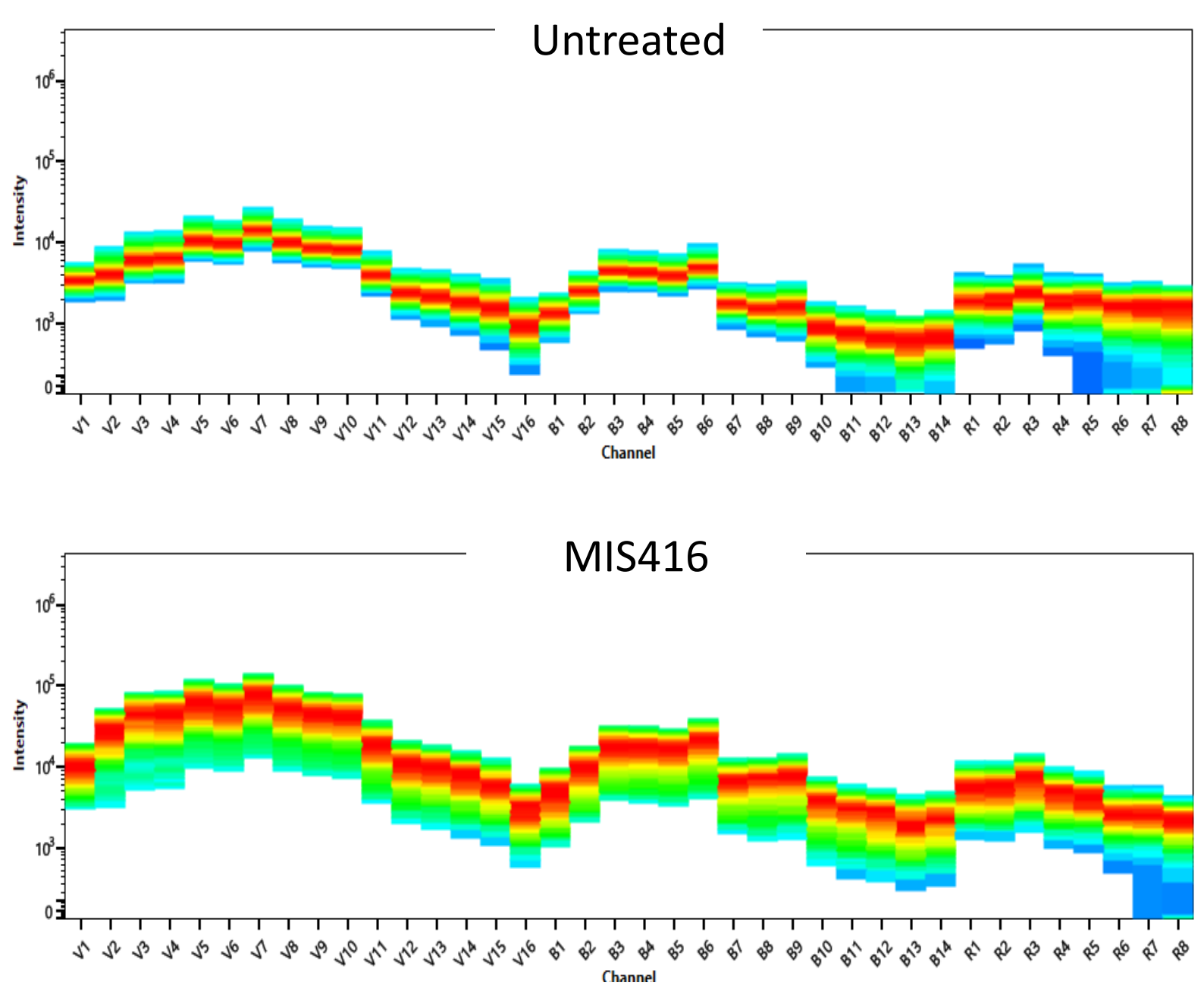

Figure 3-8 Autofluorescence signal of unstained PBMC.

The top panel is a representative sample of unstained untreated PBMC. The bottom panel is a representative sample of in vitro MIS416 stimulated PBMC. V 1-16, B 1-14, and $\mathrm{R}$ 1-8 represent the specific detectors present on the Cytek Aurora system. Intensity is the peak fluorescence intensity of signal detected in each channel. 


\section{Normalised Autofluorescence}

1.2

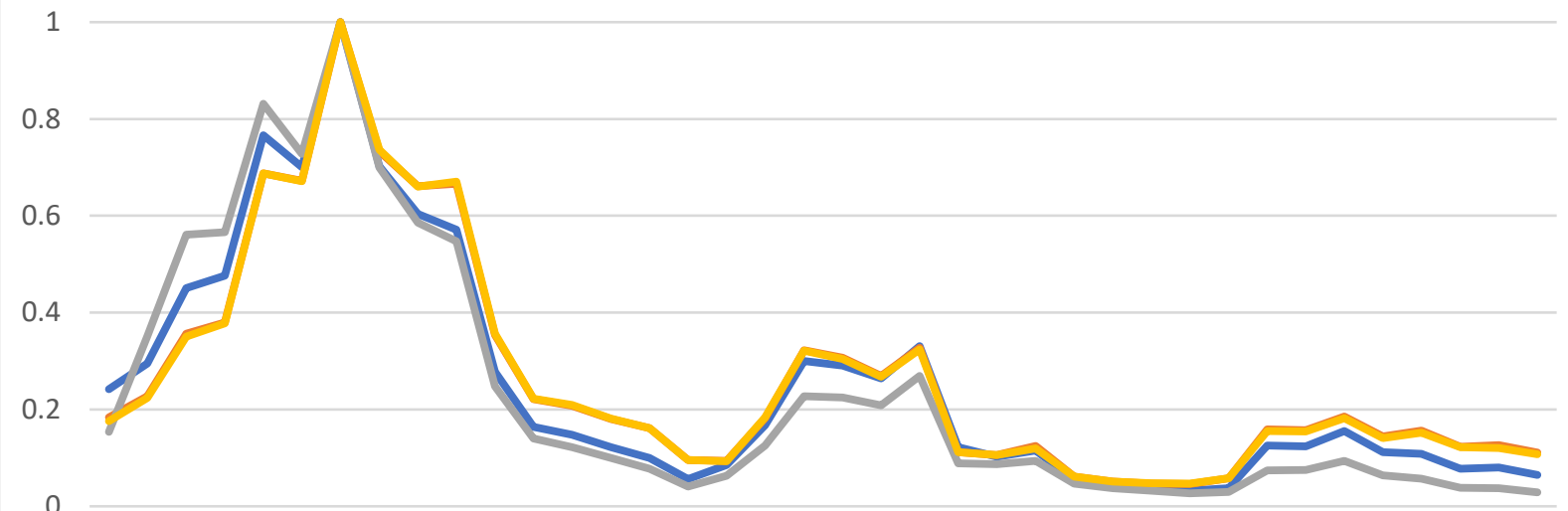

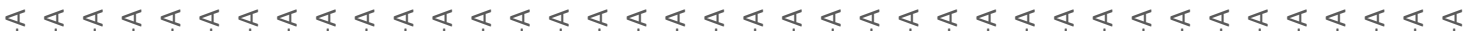

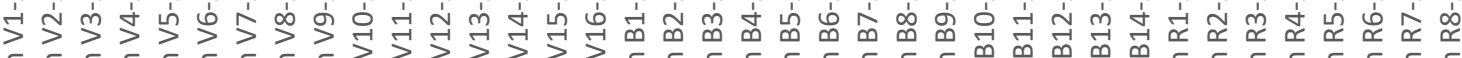

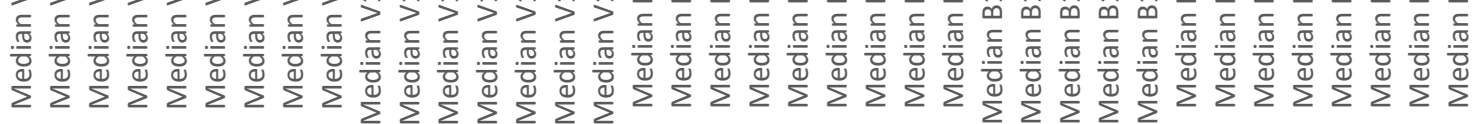

Unstained untreated monocytes _Unstained untreated Lymphocytes

Unstained MIS stimulated monocytes Unstained MIS stimulated lymphocyte

Figure 3-9 Monocyte autofluorescence by channel.

All data was normalised to brightest peak $\left(V_{7}\right)$ emission and depict unstained PBMC from a single donor. Lymphocytes and monocytes were gated by scatter properties. 


\subsubsection{Longitudinal QC.}

As the Aurora was a new instrument of unknown reliability, we decided to include additional QC steps to ensure our experimental validity. With each acquisition and over the duration of our cytometry experiment we acquired data on a sample of CS\&T beards (BD, US) from a single lot, recording emission over all instrument detectors. This was done independently from and after the daily instrument setup and calibration steps. The CS\&T beads comprise three particles of dim, medium and bright emission over a very broad detector series. We gated single bead events and extrapolated the medium bead gMFI for each of the instrument detectors. We collated data from over 30 independent runs over the course of six months. This data is depicted in Figure 310. We observed very high fidelity from the daily instrument calibration, with an average CV from blue, red, and violet lasers of 3.2\%, over all detectors (Table 3-5).

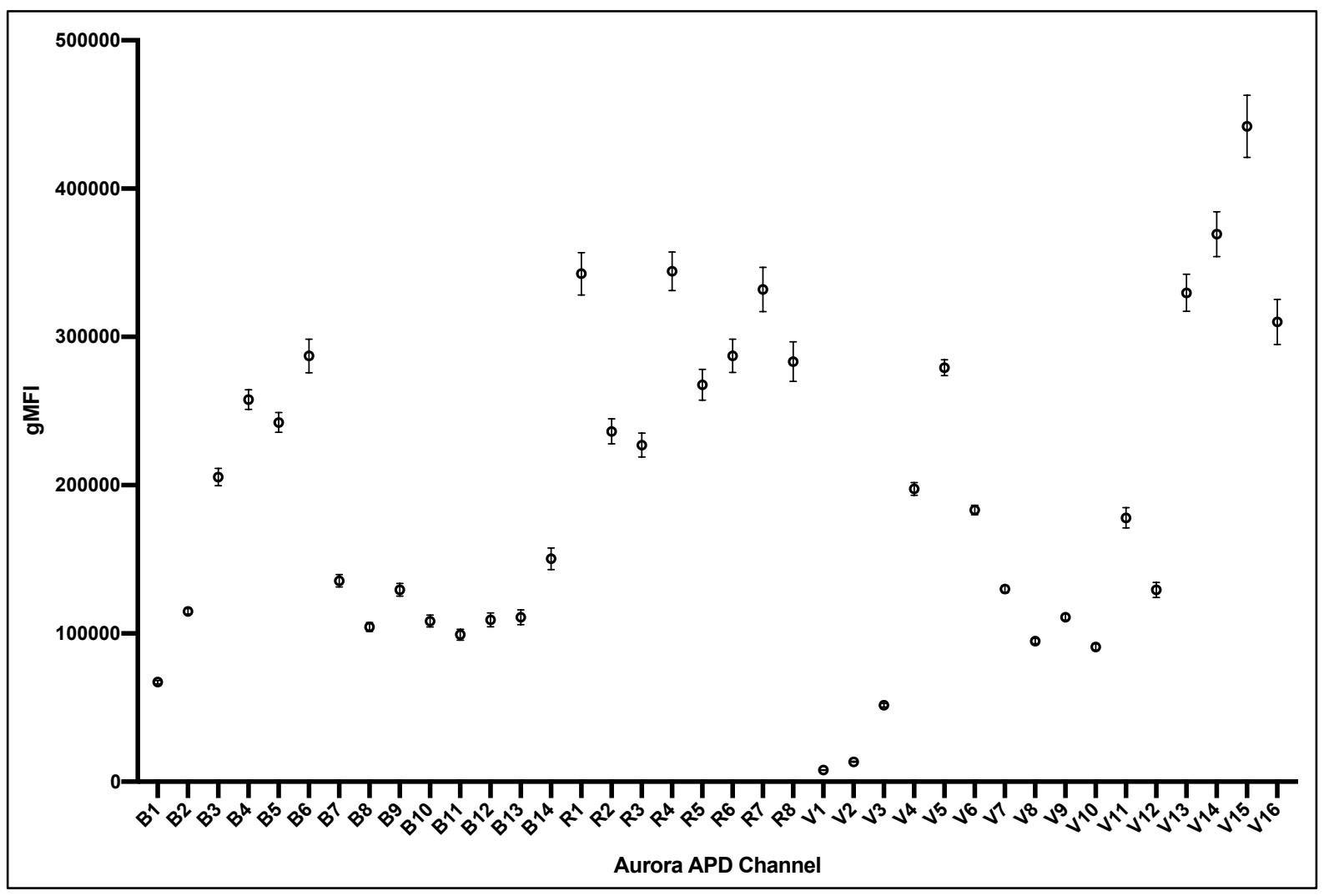

Figure 3-10 Average medium bead emission as fluorescence (gMFI) for each detector of the Aurora platform.

The data point for each channel depicts the mean and SD of 30 successive data points collected over 6 months. 
Table 3-5 Average per channel CV of the combined data series for the medium CS\&T bead collected on the Aurora platform over 6 months.

\begin{tabular}{|l|c|l|l|l|l|}
\hline \multicolumn{2}{|c|}{ Violet Laser } & \multicolumn{2}{c|}{ Blue Laser } & \multicolumn{2}{c|}{ Red Laser } \\
\hline Channel & CV & Channel & CV & Channel & CV \\
\hline V1 & 2,64 & B1 & 1,77 & R1 & 4,17 \\
\hline V2 & 2,24 & B2 & 1,60 & R2 & 3,56 \\
\hline V3 & 2,40 & B3 & 2,86 & R3 & 3,55 \\
\hline V4 & 2,13 & B4 & 2,55 & R4 & 3,78 \\
\hline V5 & 1,91 & B5 & 2,73 & R5 & 3,88 \\
\hline V6 & 1,75 & B6 & 3,94 & R6 & 3,90 \\
\hline V7 & 1,87 & B7 & 3,07 & R7 & 4,49 \\
\hline V8 & 1,73 & B8 & 2,94 & R8 & 4,71 \\
\hline V9 & 1,86 & B9 & 3,29 & & \\
\hline V10 & 2,59 & B10 & 3,71 & & \\
\hline V11 & 3,84 & B11 & 3,67 & & \\
\hline V12 & 3,92 & B12 & 4,26 & & \\
\hline V13 & 3,81 & B13 & 4,50 & & \\
\hline V14 & 4,09 & B14 & 4,82 & & \\
\hline V15 & 4,75 & & & & \\
\hline V16 & 4,92 & & & & \\
\hline \hline Average & 2,90 & Average & 3,26 & Average & 4,00 \\
\hline
\end{tabular}

3.3.3.4 Optimal permeabilisation of PBMC was obtained with Saponin.

We performed a series of experiments to select a permeabilisation buffer to use for intracellular staining. The key considerations were to maintain epitope integrity, minimise fluorescent quenching of antibody conjugates, maintain a good signal to noise ratio, and that the buffer should not alter the spectral profile of stained cells. Saponin is a longstanding permeabilisation agent and is routinely used for staining of intracellular cytokines. We selected Cytoperm (BD, USA) as a standardised formulation with proven efficacy in cytokine analysis. With phosphoproteins, saponin permeabilisation use is less common, as a cold methanol containing buffer often yields the best results. For a methanol based permeabilisation comparison we selected Perm Buffer III (BD, USA). We then performed a series of comparative staining experiments using these two buffers following manufacturer's instructions.

The FACS select resource indicated methanol permeabilisation would be required for detection of STAT nuclear epitopes (Cytobank, 2019). Our assays included STAT1 and 
STAT3. To confirm whether any signal was detectable with Cytoperm we compared it to Perm Buffer III. We found that methanol permeabilisation (Buffer III) was very effective in resolving STAT1 and STAT3 signal. Using saponin however did result in detectable STAT signals and a discernible effect (drop in gMFI) from MIS416 stimulation (Figure 3-11).

Of the four phosphoprotein markers in our analysis P65 and CREB showed effective and improved staining using saponin permeabilisation (Figure 3-12). Additionally, we observed that the methanol buffer altered the spectral profile of the CD45 APC-H7 conjugate, and of the Live/Dead Yellow viability dye (Figure 3-13). We also observed significant detrimental effects on CD4 staining (epitope loss), high numbers of cells clumping, and a loss of resolution in most markers following methanol permeabilisation.

While STAT1 detection was improved using methanol, saponin was determined to be the most reliable permeabilisation buffer as it offered consistent staining and detection for all intracellular cytokines, and for most of the phosphoprotein epitopes. 

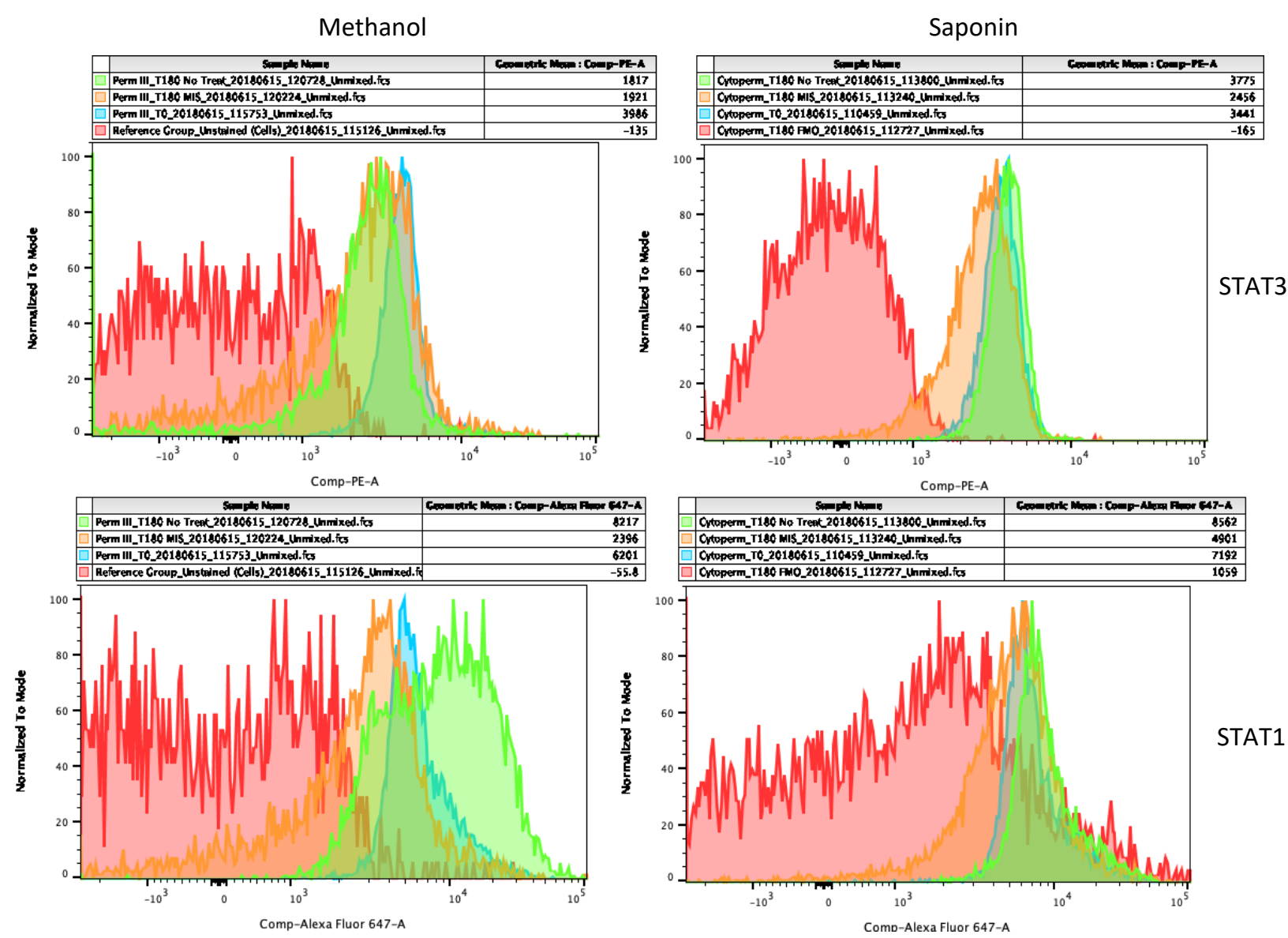

Figure 3-11 Representative donor comparison of STAT protein phosphorylation detection using methanol and saponin buffers.

Monocytes overlays of MIS416 stimulated cells. FMO (red), untreated cells (green), MIS416 stimulated (orange) and baseline unstimulated (blue). 

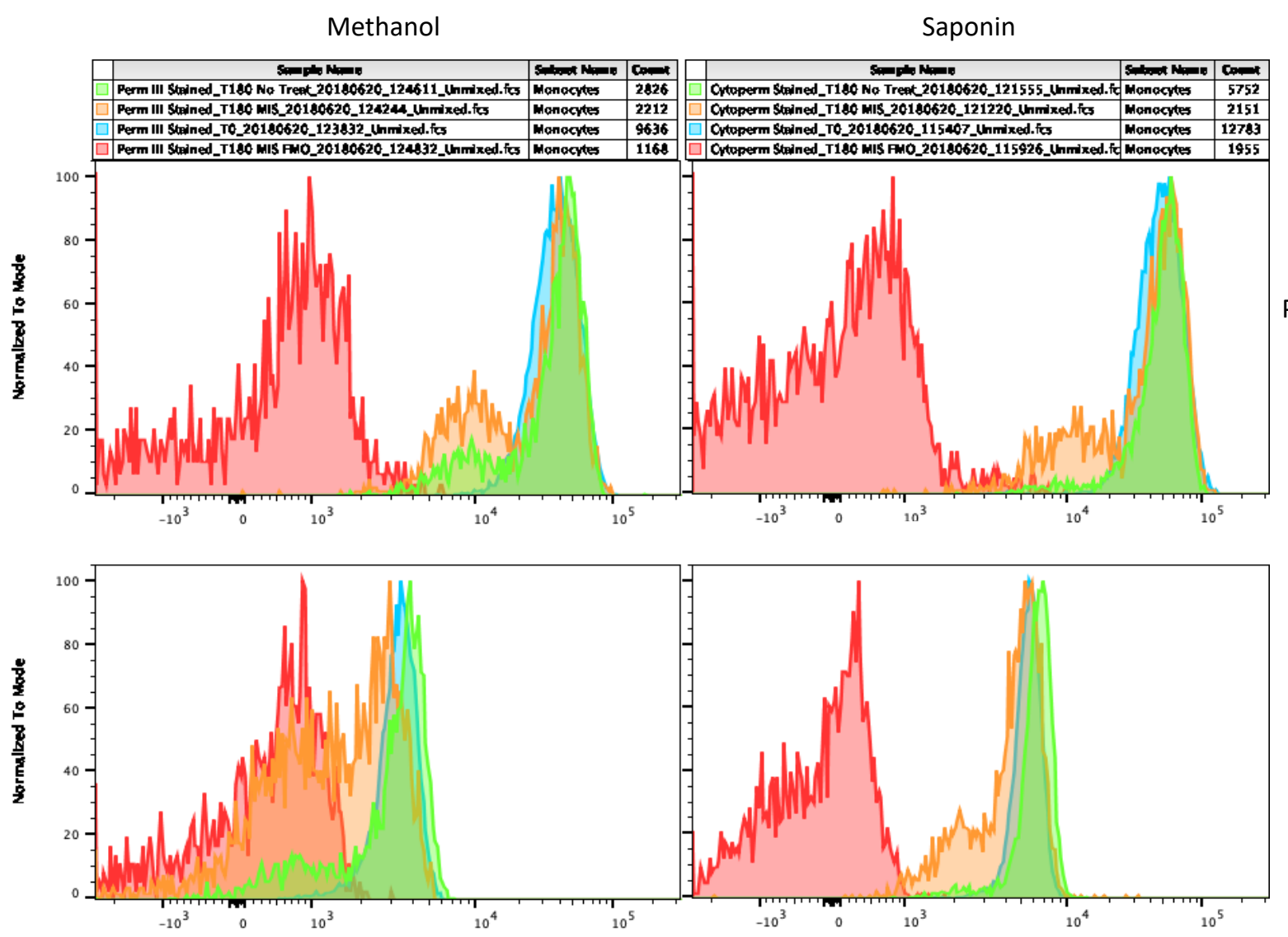

Figure 3-12 The effect of saponin and methanol containing permeabilisation buffers on monocyte phosphoprotein responses in a representative donor. 


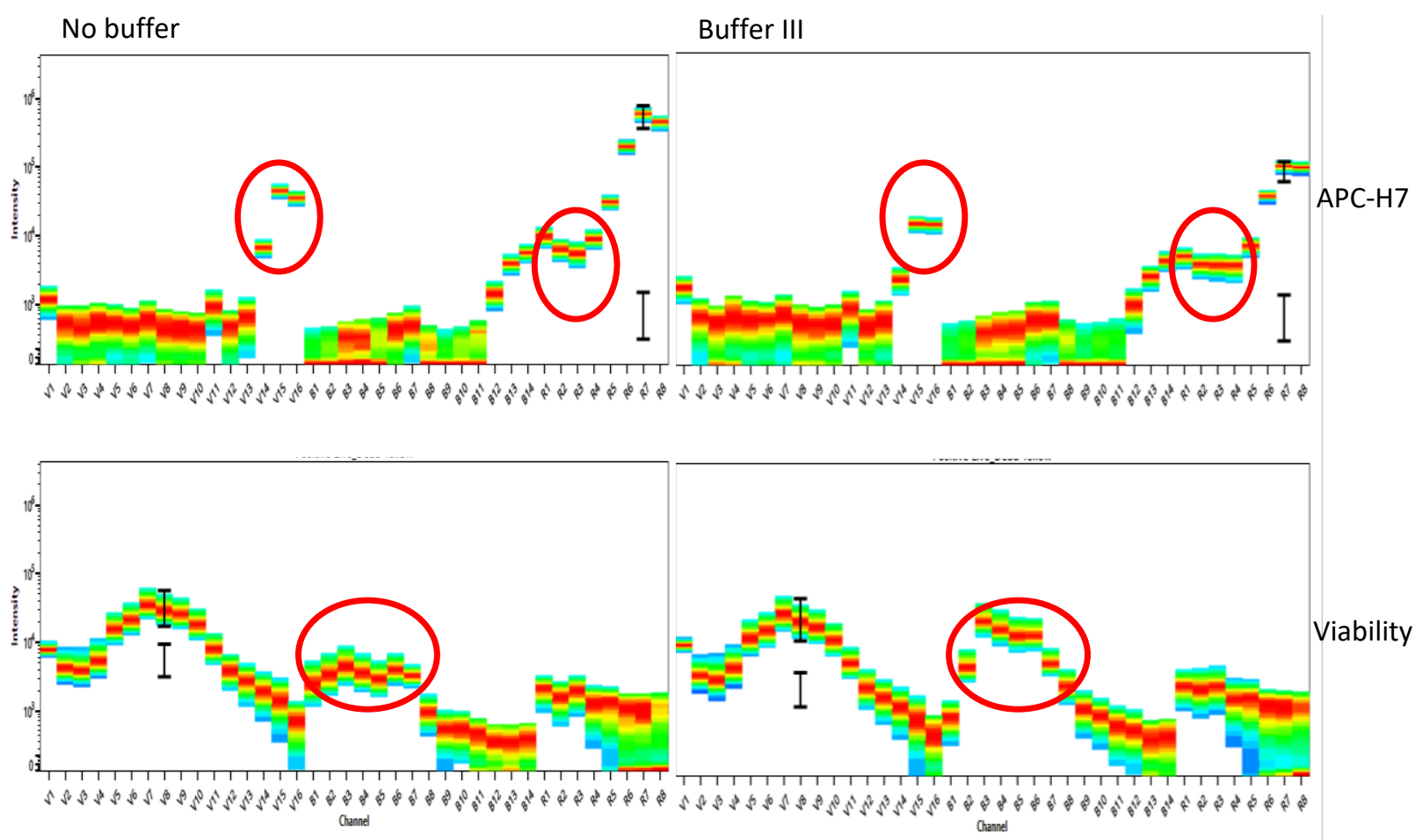

Figure 3-13 Aurora cytometer spectral emission over all channels for APC-H7 and viability dye.

Images depict the impact of methanol containing buffer III on fluorescent emission, with pattern shifts affecting spectral deconvolution. 


\subsection{Discussion.}

3.4.1.1 Sampling impacts on data quality.

All samples were stored and processed according to standard operating protocols. Where possible, instruments and reagents were kept consistent, including using single batch numbers, to limit batch variation and the need for repeat validation. Samples from the CRISP and MIS416 trials were collected, processed, and stored at two different facilities. Ultimately, all samples were held centrally in Wellington and processed for in vitro culture and cytometric data acquisition by the author. We cannot therefore preclude the introduction of sampling variation prior to in vitro processes. However, earlier work by our lab has shown the use of CPT tubes did not alter monocyte function or expression when compared to a Histopaque isolation (Johnston et al., 2015). A further significant limitation for our in vitro data comes from the assumption that differences in readout values are due to biological variation, or as a result of disease pathophysiology, which may in fact be due to technical issues. This is a common premise for longitudinal data acquisition protocols of similar studies. Ultimately, to control and report on the introduction of sampling noise was not reasonably practical and falls outside the scope for most studies of this nature.

\subsubsection{Combined cohort validation.}

We demonstrate here that there were significant differences in the group averages for readouts of some markers such as cytokines and phosphoproteins between the cohorts. Such differences may be due to sampling differences at the trial sites or due to true biological differences. All participants were recruited in New Zealand, with the MIS416 cohort comprised of participants from Wellington and Auckland, while CRISP was a Wellington only centred trial. All participants were screened to a similar standard, excluding confounding co-morbidities or treatments, and when combined, formed an age and gender match cohort of progressive MS participants. Significantly, the CRISP trial consisted of both primary and secondary progressive, while MIS416 recruited only SPMS. This was the only major apparent difference that could explain the divergent functional responses we observed. However, several phenotyping measures were also found to not show this level of variation with DR expression found to be highly consistent between the cohorts. We suggest that the observations were 
modest and likely resulted from interparticipant disease heterogeneity. It was clear that the MIS416 cohort had more outliers, but there was also double the number of participants from this study in our final cohort compared to the CRISP cohort.

\subsubsection{Panel design and optimisation limitations.}

Large cytometry panels are an exercise in compromise. No ideal combination of makers and reagents exist. Instead, we compromised on the quality of some readouts to facilitate extending the range of detected markers in each sample. Some key readouts therefore were unfortunately profiled using dim fluorescent markers. This limits the quality of the data as very dim signatures would not be discernible from background noise. Furthermore, the minimal titration of reagents and use of antibodies at manufacturer recommended volumes may result in sub-optimal staining quality, but we compromised on this given the significant time investment required to fully optimise large multicolour panels, and the general reliability of CE marked reagents we procured from reputable suppliers.

Another experimental limitation was the use of saponin as a permeabilisation buffer despite the very dim staining that resulted from STAT1 staining. Methanol permeabilisation offered better staining for STAT markers but would not have been compatible with the spectral platform. This limited the output data quality of STAT markers in our transcription factor panel.

\subsubsection{Autofluorescence.}

Our study is one of the first reported datasets where autofluorescent noise was subtracted using Spectroflow v1.o (Cytek, US) software. Our analysis did not control for autofluorescent noise, and we did not exhaustively validate the manufacturer's claims. Our data here shows that autofluorescent noise was detectable and subtracted, that all cell types had highly similar signatures and that no significant inter-individual differences were present. There is currently a lack of readily available control material of known autofluorescence intensity to use as an autofluorescence control. As such, this introduces an uncontrolled limitation. However, we feel confident that the subtraction dramatically improved the quality of dim staining epitopes in this assay. 


\subsubsection{Spectral Cytometry.}

At the time of data acquisition, the spectral cytometry platform was as yet untested. No other centre had reported on the instrument's utility and stability. Our data therefore is limited in that we accepted a new instrument with undisclosed proprietary data processing on which to acquire our dataset.

We used longitudinal controls to show that the instrument stability over time was very good. Some variation was observed in a few red and far violet channels. This was mitigated by the use of spectral fingerprints that delineate each fluorophore so that no single channel was used to report on emission for a target. The new instrument introduced an uncertainty limitation in that the spectral unmixing is proprietary. We could not therefore comment on what effect channel fluctuations have on the overall quality of the data. As these measures were within the acceptable parameters for calibrated cytometers, we suggest our data quality was on par with that of similar datasets. 


\section{Chapter 4. The effects of in vitro clozapine on dopamine receptor and cytokine expression in progressive MS.}

\subsection{Introduction.}

The neurotransmitter dopamine, a catecholamine hormone, is primarily present in the brain. Its major function as a neurotransmitter is in hormonal control, movement, and behavioural processes. In the periphery, dopamine plays a prominent role that includes homeostatic control of blood pressure, vision, olfaction, and the regulation of immune function (McKenna et al., 2002). Dopamine receptors (DR) are membrane spanning G-protein coupled receptors. They are widely expressed in the central nervous system (CNS) where these receptors' major function is in effecting the physiological role of dopamine (Beaulieu and Gainetdinov, 2011). DR expression is also systemic, so the effects of agents antagonising these receptors can range from sedation and hypotension to CNS- and immune depression (Gerson and Meltzer, 1992; Roth et al., 2003; Schmid et al., 2014). To date, five types of DR have been identified, and these five have been categorised into two classes. The most abundant of the receptors are D1 and D5, both belonging to class 1. Class 2 receptors comprise D2, D3 and D4. The opposing actions of these two receptor classes is centred around adenyl cyclase (AC), where class 1 agonism stimulates $\mathrm{AC}$ activation and cyclic adenosine monophosphate (cAMP) production, while class 2 inhibits AC and subsequent cAMP formation (Missale et al., 1998).

Dopaminergic signalling has a broad impact on the pathophysiology of diseases of the CNS, especially schizophrenia and Parkinson's where hyper- and hypoactivity of the dopaminergic system correlate to altered immune function (Basu and Dasgupta, 2000) As such, DR targeting agents have proven an effective treatment strategy for a range of health conditions including Alzheimer's, depression and schizophrenia. 
Atypical antipsychotics are thought to suppress psychosis through a transient and comparative antagonism of dopamine at D2 (Schrader et al., 2019). Clozapine is one such antagonist. Clozapine binds a wide range of neuroreceptors including both serotonin and dopamine receptors, but at therapeutic doses, acts to treat psychosis primarily through the class 2 receptors (D2 and D4)(Missale et al., 1998; Schmid et al., 2014). Because clozapine has broad receptor activity, Ribeiro and colleagues posited that clozapine may aid in disease reduction in schizophrenia by altering signalling and interacting with low affinity neuroreceptors, which together result in the dampening of the pro-inflammatory immune responses present in the diseased CNS (Ribeiro et al., 2013). This theory is supported by research indicating clozapine is an effective immunomodulatory agent capable of altering T-cell differentiation and cytokine expression (Chen et al., 2013, 2012, 2011), and it is also capable of altering cytokine expression following whole blood stimulation (Himmerich et al., 2011). It was clozapine's capacity to act on a range of neuro-immune receptors and to facilitate immunomodulation that lead to the question of whether clozapine may have therapeutic effects in other CNS diseases that have a strong inflammatory component.

To investigate the impact of atypical antipsychotics on the immune-mediated CNS disease MS, our group tested clozapine in an animal model of MS. We found that clozapine could ameliorate disease severity in a dose-dependent manner by inhibiting CNS infiltration (Green et al., 2017; O’Sullivan et al., 2014). This work also further characterized clozapine's immunomodulatory effects by showing its ability to modify bone marrow-derived macrophage responses in vitro and determining that the activity was independent of type 2 dopaminergic antagonism (O’Sullivan et al., 2014).

Following on from the work in the animal model, our group conducted a small clinical trial to determine the safety of clozapine in patients with progressive MS (La Flamme et al., 2020). The trial outcome concluded that administering clozapine to MS participants based on guidelines for schizophrenia was not suitable for people with progressive MS. All participants were withdrawn from the trial. We observed increased sensitivity to the drug, even at low doses. We concluded that the drug reaction observed in the participants may be due to changes in the drug targets (i.e. dopamine receptors) due to the underlying MS pathology. 
Previous work has shown that DR are expressed by all peripheral blood leukocytes (Arreola et al., 2016; Mikulak et al., 2014; Beaulieu and Gainetdinov, 2011; Beaulieu et al., 2015). However, most studies have been limited by their use of polyclonal antibodies and or low-resolution techniques. For monocytes, expression of all DR subtypes except D1 has been demonstrated (McKenna et al., 2002) with limited mention in published literature. McKenna et al. report that DR expression on monocytes was found to be very low. Lack of detection does not preclude the presence of a receptor, as the lack of detection of a receptor may be due to technical limitations. There is also some uncertainty in other DR expression reports, especially with regard to D1 expression at protein level in human PBMC (Arreola et al., 2016). As such the complete expression pattern of DR in PBMC remains unclear, and how this expression changes during MS is also not known.

There is a lack of information on the effects of clozapine on cytokine expression, especially by innate immune cells of the periphery. Cytokines, alongside neurotransmitters such as dopamine, have the potential to signal between the CNS and immune system. Furthermore, evidence in the literature links cytokines and neurotransmitters in relation to their actions in the CNS (Sarkar et al., 2010). It therefore becomes important to clarify clozapine's effects on DR and cytokine expression. As we undertake a repurposing of clozapine in the MS setting based on its ability to modulated DR signalling, we support this approach with our work to date (La Flamme et al., 2020), and evidence in the literature suggesting the potential benefit of dopaminergic drugs for the treatment of MS (Marino and Cosentino, 2016). To further contribute to an understanding of the immunomodulatory nature of clozapine and to elucidate how responsiveness to clozapine changes during MS, this chapter focusses on monocytes, a key innate immune cell in the periphery. 


\subsection{Aims.}

This chapter investigates the effect of low dose clozapine on the innate immune response in vitro. This study is focussed on monocytes with the expectation that these cells will allow us to determine whether clozapine alters innate cell cytokine responses and DR expression. The work is based on the hypothesis that low dose clozapine, a known immunomodulatory agent and neuroreceptor antagonist, will have a modest but demonstrable effect in reducing pro-inflammatory cytokine production and altering DR expression in PBMC in vitro. We further posit that the pro-inflammatory nature and immune activation inherent in MS pathogenesis may result in differential DR and cytokine profiles in our untreated MS cohort when compared to a group of matched healthy controls, and that clozapine exposure in vitro will result in differential responses.

\subsubsection{Specific aims.}

4.2.1.1 Assess the baseline expression pattern for D1, D2, D3, D4 and D5 on monocytes from $\mathrm{HC}$ and MS participants, and investigate if in vitro clozapine treatment alters their expression.

4.2.1.2 Determine the effect of clozapine on innate immune cytokine responses in invitro-treated monocytes by profiling IL-1 $\beta$, IL-6, IL-10, TNF $\alpha$ and INF $\gamma$.

4.2.1.3 Compare DR and cytokine expression of monocytes from $\mathrm{HC}$ and MS participants to establish if these measures are altered by MS pathology, and if MS participants have a differential response to clozapine therapy in vitro compared to healthy controls. 


\subsection{Methods.}

\subsubsection{Study participants.}

The study comprised two cohorts: a progressive MS group and a healthy group of ageand sex-matched individuals. PBMC were collected fresh and stored in liquid nitrogen until use (see Chapter 3). All MS participants were screened and enrolled as previously described (Luckey et al., 2015; La Flamme et al., 2020), and had either primary or secondary progressive disease with no significant confounding comorbidities and were not receiving any disease-modifying therapies. Subjects selected were from two clinical trial cohorts: the CRISP trial, which included healthy individuals and progressive MS ( $n=9$ /group), and the MIS416 Phase $2 b$ trial, which included only secondary progressive MS ( $\mathrm{n}=18$ selected) (see 3.3.1, demographic data). From the total MIS416 trial cohort $(n=100)$, we selected from participants who were recruited in NZ ( $\mathrm{n}=28$ available). The MIS416 participants were included to increase our analysis cohort, and we ensured age and gender were matched to the CRISP cohort.

\subsubsection{Ethical approval.}

Full ethical approval was in place before we commenced any experiments, with the CRISP trial being approved by the Central Health and Disability Ethics Committee (15/CEN/216) and the Standing Committee on Therapeutic Trials (15/SCOTT/177), and the MIS416 Phase 2b trial approved by the Uniting Care Health Human Research Ethics Committee, Western Australia (MIS416-202).

\subsubsection{Experimental procedures.}

The in vitro protocol for PBMC sample processing was standardised for all samples and detailed in Chapter 3 (see section 3.2.1.1 - 3.2.15). Briefly, individual donor PBMC were thawed, DNase treated and revived under standard conditions $\left(37^{\circ} \mathrm{C} ; 5 \% \mathrm{CO}_{2}\right)$ for 90 minutes in complete media. An aliquot was separated out (Baseline (To)), viability stained, fixed and stored for later batch antibody staining. The remaining 
cells were stimulated overnight with either clozapine (40 $\mathrm{nM})$, LPS ( $1 \mathrm{ng} / \mathrm{ml})$, both LPS and clozapine, or left untreated. An equal proportion of cells were incubated separately for DR expression, while another was incubated in the presence of a brefeldin A $(0.5 \mu \mathrm{l} / \mathrm{ml})$ \& monensin $(0.3 \mu \mathrm{l} / \mathrm{ml})$ mixture to block cytokine secretion. Following culture, cells were viability stained, fixed, batch stained with an antibody cocktail, and then washed. The brefeldin/monensin treated cells for cytokine staining were permeabilised using Cytoperm (BD, USA) just prior to antibody staining. Data were acquired on a Cytek Aurora spectral cytometer the next day.

All culture aliquots received an identical lineage marker stain (see Table 3-1, PBMC lineage stain). Additionally, cells cultured for DR expression were split, with half stained for D1,2\&5 (Table 3-1, Tube 1) and half for D3\&4 (Table 3-1, Tube 2) while cells cultured for cytokine expression were stained for cytokine reporters (Table 3-1, Tube 3). All cytometry data were gated using a common PBMC gating strategy to yield terminal monocytes (Figure 3-2).

\subsubsection{Clozapine reconstitution and storage.}

Dry clozapine (Medichem, SA, Spain) was kindly gifted by Douglas Pharmaceuticals Limited (Auckland, New Zealand), and stored sealed at $4{ }^{\circ} \mathrm{C}$ protected from light. A fresh aliquot of drug was reconstituted in o.1 M acetic acid (Sigma, St Louis, US) and once prepared, stored for no longer than two days at $4{ }^{\circ} \mathrm{C}$ protected from light.

\subsubsection{Monoclonal antibody conjugates for DR receptors.}

The DR expression profile on human PBMC is not well established; therefore, we procured monoclonal DR conjugates from reputable suppliers. Table 4-1 details the conjugate, clone and supplier details for each of the five antibodies used to profile DR expression. All antibodies were used undiluted and at the manufacturer's recommended concentration. 
Table 4-1 Dopamine receptor antibodies.

\begin{tabular}{|c|c|c|c|c|c|c|}
\hline$\underline{\text { Name }}$ & $\begin{array}{c}\text { Supplier } \\
\text { Reference }\end{array}$ & $\underline{\text { Clone }}$ & $\underline{\text { Supplier }}$ & $\underline{\underline{\text { Conjugate }}}$ & $\underline{\underline{\text { O.5x 10E6 cells }}}$ & Concentration \\
\hline D1 & 366404 & L205G1 & Biolegend & PE & $1.5 \mathrm{ul}$ & $200 \mu \mathrm{g} / \mathrm{ml}$ \\
\hline D2 & SC-5053 & B-10 & Santa Cruz & AF488 & $1.5 \mathrm{ul}$ & $200 \mu \mathrm{g} / \mathrm{ml}$ \\
\hline D3 & SC-136170 & 9F4 & Santa Cruz & AF647 & $1.5 \mathrm{ul}$ & $200 \mu \mathrm{g} / \mathrm{ml}$ \\
\hline D4 & SC-136169 & 2B9 & Santa Cruz & AF488 & $1.5 \mathrm{ul}$ & $200 \mu \mathrm{g} / \mathrm{ml}$ \\
\hline D5 & FAB82861V & 889022 & R\&D Systems & AF405 & $2.5 \mathrm{ul}$ & $100 \mu \mathrm{g} / \mathrm{ml}$ \\
\hline
\end{tabular}

4.3.3.3 Fluorescence minus (FMO) control of DR receptor expression.

We included FMO controls to ensure positive staining (expression) for each DR receptor. 


\subsubsection{Statistics.}

Statistical analyses were performed in Prism 8 (Graphpad, US). All data distributions were tested for normality, and log transformed where necessary with negative values log transformed using a correction factor. Normally distributed data was subject to an ordinary one-way analysis of variance test (ANOVA), or Mixed Effect test, with Geisser-Greenhouse post-test. Data failing normality testing was subject to a repeat measure Kruskal-Wallis test with Dunn's multiple comparison test.

To correct for multiple comparisons, we used Tukey's multiple comparison test. We applied correction but only to inter treatment group comparisons. No correction was applied in comparing stratified population differences. This approach may be less stringent but was chosen to enable future hypothesis generation.

Direct comparisons between two cohorts was performed using t-tests of the calculated mean and standard deviation (SD), except for gender distributions in the population which was by Fisher's test. Baseline corrected data was expressed as the percentage change from untreated cells, with the mean group values compared using the MannWhitney test.

In all instances p values that were at $\leq 0.05$ was taken as statistically significant, while results close to the threshold were interpreted as indicative of a 'trend' so as not to exclude potentially meaningful results.

Graphically depicted group averages are plotted as mean values, and error bars represent SD. 


\subsection{Results.}

\subsubsection{Participant demographics.}

We assessed in vitro-stimulated monocytes in PBMC cultures from two clinical trial cohorts comprising untreated progressive MS participants. Concurrently, we compared responses in PBMC cultures from an age- and sex-matched group of healthy control participants recruited locally from the Wellington region (Table 4-2).

Table 4-2 Demographic distribution of study participants.

\begin{tabular}{|c|c|c|c|}
\hline \multicolumn{4}{|c|}{ Study Population } \\
\hline Healthy & & & \\
\hline & $\mathrm{N}$ & & 9 \\
\hline & Age & & \\
\hline & & Mean & 54.33 \\
\hline & & Range & $46-66$ \\
\hline & Gender & $\mathrm{F}: \mathrm{M}(\% \mathrm{~F})$ & $7: 2(77.8)$ \\
\hline MS & & & \\
\hline & $\mathrm{N}$ & & 27 \\
\hline & & CRISP & 9 \\
\hline & & MIS416 & 18 \\
\hline & Age & & \\
\hline & & Mean & 56.07 \\
\hline & & Range & $35-70$ \\
\hline & Gender & $\mathrm{F}: \mathrm{M}(\% \mathrm{~F})$ & $\begin{array}{r}21: 6 \\
(77.8)\end{array}$ \\
\hline
\end{tabular}




\subsubsection{Clozapine in vitro dose optimisation.}

In schizophrenia, clozapine is administered to achieve a target dose of 300-450 $\mathrm{mg} /$ day but may be given at up to $900 \mathrm{mg}$ to achieve clinical response (Medscape, 2019). Post-dose therapeutic monitoring for clozapine ranges from between 0.1 - 1.0 $\mu \mathrm{g} / \mathrm{ml}$ (SAHealth, 2019) in blood plasma. Higher results of over $1.5 \mu \mathrm{g} / \mathrm{ml}$ can be considered toxic and assay values are generally reduced by up to $10 \%$ in whole blood measurements (Flanagan et al., 2003). Doses upward of at $0.1 \mu \mathrm{g} / \mathrm{ml}$ (306 nM) have been shown to reduce viability in PBMC cultures (Chen et al., 2012a, 2013, 2011) while doses higher than $100 \mu \mathrm{g} / \mathrm{ml}$ are effectively toxic to PBMC cultures (Hinze-Selch et al., 1998).

Our experiments aimed to investigate the effects of low dose clozapine on innate immune activation and expression of DR. Therefore, by using low doses in in vitro PBMC cultures, we endeavored to understand the potential effects of low dose in vivo clozapine administration on PBMC from participants enrolled in the CRISP study (starting dose of $5 \mathrm{mg} /$ day).

To establish whether clozapine negatively impacted cellular viability or cellular metabolic activity, we tested the impact over a range of doses by MTT assay and cellular viability by flow cytometry. PBMC were cultured using our optimised in vitro conditions (see 3.2.1) over a clozapine or risperidone dose range of $20 \mathrm{nM}-200 \mathrm{nM}$ or media alone, and cells were harvested after an overnight incubation. We included risperidone, another atypical antipsychotic agent, as a comparative control. PBMC were gated for single events and CD45 expression, and viable events were calculated as the relative proportion in the CD45 gate (Figure 4-1 A).

Using a single donor, we observed that clozapine doses of $>40 \mathrm{nM}$ negatively affected PBMC viability in vitro (Figure 4-1). MTT data (kindly supplied by Dr Vimal Patel), corroborated the loss in viability, as doses up to $40 \mathrm{nM}$ modestly increased metabolic activity before dropping away at higher doses. Comparatively, risperidone had a very modest change on cellular MTT readouts and no impact on viability. A repeat experiment with a second donor showed similar results. We concluded that $40 \mathrm{nM}$ was an effective dose for our in vitro setup. 

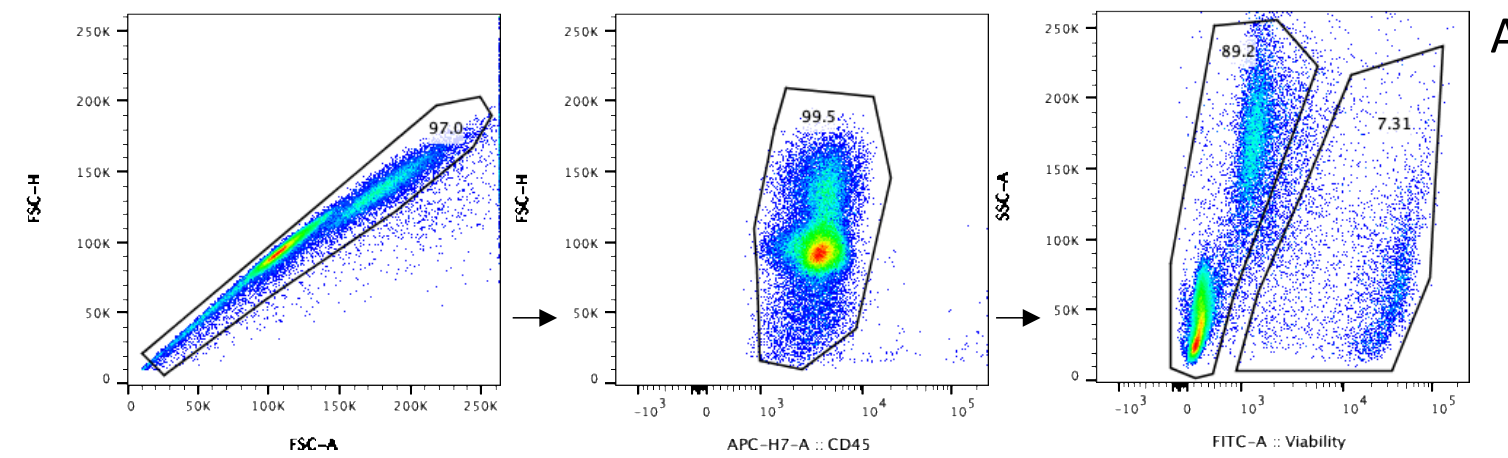

A

B

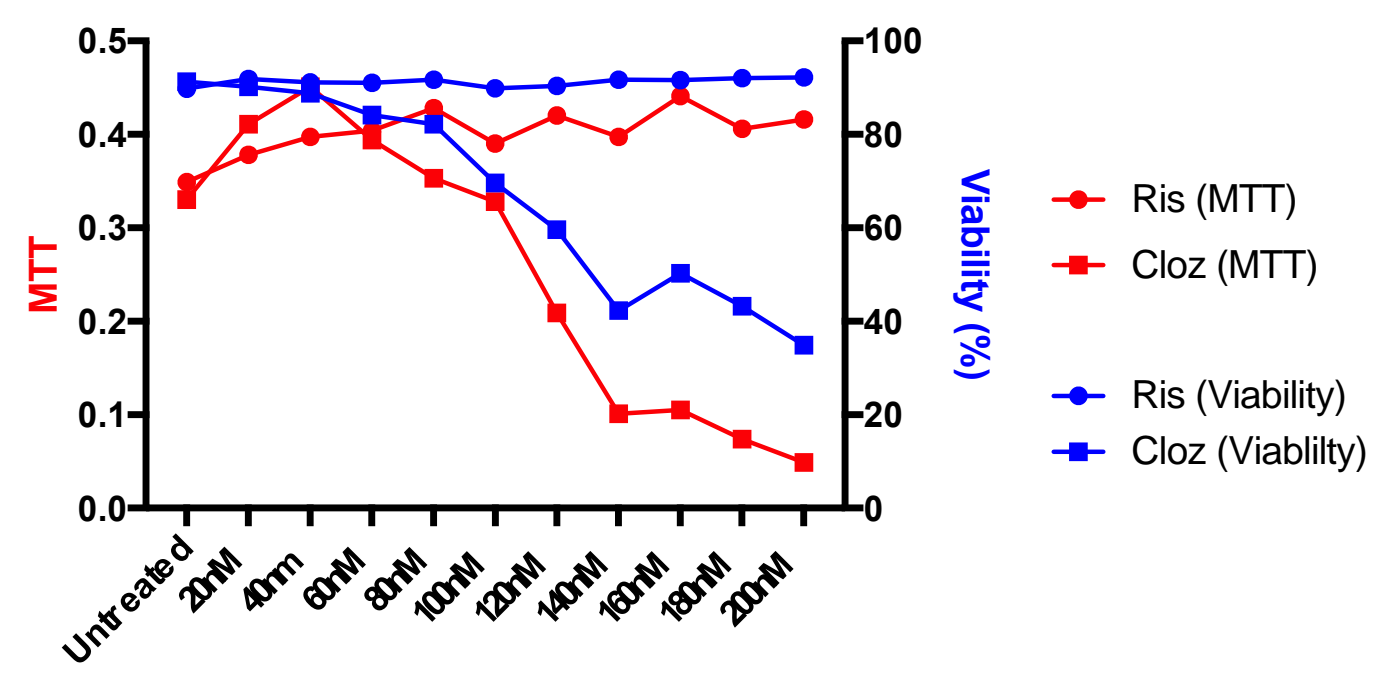

Figure 4-1 Effect of in vitro clozapine on PBMC viability.

PBMC gating strategy (Panel A). Viability was assessed cytometrically as the \% positive PBMC events from a viability stain, or by MTT assay (supplied data) over a 20-200nM dose range from overnight culture. PBMC were treated for clozapine and risperidone separately or left untreated (Panel B). Risperidone showed no change in viability, but an increase in activation (MTT), at higher doses. 


\subsection{DR expression patterns of in vitro cultured monocytes.}

Our data profiled monocytic responses using multiparameter flow cytometry to delineate immune subsets, using specific, pre-conjugated monoclonal targets. We further included cytokine response data to complement and elaborate on how immune activation affects DR expression, and how DR antagonism by clozapine effects innate immune modulation.

In this section, DR expression is inferred from the geometric mean fluorescence intensity (gMFI) of the positive staining monocyte population (Figure 4-2). While gMFI is not a direct indication of the amount of DR expressed, is follows that higher levels of expression of a given epitope allows for a greater amount of antibody binding, and the proportional increase in the signal from the fluorescent emission of that antibody conjugate. As the data here represent only cells that stained positive, we can conclude that changes in gMFI relate to altered levels of DR expression. We further compared the expression patterns between $\mathrm{HC}$ and MS participants to determine what differences MS disease processes have on DR expression.

\subsubsection{Baseline (To) DR expression.}

Baseline cells (To) were sampled prior to in vitro culture and were used to represent the in vivo state of DR expression. We compared baseline (To) expression of all DR in monocytes for all participants, observing that the total proportion of positive staining monocytes did not vary significantly for D1-4, with essentially all monocytes (>98\%) staining positive (data not shown). However, in MS participants, D5 expression was detected in only $63 \%$ of To monocytes, while in HC the value was $43 \%$ indicating that MS participants had significantly more monocytes expressing D5 at To when compared to the HC ( $\mathrm{p}=$ 0.047) (Figure 4-3). Furthermore, an inter cohort comparison of the total amount of DR expressed at To did not vary significantly, with the group average levels of D1-5 being similar for both cohorts (Figure 4-4 A-E). We did however notice a trend in D3 expression, whereby a comparison of D3 expression between $\mathrm{HC}$ and MS participants indicated a lower overall expression in the MS cohort that was just short of the definition of significant $(p=0.066)($ Figure 4-4 C). 


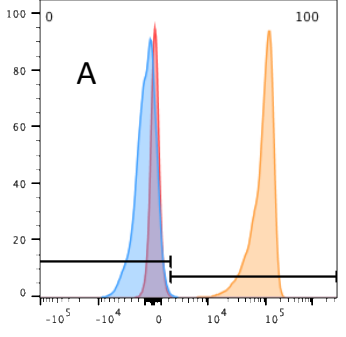

DR1 PE

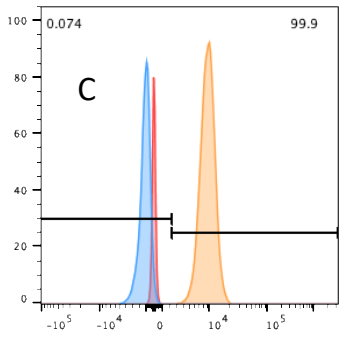

DR3 AF488

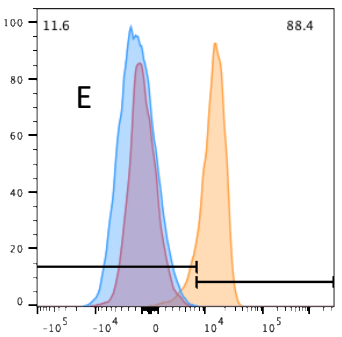

DR5 AF405

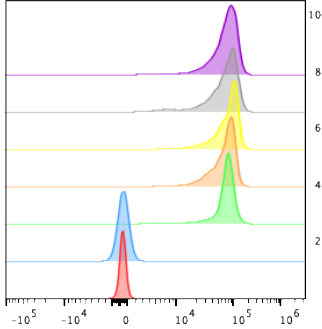

DR1 PE

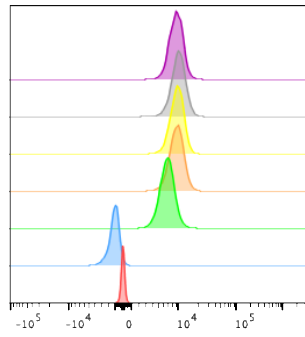

DR3 AF488

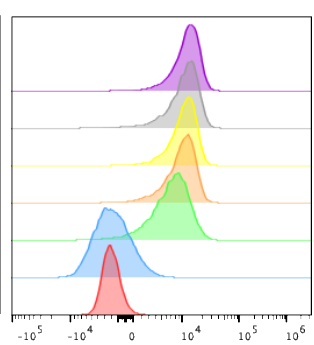

DR5 AF405

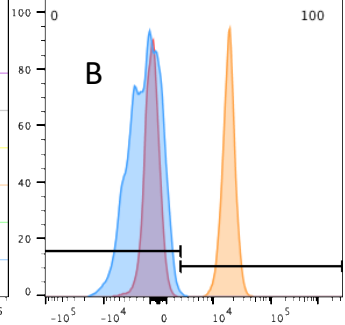

DR2 AF488

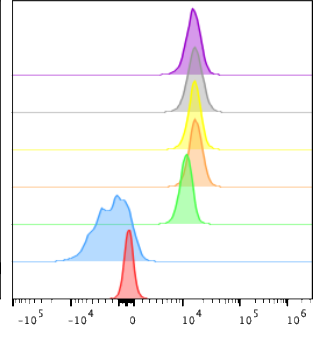

DR2 AF488

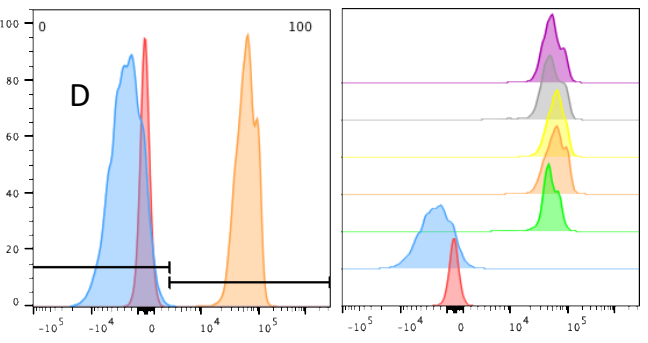

DR4 AF647

DR4 AF647

Figure 4-2 The expression of $D R$ in monocytes for all in vitro conditions.

The data is representative of a participant sample. Population colours for each different condition: unstained control(red), FMO control (blue), To baseline (green), T24 untreated (orange), T24 clozapine (yellow), T24 LPS (grey), T24 LPS\&cloz (purple). Black bisecting gate delineates positive staining events (right), from negative staining cells (left). A-E depict D1 - D5 respectively. Monocyte were gated as demonstrated in Figure 3-2. 


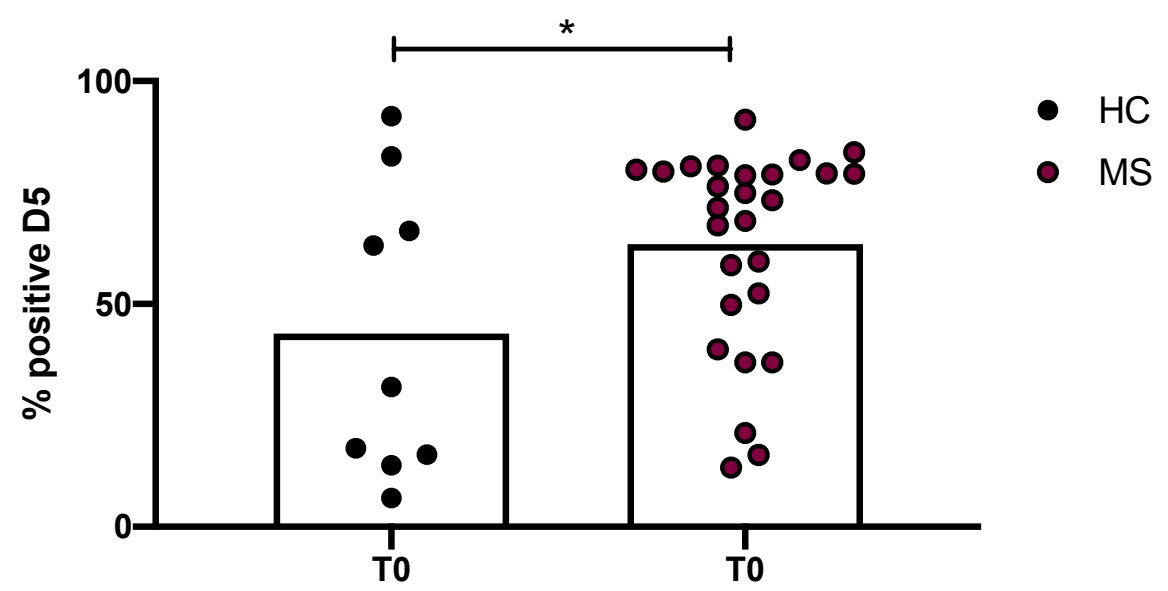

Figure 4-3 Baseline (To) proportion of D5 positive monocytes.

$*(p \leq 0.05)$ 
A

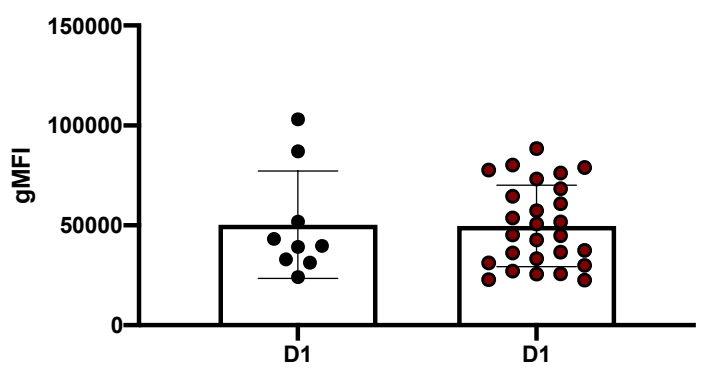

C
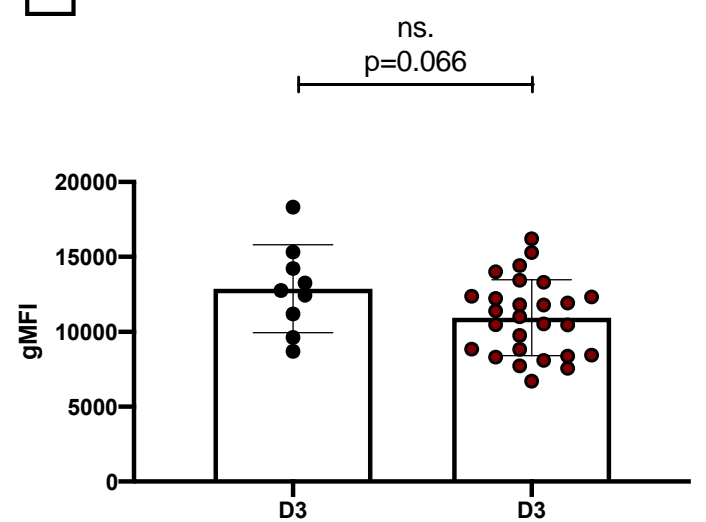

E

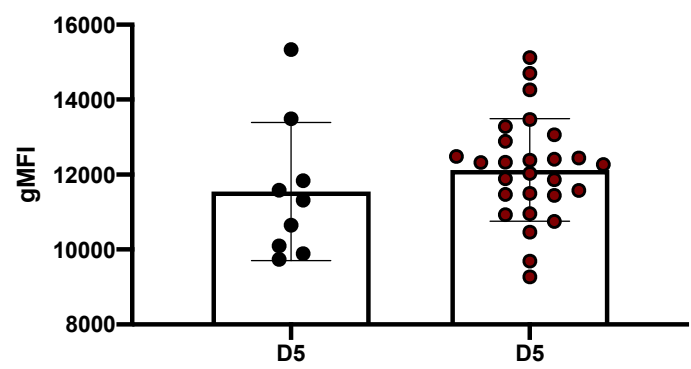

B

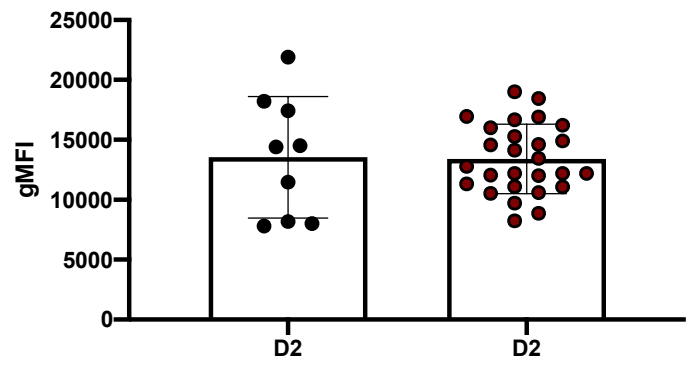

D

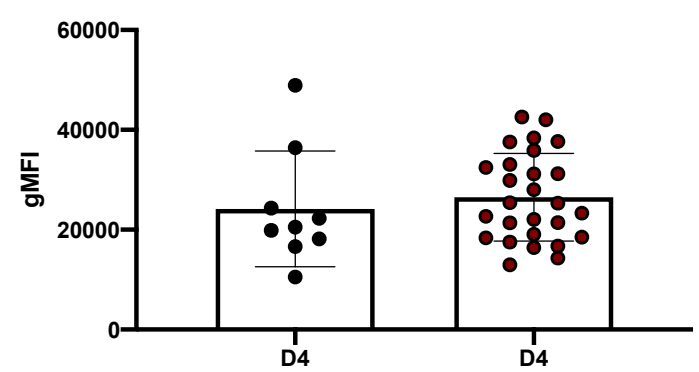

- $\mathrm{HC}$

- MS

Figure 4-4 Baseline (To) DR expression of D1-5 in HC and MS participants.

Expression as gMFI. (A-E) Bars indicate mean values with error bars indicating standard deviation. ${ }^{*}(\mathrm{p} \leq 0.05),{ }^{* *}(\mathrm{p} \leq 0.01),{ }^{* * *}(\mathrm{p} \leq 0.001),{ }^{* * * *}(\mathrm{p} \leq 0.0001)$ 


\subsubsection{Class 1 dopamine receptor expression - D1.}

4.5.2.1 Overnight incubation of PBMC resulted in the upregulation while LPS induced a downregulation of D1 expression on monocytes.

When comparing To against cells incubated overnight with media alone (T24 untreated), we observed that there was a significant upregulation of D1 expression in monocytes in the MS cohort ( $\mathrm{p}<0.0001$ ) (Figure 4-5 C). This upregulation was not reflected in the HC data (Figure 4-5 A). In LPS stimulated cells from MS patients, a significant decrease of D1 expression by monocytes resulted as compared to untreated cells (Figure 4-5 C) ( $\mathrm{p}=0.0045)$. This effect was more variable in $\mathrm{HC}$ and did not reach significance.

4.5.2.2 Clozapine treatment in vitro significantly upregulated D1 expression on monocytes.

To establish the effect of clozapine on D1 expression, we compared T24 untreated cells with those treated with clozapine (T24 clozapine). In both HC and MS cohorts, we observed the upregulation of D1 expression levels on monocytes treated with clozapine: HC ( $\mathrm{p}=0.0071$ ) (Figure 4-5 A), and MS participant ( $\mathrm{p}=0.0018$ ) (Figure 4$5 \mathrm{C})$. To determine the magnitude of the effect from the different culture conditions on monocyte D1 expression, the data were normalized to the T24 untreated samples and plotted to show the percentage change from untreated (Figure 4-5 D). This analysis highlighted the polarising effects on D1 expression from LPS and clozapine respectively. In particular, in cells that were co-cultured with LPS and clozapine (T24 LPS\&cloz), we observed that compared to LPS alone (T24 LPS), the addition of clozapine counteracted the downregulation of D1 from LPS stimulation. This effect restored expression levels back to those seen in untreated cells in MS participants (LPS\&cloz compared to LPS alone; $\mathrm{p}=0.0003$ ). This effect did not reach significance in the HC.

4.5.2.3 No difference was observed in D1 expression between HC and MS participants but the magnitude of the change in the expression of D1 varied significantly.

We compared expression levels of D1 in monocytes from HC and MS participants directly (Figure 4-5 E) and observed that group averages did not vary to any degree of 
significance. To visualise the response differential between HC and MS participants we compared the percentage change as normalised to the T24 untreated data between the cohorts (Figure 4-5 F). This comparison revealed the significant variation in response to in vitro culture for our simulation conditions, with significantly different responses between the cohorts. From these data, we conclude that in individuals with MS, D1 expression may be less well regulated. 

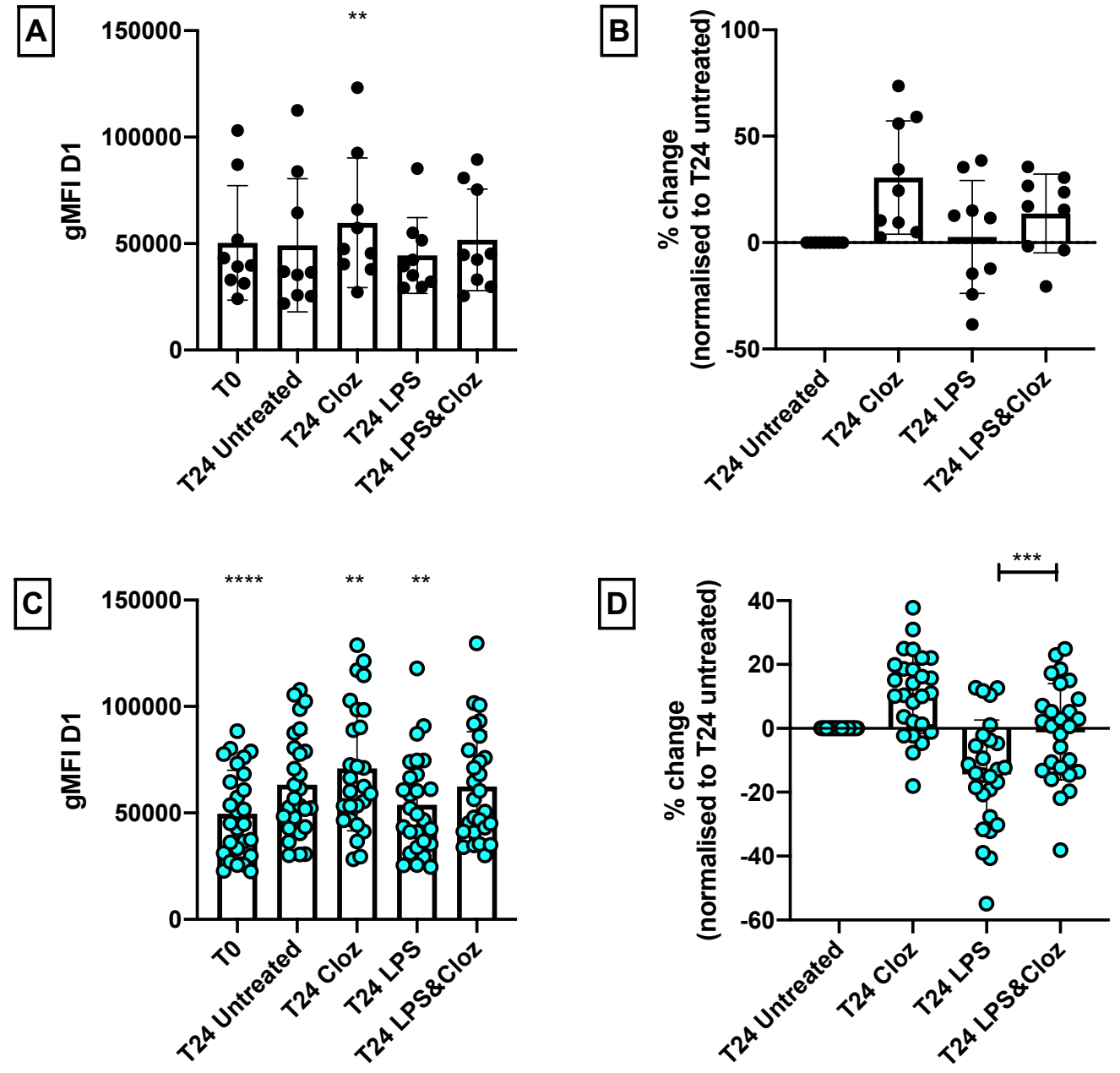

E

$\mathbf{F}$
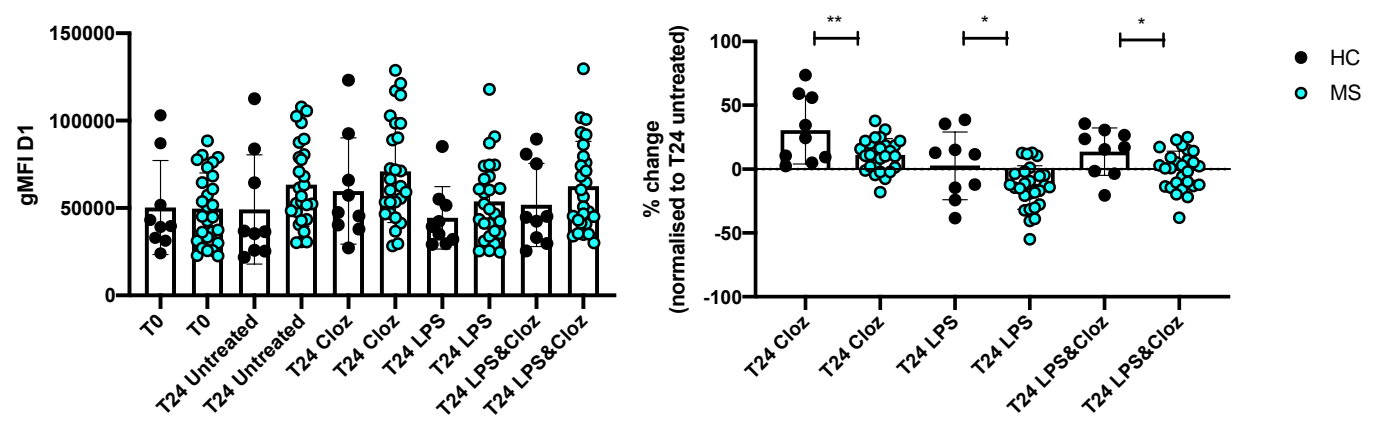

Figure 4-5 D1 expression on monocytes of all FMO positive gated cells.

Healthy control D1 expression (A) and percentage change from T24 untreated (B). MS patient D1 expression (C) and percentage change from T24 untreated (D). HC vs. MS D1 expression (E) and percentage change from T24 untreated (F). Bars indicate mean values with error bars indicating standard deviation. Unless otherwise indicated, statistically significant differences $\left(^{*}\right)$ are depicted for the calculation against the T24 untreated group. ${ }^{*}(\mathrm{p} \leq 0.05),{ }^{* *}(\mathrm{p} \leq 0.01),{ }^{* * *}(\mathrm{p} \leq 0.001),{ }^{* * * *}(\mathrm{p} \leq 0.0001)$. 


\subsubsection{Class 1 dopamine receptor expression - D5.}

D5 expression at RNA levels is ubiquitous (BioGPS, 2019), but evidence of detection at protein level is limited to specific tissues such as the neuronal structures of the limbic regions of the brain (ProteinAtlas, 2019). Unlike D1-4, we were not able to detect D5 expression on all monocytes from all conditions. Therefore, only cells where D5 expression was conclusively shown, using an FMO control to delineate positive staining events, were included in the analyses (Figure 4-2 E).

4.5.3.1 Overnight incubation in the presence or absence of LPS significantly upregulated D5 expression on monocytes.

At T24 untreated compared to To, a significant increase in the expression of D5 on monocytes was observed for both cohorts (HC: $\mathrm{p}=0.0155 ; \mathrm{MS}: \mathrm{p}<0.001$; corrected) (Figure 4-6 A\&C). After LPS treatment, we observed a significant upregulation in D5 expression compared to untreated monocytes ( $\mathrm{p}<0.0001)$ in the MS cohort (Figure 4-6 C). This effect was not found in HC cohort.

4.5.3.2 Exposure to clozapine in vitro had differential effects on D5 expression on monocytes from $\mathrm{HC}$ and MS patients.

In contrast to D1, the presence of clozapine alone did not alter D5 expression on monocytes from HC or MS patients. Moreover, clozapine did not abrogate the LPSinduced increase in $\mathrm{D}_{5}$ on monocytes from the MS patients, while it reduced D5 expression on LPS-stimulated monocytes for the HC.

4.5.3.3 Inter cohort comparison did not indicate a strong difference in D5 expression in the magnitude of change between the in vitro conditions.

In comparing $\mathrm{HC}$ and MS participant expression data (Figure 4-6 E), we noted no significant differences in the means between the conditions. While the expression of D5 on MS monocytes in the LPS\&cloz condition appeared to be higher than the HC, this difference failed to reach the significant threshold $(\mathrm{p}=0.068)$. To better understand the differences in DR expression between HC and MS cohorts, we compared normalised data (Figure 4-6 F) but found no difference in the magnitude of expression between the cohorts. Overall, we observed that D5 expression levels were 
only modestly affected in the HC, with MS participants showing strong responses to LPS. Clozapine showed limited effects on D5 expression and only in the HC cohort. 
A

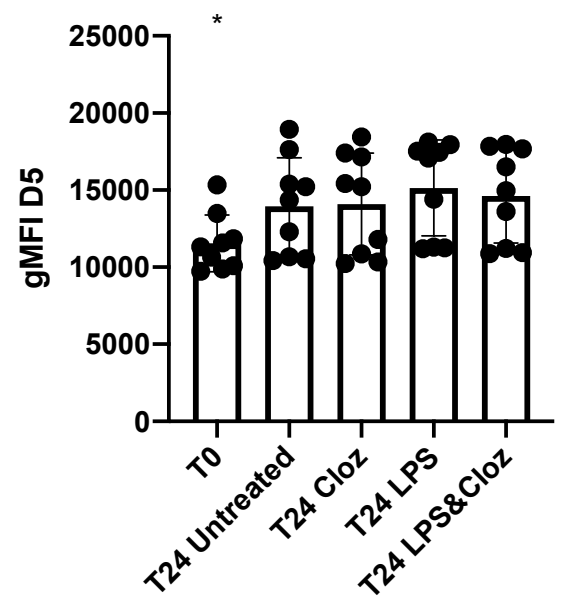

C

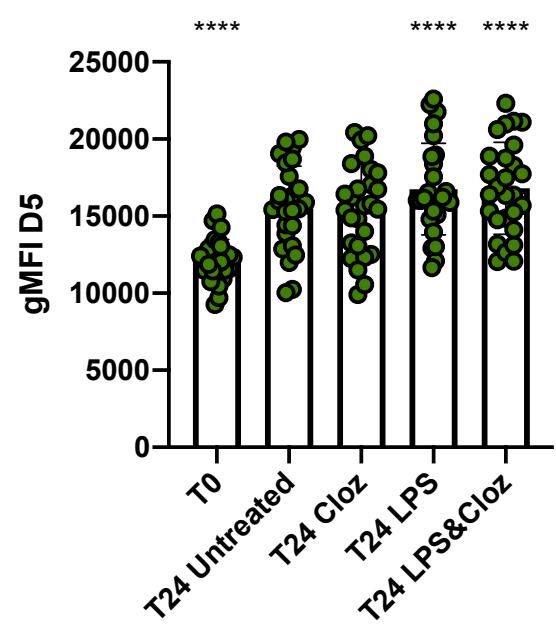

E

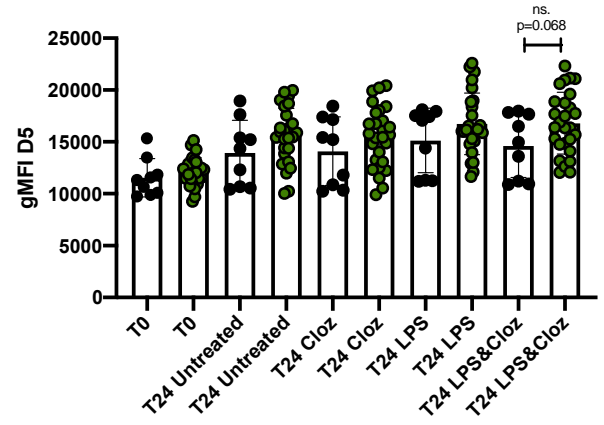

B

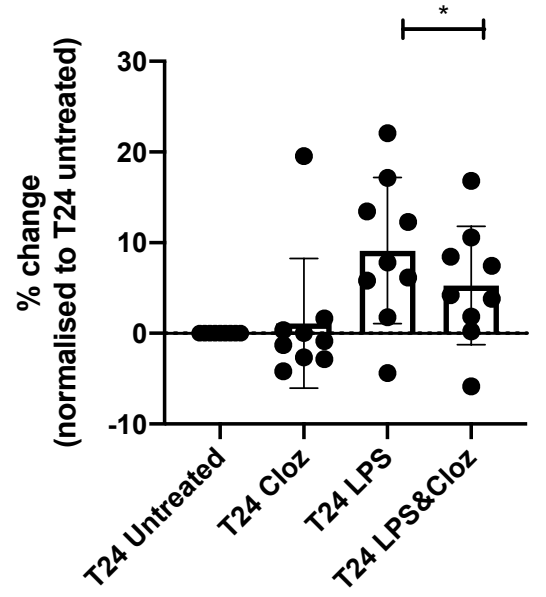

D

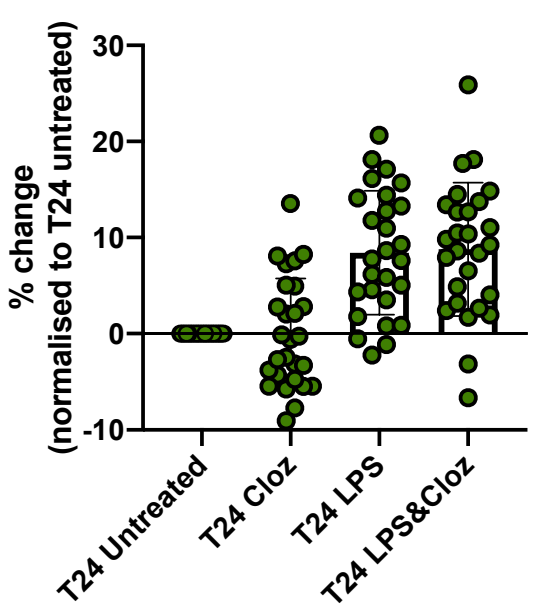

$\mathbf{F}$

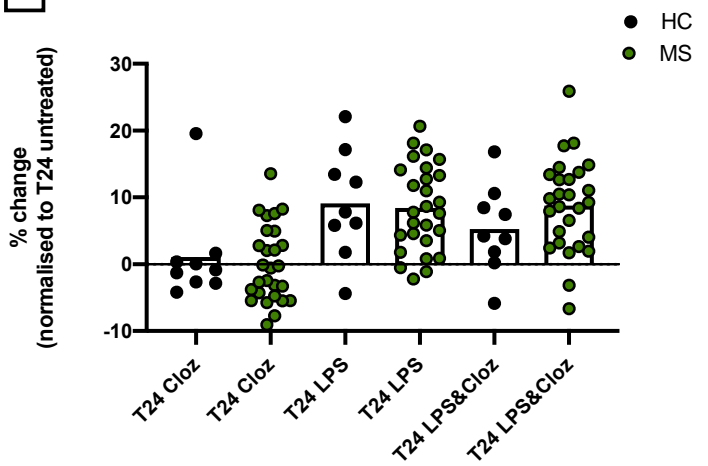

Figure 4-6 D5 expression on monocytes of all FMO positive gated cells.

Healthy control D5 expression (A) and percentage change from T24 untreated (B). MS patients D5 expression (C) and percentage change from T24 untreated (D). HC vs. MS D5 expression (E) and percentage change from T24 untreated (F). Bars indicate mean values with error bars indicating standard deviation. Unless otherwise indicated, statistically significant differences $(*)$ are depicted for the calculation against the T24 untreated group. ${ }^{*}(\mathrm{p} \leq 0.05),{ }^{* *}(\mathrm{p} \leq 0.01),{ }^{* * *}(\mathrm{p} \leq 0.001),{ }^{* * * *}(\mathrm{p} \leq 0.0001)$. 


\subsubsection{Class 2 dopamine receptor expression - D2.}

Class 2 DR are prominently expressed in the vertebrate CNS and are a common drug target for most antipsychotic drugs. D2 is much more abundant compared to other class 2 receptors (EBI, 2019), and it is the major receptor target for atypical antipsychotics, with the exception of clozapine, which has higher affinity for D4.

4.5.4.1 D2 expression was upregulated after overnight incubation but not by LPS or clozapine alone.

In MS participants, the expression of D2 on monocyte was significantly increased following an overnight incubation ( $\mathrm{p}<0.0001$ ) (Figure 4-7 C) while the increase did not reach significance in HC (Figure 4-7 A). Neither LPS nor clozapine alone altered D2 expression on monocytes from MS or HC cohorts compared to untreated monocytes. However, we did observe that co-culture with both LPS and clozapine led to a reduction in D2 expression compared to untreated in MS ( $\mathrm{p}<0.0001)$ and compared to LPS alone in HC ( $\mathrm{p}=0.026)$ (Figure 4-7 A \& C) indicating that these agents may have a compounding effect on D2 when used together.

4.5.4.2 Untreated normalisation of D2 expression data suggests clozapine, in the presence of LPS, reduced D2.

To clarify the effects of the culture conditions on D2, we normalized the response to T24 untreated values. This analysis showed that neither LPS nor clozapine alone resulted in any significant change in D2 expression. When LPS and clozapine were combined, a significant difference was observed compared to LPS alone in MS participants ( $\mathrm{p}=0.0039$ ) (Figure 4-7 D), and in HC we observed the same trend, although data failed to reach the significance threshold ( $p=0.054$ ) (Figure 4-7 B).

There was no difference in the average gMFI when HC and MS participants were compared over the range of conditions (Figure 4-7 E). Having established that an overnight incubation of PBMC resulted in the upregulation of D2 expression in monocytes, we set out to determine if there was a difference in the magnitude of this upregulation. This indicated that the magnitude of the response to co-culture was similar in both cohorts (Figure 4-7 F). Taken together, our data indicates that D2 expression is reduced modestly by combined exposure to LPS and clozapine in culture. 

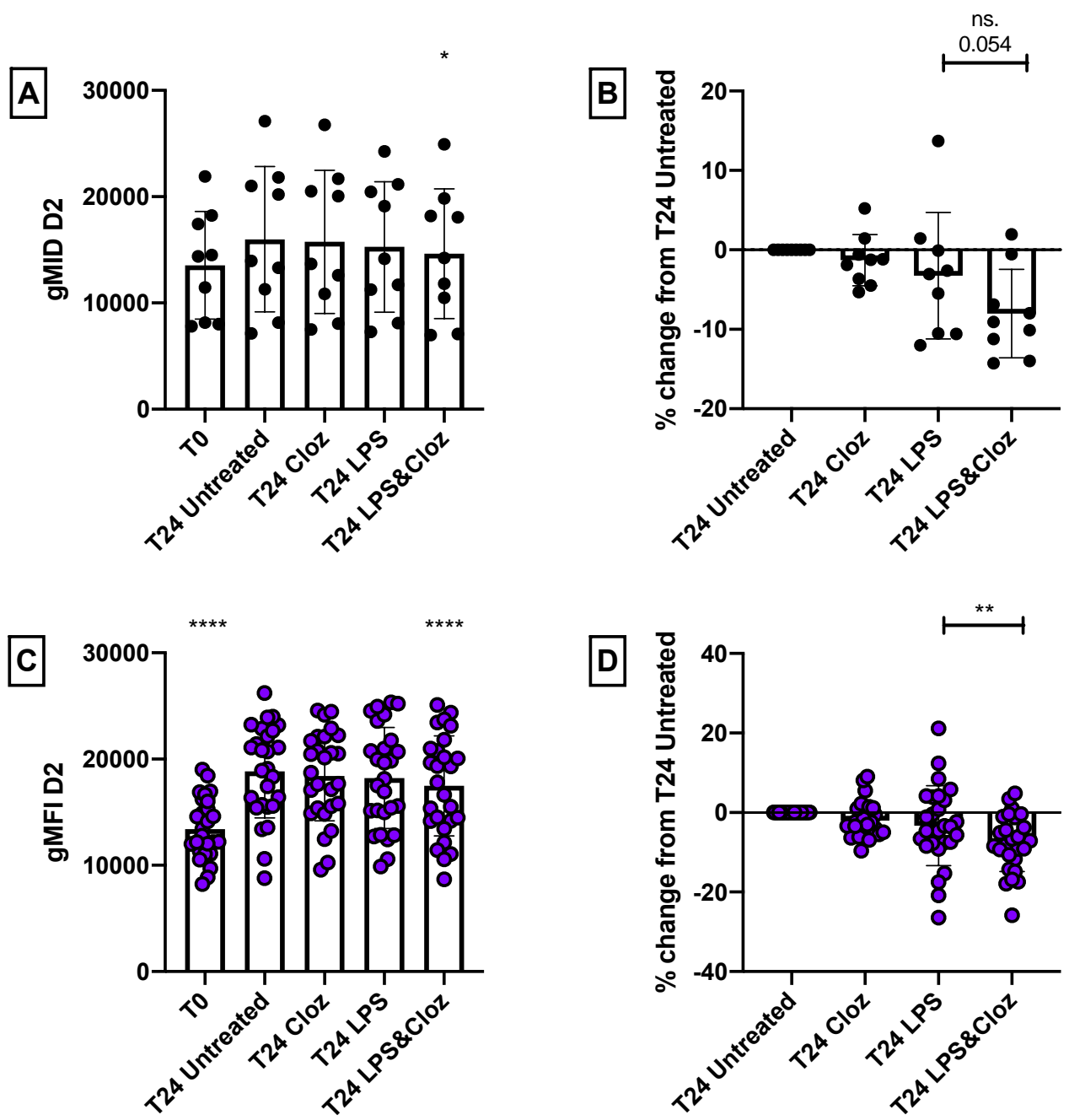

E

$\mathbf{F}$
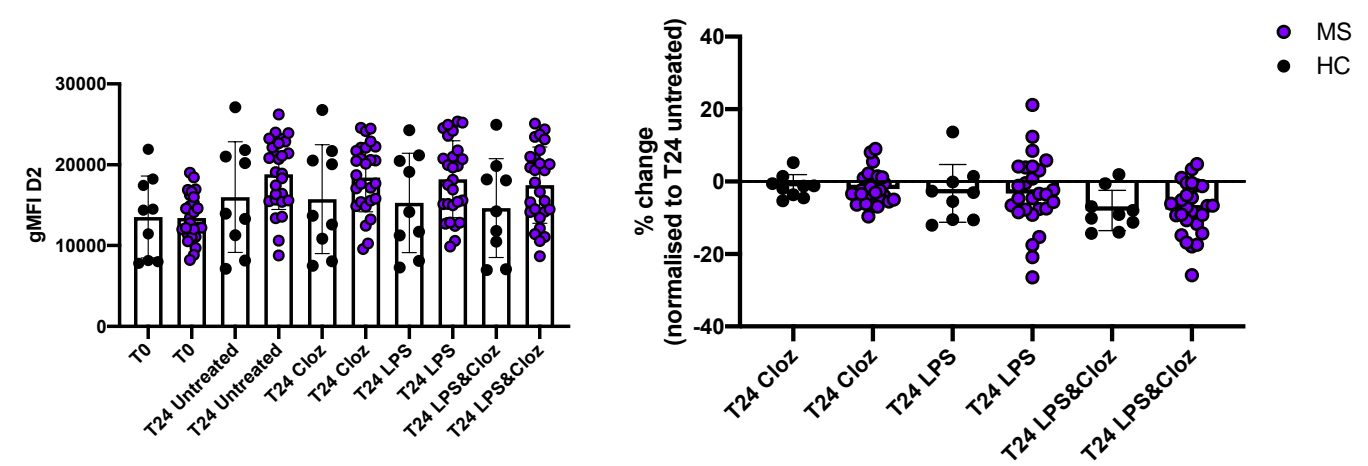

Figure 4-7 D2 expression on monocytes of all FMO positive gated cells.

Healthy control D2 expression (A) and percentage change from T24 untreated (B). MS patients D5 expression (C) and percentage change from T24 untreated (D). HC vs. MS D5 expression (E) and percentage change from T24 untreated (F). Bars indicate mean values with error bars indicating standard deviation. Unless otherwise indicated, statistically significant differences $(*)$ are depicted for the calculation against the T24 untreated group. ${ }^{*}(\mathrm{p} \leq 0.05),{ }^{* *}(\mathrm{p} \leq 0.01),{ }^{* * *}(\mathrm{p} \leq 0.001),{ }^{* * * *}(\mathrm{p} \leq 0.0001)$. 


\subsubsection{Type 2 dopamine receptor expression - D3.}

4.5.5.1 D3 expression was upregulated on MS but not $\mathrm{HC}$ monocytes following in vitro culture in the presence or absence of LPS.

The average expression of D3 on HC monocytes over the range of culture conditions did not vary significantly from the values observed at baseline (To) (Figure 4-8 A). In contrast, the level of D3 expression on MS monocytes was significantly increased in T24 untreated cells compared to To expression ( $\mathrm{p}<0.0001$ ) (Figure 4-8 C), and in LPS-stimulated monocytes, D3 was further increased compared to T24 untreated ( $\mathrm{p}=$ 0.0289).

4.5.5.2 The effect of clozapine on D3 was detected in co-culture with LPS.

Although the addition of clozapine to cultures stimulated with LPS or untreated, did not alter the expression level of D3 in either cohort, we normalized the expression data to untreated values to assess whether clozapine altered the magnitude of change. In both cohorts we observed that the presence of clozapine with LPS significantly decreased D3 expression compared to LPS alone, indicating a reduction in the magnitude of the response (MS: $\mathrm{p}=0.015$; HC: $\mathrm{p}=0.039$ ) (Figure 4-8 B\&D).

With D3 we observed only modest change in expression, with the strongest change being an upregulation in expression in MS patients after overnight incubation. LPS stimulation also resulted in the upregulation of D3 expression in MS, and it appears that the magnitude of the response to LPS may be somewhat abrogated by clozapine, which reduced expression to that observed on untreated cells. Furthermore, we observed a trend suggesting that MS participants had lower levels of D3 expression at baseline when compared to HC ( $\mathrm{p}=$ o.066). Together, these results suggest that MS monocytes may be more responsive to conditions that mediate changes in $\mathrm{D}_{3}$ expression. 
A

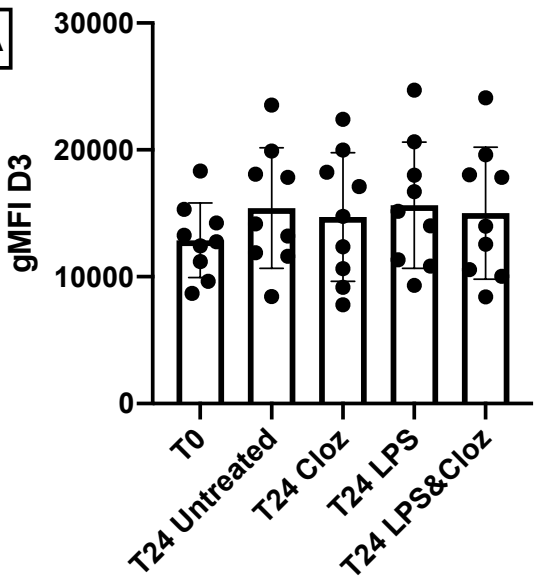

C

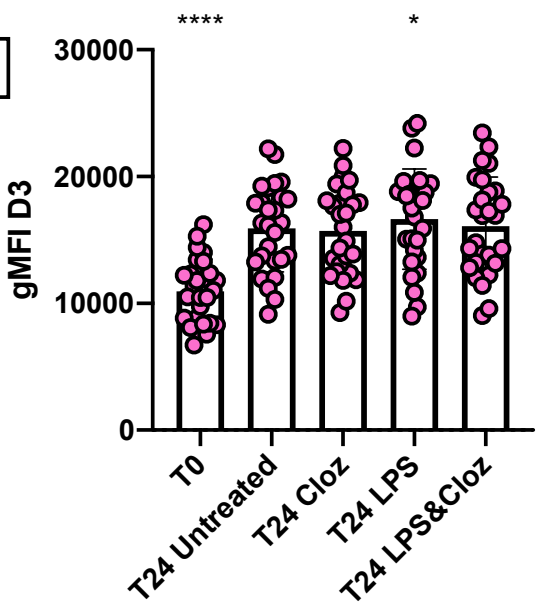

E

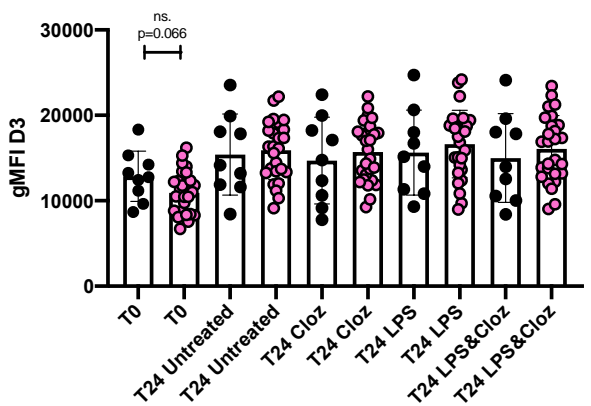

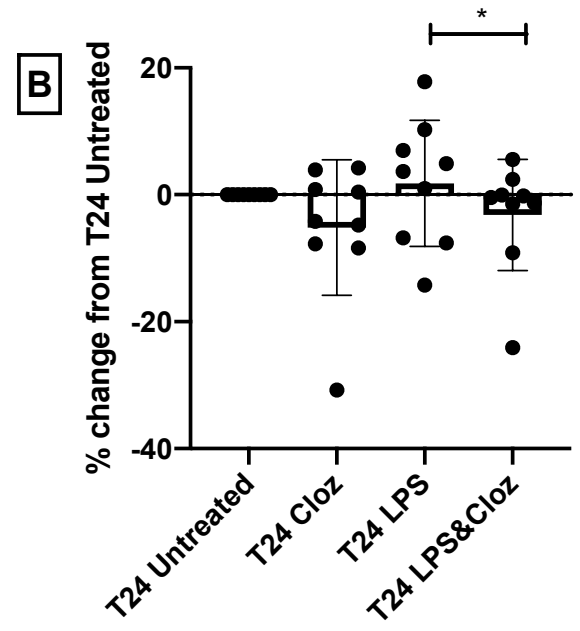

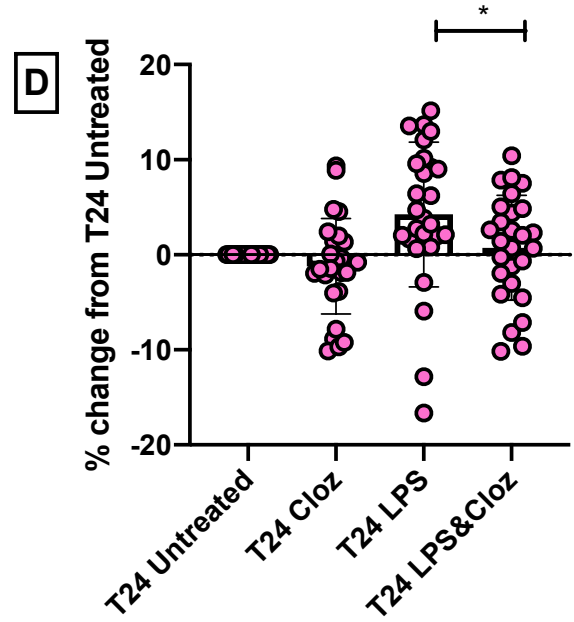

$F$

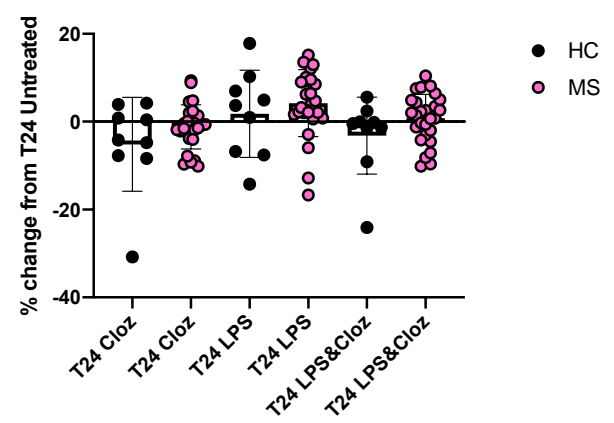

Figure 4-8 D3 expression on monocytes of all FMO positive gated cells.

Healthy control D3 expression (A) and percentage change from T24 untreated (B). MS patients D3 expression (C) and percentage change from T24 untreated (D). HC vs. MS D3 expression (E) and percentage change from T24 untreated (F). Bars indicate mean values with error bars indicating standard deviation. Unless otherwise indicated, statistically significant differences $\left(^{*}\right)$ are depicted for the calculation against the T24 untreated group. ${ }^{*}(\mathrm{p} \leq 0.05),{ }^{* *}(\mathrm{p} \leq 0.01),{ }^{* *}(\mathrm{p} \leq 0.001),{ }^{* * *}(\mathrm{p} \leq 0.0001)$. 


\subsubsection{Type 2 dopamine receptor expression - D4.}

After overnight culture, D4 expression significantly increased on monocytes from MS participants ( $\mathrm{p}<0.0001$ ) (Figure 4-9 C) but was only marginally changed in HC (Figure 4-9 A). Upon LPS stimulation, monocytes from MS participants had a strong reduction in D4 expression ( $\mathrm{p}<0.0001$ ) (Figure 4-8 C), which was maintained when clozapine was also present $(\mathrm{p}<0.0001)$. No effect on D4 was observed in the HC cohort although culture with both clozapine and LPS caused a modest but significant decrease from the level detected in untreated cells $(p=0.025)$.

We also observed that incubation with clozapine alone had no discernable effect on D4 in either cohort (Figure 4-9 A \& C) nor was the gMFI of D4 for HC compared to MS participants significantly different (Figure 4-9 E), with the response magnitude also very similar (Figure 4-9 F). 

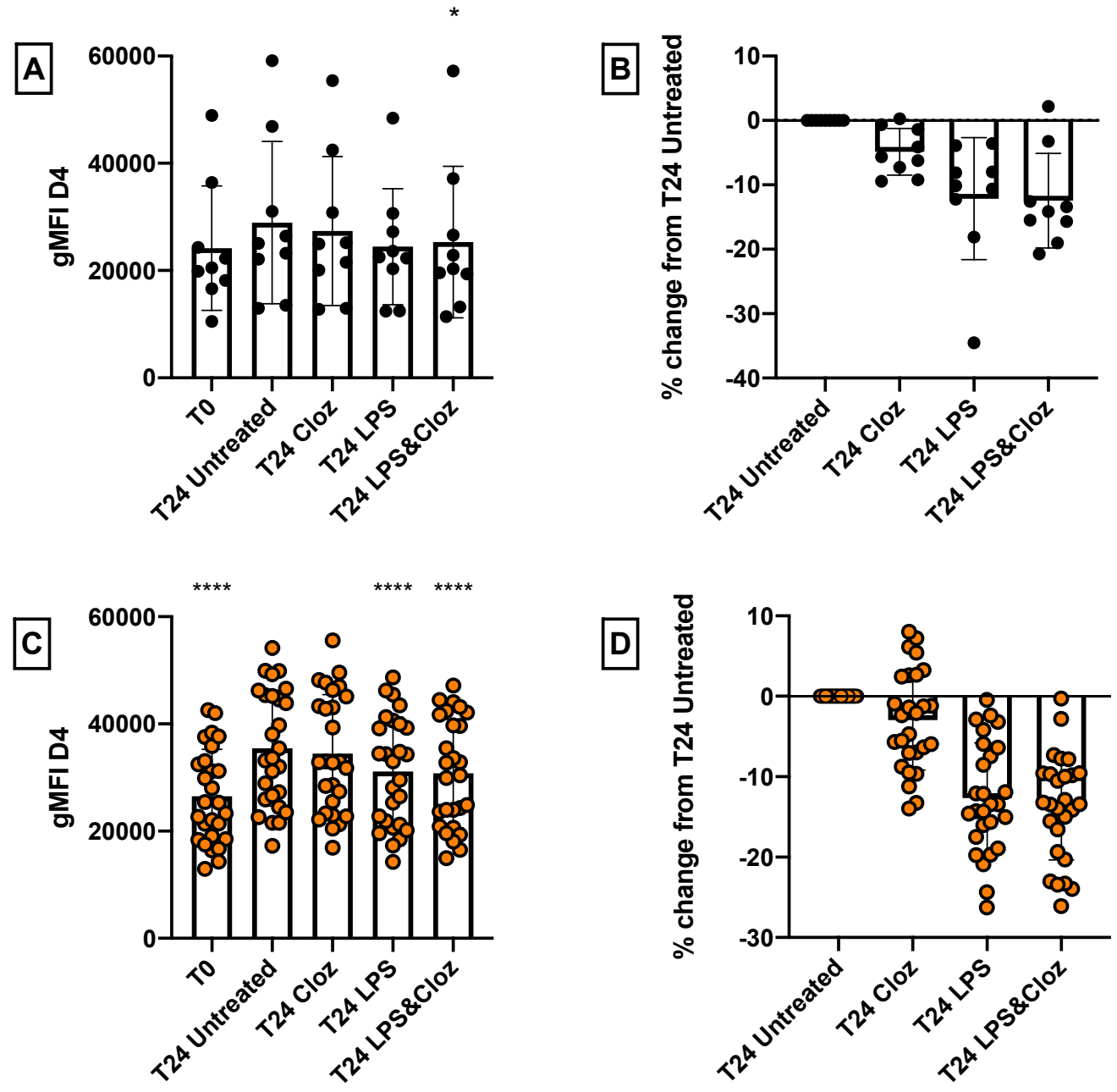

E
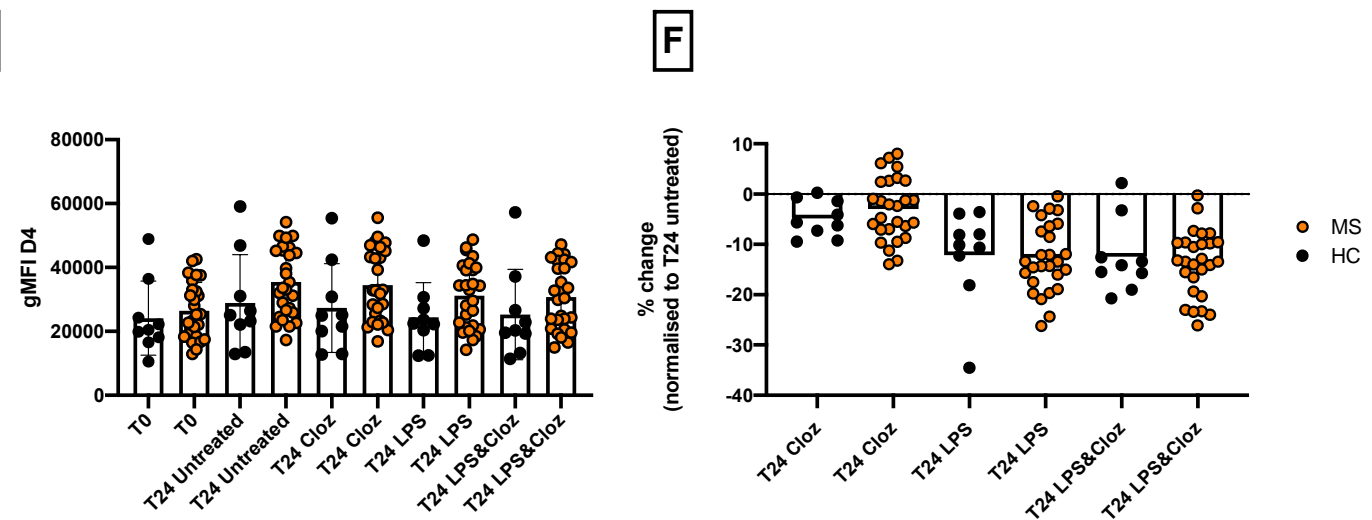

Figure 4-9 D4 expression on monocytes of all FMO positive gated cells.

Healthy control D4 expression (A) and percentage change from T24 untreated (B). MS patients D4 expression (C) and percentage change from T24 untreated (D). HC vs. MS D4 expression (E) and percentage change from T24 untreated (F). Bars indicate mean values with error bars indicating standard deviation. Unless otherwise indicated, statistically significant differences $(*)$ are depicted for the calculation against the T24 untreated group. ${ }^{*}(\mathrm{p} \leq 0.05),{ }^{* *}(\mathrm{p} \leq 0.01),{ }^{* * *}(\mathrm{p} \leq 0.001),{ }^{* * * *}(\mathrm{p} \leq 0.0001)$. 


\subsection{In vitro clozapine and its effects on intracellular cytokine expression.}

In the preceding section we investigated whether DR expression was altered by in vitro culture and stimulation with LPS in the presence or absence of clozapine. To further understand clozapine's immunomodulatory effects, we assessed the production of five cytokines from the same participants and cultures.

\subsubsection{Intracellular expression of IL-6.}

The proportion of monocytes expressing IL-6 was nearly $100 \%$ for the majority of participants. None of the conditions resulted in a significant variation in the proportion of positive events in either cohorts (Figure 4-10 A \& E). When the level of IL-6 (i.e. gMFI) was assessed, we observed a significant and many fold increase in IL6 expression following LPS stimulation. In both HC and MS cohorts, LPS induced on average over a 1000 fold increase in IL-6 compared to untreated cells (Figure 4-10 D \& H). In MS participants (Figure 4-10 F), stimulation with LPS (p < 0.0001) and LPS\&cloz ( $\mathrm{p}<0.0001)$ resulted a large increase in IL-6, with a similar increase observed in HC for LPS ( $p=0.001)$ and LPS\&cloz ( $\mathrm{p}=0003$ ) (Figure 4-10 B), as compared to the expression by unstimulated cells respectively.

In the presence of clozapine alone, we observed a significant reduction in IL-6 expression in MS participants ( $\mathrm{p}=0.042)$ (Figure 4-10 G). This effect was most prominent in participants with very high IL-6 expression in untreated culture, as demonstrated in individual response plots (Figure 4-10 G). This effect was not observed in the HC population. Additionally, the addition of clozapine did not alter LPS-stimulated IL-6 levels in either cohort. Finally, inter-cohort comparisons of the proportion of IL-6 positive cells (Figure 4-10 I), total IL-6 expression (Figure 4-10 J), and data normalised to T24 Untreated (Figure 4-10 K) did not show any significant variation between the cohorts. 

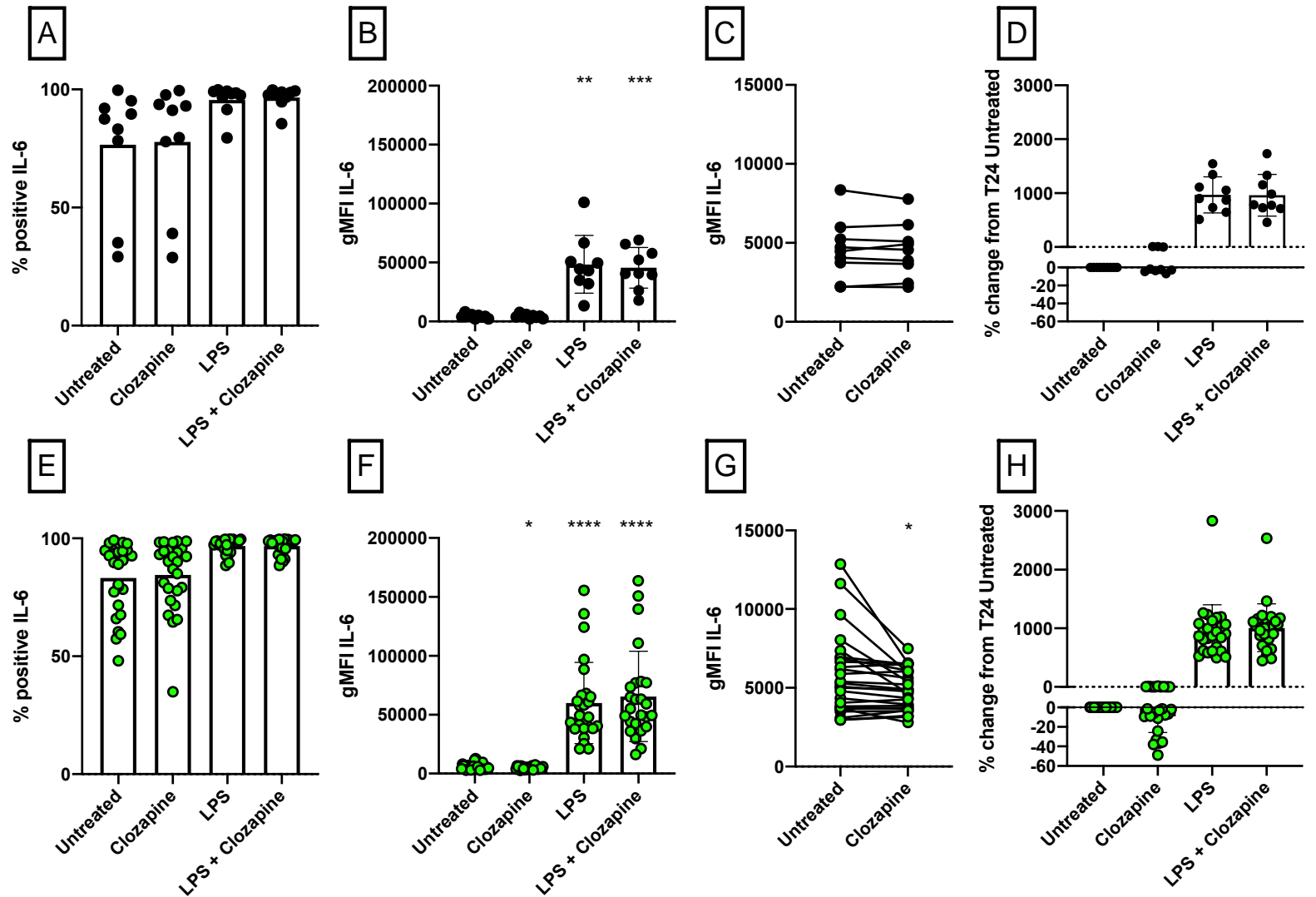

1

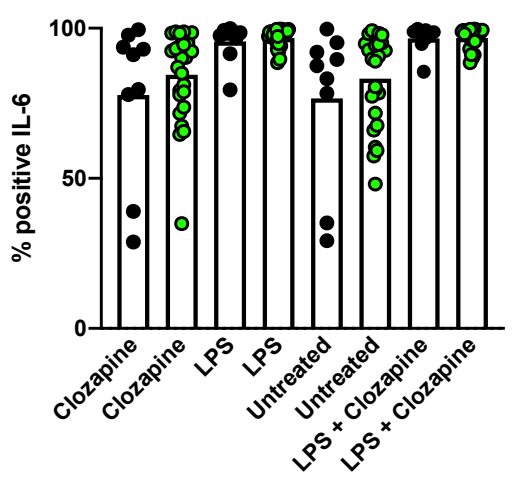

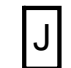

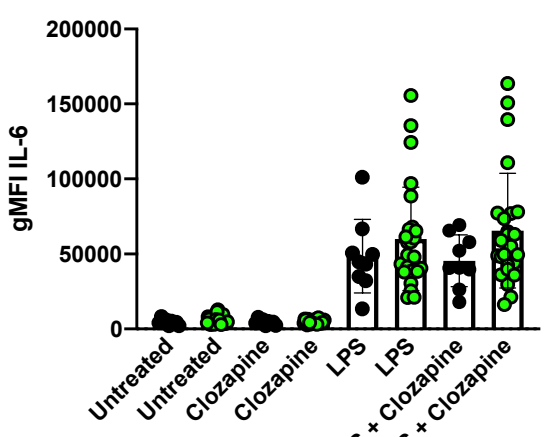

$s^{s^{x}} p^{s^{x}}$

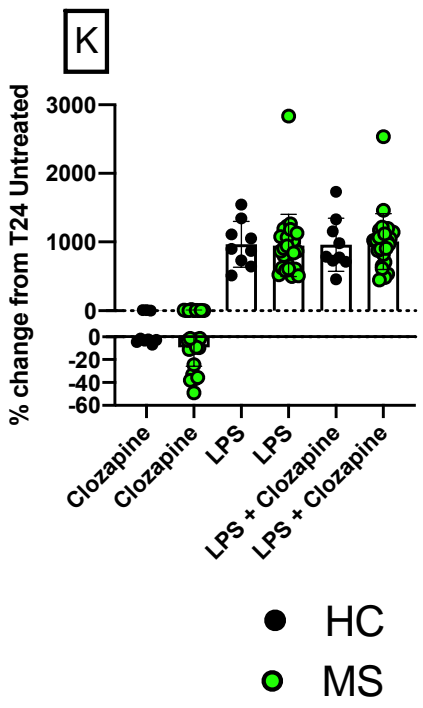

Figure 4-10 IL-6 expression in monocytes over all in vitro conditions.

Proportion of positive staining cells (A \& E), total IL-6 expression (B \& F), individual participant response to Clozapine ( $\mathrm{C} \& \mathrm{G}$ ), proportional change in expression normalised to untreated (D \& H), proportion of positive staining cells $\mathrm{HC}$ vs. MS (I), Il-6 expression HC vs. MS (J) and normalised to untreated expression HC vs MS (K). Unless otherwise indicated, statistically significant differences $\left(^{*}\right)$ are depicted for the calculation against the T24 untreated group. ${ }^{*}(p \leq 0.05),{ }^{* *}(p \leq 0.01),{ }^{* * *}(p \leq 0.001)$, **** $(\mathrm{p} \leq 0.0001)$. 


\subsubsection{Intracellular expression of IL-1ß.}

466.2.1 Even untreated cells produced IL-1 $\beta$, and the proportion and amount were strongly increased by LPS stimulation.

In both cohorts, the average proportion of IL-1 $\beta$-expressing monocytes following untreated overnight culture was 50\% (Figure 4-11 A \& E). This proportion increased following LPS stimulation to where we could detect IL-1 $\beta$ in nearly all cells. This strong increase was significant in both MS $(\mathrm{p}<0.0001)$ and $\mathrm{HC}(\mathrm{p}=0.0001)$ cohorts. Similarly, LPS stimulation resulted in a strong increase in total IL-1 $\beta$ in HC (p = o.ooo9) and MS (p < 0.0001) monocytes (Figure 4-11 B \& F).

4.6.2.2 In MS, clozapine reduced the proportion and amount of IL-1 $\beta$ producing monocytes.

In MS participants but not $\mathrm{HC}$, clozapine treatment led to a reduction in the proportion of IL-1 $\beta$ positive cells ( $\mathrm{p}<0.0001$ ) (Figure 4-11 E) as well as the total amount of IL-1 $\beta$ produced ( $\mathrm{p}<0.0001$ ) (Figure 4-11 F) compared to untreated. This was most clearly depicted when individual participant responses were plotted (Figure 4-11 G), which showed that those participants with the highest IL-1 $\beta$ expression in untreated cells, showed the greater reduction by clozapine.

4.6.2.3 Clozapine could reduce the magnitude of the IL-1 $\beta$ upregulation induced by LPS.

To grasp the magnitude of the response differential that was induced by clozapine, and to determine if the drug could reduce expression in the presence of LPS, we calculated the proportional change in expression compared to untreated cells. Comparing LPS with and without clozapine revealed that clozapine significantly reduced the magnitude of the IL-1 $\beta$ response induced by LPS, and this effect occurred in MS ( $p=$ o.001) and $\mathrm{HC}(\mathrm{p}=0.019)$ participants. No significant differences were detected when comparing HC and MS IL-1 $\beta$ responses (Figure 4-11 K). 
A

B D

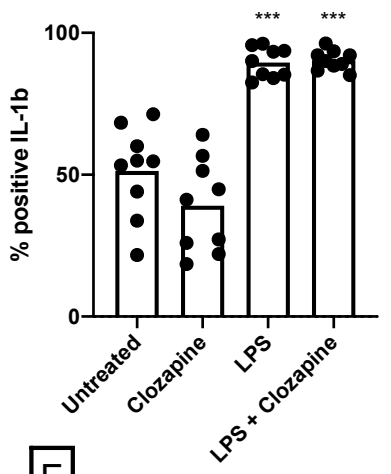

E

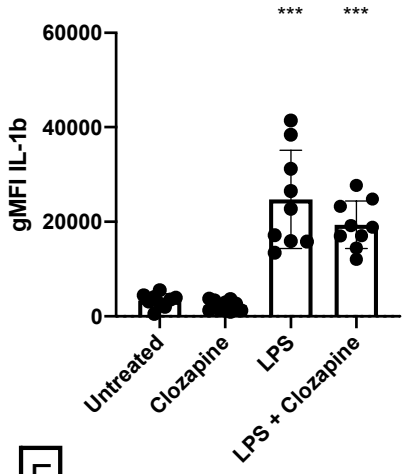

$\mathrm{F}$
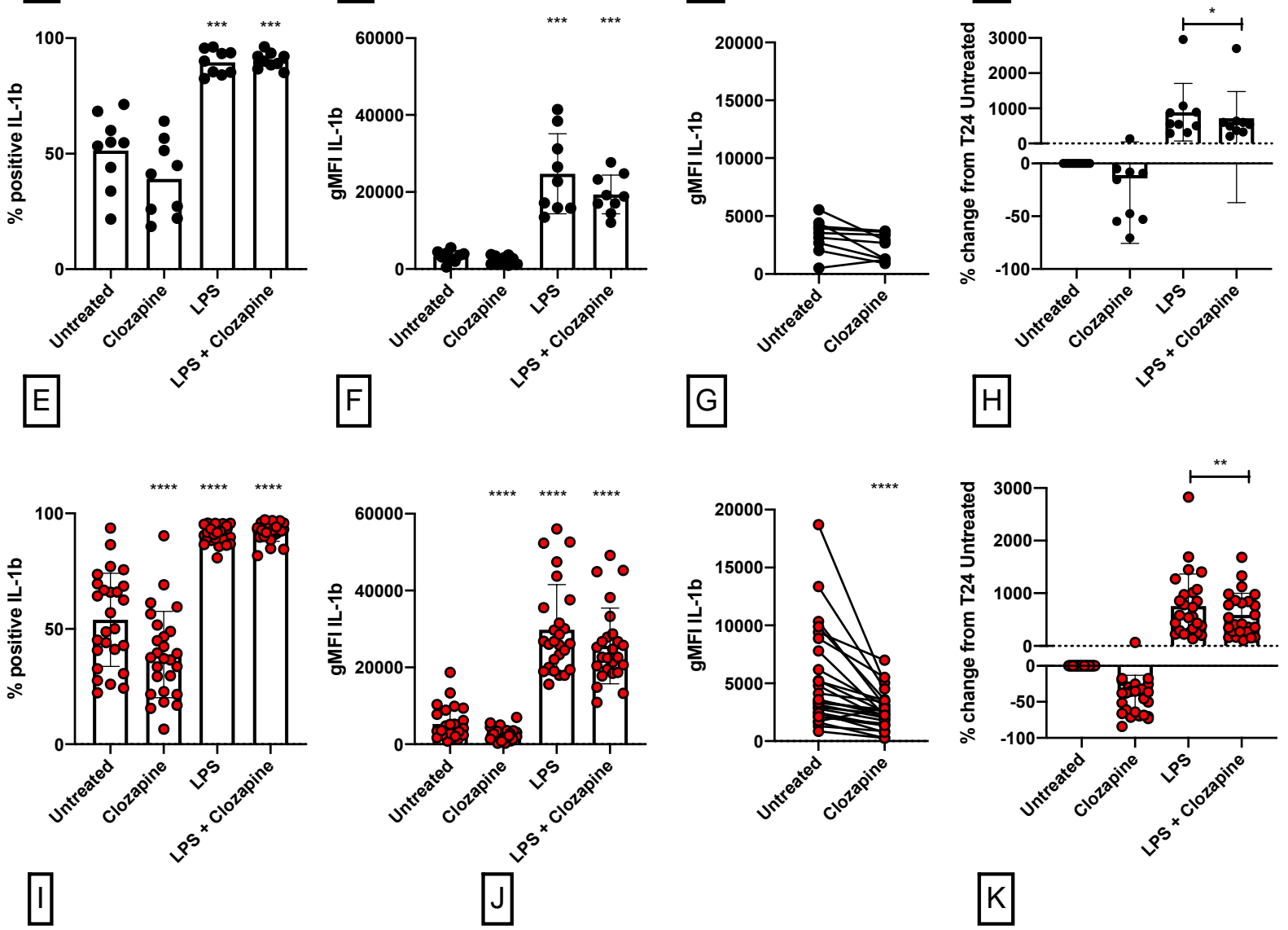

ॠ
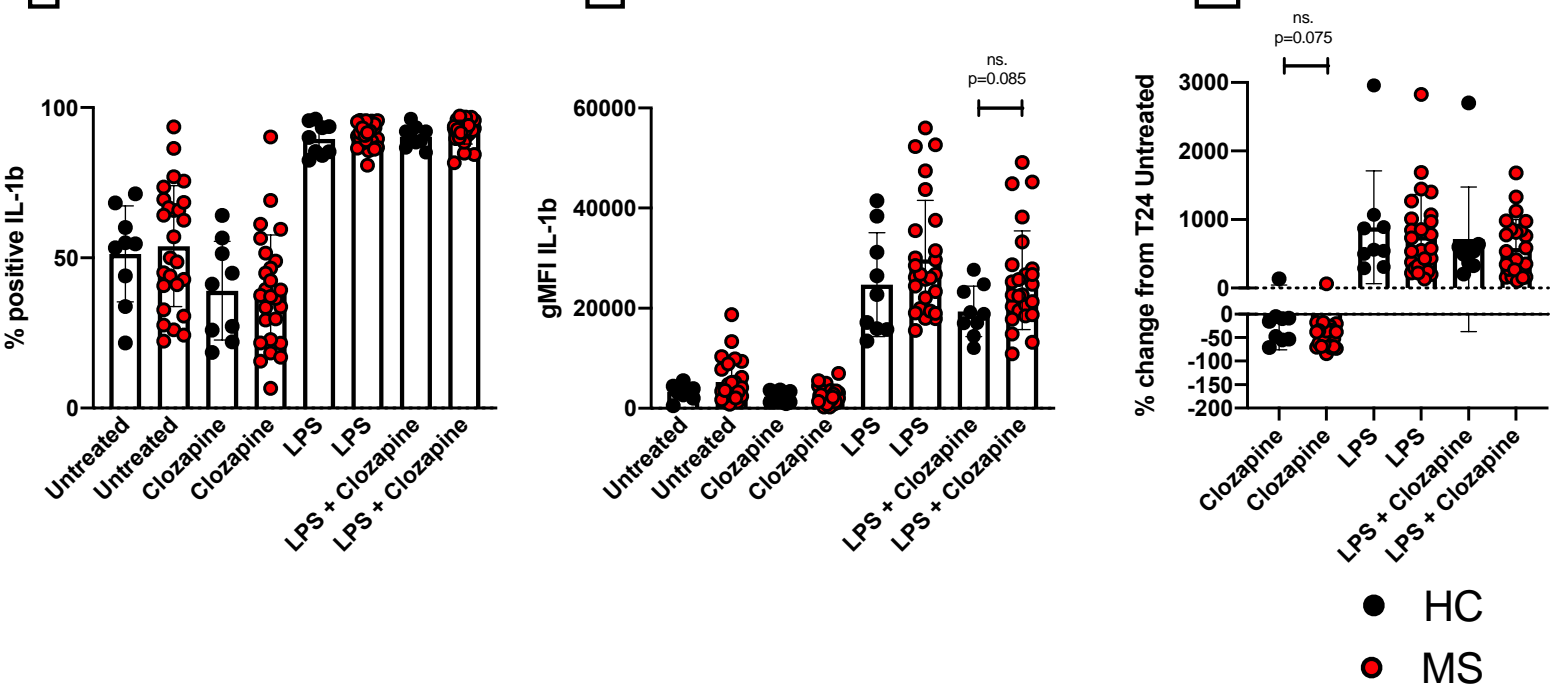

Figure 4-11 IL-1 $\beta$ expression in monocytes over all in vitro conditions.

Proportion of positive staining cells (A \& E), total IL-1 $\beta$ expression (B \& F), individual participant response to Clozapine ( $\mathrm{C} \& \mathrm{G}$ ), proportional change in expression normalised to untreated (D \& H), proportion of positive staining cells HC vs. MS (I), IL-1 $\beta$ expression HC vs. MS (J) and normalised to untreated expression HC vs MS (K). Unless otherwise indicated, statistically significant differences $(*)$ are depicted for the calculation against the T24 untreated group. ${ }^{*}(\mathrm{p} \leq 0.05),{ }^{* *}(\mathrm{p} \leq 0.01),{ }^{* * *}(\mathrm{p} \leq 0.001)$, **** $(\mathrm{p} \leq$ 0.0001). 


\subsubsection{Intracellular expression of TNFa.}

4.6.3.1 LPS induced a strong increase in TNFa production, which was reduced by clozapine.

While some participants clearly had very low proportions of monocytes expressing TNFa in untreated culture (Figure 4-12 A \& E), no significant differences in the intra cohort averages for the proportion of cytokine-producing cells was detected for any in vitro condition. Instead, stimulation with LPS significantly altered TNFa expression by monocytes in the MS ( $\mathrm{p}<0.0001)$ and HC cohort $(\mathrm{p}=0.0043)$. In contrast to LPS alone, clozapine-treated monocytes showed a significant reduction in the total amount of TNFa produced in MS participants when compared to untreated cells ( $p=0.022$ ) (Figure 4-12 F), and this reduction was not observed in the HC. Furthermore, an individual participant response plot (Figure 4-12 G) highlights that this effect was most pronounced in individuals where TNFa was highest in untreated monocytes.

A significant increase was also observed in cultures with both LPS and clozapine in both MS participants $(\mathrm{p}<0.0001)$ and HC $(\mathrm{p}=0.0009)$, but the level of TNFa was similar to that of monocytes stimulated with LPS alone suggesting clozapine did not affect LPS-stimulated TNFa (Figure 4-12 B \& F).

4.6.3.2 Constitutive TNFa was significantly higher in MS participants when compared to $\mathrm{HC}$.

In comparing TNFa responses between $\mathrm{HC}$ and MS participants, it was evident that MS participants had more TNFa-positive cells and higher TNFa expression in monocytes when compared to HC (Figures 4-12 I \&J). This increase persisted over all conditions. When the expression of TNFa was normalised to untreated expression levels, a comparison between the cohorts showed that the reduction in TNFa effected by clozapine was significantly greater in MS participants compared to HC ( $\mathrm{p}=0.022)$, further supporting the possibility of a differential effect of clozapine in MS. 
A

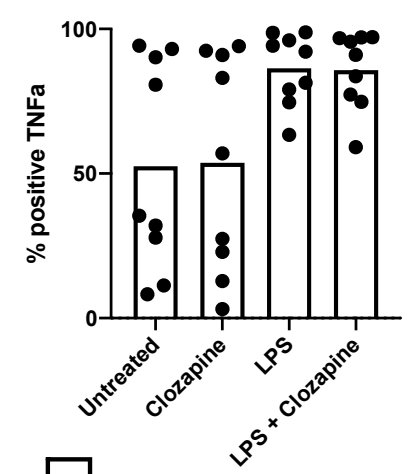

E

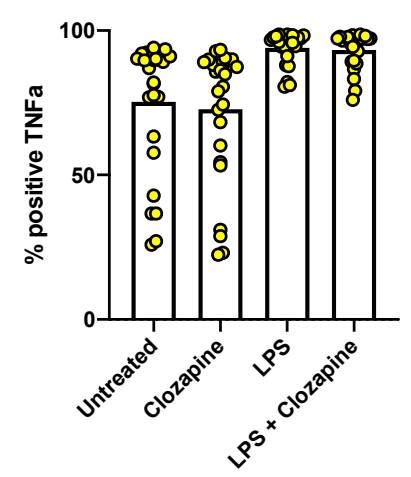

I

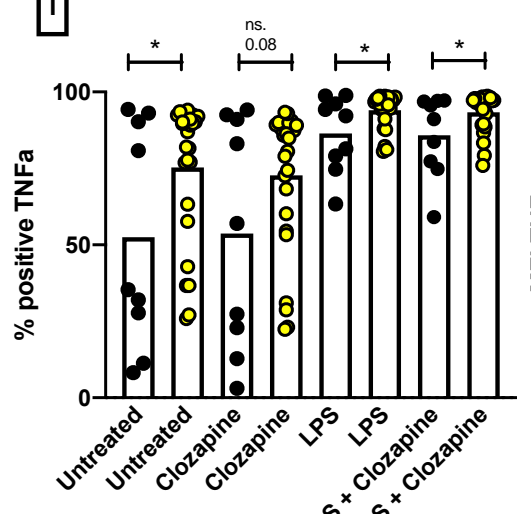

$2^{5^{x}} 2^{5^{x}}$

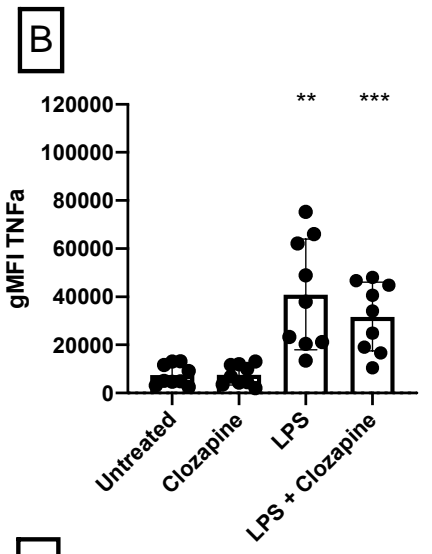

$\mathrm{F}$
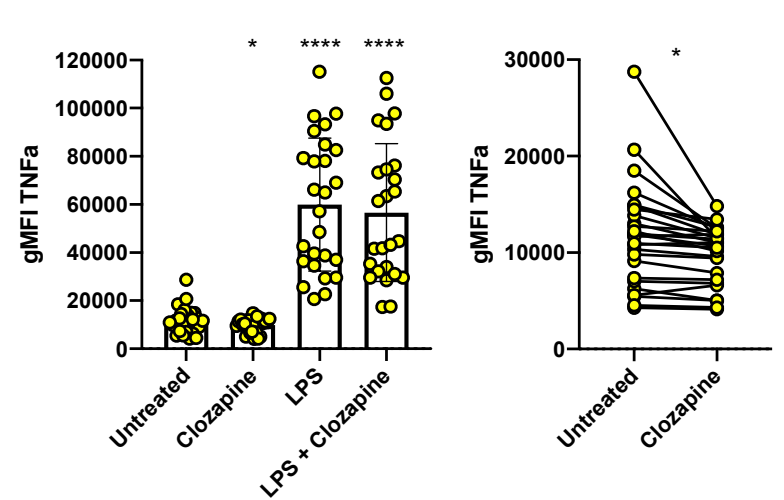

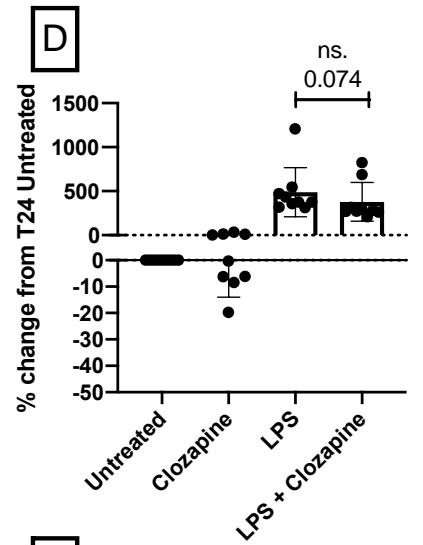

$\mathrm{H}$
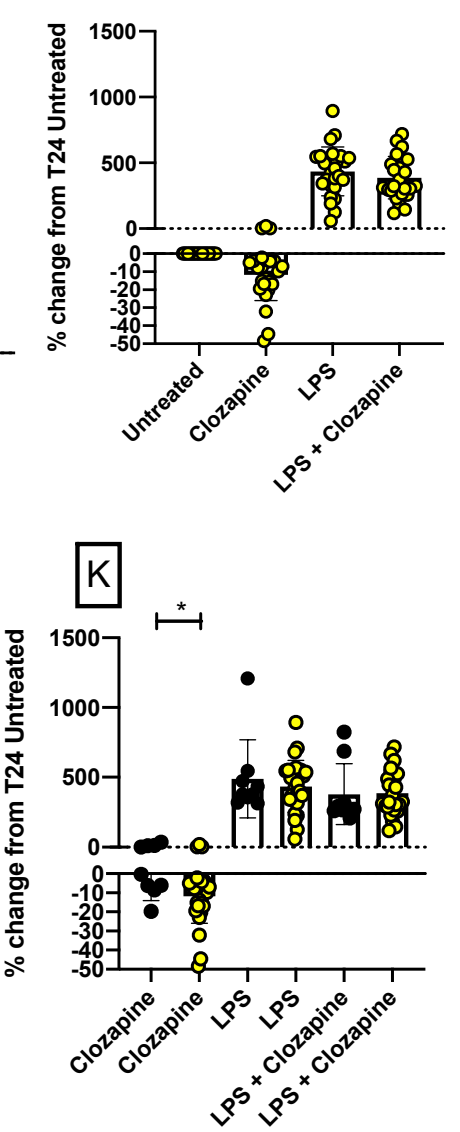

- $\mathrm{HC}$

- MS

Figure 4-12 TNFa expression in monocytes over all in vitro conditions.

Proportion of positive staining cells (A \& E), total TNFa expression (B \& F), individual participant response to Clozapine ( $\mathrm{C} \& \mathrm{G}$ ), proportional change in expression normalised to untreated (D \& H), proportion of positive staining cells $\mathrm{HC}$ vs. MS (I), TNFa expression HC vs. MS (J) and normalised to untreated expression HC vs MS (K). Unless otherwise indicated, statistically significant differences $\left(^{*}\right)$ are depicted for the calculation against the T24 untreated group. ${ }^{*}(\mathrm{p} \leq 0.05),{ }^{* *}(\mathrm{p} \leq 0.01),{ }^{* * *}(\mathrm{p} \leq 0.001)$, **** $(\mathrm{p} \leq 0.0001)$. 


\subsubsection{Intracellular expression of INFy.}

On average, between $30-36 \%$ of monocytes stained positive for INFY, which remained consistent irrespective of the addition of LPS or clozapine to the cultures and was observed in all participants (Figure 4-13 A \& E). However, the total amount of INFY expressed by the monocyte population was significantly increased in cultures with LPS (HC: p = 0.025; MS: o<0001) (Figure 4-13 B \& F). The addition of clozapine did not appear to affect the expression of INF $\gamma$ in monocytes compared to untreated, and while a significant increase in INF $\gamma$ was detected in the combined LPS and clozapine cultures, the level was reduced compared to LPS alone. This was most prominent when data were normalised to expression levels seen at baseline and depicted as the proportion change in total expression (Figure 4-13 D \& H). We observed this effect in both MS ( $\mathrm{p}=0.0051)$ and $\mathrm{HC}(0.0078)$ participants. In contrasting data between the $\mathrm{HC}$ and MS cohort, we found no significant difference in either the total expression, the proportion of cells, nor the magnitude of response for INFY (Figure 4-13 I \& J). 
A

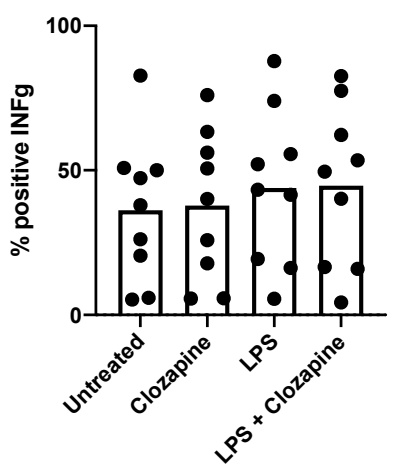

E

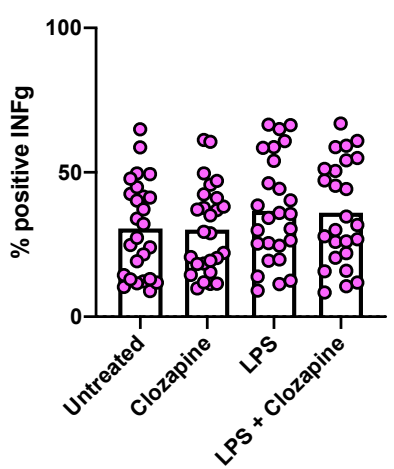

๑
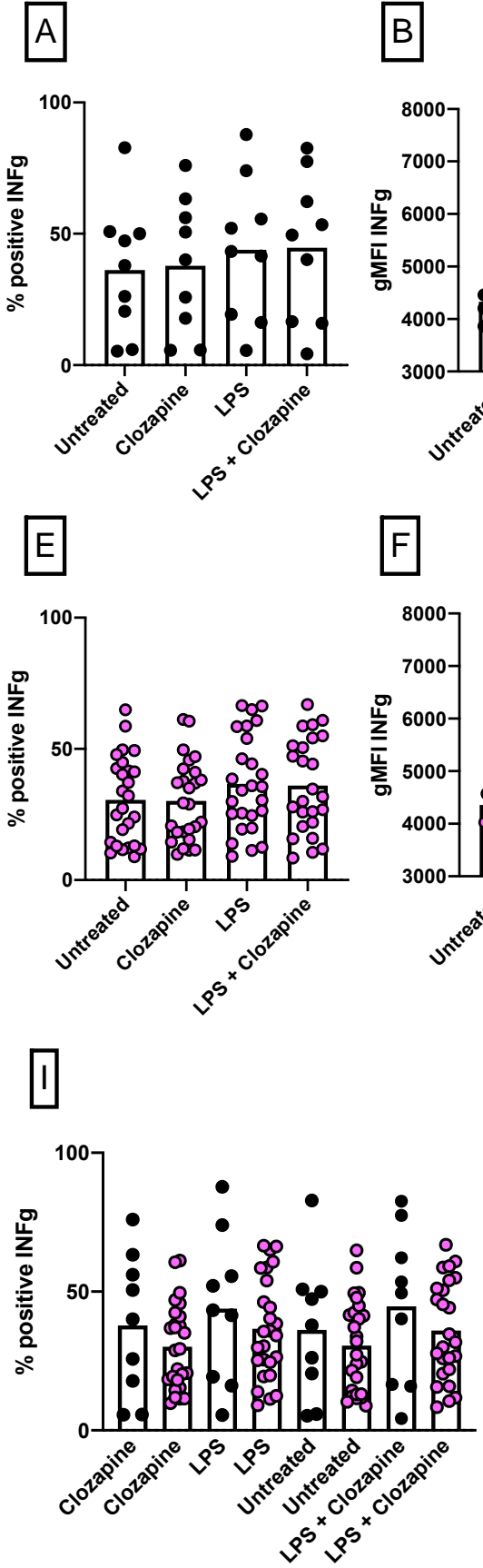
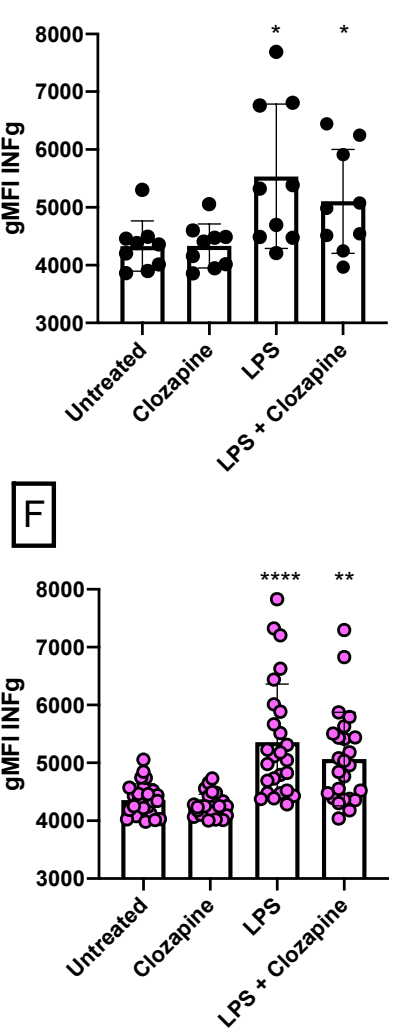

๑
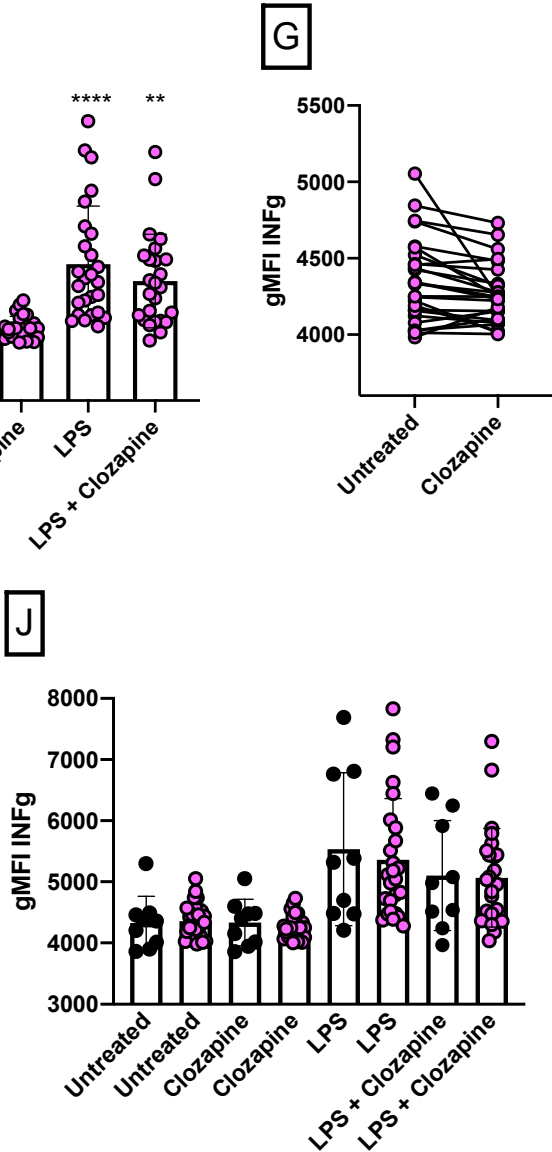
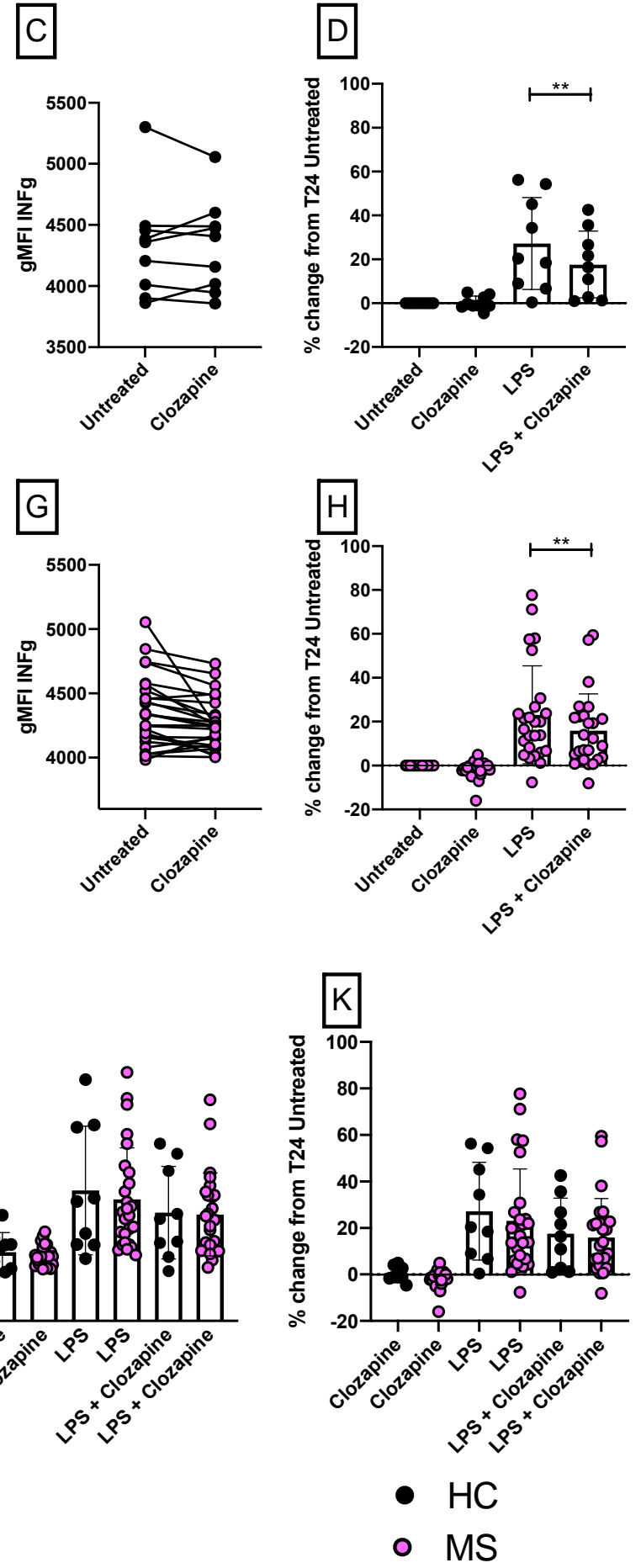

Figure 4-13 INFy expression in monocytes over all in vitro conditions.

Proportion of positive staining cells (A \& E), total INFY expression (B \& F), individual participant response to Clozapine ( $\mathrm{C} \& \mathrm{G}$ ), proportional change in expression normalised to untreated (D \& H), proportion of positive staining cells $\mathrm{HC}$ vs. MS (I), INF $\gamma$ expression HC vs. MS (J) and normalised to untreated expression HC vs MS (K). Unless otherwise indicated, statistically significant differences $\left(^{*}\right)$ are depicted for the calculation against the T24 untreated group. ${ }^{*}(\mathrm{p} \leq 0.05),{ }^{* *}(\mathrm{p} \leq 0.01),{ }^{* * *}(\mathrm{p} \leq 0.001)$, $* * * *(\mathrm{p} \leq 0.0001)$. 


\subsubsection{Intracellular expression of IL-10.}

The average proportion of IL-10 positive cells increased modestly but significantly in HC cultures incubated overnight with LPS ( $\mathrm{p}=0$.0335) or when co-cultured with LPS and clozapine ( $\mathrm{p}=0.0238$ ) (Figure 4-14 A) but this increase was not observed in the MS population, where the proportion of positive staining cells remained unchanged over all conditions. When the level was assessed, we found that IL-10 production was significantly increased by LPS compared to untreated cells for both cohorts (Figure 414 B \& F), and the addition of clozapine showed a similar increase; however, the magnitude of response was no different between LPS and the co-cultured cells (Figure 4-14 D \& H \& K). Additionally, clozapine alone had no effect on IL-10 production (Figure 4-14 A \& E), and the individual response plots between untreated cells and clozapine also did not indicate any difference in IL-10 expression (Figure 4-14 C \& G). Furthermore, inter-cohort comparisons did not show any differences in the proportion (Figure 4-12 I), total IL-10 produced (Figure 4-14 J), or magnitude of response (Figure 4-14 K). 

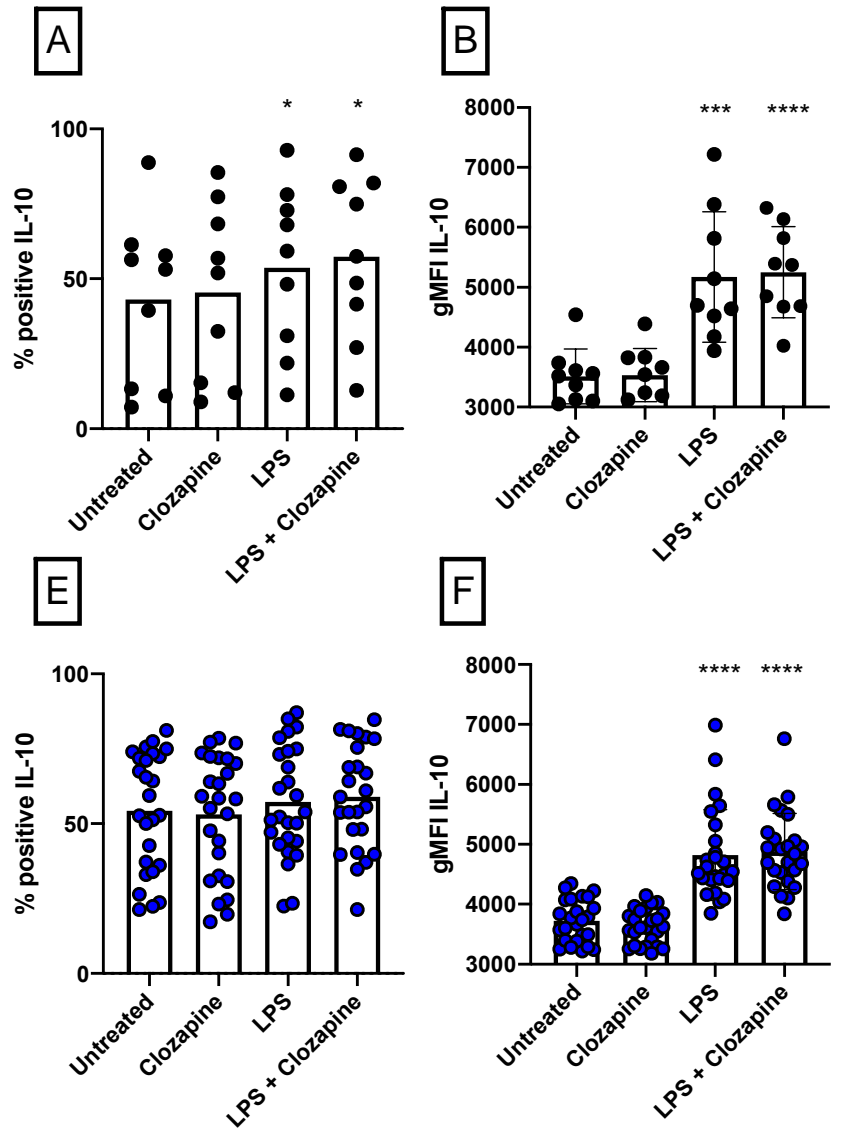

1

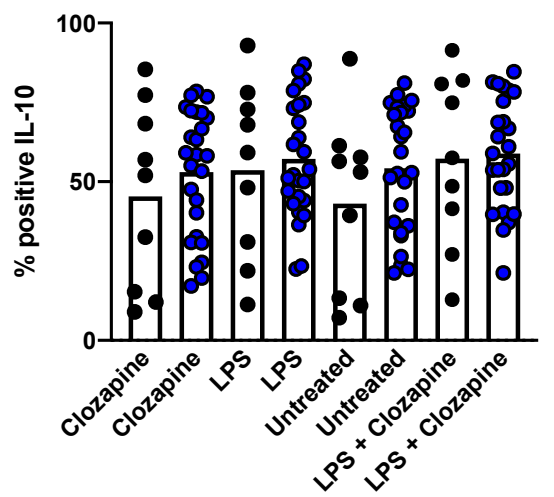

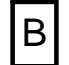

C

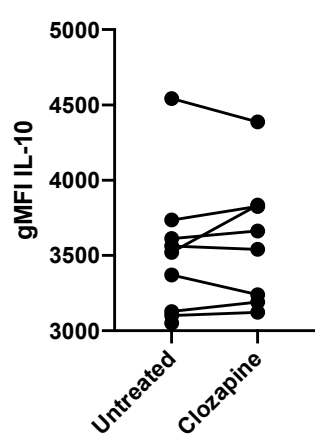

$\mathrm{G}$
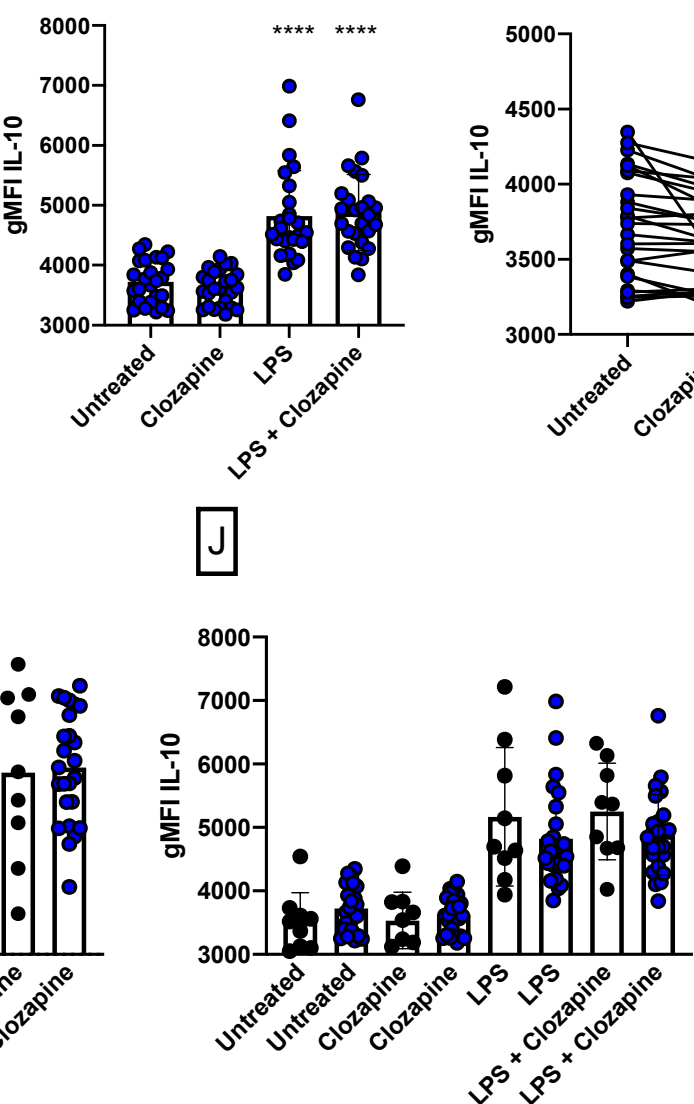
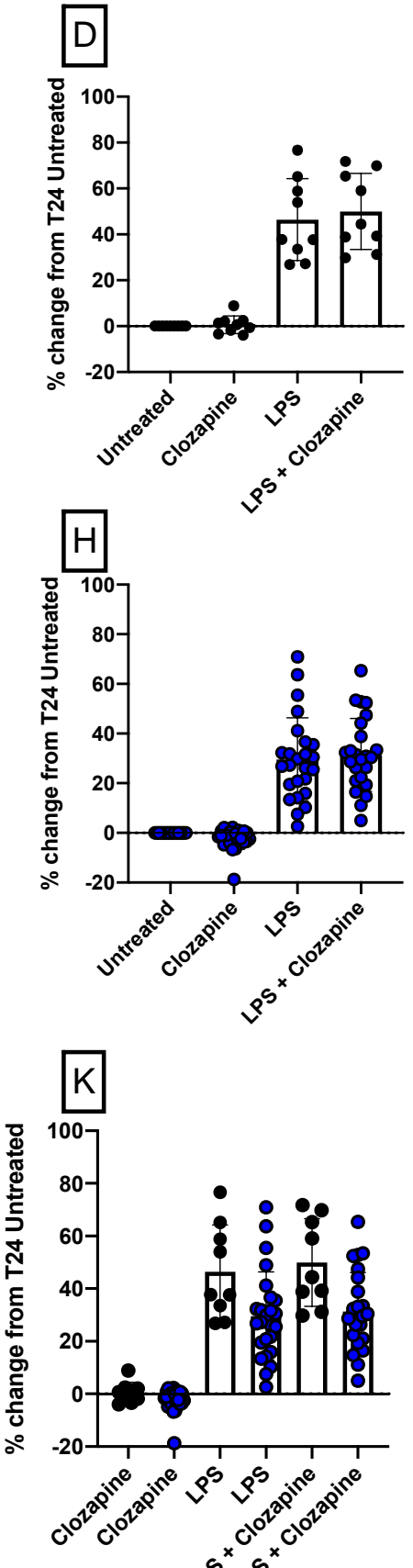

$8^{s^{x}} p^{s^{x}}$

- $\mathrm{HC}$

- MS

Figure 4-14 IL-10 expression in monocytes over all in vitro conditions.

Proportion of positive staining cells (A \& E), total IL-10 expression (B \& F), individual participant response to Clozapine ( $\mathrm{C} \& \mathrm{G}$ ), proportional change in expression normalised to untreated (D \& H), proportion of positive staining cells HC vs. MS (I), IL-10 expression HC vs. MS (J) and normalised to untreated expression HC vs MS (K). Unless otherwise indicated, statistically significant differences $\left(^{*}\right)$ are depicted for the calculation against the T24 untreated group. ${ }^{*}(\mathrm{p} \leq 0.05),{ }^{* *}(\mathrm{p} \leq 0.01),{ }^{* *}(\mathrm{p} \leq 0.001)$, $* * * *(\mathrm{p} \leq 0.0001)$. 


\subsubsection{Clozapine treatment abrogates the strong correlation between D1 and IL-1ß.}

In the preceding sections we observed that clozapine could modulate inflammatory signalling in monocytes by reducing both IL-1 $\beta$ and IL-6 expression, as well as in inducing the expression of D1 by those same cells. To better model the interaction and overall effect of the drug on innate immune cell responses we performed linear regression analysis of these three output variables. Using D1 as the dependent variable, we plotted the relationship between cytokine and DR expression (Figure 415). We observed a correlation in untreated monocytes (T24) for IL-1 $\beta$ ( $p=0.042$, CI $\left.+0.0056-0.0283, \mathrm{R}^{2}=0.162\right)$ and IL-6 $\left(\mathrm{p}=0.0045, \mathrm{CI}+0.020-0.0988, \mathrm{R}^{2}=0.290\right)$ to D1 expression, indicating those cells with high cytokine production also strongly expressed D1. Furthermore, when treated with clozapine, the correlation between IL6 and D1 remained $\left(\mathrm{p}=0.0242, \mathrm{CI}=0.0025-0.0337, \mathrm{R}^{2}=0.194\right)$, although it was not strong. In contrast, the correlation between IL-1 $\beta$ and D1 was abolished by clozapine treatment (Figure 4-15 B), and we observed that in individuals with high IL$1 \beta$, treatment with clozapine reduced IL-1 $\beta$ levels to those of untreated low D1 expressors (Figure 4-15 B).

In LPS treated cultures, a strong correlation was also observed between IL-6 ( $\mathrm{p}=$ $\left.0.0035, \mathrm{CI}+0.435-1.961, \mathrm{R}^{2}=0.3144\right)$, and IL-1 $\beta$ ( $\mathrm{p}=0.0051, \mathrm{CI}+0.1646-0.8250$, $\mathrm{R}^{2}=0.2946$ ) and D1 expression (Figure 4-15 C\&D). LPS induced a decrease in D1, with a concurrent increase in cytokine expression yet despite this, the correlations persisted. Furthermore, the addition of clozapine to LPS culture did not have a major impact. We conclude that this work suggests a strong relationship between the expression of IL-1 $\beta$, IL-6, and D1 under pro-inflammatory conditions.

It is apparent from these data that exposure to clozapine strongly limits IL- $1 \beta$ in MS, while also increasing D1 expression, and while the effects may be less pronounced on IL-6, clozapine was still able to effect its expression under the modest proinflammatory conditions modelled by our untreated culture conditions. We suggest that D1 expression changes are therefore not necessarily required to establish the pro- 
Chapter 4

inflammatory state seen in untreated cells, but that a change in its expression goes hand in hand with alterations to immune cell activation. 

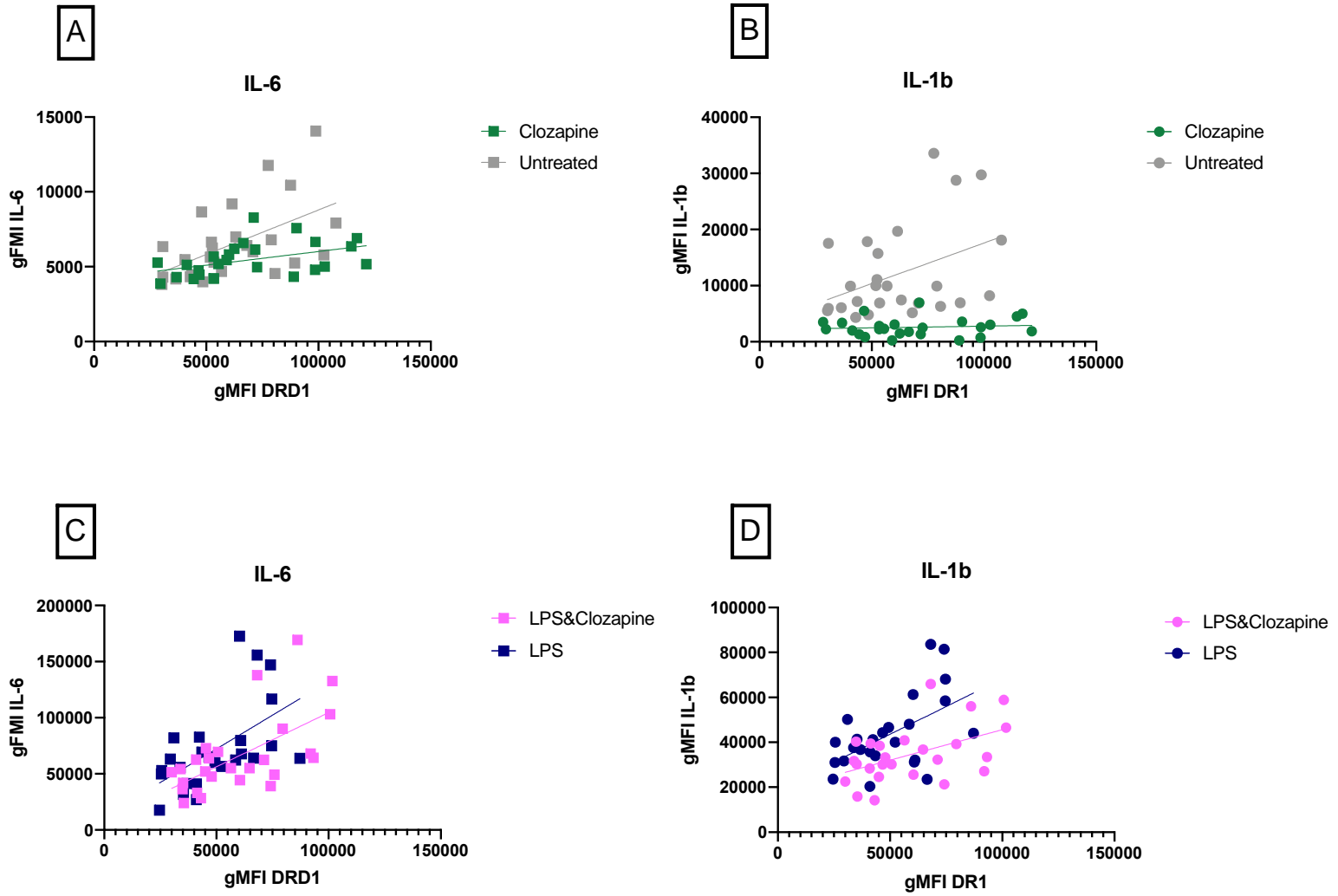

Figure 4-15 Linear regression of cytokine and D1 (dependent variable) expression for monocytes from the MS cohort.

Each symbol represents an individual participant, with a trend line from the regression calculation. Effects of clozapine vs. untreated on IL-6 expression (A) and IL-1 $\beta$ (B). The effects of clozapine in the presence of LPS for IL-6 (C) and IL-1 $\beta$ (D). 


\subsection{Discussion.}

Very little is known about the impact of dopamine receptor expression and signalling on innate immune cells, as most studies to date have focussed on the impact on $\mathrm{T}$ cells (Levite, 2012). Previous research has reported the expression of DR on most PBMC, but detailed studies of DR expression on monocytes are absent. Dopamine signalling in this context is very dependent on factors such as concentration, immune subset, and the specific receptor triggered, all of which can significantly alter the response outcomes of cells (Levite, 2012). Additionally, clozapine is not solely a DR antagonist but has wide affinity for a range of other receptors (Seeman, 2014). Thus, DR antagonism by clozapine in the context of immune modulation is a complex and difficult research topic.

We set out to investigate the effect of low dose clozapine on the innate immune response in vitro. Our hypothesis was that low dose clozapine would have a modest but demonstrable inhibitory effect on pro-inflammatory cytokine production and alter DR expression in PBMC in vitro. These effects were anticipated in our model as we considered the untreated overnight culture representative of a modest proinflammatory state given the data showed the induction of a low level of proinflammatory cytokines (IL-6 \& IL-1 $\beta$ ) in these cells.

Our results supported this hypothesis and showed a reduction of IL-1 $\beta$, IL-6, and TNFa, but not INF $\gamma$ or IL-10 by clozapine by the monocytes of treatment-naïve participants with MS. We further demonstrated the effects of clozapine in the presence of highly inflammatory TLR4 (LPS) signalling, where clozapine also mediated a reduction in pro-inflammatory cytokine production. We further posited that the pro-inflammatory nature and immune activation inherent from MS pathogenesis may result in differential DR and cytokine profiles in our untreated MS cohort when compared to a group of match healthy controls. This differential response was demonstrated with our TNFa and D1 expression results. While our broad aims and hypotheses were met, we also observe several unexpected and intriguing results that merit discussion. These findings are summarised in Table 4-3. 
Table 4-3 Summarised results of the effects of in vitro culture conditions on monocytes.

\begin{tabular}{|c|c|c|}
\hline & MS & $\mathrm{HC}$ \\
\hline $\begin{array}{l}\text { Untreated } \\
\text { cells }^{1}\end{array}$ & $\begin{array}{l}\Uparrow \mathrm{D} 1-4 \text { expression } \\
\Uparrow \mathrm{D} 5 \text { expression } \\
\Uparrow \% \mathrm{D} 5+\text { cells }\end{array}$ & đD5 expression \\
\hline $\begin{array}{c}\text { LPS } \\
\text { stimulation }\end{array}$ & $\begin{array}{l}\Downarrow \text { D1 } \\
\Uparrow \text { D3\&D5 expression } \\
\Downarrow \text { D4 expression } \\
\Uparrow \text { All cytokines }\end{array}$ & All cytokines \\
\hline $\begin{array}{l}\text { Clozapine } \\
\text { treatment }\end{array}$ & $\begin{array}{l}\text { D1 } \\
\Downarrow \mathrm{IL}-6, \mathrm{IL}-1 \beta, \mathrm{TNF} \alpha\end{array}$ & D1 \\
\hline $\begin{array}{c}\text { LPS \& } \\
\text { Clozapine } \\
\text { Co-culture }\end{array}$ & $\begin{array}{l}\Downarrow \mathrm{D} 2,4 \text { expression } \\
\Uparrow \mathrm{D} 5 \text { expression } \\
\Uparrow \text { All cytokines }\end{array}$ & $\downarrow D 2,4$ expression \\
\hline
\end{tabular}

1Untreated cells changes are depicted as they relate to baseline expression levels. All other changes represent expression as compared to untreated cultures.

\subsubsection{Dopamine receptors.}

The evidence of the effects on class 1 DR signalling by immune cells of the periphery is mounting. To date, the sole focus of this area has been on adaptive immune cells, particularly T cells (Ghosh et al., 2003; Marino and Cosentino, 2016; Melnikov et al., 2016). Our work used a range of monoclonal antibodies targeting all known DR to profile their expression in a progressive MS cohort. It is the first study to detail DR expression by clearly defined monocytes, while also describing the impact of clozapine 
on DR expression. Furthermore, we combined DR expression data with data on the expression of cytokines by monocytes, for a comprehensive outline of the immunomodulation effected by clozapine on this immune cell subset. While overlapping datasets have allowed some authors to compile a picture of DR expression in leukocytes (Levite, 2012), no single study has offered data to the sensitivity and specificity of our work. With the impact on DR signalling being contextual, it is reasonable to assume that clozapine concentration would have a differential impact on cell responses. Here, we used a single low dose of drug, optimised to model possible levels seen in the periphery following low dose oral administration while limiting negative effects on cellular viability.

We found that clozapine most prominently affected the expression of D1 on all viable monocytes in both the participant cohorts. The cells showed consistent D1 positivity, irrespective of in vitro manipulation. This was contrary to early reports where D1 could not be detected in this immune subset (McKenna et al., 2002). We also observe changes in the level of expression of D1 and noted a significant increase in total D1 expression after an overnight culture step. This was also observed for all other DR tested, but only in the MS cohort. An increase in DR expression following in vitro culture has been demonstrated previously and was likely due to the increase in subset maturation of monocytes (Coley et al., 2015). Coley et al. also showed expression of D1 by monocytes using a flow cytometry approach, thus substantiating our finding that D1 is expressed by monocytes.

Our results further show that clozapine treatment resulted in a significant increase in D1 expression by monocytes, with the overall level of D1 increased in both cohorts compared to untreated cells. Furthermore, in MS participants, clozapine could reverse the observed reduction in D1 from LPS stimulation while this effect was not observed in the HC. In addition to this finding we detected a strong correlation between proinflammatory cytokines (esp. IL-1 $\beta$ and IL-6) production and D1 expression in people with MS.

Our data focussing on the responses of monocytes offer new insights. A previous study found that dopamine inhibits anti-CD3 stimulated cytokine release, but that the addition of clozapine as a DR antagonist could not significantly impact this induction 
nor affect protein tyrosine kinase expression in activated T-cell cultures (Ghosh et al., 2003). Furthermore, our group has demonstrated that in an animal model of MS, the protective effects of clozapine were not mediated through direct alteration of specific CD4+ T-cells subsets (Zareie et al., 2017). Instead it is possible that an alternative immune subset, namely monocytes, may be more affected by clozapine and thus promote an anti-inflammatory response. Adding to this idea is a report in which the authors used mRNA interference of D1 to demonstrate that this DR was essential for dopamine driven anti-inflammatory signalling (Yan et al., 2018). Yan et al. showed signalling through D1 is required to inhibit inflammasome activation and the subsequent reduction of IL-1 $\beta$ production by LPS-primed bone marrow derivedmacrophages. Together with our findings, this work suggests that clozapine, which we found increased the expression of D1, may enable improved signalling by endogenous dopamine through D1, inhibiting the inflammasome and reducing IL-1 $\beta$ production by monocytes. Further work is required to confirm this mechanism, and while assessing changes in endogenous dopamine in our model would add to our understanding of clozapine's mechanism. Unfortunately, dopamine has a very short half-life (under 2 minutes in plasma) which limits its measurement in stored culture supernatants.

In the CNS at therapeutic doses, clozapine transiently occupies D2, facilitating neuronal activity that increases synaptic dopamine (Seeman, 2014). This increase in dopamine by transient occupation then results in the drug's antipsychotic action while limiting parkinsonian motor side effects. We note a recent report in literature that D2 was upregulated following clozapine exposure in cell line models, which the authors suggested was due to increased translocation of receptor to the cell surface (Schrader et al., 2019). Our data showed upregulation of D2 expression following overnight culture, but we observed that low dose clozapine alone had no significant effect on D2 expression by monocytes. Detection of changes to D2 expression may be limited in our model by the use of a low dose and from clozapine having a significantly reduced capacity to increase D2 expression as compared to other atypical antipsychotic drugs (Schrader et al., 2019). Schrader and colleagues further suggest D2 upregulation is caused by a small proportion of drug that enters the cell to induce D2 upregulation. Our low dose model may not provide sufficient bioavailable agent to mimic the actions 
seen by Schrader et al. Furthermore, from our study, D2 does not appear to be the major target through which clozapine exerts its immune modulating effects.

The effects of clozapine may be beneficial in MS if in vivo administration of the drug has similar immune modulating outcomes as those described here. In MS pathology, it is accepted that immune cells are present and contributing at the sites of inflammation in the CNS, and that the periphery may be more pro-inflammatory than in healthy people (Krei et al., 1991; Mikova et al., 2001; Tsukada et al., 1991). Clozapine treatment in MS may therefore be beneficial, as we demonstrate here that clozapine can dampen cytokine expression under pro-inflammatory conditions.

\subsubsection{Intracellular cytokines.}

The inflammatory mediators IL-1 $\beta$, IL-6, TNFa, INF $\gamma$ and IL-10 are known to play a key role in neuroinflammation and signalling in the brain (Bobermin et al., 2018) and as such were selected for our analysis. The effects of LPS stimulation in cultured cells were as expected: a significant increase in all of these mediators was observed following overnight stimulation. This expected response set a baseline, from which we were able to evaluate the effects of clozapine. Our data revealed a modest immunomodulatory effect by the drug when used at low concentration and especially when cells were in an inflammatory environment (i.e. stimulated with LPS or from people with MS). In MS participants, we noted a modest but significant reduction by clozapine in the amount of IL-6, IL-1 $\beta$ and TNFa expressed compared to untreated cells. This appeared most apparent in participants who had higher cytokine levels. Neither of these observations were noted in HC. Furthermore, IL-1 $\beta$ levels in MS participants were also reduced by clozapine in the presence of LPS, although this was not observed for IL-6, and failed to reach significance for TNFa cultures.

Of the 5 cytokines tested only levels of TNFa were consistently altered in MS participants when compared to HC. We observed both the proportion and total amount (i.e. gMFI) of this cytokine produced was significantly higher in MS participants. Moreover, baseline levels were elevated, indicating higher constitutive TNFa expression in MS. Reports in the literature demonstrate that monocyte derived TNFa is increased in SPMS over RRMS, with MS patients also having higher levels 
than healthy controls (Filion et al., 2003; Maimone et al., 1991; Sharief and Hentges, 1991; Trotter et al., 1991). This is in agreement with our findings. Although reducing TNFa has proven highly effective in other autoimmune conditions such as rheumatoid arthritis, this strategy failed in phase two trials for patients with MS (LENERCEPT, 1999) and even led to worsening of disease. Subsequent investigations have shown TNFa has a central role in CNS remyelination (Arnett et al., 2001), which is essential for CNS repair and recovery post relapse. The effect of clozapine was also found to be very pronounced in our MS cohort, with the magnitude of the response to clozapine being significantly stronger, and clozapine effecting a greater reduction in TNFa levels compared to HC. While dramatically reducing TNFa levels during MS has been shown to lead to poorer outcomes, we believe that the clozapine-mediated reduction in TNFa is more subtle than that of TNFa-blocking agents and is due to the anti-inflammatory effects of clozapine.

PBMC from patients in the relapsing phase of RRMS have been shown to produce more INFY than participants in remission, and in healthy controls (Melnikov et al., 2016). Additionally, previous studies have shown that clozapine in the presence of LPS can differentiate schizophrenic patients from healthy controls in terms of their INF $\gamma$ response to in vitro stimulation (Krause et al., 2013; Reale et al., 2011). At therapeutic doses, clozapine is further known to reduce INF $\gamma$ production by PBMC (Chen et al., 2012b). Our data shows that clozapine has the capacity to effect a reduction in INFY expression in co-cultured with LPS and normalised to untreated cells. This suggests that clozapine has the capacity to affect INFY expression but only in activated cell since this effect was not observed in the absence of LPS. The effect of altering INF $\gamma$ in MS is complex. The type 1 interferon pathway is a longstanding and effective therapeutic target in RRMS, but it remains unclear exactly why it is effective (Reder and Feng, 2014). Early clinical trials with INF $\gamma$ showed a striking increase in MS exacerbations from INF $\gamma$ therapy (Panitch et al., 1987). In contrast, work in our lab has shown that disease protection in an animal model of MS is dependent on INFY (O’Sullivan et al., 2014) and animals deficient in INF $\gamma$ suffer a more severe disease in this model (Ferber et al., 1996). Furthermore, recent studies have suggested that a subset-specific effect of INFY on innate cells may be neuroprotective (White et al., 2018). Overall, while there is evidence that interferons have positive benefits in MS, 
what role INF $\gamma$ has in the therapeutic effects of clozapine during MS remains to be established.

IL-10 production has been shown to be increased by high dose clozapine in co-culture with LPS compared to LPS alone, as well as clozapine inducing an increase in IL-10 in PBMC cultures stimulated through TLR3 (Al-Amin et al., 2013). In contrast, we did not detect an upregulation in IL-10 by clozapine. This difference may be due to the much lower dose in our cultures. While the reduction in the amount of proinflammatory cytokine by clozapine seen in our data supports a dampening of inflammatory processes, we cannot attribute this reduction to any observed change in IL-10. It is possible that at this concentration, clozapine's immunomodulatory effect is in reducing pro-inflammatory cytokine production rather than instigating an antiinflammatory cascade through IL-10.

Lastly, this study is the first to evaluate the effect of low dose clozapine on monocyte cytokine responses; however, a previous study has investigated the impact of clozapine on monocytes, but on immortalised mouse cell lines and with doses of clozapine that was in excess of a 1000 fold of our use (Contreras-Shannon et al., 2013). This previous study showed that very high doses of clozapine detrimentally affected cellular ATP production and viability, with an increase in culture supernatant cytokines when administered at $>50 \mu \mathrm{M}$. Because we found high doses greatly reduced cellular viability, and our work was geared toward maintaining viability and limiting cytotoxicity, it is difficult to form a comparison between these two studies. 


\subsubsection{Conclusion.}

In animal models of MS, dopamine agonism appears to drive MS pathogenesis (Prado et al., 2018). Coupled to this, is the increased presence of immune cells in the CNS during MS, which is an environment rich in dopamine. Together with our findings, this research supports DR antagonism as a drug option with which to alter activated immune cells in the CNS. Although few dopaminergic agents have been studied in the context of MS therapeutics, changes in DR expression in PBMC from MS participants and a disparity in PBMC responses between $\mathrm{HC}$ and MS participants, are known (Giorelli et al., 2005). Effective drug interventions in RRMS, specifically INF $\beta$ therapy, are known to change DR expression on immune cells of the periphery (Zaffaroni et al., 2008). The potential of dopamine-mediated interventions being effective in MS is supported by recent work (Marino and Cosentino, 2016). Overall, our study shows significant immunomodulation and alteration of DR expression by clozapine as well as a differential clozapine-mediated response by monocytes from people with MS when compared to healthy controls. These novel results shed light on how MS changes neuroimmune signalling pathways, and highlights the potential of clozapine as a novel treatment approach in progressive MS. 


\subsection{Limitations.}

The DRD5 used in our assay was conjugated to $\mathrm{AF} 405$, which is a very dim fluorophore. This issue may affect our ability to reliably detect dim levels of expression even on our high-resolution cytometry platform. Even though we reported that not all monocytes express D5, and that the proportion of cells positive for D5 was significantly increased in the MS cohort at baseline, these results should be interpreted with caution. While Prado et al. similarly showed a significant increase in the number of DR (1-5) expressing cells, particularly in non-classical monocytes for MS participants over HC (Prado et al., 2018), our result was contrary to these findings as we showed D1-4 positivity in all monocyte from all participants and did not observe any difference between the cohorts for D1-4. The differences between our data and Prado et al. are likely due to differences in cytometry platform resolution combined with the use of different antibodies to these target epitopes.

Our experimental design did not allow us to detect the direct cause of alterations in DR expression such as whether they were due to receptor cycling between the surface and an internal compartment or if our in vitro conditions effected upstream changes that resulted in differential expression of the marker. Future work should include additional measures to help establish the impact of the changes observed in our model. Our data acquisition techniques also did not allow for a comparison of the expression of the density of DR relative to each other. Future directions should be geared to allow for the detection of receptor density expression relative to one another as this can aid in determining the relative importance of the different receptors in this context.

For our clozapine dose optimisation, we did not consider the toxicity effects of clozapine doses over time, which may impact PBMC viability in vitro. However, our use of a low dose and the dose titration data indicating that low doses had no significant effects on viability, suggests a negligible effect of toxicity from an overnight culture.

We report here data on intracellular cytokine expression at a single time point (T24); however, monocyte production of these cytokines may be optimally detected at 
different times. Because multiple timepoint sampling was experimentally prohibitive, we chose the best overall time point (i.e. T24). We also counteracted some of this limitation by using agents that impeded the secretion of cytokines by interfering with the cellular Golgi apparatus thus retaining them inside the cell such that early and later produced cytokines were present at the single sample timepoint.

Our data here of on the effects on monocytes does not differentiate between direct and indirect actions. The cultures comprised PBMC with monocyte data collated from cytometric gating. This limits the interpretation of the data, as clozapine may act both directly and indirectly through the action of other cells in culture. 


\subsection{Summary.}

The ability of clozapine to alter the peripheral and therefore, potentially the CNS environment, make it an intriguing therapeutic option in treating progressive MS. We demonstrated modulation of dopamine receptors and cytokine expression by monocytes, the key immune effector cells that have a well-established role in autoimmune pathogenesis and disease outcome. Monocytes from MS participants showed a clear differential pattern in cytokine responses and DR expression following in vitro stimulation and exposure to clozapine. Our data offers additional areas of research in which to explore alternative strategies for the treatment of progressive MS. 


\section{Chapter 5. Transcription factor and cytokine responses following MIS416 treatment in vitro.}

\subsection{Introduction.}

MIS416 comprises a large, non-soluble microparticle suspension that must be internalised by phagocytosis to reach its targets. MIS416 has been demonstrated to be safely administered intravenously at up to $500 \mu \mathrm{g}$ / week (Luckey et al., 2015). Uptake of MIS416 by phagocytic cells induces a characteristic cytokine profile resulting in IL$1 \beta, \mathrm{IL}-10, \mathrm{TNF} \alpha$, and INF $\gamma$ production in human PBMC cultures in vitro (Girvan et al., 2011).

Monocytes are key cytokine-producing cells (Nathan, 1987; Xing and Remick, 2003) and have been well documented to produce a range of inflammatory (IL-6, IL-1 $\beta$, TNFa, IL-12) and anti-inflammatory cytokines (IL-10) in patients with MS (Filion et al., 2003; Liu et al., 2001). Activated monocytes are reported to be present in MS lesions (Kouwenhoven et al., 2001) and the pathogenicity of cytokines following immune activation is an established contributor to MS pathology (Rudick and Ransohoff, 1992). Furthermore, there are reports of altered cytokine levels and proportion of cytokine-producing cells present in the blood of patients with MS (Navikas and Link, 1996).

The major source of MIS416-induced cytokines in humans following MIS416 induction has not been shown. Monocytes are the likely source as they are early innate immune responder cells, acting through cytokine production and antigen presentation (Murphy, 2012). One potential benefit of MIS416 therapy is through altering cytokine profiles in patients (i.e. immunoregulation). Upregulation of cytokines by monocytes may contribute to inflammation in MS (Filion et al., 2003). Drug induced cytokine alterations have been observed to be beneficial in the treatment of early stage disease, as treatments such as interferon beta are known to increase IL-10 production, a known inflammation suppressing immunomodulator (Liu et al., 2001) . Altering cytokine profiles can therefore dampen immune responses or establish a more anti- 
inflammatory environment. This may be an effective treatment strategy if the resulting immunosuppression lessens MS disease activity.

Exposure to MIS416 engages extensive cellular signalling machinery which then results in the production and secretion of cytokines. Several key molecular steps, such as the phosphorylation of transcription factors (TF), are central to this process, and these steps can be detected and used to interrogate the signal pathways involved downstream of MIS416 activation.

MIS416 comprises two pathogen-associated molecular patterns (PAMP) namely CpG and MPD, targeting TLR9 and NOD2 respectively. TLR9 agonism from CpG results in TNF $\alpha$ and IL-6 induction via nuclear factor kappa B (NFאB) activation and associated transcriptional activity (Tsujimura et al., 2004). NOD2 agonism induces IL-10, also in response to $\mathrm{NF \kappa B}$ activation (Macho Fernandez et al., 2011). In profiling protein phosphorylation of the TF central to the MIS416 response, we can gain insight into the drug's mechanism of action and whether those pathways are substantially altered in progressive MS. Furthermore, the ability of MIS416 to alter cellular immune homeostasis by modulating cellular $\mathrm{NF} \kappa \mathrm{B}$-mediated signalling responses can be investigated.

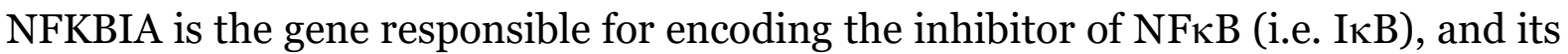
expression is, in part, regulated by un upstream promotor. Miterski et al. identified an insertion of 8 base pairs in the promotor of NFKBIA which occurred at a lower frequency in progressive MS compared to control subjects, suggesting a putative protective role for this allele (Miterski et al., 2002). Because the effect of the NFKBIA promoter insertion on function is unknown, we propose to stratify participants based on their NFKBIA mutation status to observe what effect the mutation may have on TF phosphorylation and cytokine expression.

Our work in this chapter was based on the premise that in vitro exposure of human PBMC to MIS416 would activate NFאB-mediated signalling and cytokine production in monocytes and that these responses would be shaped by the presence of NFKBIA promoter mutations. 


\subsection{Aims.}

This chapter investigates the specific effects of MIS416 treatment on PBMC in vitro with a particular focus on the effects in monocytes. In focussing on monocytes, we aimed to determine if there were inherent differences in the innate response to MIS416 during MS. Additionally, because previous research has indicated that a mutation in the promotor region of the inhibitor for $\mathrm{NF \kappa B}$ may impact disease outcome in progressive MS, we stratified individuals from our MS and HC cohort further, using their sequencing data for this promotor region to explore the effect of genotype on cytokine production and innate signalling pathways. In this chapter we tested the hypothesis that monocytes from people with progressive MS respond differently to MIS416 in vitro compared to those from HC, and that the pattern of MIS416-induced responses correlates to mutational status. To test this hypothesis, we addressed three specific aims.

\subsubsection{Specific Aims.}

5.2.1.1 To profile and compare the phosphorylation status of NFkB p65, CREB, STAT1 and STAT3 proteins in monocytes from $\mathrm{HC}$ and MS participants that were stimulated in vitro with MIS416.

5.2.1.2 To determine if in vitro-stimulated monocyte responses vary between $\mathrm{HC}$ and MS participants by profiling the cytokines IL-1 $\beta$, IL-6, IL-10, TNF $\alpha$ and INF $\gamma$.

5.2.1.3 To elucidate the effect of a mutation in the promotor region for the NFKBIA gene on cytokine production and signal pathways in monocytes from healthy and progressive MS subjects. 


\subsection{Methods.}

\section{5•3.1 Study participants.}

The study comprised two cohorts: a progressive MS group and a healthy group of ageand sex-matched individuals. PBMC were collected fresh and stored in liquid nitrogen until use. All MS participants were screened and enrolled as previously described (Luckey et al., 2015; La Flamme et al., 2020) and had either primary or secondary progressive disease with no significant confounding comorbidities and were not receiving any disease-modifying therapy. Subjects selected were from two clinical trial cohorts - the CRISP trial, which included healthy individuals and progressive MS ( $\mathrm{n}=$ 9/group), and the MIS416 Phase $2 \mathrm{~b}$ trial which included only secondary progressive MS ( $n=18$ selected) (see 3.3.1, demographic data). From the total MIS416 trial cohort we only selected from participants who were recruited in NZ ( $n=28$ available). Selection was based on their NFKBIA mutation status to include participants with homozygous $(n=2)$ heterozygous $(n=7)$, and wild type $(n=9)$ mutational status, while also ensuring age and gender averages matched to the CRISP cohort.

\subsubsection{Ethical approval.}

Full ethical approval was in place before we commenced any experiments, with the CRISP trial being approved by the Central Health and Disability Ethics Committee (15/CEN/216) and the Standing Committee on Therapeutic Trials (15/SCOTT/177), and the MIS416 Phase 2b trial approved by the Uniting Care Health Human Research Ethics Committee, Western Australia (MIS416-202).

\section{5•3.3 Experimental procedures.}

The in vitro protocol for PBMC sample processing was standardised for all samples and detailed in Chapter 3 (see section 3.2.1.1 - 3.2.15). Briefly, individual donor human PBMC were thawed, DNase treated and revived under standard in vitro conditions $\left(37^{\circ} \mathrm{C} ; 5 \% \mathrm{CO}_{2}\right)$ for 90 minutes in complete media. Cells were then 
stimulated with either MIS416 ( $5 \mathrm{ug} / \mathrm{ml}$ ), LPS ( $1 \mathrm{ng} / \mathrm{ml})$, or left untreated. After 3 hours an aliquot of cells was removed, viability stained, and immediately fixed for TF analysis. The remaining cells were incubated overnight in the presence of a brefeldin $\mathrm{A}(0.5 \mathrm{ul} / \mathrm{ml}) \&$ monensin $(0.3 \mathrm{ul} / \mathrm{ml})$ mixture. Following they overnight culture, the cells were viability stained, fixed, permeabilised using Cytoperm Buffer (Becton Dickenson, USA), stained with antibodies, and washed. Data were acquired on a Cytek Aurora spectral cytometer the following day.

All culture aliquots received an identical lineage marker stain (see Table 3-1, PBMC lineage stain). Additionally, cells cultured for $\mathrm{TF}$ expression were stained with $\mathrm{TF}$ reported markers (Table 3-1, Tube 4) while cells cultured for cytokine expression were stained for cytokine reporters (Table 3-1, Tube 3). All cytometry data were gated using a common PBMC gating strategy to yield terminal monocytes (Figure 3-2).

\section{5•3.4 NKFBIA mutation analysis.}

Sanger sequencing for the promotor region of the NFKBIA gene was performed on the purified DNA for all our participants by our collaborators. For the CRISP trial participants, DNA was extracted with the DNeasy Blood and Tissue Kit, (Qiagen, US) and shipped as dehydrated pellets for sequencing. All MIS416 phase 2b trial participants had mutational analysis for the NFKBIA gene performed previously as part of the trial protocol. Primer sequences and PCR conditions were as previously described (Miterski et al., 2002).

\section{$5 \cdot 3 \cdot 5$ Statistics.}

Statistical analyses were performed in Prism 8 (Graphpad, US). All data distributions were tested for normality, and log transformed where necessary with negative values log transformed using a correction factor. Normally distributed data was subject to an ordinary one-way analysis of variance test (ANOVA), or Mixed Effect test, with Geisser-Greenhouse post-test. Data failing normality testing was subject to a repeat measure Kruskal-Wallis test with Dunn's multiple comparison test. 
To correct for multiple comparisons, we used Turkey's multiple comparison test. We applied correction but only to inter treatment group comparisons. No correction was applied in comparing stratified population differences. While this approach was less stringent, it was appropriate as it enabled for future hypothesis generation.

Direct comparisons between two cohorts was performed using t-tests of the calculated mean and SD, except for gender distributions in the population which was by Fisher's test. Baseline corrected data was expressed as the percentage change from untreated cells, with the mean group values compared using the Mann-Whitney test.

All correlation analysis was performed using Pearson's correlation matrixes, and we used linear regression to demonstrate the relationship between ELISA, gMFI and percentage positive cell data. $\mathrm{R}^{2}=$ coefficient of determination (i.e. 'goodness of fit'), $\mathrm{r}=$ Pearson $\mathrm{r}, \mathrm{CI}=$ confidence interval.

In all instances $\mathrm{p}$ values at $\leq 0.05$ were taken as statistically significant. Results close to the threshold were interpreted with caution so as not to exclude potentially meaningful results.

All graphically depicted group averages were plotted as mean values, and error bars represent SD. 


\subsection{Results.}

\subsubsection{Participant demographics.}

To investigate if monocyte responses to MIS416 were altered during progressive MS, we assessed stimulated monocytes in PBMC cultures from two New Zealand based clinical trial cohorts comprising untreated progressive MS participants. Concurrently, we compared responses in PBMC cultures from an age- and sex-matched group of healthy control participants recruited locally from the Wellington region (Table 5-1).

Table 5-1 Demographic distribution of study participants.

\begin{tabular}{|c|c|c|c|}
\hline \multicolumn{4}{|c|}{ Study Population } \\
\hline Healthy & & & \\
\hline & $\mathrm{N}$ & & 9 \\
\hline & Age & & \\
\hline & & Mean & $54 \cdot 33$ \\
\hline & & Range & $46-66$ \\
\hline & Gender & $\mathrm{F}: \mathrm{M}(\% \mathrm{~F})$ & $7: 2(77.8)$ \\
\hline MS & & & \\
\hline & $\mathrm{N}$ & & 27 \\
\hline & & CRISP & 9 \\
\hline & & MIS416 & 18 \\
\hline & Age & & \\
\hline & & Mean & 56.07 \\
\hline & & Range & $35-70$ \\
\hline & Gender & $\mathrm{F}: \mathrm{M}(\% \mathrm{~F})$ & $21: 6(77.8)$ \\
\hline
\end{tabular}




\subsubsection{Protein phosphorylation responses to MIS416 in vitro.}

$\mathrm{NF}_{\kappa \mathrm{B}}$ activation is one of the key transcription factors mediating the response to MIS416. To better understand the cellular response to NFкB-mediated signalling, we profiled the phosphorylation of transcription factors (NFאB p65, CREB, STAT1, and STAT3) that are key to the induction of cytokine expression and activation in monocytes.

5.4.2.1 Determining MIS416 uptake by monocytes.

MIS416 must be internalised by the cell before it can make contact with its intravesicular and cytosolic receptors, so we conducted a time course with fluorescently labelled MIS416 conjugated to FITC to determine the optimal time point. We found that after 3 hours approximately 60\% of the monocytes in a PBMC culture had taken up MIS416 (Figure 5-1). Since protein phosphorylation is generally a rapid process, with soluble targets such as LPS causing a detectable increase after only fifteen minutes (Nolan and Condello, 2013), it was anticipated that NFкB phosphorylation and other downstream signal pathways would occur by three hours post exposure to MIS416. Indeed, a time course for the detection of phosphorylated NFאB, CREB, STAT1, and STAT3, showed no difference between untreated and MIS416-treated monocytes at 15 and 60 minutes but did show a detectable decrease in the fluorescent intensity of monocytes after 180 minutes (Figure 5-2). Using this system, we aimed to identify the MIS416-induced effects on the signalling pathways in monocytes, and if these effects were altered during progressive MS. 
A
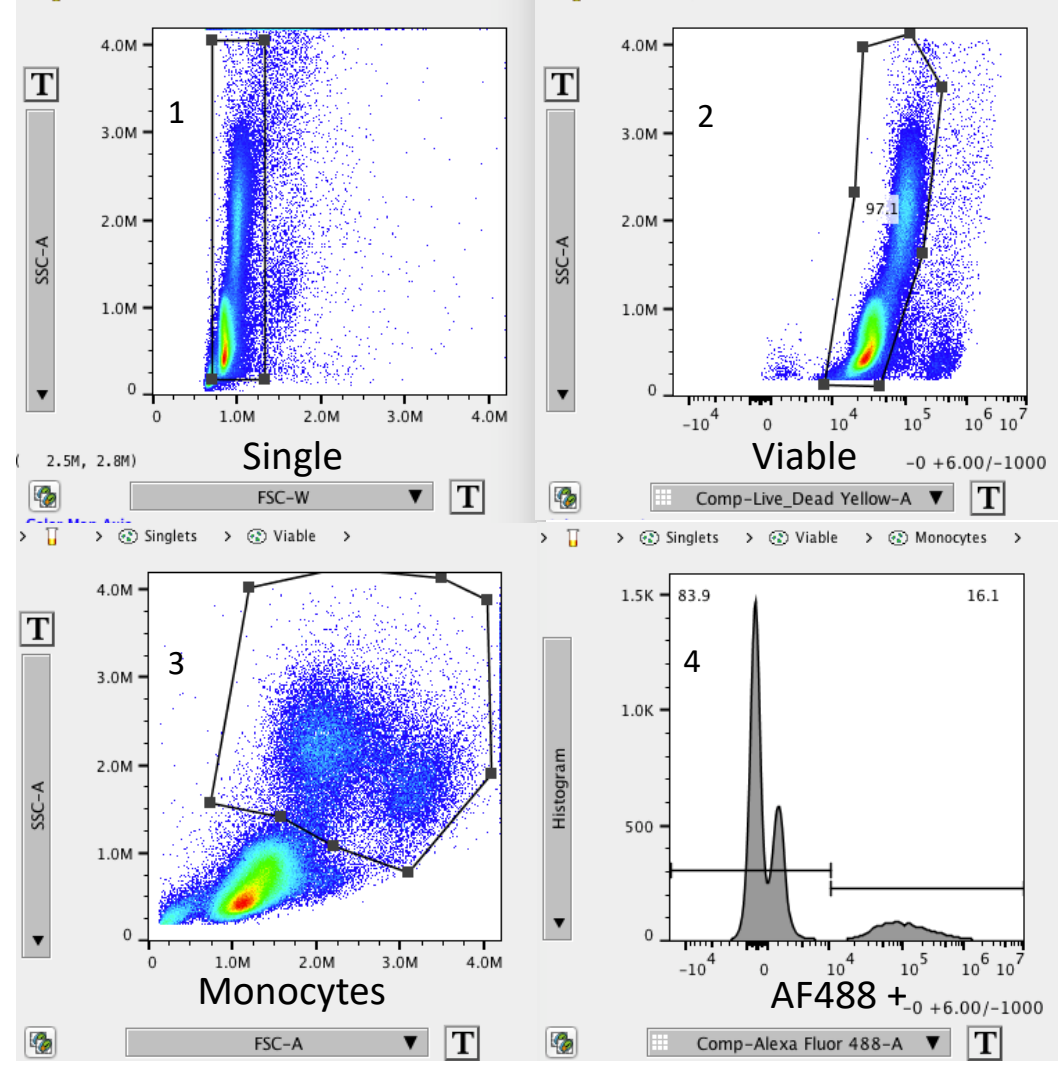

B

AF488 MIS418 uptake (monocytes)

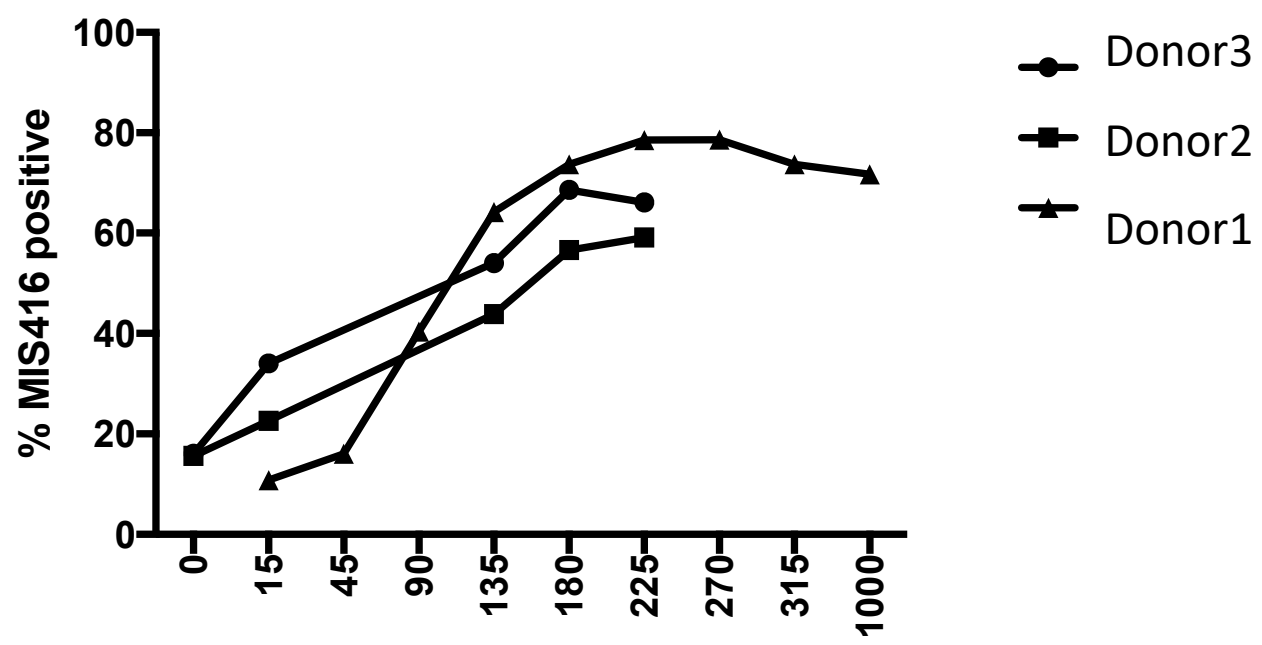

Time (min)

Figure 5-1 Time series of fluorescent MIS416 uptake by monocytes from 3 healthy donors.

(A) Gating strategy sequence 1-4. (B) The proportion of positive plotted over a sampling time series. Donor 1 had an extended sample series while donor 2 and 3 ended at 225 minutes. 


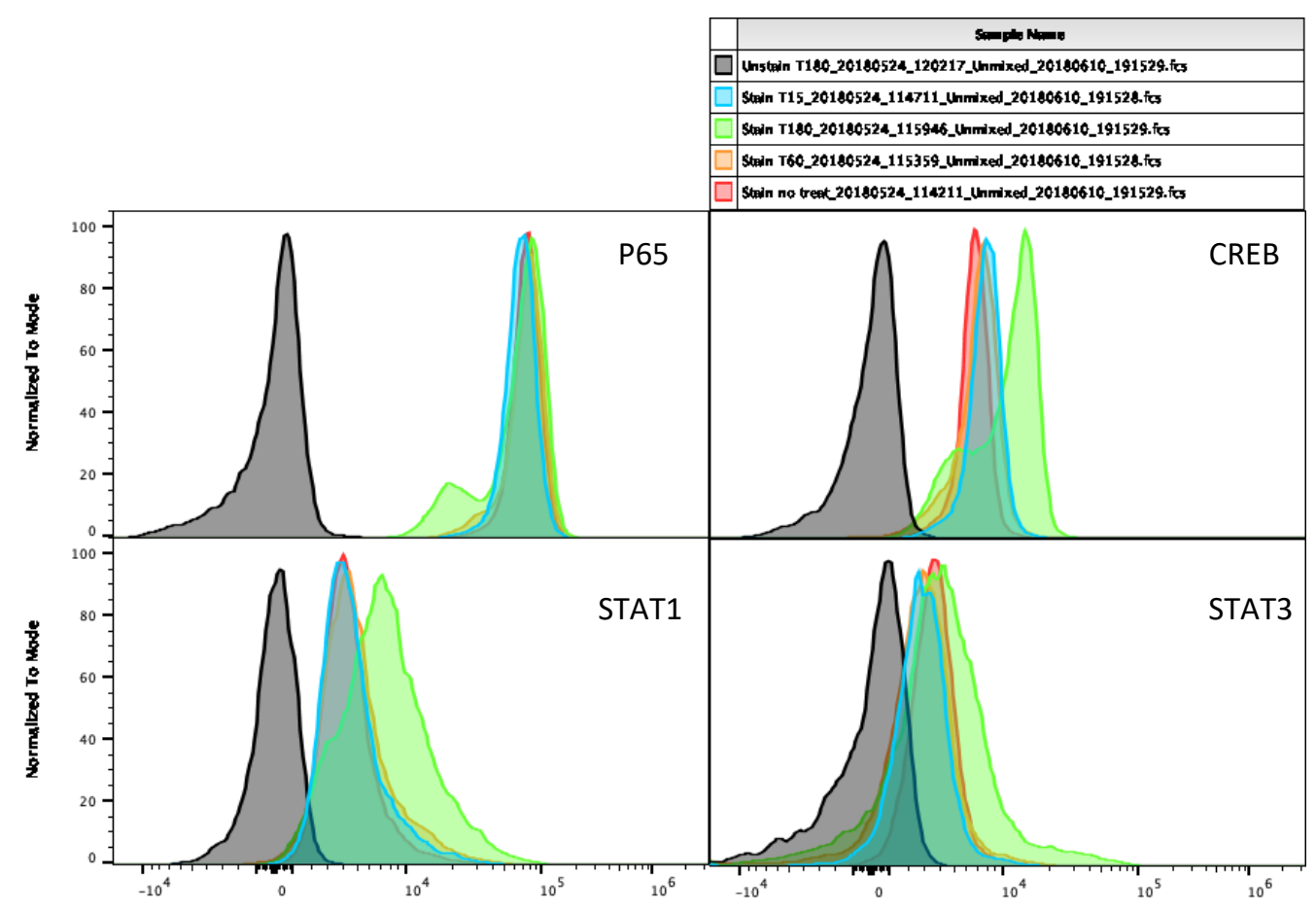

Figure 5-2 Phosphorylation of STAT1, STAT3, CREB and p65 in monocytes stimulated with MIS416 over a time series.

15 minutes (blue), 60 minutes (red) 180 minutes (green) of MIS416 stimulation. 
5.4.2.2 5529 is a phosphorylation site in the transactivation domain of $\mathrm{p} 65$ and is used to profile activated $\mathrm{NF \kappa B}$.

The transactivation domain is responsible for interacting with the p65 inhibitor of $\mathrm{NF} \kappa \mathrm{B}(\mathrm{I} \kappa \mathrm{B})$. Upon activation and release from $\mathrm{I} \kappa \mathrm{B}$, the $\mathrm{S} 529$ site is phosphorylated to increase transcriptional activity. The monoclonal antibody K10-895.12.50 recognises phosphorylated S529 (pS529) on the human NFאB p65 subunit. Intracellular flow cytometric detection of a fluorescent conjugate to this antibody thus represents relative, unbound, and active $\mathrm{NF} \kappa \mathrm{B}$ with high transcriptional activity.

5.4.2.3 Three hours of in vitro culture with MIS416 reduced $\mathrm{NF}_{\kappa} \mathrm{B}$ activity in healthy participants.

In the HC cohort, the total proportion of monocytes expressing activated p65 did not vary significantly between the in vitro conditions, and active p65 was detected in essentially all viable monocytes (Figure 5-3). In contrast to the proportion of monocytes expressing activated p65, the level of activated p65 as assessed by the geometric mean fluorescent intensity (gMFI) was reduced in HC monocytes exposed to MIS416 for three hours in culture (T3 MIS) and was significantly lower than baseline (To) measures (Figure 5-3). Compared to untreated cells (T3 untreated), there was a modest treatment effect, but it did not reach statistical significance $(\mathrm{p}=$ o.07). Individual participant plots emulate this effect.

5.4.2.4 In progressive MS, there was an overall reduction in the proportion and activation of $\mathrm{NF \kappa B}$ in monocytes.

The total proportion of monocytes expressing active p65 in the MS cohort was reduced at T3 in both the untreated and MIS416-treated cultures to a similar extent (Figure 53). This reduction in activated p65 after 3 hours of culture was also evident when the level of activated p65 was assessed (i.e. gMFI). When the levels of activated p65 in monocytes from HC and MS individuals were directly compared, it was clear that at To and T3, irrespective of treatment, monocytes from MS participants had significantly less active p65. To determine if there was a differential response to MIS416 by HC and MS monocytes under in vitro conditions, the percentage change in activated p65 gMFI was compared. This analysis illustrated that while the levels were 
Chapter 5

lower in MS monocytes, the magnitude of the MIS416-mediated response was the same as in HC monocytes. 
A

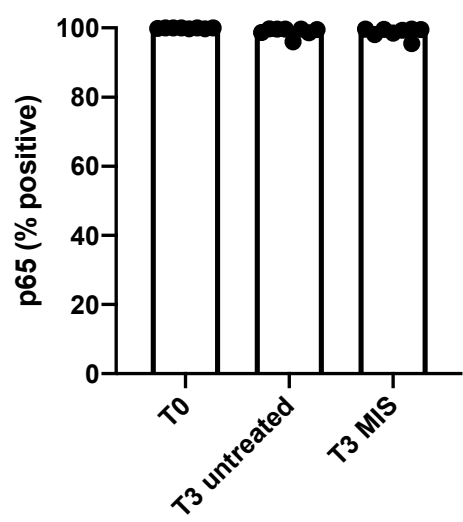

D
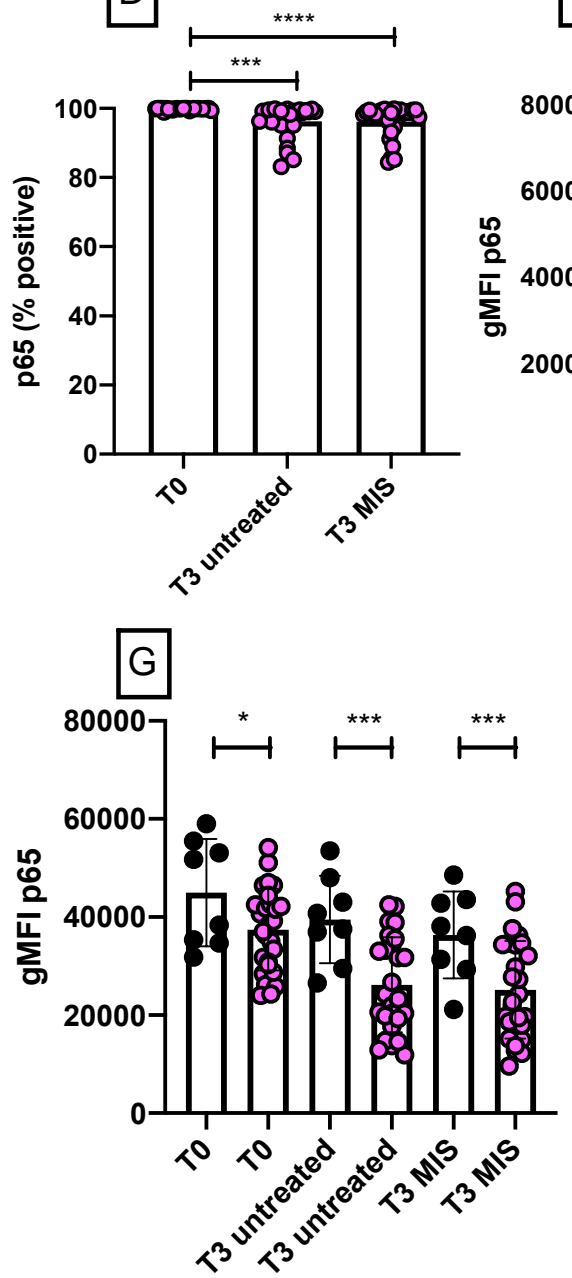

$B$

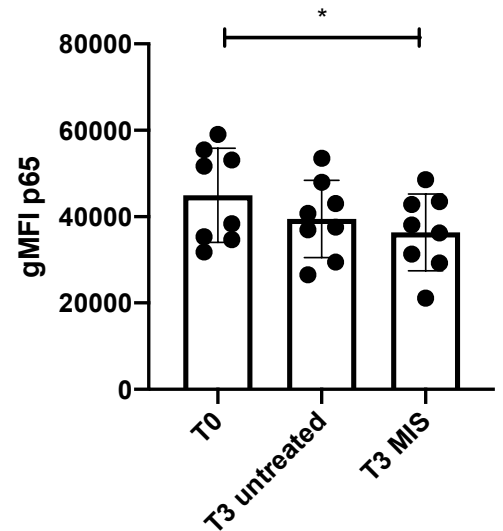

E
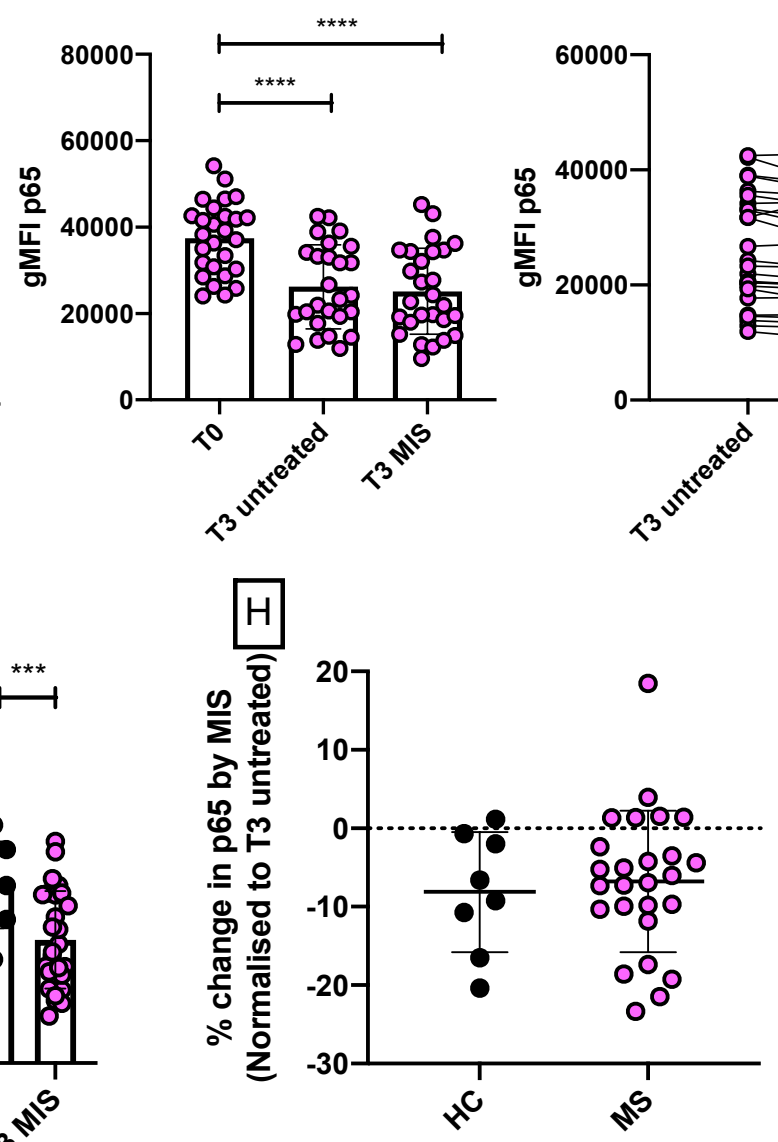

C

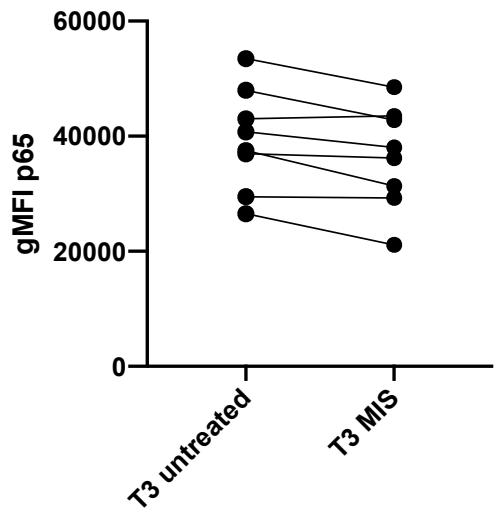

$\mathrm{F}$

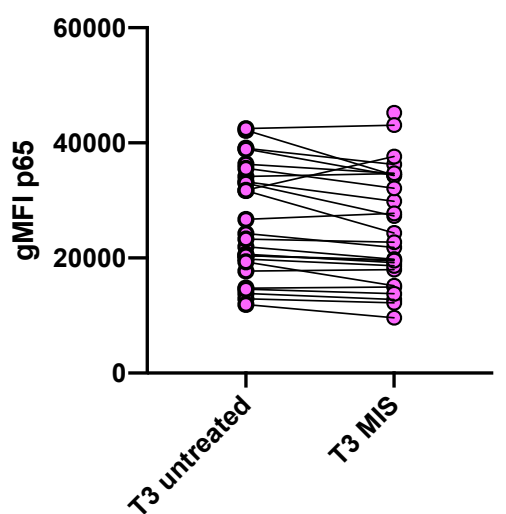

- $\mathrm{HC}$

$\circ \mathrm{MS}$

Figure 5-3 The mean proportion (A \& D) and level of activated NFkB p65 in monocytes.

Individual participants are indicated by a coloured circle, HC (black), MS (pink). (AC) depicts HC only data. (D-F) MS participant only data. (G \& H) a direct comparison between the cohorts. Bars indicate group mean, with error bars show SD. ${ }^{*}(\mathrm{p} \leq 0.05)$, ${ }^{* *}(\mathrm{p} \leq 0.01),{ }^{* * *}(\mathrm{p} \leq 0.001),{ }^{* * * *}(\mathrm{p} \leq 0.0001)$. 
5·4.2.5 MIS416 stimulation may alter STAT1 phosphorylation.

STAT1 is a ubiquitous protein that regulates interferon-induced genes (Zakharova et al., 2003). Exposure to INFY results in the phosphorylation at residue Y701, nuclear translocation, and DNA interaction (Darnell, 1997). The phosphorylated site (pY701) is detected by the monoclonal antibody 4a-pSTAT1. Cytometric detection of fluorescently labelled pY701 antibody is therefore primarily, but not restricted to, an indication of INF $\gamma$ response and activation. STAT1 is also activated by other INF proteins including IFNa \& IFN $\beta$, immune factors such as cytokines (IL-10, IL-6, IL11) as well as receptor tyrosine kinases and G-protein coupled receptors (Zakharova et al., 2003).

5.4.2.6 MIS416 treatment in vitro significantly reduced STAT1 activation in monocytes.

In the HC cohort, the total proportion of activated STAT1-expressing monocytes did not vary to any significant degree between the in vitro conditions (Figure 5-4). In contrast to the proportion of positive cells, we detected a significance decrease in the total amount of activated STAT1 (gMFI) present at T3, but only when cells had been treated with MIS416 (T3 MIS). This effect was observed in both the HC and MS cohorts. Furthermore, we observed that in MS participants, MIS416 treatment also reduced the total proportion of monocytes expressing activated STAT1.

5.4.2.7 In progressive MS, there is a reduction in the activity of STAT1.

Comparing MS with HC participates highlighted a significant reduction in the amount of active STAT1 in MS participants at To. Untreated cells at T3 showed a similar trend but did not reach the threshold of significance $(\mathrm{p}=0.06)$ (Figure 5-4). To establish if there was a difference in the magnitude of response between MS and HC participants following MIS416 stimulation, we compared the fold change in activated STAT1 between the cohorts but did not observe any significant difference in the magnitude of MIS416-mediated change. 
A

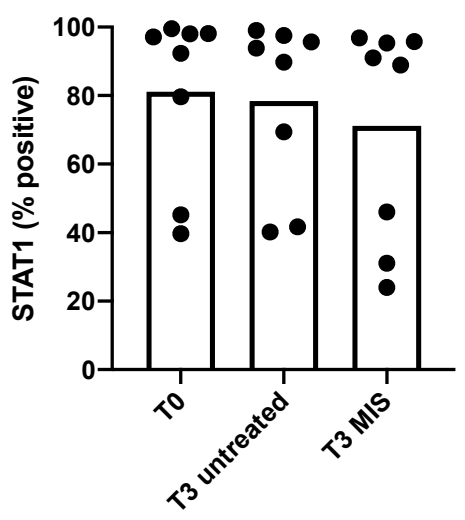

D

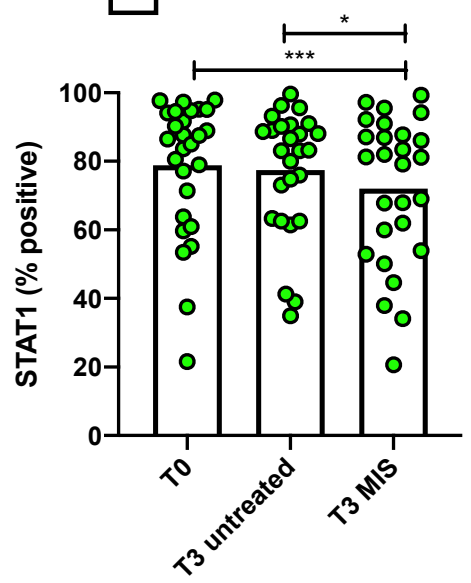

$\mathrm{G}$

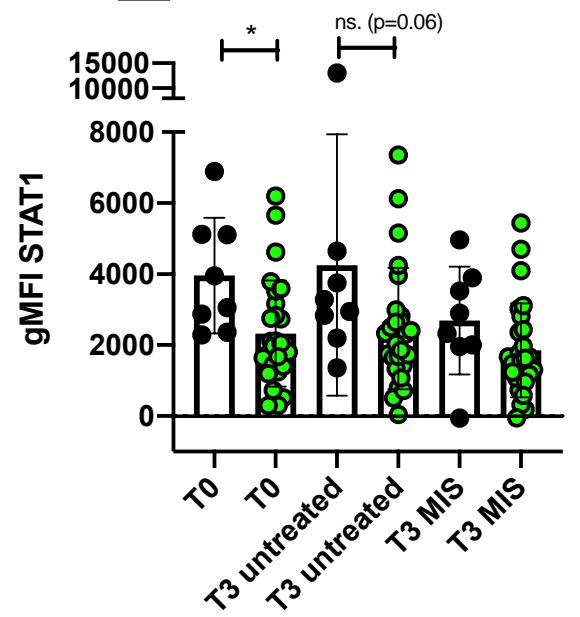

B

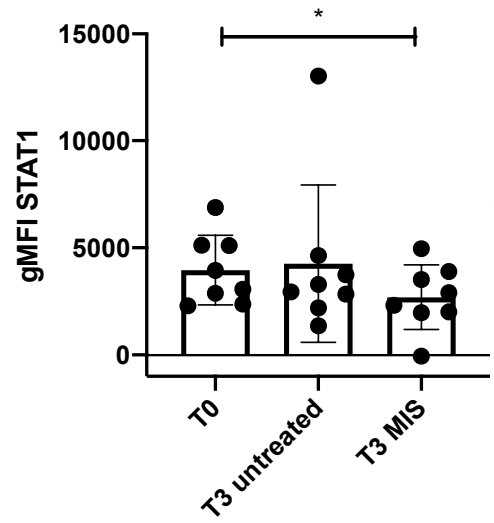

E

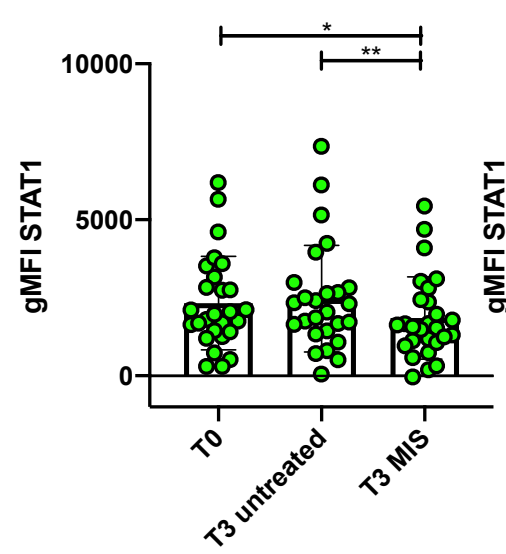

$\mathrm{H}$

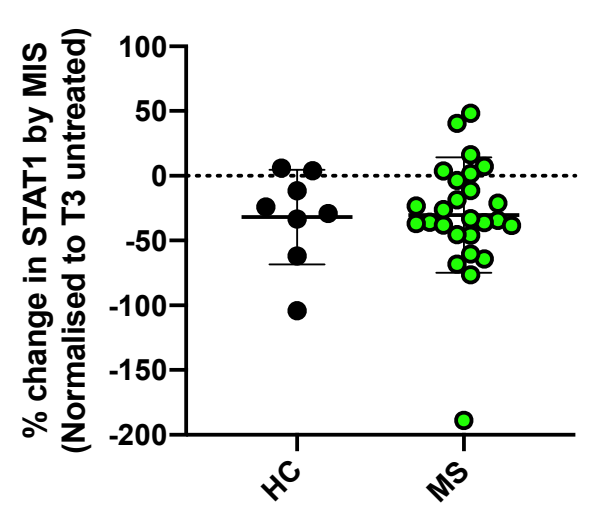

C

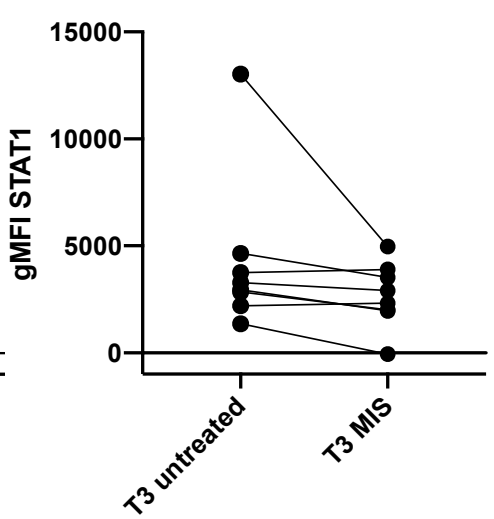

$\mathrm{F}$

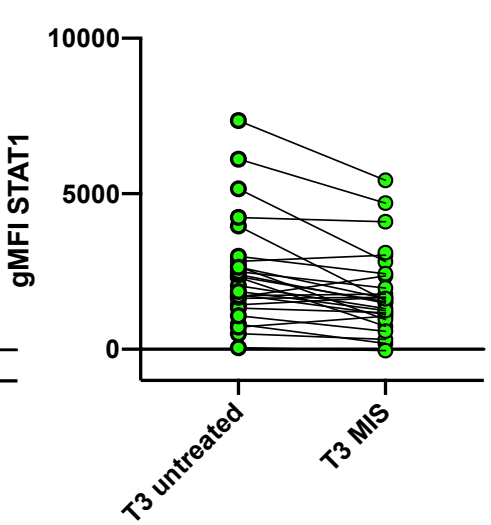

- $\mathrm{HC}$

- MS

Figure 5-4 The mean proportion (A \& D) and level of activated STAT1 in monocytes.

Individual participants are indicated by a coloured circle, HC (black), MS (green). (AC) depicts HC only data. (D-F) MS participant only data. (G \& H) a direct comparison between the cohorts. Bars indicate group mean, with error bars show $\mathrm{SD} .{ }^{*} \mathrm{p} \leq 0.05$, ${ }^{* *} \mathrm{p} \leq 0.01,{ }^{* * *} \mathrm{p} \leq 0.001,{ }^{* * * *} \mathrm{p} \leq 0.0001$. 
5.4.2.8 STAT3 is crucial mediator of TNFa signalling.

STAT3 is transcription factor that is essential for cellular differentiation, apoptosis and growth as it mediates signals from receptors to a range of different cytokines (Forbes et al., 2016) including: IL-10, IL-6, IL-12 and INF. In monocytes, STAT3 signalling is crucial in the downregulation of TNFa synthesis (de Jong et al., 2012). Y705 is a tyrosine residue site on STAT3 that is phosphorylated to activate $\mathrm{STAT}_{3}$ in response to IL-6, INF and epidermal growth factor (EGF) (Zhong et al., 1994). Detection of pY705 STAT3 by intracellular staining with the 4/P-STAT3 monoclonal antibody indicates activated STAT3.

5.4.2.9 MIS416 treatment significantly reduces STAT3 activity in monocytes.

We observed that in both cohorts, the total proportion of activated STAT3-expressing monocytes was reduced at T3 MIS, while untreated cells showed no significant change from To (Figure 5-5). Furthermore, this reduction was reflected in the levels of active STAT3 detected, with MIS416 treatment causing a significant reduction in both cohorts. The variation in STAT3 proportion and activity between HC and MS cohorts did not reach significance nor did the magnitude of the treatment effect at $\mathrm{T}_{3}$ between $\mathrm{HC}$ and MS, indicating that STAT3 activation in monocytes from both MS and HC cohorts was similar. 
A
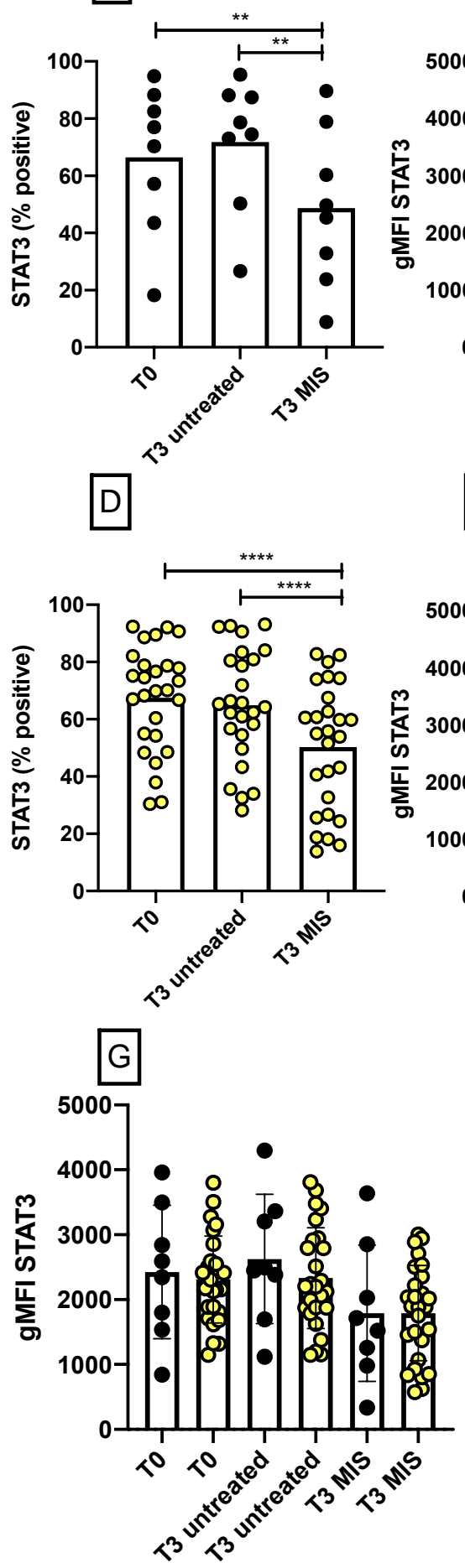

B
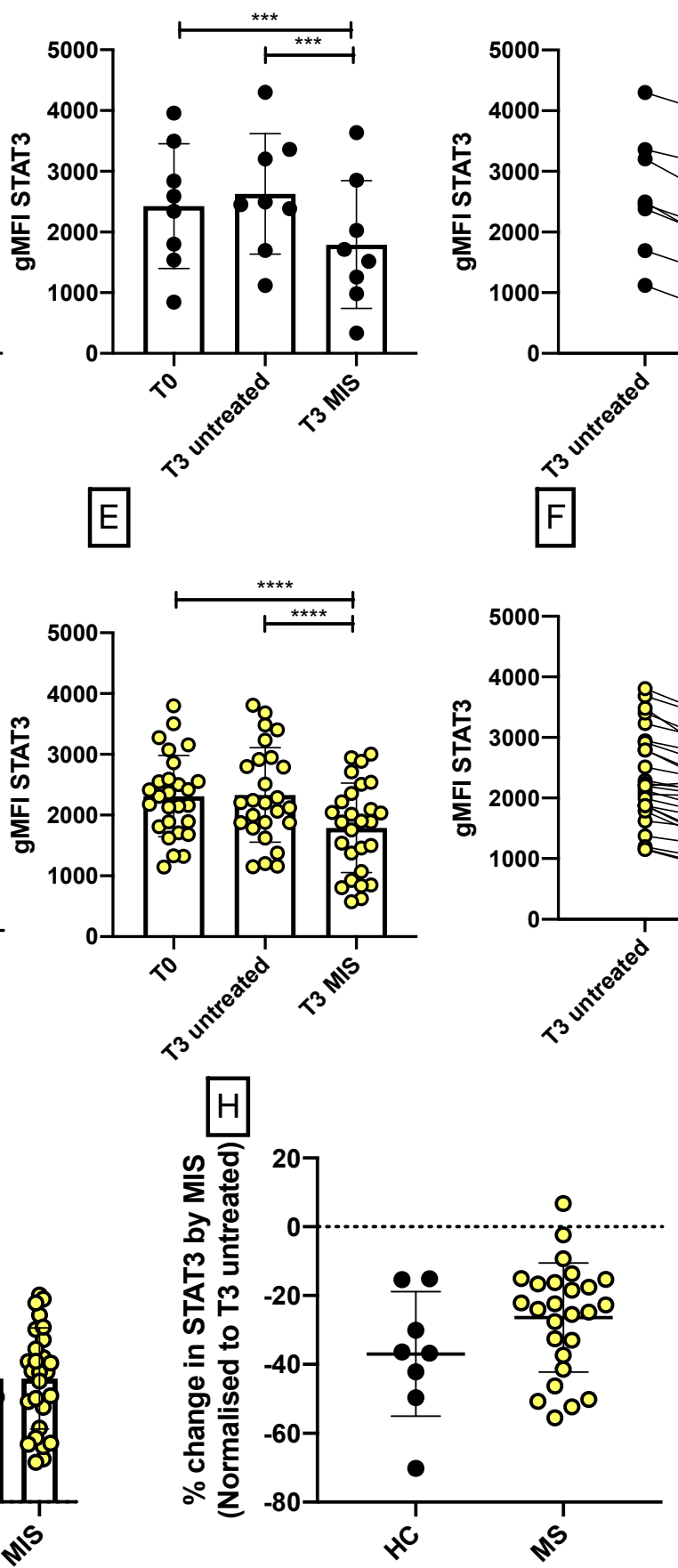

C
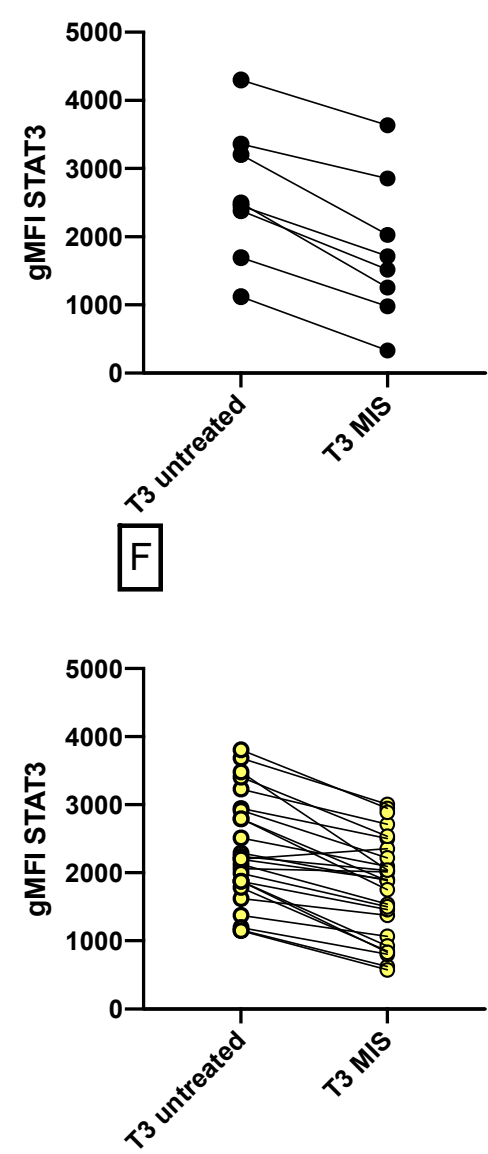

- $\mathrm{HC}$

- MS

Figure 5-5 The mean proportion (A \& D) and level of activated STAT3 in monocytes.

Individual participants are indicated by a coloured circle, HC (black), MS (yellow). (AC) depicts HC only data. (D-F) MS participant only data. (G \& H) a direct comparison between the cohorts. Bars indicate group mean, with error bars show SD. * $(p \leq 0.05)$, ** $(\mathrm{p} \leq 0.01),{ }^{* * *}(\mathrm{p} \leq 0.001),{ }^{* * * *}(\mathrm{p} \leq 0.0001)$. 
5.4.2.10 CREB activation, the counterpoint to p65 activity.

In addition to the effects of MIS416 on p65 activity, we measured adenosine $3^{\prime}, 5^{\prime}-$ monophosphate response element-binding (CREB) protein phosphorylation. CREB in part, functions in the regulation of immune responses by inhibiting $\mathrm{NF}_{\kappa} \mathrm{B}$ activation, promoting proliferation and survival (Park et al., 2005). The J151-21 antibody targets the pS133 site of CREB protein, and detection with a fluorophore conjugate indicates activated CREB protein.

5.4.2.11 Early CREB response was not significantly altered.

The total proportion of monocytes expressing activated CREB was not significantly different in the in vitro conditions of the HC cohort (Figure 5-6). In the MS cohort, we observed a modest decrease in the total CREB positive cells at T3 compared to To, independent of any treatment effect, but this modest effect was not found when the level of activated CREB (i.e. gMFI) was assessed. Moreover, three-hour treatment with MIS416 did not have a discernible effect in either cohort.

5.4.2.12 MIS416 in vitro responses indicate large a variation in CREB activation for individual participants.

To observe if there was a difference in the magnitude of CREB activation between HC and MS participants, we normalised expression to untreated T3 expression levels. While the difference between the cohorts was not significant, we observed that both cohorts appeared to have large variation with some individuals upregulating CREB activity, while others reducing it after MIS416 stimulation. 
A

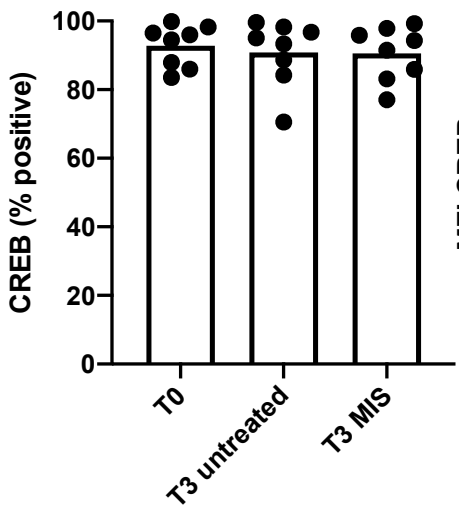

D
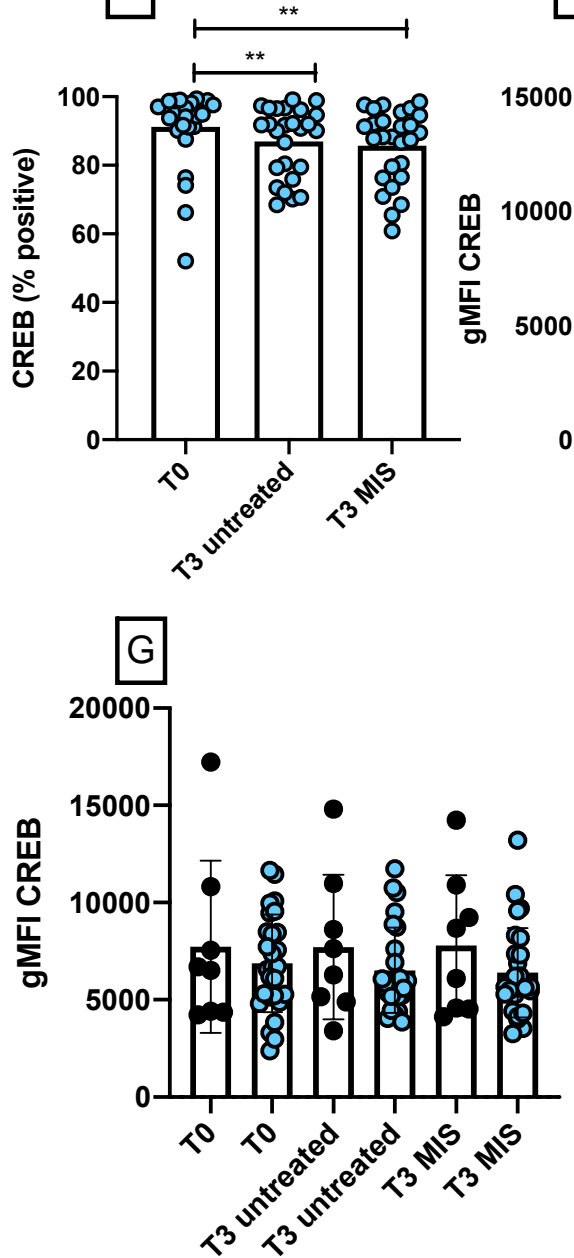

$B$

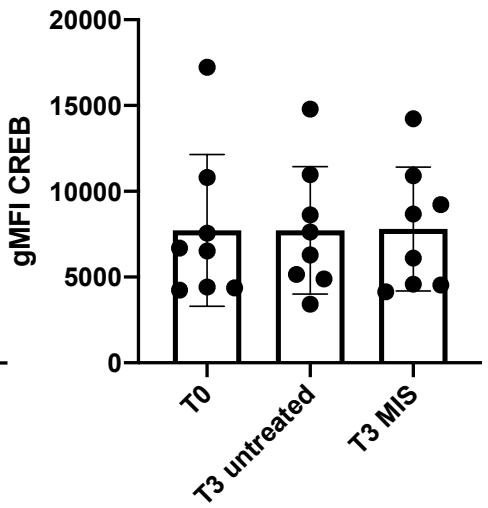

E
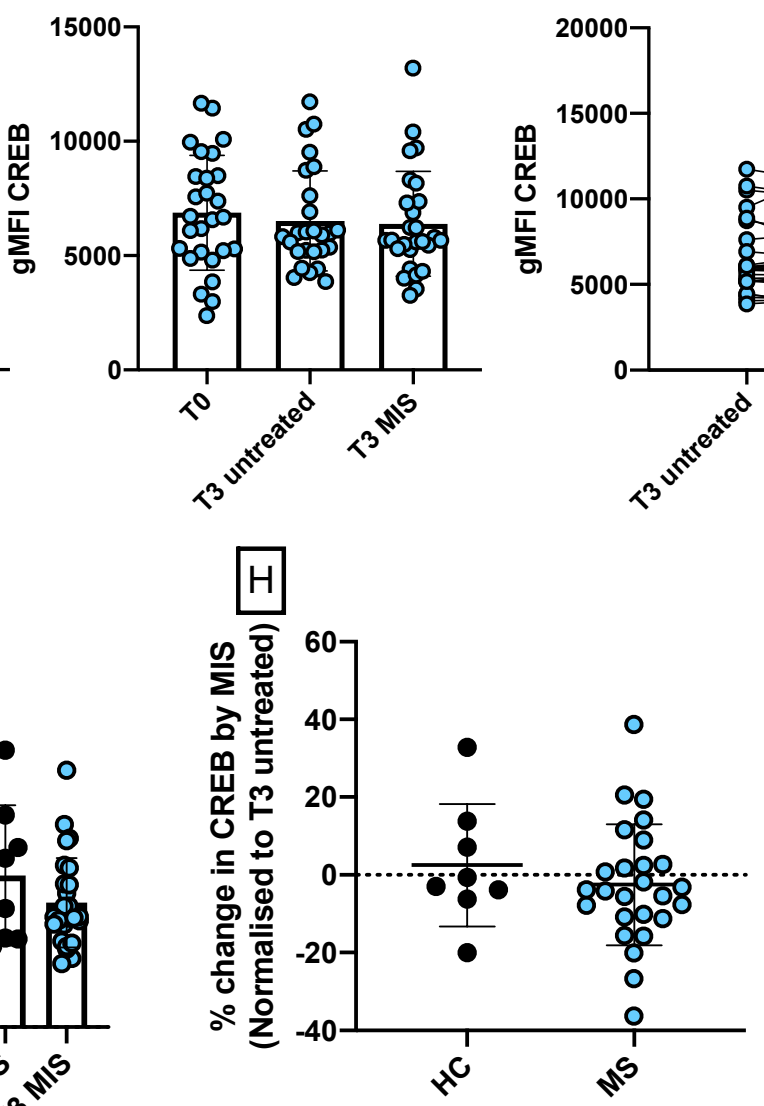

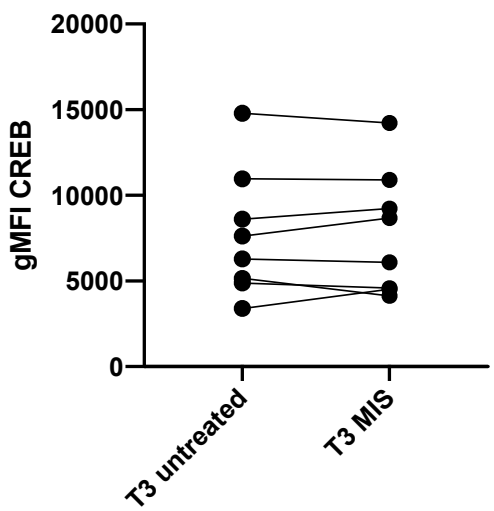

$\mathrm{F}$

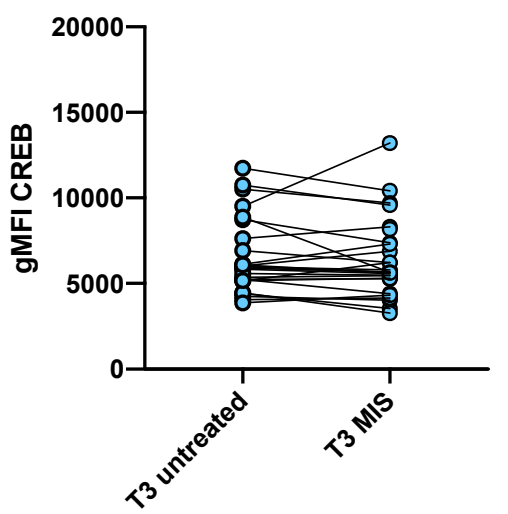

- $\mathrm{HC}$

○ MS

Figure 5-6 The mean proportion (A \& D) and level of activated CREB in monocytes.

Individual participants are indicated by a coloured circle, HC (black), MS (blue). (AC) depicts HC only data. (D-F) MS participant only data. (G \& H) a direct comparison between the cohorts. Bars indicate group mean, with error bars show SD. * ( $1 \leq 0.05)$, ${ }^{* *}(\mathrm{p} \leq 0.01),{ }^{* * *}(\mathrm{p} \leq 0.001),{ }^{* * * *}(\mathrm{p} \leq 0.0001)$. 


\subsubsection{The cytokine response profile of monocytes treated with MIS416.}

To establish if monocytes from HC and MS participants had a different cytokine response to in vitro MIS416 stimulation, we profiled IL-1 $\beta$, IL-6, IL-10, TNFa and INFY expression. These cytokines have previously been described as key response mediators to MIS416, and together, offer a diverse view of traditionally pro- and antiinflammatory response proteins.

5.4.3.1 A concentration of $5 \mu \mathrm{g} / \mathrm{ml}$ MIS416 induced sufficient cytokine expression in overnight culture for effective intracellular detection.

Our collaborators have previously done extensive optimisation of in vitro MIS416 use (unpublished data). We used their work as starting point to select the best concentration. We then confirmed which concentration induced a sufficient cytokine signal to enable effective detection in our system. Figure 5-7 shows the results from the concentration range experiment with MIS416, showing that IL-1 $\beta$, IL-6, and TNF $\alpha$ were detectable over a wide range of MIS416 concentrations following overnight culture. Next, we confirmed these findings with two different donors using two doses of MIS416 ( 0.5 and $5 \mu \mathrm{g} / \mathrm{ml}$ ) and found that $5 \mu \mathrm{g} / \mathrm{ml}$ MIS416 reliably induced cytokine responses that could be measured using standard intracellular cytometry techniques (Figure 5-8).

5.4.3.2 Low dose LPS results in a good cytokine response to act as control for the induction of monocytic cytokine production.

LPS was selected as an activation control for cytokine induction to profile the response alongside MIS416. We aimed for low dose LPS stimulation, as the overnight incubation required for optimal IL-10 induction from LPS could result in cell death at high concentrations. We started by profiling cytokine production (IL-6, IL-1 $\beta$ and TNFa) and observed that over an LPS dose series ranging from $0.1 \mathrm{ng} / \mathrm{ml}$ to $200 \mathrm{ng} / \mathrm{ml}$ the proportion of recorded monocytes was not significantly reduced following $6 \mathrm{~h}$ stimulation (Table 5-2). In PBMC cultured overnight with $1 \mathrm{ng} / \mathrm{ml}$ or $10 \mathrm{ng} / \mathrm{ml} \mathrm{LPS}$ respectively, we observed the higher dose to result in a reduction in viable monocytes (Table 5-3). We also observed that lower doses of LPS resulted in better cytokine 
Chapter 5

detection, with the lower doses yielding higher gMFI values for the three early response cytokines, and $1 \mathrm{ng} / \mathrm{ml}$ giving the best signal to noise ratio (Figure 5-9). 

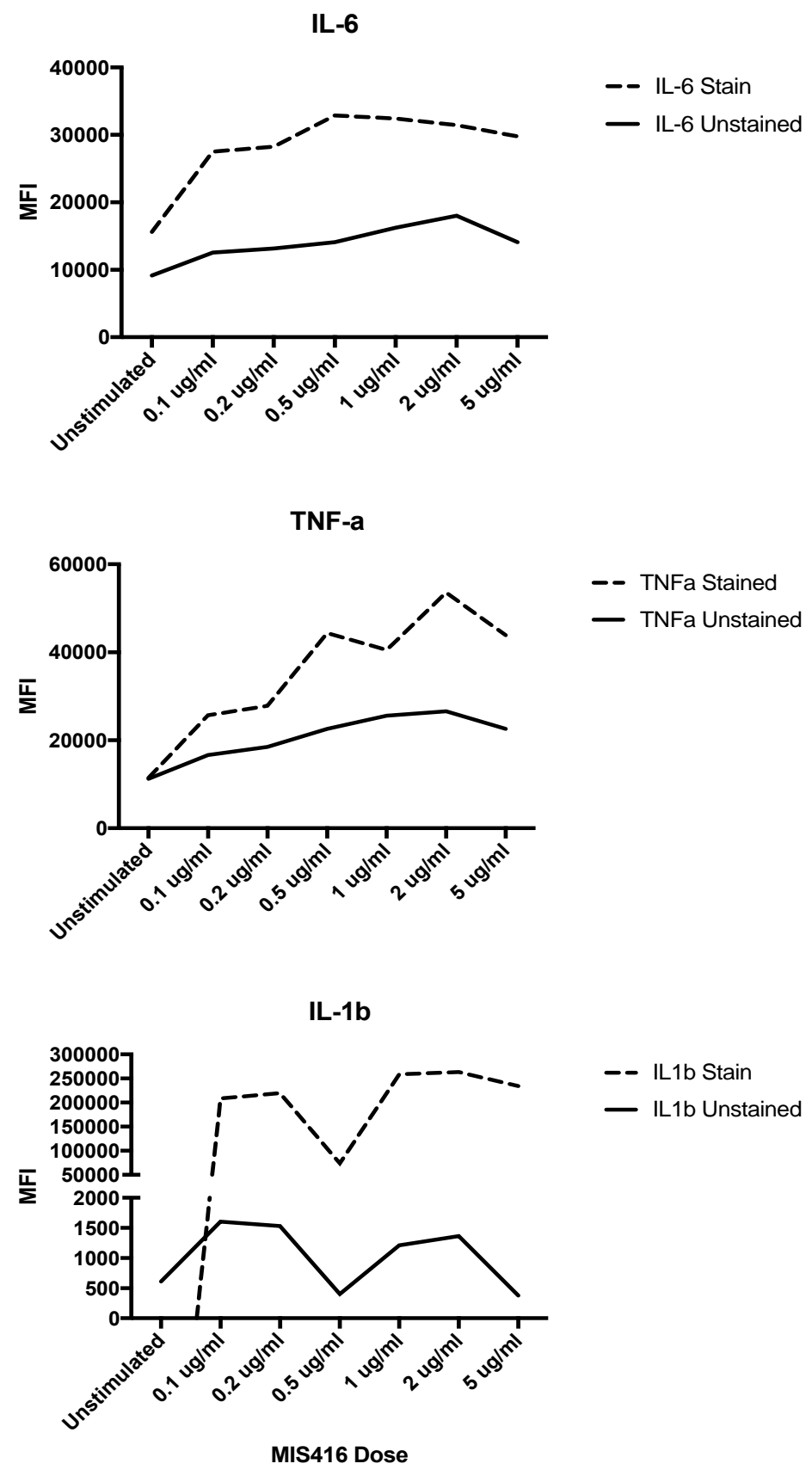

Figure 5-7 MIS416 response curve of cytokine expression.

Cytokine expression as gMFI is depicted over a MIS416 dose range.

Cells incubated overnight. 


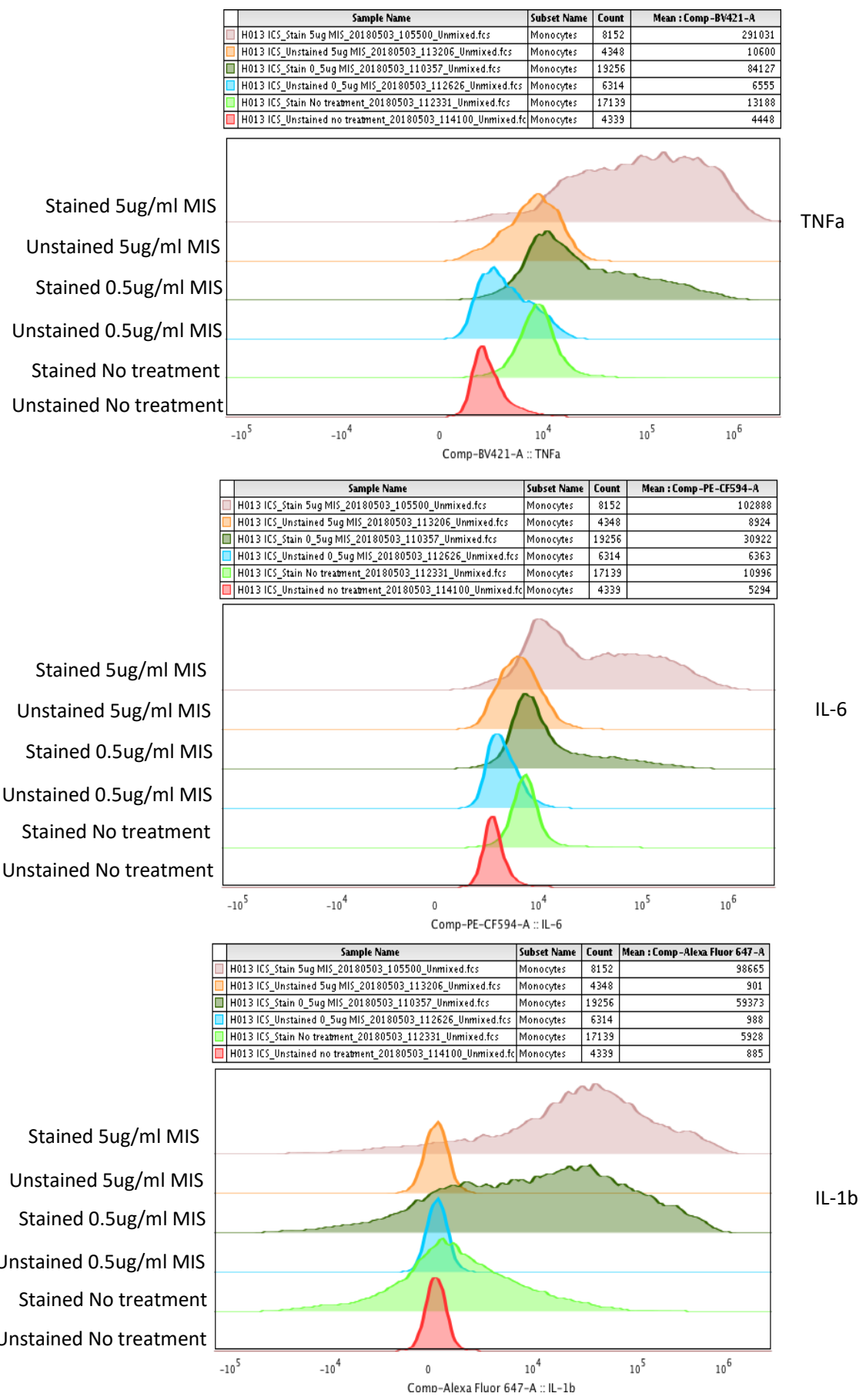

Figure 5-8 Effect of MIS416 on in vitro cytokine production in cells stimulated overnight.

Representative histogram layouts of stained and unstained gated monocytes for three stimulation conditions $(0.5 \mathrm{ug} / \mathrm{ml}$, $5 \mathrm{ug} / \mathrm{ml} \mathrm{MIS} 416$, or untreated) and their expression of TNF $\alpha$, IL-6 and IL-1 $\beta$ in a single donor. 
Table 5-2 Single donor stimulation of

PBMC over an LPS dose range.

\begin{tabular}{|l|r|}
\hline & $\begin{array}{l}\text { Monocytes } \\
(\%)\end{array}$ \\
\hline Media only & 7.94 \\
\hline Brefeldin A \& Monensin & 6.65 \\
\hline $0.1 \mathrm{ng} / \mathrm{ml}$ LPS & 4.35 \\
\hline $1.0 \mathrm{ng} / \mathrm{ml} \mathrm{LPS}$ & 2.94 \\
\hline $10 \mathrm{ng} / \mathrm{ml}$ LPS & 2.79 \\
\hline $100 \mathrm{ng} / \mathrm{ml}$ LPS & 4.52 \\
\hline $200 \mathrm{ng} / \mathrm{ml} \mathrm{LPS}$ & 4.69 \\
\hline
\end{tabular}

Data depicts the proportion of monocytes

from all CD45 positive single cell events

for cells incubated overnight.

Table 5-3 Cell proportions following overnight LPS stimulation.

\begin{tabular}{|c|c|c|c|c|c|c|}
\hline Donor & \multicolumn{3}{|c|}{ H013 } & \multicolumn{3}{|c|}{$\mathrm{H} 022$} \\
\hline Dose & Untreated & $\begin{array}{l}1 \mathrm{ng} / \mathrm{ml} \\
\text { LPS }\end{array}$ & $\begin{array}{l}10 \mathrm{ng} / \mathrm{ml} \\
\text { LPS }\end{array}$ & Untreated & $\begin{array}{l}1 \mathrm{ng} / \mathrm{ml} \\
\text { LPS }\end{array}$ & $\begin{array}{l}10 \mathrm{ng} / \mathrm{ml} \\
\text { LPS }\end{array}$ \\
\hline Monocyte & 14,30 & 5,51 & 4,77 & 19,70 & 8,42 & 4,44 \\
\hline NK-cells & 5,48 & 7,24 & 7,04 & 12,40 & 11,20 & 11,50 \\
\hline B-cells & 3,51 & 5,62 & 7,00 & 6,54 & 6,59 & 5,75 \\
\hline T-cells & 76,70 & 81,60 & 81,20 & 61,30 & 73,80 & 78,30 \\
\hline
\end{tabular}

Data depicts the major immune cells subsets of the PBMC as a proportion of total viable CD45 positive cells. 


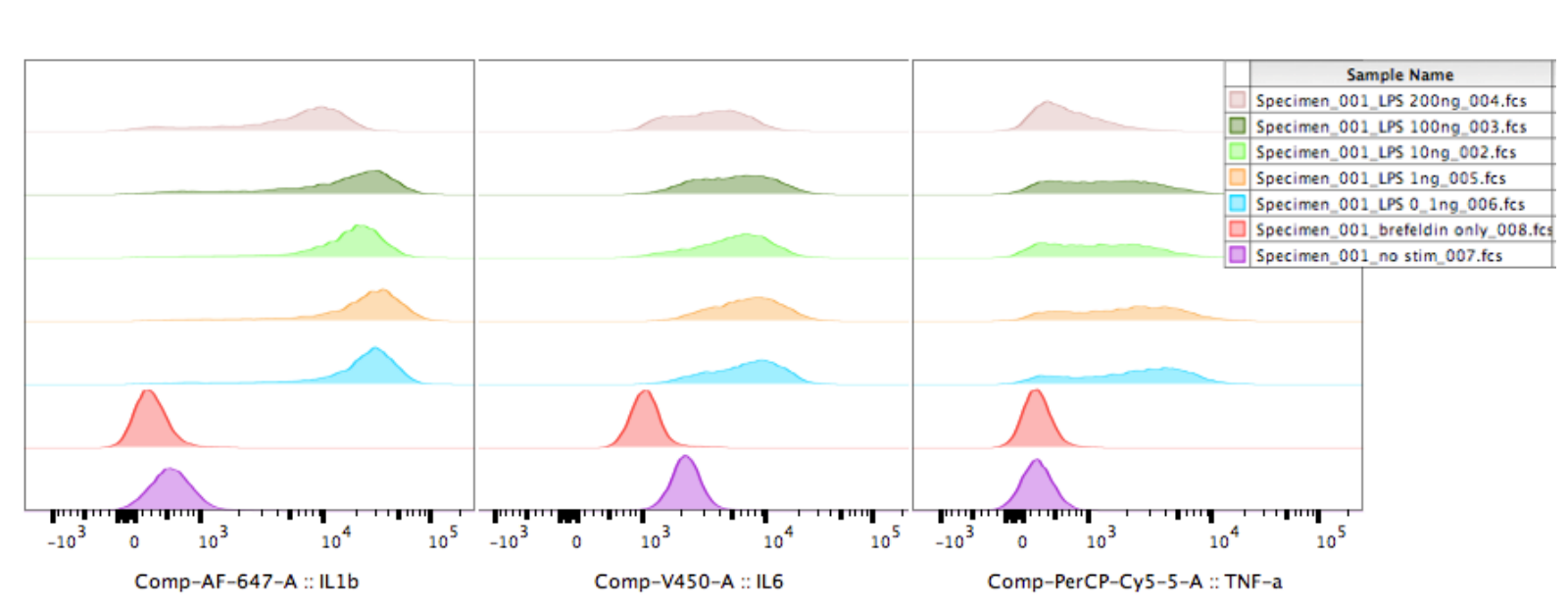

Figure 5-9 Representative, single donor LPS stimulation series.

PBMC were incubated over a range of LPS doses and stained for cytokine expression.

Viable monocytes demonstrate cytokine staining at lower LPS doses with $1 \mathrm{ng} / \mathrm{ml}$ (Orange) indicating the best signal to noise ratio. 
5.4.3.3 The profile of IL-1 $\beta$ expression in in vitro cultured monocytes was characteristic of a response to TLR agonism.

Overnight exposure to either LPS or MIS416 resulted in a strong IL-1 $\beta$ response in monocytes, indicating cellular immune activation (Figure 5-10). In both cohorts, LPS (HC: $\mathrm{p}=0.0035 ; \mathrm{MS}: \mathrm{p}<0.0001$ ) and MIS416 (HC: $\mathrm{p}=0.0179 ; \mathrm{MS} \mathrm{p}<0.0001$ ) yielded a significant increase in the proportion of IL-1 $\beta^{+}$cells, up from $51 \%$ in untreated cells, to nearly 90\% following either LPS or MIS416 stimulation. No significant difference in the proportion of IL-1 $\beta^{+}$cells was observed between LPS and MIS416 for either cohort, nor did inter cohort comparisons of the proportion of IL-1 $\beta^{+}$ cells show significant variation.

5.4.3.4 Monocytes induced a strong IL-1 $\beta$ response following in vitro exposure to MIS416.

Inter group analysis of the HC participants showed that the total amount (gMFI) of IL-1 $\beta$ expressed by monocytes was significantly higher for LPS ( $p=0.0009)$ and MIS416 ( $\mathrm{p}=0.005$ ) compare to untreated cells. The data shows that both LPS and MIS416 induced a strong IL-1 $\beta$ response in all HC participants, but that the magnitude of the response between the two agents did not vary greatly (Figure 5-10D).

5.4.3.5 MS participants were more responsive in terms of IL-1 $\beta$ induction by MIS416 compared to LPS.

In the MS cohort, the data showed a strong response to stimulation. MS participants responses were significantly stronger to MIS416 $($ mean $=52078.2)$ than LPS (mean = 29822.2) ( $\mathrm{p}=0.0126)$ (Figure 5-10). The magnitude of response to MIS416 in MS participants over untreated values was also significantly higher than to LPS ( $\mathrm{p}=$ 0.0051). This effect was not observed in the HC. However, when contrasting HC with MS, we did not observe a significant difference in the levels of IL-1 $\beta$ for any of the in vitro conditions for either the proportion of IL-1 $\beta^{+}$cells, nor the total amount of L-1 $\beta$ detected (Figure 5-10 I\&J). 

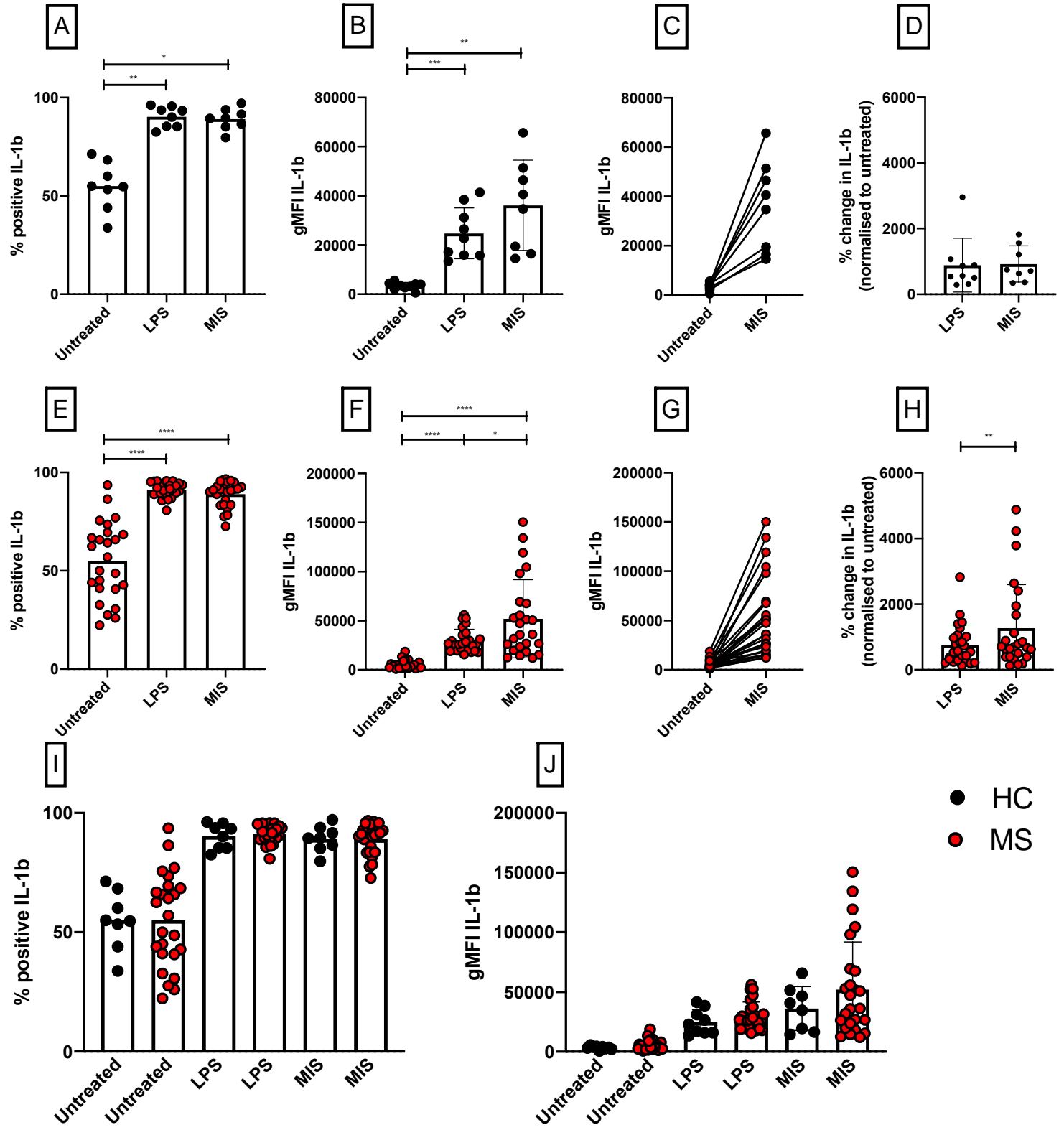

Figure 5-10 The mean difference in the proportion (A, E \& I) and relative expression of IL-1 $\beta$ in monocytes following overnight in vitro stimulation.

Each dot represents an individual participant's response, with HC (black) comparison to MS (red). (A-D) HC only data. (E-H) MS participants only data. (I \& J) comparison of MS to HC. Bars indicate mean with SD. ${ }^{*}(\mathrm{p} \leq 0.05),{ }^{* *}(\mathrm{p} \leq 0.01),{ }^{* * *}(\mathrm{p} \leq 0.001)$, $* * * *(\mathrm{p} \leq 0.0001)$. 
5.4.3.6 LPS and MIS416 upregulated the expression of IL-6 in monocytes, but HC and MS cohort did not differ in their IL-6 responses to either stimulation.

In HC ( $\mathrm{p}=0.0022)$ and MS $(\mathrm{p}<0.0001)$ participants, LPS induced a significant increase in the proportion of IL-6 $6^{+}$cells compared to untreated cells (Figure 5-11). While for MIS416, it was only in the MS cohort that we observed a significant increase in the positive cell proportion when compared to untreated cells $(\mathrm{p}=0.040)$.

Both cohorts showed a similar response in terms of total IL-6 (gMFI) produced by monocytes in response to LPS (HC: $\mathrm{p}=0.0019$; MS: $\mathrm{p}<0.0001$ ) and MIS416 (HC: $\mathrm{p}$ $=0.0323$; MS: $\mathrm{p}=0.0003$ ) compared to untreated. Additionally, monocytes from both cohorts expressed significantly more IL-6 when stimulated by LPS than MIS416. This effect was also reflected in the magnitude of the IL-6 response between LPS and MIS416, in that when normalised to untreated levels, LPS showed a striking increase in IL-6 compared to MIS416 (HC: $\mathrm{p}=0.0078$; MS: $\mathrm{p}<0.0001$ ). However, neither the proportional change in cells expressing IL-6 nor the total amount produced was significantly different when the MS and HC cohorts were directly compared (Figure 5$11 \mathrm{I} \& J)$. 
A

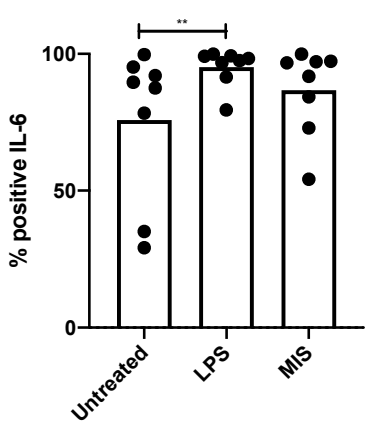

E
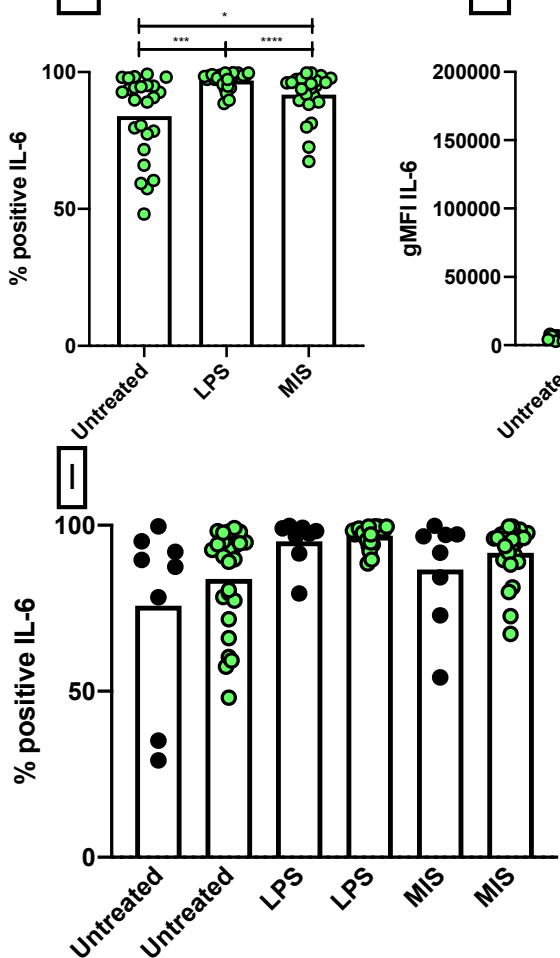

B

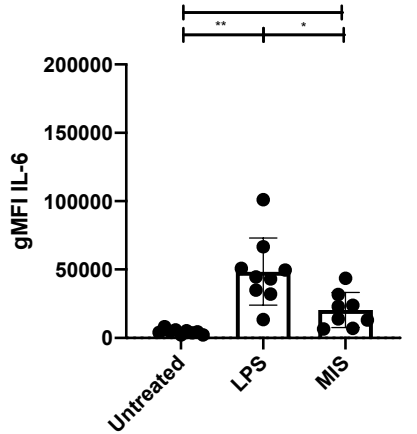

F

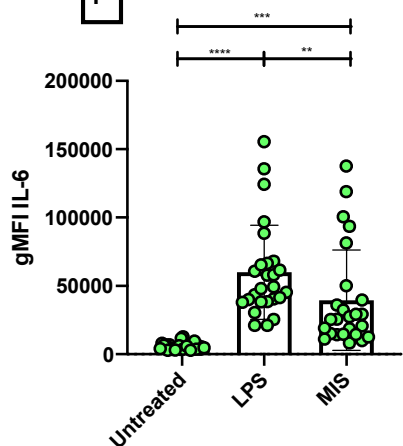

C

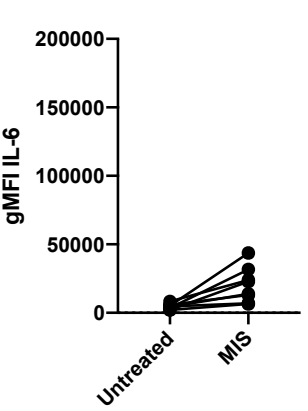

G

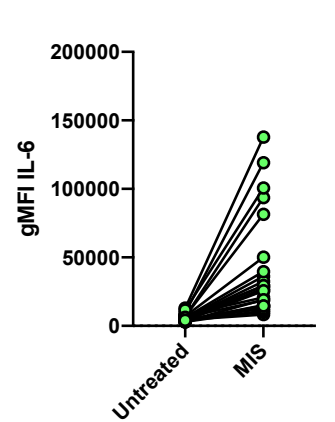

D

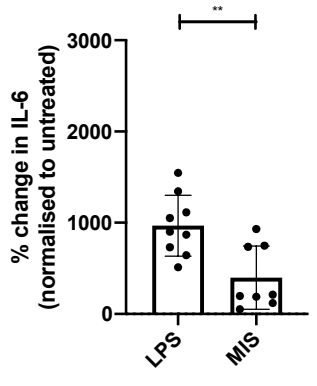

$\mathrm{H}$

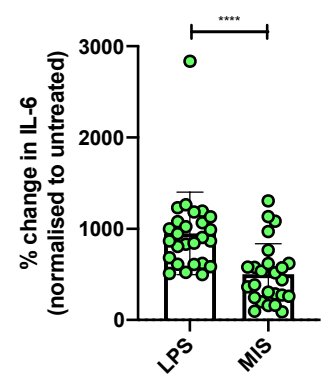

Figure 5-11 The mean difference in the proportion (A, E \& I) and relative expression of IL-6 in monocytes following overnight in vitro stimulation.

Each dot represents an individual participant's response, with HC (black) comparison to MS (green). (A-D) HC only data. (E-H) MS participants only data. (I \& J) comparison of MS to HC. Bars indicate mean with SD. * $(p \leq 0.05),{ }^{* *}(p \leq 0.01),{ }^{* * *}$ $(\mathrm{p} \leq 0.001),{ }^{* * * *}(\mathrm{p} \leq 0.0001)$. 
5.4.3.7 IL-10 expression in monocytes was not as distinct as observed for the other cytokines detected.

We observed that IL-10 expression was limited to a subset of cells, and that when all monocytes were analysed, that there was no significant difference in the proportion of positive staining monocytes expressing IL-10 over all treatment conditions over all participants. We concluded that IL-10 production in vitro only occurs in a subset of reactive cells. This then can obscure the amount of IL-10 signal when the entire monocyte population for an individual participant is assessed as a whole, such that it appears as though no cytokine is produced in the cells for some participants. To correct for this an independent FMO control was used for each participant to select only the cells that stained positive for IL-10.

5.4.3.8 The proportion of IL-10-expressing monocytes did not increase significantly following an overnight in vitro stimulation with LPS or MIS416.

In the $\mathrm{HC}$ population, we observed no difference in the proportion of $\mathrm{IL}-1 \mathrm{O}^{+}$cells following either LPS or MIS416 stimulation (Figure 5-12). The proportion of cells staining positive for IL-10 varied modestly between LPS and MIS416 in the MS cohort ( $\mathrm{p}=0.0216$ ), but not when compared to untreated cells. Together this indicated that neither cohort had an altered ability to produce IL-10, and that neither stimulation significantly altered this priming either.

5.4.3.9 In IL-10-expressing cells, the total amount made was significantly increased following in vitro stimulation, with MIS416 inducing significantly more IL-10 compared to LPS.

While the proportion of IL-10-producing cells did not appear to vary, we observed that the total amount of IL-10 detected was increased compared to untreated cells, in HC ( $\mathrm{p}=0.0004)$ and MS ( $\mathrm{p}<0.0001)$ cohorts following LPS stimulation, and also for MIS416 stimulation (HC: $\mathrm{p}=0.0027$; MS: $\mathrm{p}<0.0001$ ) (Figure 5-12). MIS416 induced significantly more IL-10 compared to LPS, and when normalised to untreated cells, showed a striking increase in both HC $(\mathrm{p}=0.0078)$ and MS $(\mathrm{p}<0.0001)$ cohorts. We did not observe any significant difference in IL-10 expression when the HC and MS cohorts were compared directly (Figure 5-12 I\&J). 
A

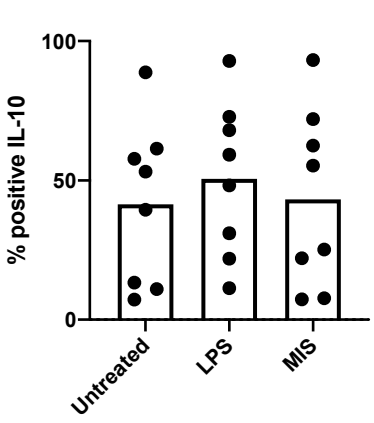

E
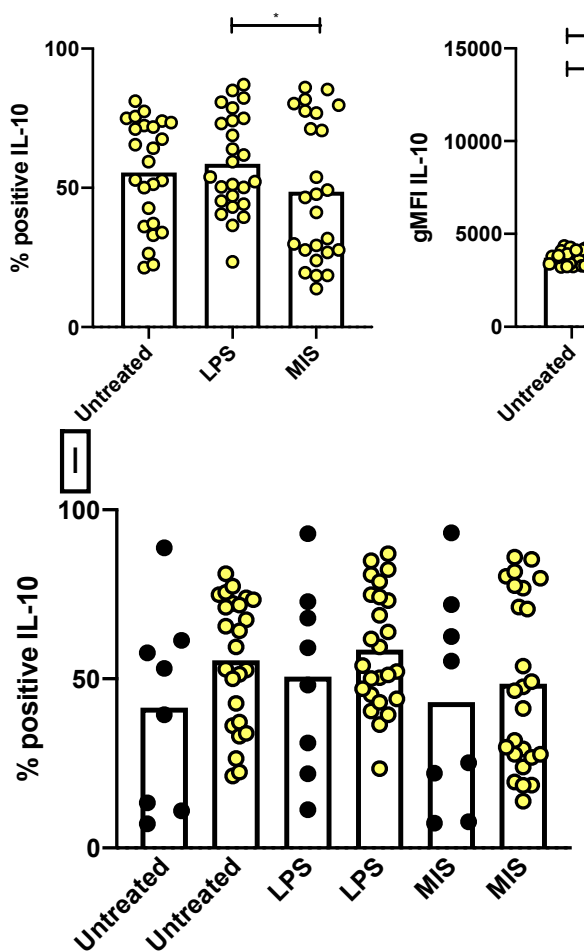

C

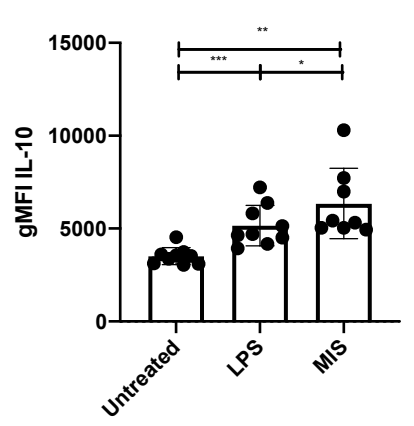

F
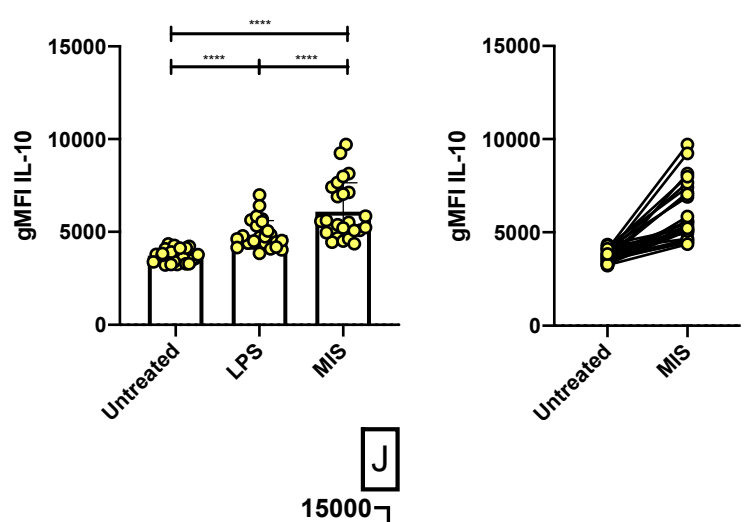

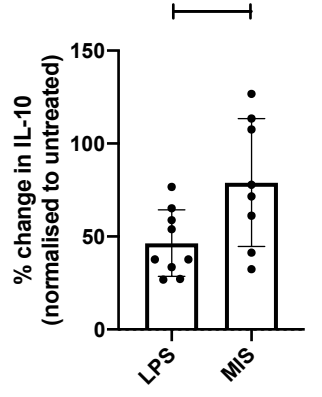

G
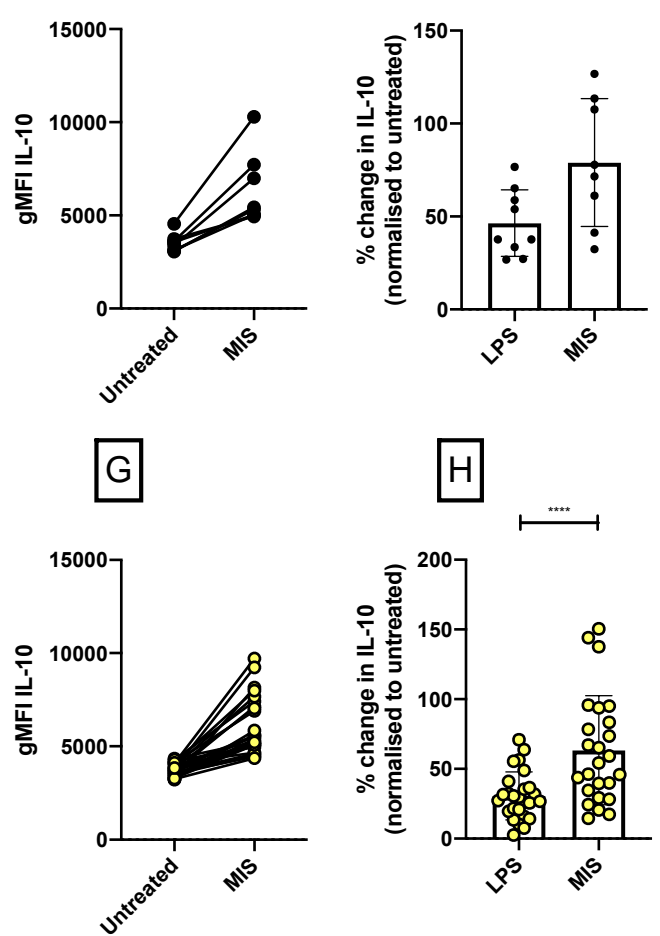

Figure 5-12 The mean difference in the proportion (A, E \& I) and relative expression of IL-10 in monocytes following overnight in vitro stimulation.

Each dot represents an individual participant's response, with HC (black) comparison to MS (yellow). (A-D) HC only data. (E-H) MS participants only data. (I \& J) comparison of MS to HC. Bars indicate mean with SD. ${ }^{*}(\mathrm{p} \leq 0.05),{ }^{* *}(\mathrm{p} \leq 0.01),{ }^{* * *}$ $(\mathrm{p} \leq 0.001),{ }^{* * * *}(\mathrm{p} \leq 0.0001)$. 
5.4.3.10 The pattern of INFy expression in monocytes was similar to IL-10.

An FMO control was also used to gate monocytes that were $\mathrm{INF}^{+}$since the total proportion of INF $\gamma$ producing cells appeared nearly equal across all in vitro conditions (Figure 5-13). The aim of our assay setup was not to induce a typical INF $\gamma$ PBMC response; instead, it was included to complete the panel of known MIS416 response cytokines. INF $\gamma$ is typically detected in T-cell or NK cell response assays but because of the importance of INF $\gamma$ in mediating the protective effects of MIS416, we wanted to confirm whether MIS416 could directly induce expression of this cytokine in monocytes.

5.4.3.11 INFy response in monocytes stimulated with MIS416 was significantly increased compared to untreated and LPS stimulation.

In INF $\gamma$-responsive monocytes, the INF $\gamma$ signal was significantly higher for $\mathrm{HC}(\mathrm{p}=$ o.0266) and MS ( $<$ < 0.0001) in MIS416, and for HC ( $\mathrm{p}=0.0148)$ and MS ( $<<0.0001)$ in LPS cultures compared to untreated. MIS416 stimulation led to significantly increased levels of INF $\gamma$ in HC (p = o.0439) and MS ( $<$ o.0001) monocytes when compared to LPS alone (Figure 5-13), but direct comparisons did not indicate a difference in the INF $\gamma$ response between the cohorts. 
A

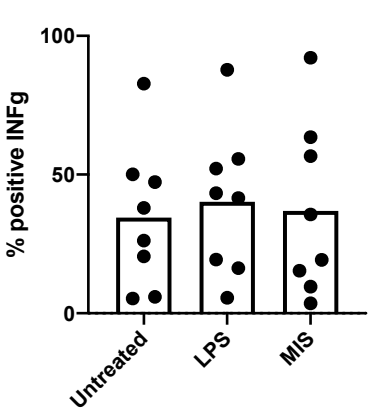

E
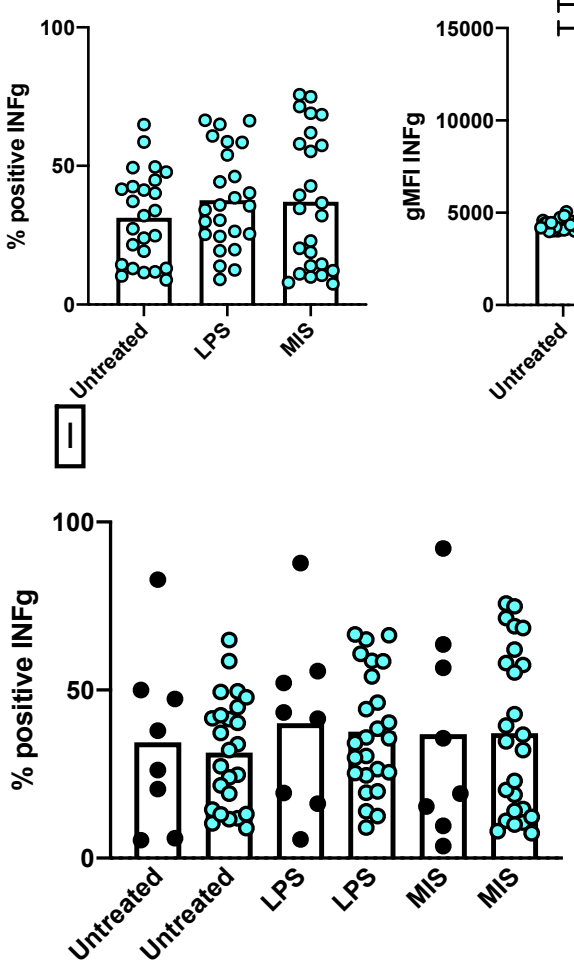

F
B
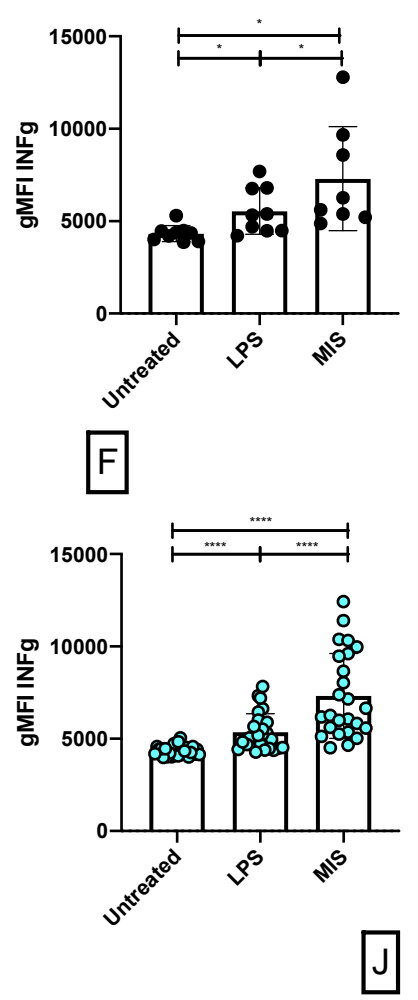

J

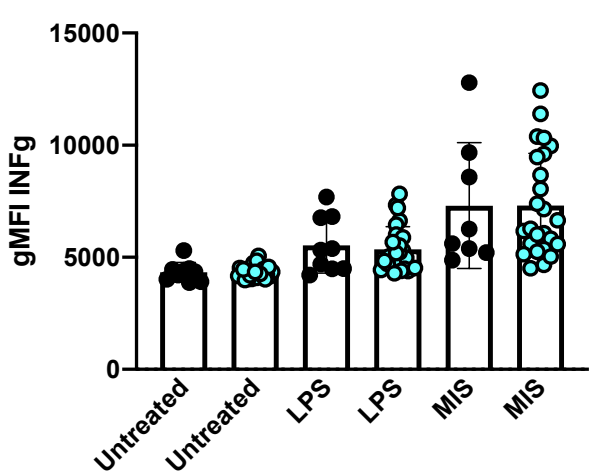

$D$

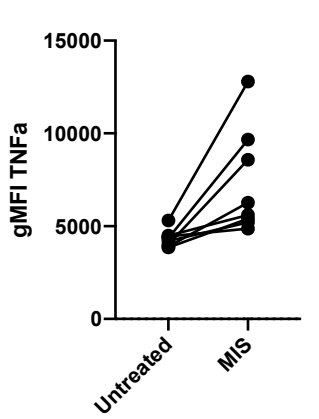

$\mathrm{G}$
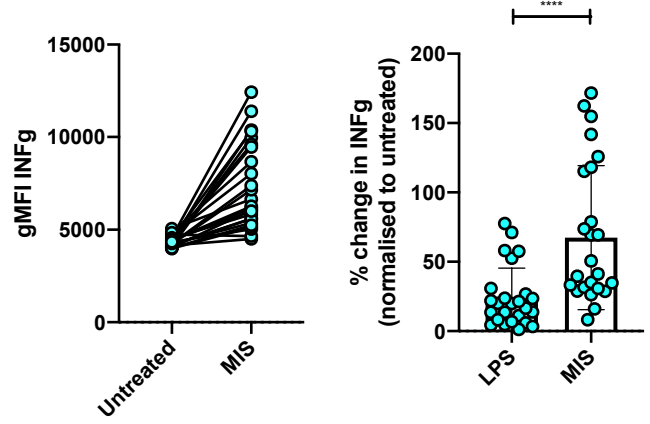

- $\mathrm{HC}$

- MS

Figure 5-13 The mean difference in the proportion (A, E \& I) and relative expression of INFY in monocytes following overnight in vitro stimulation.

Each dot represents an individual participant's response, with HC (black) comparison to MS (teal). (A-D) HC only data. (E-H) MS participants only data. (I \& J) comparison of MS to HC. Bars indicate mean with SD. ${ }^{*}(\mathrm{p} \leq 0.05),{ }^{* *}(\mathrm{p} \leq 0.01),{ }^{* * *}(\mathrm{p} \leq 0.001)$, **** $(\mathrm{p} \leq 0.0001)$. 
5.4.3.12 MIS416 effects a strong increase in the proportion of monocytes expressing TNFa.

In vitro cultures with LPS and MIS416 showed an increase in the proportion of $\mathrm{TNFa}^{+}$ cells, with MIS416 inducing a significant increase compared to untreated cells in both cohorts (HC: $\mathrm{p}=0.0005$; MS: $\mathrm{p}<0.0001$ ) (Figure 5-14). We observed that in the MS cohort, LPS also resulted in a dramatic proportional increase in TNFa production over untreated ( $\mathrm{p}=0.0005)$, and that there was a modest but significant difference between LPS and MIS416 in terms of TNFa induction $(\mathrm{p}=0.0056)$.

5.4.3.13 MIS416 treatment induced higher levels of TNFa than LPS.

In HC ( $\mathrm{p}=0.0043)$ and MS cohorts ( $\mathrm{p}<0.0001)$, LPS significantly increased TNFa levels in monocytes (gMFI), and MIS416 also significantly increased TNFa production in $\mathrm{HC}(\mathrm{p}=0.0354)$ and MS ( $\mathrm{p}<0.0001)$ when compared to untreated cells (Figure 514). However, in the MS group, MIS416 induced a significantly greater TNFa response compared to LPS ( $\mathrm{p}<0.0001$ ). This difference was also present in HC participants $(\mathrm{p}=0.08)$ and was significant when the magnitude of the change was assessed after normalisation to untreated cells (HC: $\mathrm{p}=0.0156$; MS: $\mathrm{p}<0.0001$ ).

5.4.3.14 Patients with progressive MS had a higher basal level of TNFa compared to HC.

In untreated cells from our in vitro cultures, we observed the levels of TNFa (gMFI) to be significantly higher in the MS cohort $(\mathrm{p}=0.0372)$, indicating higher basal levels of TNFa induction (Figure 5-14). This was substantiated by an increase in the proportion of positive staining cells, in both untreated $(\mathrm{p}=0.0341)$ and LPS $(\mathrm{p}=$ o.018) cultures of the MS cohort, when compared to the HC. 
A

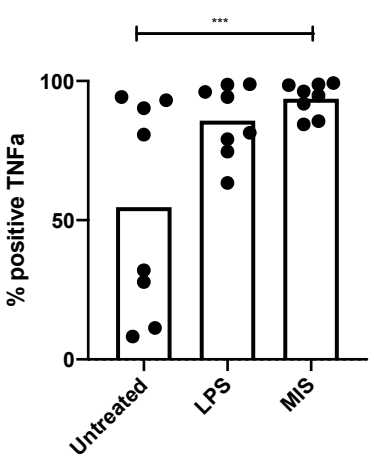

E
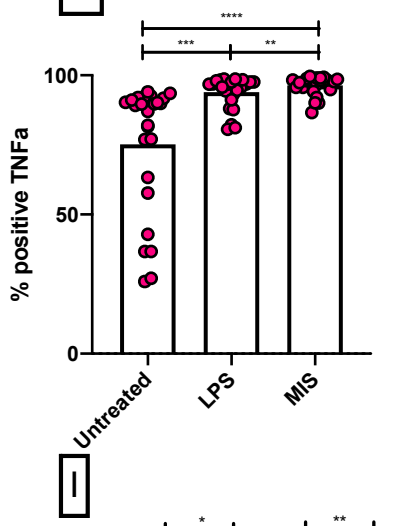

$B$

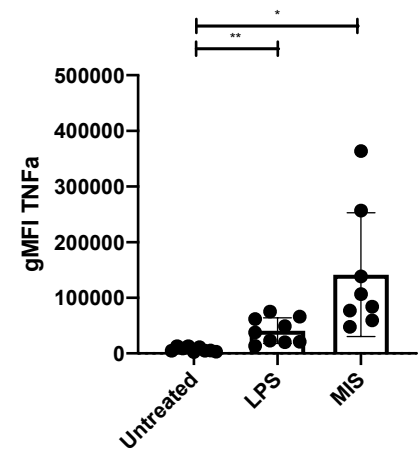

F

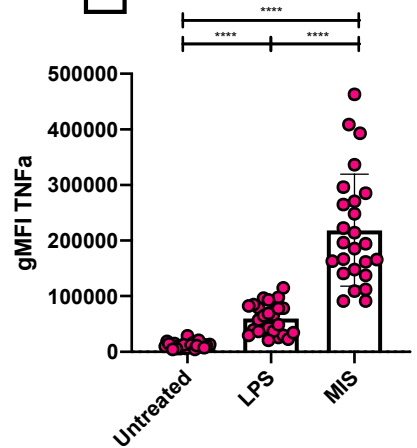

C

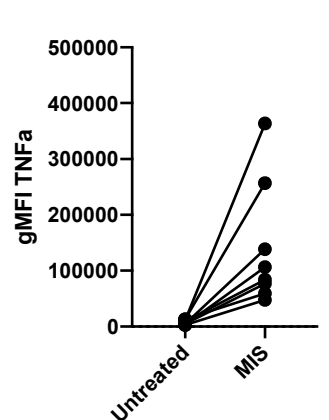

G
$D$

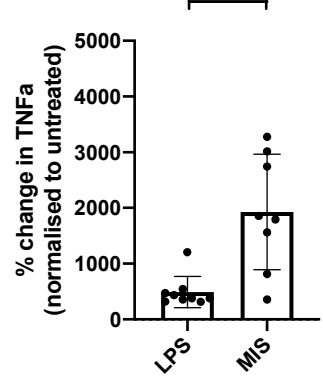

$\mathrm{H}$
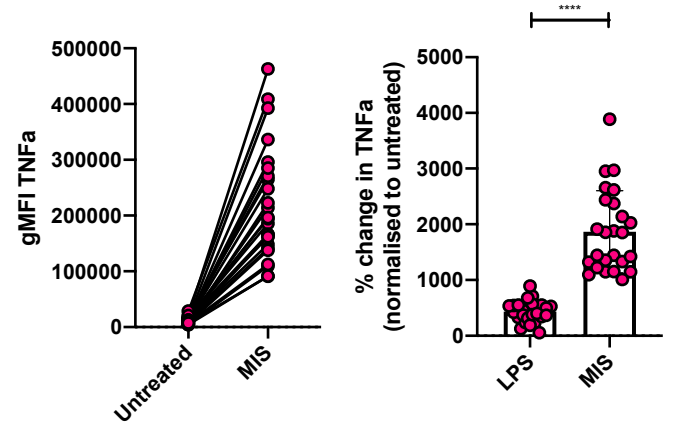
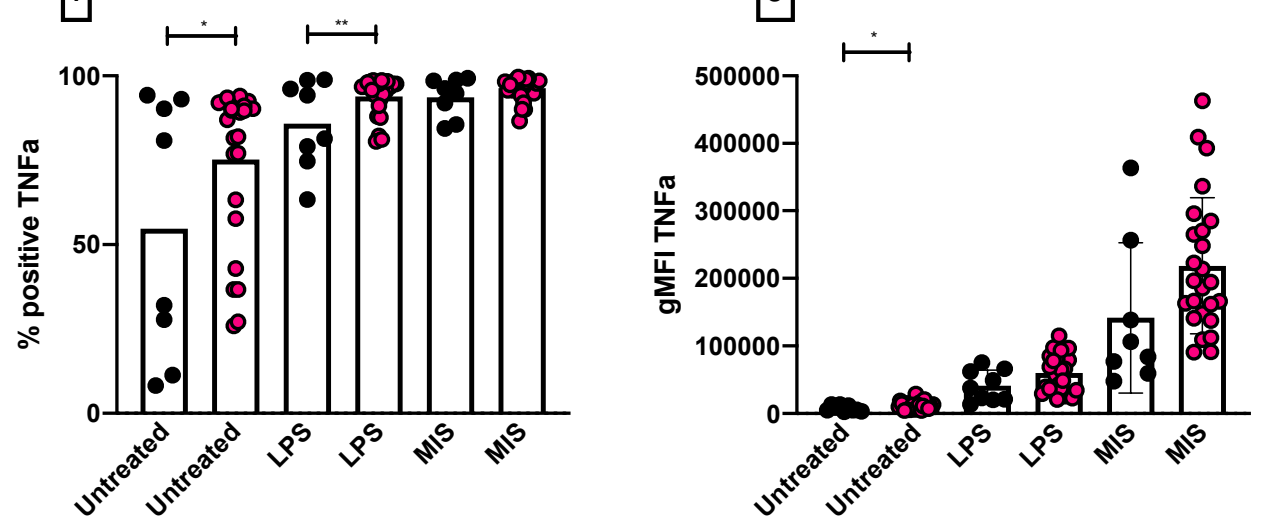

- $\mathrm{HC}$

- MS

Figure 5-14 The mean difference in the proportion (A, E \& I) and relative expression of TNFa in monocytes following overnight in vitro stimulation.

Each dot represents an individual participant's response, with HC (black) comparison to MS (red). (A-D) HC only data. (E-H) MS participants only data. (I \& J) comparison of MS to HC. Bars indicate mean with SD. ${ }^{*}(\mathrm{p} \leq 0.05),{ }^{* *}(\mathrm{p} \leq 0.01),{ }^{* * *}(\mathrm{p} \leq 0.001)$, **** $(\mathrm{p} \leq 0.0001)$. 


\subsubsection{Cytokine levels in supernatants corroborated the induction of both a pro- and anti-inflammatory response by MIS416 in PBMC cultures in vitro.}

To further substantiate the cytokine profile induced by MIS416 in vitro, we measured total IL-1 $\beta$ and IL-10 in the tissue culture supernatants. The culture supernatants were collected and stored as part of our in vitro experimental procedures, and total cytokine detection was performed using ELISA kits. The supernatants analysed were generated from the phenotyping work (Chapter 4) that ran concurrently with the intracellular cytokine assays. Thus, the culture conditions and clinical material were identical except that no brefeldin A or monensin were added to stop protein transport.

5.4.4.1 MIS416 induced IL-10 and IL-1 $\beta$ secretion in the monocyte cultures.

Monocytes from HC and MS cohorts produced IL-10 and IL-1 $\beta$ in response to both LPS and MIS416 (Figure 5-15). IL-10 was significantly increased in response to LPS for $\mathrm{HC}(\mathrm{p}=0.0002)$ and MS ( $\mathrm{p}=0.0399)$ cohorts compared to untreated supernatants while for MIS416, IL-10 levels only reached significance in the HC cohort ( $p=0.0331)$.

IL-1 $\beta$ was significantly increased in supernatants from HC (p = 0.0006) and MS $(\mathrm{p}<$ o.0001) monocytes after LPS stimulation, and in HC ( $\mathrm{p}=0.0052)$ and in MS $(\mathrm{p}=$ 0.0002) monocytes from MIS416 cultures compared to untreated. Furthermore, the total amount between the stimulations did not vary to any degree of significance (Figure 5-15). We also observed no significant difference when cytokine production from the HC and MS cohorts were compared. 
A
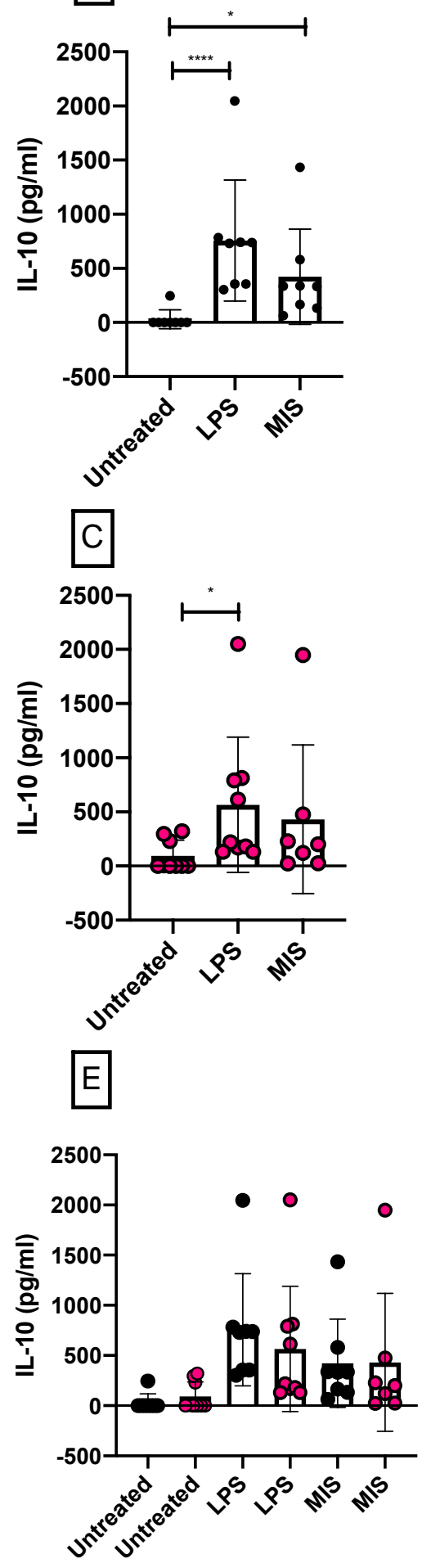
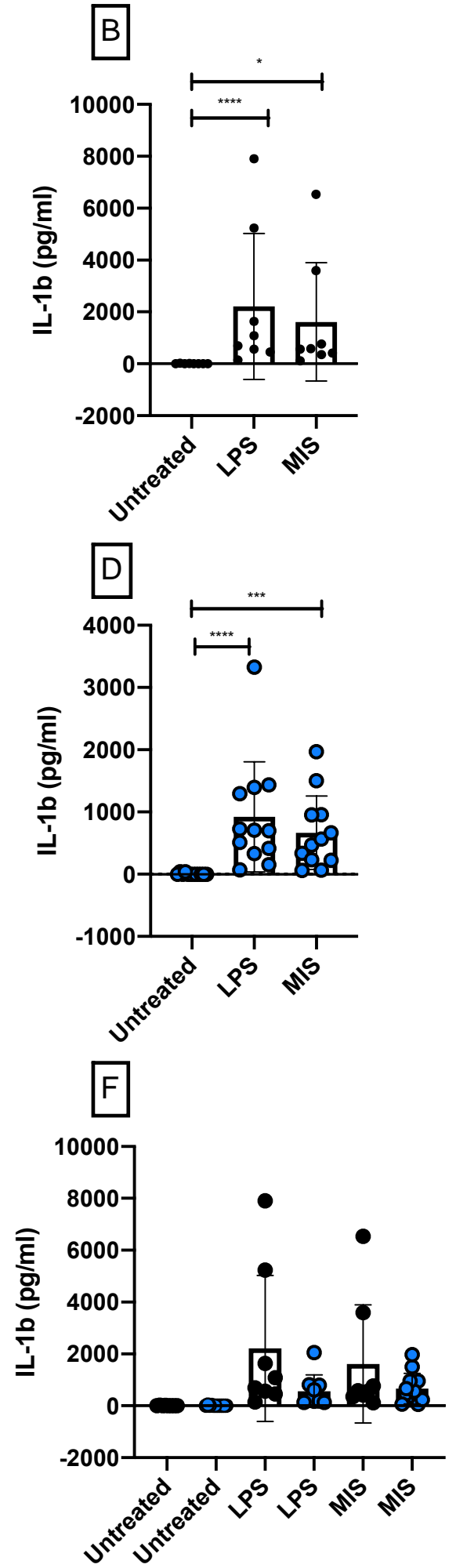

Figure 5-15 The mean total cytokine detected by ELISA in tissue culture supernatants from concurrent in vitro experiments.

HC (black) total IL-10 (A) and IL-1 $\beta$ (B) in culture supernatant. MS (coloured) total IL-10 (C) and IL-1 $\beta$ (D) in culture supernatant. Comparative plots of HC and MS participants for IL-10 (E) and IL-1 $\beta(\mathrm{F}) .{ }^{*}(\mathrm{p} \leq 0.05),{ }^{*}(\mathrm{p} \leq 0.01),{ }^{* *}(\mathrm{p} \leq 0.001)$, $* * * *(\mathrm{p} \leq 0.0001)$. 
5.4.4.2 No correlation was observed between intracellular cytokine production (flow cytometry) and secreted cytokine levels (ELISA).

To establish if there were a correlation between the total amount of cytokine produced, the intracellular cytokine readouts, and the percentage positive cells, a Pearson's correlation matrix of the three output measures was performed (Figure 5-16). As we had previously established that there was no difference in IL-10 (Figure 5-12) nor IL$1 \beta$ (Figure 5-10) between $\mathrm{HC}$ and MS cohorts, we combined the participants into a single cohort.

We observed that for IL-10, there was a strong correlation $(\mathrm{p}=0.0003 ; \mathrm{r}=0.791, \mathrm{CI}$ $=0.4850$ to 0.9241 ) between the proportion of positive cells and their expression (gMFI) from LPS stimulation (Figure 5-16 B), as well as from MIS416 stimulation (Figure 5-16 A) ( $\mathrm{p}=0.0005 ; \mathrm{r}=0.855, \mathrm{CI}=.0 .6094$ to 0.9508$)$. We found no correlation between the ELISA data and any other output measuring IL-10.

For IL-1 $\beta$ we observed a positive correlation $(\mathrm{p}=0.001, \mathrm{r}=0.67, \mathrm{CI}=0.3311$ to 0.8604 ) between the proportion of IL-1 $\beta$ positive cells and their IL-1 $\beta$ production (as gMFI) for LPS stimulated cells (Figure 5-13 D), and further strong correlation ( $\mathrm{p}=$ $0.0004 ; \mathrm{r}=0.732, \mathrm{CI}=0.4164$ to 0.8903 ) for MIS416 stimulated cells (Figure 5-16 C). Interestingly, we also observed a modest inverse correlation $(\mathrm{p}=0.038, \mathrm{r}=-0.47, \mathrm{CI}$ $=-0.7534$ to -0.02993 ) between the proportion of IL-1 $\beta$ positive cells and the total IL$1 \beta(\mathrm{pg} / \mathrm{ml})$ for LPS (Figure 5-16 D).

To profile the relationship between the three output measures for IL-1 $\beta$, we then conducted a linear correlation of the total supernatant cytokine values (media) to the two cytometry readouts (ICC and \%Pos). This analysis revealed that in LPS stimulated cultures, very high total cytokine levels predicted a decrease in the proportion (\% positive) of cytokine producing monocytes $\left(\mathrm{R}^{2}=0.271, \mathrm{p}=0.038\right.$, slope $\mathrm{Y}=$ $0.001020 * \mathrm{X}+93.20$ ) (Figure $5-17$ ) as suggested by the modest inverse correlation. 


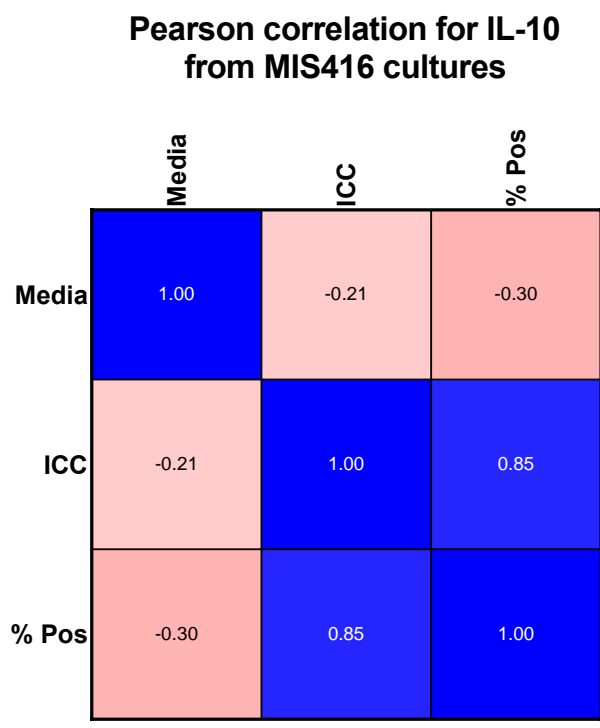

Pearson correlation for IL-1b from MIS416 cultures

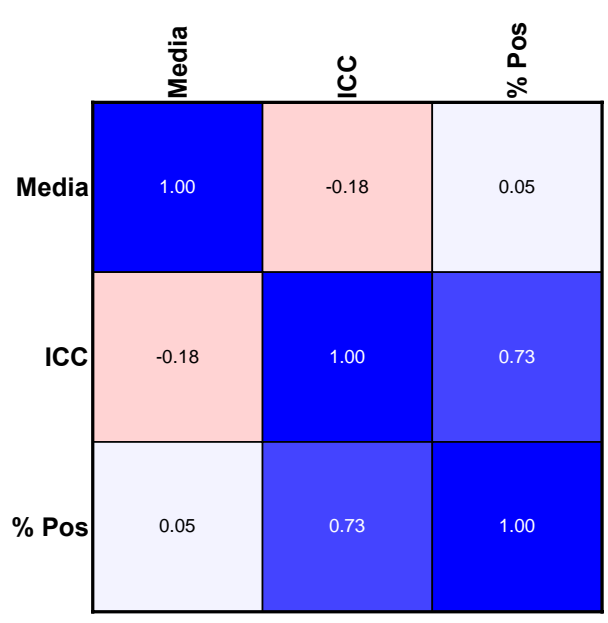

$A$

Pearson correlation for IL-10

from LPS cultures

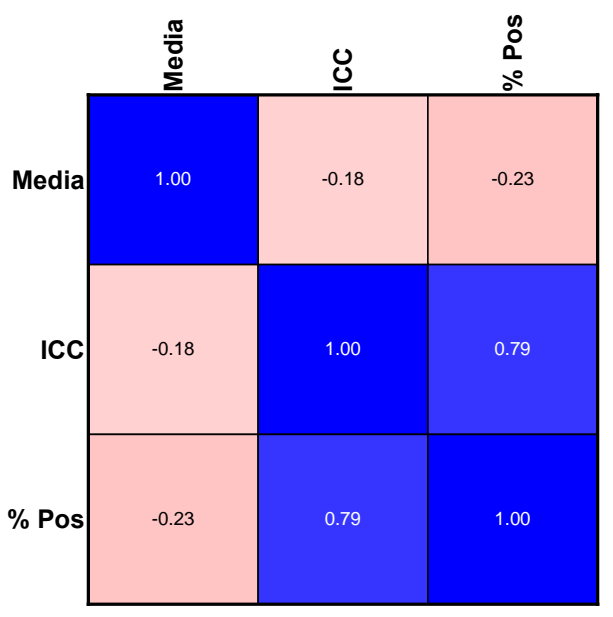

Pearson correlation for IL-1b from LPS cultures
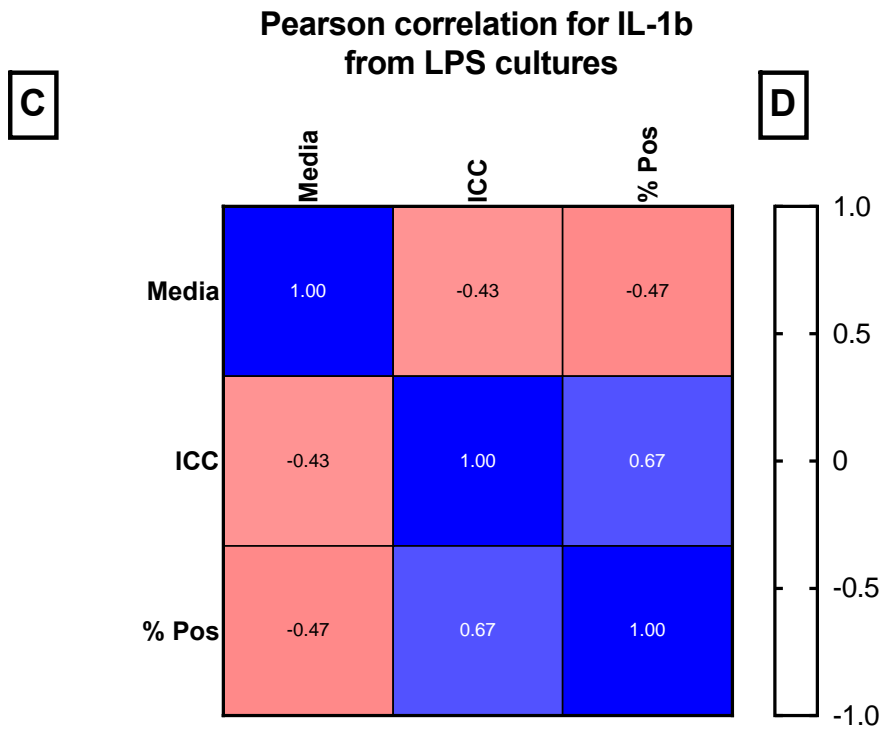

Figure 5-16 Pearson's Correlation of cytokine expression.

IL-10 (A\&B) and IL-1 $\beta$ (C\&D) for total cytokine produced from MIS416 (A\&C) and LPS (B\&D) in tissue culture supernatants (Media), from intracellular cytokine (ICC), and for the percentage positive (\%Pos) cells. Colour gradient indicates the correlation, ranging from no correlation ( $\mathrm{o}=$ white), either towards a more positive correlation ( 1 $=$ blue), or a more inverse correlation $(-1=$ red $)$. 


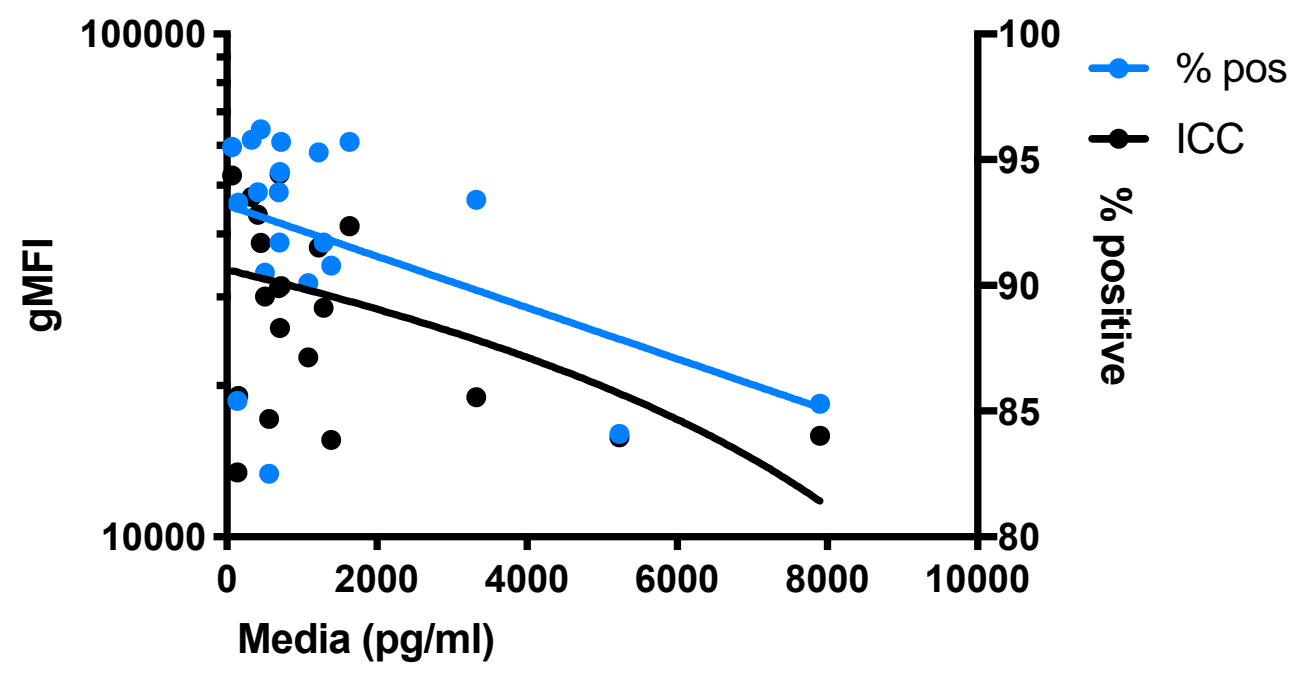

Figure 5-17 Linear regression of total cytokine expression.

Total cytokine (Media) as dependent variable, indicating its relationship to total expression (gMFI) and the proportion of positive cells (\% positive) as readouts for monocytes stimulated with LPS. 


\subsubsection{The effect of an NFKBIA promotor mutation on cytokine and transcription factor response.}

NFKBIA gene alterations, especially in the promotor region, could putatively impact the overall activity of the $\mathrm{NF \kappa B}_{\mathrm{B}}$ pathway. For this we reviewed Sanger sequencing readouts of a NFKBIA promoter polymorphism on our patients that were analysed and provided by our collaborators at the University of Queensland in Brisbane, Australia. Participants were then grouped based on either wild type (wt), heterozygous (hetero), or homozygous (homo) results for the 8bp insertion (-708ins8) into the promoter region. Having established several response differentials in cytokine and protein phosphorylation between HC and MS cohorts, we used this mutational status to stratify participants to determine if the NFKBIA promotor mutation was involved in effecting the magnitude of responses from $\mathrm{NF}_{\kappa} \mathrm{B}$ induction as modelled here using MIS416 in vitro.

5.4.5.1 Stratification of participants by genotype did not show significant group variation for cytokine or protein phosphorylation.

We observed no significant effect on the cytokine or TF readouts between LPS, MIS416 or untreated cells in vitro when participants were stratified by genotype and into HC or MS cohort (data not shown). We found that this additional stratification of our two cohorts into a further three subclasses based on genotype, diluted the power to detect any variation, so reworked the analysis to stratify participants based purely on genotype.

5.4.5.2 Active p65 in treatment-naïve monocytes was increased in a combined analysis for participants with a homozygous NFKBIA promoter mutation.

Analysis of the levels of activated p65 in monocytes from all participants stratified by NFKBIA promoter mutation indicated that homozygous individuals had a modest but significant $(\mathrm{p}=0.044)$ increase in $\mathrm{NF} \kappa \mathrm{B}$ activity compared to wild type individuals (Figure 5-18). 
5.4.5.3 Active CREB in monocytes treated with MIS416 was increased in a combined analysis for participants with a homozygous NFKBIA promoter mutation.

While CREB expression for other in vitro conditions did not show any significant variation following mutation stratification, we observed that in MIS416-treated monocytes (T3 MIS), those participants who harboured homozygous NFKBIA promoter mutations showed a modest and significant $(\mathrm{p}=0.038)$ increase in their level of activated CREB (Figure 5-18).

5.4.5.4 Cytokine levels were unaffected, irrespective of NFKBIA mutation status, but modest trends were observed in IL-1 $\beta$ and IL-6.

In monocytes treated overnight with MIS416 (T24 MIS), we noted a trend that failed to meet the definition of statistically significant, whereby participants who harbour a homozygous mutation appeared to induce a stronger IL-6 response compared to wild type (ns. p=0.08) (Figure 5-18).

We also noted a trend where in untreated cells following overnight culture, IL-1 $\beta$ appears to be reduced in participants who harbour a heterozygous mutation, compared to wild type (ns. $\mathrm{P}=0.07$ ) (Figure 5-18).

5.4.5.5 NFKBIA promoter mutations appeared to result in a stronger cytokine response to MIS416 in the peripheral blood plasma.

Plasma samples were collected from all participants who were enrolled in the MIS416 phase $2 \mathrm{~b}$ trial and analysed for the presence of a rage of measures including serum immune factors and cytokines namely IL-1 $\beta$, IL-6, IL-8, TNFa, INF $\gamma$, IL-12p70, CXCL10 and Neopterin. These plasma samples were collected at baseline (C1D1) and on multiple routine visits (e.g. 23 days post dose 1 (C1D23)) as part of the trial protocol, and total levels were measured by cytometric bead array by our collaborators at Innate Immunotheraputics in Auckland. Using this externally generated data, we compared the plasma levels of these factors in individuals who received MIS416 where their mutation status for the NFKBIA promoter was known, and where data from both their baseline untreated (C1D1) and their first post treatment follow-up visit (C1D23) was available.

We observed three interesting patterns in the response to MIS416, whereby participants stratified by their mutation status, appeared to show a differential 
response to treatment. In particular, neopterin levels were increased in heterozygous individuals who received MIS416, as detected in a post dose sample (C1D23), and compared to WT. Although this finding failed to reach statistical significance (ns. $p=$ o.066) (Figure 5-19). Interestingly, this difference was absent in the baseline (C1D1) measure. A similar pattern in CXCL1o levels post-treatment showed a stronger response in heterozygous individuals compared to WT (ns. $p=0.11$ ). Furthermore, IL-12p7o levels were significantly reduced in heterozygous individuals compared to WT $(\mathrm{p}=0.039)$ at C1D23. While this difference was present at baseline, it failed to reach significance (ns. $\mathrm{p}=0.10$ ). 


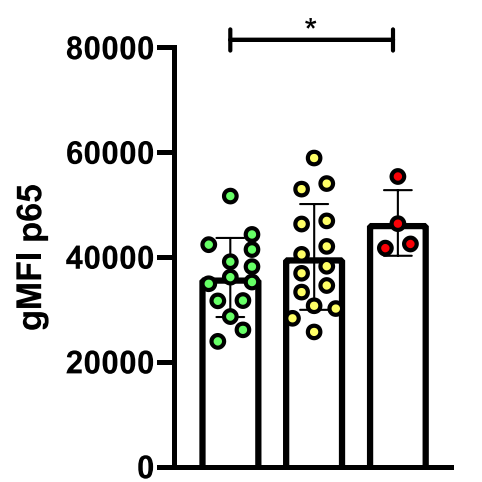

T0 (untreated baseline)

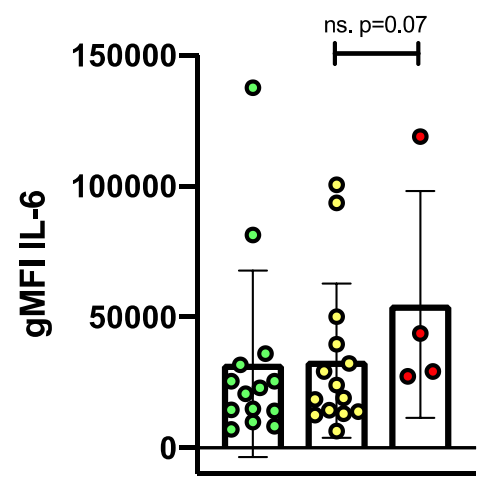

T24 MIS

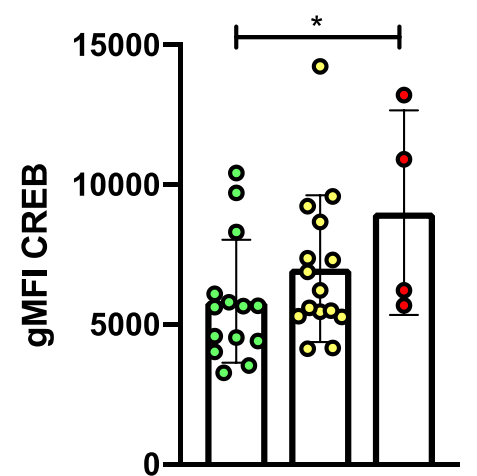

T3 MIS

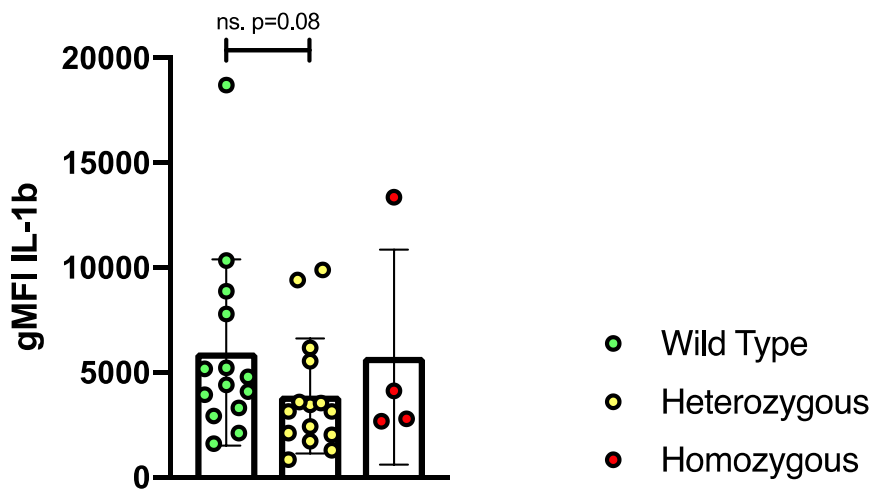

T24 Untreated

Figure 5-18 The mean difference in total marker expression (gMFI) for NFkB p65, CREB, IL-6 and IL-1 $\beta$ in monocytes.

Data represent HC and MS participants combined and stratified by NFKBIA mutation status. Wild type (green), heterozygous (yellow), and homozygous (red) for insertion (-708ins8). * ( 1 < 0.05), ns. = not significant with error bars indicate SD. 

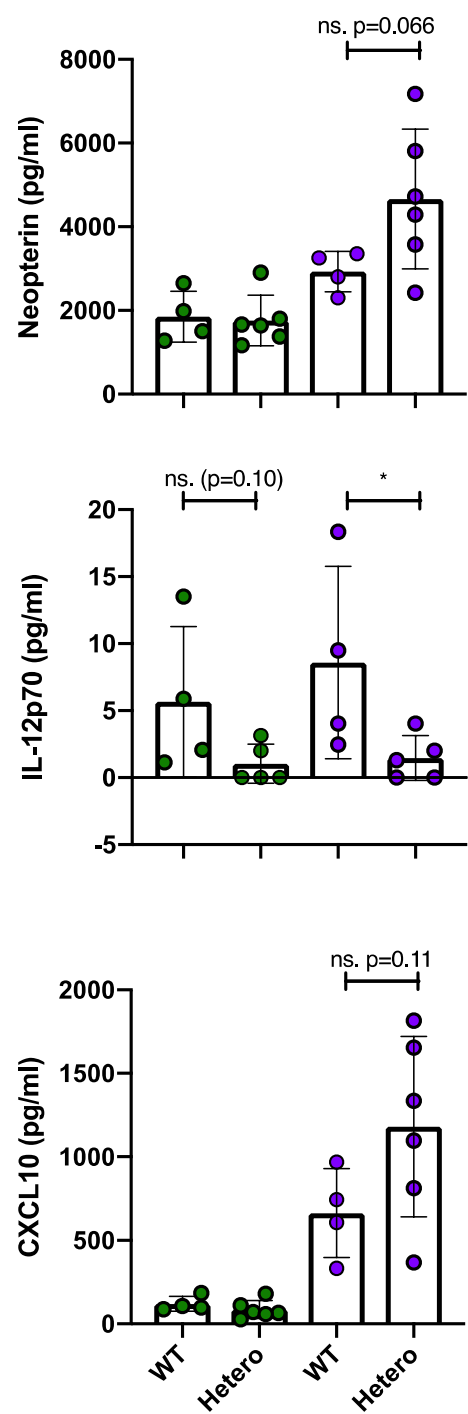

- C1D1 (Baseline)

- C1D23

Figure 5-19 Mean serum immune factor response for MS participants stratified by NFKBIA promotor mutation.

Each circle represents an individual participant readout. Baseline untreated values (C1D1) are contrast with MIS416 treated (C1D23) follow up to indicate the mutation effects. 


\subsubsection{The effect of overnight incubation on transcription factor phosphorylation.}

While we initially established three hours in vitro as a suitable time point for detecting alterations in MIS416-induced TF phosphorylation, in a smaller subset of participants, we extended the culture overnight to observe the effect of prolonged activation on PBMC cultures. There was however no untreated control included at this timepoint, which limits interpretation of the effects described here.

5.4.6.1 Activated $\mathrm{NF \kappa B}$ p65 levels increased in monocytes after overnight stimulation with MIS416.

We observed a modest but significant increase in the total proportion of monocytes expressing activated $\mathrm{NF \kappa B}$ p65 in MS but not $\mathrm{HC}$ participants (Figure 5-20). Monocytes from both cohorts, however, had significantly increased amounts of active NFאB p65 compared to T3 MIS (HC: $\mathrm{p}=0.043$; MS: $\mathrm{p}=<0.0001)$. No difference in the proportion or amount of activated $\mathrm{NF}_{\kappa} \mathrm{B}$ p65 was found when MS and HC were compared.

5.4.6.2 In contrast to the increased active p65 levels, CREB activity was reduced after overnight stimulation.

In MS participants, we observed a significant reduction in CREB at T24 MIS compared to T3 MIS, firstly from a reduction in the total proportion of cells staining positive ( $p$ $=0.0002)$, as well as the mean level of active CREB in monocytes $(p=0.0022)$ (Figure 5-21). In HC participants, a similar trend was present, but it did not reach statistical significance. We also observed no significant difference when HC and MS data were compared. 

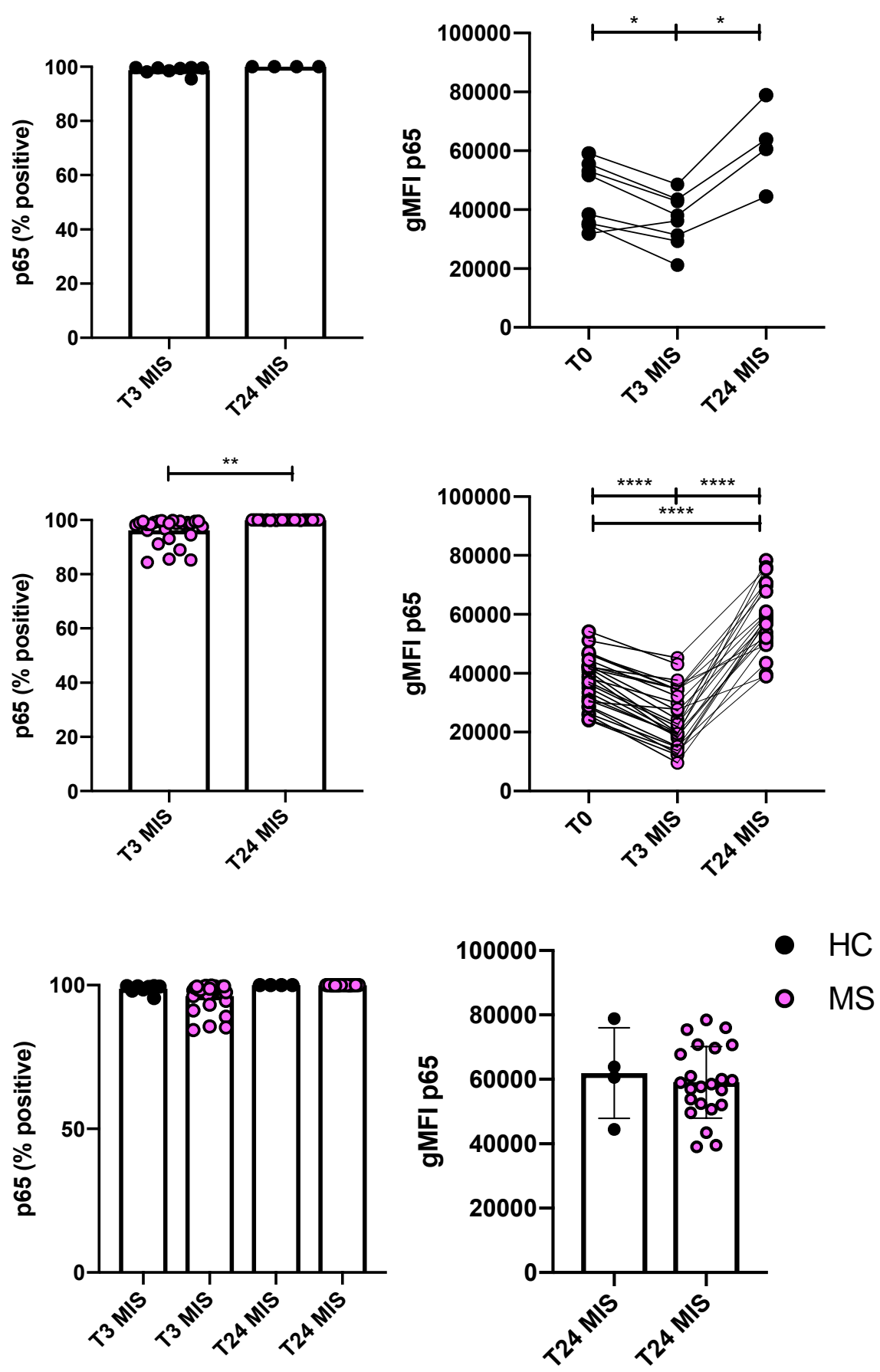

Figure 5-20 The mean proportion, and level of activated NFKB p65 in monocytes.

Individual participants are indicated by a coloured circle, HC (black), MS (pink). First row depicts the HC data, the second MS participant data, and direct comparisons between cohorts in the third row. Bars indicate group mean, with error bars show SD. * $(\mathrm{p} \leq 0.05),{ }^{* *}(\mathrm{p} \leq 0.01),{ }^{* *}(\mathrm{p} \leq 0.001),{ }^{* * * *}(\mathrm{p} \leq 0.0001)$. 

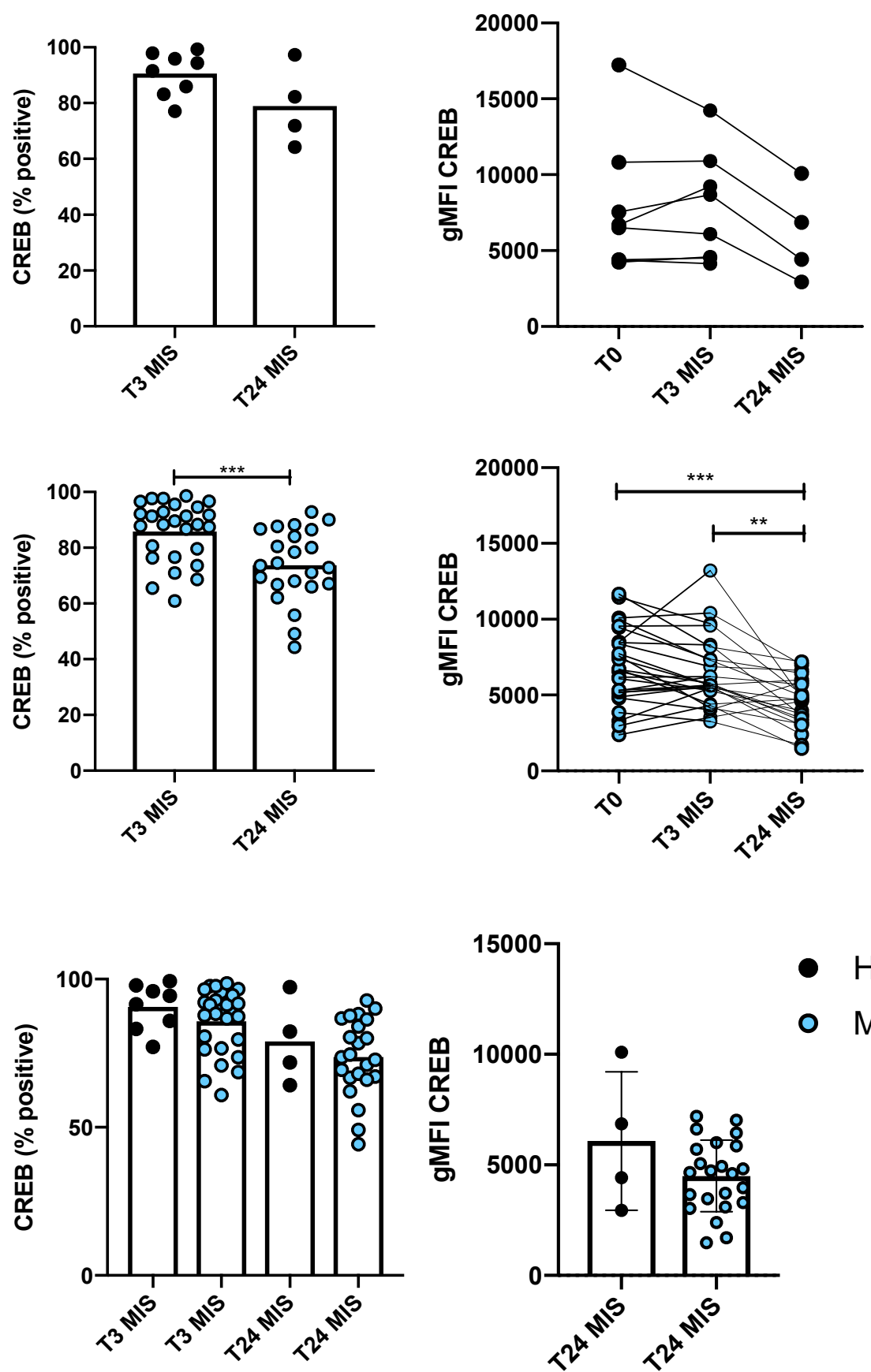

- $\mathrm{HC}$

- MS

Figure 5-21 The mean proportion, and level of activated CREB in monocytes.

Individual participants are indicated by a coloured circle, HC (black), MS (blue). First row depicts the HC data, the second MS participant data, and direct comparisons between cohorts in the third row. Bars indicate group mean, with error bars show SD. $*(\mathrm{p} \leq 0.05),{ }^{* *}(\mathrm{p} \leq 0.01),{ }^{* * *}(\mathrm{p} \leq 0.001),{ }^{* * *}(\mathrm{p} \leq 0.0001)$. 
5.4.6.3 Extending MIS416 cultures resulted in decreased STAT1 activation in monocytes from MS participants.

While the proportion of cells expressing active STAT1 did not appear to change, we observed that extending MIS416 cultures from T3 to T24 effected a significant further reduction in the amount of active STAT 1 in monocytes for the MS cohort ( $p=0.005)$ (Figure 5-22). Data for HC did not reach significance for any comparisons, but we observed a trend for MS participants to show a larger reduction in active STAT1 levels compared to HC at T24 MIS (ns. p = 0.09).

5.4.6.4 STAT3 levels in monocytes were largely unaffected following an extension in MIS416 culture.

We observed that the total levels of active STAT3 at T24 MIS did not vary significantly for either MS or HC participants, and that the proportion of STAT3 expression in HC participants also remained largely unchanged when compared to T3 MIS (Figure 523). In contrast, we observed that more monocytes expressed STAT3 at T24 MIS compared to T3 MIS, but that none of the direct cohort comparisons indicated a difference between $\mathrm{HC}$ and MS at this sample time. 

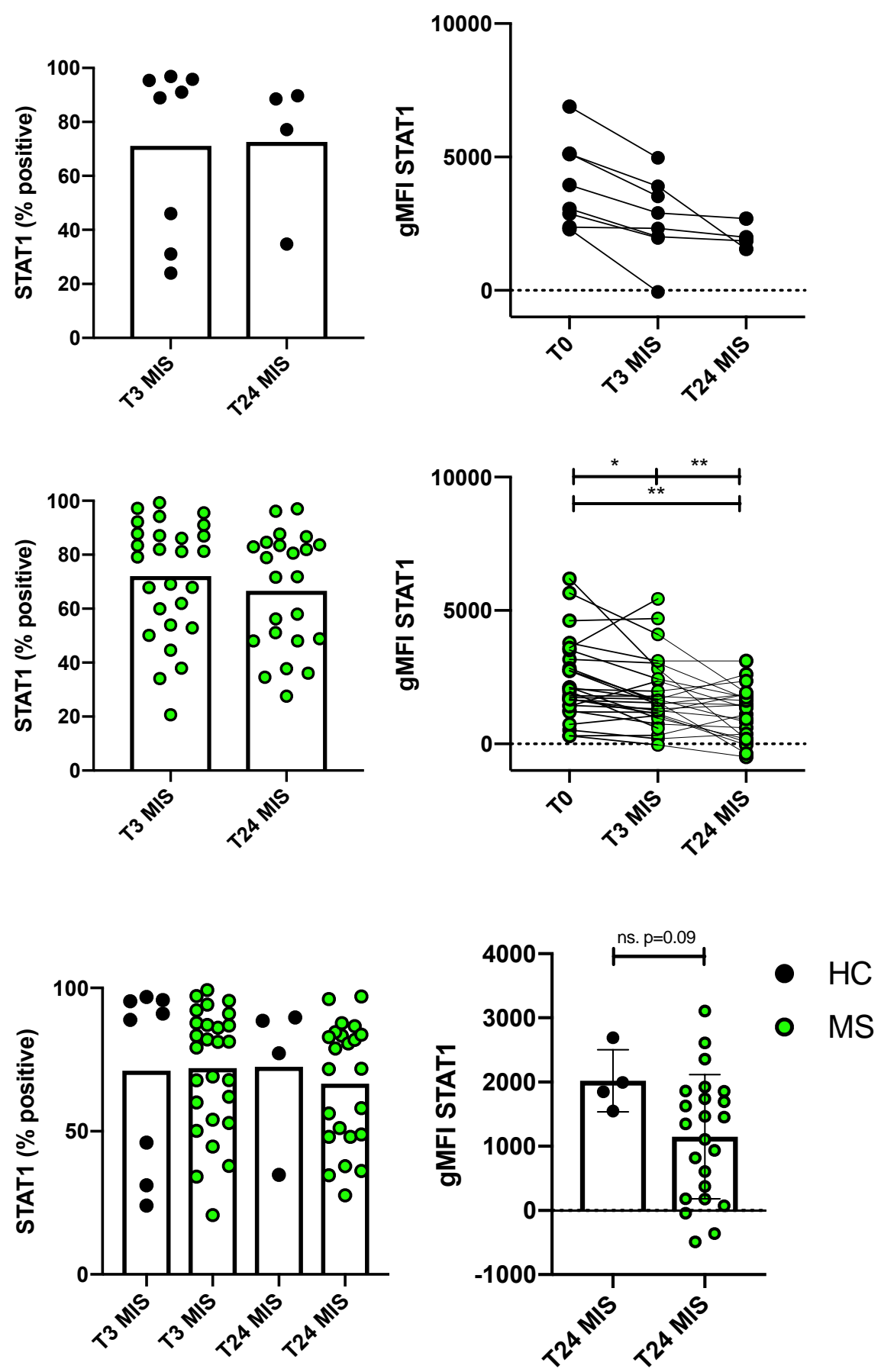

Figure 5-22 The mean proportion, and level of activated STAT1 in monocytes.

Individual participants are indicated by a coloured circle, HC (black), MS (green). First row depicts the HC data, the second MS participant data, and direct comparisons between cohorts in the third row. Bars indicate group mean, with error bars show SD. * $(\mathrm{p} \leq 0.05),{ }^{* *}(\mathrm{p} \leq 0.01),{ }^{* *}(\mathrm{p} \leq 0.001),{ }^{* * * *}(\mathrm{p} \leq 0.0001)$. 

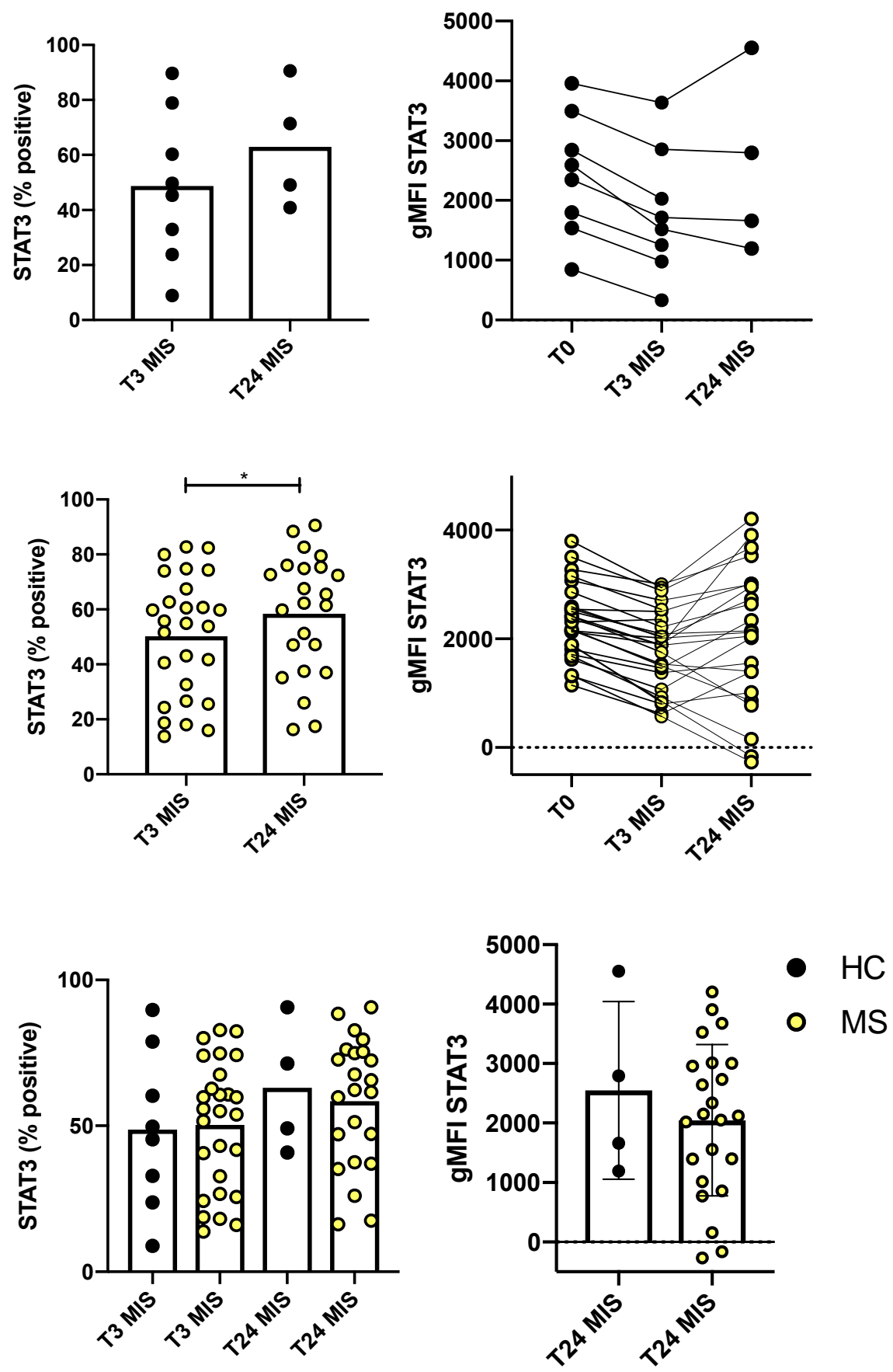

Figure 5-23 The mean proportion, and level of activated STAT3 in monocytes.

Individual participants are indicated by a coloured circle, HC (black), MS (yellow). First row depicts the HC data, the second MS participant data, and direct comparisons between cohorts in the third row. Bars indicate group mean, with error bars show SD. $*(p \leq 0.05),{ }^{* *}(p \leq 0.01),{ }^{* * *}(p \leq 0.001),{ }^{* * * *}(p \leq 0.0001)$. 


\subsection{Discussion.}

The highly conserved cellular signalling cascade initiated by MIS416 stimulation ensures that the first effect of the drug on cellular function would be a noticeable impact on the cellular transcriptional machinery governing what is a highly conserved response to PAMP. Here we demonstrate this effect in profiling the TF response of monocytes and show how the activation of these cells then ultimately culminates in a demonstrable and overt cytokine response.

\subsubsection{NFKB in the MS disease state and the effects from MIS416 induction.}

The ubiquitous transcription factor $\mathrm{NF} \kappa \mathrm{B}$ is a complex regulatory protein controlling the inducible expression of several genes. In the immune system, it plays a key role in modulating immune-regulatory genes and regulating expression of cytokines and other cell signalling regulators in response to a range of stimuli such as microbial pathogens and cellular stress (Sun and Andersson, 2002). NFкB is directly involved in the molecular mechanisms of a range of immunopathological conditions such as cancer and inflammatory disorders as well as MS, often through constitutive activation (Yan et al., 2018).

$\mathrm{NF}_{\kappa} \mathrm{B}$ transcriptional activity is restricted by its association with an inhibitor protein $\mathrm{I} \kappa \mathrm{B}$, and it is only after the ubiquitination, phosphorylation and proteolysis of $\mathrm{I} \kappa \mathrm{B}$ that

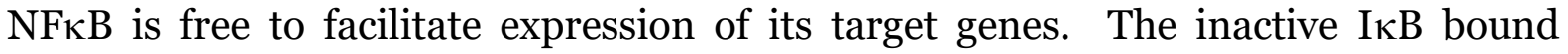
complex is continually shuffled between the cytoplasm and the nucleus, but the strong nuclear export signal on IאB ensures a cytoplasmic majority (bu.edu, 2019). Free,

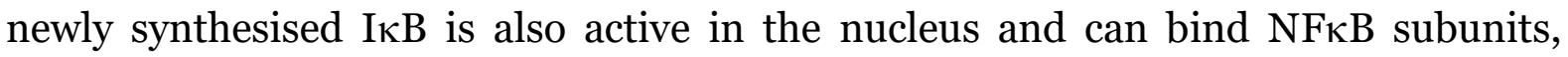
resulting in their release from the promoter sites and restricting transcriptional activity through transportation of $\mathrm{NF \kappa B}$ back to the cytoplasm (bu.edu, 2019). While increased activation of $\mathrm{NF}_{\kappa} \mathrm{B}$ leads to the up-regulation of expression of inflammatory mediators and immune responses, the full complex is required to fully realise an immune response. $\mathrm{NF \kappa B}$ activation is a circular system, where the promotor for the 
I $\mathrm{B}$ subunit is transcriptionally activated by $\mathrm{NF} \kappa \mathrm{B}$ itself thereby forming negative feedback.

$\mathrm{NF}_{\kappa} \mathrm{B}$ immune regulation is subject to the development of tolerance. An example of this tolerogenic response was reported by Bohuslave et al. when they demonstrated that exposure of an endotoxin (i.e. LPS) to monocytes/macrophages cultures induced $\mathrm{NF} \kappa \mathrm{B}$ activation, and that low levels of previous endotoxin exposure resulted in tolerance where $\mathrm{NF} \kappa \mathrm{B}$ subunits crowded $\mathrm{TNFa}$ response promoter regions, reducing the amount of that cytokine produced (Bohuslav et al., 1998). Specific NFאB subunits have been shown to regulate specific parts of the innate immune response (Bohuslav et al., 1998). Wang et al. described how stimulation by TNFa in vitro results in the phosphorylation of $\mathrm{S}_{5} 29$ site, increasing transcriptional activity independent from nuclear translocation (Wang and Baldwin, 1998). The same group later showed that IL-1 $\beta$ stimulation induces p529 site phosphorylation (Wang et al., 2015), maximally after 15 minutes which then deteriorates after $2 \mathrm{~h}$.

Our data focused on MIS416-induced TF phosphorylation as it was induced either by direct MIS416 stimulation or by downstream cytokine-driven responses. The most surprising finding from our data was that we did not detect a direct increase in $\mathrm{NF}_{\kappa} \mathrm{B}$ from early MIS416 stimulation. Instead, a reduction in activity was observed, independent of MIS416 stimulation in the MS cohort. This finding implies that at T3 dephosphorylation (or inhibitor binding) of p65 may have occurred. TLR-mediated signalling in sterile inflammation, such as from surgery, has previously been found to result in reproducible dephosphorylation of p65 in monocytes (Schulz et al., 2012) at $1 \mathrm{~h}$ post-op. It is not possible from our data to conclude when and if MIS416 had a significant effect that directly activates p65, although given the known effects of MIS416, it is very likely. While a T3 timepoint was found to be optimal for MIS416 internalisation, subsequent phosphorylation would be rapid and could only be established through sampling over a tight time series in several participants. The scope here was not to determine this optimal time point, but to establish what if any response differentials there were from participant stratification. As such, we reiterate our observation of a significant reduction in $\mathrm{NF \kappa B}$ activity in $\mathrm{MS}$ that was already present in untreated cells prior to in vitro manipulation. 
When we extended out incubations overnight with MIS416 we observed that NFкB activity did significantly increase when compared to T3. Unfortunately, we cannot ascribe this increase to MIS416 given the lack of an untreated control for the later time point. We also observed that NFאB activation levels at T24 MIS in MS participants were comparable to those seen in HC. This observation, then, may offer avenues of further investigation. It would be valuable to establish what $\mathrm{NF} \kappa \mathrm{B}$ activation levels are following in vivo administration, and to what extent $\mathrm{NF \kappa B}$ activation corresponds to any clinical disease course and response to treatment. We have also not shown when MIS416 optimally induced NFאB activation, and this time point may be valuable to establish, as it will add context to dosing and drug administration.

\subsubsection{Late CREB activation juxtaposed NFkB activation.}

In contrast to the stark changes observed in $\mathrm{NF \kappa B}$, we only observed modest changes in CREB expression in monocytes. CREB induction was also not attributable to MIS416. Active CREB levels showed no variation for either cohort irrespective of in vitro condition, or in the proportion of positive monocytes in the $\mathrm{HC}$ population. The only change we noted was in the proportion of CREB expressing cells in MS participants at T3. In a study of the response to traumatic injury cause by surgery, Gaudillière et al. noted the reproducible dephosphorylation of CREB in monocytes at $1 \mathrm{~h}$ and $24 \mathrm{~h}$ post operatively and likened this response in part due to the multifactorial response to sterile inflammation and TLR signalling (Gaudillière et al., 2014). As we observed a reduction, which appeared independent of our combined TLR9/NOD2 signal, and without having a single agent TLR as control, we are unable to conclude why the drop is observed. It is however possible that the modest pro-inflammatory conditions solicited by rich media from our cultures are in part a reason for the dephosphorylation of CREB in monocytes, given the modest inflammatory activation of cells seen in our in vitro culture.

As with $\mathrm{NF \kappa B}$, we noticed the most dramatic alteration in CREB activation when we extended MIS416 cultures overnight. We observed a distinct drop in active CREB in monocytes at T24 MIS in comparison to T3 MIS. This decrease also corresponded 
with an increase in active p65 and suggests that competitive binding between active CREB and p65 sites may be responsible for this observation. With direct competition for CREB-binding protein known to result in downregulation of the other, high p65 levels can explain the observed decrease in CREB at T24 MIS. This effect was also noted in a subpopulation of participants at T3 MIS, in whom active CREB increased following MIS416 treatment at $\mathrm{T}_{3}$, to contrast to the consistent observation of a groupwide reduction in active p65.

\subsubsection{STAT activation was directly impacted by MIS416 induced cytokine} signals.

While CREB and NFкB changes could not be ascribed to MIS416, we were able to show that STAT1 activity was significantly reduced in monocytes from patients with MS in response to MIS416 in vitro. Furthermore, we also demonstrated a significant STAT1 activation differential between $\mathrm{HC}$ and MS cohorts. A decrease at baseline of STAT1 phosphorylation in MS patients has been observed previously (Canto et al., 2018), although this was in a relapsing-remitting disease cohort. STAT1 activity is essential for an optimal immune response, and the reduced levels of active STAT1 in MS participants is therefore likely to be related to the underlying pathology and indicates an aberrant response to INF in MS (Feng et al., 2002). In autoimmune conditions such as rheumatoid arthritis the lack of response to INF $\gamma$ stimulation correlate to a lack of response to therapy (Kuuliala et al., 2016). Further MS cohort stratification and correlation analysis may be valuable to establish MIS416 clinical response and pSTAT1 levels are interrelated.

STAT3 is a crucial signalling hub mediating pro- and anti-inflammatory activity in monocytes through IL-6 or IL-10 signalling (Niemand et al., 2003). LPS, as expected, induced a strong TNFa and IL-6 response in monocytes from our participants. MIS416 stimulation resulted in a striking increase in TNFa, and a more modest response in IL-6. We show that MIS416 induces a direct decrease in the proportion and activation of STAT3 in monocytes. It is reasonable to conclude that the ability of MIS416 to induce such a strong TNFa response may be linked to the reduced STAT3 activity observed from MIS416 stimulation, as it has been shown that the inhibition of 
STAT3 activity in monocytes restores the cells' ability to produce TNFa following a suppressed state and sterile inflammatory environment post cardiac surgery (de Jong et al., 2012). De Jong et al. also noted that the inhibition of STAT3 under conditions of sterile inflammation may be due to an increase in IL-10 present in post-operative serum. We show that STAT3 activity returns to baseline levels from MIS416 stimulation in overnight cultures, and that those same cultures had high IL-10 production in monocytes. The early STAT3 response seen in our data could not be due to IL-10 but may instead be tied to the direct effects of MIS416 stimulation on monocytes resulting from early TNFa release. IL-6 in MIS416-treated monocytes was found to be significantly lower than those treated with LPS. De Jong et al. noted that STAT3 inhibition was not able to restore IL-6 synthesis following sterile inflammation. The reduced IL-6 response from MIS416 stimulation is likely ameliorated by the increase in IL-10 mediated through STAT3 signalling. Our IL-6 response data therefore indicates how a single TLR agonist LPS, induces a strong inflammatory response while the immunomodulatory nature of a combined TLR and NOD2 signal from MIS416 is less pro-inflammatory.

It is also important to note in terms of STAT activation we did not profile the effects of non-canonical (direct TLR9) pathways following MIS416 induction. The activity of STAT profiled by tyrosine phosphorylation speaks to canonical pathway activity and therefore, to MIS416-induced cytokine signalling. To further investigate noncanonical STAT activity, serine residue phosphorylation could be investigated (Bezbradica and Schroder, 2014).

\subsubsection{Increased IL-10 in MIS416-treated cells resulted in an altered immune milieu.}

Central to the proposed efficacy of MIS416 is the upregulation of IL-10 following stimulation. There is longstanding evidence whereby treatment efficacy in established MS therapies correlates with increased IL-10 production (Liu et al., 2001). The effect of the upregulation of other pro-inflammatory cytokines, such as those produced during sterile inflammation, also effects the inactivation of innate immune cells, thereby providing negative feedback (de Jong et al., 2012). This may be a mechanism 
by which MIS416 could effect a reduction in inflammation and achieve therapeutic efficacy. NFKB, CREB and STAT are all critical in the regulation and induction of IL10 (Iyer and Cheng, 2012) and we've demonstrated conclusively that MIS416 has the capacity to modulate this important inflammation-dampening cytokine.

\subsubsection{MIS416 may induce immune tolerance.}

Adding to this, there is emerging evidence of a tolerising effect of MIS416 as such a tolerogenic immunomodulatory state has been shown to be effective in controlling MS disease in model organisms by our lab (White, 2015). It has been proposed that the presence of macrophages in the CNS containing peptidoglycan antigen contribute to the inflammatory state seen in MS pathology (Schrijver et al., 2001). MIS416 may be beneficial in modulating the immune system to a more tolerogenic state when administered regularly, as MDP-induced tolerance could limit TNFa production (Castellaneta et al., 2009), while potentially also inducing cross-tolerance to TLR products (Hedl et al., 2007). It must be noted that direct anti-TNF therapies are not a viable target for MS, and MS disease is in fact a contraindication for anti-TNF agents as TNFa neutralising antibodies are associated with adverse demyelinating events (Sedger and McDermott, 2014).

\subsubsection{NFkB inhibitor mutations offer insight into effects of NFkB immune variants on MS pathogenesis.}

The NFKBIA gene encodes the alpha subunit of the IKK complex (IкB) (Weizmann Institute, 2019), which forms part of the inhibitor IкB that is responsible for binding inactive $\mathrm{NF \kappa B}$ in the cytoplasm. $\mathrm{NF \kappa B}$ genes have long been considered candidate genes in MS pathogenesis given their central role in immune homeostasis. Our collaborators in Brisbane have previously shown that there is increased constitutive activation of $\mathrm{NF \kappa B}$ in the peripheral blood cells from MS patients compared to controls (Yan et al., 2018). Our cytometry data has however shown the opposite, with HC having higher levels of activated NFKB compared to our MS cohort. While the 
experimental conditions and techniques are not directly comparable, what we can conclude is that $\mathrm{NF \kappa B}$ activation in MS is certainly altered.

There were also early indications from the MIS416 phase 2B trial cohort, where sequencing information for NFKBIA was assessed against clinical immune outcome parameters, that mutational status may impact clinical response to MIS416 and that participants with mutations were disproportionally represented in a clinical 'nonresponder' group (McCombe et al., 2017). We therefore posited that mutations in the promoter for NFKBIA would result in less of the inhibitor molecule $\mathrm{I} \kappa \mathrm{B} \alpha$, resulting in constitutive activation of $\mathrm{NF \kappa B}$ that is detectable as an increase in $\mathrm{NF} \kappa \mathrm{B}$ activity. Our stratification of participants by mutations status offers modest support for this hypothesis. The impact of NFKBIA promoter mutations showed that in a combined analysis of all participants how homozygous mutation burden increased constitutive NFאB p65 activity in monocytes, and that participants may respond differentially in terms of their MIS416 induced cytokines. We also demonstrate using blood plasma immune factor data, that the effect of the mutation results in a trend whereby heterozygous mutation carriers in the MS cohort appear to effect a greater response to MIS416 over wild type individuals. 


\subsection{Limitations.}

A key limitation to our study is the small sample size of our HC cohort, which limits the power to detect only the strongest effects induced by our experimental setup. This is compounded in the stratification of participants into their NFKBIA promoter mutation groups as this further diluted sample sizes. However, our aims in conducting this work was of an exploratory nature so these limitations were accepted as part of our experimental approach.

We showed the effects of in vitro stimulation on TF phosphorylation and cytokine expression. Experimentally, these markers may have disparate optimal detection and activation time points that we were not able to meet using our selected sampling criteria. What we aimed for here was a comprehensive overview that yielded new avenues of investigation, which could then be targeted with a more focussed experimental model.

We were not able to include a comparative untreated control in our setup with which to contrast the effects of MIS416 on phosphorylation markers in the T24 cultures, thus limiting the interpretation and utility of this dataset.

We aimed to extrapolate what effects a known polymorphism in the promoter of NFKBIA had on the immune response using MIS416 in vitro stimulation as a model system. It has not been previously established what effect the mutation has at the gene level. As the impact on the overall system is likely to be complex, interpretation is limited to a potential impact on immune function. MIS416 is not an ideal model system with which to profile the effect of the mutation. MIS416 may activate NFאB canonically or non-canonically, and given the importance of these pathways, redundancy systems would likely impact cytokine or protein phosphorylation in this model, obscuring data interpretation. While we showed an effect on both in vitro and ex vivo immune factors from the mutation, we cannot therefore conclude its impact on MS pathogenesis or MIS416 treatment. 


\section{$5 \cdot 7$ Summary.}

MIS416 is a powerful immune-stimulating agent targeting myeloid cells of the peripheral blood, particularly monocytes, to modulate their cytokine output mediated by alterations in NFKB transcription.

This study revealed several intriguing results that aid in our understanding of the impact of MIS416 therapy on protein phosphorylation and cytokine production in vitro. We report here a clear discernible difference in the response to transcription factor phosphorylation and cytokine expression between MS and healthy people, with some of our findings in agreement with data reported in other studies.

We believe that that our broad aims were achieved, and the major part of our hypothesis, which tested whether monocytes from progressive MS treated with MIS416 in vitro showed differential responses compared to HC, was met. We were not able to conclusively describe the effect of NFKBIA promoter mutations in healthy or MS patients, although a pooled analysis revealed a positive relationship between having the mutation and enhanced cytokine production. While these findings are preliminary, they warrant further exploration. 


\section{Chapter 6. t-SNE \& SPADE of multicolour cytometry data.}

\subsection{Introduction.}

Our work in the preceding chapters has focussed on monocytes and in using in vitro culture to profile the phenotype and function of this innate cell in health and progressive MS. During the experimental data acquisition steps, we used PBMC as a sample source. We felt that the presence of other immune cells in our culture would better model responses of monocytes to those in the periphery in vivo, and that the isolation of monocytes would alter the nature of the cells due to the additional purification steps. To profile monocytes, we relied on a series of 4 stains with overlapping PBMC phenotyping markers. We acquired on average 200,000 events for each stain and had different sampling timepoints over twenty-seven participants. Together this work resulted in a large dataset. Fully profiling monocytes required analysis of only a small part of the dataset and there remained a wealth of information to explore. While responses of other immune cells to our model system were pertinent, the extensive work required to process it falls outside the limits of this thesis. We therefore set out to explore this dataset using faster bioinformatic tools with the aim of gleaning insight on the responses of other immune cells in the samples.

Clustering and dimensionality reduction are bioinformatics approaches that have been well validated for use in processing cytometry data (Eshghi et al., 2019). Following our initial exploration into the use of a clustering algorithm (i.e. SPADE) in Chapter 2, we realised a similar approach would be limiting in that handling a much larger dataset would be restrictive: requiring higher computing time and capacity. To overcome this, we also implemented the use of $t$-distributed stochastic neighbour embedding ( $t$-SNE). $t-S N E$ is one of the best bioinformatic tools for visualising and reducing high dimensional data (Hinton and van der Maaten, 2008). It retains the structure of the data (Amir et al., 2013), while reducing the dimensions to two, and plots datapoints that are similar to each other in terms of dimensional likeness 
adjacent in the dimensionality reduced space. While not a form of clustering analysis, it creates visually similar results.

We therefore set out to mine the dataset from chapters 4 and 5 by performing a further analysis using hi-dimensional tools. Our hypothesis was that given the use of the same dataset, unsupervised clustering and dimensionality reduction tools should offer new insights, but also yield the same findings from earlier analysis. We further posited that clustering analysis on monocytes specifically should describe the heterogeneity of this subset in culture, and that in contrasting $\mathrm{HC}$ and MS data, that this heterogeneity should yield large differences given the underlying pathology of the MS participants. 


\subsection{Aims.}

Our aims were to show the substantial value that lay in our untapped data, to generate new hypotheses, and gain insight pertinent to our established work. To achieve this, we set out with the following specific aims:

\subsubsection{Specific aims.}

6.2.1.1 To perform an analysis of in vitro cytometry data using dimensionality reduction and clustering algorithms.

6.2.1.2 To explore the output data from SPADE and t-SNE algorisms to identify new as well as previously identified trends.

6.2.1.3 With the use of clustering tools, describe the heterogeneity of in vitro treated monocytes and establish the relationship of subpopulations to cytokine production.

6.2.1.4 Establish future directions by profiling phenotype and function of other immune subsets present in the dataset. 


\subsection{Methods.}

Our methods comprised implementing t-SNE and SPADE analysis of a dataset. The experimental methods used to acquire the dataset are detailed in Chapter 3 . Cytometric gating was performed according to the PBMC gating strategy (see Figure 3-2). For the bioinformatics analysis we used the t-SNE analysis tool included in the Flowjo v10 package (BD, US). We based the t-SNE analyses on a guide kindly provided by the Cytometry Core Facility at the University of Sydney (Ashhurst, 2017). For SPADE, we used the SPADE3 (Qiu et al., 2011) package in MATLAB v. R2018b (MathWorks, US).

Prior to any bioinformatic tool use, we performed processing and clean-up steps for .fcs files. This included standard gating to exclude debris from files, such as dead cells, and for all PBMC, CD45 negative events. We further ensured that all events were compensated, on scale, and indexed.

\subsection{1 t-SNE methods.}

t-SNE outputs result in a representation of the average expression and proportion of subset markers. To compile a representative data file for the runs, we first concatenated samples into treatment sub files. This included ensuring that these new files were representative of all participants, and that they contained a proportional number of events by downsampling each individual data file to a set minimum. We then collated an equal number of total events from both cohorts into new files. The result was 10 new combined treatment files (e.g. Untreated, LPS, Clozapine, LPS\&cloz and MIS416) over the two cohorts. Another proportional downsample and concatenation of these files yielded in a final master file that contained equal and proportional data of the entire dataset of in vitro conditions and participants.

The output from t-SNE analyses are stochastic and result in unique plots for each run. While separate runs on the data would generate highly comparable results, the different appearance of the plots could be misleading. Where possible, we performed a single analysis on all data to keep our graphic output consistent. 
6.3.1.1 t-SNE of cytokine expression in monocytes.

We generated a t-SNE plot from a combination of all participants monocyte data for all treatment conditions. The run specific parameters included all PBMC lineage markers FSC-H and SSC-H but not CD45 or viability. Prior to the generation of new files for t-SNE analysis, we gated the events using our PBMC gating strategy (Figure 3-2) to obtain single viable monocytes. The final t-SNE files for each treatment contained on average 1,000 events from MS participants, while for $\mathrm{HC}$ 3,000 were collated to equal the proportional contribution from the cohorts.

\subsubsection{2 t-SNE of transcription factor (TF) data.}

TF expression in monocytes was modest to low for most of the markers, and a further reduction of their expression resulted from our in vitro culture. Furthermore, we could not combine datasets for TF with any other output, such as cytokine production, given the divergent sampling time-points. As such, we generated a limited profile of TF expression in monocytes. For the plots we initially downsampled all files to 5000 events. These files were then then concatenated into cohort and treatment specific subfiles, while ensuring an equal proportion of MS and HC events were present. tSNE was performed using standard FlowJo parameters using SSC-H, FSC-H, lineage markers, but not CD45 or viability.

\subsubsection{3 t-SNE of DR expression of monocytes and in the PBMC.}

We combined two cytometry stains containing overlapping lineage markers but with different DR receptors. In total the analysis contained over 350 files. We downsampled MS participants and $\mathrm{HC}$ to equal the proportional weight for the cohorts. We concatenated all files from panels 1 and 2 to generate a dataset of all DR expression. t-SNE was run using standard pre-set FlowJo parameters of all lineage markers, SSC-H, and FSC-H but without viability, CD45 or DR expression. We included a numerical keyword identifier in the master file to distinguish the respective treatment, cohort contributions and panel origin. 


\subsubsection{SPADE methods.}

SPADE offers simple two-dimensional visualisation of cytometry data depicting marker behaviour as a tree structure formed from hierarchical clustering. The analysis consists of five steps (Figure 6-1) and processes data without the need for a user specified order.

(i) Cytometry data

\section{Density-dependent downsampling}

(ii) Downsampled data

Agglomerative clustering

(iii) Clustering result

Minimum spanning tree construction

(iv) SPADE tree

Upsampling

(v) Colored tree showing cellular heterogeneity
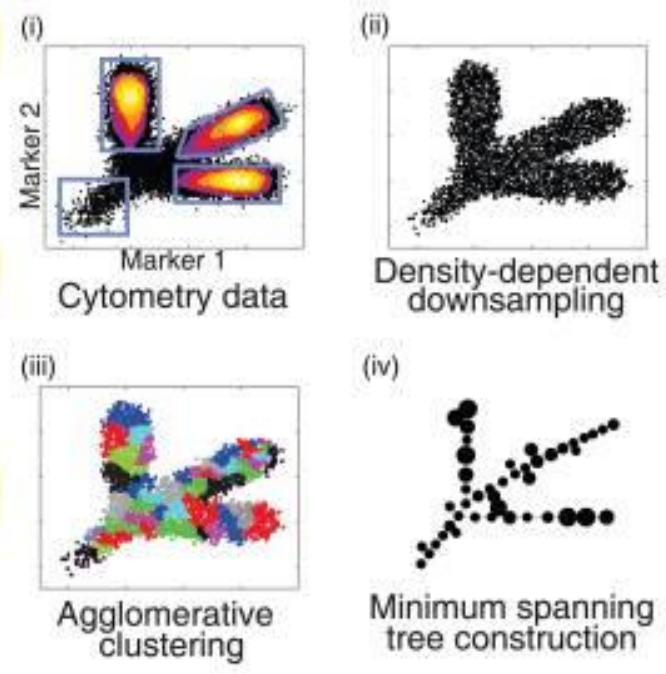

(iv)

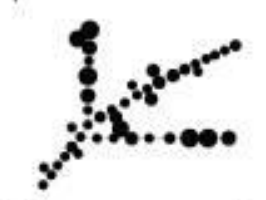

Minimum spanning tree construction

(v) Marker 1 intensity Marker 2 intensity

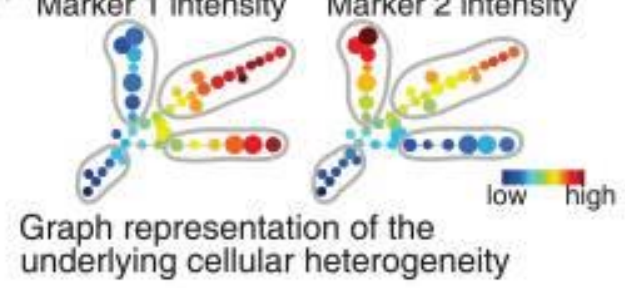

Figure 6-1 SPADE analysis data-flow for a simulated dataset.

(i) Four simulated populations comprise the sample. (ii) Density dependent downsampling results. (iii) Clustering of downsampled data. (iV) Spanning tree construction connects clusters. (v) Heatmaps for two simulated median marker intensities. Copied with permission from Qui et al. 2011.

SPADE plots are analysed as an interpretation of the tree structure and is based on three factors: clusters (nodes), edges between clusters, and the cluster location (edges from other clusters). Edges are the connections between clusters and the size of each cluster is proportional to the number of events within that cluster. While the length of the edge is not informative, the number of edges between two nodes is representative of the cluster similarity, with fewer edges between more similar 
clusters. In general, we stipulated only the standard lineage phenotyping markers in the tree construction.

We generated SPADE trees with concatenated files containing all participant samples for each treatment condition, and a pooled file for all conditions. In general, we downsampled each participant file in Flowjo (BD, US) such that the total concatenated file comprised an equal proportion of events from each participant.

The software enables a description of markers not included in the tree construction by interpolating how similar in weight a marker is to those used to define the phenotype. Where a heatmap overlay of a marker is highly correlated to the ones used to build the tree then the numerical range of the colour bar would be comparable to the range of axis in the pooled file, or within less than 5 -fold difference. SPADE colour bar range is depicted in our data as the average expression (or proportion) of events over the nodes where the $5^{\text {th }}$ and 95 percentiles were used to construct this range.

\subsubsection{SPADE of cytokine expression by monocytes.}

We used the standard pre-set functions of the SPADE 3 package with a total cluster size of 20. The plots all therefore comprise 20 clusters. We included an Arcsine normalisation factor of 5 to allow for a comparison of the expression scales between the t-SNE and raw data SPADE trees. Data normalisation corrects for channel specific differences in spreading of negative events from fluorophore combinations, compensation, and instrument setup.

Where the analysis used t-SNE parameters, those plots represent a clustering analysis of dimensionality reduced data (Diggins et al., 2015). We also complied a SPADE tree using raw cytometry data, i.e. without t-SNE pre-processing, to validate clustering on non-reduced data. We used lineage markers in their construction, as treatment conditions were known to significantly and differentially impact cytokine expression. As such, cytokine markers did not contribute directly to the overall tree structure, but we could then interpolate how expression of any cytokine is distributed within the monocyte compartment. 
6.3.2.2 SPADE of DR expression by monocytes.

For DR expression we also used the standard pre-set functions with a total cluster size of 20, however, no data normalisation (i.e. arcsine) was used. The analysis here again included only lineage phenotyping markers to generate a clustering representative of the subset phenotype independent of DR expression. This was done as not all samples contained all DR markers. Therefore, DR markers did not contribute directly to the overall tree structure, but we can interpolate how expression of any DR is distributed within the monocyte compartment. 


\subsection{Results.}

\subsection{1 t-SNE of cytokine expression by monocytes.}

We performed t-SNE analysis of monocytes to demonstrate cytokine expression responses in our in vitro model. The resulting image depicts the entirety of the monocyte dataset from our cytokine expression experiments (Figure 6-2, Row A). To better understand the dimensional reduction that this image represents, we overlaid the expression of common monocyte expression markers as well as the expression of cytokines within the new plot (Figure 6-2, Row B). This highlights the common phenotypic profile of monocytes in vitro, with the entire subset being positive for HLADR and CD45, while CD14 expression varies within the treatment conditions. We note here that CD16 expression does not define a unique high expressing subset of mature cells. This absence suggests our in vitro model abrogated this subpopulation which is usually present in the PB. We also noted expression of CD123 in most cells, with hotspots likely accounting for the presence of dendritic cells within the gating.

Extrapolating further from the same base plot, we reviewed the contribution each treatment condition had to the overall data, and the specific contribution from either HC or MS cohort. Having included a unique identifier during the concatenation of the master file allowed for the separation of the treatment specific data. The treatment effects are depicted here as density plots of events (Figure 6-2, Row C). These plots highlight the differences in monocyte population heterogeneity between the HC and MS cohort in that, within the monocyte compartment, significant differences in subset phenotype and expression occurred, that resulted in a different density distribution for the various treatment conditions and for the respective cohorts. While there were areas where phenotypically similar cells were present in both cohorts, there also exists a substantive shift in monocyte characteristics that was putatively due to MS pathology. Specifically, in untreated cells, we noted the expansion of events that corroborate with the TNFa positive space for the MS cohort (red arrow). MIS416 also induced a greater number of monocytes to produce IL-6 in the MS cohort when compared to the HC (blue arrow). This is supported by from our findings in Chapter 
5 of this thesis, that showed different expression and proportions of positive cells between the cohorts.

New insights offered by the plots indicate that unusually, clozapine treatment resulted in the downregulation of CD45 in a subset of HC monocytes (green arrow), which was not present in the MS cohort. There was also a subpopulation of non-responder cells in terms of IL-1 $\beta$ induction with these cells occurring in all treatment conditions, even MIS416, suggesting IL-1 $\beta$ induction was not universal (purple arrow). We also see here that IL-10 and INFy induction occurred at a low level in most cells, even untreated, and that there were distinct patches of cells that either up- or downregulate expression following LPS stimulation (orange arrows). 
A

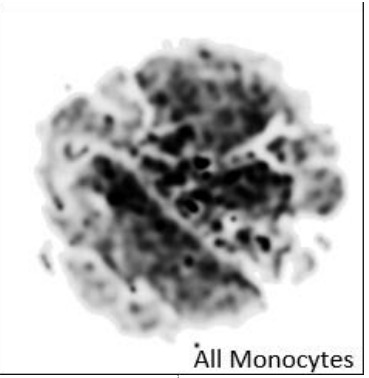

B

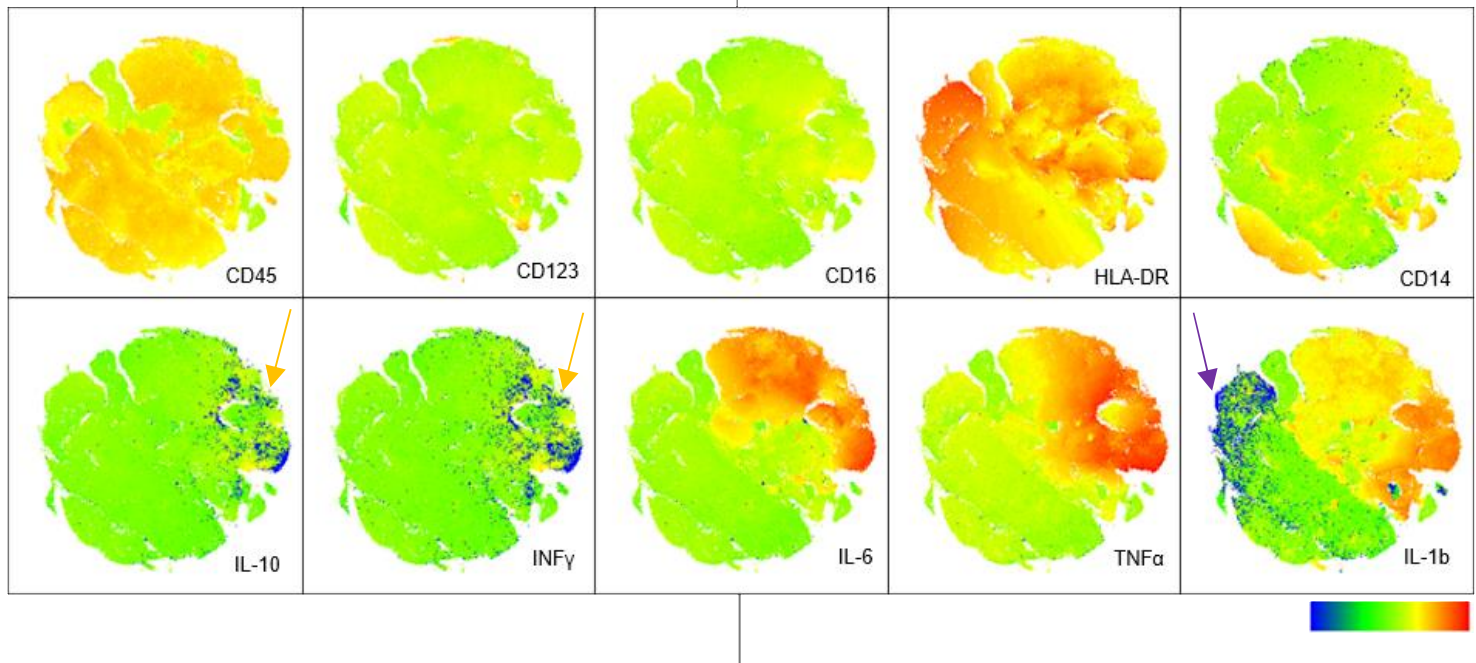

C

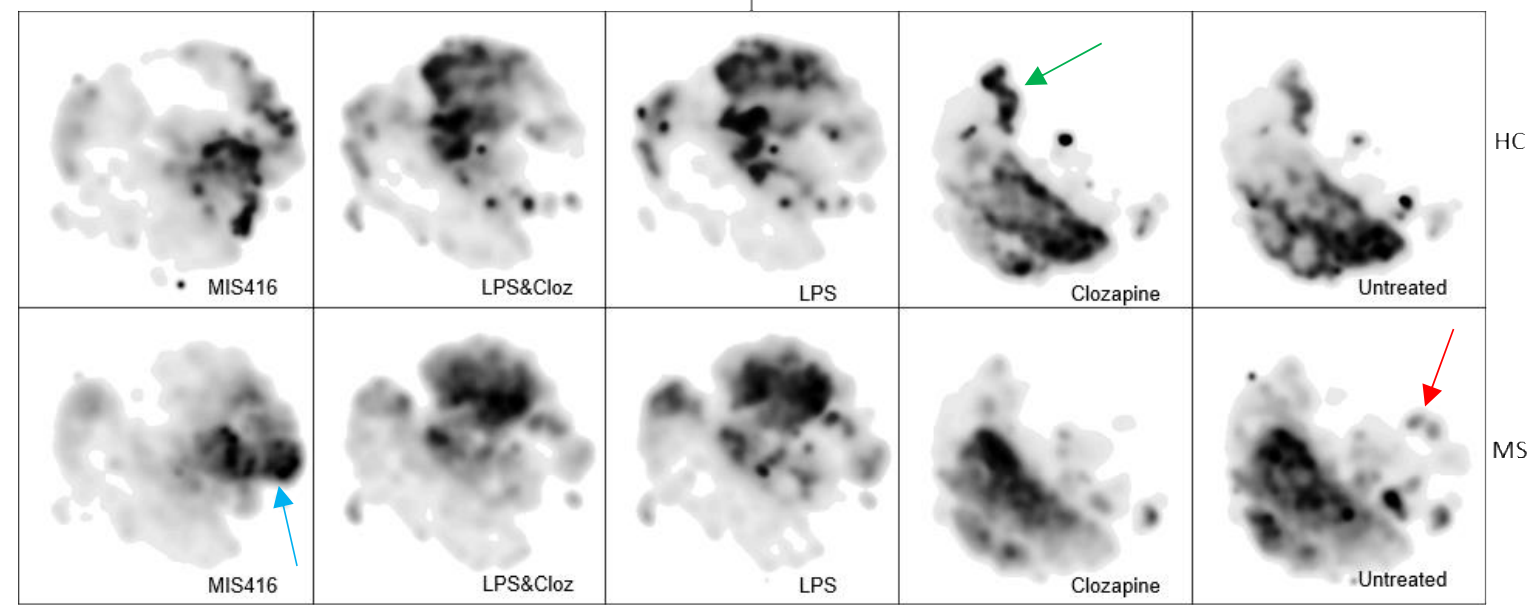

Figure 6-2 t-SNE plot representative of all in vitro monocytes (Row A).

Cell marker expression for all monocytes as overlay heatmaps of surface markers and cytokines (Row B). Density plots of all monocytes only depicting events from each relevant in vitro condition and separated by $\mathrm{HC}$ and MS participants (Row C). 
6.4.1.1 SPADE analysis using t-SNE parameters demonstrates cytokine expression by monocytes.

While t-SNE plots are great at reducing the dimensionality of data, they are not well suited to clustering. To interrogate the heterogeneity of the monocyte population we performed SPADE analysis using the generated t-SNE parameters. This allows for a clustering analysis of the dimensionally reduced cytometry data and is depicted in Figure 6-3.

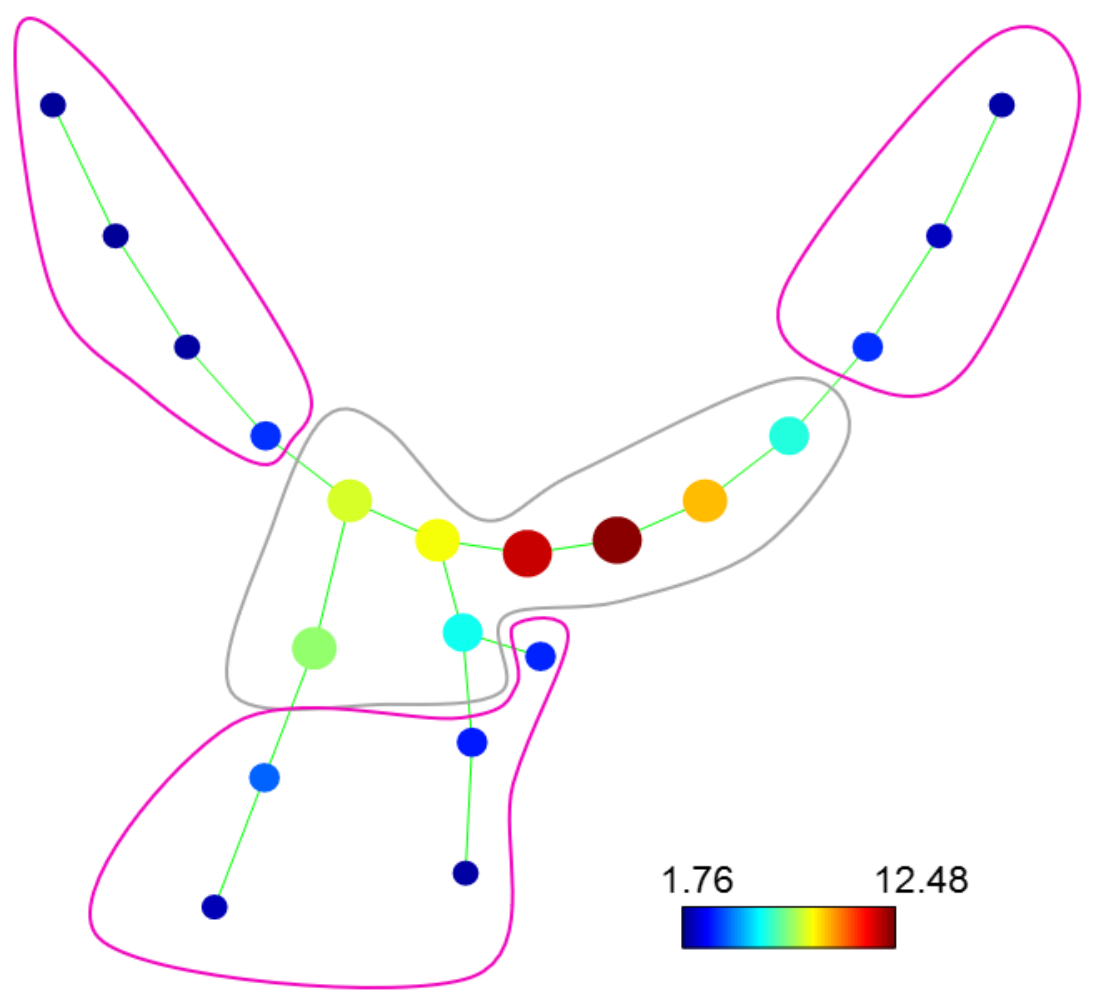

Figure 6-3 SPADE analysis plot using t-SNE parameters of all concatenated in vitro monocyte data.

The nodes depict monocyte clusters with a heatmap overlay depicting the proportional frequency of events within each node. Eight central nodes that are similar in terms of surface marker expression are outlined in silver, with three groupings of outlier nodes outlined in purple. All data were corrected with Arcsine correction of 5 . 
Peripheral monocytes are commonly classified into three subsets based on CD14 and CD16 expression, but t-SNE and clustering analysis showed that in vitro manipulation and stimulation substantively alter the cells away from this limited phenotypic definition. Figure 6-2 suggests that within the monocyte compartment, several distinct clusters of subsets are present, and these subsets respond differentially to in vitro manipulation. From Figure 6-3, we calculated that the central monocyte cluster comprised of roughly 69\% (of all clusters) and were similar in phenotype and size (silver clusters), with the remaining 31\% representing outliers (purple clusters).

Even though the outliers were a minor fraction they are an important subset. Phenotypically the outlier clusters were diverse, and had some common features including a significant upregulation of HLA-DR, a loss of CD14, and low cytokine expression. This was demonstrated when we generated a plot of the outlier clusters as an overlay onto our t-SNE plot (Figure 6-4), furthermore we bisected the clusters into untreated (encircled in brown), treated, and by cohort (Figure 6-4C). We noted the untreated fraction of cells had higher HLA-DR expression. These cells expressed nearly three times more HLA-DR $(\mathrm{gMFI}=221,423)$ than their central cluster counterparts $(\mathrm{gMFI}=76,024)$ while comparatively producing very low levels of cytokine (Figure 6-4B). CD14 expression was also reduced. When we compared the distribution between HC and MS participants using density plots, we observe only modest differences in LPS and MIS416 responses but noted that clozapine and untreated in vitro data retained distinct shifts between the cohorts. This indicates that the outlier cells responded in a similar fashion to LPS and MIS416, independent of pathology, but that their naïve and clozapine responses likely retained patterns associated by disease pathology. Further phenotypic exploration of these cells may describe their function but would require more extensive phenotyping work.

Surprisingly, cells treated with MIS416 comprised only 4\% of the outliers, suggesting MIS416 responder cells in general do not cluster within the outlier subpopulation, and that MIS416 induction was specifically targeting the larger cluster containing classical monocytes. Given that MIS416 is known to transiently increase intermediate monocyte populations in the $\mathrm{PB}$, we suggest that those cells must arise from classical CD14 cells rather than elsewhere, as the data here points to classical monocytes responding to the drug. 
The central cluster of nodes from the SPADE tree (Figure 6-3) is depicted as an overlay onto the t-SNE plot (Figure 6-5), again with the untreated cells highlighted in brown. Within this larger central cluster, we observe the contribution of classical monocyte responders to treatment and untreated (encircled) events. Phenotypically this central cluster comprised two populations, with either dim or bright CD14 expression. HLADR appeared uniform, while CD16 appeared to be increased in the events within the treatment responder events. TNFa, IL-6 and IL-1 $\beta$ was strongly upregulated in response to stimulation, and in contrasting MS and $\mathrm{HC}$, we noted changes that were effected by MS disease pathology on monocytes from our in vitro experiments (Figure 6-5C). The shift in the density of events depicted here are indicative of the differential responses of monocytes between the cohorts. This follows as the events occupied different areas of the plot. The density distribution between the cohorts again suggested distinct responses between the cohorts, particularly to MIS416. 
A

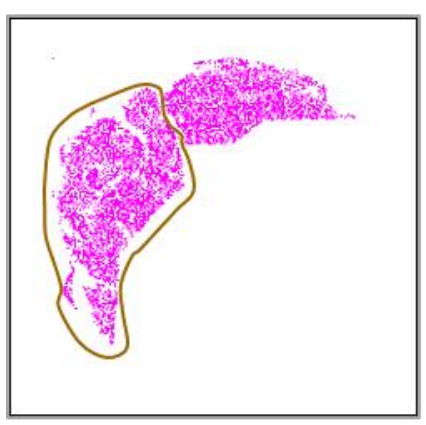

Outlier monocytes
B

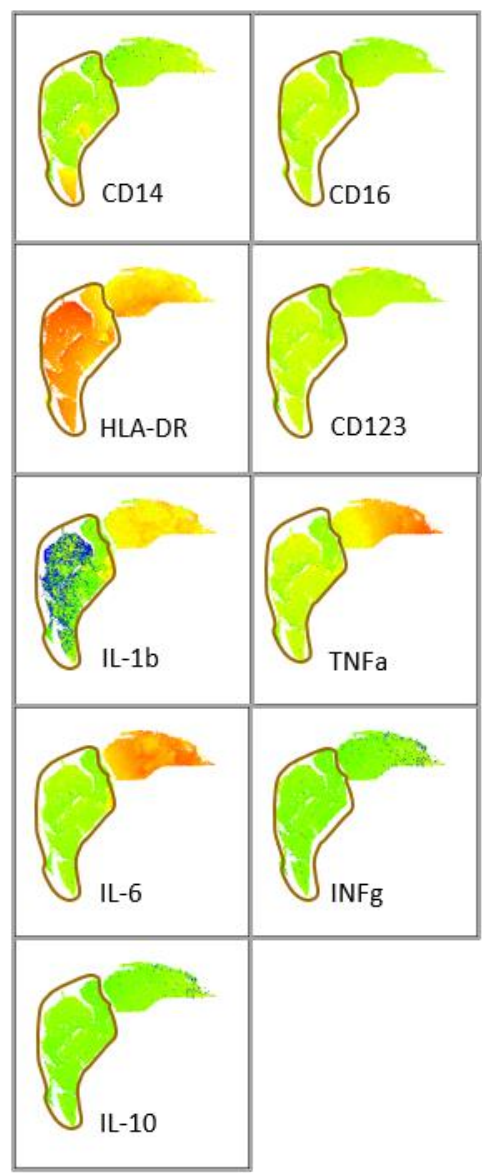

Phenotype

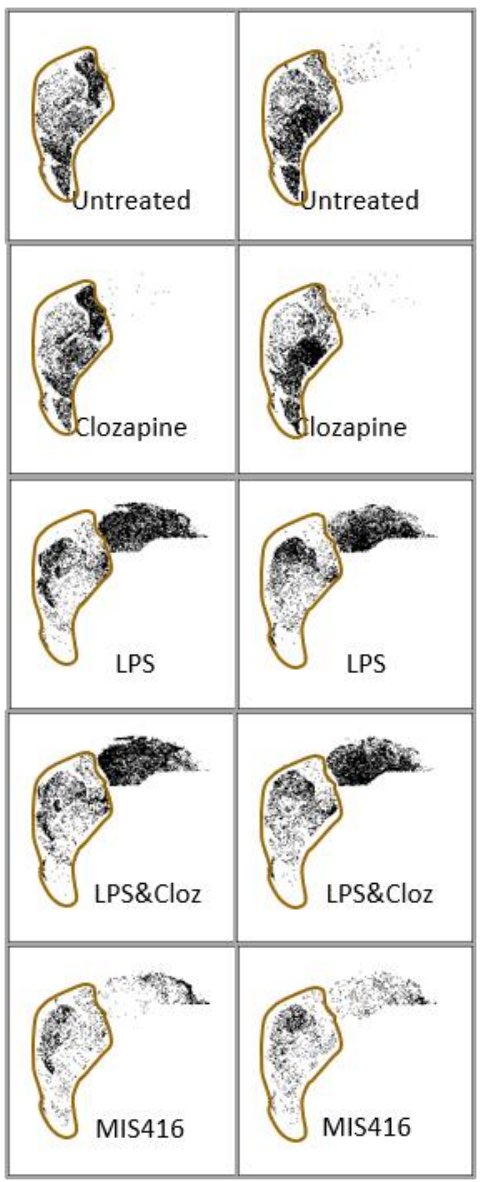

$\mathrm{HC}$

C 
A

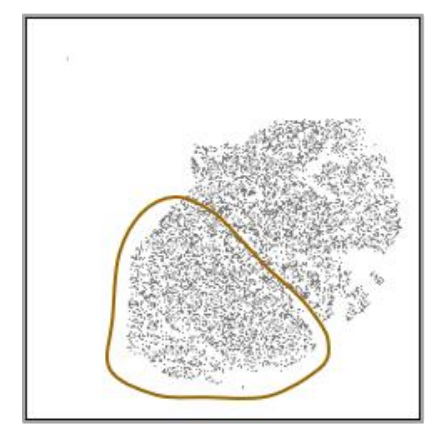

Phenotype

B

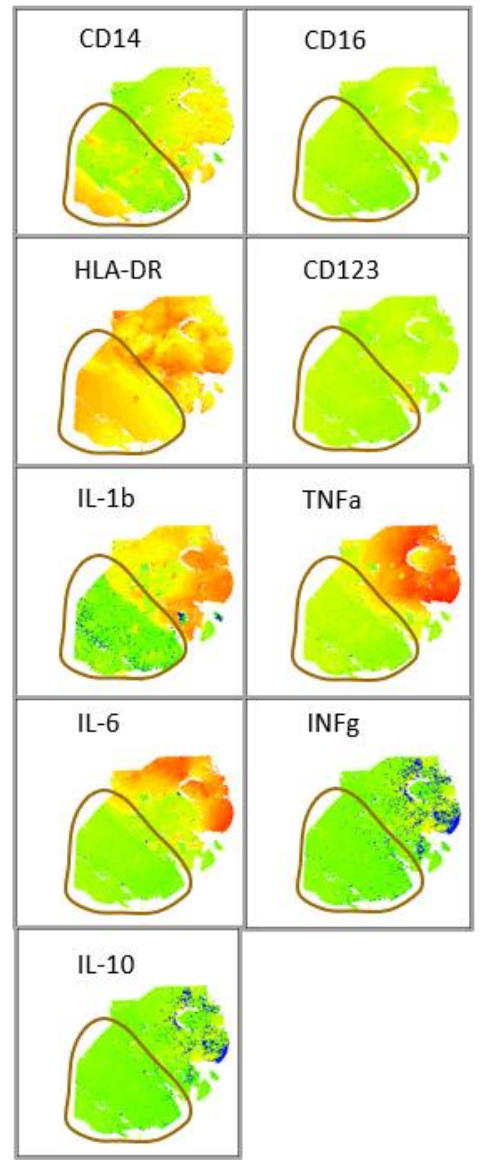

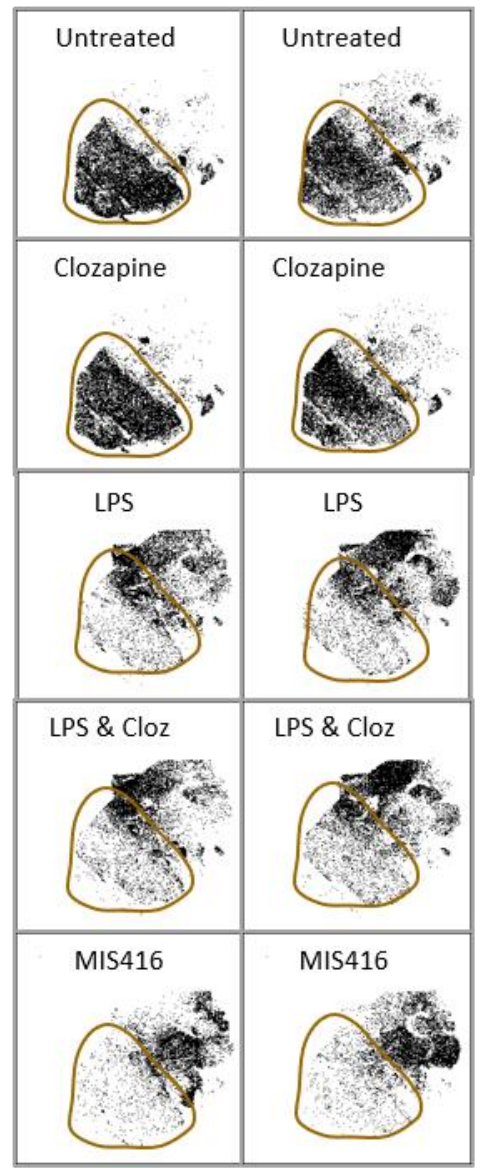

MS

Figure 6-5 t-SNE plot overlay of central clustered monocyte data.

SPADE analysis of monocytes indicated a central cluster (Figure 6-3). The events are depicted here by treatment condition and cohort, using the initial t-SNE generated plot as overlay. (A) All central cluster events as treated and untreated (brown), (B) heatmap overlays of common marker expression, and (C) density distribution of events by treatment and cohort. 


\subsubsection{SPADE of cytokine expression by monocytes.}

6.4.2.1 SPADE analysis of unprocessed monocyte data.

To further build on the monocyte clustering analysis we performed a SPADE analysis without first doing the dimensionality reduction (i.e. $\mathrm{t}-\mathrm{SNE}$ ) steps to generate the tree structure in Figure 6-6. This contrast directly with the analysis that generated Figure 6-3 and highlight the synergy between the two approaches. Performing SPADE analysis alone (without a preceding t-SNE) allows for the interrogation of the expression of cytokines within the amalgamated file for both $\mathrm{HC}$ and MS populations and by treatment condition. We again found a central series of clusters (Figure 6-6, silver highlight) representing monocytes that showed a very high level of overlap to the data from the SPADE plot of t-SNE parameters (Figure 6-3). In fact, comparing the central cluster data from the t-SNE and non-t-SNE SPADE plots showed these nodes comprised equal proportions of events from the respective treatment conditions (Table 6-1).

Table 6-1 Proportional composition of central cluster events by treatment condition.

\begin{tabular}{|c|l|l|l|}
\hline \multirow{5}{*}{ HC } & $\begin{array}{c}\text { In Vitro } \\
\text { Treatment }\end{array}$ & $\begin{array}{c}\text { SPADE of t- } \\
\text { SNE } \\
\text { parameters }\end{array}$ & $\begin{array}{c}\text { SPADE } \\
\text { without t- } \\
\text { SNE }\end{array}$ \\
\hline \multirow{5}{*}{ MS } & Untreated & $10.8 \%$ & $10.6 \%$ \\
\cline { 2 - 4 } & Clozapine & $10.9 \%$ & $10.7 \%$ \\
\cline { 2 - 4 } & LPS & $9.90 \%$ & $9.88 \%$ \\
\cline { 2 - 4 } & LPS \& Cloz & $10.0 \%$ & $10.0 \%$ \\
\hline \hline \multirow{4}{*}{ MIS416 } & $10.5 \%$ & $11.0 \%$ \\
\cline { 2 - 4 } & Untreated & $8.77 \%$ & $9.14 \%$ \\
\cline { 2 - 4 } & Llozapine & $10.6 \%$ & $10.3 \%$ \\
\cline { 2 - 4 } & LPS \& Cloz & $10.7 \%$ & $10.5 \%$ \\
\cline { 2 - 4 } & MIS416 & $8.98 \%$ & $8.87 \%$ \\
\hline
\end{tabular}

A contrast of the data from the two SPADE

plots in Figure 6-3 and Figure 6-6 to demonstrate SPADE analysis with or without a preceding t-SNE step. 


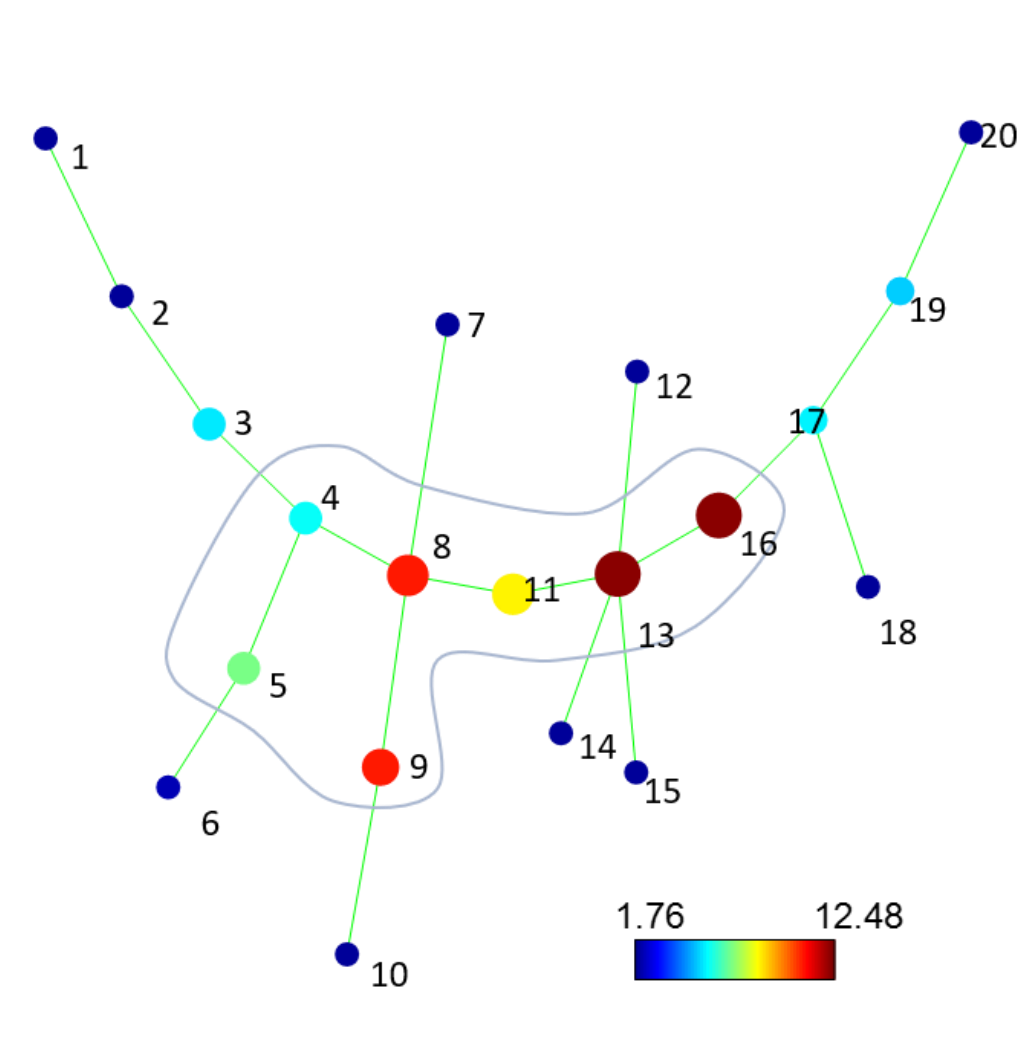

Figure 6-6 SPADE analysis plot using lineage markers of all concatenated in vitro monocyte data.

The nodes depict monocyte clusters as a heatmap of the proportional cell frequency in each node. Seven central nodes that are similar in terms of surface marker expression are highlighted in silver, with outlier nodes in purple. All data corrected with Arcsine correction of 5 .

With this new plot (Figure 6-6) we could then then visualise expression of cytokines and establish whether there were subpopulations of responder cells within the monocyte compartment. To this aim, we contrasted each cytokine for LPS and MIS416 stimulation to the untreated cells for both HC and MS cohort (Figures 6-7 to Figure 611). For TNFa (Figure 6-7) we observed a broad and general induction of cytokine response from most clusters, and that several high-frequency nodes of the central cluster $(8,11,13)$ and outside it (3 \& 17) had only a very modest response to either LPS or MIS416 stimulation. Generally, cytokine-producing cells were those most phenotypically diverse, as represented in the outlier nodes. We also observed from untreated data, where nodes were already expressing TNFa (nodes 6, 10, 16, 19), that those went on to produce the most cytokine following subsequent simulation. 
Together this suggests TNFa induction by both agents occurs in a diverse subset of monocytes and that there are a substantive proportion of relatively homogenous cells producing much less TNFa than their counterparts. The plot also shows that MIS416 induces more TNFa than LPS stimulated cells in the HC, which did not reach the definition of significance using conventional analysis (see Chapter 5) but is apparent from this clustering analysis.

Similarly, the cluster plots of IL-1 $\beta$ (Figure 6-8) and IL-6 (Figure 6-9) depict cytokine expression over the SPADE tree. Generally, the outlier nodes here also produced the most cytokine with a distinct population of dim or low producing cells $(3,8,13,17)$. When the expression differs, it can be described due to the nature of the induction/stimulation method, as LPS was a stronger inducer of IL-6, and MIS416 of IL-1 $\beta$ respectively. Again, these differences are reflected in the SPADE plots.

For INFY (Figure 6-10) and IL-10 (Figure 6-11) the plots were difficult to interpret owing to their dim expression. Our conventional analysis required an FMO gating control to delineate positive staining cells from negative. SPADE plots only show the average expression of the marker for the cells in it - which with these dim markers resulted in lower resolution. We did however deduce that expression of these two cytokines was distinctively limited to a very few clusters ( $13 \& 16)$, which also showed limited expression of the pro-inflammatory cytokines. A further contrast in expression was that the outlier populations we show to produce IL-6 and IL-1 $\beta$ and TNFa, did not make IL-10 or INFY. This finding suggests that following in vitro culture, distinct populations of monocyte produce pro-inflammatory cytokines such as IL-6, IL-1 $\beta$ and TNFa, while others express IL-10 and INF $\gamma$ and indicates that the monocyte population in vitro is highly heterogeneous, phenotypically distinct, and possesses differential response properties.

Lastly, the data in these plots corroborated our results from Chapter 5: for example, the expression of TNFa. Contrasting SPADE plots of MIS416 and LPS showed significantly more TNFa produced compared to LPS, and we noted untreated MS participants had significantly more TNFa induction at baseline and following LPS stimulation that HC. 


\section{TNF $\alpha$}

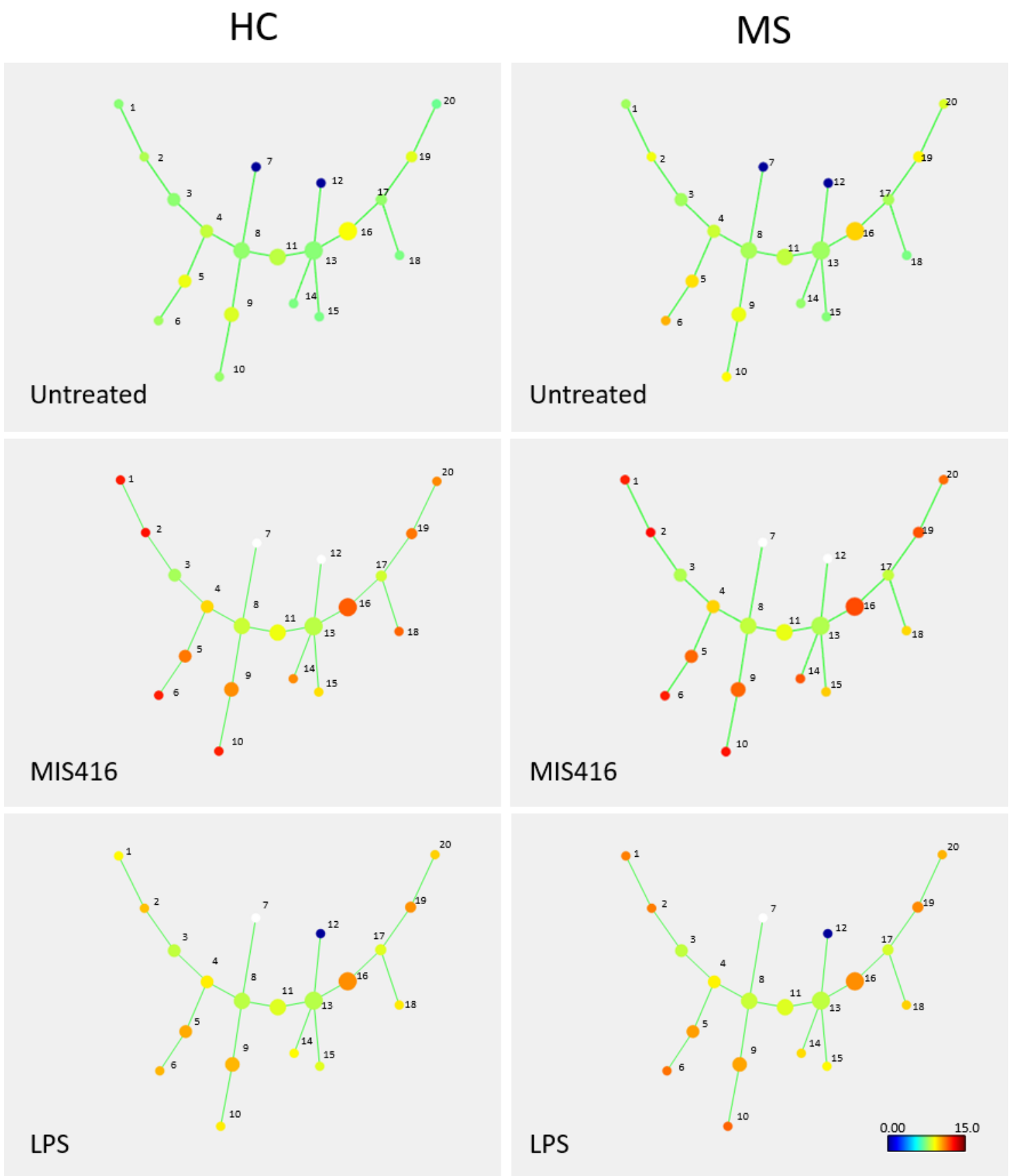

Figure 6-7 TNFa expression comparison between HC and MS cohorts for LPS, MIS416 and untreated cells.

Expression heatmap represent transformed and corrected data of all plots ranging from undetected to the $95^{\text {th }}$ percentile. 


\section{IL-1及}

$\mathrm{HC}$

MS
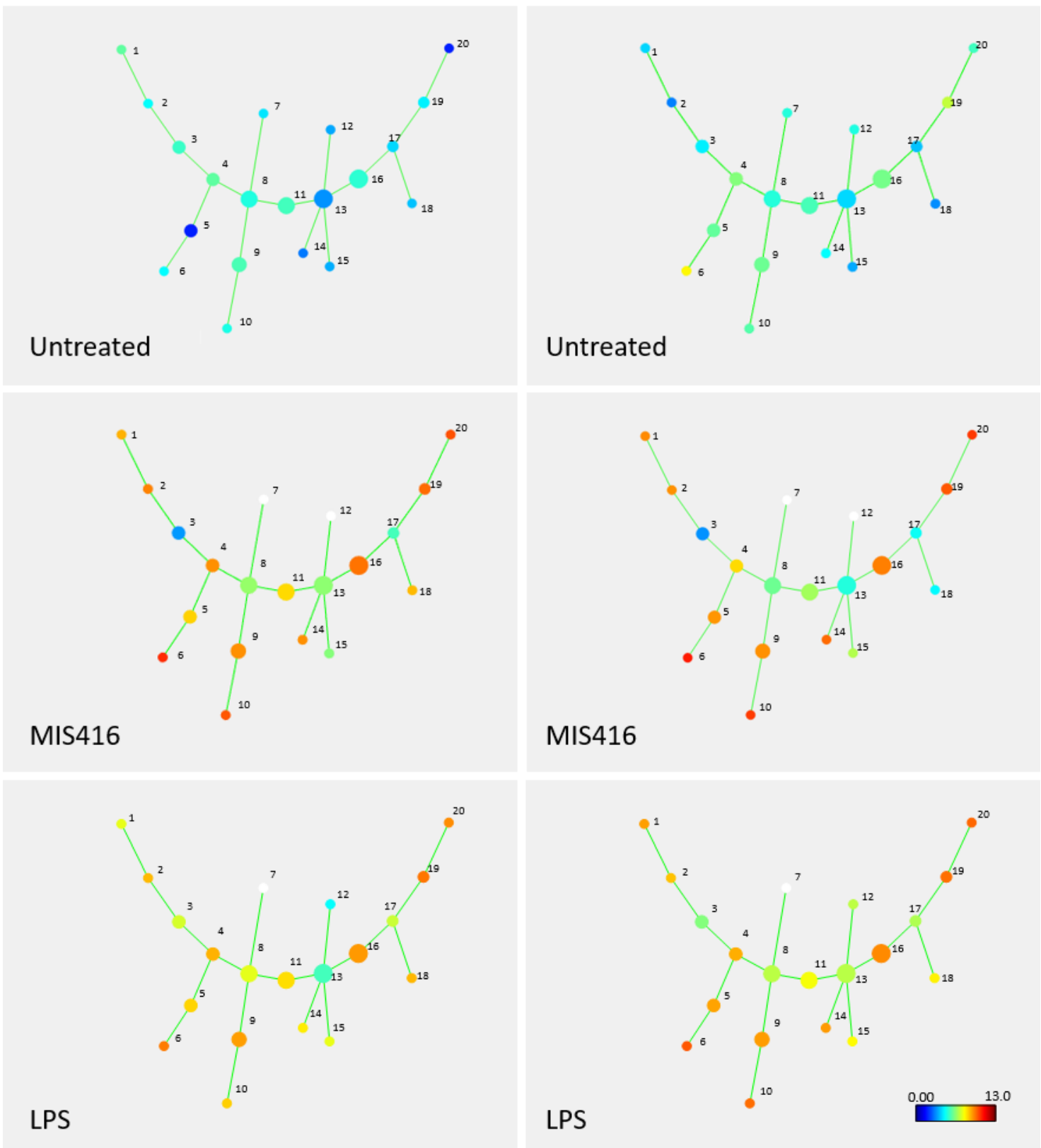

Figure 6-8 IL-1 $\beta$ expression comparison between $\mathrm{HC}$ and MS cohorts for LPS, MIS416 and untreated cells.

Expression heatmap represent transformed and corrected data of all plots ranging from undetected to the $95^{\text {th }}$ percentile. 


\section{IL-6}

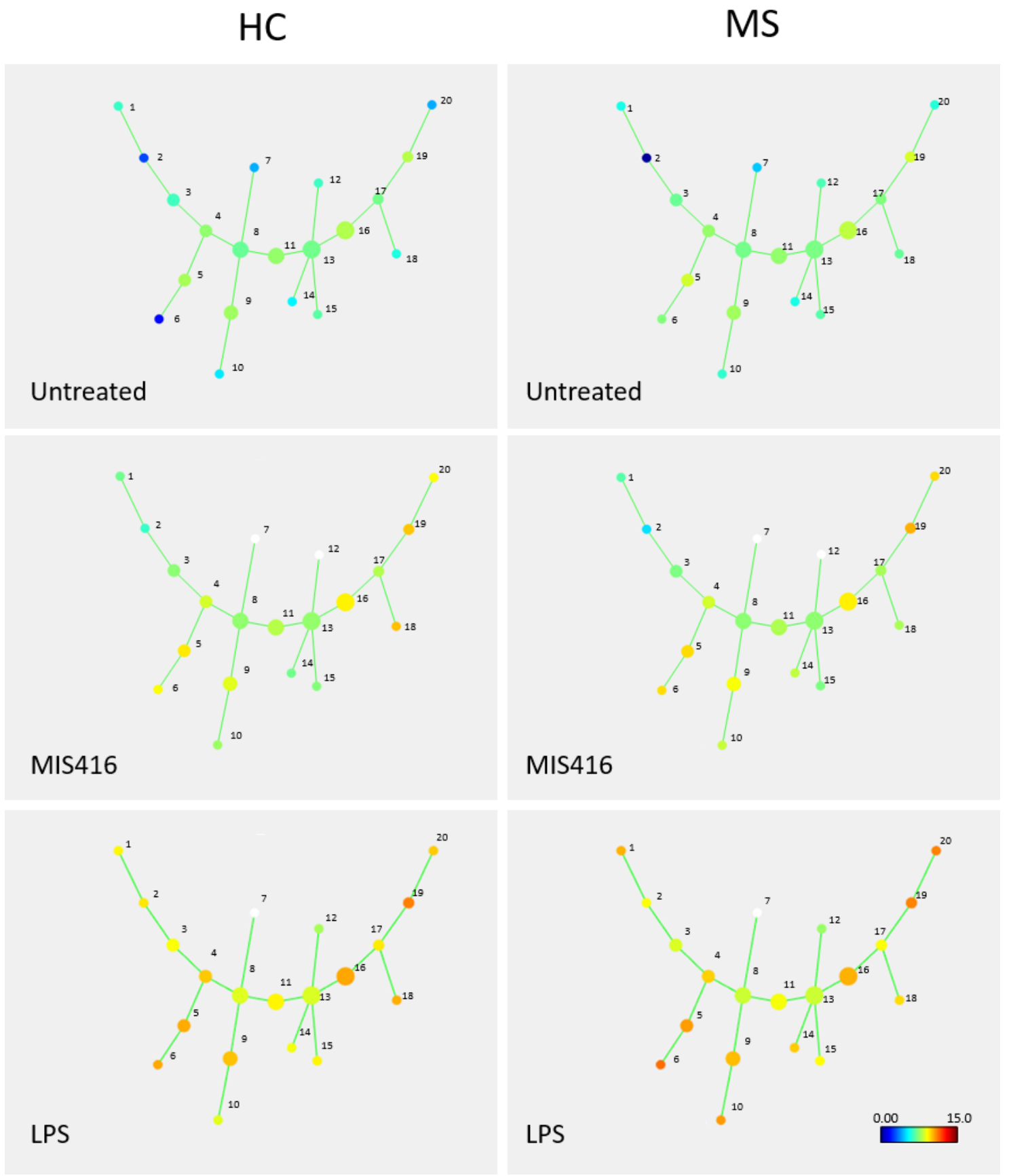

Figure 6-9 IL-6 expression comparison between HC and MS cohorts for LPS, MIS416 and untreated cells.

Expression heatmap represent transformed and corrected data of all plots ranging from undetected to the $95^{\text {th }}$ percentile. 


\section{INFY}

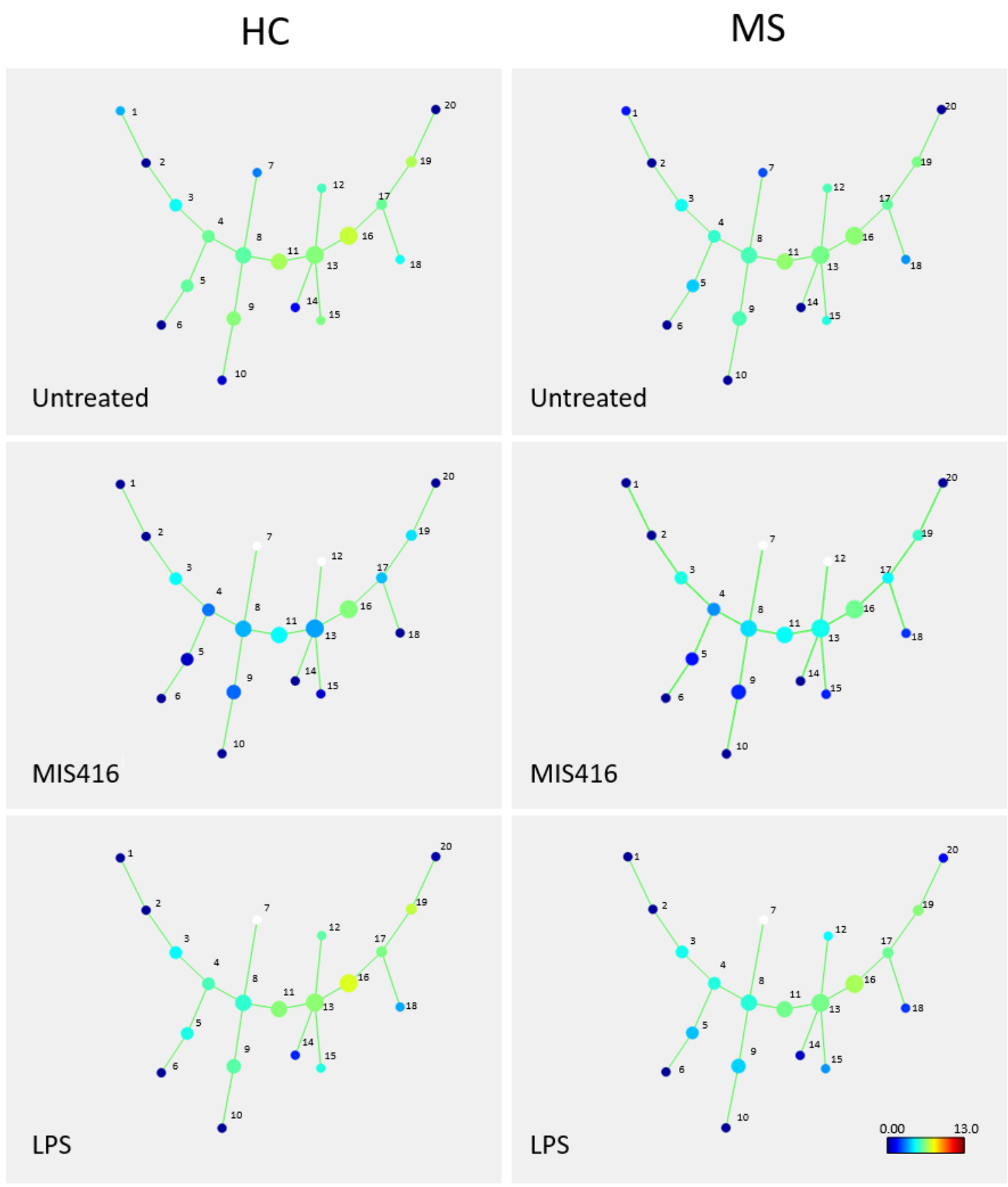

Figure 6-10 INFy expression comparison between $\mathrm{HC}$ and MS cohorts for LPS, MIS416 and untreated cells.

Expression heatmap represent transformed and corrected data of all plots ranging from undetected to the $95^{\text {th }}$ percentile. 


\section{IL-10}

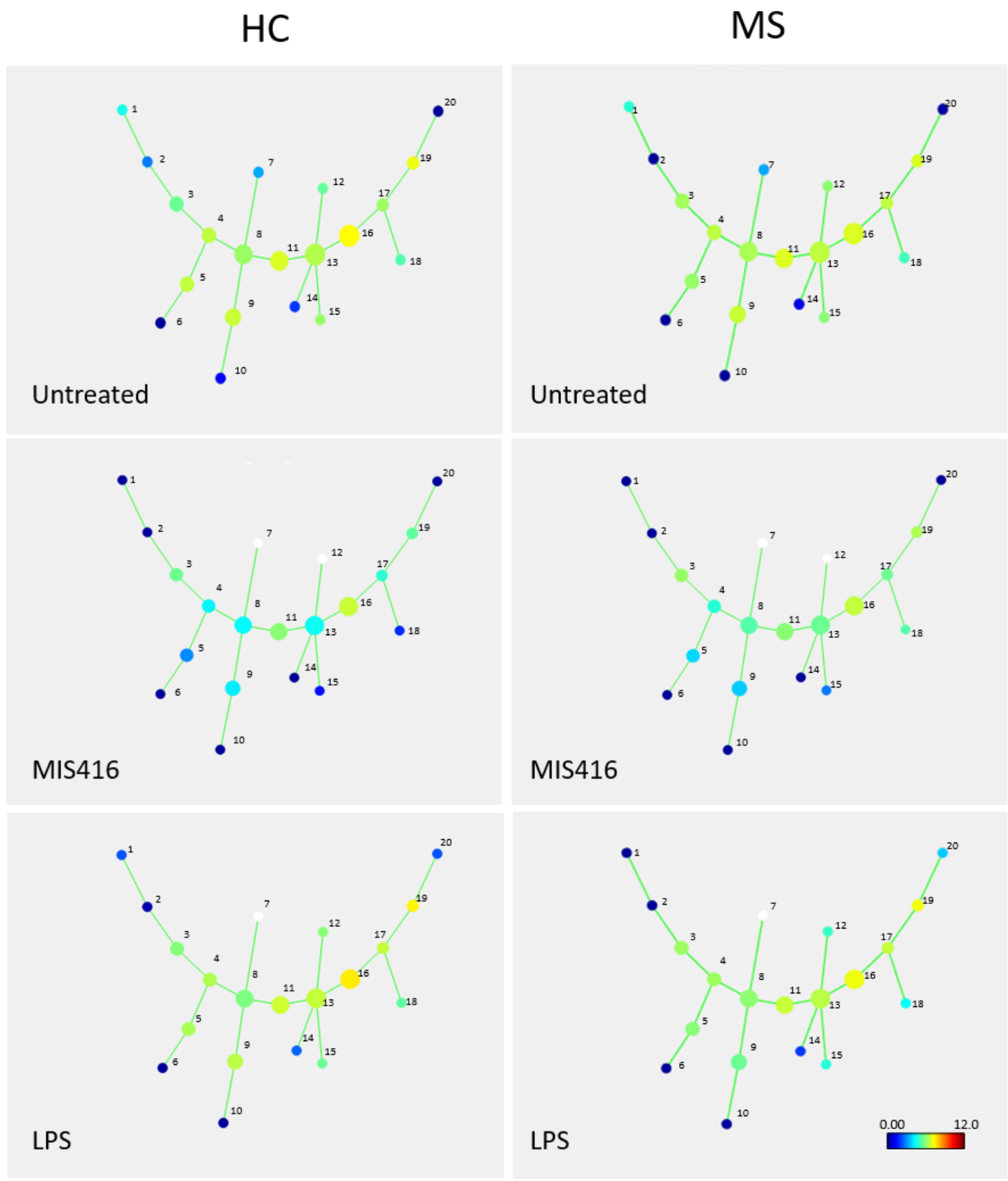

Figure 6-11 IL-10 expression comparison between $\mathrm{HC}$ and MS cohorts for LPS, MIS416 and untreated cells.

Expression heatmap represent transformed and corrected data of all plots ranging from undetected to the $95^{\text {th }}$ percentile. 


\subsection{3 t-SNE of transcription factor expression in monocytes.}

We performed t-SNE analysis to visualise TF expression and establish whether expression was by all monocytes, or by subpopulations within it. To this end, we generated Figure 6-12 which depicts monocytes representing all conditions for all participants and TF expression in these cells as a heatmap overlay. From this analysis we observed that TF expression for P65 and CREB were distinctly positive in all cells, with low expression of some cells aligning with the decrease in expression observed at T3 in our cultures.

For STAT1 most cells were positive, but there were also a minority of events producing much higher amounts. We described in Chapter 5 that HC participants showed higher STAT1 expression, and they are depicted here in the collated data t-SNE image as modestly warmer events. In general, the data did not indicate a differential response across monocytes in terms of STAT1.

STAT3 expression was difficult to interpret as unfortunately the plot showed poor resolution, likely exacerbated by technical limitations from dim staining. In contrasting the expression here to our data in Chapter 5 , we note that we observed a broad range in the proportion of positive staining events for STAT3. Some participants showed as low as 20\% positive staining, while most were high. This broad range of expression of STAT3 results in the poor expression resolution of the t-SNE plot. We conclude that TF expression in monocytes appeared uniform, indicating that the response from monocytes in our models was homogenous, and that no distinct cluster of responder cells were apparent. Furthermore, given the absence of subset heterogeneity we deemed this data unsuited to clustering analysis with SPADE.

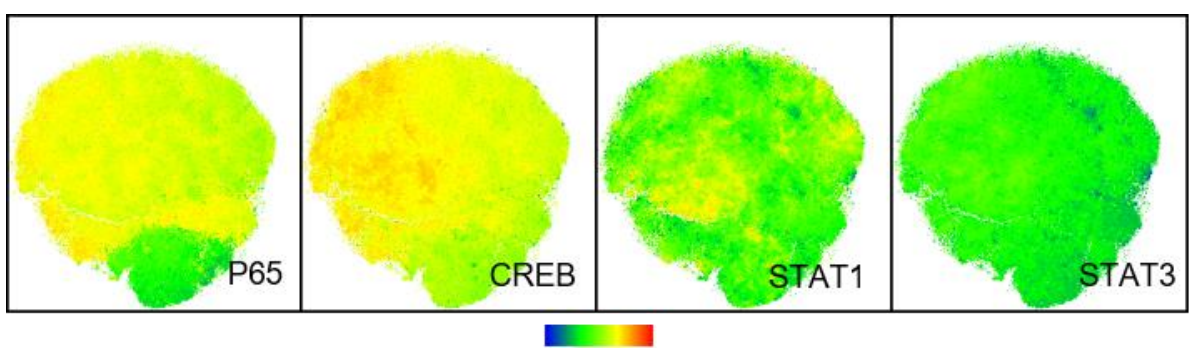

Figure 6-12 t-SNE plot of TF expression by monocytes for all participants and all conditions. 


\subsubsection{Resolving the loss of P65 in monocytes.}

Having observed a loss of P65 in our data, and with the t-SNE plot showing a grouping of low expressing events, we set out to investigate expression of this marker and the impact of our culture. We plotted the density distribution of monocytes at baseline and $\mathrm{T}_{3}$ to highlight the response differences between HC and MS participants (Figure 6-13A). The distribution differences at To between HC and MS speak to a difference in monocyte subset characteristics induced by disease processes. Given that basic phenotyping lineage markers were used to construct these plots, we suggest that more detail monocyte phenotyping may clarify MS specific monocyte changes. Furthermore, the differences between $\mathrm{HC}$ and MS plots persisted at T3, suggesting that culture and MIS416 both induce different responses in HC and MS participants.

In comparing the plots within a cohort, we observed an overlap of the distribution of events between T3 untreated and T3 MIS416 for both, suggesting a degree of similarity and that this time point was likely not optimal for MIS416 induced subset changes. We are further limited in our interpretation of the characteristics of these differences, as generally $\mathrm{TF}$ expression was low resulting in low resolution. However, P65 expression was a robust marker where we detected good signal. From this we plotted P65 as heatmap overlay by treatment condition to contrast its expression between HC and MS participants (Figure 6-13B). We observed a reduction in $\mathrm{P} 65$ following in vitro culture, that reflects the reduction detailed in Chapter 5 which we described as generalised reduction in TF expression, specifically in MS participants at T3. What the t-SNE plot clarifies is that the observed reduction is driven by the expansion of a subset of cells producing less active TF, and not a generalised reduction. To highlight this finding, we plotted only data from MS participants (Figure 6-13C), which demonstrated that this sub-population is distinguished by a distinct loss of CD14, as well as a loss of granularity i.e. internal complexity (Figure 6-12C).

These observations suggest that MS participant responses were distinct from those of $\mathrm{HC}$, and that at baseline there already existed disease specific phenotypic differences. We suggest that future work focussing on monocyte TF expression in MS could be valuable, but that highly detailed subset markers should be included to identify their nature. 
A

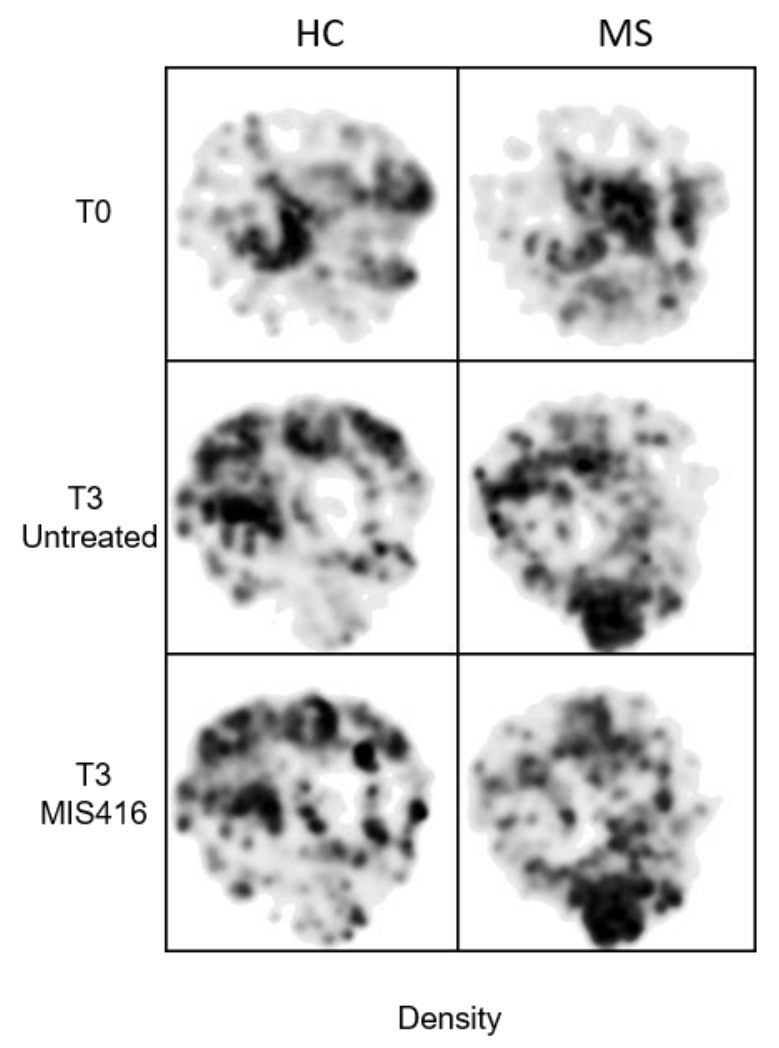

B

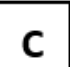

MS

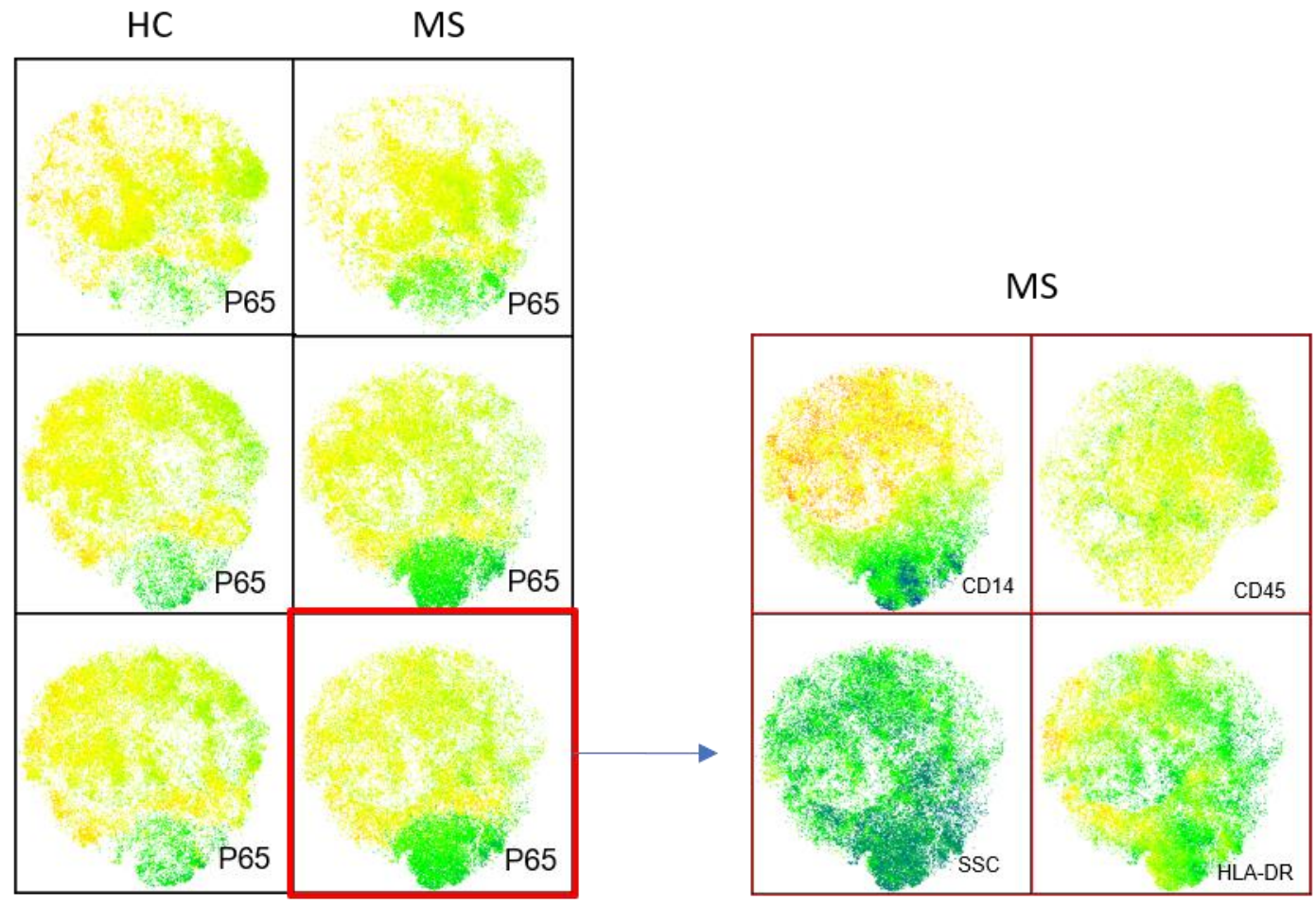

Figure 6-13 t-SNE analysis of TF expression in monocytes from all participants and all conditions.

(A) density overlay of events by treatment condition for HC and MS participants. (B) expression of P65 by HC and MS participants. (C) MS participant only profile of monocytes at T3 and MIS416 treated 


\subsubsection{Dopamine receptor expression by monocytes}

\subsubsection{1 t-SNE of monocytes}

We performed t-SNE analysis on gated monocytes to depict their expression of DR. We found this was best demonstrated by D1 expression for MS participants as depicted in Figure 6-14. In contrasting expression from baseline and single agent exposure we corroborated our findings as in our data from Chapter 4, where we showed a significant upregulation in D1 expression in culture and following clozapine treatment in monocytes while LPS was observed to reduced expression. A limitation of the tSNE analysis is that the individual participant expression data becomes lost, so we only see the group average changes. However, the analysis does indicate D1 expression in monocytes was not universal, that subsets of high and low expressing cells exists within a respective treatment condition. We observe for example that D1 reduction by LPS was not generalised, that some cells retain high levels, and that that there was an expansion of cells that show lowered D1.

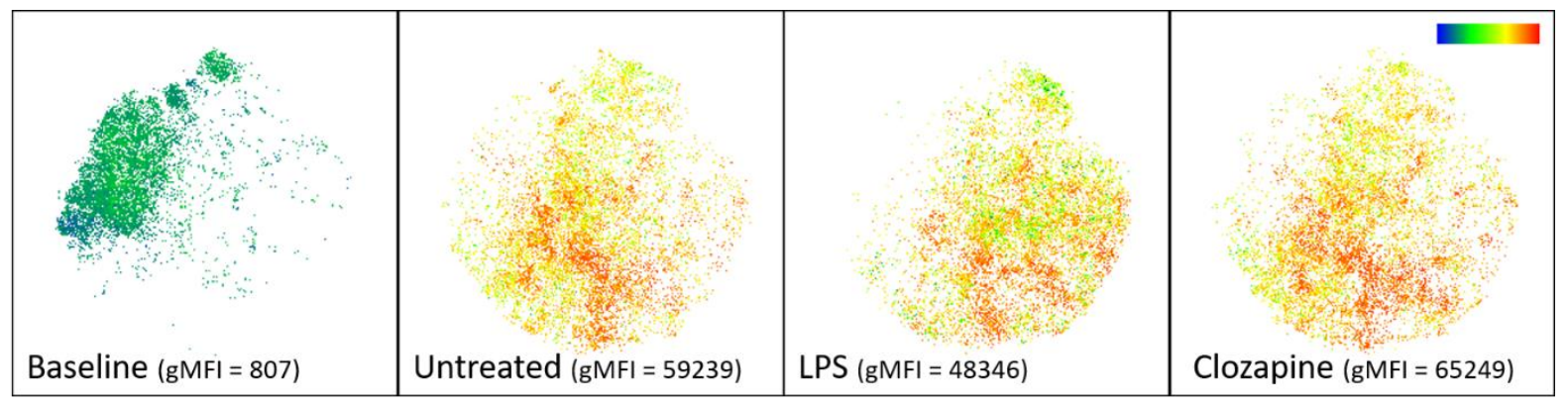

Figure 6-14 Heatmap overlay of Monocyte D1 expression in MS participants.

\subsubsection{SPADE of DR expression by monocytes.}

To establish if clustering analysis offered an insight into DR expression in monocytes, we performed SPADE analysis of the DR dataset. Figure 6-15 depicts this analysis and represents all data from all conditions and representative of all participants. The plot details the expression as gMFI of DR1,3 and 5. In it we observe a similar profile of expression for DR1 and DR3 in that the same clusters express the respective markers, while others do not. This suggests that these markers were relatively conserved in terms of their expression by different monocyte subsets. 
For DR5, we note generally dim expression of this marker over half the dataset and that high levels of DR5 expression were limited to only a minor subset of cells. We also observed that those cells expressing low DR1 and DR3 were expressing low levels of DR5. Unfortunately, as DR2 and DR4 data were acquired with the same fluorophore conjugate, their expression over the collated dataset could not be resolved in this analysis.

\subsubsection{Phenotypic diversity of in vitro cultured monocytes.}

Like t-SNE plots, SPADE trees are informed by the contribution from the respective treatment files. Figure 6-16 demonstrates, as an event frequency heatmap, the proportion of events that each treatment file contributed to the overall plot structure. A SPADE analysis sets out to over cluster, i.e. generate more nodes than there are subsets within the sample. By generating plots with many clusters, we can then interpret the similarity of nodes by their distance to the nearest neighbour. What Figure 6-16 demonstrates is that from baseline, monocytes in the periphery expanded significantly following culture and that the compartment contains a broad subset of cells that is both phenotypically and functionally distinct. This suggests the monocyte compartment for our model consists of subsets of cells at various stages of response and activation, that contract and expand reflexively to the environment. Lastly, the plots also show that co-culture with two agents (LPS\&cloz) generated the biggest diversity in terms of subset distribution. 

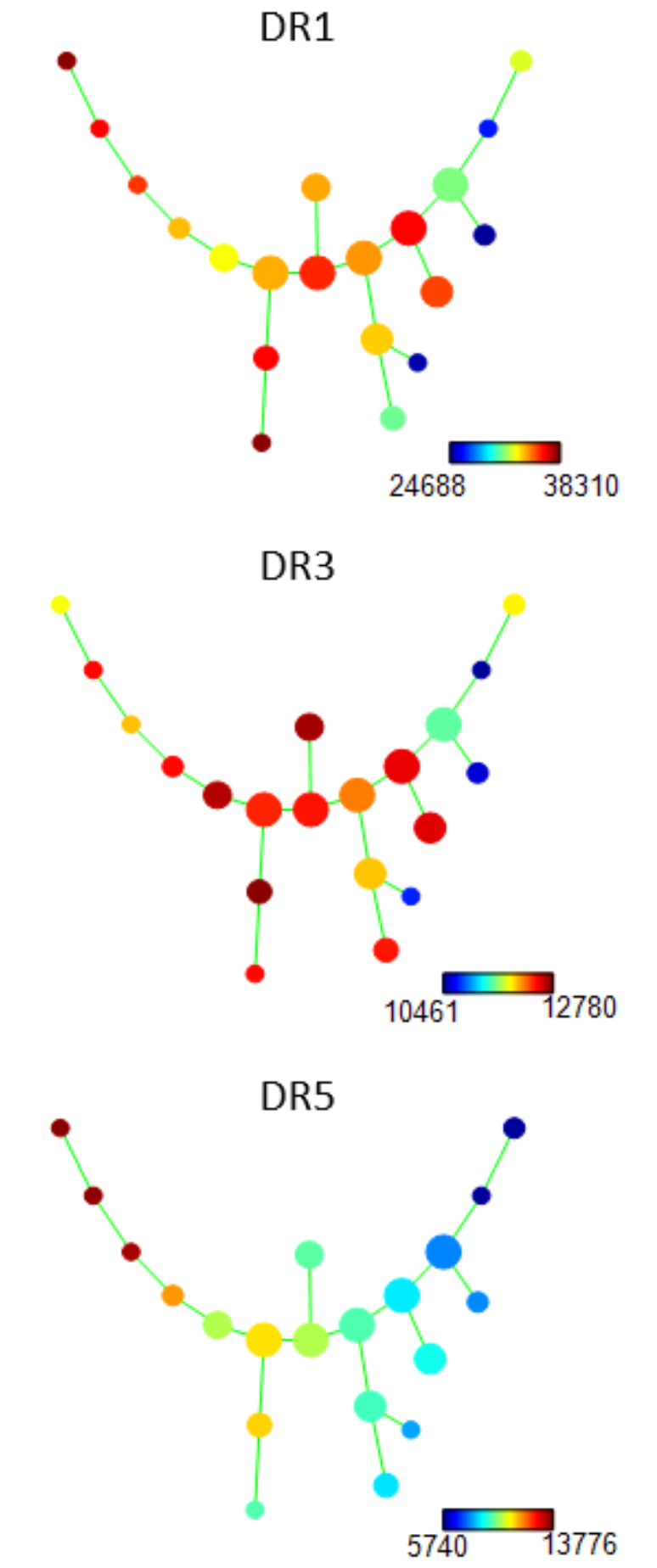

Figure 6-15 Clustering analysis of all in vitro monocytes and expression (gMFI) of DR1, DR3 and DR5.

Heat map ranges indicate $5^{\text {th }}$ and $95^{\text {th }}$ percentile of expression. 

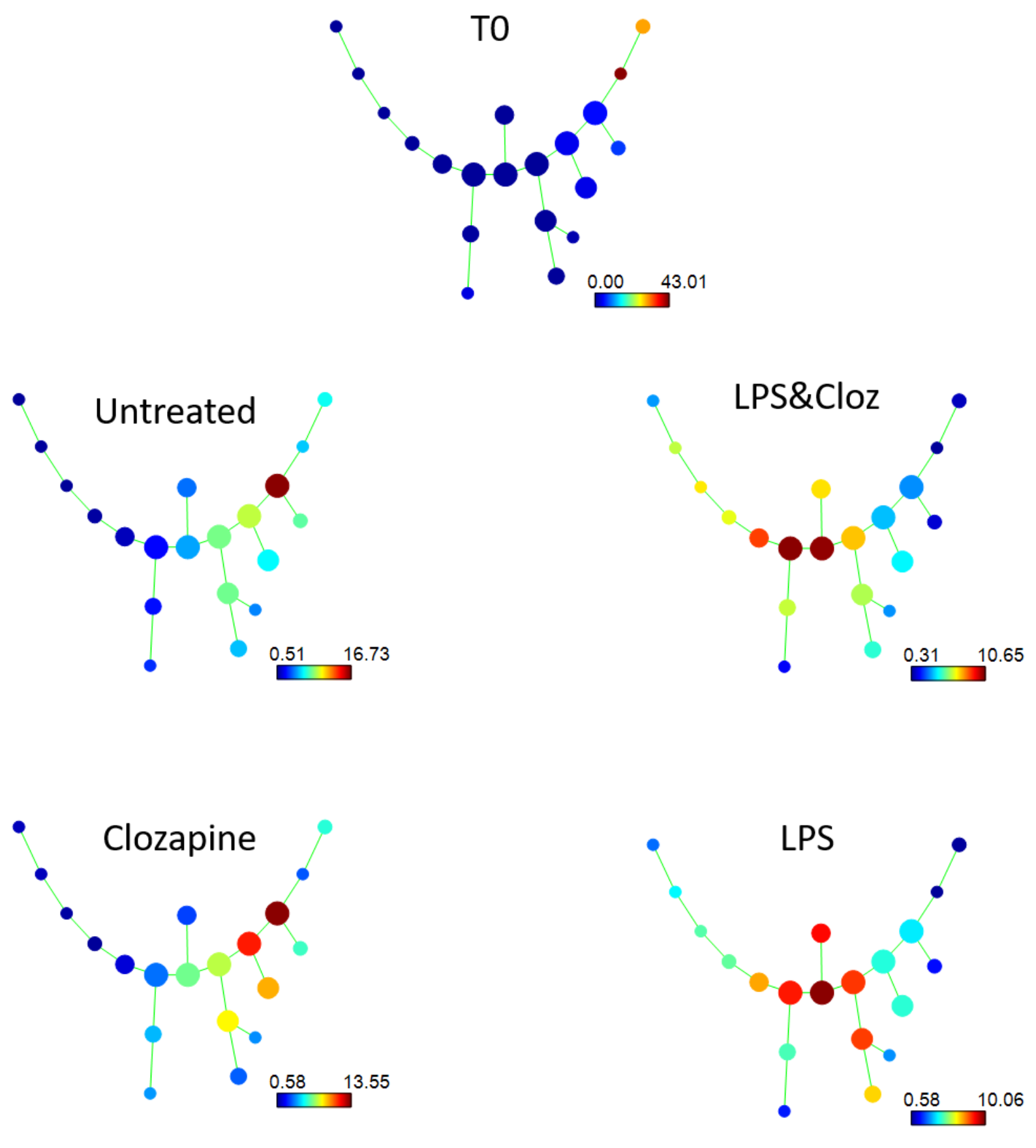

Figure 6-16 SAPDE analysis of monocyte diversity.

Data is depicted using the contribution from each treatment condition sample file to the overall structure. MS participant only data. Heatmap data indicate the proportional contribution (\%) of from each data file to the respective nodes. 
6.4.4.4 DR expression as a clustering analysis for MS participants.

We compared DR expression using SPADE in our MS cohort, for each respective treatment condition against the untreated control, by stipulating the untreated data as a reference file. This highlighted the effects of the various in vitro conditions on DR expression, as the generated plots show expression normalised to the untreated data (Figure 6-17). Class 1 DR, i.e. DR1 and DR5, showed increased expression in the presence of clozapine. We also note that LPS downregulated DR1 levels, while conversely, LPS induced an increase in DR5 expression. The generalised response to clozapine and LPS across most nodes suggests a uniformity in monocyte reactions to these agents respectively, especially for DR1 expression. The plots further show that co-culture with clozapine and LPS seemed to modestly increase DR5 expression, while for DR1 co-culture, that clozapine overcame the downregulation of expression induced by LPS alone in select subpopulation of monocytes.

Furthermore, class 2 DR expression, i.e. DR2-4, suggest a generalised downregulation of expression across all in vitro conditions. The uniformity of this response indicates a high degree of similarity in the regulation of class 2 expression within our model. While conventional subset averages found no change in class 2 expression from clozapine (see Chapter 4 ), we note that for all three markers there were a small subset of cells within the monocyte compartment that clearly upregulate expression. The plots also substantiate our findings that class 2 expression was significantly reduced in co-cultures of LPS \& clozapine. Interestingly, this seems to be primarily a response to LPS, as the clozapine responder nodes in single culture are absent, and LPS alone resulted in a general reduction of expression. 
Clozapine
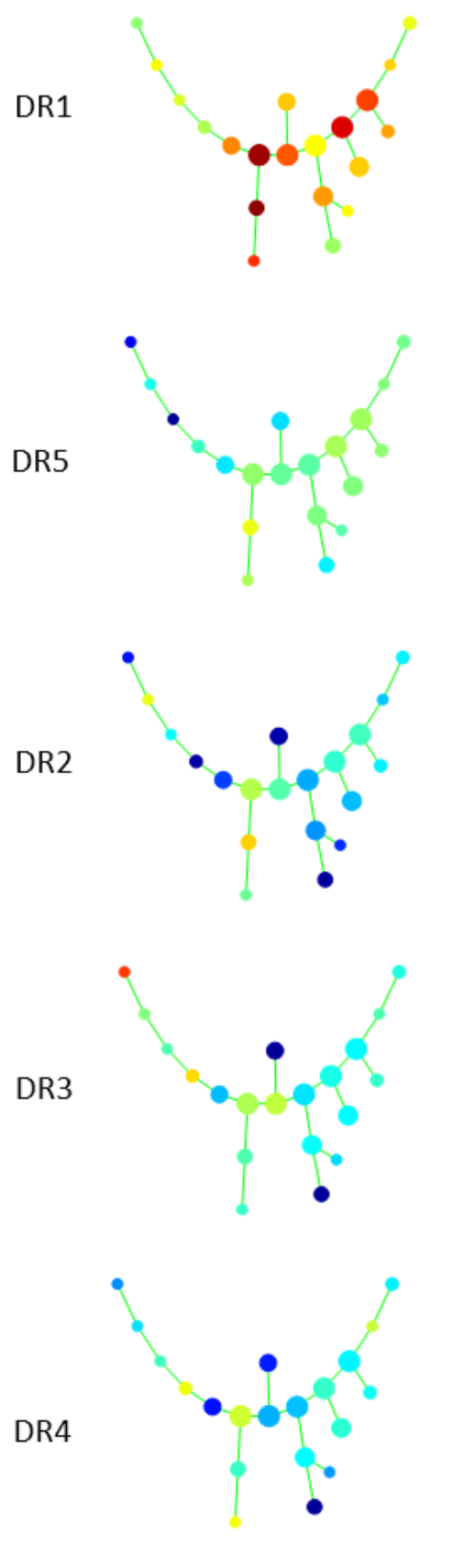

LPS
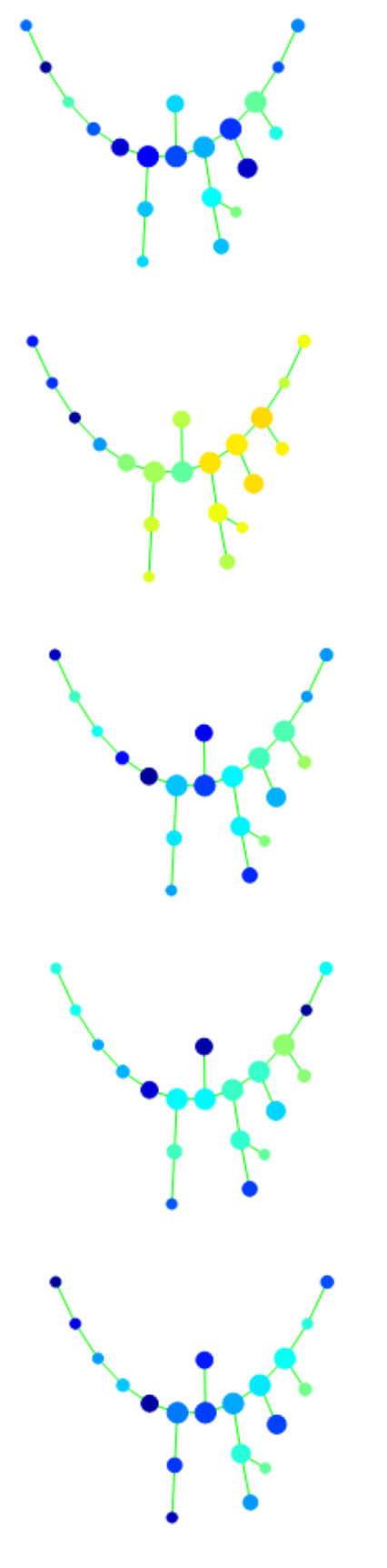

LPS\&Cloz
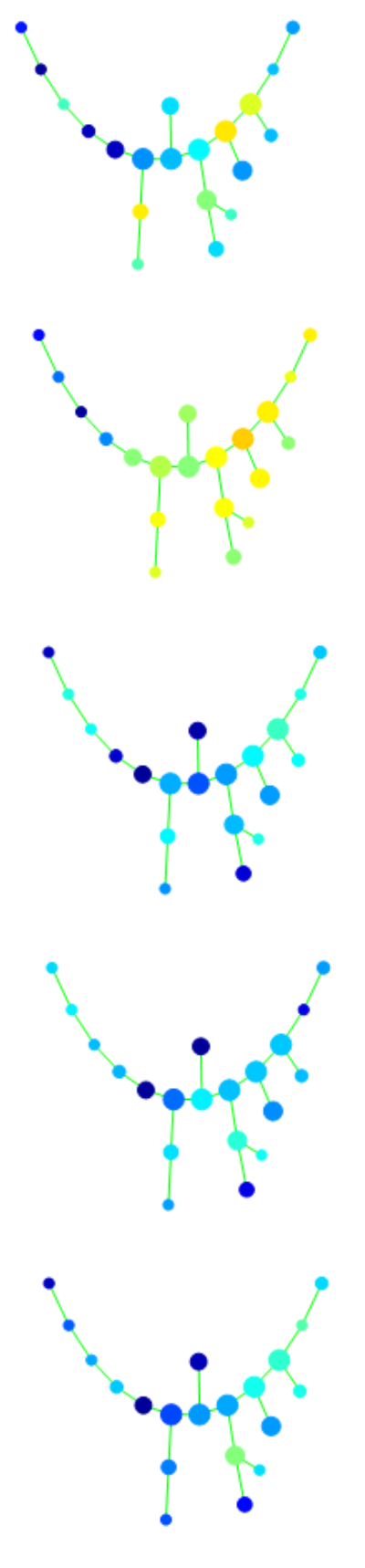

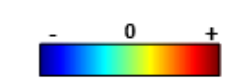

Figure 6-17 SPADE analysis depicting normalised DR expression in monocytes for MS participants.

Each row shows data normalised to untreated levels of expression for D1-5 respectively, with columns depicting data by treatment condition. Expression depicted as a heatmap relative to untreated levels, where light green indicates no change, lowered expression in blues, and increases in the red range. 
6.4.4.5 SPADE for the comparison of DR expression in HC and MS participants.

To compare the expression of DR from monocytes between HC and MS cohorts we reanalysed the dataset with standard SPADE but included an arcsine normalisation factor of 5 to make expression ranges comparable (Figure 6-18). We had previously observed no significant difference in the average expression of DR at baseline using conventional group statistics (Chapter 4), although a trend showed DR3 may be lower in MS. Figure 6-18 shows that MS participants appear to have reduced expression of DR3 at To, and that this loss was not specific to a subset of cells, but rather a generalised loss of expression. This, also in Figure 6-18, contrasts with DR1, which by SPADE analysis and conventional statistics showed a high degree of similarity in its expression between the cohorts.

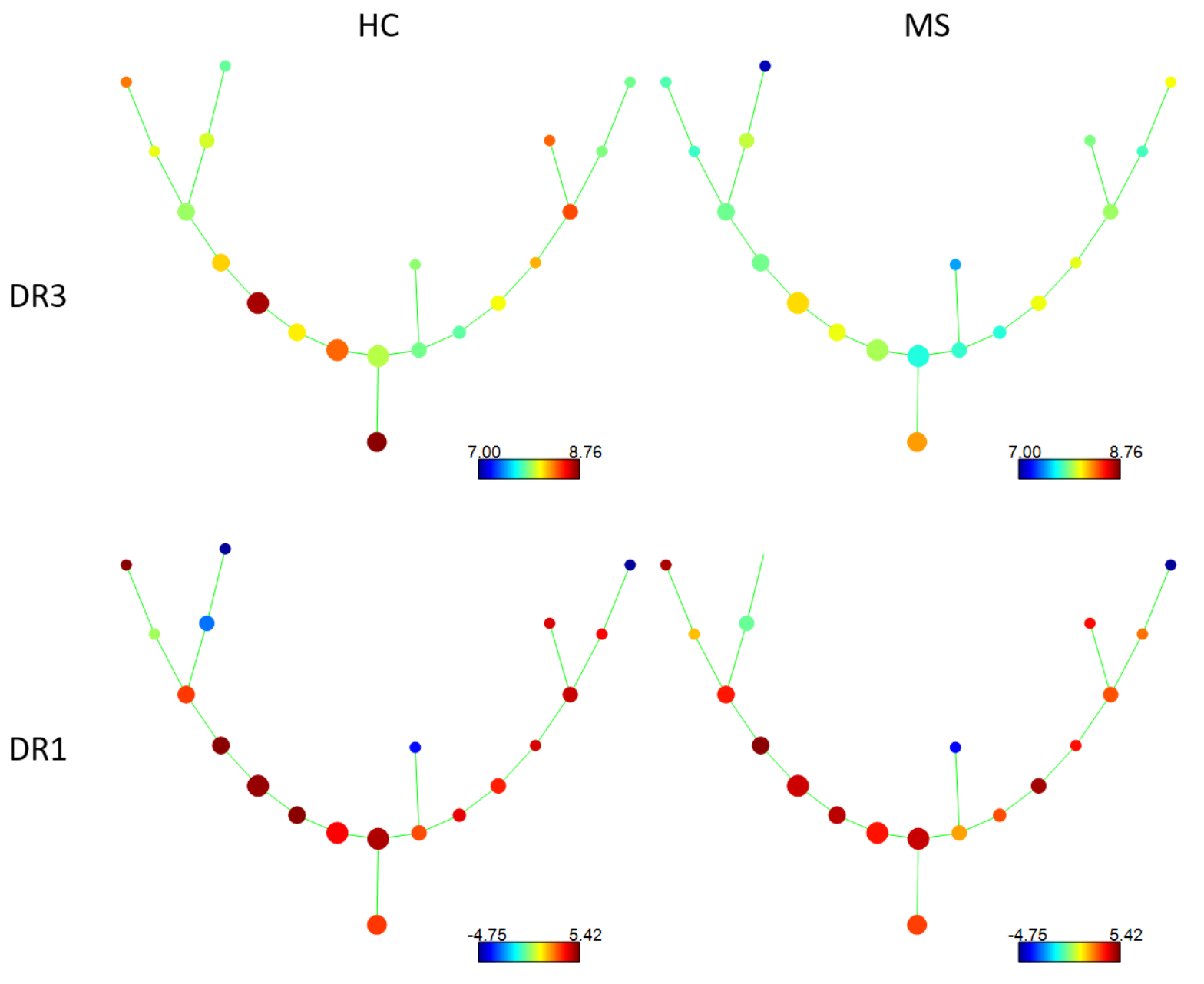

Figure 6-18 Inter-cohort comparison of DR expression for DR3 and DR5 at To.

Data depicts an arcsine normalisation of expression for each marker respectively. 
6.4.4.6 SPADE of t-SNE parameters for monocytes.

A SPADE analysis of the t-SNE generated, dimensionality-reduced data of DR expression by monocytes was performed and is depicted in Figure 6-19. This clustering output depicts the frequency of events within each node for the entire dataset. We observe that, irrespective of the differences noted in this chapter for in vitro culture and the impacts on cytokine and DR expression, monocytes remained a phenotypically similar collection of cells, represented by roughly $70 \%$ of events that comprise the core subset. We conclude this section with suggesting that the inclusion of additional monocyte-specific activation and phenotyping markers could likely better inform as to the heterogeneity of this subset. Here we were limited by the use of standard PBMC typing markers, that essentially designate monocytes as CD3-, HLA-DR+, CD14+ cells.

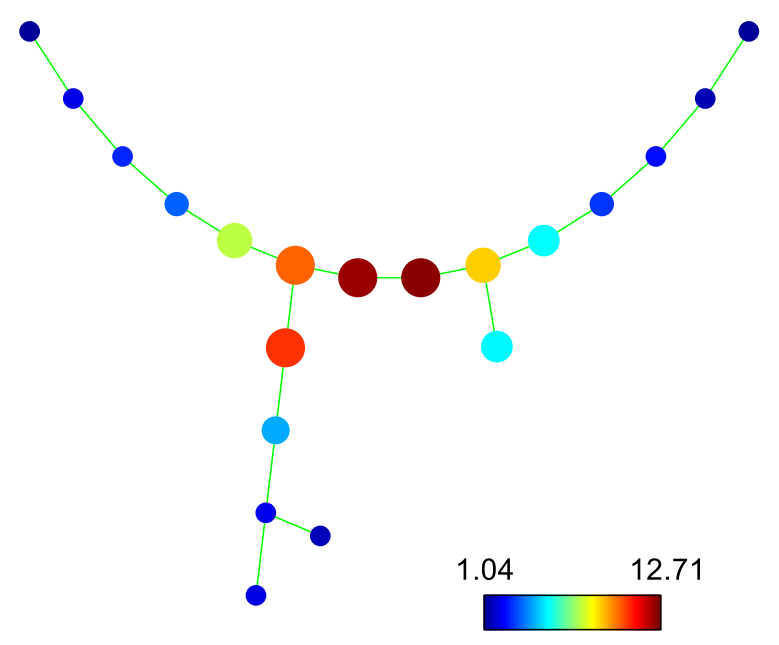

Figure 6-19 Density distribution SPADE analysis of $t-S N E$ parameters for monocytes. 


\subsubsection{Whole PBMC analysis by high dimensional cytometry tools.}

We caveat this next data section by emphasising that all our PBMC work was geared to inducing responses and optimally detecting monocytes. This limits detection in other subsets, particularly for TF, as expression of these marker are best detected under optimised stimulation and culture conditions. Our culture was however not insular, and the dynamics that arise from complex stimulation agents such as MIS416 and clozapine invariably have broad subset changing effects. The analysis of monocytes detailed in chapters 4 and 5 of this thesis, could therefore also be performed on all other cells of the PBMC. However, such detail was outside the limits of this thesis. To fully understand this complex dataset, we explored the other immune cell populations in our data, using multidimensional tools to concisely illustrate cytokine and DR expression by all of these subsets. Our aim here is to capture a bird's eye view of the immune interactions and determine the value of further in-depth analysis of the data.

\subsubsection{DR expression in the PBMC.}

We performed a t-SNE analysis to depict the PBMC as a dimensionality reduced sample. Figure 6-20A represents the analysis of all PBMC for all conditions. Using our gating strategy from Chapter 3, we then manually gated the major PBMC cell fractions and overlaid each subset onto the t-SNE plot (Figure 6-20B). From the gating we calculated the proportional composition of each subset as a fraction of all PBMC (Table 6-2). On average, $\mathrm{T}$ cells comprised over $60 \%$ of cells recorded in the samples, while monocytes, the primary data source for this thesis, were $12 \%$. This distribution was also reflected in the density distribution t-SNE (Figure 6-20A). 


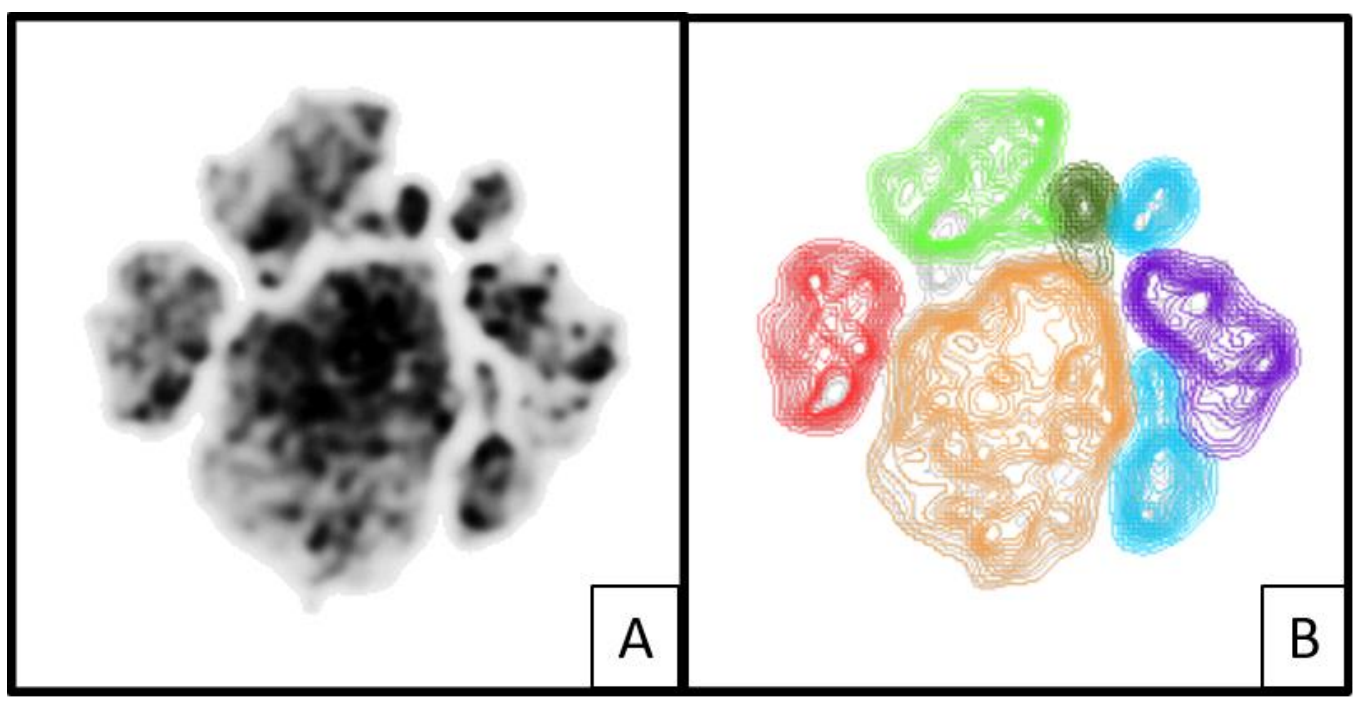

Figure 6-20 t-SNE of all PBMC data depicted as a density overlay of all PBMC (A) and displaying manually gated subsets (B).

Each subset is colour coded as outlined by Table 6-2.

Table 6-2 t-SNE overlay of the proportional distribution of the PBMC.

\begin{tabular}{|c|c|c|}
\hline Subset Name & $\begin{array}{c}\text { Frequency } \\
\text { of Total (\%) }\end{array}$ \\
\hline & B cells & 12.0 \\
\hline CD8+ T cells & 15.3 \\
\hline CD4+ T cells & 45.6 \\
\hline Monocytes & 12.1 \\
\hline NK cells & 9.36 \\
\hline Double negative T cells & 1.78 \\
\hline ALL PBMC & 100.0 \\
\hline
\end{tabular}

We next profiled the expression of DR across all cells of the PBMC using the same tSNE analysis. With this, we calculated the level of expression (gMFI) of DR1 - 5 for all PBMC and noted that all cells showed significantly increased expression compared to the average of FMO controls (Table 6-3). This indicates positive staining for all DR in all cells. 
Table 6-3 Expression (gMFI) of DR for all PBMC by treatment condition.

\begin{tabular}{|c|c|c|c|c|c|c|}
\hline & & D1 & D2 & D4 & D3 & D5 \\
\hline \multirow{2}{*}{$\underline{\text { Baseline }}$} & $\mathrm{HC}$ & 12513 & 5894 & 12735 & 5105 & 3735 \\
\hline & MS & 16334 & 7001 & 16303 & 5456 & 5955 \\
\hline \multirow{2}{*}{ Untreated } & $\mathrm{HC}$ & 11906 & 5844 & 12864 & 4966 & 4452 \\
\hline & MS & 17258 & 7171 & 17071 & 5716 & 6943 \\
\hline \multirow{2}{*}{ Clozapine } & $\mathrm{HC}$ & 12416 & 5758 & 12794 & 4789 & 4663 \\
\hline & MS & 17317 & 7227 & 17483 & 5687 & 7419 \\
\hline \multirow{2}{*}{$\underline{\text { LPS }}$} & $\mathrm{HC}$ & 12119 & 5807 & 12774 & 4946 & 4559 \\
\hline & MS & 16325 & 7118 & 17029 & 5677 & 6994 \\
\hline \multirow{2}{*}{ LPS\&cloz } & $\mathrm{HC}$ & 11893 & 5784 & 13212 & 4936 & 4748 \\
\hline & MS & 16741 & 7083 & 17297 & 5666 & 7462 \\
\hline FMO & ALL & 146 & 79 & 60 & 181 & 136 \\
\hline
\end{tabular}

Having included a numerical keyword in the analysis allowed for a visualisation of events by specific treatment condition and cohort and this aids in validating the use of the plots as data interpretation tools. Using this, we demonstrate in Figure 6-21 the loss of DR1 expression in monocytes stimulated with LPS as compared to untreated cells. This corroborates with the same finding in Chapter 4. Furthermore, it appears as though this effect was subset specific, as no other subset reduction was observed. This was not surprising given that LPS is a specific TLR4 agonist and monocytes were the predominant innate subsets in the PBMC that express this PRR (i.e. TLR) (see Chapter 1).

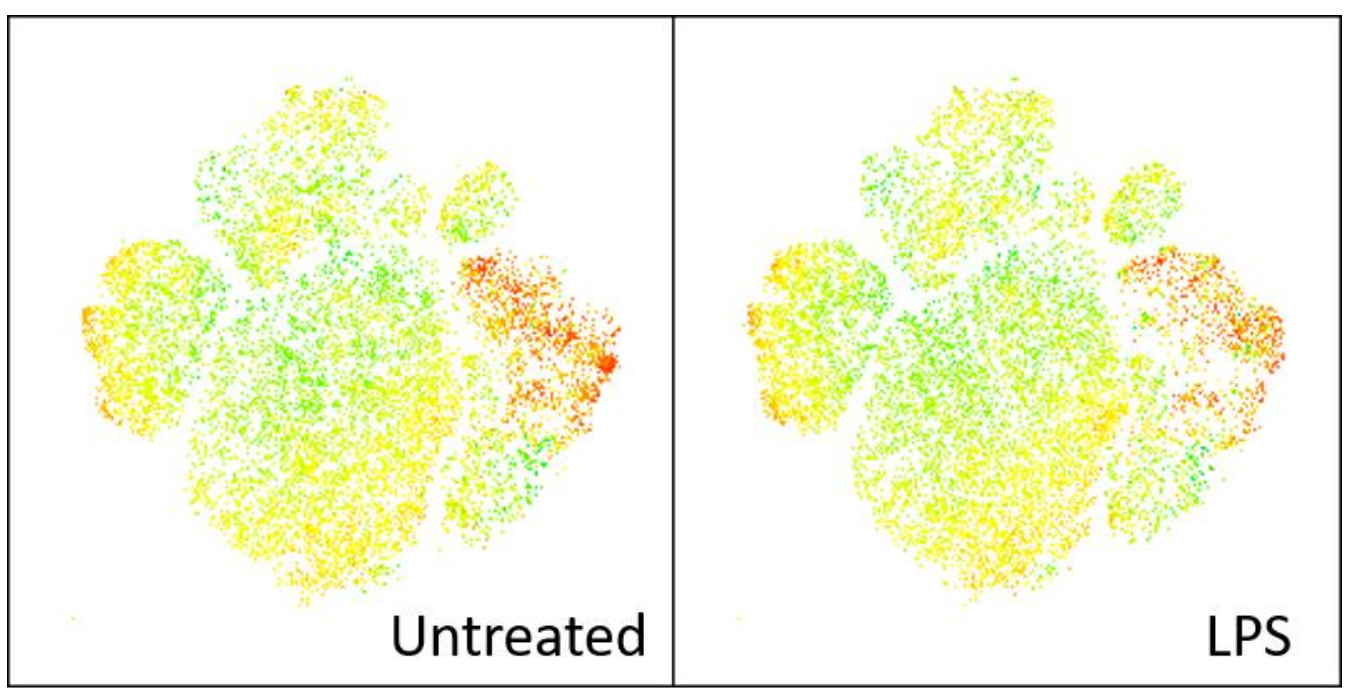

Figure 6-21 t-SNE plot of PBMC from MS participants and the effects on DR1 expression in untreated and LPS cultures respectively. 


\subsubsection{2 t-SNE of the PBMC expression of DR.}

To depict expression of DR1 - 5 respectively across the PBMC we required two new $\mathrm{t}$ SNE plots (Figure 6-21). During our data acquisition we stained for D1, D3 and D5 together (Stain 1), and D3 and D4 together (Stain 2) while using overlapping lineage markers. This requires the use of separate t-SNE analysis to avoid the introduction of background noise from 'empty' channels into the plots. From this we observed that both plots resulted in a similar subset distribution across the two t-SNE dimensions. We note a distinct clustering of the major immune subsets with T cells in the majority. We then plotted, as heatmap overlays, expression of DR within each stain. This depicts a representative outlay of DR expression across all events (Figure 6-22).

In analysing the plots, we note that comparisons between the plots are not accurate as they are not normalised to an expression control. This would require the inclusion of a sample of known DR expression density. D2 and D4 were however detected with the same fluorophore (AF488). While we cannot exclude affinity and emission differences it appears that D4 may be more abundant than D2, but this would need to be verified. Even though we cannot compare expression between the DR plots we can compare the range of expression within a subset for each DR marker respectively. This is can be observed particularly in monocytes, which show a broad variation in expression that results from in vitro culture.

A new insight from the t-SNE plots over conventional analyses is that DR expression was detected across all cells of the PBMC. We note that D1-3 expression appeared generally homogenous across the majority of PBMC subsets (with the exclusion of monocytes), but that a subpopulation of DR bright B cells were present that may warrant further investigation. Expression of D4 also appears to be bimodal for most subsets. The observations from D4 expression and for B cells may be due to an effect induced by our in vitro culture conditions and is an insight that may be explored in future work. 
Stain 1

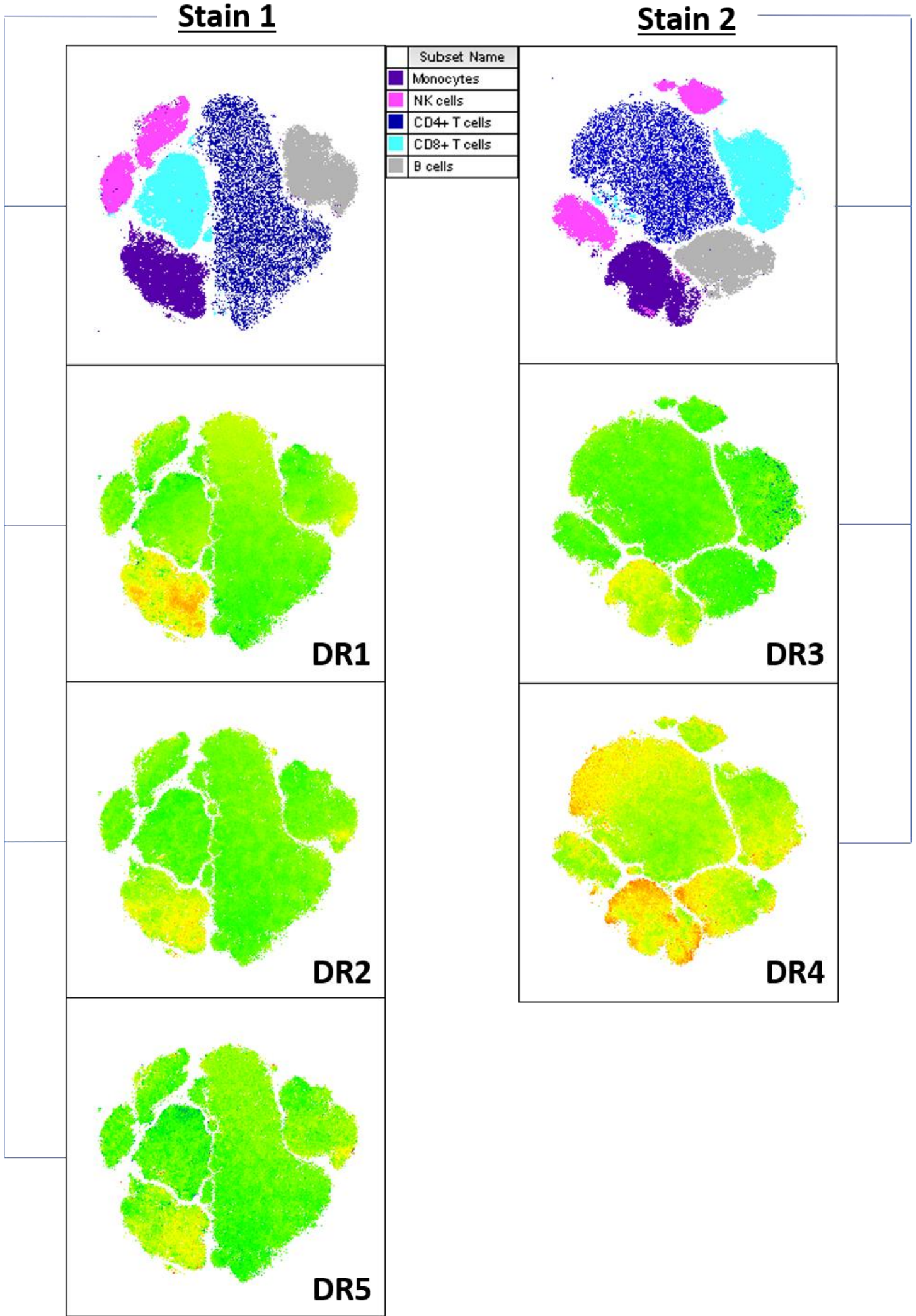

Figure 6-22 t-SNE plots of DR expression by the PBMC.

Each stain depicts a separately acquired cytometry dataset with overlapping lineage markers. The top row depicts manually gated lineage subsets overlays for each stain. DR expression is depicted as a heatmap overlay of expression for each stain. 


\subsubsection{Cytokine expression in the PBMC.}

We compiled a t-SNE analysis on the dataset for all in vitro cytokines (Figure 6-22). This aided in the visualisation of cytokine expression across all cell types and represent the average cytokine production capacity of the PBMC in our model. The generated plot yielded a distinct clustering of the major immune subsets over the two parameters. The depicted heatmap overlays of cytokine expression was constructed by manually adjusted the scale of each cytokine such that negative events reflected the average level of expression from the FMO control. This allows for a better visualisation of the average expression across a subset. We also list the average level of expression from the t-SNE gated subsets in Table 6-4.

From this visualisation, we observed most cells had cytokine producing capacity. Monocytes were the major cytokine-producing subset, which is not surprising given they were the primary target of the in vitro stimulations. Generally, we note that for CD8 T cells, there was a large proportion of non-responder cells. This was anticipated given the absence of a T-cell-specific ligand. Interestingly, we found modest induction of IL-1 $\beta$ and IL-6 in some CD4 T cells, B cells and some NK cells. These cells were not specifically targeted, and induction was likely due to indirect or by-stander activation during the culture conditions. This cytokine production may also be due to the presence of debris from dead cells such as DNA in the culture and would have resulted in TLR signalling induction. We suggest this explains the modest pro-inflammatory response observed in from our model. Furthermore, we observed very low levels of INFY and IL-10 across all subsets. The lack of resolution for these analyses is likely due to dim expression and technical limitations from using dim conjugates for these two cytokines. 


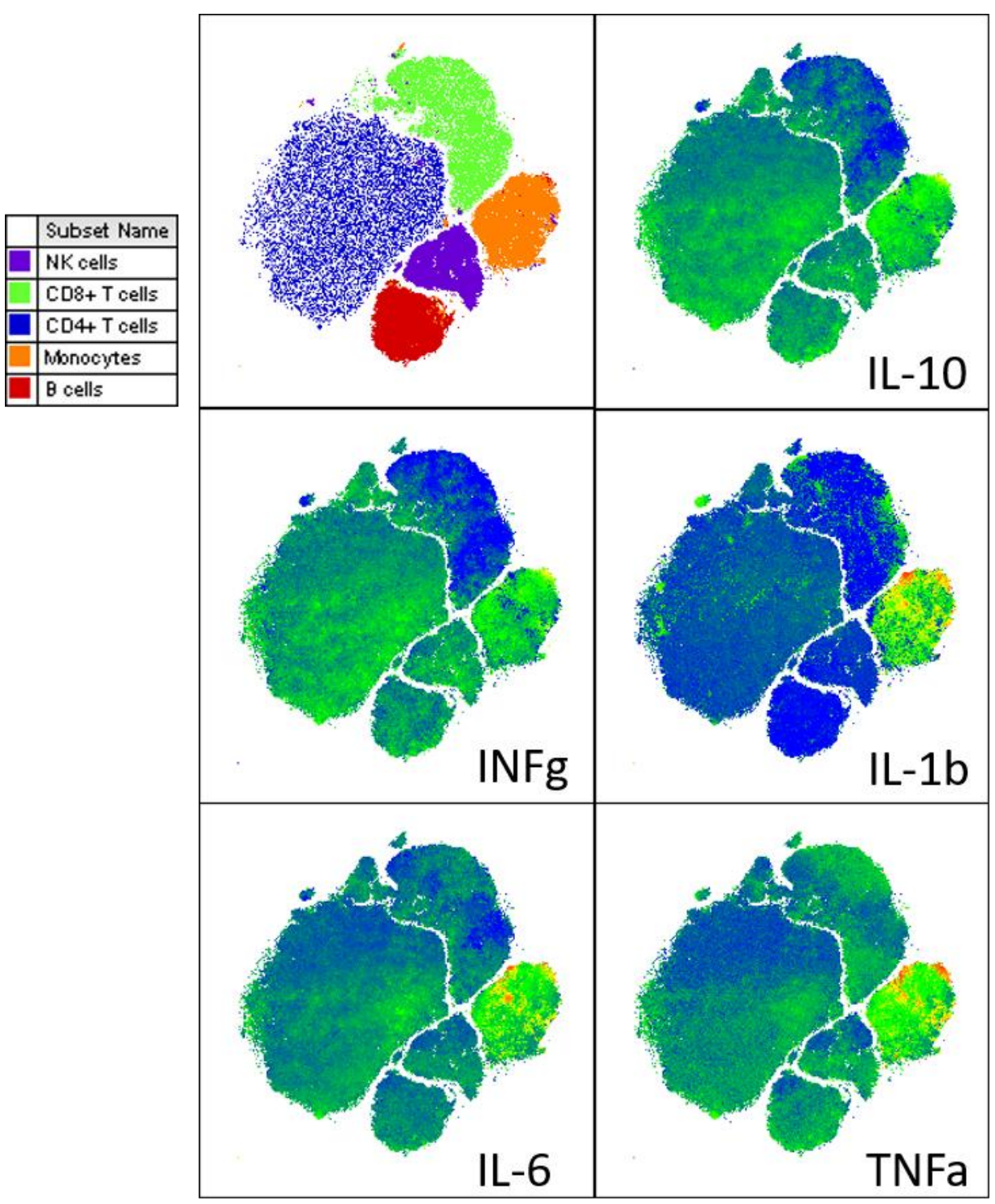

Figure 6-23 t-SNE analysis of cytokine expression by the PBMC.

The first plot depicts an overlay of manually gated immune cell subsets (top left). Other images show heatmap overlays of individual cytokine expression for pooled data from all in vitro conditions overlaid onto the t-SNE plot.

Table 6-4 Heatmap of average expression (gMFI) of cytokines for gated immune subsets from all events.

\begin{tabular}{|c|c|c|c|c|c|c|c|c|c|c|}
\hline & FMO & B cells & FMO & CD8 T cells & FMO & Monocytes & FMO & CD4 T cells & FMO & NK cells \\
\hline INFg & 40,8 & 1153 & -422 & $-96,8$ & -682 & 1899 & 377 & 1374 & 216 & 1307 \\
\hline IL-1b & -330 & $-41,9$ & 189 & 308 & -300 & 6340 & 485 & 625 & 216 & 1307 \\
\hline TNFa & 669 & 4150 & 1047 & 3503 & 317 & 22301 & 604 & 2661 & 707 & 3238 \\
\hline IL-10 & 181 & 1346 & -151 & 338 & -551 & 2246 & 273 & 1358 & 197 & 1212 \\
\hline IL-6 & $-10,5$ & 2212 & -255 & 1373 & -344 & 12058 & 53,7 & 2090 & $-43,9$ & 1867 \\
\hline
\end{tabular}


We can conclude that cytokine expression in this data set may be limited in resolution such that interpretation of expression changes in other subsets may be more challenging than the clear changes observed in monocytes. For IL-1 $\beta$ specifically it appears as though most cells, excluding monocytes, made very little. Other markers did however show positive signal, and we can suggest that this expression indicates that $\mathrm{T}$ cells may be a valuable source of in vitro response data. The broad cytokine induction seen in the PBMC in our models suggests that by making alterations to our in vitro culture system, that the effects of clozapine or MIS416 could also be modelled in other subsets.

\subsubsection{Effect of clozapine on TNFa expression.}

The use of t-SNE on whole PBMC was able to replicate some of our findings from results in Chapter 4. There, the most prominent effect of clozapine therapy in in vitro treated MS participants was on the expression of TNFa. This was due to the higher basal level of this cytokine being present in untreated MS participants, and we demonstrated the drugs ability to lower TNFa production. Here we depict those findings with the use of t-SNE plots. In untreated MS participants we observe a population of monocytes actively expressing TNFa (arrows). These cells are absent in the clozapine treated sample, and the average gMFI of the monocyte subset in this tSNE plot dropped from 12895 to 10308, suggesting either their absence, or a downregulation and redistribution during clozapine treatment. Furthermore, the tSNE shows this effect was only in monocytes, as the general level of expression of TNFa across other subsets remained unchanged (Table 6-5). 
Table 6-5 Effects of clozapine on

TNFa expression by subset.

\begin{tabular}{|l|l|r|}
\hline \multirow{2}{*}{ Subset } & Treatment & \multicolumn{1}{l|}{$\begin{array}{l}\text { TNF } \boldsymbol{~} \\
\text { (gMFI) }\end{array}$} \\
\hline \multirow{2}{*}{ B cells } & Untreated MS & 4048 \\
\cline { 2 - 3 } & Cloz MS & 3873 \\
\hline \multirow{2}{*}{$\begin{array}{l}\text { CD8+ T } \\
\text { cells }\end{array}$} & Untreated MS & 4387 \\
\cline { 2 - 3 } Monocytes & Cloz MS & 4265 \\
\cline { 2 - 3 } & Untreated MS & 12077 \\
\cline { 2 - 3 } & Cloz MS & $* 10636$ \\
\hline \multirow{2}{*}{ CD4 T cells } & Untreated MS & 3405 \\
\cline { 2 - 3 } & Cloz MS & 3351 \\
\hline \multirow{2}{*}{ NK cells } & Untreated MS & 3608 \\
\cline { 2 - 3 } & Cloz MS & 3547 \\
\hline
\end{tabular}

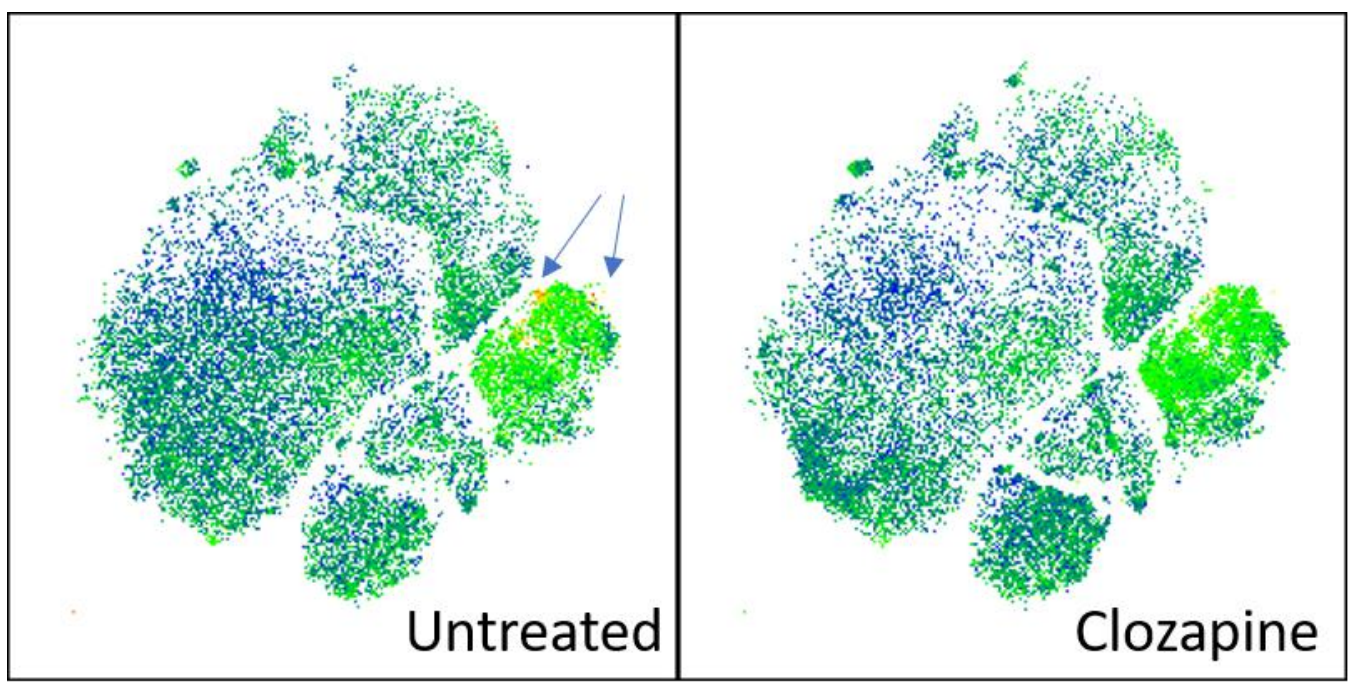

Figure 6-24 Heatmap of TNFa expression in the PBMC of MS participants by t-SNE analysis.

Arrows indicate high expression by monocytes.

\subsubsection{The effects of MIS416 on TNFa expression.}

In Chapter 5 we showed the effects of MIS416 treatment on monocytes in vitro. Using the t-SNE analysis, we can see that the tools emulate those observations by using TNFa expression as an example. TNFa was significantly upregulated in MS participants over HC in untreated cells (Figure 6-24). MIS416 further induced TNFa expression in monocyte, but this induction was significantly stronger in MS participants which also had an already increased basal level of TNFa induction (Figure 6-25). From our plot we further observe that TNFa induction was not just limited to the monocyte 
compartment. Here we observe a modest increase in expression in B cells, NK cells as well as CD4 $\mathrm{T}$ cells and note this effect far less prominent in $\mathrm{HC}$ cohort.

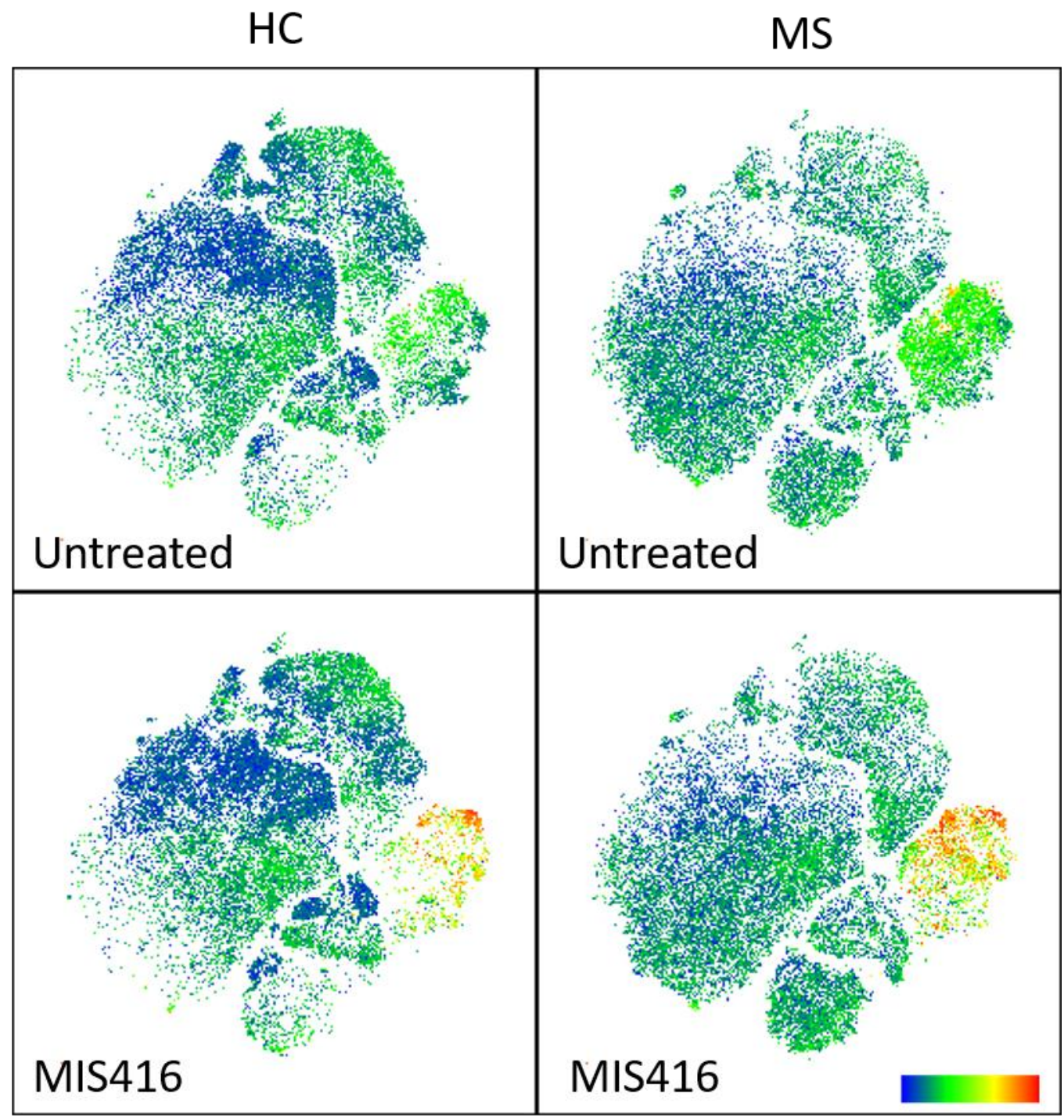

Figure 6-25 t-SNE plot of TNFa expression in untreated and MIS416 treated PBMC. 


\subsection{Discussion.}

The aim of this chapter was to create an overview of a cytometry dataset of PBMC samples, acquired to profile monocyte phenotype and function. To establish the value of the untapped dataset and explore future analysis directions we performed a series of dimensionality reduction and clustering analyses to give a broad overview of its contents. We also set out to establish if the tools could provide further insight into the responses observed in monocyte phenotype and function from earlier analyses. The results showed that monocytes in our model were a highly heterogenous population, and that these cells have distinct response differentials in MS pathology. Furthermore, we have shown substantive changes to the PBMC in MS participants in terms of phenotype and function and added to the insights gleaned from our conventional statistical approaches. These findings do not warrant discussion as they only serve as a prelude to future work. Here we discuss the limitations and the merits and review the use of high dimensional tools in cytometry to substantiate their utility in analysing cytometry datasets.

Sequential gating has formed and integral part of the analysis tools for this thesis. Unfortunately, this method is very limiting, being unable to visualise the data as a whole and only displays at most three parameters. The subjective nature of the method also may result in the loss of information and is highly time consuming. There exists a broad range of complementary tools that have aimed to overcome these limitations. Inherently these tools are considered exploratory, and as demonstrated by this thesis, any findings must be substantiated by conventional analyses or be confirmed by targeted testing.

\subsection{1 t-SNE.}

t-SNE processing is well established as a tool that facilitates the identification of immune subpopulations (Mair et al., 2015). t-SNE is an unsupervised bioinformatics method resulting in a display of the data in only two dimensions. A t-SNE map contains all events with the proximity of events directly related to their relationship in multidimensional space. While data are resolved to single event level, datapoints may 
overlap, so the effect of excessive input can result in obscured data. Unlike SPADE, user defined clusters are not an input parameter. This significantly increases the computation cost required for each run. To limit data overlap, and reduce computing requirements, it has been suggested that t-SNE analysis data input remains below 200,000 events (Ashhurst, 2017). t-SNE is however not a clustering tool. While clusters may present, they are not assigned, and subsequent processing by gating or another tools such as SPADE is required to group events into clusters.

In creating t-SNE plots on already gated monocytes we limited a portion of the utility of the application. Within the PBMC sample there would be many heterogeneous cell subsets that a t-SNE could depict as individual compartments. What we found when we performed t-SNE on gated monocytes was that their common expression patterns resulted in a very homogenous 'pancake' shaped plot with few to no distinct areas, which was likely because these cells were already selected for this phenotype. The reason for implementing a pre-gating strategy was that that it allowed for the analysis of distinct treatment subpopulations to show the differences that culture conditions have on the monocyte population. How for example stimulation with LPS dramatically changed the nature, size and expression of protein by monocytes such that they occupy a distinctly different area of the overall map. Furthermore, the very high number of events from our larger dataset required measures to limit the total input events, as running t-SNE on files with event numbers exceeding 200,000 would result in the overlapping of events that can obscure the plot. t-SNE plots also cannot stratify populations that are conventionally identified by hierarchical bifurcate gates (Eshghi et al., 2019). While this largely applies to continuous maker expression such as CD45RA by T cells, it was a limitation worth consideration.

One way to overcome the limitations of t-SNE was by using a subsequent clustering analysis by SPADE on the t-SNE generated files (Anchang et al., 2016). This method used the two new t-SNE parameters as the basis for clustering, resulting in a clustering of dimensionality reduced data. We found this to be a highly effective strategy that limited the total data input and processing time. 


\subsubsection{SPADE.}

Cluster analysis was one of the early complementary tools to arise. Cluster analysis facilitates data visualisation using coloured heat maps (Eisen et al., 1998). The analysis clusters variables, such as protein markers, in an unsupervised bioinformatics approach which treats all variables as equal, aiming to group events by similarity (Bashashati and Brinkman, 2009). This approach has longstanding use in flow cytometry research (Kitsos et al., 2007; Murphy, 1985; Wilkins et al., 2001).

SPADE is one such form of cluster analysis (Qiu et al., 2011). SPADE projects the data as a tree structure that reflects all dimensions. It has been successfully used in clinical haematological research with seminal work detailing the healthy human bone marrow continuum to a level of detail that reflects the current understanding of haematopoiesis (Bendall et al., 2011). SPADE was also validated against expert based gating approaches, with consistent results (Qiu et al., 2011)

The major limitation of SPADE is that it is not a stand-alone tool, but a complementary one. As a computational algorism, the time to calculate is also heavily influenced by the number of events in the sample, with large input event numbers significantly extending computation time. Built into the tool is a density depended downsampling step to limit the total number of events and make analysis of larger files reasonable. Downsampling randomly retains a more limited number of events from high density populations, while rare populations are not affected. Fortunately, the SPADE3 algorithm is not stochastic, such that repeat analysis result in reproducible plots. This is an inherent feature that is achieved by the software identifying the event with the highest local density (i.e high number of cells within its neighbourhood) as the clustering start point (Qiu, 2017).

SPADE analysis has added substantially to our data interpretation. Specifically, in understanding monocyte subset heterogeneity. One of the key limitations in our approach was the use of lineage gating to terminal monocytes that formed the starting sample for some of our SPADE analysis. This may introduce bias in that it subverts the utility of the tool in clustering monocytes independently. However, Qui et al. have shown that SPADE clustering and conventional gating resulting in greater than $95 \%$ 
concordance in calling terminal monocytes (Qiu et al., 2011), with similar findings by others (Mukherjee et al., 2015). We implemented terminal gating to limit the initial input data, as our dataset consisted of over 350 files, many in excess of 200,000 events. Ultimately, we found that the overlap of SPADE data findings with our conventional analysis approaches supported this choice. 


\subsection{Conclusion.}

The use of sequential gating and subset analysis remain the standard method with which cytometry data is analysed. However, this approach is subjective and very time consuming. The complexity of larger multicolour panels, coupled with a range of treatment conditions, participants, and culture results in potentially millions of permutations that would need to be analysed. We have shown here that the findings from our conventional analysis approach was present and evident with the use of high dimensional bioinformatics tools validating this complementary data handling approach. We also set out to establish if there were other observations in the dataset that were overlooked using only standard cytometry analysis and found extensive monocytes subset heterogeneity. We further went on to show the remaining value of the dataset, gleaning new insights and demonstrating the value of preforming additional analysis of the PBMC. Our work in this chapter has only started to explore the breadth of the dataset, with new avenues of analysis and research opened, facilitating future directions from this work. 


\section{Chapter 7. Summary and future directions}

\subsection{Introduction.}

This thesis has examined the phenotype and function of monocytes, by using ex vivo peripheral blood cells to identify if and how progressive MS alters monocytes. The peripheral immune system is highly polymorphic, and peripheral blood as a sample offers a good overview of the state of the immune system at the time of collection. This analysis has allowed us the opportunity to gain valuable insight but is limited by the individual heterogeneity of this population, which complicated the data interpretation. Flow cytometry was the main tool used for our data collection, and it allowed us to resolve the complexity of the peripheral immune system by reducing the data to the single cell level and using each cell to build an understanding of the whole network of cells.

Understanding the function of monocytes in this immune network was the foundation for this work. As a basis, we set out to understand single cell responses, so we undertook a broad review of the immune system and its cellular components in Chapter 1. There, we outlined two arms of the immune response: the innate and the adaptive systems. Substantial contributions have been made by others to describe the adaptive biology of MS. As a result of this, our research direction pivoted to the innate arm, so that we could offer novel insights into MS pathology. To this end, we targeted monocytes through their innate and other surface receptors to model their responses. Initially, we sought to determine if there were detectable changes in monocyte numbers and phenotype in the periphery. Chapter 2 showed that these cells, unlike most others, appeared to be unaltered in progressive MS. This led us to examining what functional changes were present, with data from Chapter 4 and Chapter 5 showing monocyte responses in terms of cytokine and transcription factor expression, and we contextualised monocytes by profiling their dopamine receptor expression, to show their key role in neuroimmune signalling. Finally, in Chapter 6, we examined 
the heterogeneity of the monocyte compartment with high dimensional tools, to demonstrate the depth of this immune cell subset and how the compartment shows differential responses to different therapeutic agents. We concluded this work by reintroducing these cells into the immune network, with an analysis of all mononuclear cells, to contextualise their responses and to show the bystander effects that occur when monocytes respond to stimulation.

To reiterate the key findings of this thesis, we summarise here our observations from each data chapter.

\subsection{Chapter 2. Immune phenotyping in progressive MS.}

In Chapter 2, we investigated the whole blood phenotype of people with progressive MS and compared their data to those of a healthy control group. We demonstrated substantive changes in the absolute number and proportion of nearly all types of immune cells in people with MS. Not surprising, T cells were highly altered as these adaptive cells are known to be key drivers of MS pathology and are readily profiled by standard cytometry methods. Our data showed strong parallels to work by others, with a contraction of $\mathrm{CD} 8^{+} \mathrm{T}$ cells resulting in an altered $\mathrm{CD} 4^{+}$and $\mathrm{CD} 8^{+}$ratio in the periphery. Our B-cell findings also reflected established work, as we observed a contraction in naïve and memory subsets, and an expansion in the transitional compartment. We further showed a reduction in pDC cell proportions in the periphery, which has previously been described in progressive patients. We feel these parallels validated our approach and added weight to those findings where we diverged with those in the literature.

Surprisingly, our whole blood analysis showed no significant changes to the phenotype of monocytes in our MS cohort. Previous work in our lab had shown that the proportion of $\mathrm{CD}_{16}{ }^{+}$monocytes as a proportion of PBMC increased in a cohort of untreated MS participants comprising primarily relapsing-remitting patients, while other groups had similarly shown that the composition of subsets of monocytes may be altered in MS, noting that an increase in non-classical cells occurs with an associated reduction in classical cells. The absence of any change in our monocyte 
data may have been due to our MS cohort comprising solely progressive patients, yet we noted several of our findings in other immune subsets reflect those observed in relapsing disease. This finding led to the question of whether monocytes may instead differ in their functional capacity in progressive disease. To test this question, we expanded our hypotheses, and sought to define the function and response profiles of monocytes in progressive MS. We tested these hypotheses using an in vitro model, using PBMC as sample source, and with a new spectral flow cytometry method outlined in Chapter 3.

\subsection{Chapter 3. Materials and Methods for in vitro culture.}

To improve the power of the in vitro work we combined samples from two cohorts of progressive MS participants and validated this approach by contrasting cytometry output measures between the cohorts. While we noted significant differences in terms of pro-inflammatory cytokine responses, these differences were modest and contrasted to the absence of differences in general immune phenotype, age, or sex between the cohorts. Our work was also one of the first of its kind to use a new spectral platform to perform cytometry research in the clinical trial space. We showed the broad utility and stability of the platform, outlined methods, and showed broad quality control and instrument validation data. The use of spectral cytometry proved to be an excellent approach over conventional multicolour work, with increased data resolution, ease of use, and of the ability to collect a large high-dimensional dataset.

\subsection{Chapter 4. The effects of in vitro clozapine on dopamine receptor and cytokine expression in progressive MS.}

The analysis of the data investigating monocyte function was then compiled into three subsequent chapters. Chapter 4 discussed the merits of repurposing clozapine, an atypical antipsychotic drug, as a treatment for people with progressive MS. Central to this chapter was the expression of dopamine receptors in the periphery. Very little detail on DR expression on human immune cells is available in the literature, and as clozapine is a DR antagonist, we aimed to determine baseline expression and the 
effects of culture conditions on DR expression. Monocytes are a major source of cytokines in response to damage-associated molecular patterns that arise during the inflammatory and neurodegenerative pathology of MS. Additionally, because work by others has demonstrated clozapine's effectiveness at altering inflammatory cytokine expression, monocytes formed the major focus in terms of immune subsets analysed. We found that clozapine had an inhibitory effect on cytokine expression and altered DR expression on monocytes in vitro. Our results showed that clozapine's antiinflammatory effect in our model was associated with a reduced expression of proinflammatory cytokines (IL-6, IL-1 $\beta$ \& TNF $\alpha$ ). Furthermore, these findings suggest that clozapine treatment may be beneficial for the treatment of MS, as clozapine could dampen the expression of cytokine associated with MS pathology.

Clozapine also induced a significant increase in the expression of D1. To demonstrate what association there was between D1 and cytokine expression, we performed a correlation analysis of D1, IL-1 $\beta$, and IL-6 to D1 expression. From this we observed that D1 was not necessarily required to establish the pro-inflammatory state seen in untreated culture, but that a change in its expression happened alongside alterations to immune cell activity. Interestingly, we observed that MS participant monocytes were much more susceptible to DR expression changes from our culture compared to the HC. Following the modest pro-inflammatory effects of overnight culture, all DR were increased in the MS cohort but only D5 increased in HC monocytes. Under highly inflammatory LPS stimulation, the HC showed no substantial change in DR, while significant effects were noted in the MS cohort. Clozapine's effect on D1 was, however, noted in all participants. Together, these data offer valuable insight towards understanding clozapine's impact on dopamine and inflammatory signalling in the periphery, while also demonstrating the changes MS pathology has on these pathways.

The next chapter focused on the use of MIS416 as a treatment for patients with progressive MS. The experiments for this work were run concurrently, using the same methods, controls, and samples as those used in Chapter 4. 


\subsection{Chapter 5. Transcription factor and cytokine responses following MIS416 treatment in vitro.}

In Chapter 5, we studied the use of a drug, MIS416, for the treatment of patients with progressive MS. Our work used in vitro culture to model the peripheral innate system response to the drug. The microparticle suspension MIS416 stimulates intracellular PRR (i.e. TNR9 and NOD2), and treatment results in a distinctive cytokine response that is mediated by the NF- $\mathrm{kB}$ signalling cascade. Monocytes were the key target, owing to their phagocytic function and PRR expression. While MIS416's cytokine response profile had been reported previously, the exact subset responsible for cytokine production had not been described and therefore, was a key objective. Furthermore, we aimed to establish if there were any significant differences between the response of monocytes from HC and MS participants to MIS416.

In our data, we observed that the levels of TNFa were significantly increased in MS participants over those of the $\mathrm{HC}$ at baseline, indicating MS participants likely had higher constitutive expression of this cytokine. MS participants also produced more IL-1 $\beta$ following MIS416 treatment as compared to LPS, which was not observed in the HC. These findings indicated that people with progressive MS have distinctive immune profiles and drug response patterns compared to healthy subjects. We also concluded that monocytes were a key target for MIS416, and that their increased production of cytokines could be a route of treatment efficacy 1) through tolerisation mechanisms (TNFa), 2) by reducing inflammation through upregulation of antiinflammatory cytokines (IL-10), or 3) through negative feedback from proinflammatory cytokine release (IL-6 \& IL-1 $\beta$ ).

In this chapter, we also used transcription factor activation as an output measure to describe MIS416 molecular signalling. We attempted to capture the molecular effects of the drug by detecting the phosphorylation of a series of key TF. Our major indicator of MIS416 activity was using phosphorylated NFkB p65. We had previously determined three hours in vitro culture as an optimal time at which monocytes internalised MIS416. However, we could not show an increase in p65 from MIS416 at 
that time. TF staining proved to be technically challenging, and we concluded that a more targeted approach would be required to identify optimal MIS416 TF activity.

Overall, this data yielded new research directions: we observed that MS participants had significantly less active p65 at T3 as compared to the $\mathrm{HC}$, and that in vitro culture resulted in a reduction of the active molecule, likely from dephosphorylation, in all participants. Our collaborators at the University of Queensland (Brisbane, Australia) have previously shown that there was increased constitutive activation of NF- $\kappa \mathrm{B}$ in the peripheral blood cells from MS patients compared to controls, but we could not determine whether our observations were relevant to those findings. Other TF markers we profiled were STAT1 and STAT3, for both of which we observed an MIS416 treatment effect that resulted in a reduction in active TF protein in both cohorts. Moreover, for STAT1 alone at baseline, MS participants were found to have had significantly less active protein than their HC counterparts. Both STAT1 and STAT3 are key signalling nodes for immune cell cytokine response. In combining these measures with our data on INFY and TNFa, we started to explore the interrelationship of aberrant cytokine responses in MS to cellular singling through STAT. Together, the data demonstrated that MIS416 and our in vitro model, may be effective tools with which to explore these interactions.

We concluded the chapter by comparing the effect of the NFKBIA promotor mutation on serum cytokines from participants who received MIS416 in vivo as part of a phase 2B clinical trial. We showed that an $8 \mathrm{bp}$ insertion into IkBa increased constitutive $\mathrm{NF}-\kappa \mathrm{B}$ activity, and that a differential response to treatment may result with this genotype as heterozygous individuals tended to show a greater response to MIS416 over wild type individuals. In concluding this work, we suggest that responses to MIS416 in individuals is highly polymorphic. We observed that disease heterogeneity, phenotype, and genotype significantly affect drug response outcomes. Together our data contributes to the understanding of who may befit from MIS416 therapy and helps to clarify why the drug failed to show any treatment effect during a phase $2 \mathrm{~B}$ clinical trial. 


\subsection{Chapter 6. t-SNE \& SPADE of multicolour cytometry data.}

Chapter 6 formed our concluding results chapter. The analysis in the preceding in vitro chapters focussed only on monocytes, to the exclusion of a large amount of information available in the PBMC samples. Performing a similar style of analysis, using conventional gating and statistics, would be very time consuming and would have exceeded the limits of this thesis. Furthermore, this type of analysis is also known to be subjective and targeted and can result in a loss of information.

Despite these limitations, it was important to establish what value lay in the remaining data, as a primary objective for this thesis was in the generation of new hypotheses for future research by our group. We therefore set out to explore our in vitro dataset with the use of adjunct tools by performing high-dimensional analysis. For this we used two tools: a clustering analysis tool (i.e. SPADE) and a dimensionality reduction tool (i.e. t-SNE). Individually, and in combination, their use allowed for the visualisation of the entire dataset, and generated data that underpinned our conventional analysis and yielded new insights specifically by describing immune subset heterogeneity.

Firstly, we focussed on monocytes and showed substantial subset heterogeneity and cytokine induction by this subset in response to treatment and stimulation. Cluster analysis was used to demonstrate the changes the compartment undergoes as a result of in vitro culture. Our experiments showed that the monocyte compartment formed a cluster of events, similar in their expression patterns, which comprised 70\% of the cells. These showed homogenous responses to cytokine induction. In contrast, there were also several outlier clusters that showed distinctive phenotype and cytokine responses. Furthermore, MS participants profiles appear distinct compared to the HC, likely demonstrating an MS disease-induced change, mostly in the outlier clusters. Overall, we concluded that MS participants appeared to have more and stronger responses to our in vitro system and that these were over-represented in the outlier cells. When paired with data on TF expression, we observe that a minor fraction of monocytes also showed alter $\mathrm{TF}$ expression, indicating that their signalling processes were altered. Together, this data points to a minor subset of monocytes present only 
in MS participants. The contrast in profile, expression, and function of these cells in the MS cohort to the HC suggests they may have a role in MS disease pathogenesis.

Next, we explored the remaining information held in our dataset by compiling an analysis of all PBMC. With the model targeting monocytes, we observed that these cells were indeed the major population affected. However, we were surprised to observe changes in the phenotype and cytokine expression in other cells of the PBMC, indicating a bystander effect. Our data confirms the expression of all DR types by all cells of the PBMC, and an interesting difference in expression intensity in B cells suggests they may be a subset where DR signalling is important. D4 expression was also observed to be highly varied which suggests this marker expression is worth analysing using conventional gating and statistical approaches to clarify the conditions that altered its expression. However, to clearly visualise the expression changes across the PBMC, we would need to exclude monocytes from the analysis or perform a persubset analyses. Such an approach would then better describe cytokine expression by non-monocytes, as induction was observed but did not appear significant due to a skew in image scaling from the very high levels of expression in monocytes.

Together, we showed that t-SNE and SPADE analysis tools were informative, complementary, and added substantial value when applied to our data. Their use resulted in the verification of the finding from our previous chapters and generated new avenues for research. 


\subsection{Future directions.}

While this work is intended to be a stand-alone thesis, the nature of the investigations were exploratory. Several of our findings were of clinical importance and from those findings we can outline new directions of research. Furthermore, the projects were designed to align with the ongoing clinical research of MIS416 and clozapine respectively, both for the treatment of progressive MS.

Our clozapine work showed the drug increased the expression of D1. We posited that higher expression of D1 may enable improved signalling by endogenous dopamine through D1, thus inhibiting the inflammasome and reducing IL-1 $\beta$ production by monocytes. This finding would be a valuable future direction to explore. Initially the upregulation of D1 by clozapine should be confirmed in a validation cohort or cell lines. Further work could then confirm clozapine's effects on the inflammasome. Assessing changes in endogenous dopamine using our model may aid in the understanding of clozapine's mechanism. Unfortunately, dopamine has a very short half-life (under 2 minutes in plasma), which limited its measurement in stored culture supernatants from our experiments.

Our correlation analysis between high D1 and cytokine expression lead to the conclusion that changes in D1 expression occur in response to changes in immune activation and should be better defined. Clearly D1 expression has a role in immune activation and determining if this is as a signalling node or a potential biomarker that relate to specific immune state would aid in future treatment investigations. Furthermore, D4 expression in the PBMC showed a high degree of variability in our model, and when coupled with the findings that B cell expression of other DR should was also altered, points to a starting point for future work.

During the course of this work, the phase $2 \mathrm{~b}$ trial results were released and showed the MIS416 treatment did not lead to any significant clinical improvement. In analysing some of the trial output measures, we showed treatment responses in participants may differ as a result of genetic polymorphism. This contributed to our understanding of the failure of the drug in phase $2 b$ and suggests alternative analysis strategies may 
shine new light on the trial outcomes. Participant stratification by genotype for known $\mathrm{NF \kappa B}$ alterations may lead to the identification of differential responders within the larger trial cohort. We concluded that MIS416 was not the ideal molecule with which to show this proof and suggest a more targeted approach such as through single agent NOD or TLR stimulation in cell line models. With the MIS416 work, we also did not plan to profile TF changes in overnight culture, but we had spare clinical material on some participants that was used to demonstrate this as a proof of concept. Unfortunately, no untreated controls were available. The data showed that TF expression can be substantially increased in the presence of MIS416, but whether the increase in TF expression is from culture or MIS416 remains to be resolved. Future work that establishes when innate immune stimulation effects an increase in active TF levels would add significantly to understanding the mechanisms and dosing of MIS416.

Lastly, we compiled an overview of the entire dataset for cytokine, DR, and TF expression in the PBMC. Our work also went on to detail DR expression in the monocyte compartment in great detail. We demonstrated the changes in DR expression monocytes undergo in culture and we describe previously unreported expression data of DR in human PBMC. We further show that D3 expression may also be altered in MS, an insight that only came to light with the use of high dimensional analysis; however, further work would need to validate this finding. Together, these findings highlighted the substantial data that remains to be mined in our dataset. We showed that a broad, multi-immune subset data acquisition strategy increases the value of targeted in vitro work, as the complex interaction of stimulated immune cells can then be viewed with high dimensional analysis tools. Spectral cytometry was instrumental in facilitating this work, and our work leads the field in its implementation in clinical immune data analysis. 


\section{Bibliography.}

Abeles, R.D., McPhail, M.J., Sowter, D., Antoniades, C.G., Vergis, N., Vijay, G.K.M., Xystrakis, E., Khamri, W., Shawcross, D.L., Ma, Y., et al. (2012). CD14, CD16 and HLA-DR reliably identifies human monocytes and their subsets in the context of pathologically reduced HLA-DR expression by CD14(hi) /CD16(neg) monocytes: Expansion of CD14(hi) /CD16(pos) and contraction of CD14(lo) /CD16(pos) monocytes in acute liver failure. Cytom Part J Int Soc Anal Cytol 81, 823-834.

Aguirre-Gamboa, R., Joosten, I., Urbano, P.C.M., van der Molen, R.G., van Rijssen, E., van Cranenbroek, B., Oosting, M., Smeekens, S., Jaeger, M., Zorro, M., et al. (2016). Differential Effects of Environmental and Genetic Factors on T and B Cell Immune Traits. Cell Reports 17, 2474-2487.

Ajami, B., Bennett, J.L., Krieger, C., McNagny, K.M., and Rossi, F.M.V. (2011). Infiltrating monocytes trigger EAE progression, but do not contribute to the resident microglia pool. Nat Neurosci 14, 1142-1149.

Akira, S., and Hemmi, H. (2003). Recognition of pathogen-associated molecular patterns by TLR family. Immunol Lett 85, 85-95.

Al-Amin, M., Uddin, M.M.N., and Reza, H.M. (2013). Effects of Antipsychotics on the Inflammatory Response System of Patients with Schizophrenia in Peripheral Blood Mononuclear Cell Cultures. Clin Psychopharm Neu 11, 144-151.

Alla, S., Pearson, J., Debernard, L., Miller, D., and Mason, D. (2014). The increasing prevalence of multiple sclerosis in New Zealand. Neuroepidemiology 42, 154-160.

Alla, S., Pearson, J.F., Taylor, B.V., Roxburgh, R., Clarke, G., Miller, D.H., Richardson, A., Abernethy, D.A., Willoughby, E., Sabel, C.E., et al. (2015). An investigation of the relationship between latitude and multiple sclerosis severity in New Zealand. Multiple Scler Houndmills Basingstoke Engl 22, 705-707.

Amato, M.P., Portaccio, E., Goretti, B., Zipoli, V., Battaglini, M., Bartolozzi, M.L., Stromillo, M.L., Guidi, L., Siracusa, G., Sorbi, S., et al. (2007). Association of Neocortical Volume Changes With Cognitive Deterioration in Relapsing-Remitting Multiple Sclerosis. Arch Neurol-Chicago 64, 1157.

Amir, E.D., Davis, K.L., Tadmor, M.D., Simonds, E.F., Levine, J.H., Bendall, S.C., Shenfeld, D.K., Krishnaswamy, S., Nolan, G.P., and Pe'er, D. (2013). viSNE enables visualization of high dimensional single-cell data and reveals phenotypic heterogeneity of leukemia. Nat Biotechnol 31, 545-552.

Anchang, B., Hart, T.D.P., Bendall, S.C., Qiu, P., Bjornson, Z., Linderman, M., Nolan, G.P., and Plevritis, S.K. (2016). Visualization and cellular hierarchy inference of single-cell data using SPADE. Nat Protoc 11, 1264-1279.

Ancuta, P., Rao, R., Moses, A., Mehle, A., Shaw, S.K., Luscinskas, F.W., and Gabuzda, D. (2003). Fractalkine Preferentially Mediates Arrest and Migration of CD16+ Monocytes. J Exp Medicine 197, 1701-1707.

Andersen, M.N., Al-Karradi, S.N.H., Kragstrup, T.W., and Hokland, M. (2016). Elimination of erroneous results in flow cytometry caused by antibody binding to Fc receptors on human monocytes and macrophages. Cytom Part A 89, 1001-1009. 
Annacker, O., Asseman, C., Read, S., and Powrie, F. (2003). Interleukin-10 in the regulation of T cell-induced colitis. J Autoimmun 2O, 277-279.

Arnett, H.A., Mason, J., Marino, M., Suzuki, K., Matsushima, G.K., and Ting, J.P.-Y. (2001). TNFa promotes proliferation of oligodendrocyte progenitors and remyelination. Nat Neurosci 4, 1116-1122.

Arreola, R., Alvarez-Herrera, S., Pérez-Sánchez, G., Becerril-Villanueva, E., CruzFuentes, C., Flores-Gutierrez, E.O., Garcés-Alvarez, M.E., Cruz-Aguilera, D.L. de la, Medina-Rivero, E., Hurtado-Alvarado, G., et al. (2016). Immunomodulatory Effects Mediated by Dopamine. J Immunol Res 2016, 1-31.

Ascherio, A., Munger, K.L., and Lünemann, J.D. (2012). The initiation and prevention of multiple sclerosis. Nat Rev Neurology 8, 602-612.

Ashhurst, T. (2017). Using tSNE (t-distributed nearest neighbour embedding) in FlowJo 10.2 and Matlab.

Bach, M.A., Phan-Dinh-Tuy, F., Tournier, E., Chatenoud, L., Bach, J.F., Martin, C., and Degos, J.D. (1980). Deficit of suppressor T cells in active multiple sclerosis. Lancet Lond Engl 2, 1221-1223.

Baecher-Allan, C., Wolf, E., and Hafler, D.A. (2006). MHC class II expression identifies functionally distinct human regulatory T cells. J Immunol Baltim Md 1950 176, 4622-4631.

Bankey, P.E., Banerjee, S., Zucchiatti, A., De, M., Sleem, R.W., Lin, C.-F.L., MillerGraziano, C.L., and De, A.K. (2010). Cytokine induced expression of programmed death ligands in human neutrophils. Immunol Lett 129, 100-107.

Bar-Or, A. (2008). The immunology of multiple sclerosis. Semin Neurol 28, 29-45.

Bar-Or, A., Nuttall, R.K., Duddy, M., Alter, A., Kim, H.J., Ifergan, I., Pennington, C.J., Bourgoin, P., Edwards, D.R., and Yong, V.W. (2003). Analyses of all matrix metalloproteinase members in leukocytes emphasize monocytes as major inflammatory mediators in multiple sclerosis. Brain 126, 2738-2749.

Barr, T.A., Shen, P., Brown, S., Lampropoulou, V., Roch, T., Lawrie, S., Fan, B., O'Connor, R.A., Anderton, S.M., Bar-Or, A., et al. (2012). B cell depletion therapy ameliorates autoimmune disease through ablation of IL-6-producing B cells. J Exp Medicine 209, 1001-1010.

Bashashati, A., and Brinkman, R.R. (2009). A survey of flow cytometry data analysis methods. Adv Intel Soft Compu 2009, 584603.

Basu, S., and Dasgupta, P.S. (2000). Dopamine, a neurotransmitter, influences the immune system. J Neuroimmunol 102, 113-124.

BD (2019). BD CTP Tube.

Https://www.Bdbiosciences.Com/Ds/Ab/Others/PI_CPT_heparin_March_2016_V DP40105-07_web_500010323.Pdf.

Beaulieu, J.-M., and Gainetdinov, R.R. (2011). The physiology, signaling, and pharmacology of dopamine receptors. Pharmacol Rev 63, 182-217.

Beaulieu, J.-M., Espinoza, S., and Gainetdinov, R.R. (2014). Dopamine receptors IUPHAR Review 13. Brit J Pharmacol 172, 1-23. 
Belbasis, L., Bellou, V., Evangelou, E., Ioannidis, J.P.A., and Tzoulaki, I. (2015). Environmental risk factors and multiple sclerosis: an umbrella review of systematic reviews and meta-analyses. Lancet Neurology 14, 263-273.

Bendall, S.C., Simonds, E.F., Qiu, P., Amir, E.D., Krutzik, P.O., Finck, R., Bruggner, R.V., Melamed, R., Trejo, A., Ornatsky, O.I., et al. (2011). Single-cell mass cytometry of differential immune and drug responses across a human hematopoietic continuum. Sci New York N Y 332, 687-696.

Berzins, S.P., Smyth, M.J., and Baxter, A.G. (2011). Presumed guilty: natural killer T cell defects and human disease. Nat Rev Immunol 11, 131-142.

Bevan, M.J. (1976). Minor H antigens introduced on H-2 different stimulating cells cross-react at the cytotoxic T cell level during in vivo priming. $\mathrm{J}$ Immunol Baltim $\mathrm{Md}$ 1950 117, 2233-2238.

Bezbradica, J.S., and Schroder, K. (2014). TRAF6 is a nexus for TLR-STAT1 crosstalk. Immunol Cell Biol 92, 737-738.

Biasi, S.D., Simone, A.M., Nasi, M., Bianchini, E., Ferraro, D., Vitetta, F., Gibellini, L., Pinti, M., Giovane, C.D., Sola, P., et al. (2016). iNKT Cells in Secondary Progressive Multiple Sclerosis Patients Display Pro-inflammatory Profiles. Front Immunol 7, 555 .

BioGPS (2019). DRD5 (dopamine receptor D5).

Http://www.Biogps.Org/\#goto=genereport\&id=1816.

Blumenfeld, S., Staun-Ram, E., and Miller, A. (2016). Fingolimod therapy modulates circulating B cell composition, increases B regulatory subsets and production of IL10 and TGF $\beta$ in patients with Multiple Sclerosis. J Autoimmun 70, 40-51.

BMJ (2019). Migration, age, and MS . . . and other stories. Bmj 365, 12412.

Bobermin, L.D., Silva, A. da, Souza, D.O., and Quincozes-Santos, A. (2018).

Differential effects of typical and atypical antipsychotics on astroglial cells in vitro. Int J Dev Neurosci Official J Int Soc Dev Neurosci 69, 1-9.

Bohuslav, J., Kravchenko, V.V., Parry, G.C., Erlich, J.H., Gerondakis, S., Mackman, N., and Ulevitch, R.J. (1998). Regulation of an essential innate immune response by the p5o subunit of NF-kappaB. J Clin Invest 102, 1645-1652.

Bose, G., Atkins, H.L., Bowman, M., and Freedman, M.S. (2018). Autologous hematopoietic stem cell transplantation improves fatigue in multiple sclerosis. Mult Scler J 25, 135245851880254 .

Brinkmann, V., Davis, M.D., Heise, C.E., Albert, R., Cottens, S., Hof, R., Bruns, C., Prieschl, E., Baumruker, T., Hiestand, P., et al. (2002). The Immune Modulator FTY720 Targets Sphingosine 1-Phosphate Receptors. J Biol Chem 277, 21453-21457.

Brown, G.C., and Bal-Price, A. (2003). Inflammatory Neurodegeneration Mediated by Nitric Oxide, Glutamate, and Mitochondria. Mol Neurobiol 27, 325-355.

Brück, W. (2005). The pathology of multiple sclerosis is the result of focal inflammatory demyelination with axonal damage. J Neurol 252, v3-v9.

Büdingen, H. von, Bar-Or, A., and Zamvil, S. (2011). B cells in multiple sclerosis: connecting the dots. Curr Opin Immunol. 2011 Dec; 23(6): 713-720.

bu.edu (2019). NF-kB Transcription Factors.

Http://www.bu.edu/nf-kb/ 
Burdo, T.H., Soulas, C., Orzechowski, K., Button, J., Krishnan, A., Sugimoto, C., Alvarez, X., Kuroda, M.J., and Williams, K.C. (2010). Increased monocyte turnover from bone marrow correlates with severity of SIV encephalitis and CD163 levels in plasma. Plos Pathog 6, e1000842.

Burt, R.K., Balabanov, R., Burman, J., Sharrack, B., Snowden, J.A., Oliveira, M.C., Fagius, J., Rose, J., Nelson, F., Barreira, A.A., et al. (2019). Effect of

Nonmyeloablative Hematopoietic Stem Cell Transplantation vs Continued DiseaseModifying Therapy on Disease Progression in Patients With Relapsing-Remitting Multiple Sclerosis: A Randomized Clinical Trial. Jama 321, 165-174.

Canto, E., Isobe, N., Didonna, A., Group, M.-E.S., Hauser, S.L., and Oksenberg, J.R. (2018). Aberrant STAT phosphorylation signaling in peripheral blood mononuclear cells from multiple sclerosis patients. J Neuroinflamm 15, 72.

Carr, E.J., Dooley, J., Garcia-Perez, J.E., Lagou, V., Lee, J.C., Wouters, C., Meyts, I., Goris, A., Boeckxstaens, G., Linterman, M.A., et al. (2016). The cellular composition of the human immune system is shaped by age and cohabitation. Nat Immunol 17, 461-468.

Casadevall, A., and Pirofski, L. -a (2004). New Concepts in Antibody-Mediated Immunity. Infect Immun 72, 6191-6196.

Castellaneta, A., Sumpter, T.L., Chen, L., Tokita, D., and Thomson, A.W. (2009). NOD2 ligation subverts IFN-alpha production by liver plasmacytoid dendritic cells and inhibits their T cell allostimulatory activity via B7-H1 up-regulation. J Immunol Baltim Md 1950 183, 6922-6932.

Chari, D.M. (2007). International Review of Neurobiology. Int Rev Neurobiol 79, 589-620.

Chen, K., Wang, J.M., Yuan, R., Yi, X., Li, L., Gong, W., Yang, T., Li, L., and Su, S. (2016). Tissue-resident dendritic cells and diseases involving dendritic cell malfunction. Int Immunopharmacol 34, 1-15.

Chen, M.-L., Tsai, T.-C., Lin, Y.-Y., Tsai, Y.-M., Wang, L.-K., Lee, M.-C., and Tsai, F.M. (2011a). Antipsychotic drugs suppress the AKT/NF- $\kappa B$ pathway and regulate the differentiation of T-cell subsets. Immunol Lett 14O, 81-91.

Chen, M.-L., Tsai, T.-C., Wang, L.-K., Lin, Y.-Y., Tsai, Y.-M., Lee, M.-C., and Tsai, F.M. (2011b). Risperidone modulates the cytokine and chemokine release of dendritic cells and induces TNF- $\alpha$-directed cell apoptosis in neutrophils. Int Immunopharmacol 12, 197-204.

Chen, M.-L., Tsai, T.-C., Wang, L.-K., Lin, Y.-Y., Tsai, Y.-M., Lee, M.-C., and Tsai, F.M. (2012). Clozapine inhibits Th1 cell differentiation and causes the suppression of IFN- $\gamma$ production in peripheral blood mononuclear cells. Immunopharm Immunot 34, 686-694.

Chen, M.-L., Wu, S., Tsai, T.-C., Wang, L.-K., and Tsai, F.-M. (2013). Regulation of macrophage immune responses by antipsychotic drugs. Immunopharm Immunot 35, 573-580.

Chiarini, M., Serana, F., Zanotti, C., Capra, R., Rasia, S., Rottoli, M., Rovaris, M., Caputo, D., Cavaletti, G., Frigo, M., et al. (2011). Modulation of the central memory and Tr1-like regulatory $\mathrm{T}$ cells in multiple sclerosis patients responsive to interferonbeta therapy. Mult Scler J 18, 788-798. 
Christensen, J.R., Börnsen, L., Ratzer, R., Piehl, F., Khademi, M., Olsson, T., Sørensen, P.S., and Sellebjerg, F. (2013). Systemic Inflammation in Progressive Multiple Sclerosis Involves Follicular T-Helper, Th17- and Activated B-Cells and Correlates with Progression. Plos One 8, e57820.

Chuluundorj, D., Harding, S.A., Abernethy, D., and La Flamme, A.C. (2014). Expansion and preferential activation of the CD14(+)CD16(+) monocyte subset during multiple sclerosis. Immunol Cell Biol 92, 509-517.

Chuluundorj, D., Harding, S.A., Abernethy, D., and La Flamme, A.C. (2016). Glatiramer acetate treatment normalized the monocyte activation profile in MS patients to that of healthy controls. Immunol Cell Biol 95, 297-305.

Claes, N., Fraussen, J., Stinissen, P., Hupperts, R., and Somers, V. (2015). B Cells Are Multifunctional Players in Multiple Sclerosis Pathogenesis: Insights from Therapeutic Interventions. Front Immunol 6, 642.

Coley, J.S., Calderon, T.M., Gaskill, P.J., Eugenin, E.A., and Berman, J.W. (2015). Dopamine Increases CD14+CD16+ Monocyte Migration and Adhesion in the Context of Substance Abuse and HIV Neuropathogenesis. Plos One 1O, e0117450.

Comabella, M., Lünemann, J.D., Río, J., Sánchez, A., López, C., Julià, E., Fernández, M., Nonell, L., Camiña-Tato, M., Deisenhammer, F., et al. (2009). A type I interferon signature in monocytes is associated with poor response to interferon- $\beta$ in multiple sclerosis. Brain 132, 3353-3365.

Comi, G., Group, the E.T. of M.S.S., Filippi, M., Barkhof, F., Durelli, L., Edan, G., Fernández, O., Hartung, H.-P., Seeldrayers, P., Sørensen, P.S., et al. (2001). Effect of early interferon treatment on conversion to definite multiple sclerosis: a randomised study. Lancet 357, 1576-1582.

Confavreux, C., Vukusic, S., Moreau, T., and Adeleine, P. (2000). Relapses and Progression of Disability in Multiple Sclerosis. New Engl J Med 343, 1430-1438.

Contreras-Shannon, V., Heart, D.L., Paredes, R.M., Navaira, E., Catano, G., Maffi, S.K., and Walss-Bass, C. (2013). Clozapine-induced mitochondria alterations and inflammation in brain and insulin-responsive cells. Plos One 8, e59012.

Cooper, M.A., Fehniger, T.A., and Caligiuri, M.A. (2001). The biology of human natural killer-cell subsets. Trends Immunol 22, 633-640.

Cormican, S., and Griffin, M.D. (2020). Human Monocyte Subset Distinctions and Function: Insights From Gene Expression Analysis. Front Immunol 11, 1070.

Correale, J., and Villa, A. (2007). The blood-brain-barrier in multiple sclerosis: Functional roles and therapeutic targeting. Autoimmunity 4O, 148-160.

Crispín, J.C., Oukka, M., Bayliss, G., Cohen, R.A., Beek, C.A.V., Stillman, I.E., Kyttaris, V.C., Juang, Y.-T., and Tsokos, G.C. (2008). Expanded Double Negative T Cells in Patients with Systemic Lupus Erythematosus Produce IL-17 and Infiltrate the Kidneys. J Immunol 181, 8761-8766.

Cytek (2019). Cytek Aurora Fluorochrome Selection Guidelines. Https://cancer.columbia.edu/sites/hiccc.columbia.edu/files/Fluorochrome\%20selec tion\%20guide\%20for\%20Aurora.pdf

Cytobank (2019). Facselect.

Https://Www.Cytobank.Org/Facselect 
Das, J., Sharrack, B., and Snowden, J.A. (2019). Autologous Haematopoietic Stem Cell Transplantation in Multiple Sclerosis: a Review of Current Literature and Future Directions for Transplant Haematologists and Oncologists. Curr Hematol Malig R 14, 127-135.

Davies, L.C., Jenkins, S.J., Allen, J.E., and Taylor, P.R. (2013). Tissue-resident macrophages. Nat Immunol 14, 986-995.

Dendrou, C.A., Petersen, J., Rossjohn, J., and Fugger, L. (2018). HLA variation and disease. Nat Rev Immunol 18, 325-339.

Dhib-Jalbut, S. (2002). Mechanisms of action of interferons and glatiramer acetate in multiple sclerosis. Neurology $58, \mathrm{~S}_{3}-\mathrm{S} 9$.

Diggins, K.E., Ferrell, P.B., and Irish, J.M. (2015). Methods for discovery and characterization of cell subsets in high dimensional mass cytometry data. Methods San Diego Calif 82, 55-63.

Doshi, A., and Chataway, J. (2016). Multiple sclerosis, a treatable disease. Clin Med 16, s53-s59.

Dutta, R., and Trapp, B.D. (2010). Mechanisms of neuronal dysfunction and degeneration in multiple sclerosis. Prog Neurobiol 93, 1-12.

Dutta, R., McDonough, J., Yin, X., Peterson, J., Chang, A., Torres, T., Gudz, T., Macklin, W.B., Lewis, D.A., Fox, R.J., et al. (2006). Mitochondrial dysfunction as a cause of axonal degeneration in multiple sclerosis patients. Ann Neurol 59, 478-489.

Ear, T., and McDonald, P.P. (2008). Cytokine generation, promoter activation, and oxidant-independent NF- $\kappa \mathrm{B}$ activation in a transfectable human neutrophilic cellular model. Bmc Immunol 9, 14.

EBI (2019). Dopamine D2 receptor InterPro. Https://Www.Ebi.Ac.Uk/Interpro/Beta/Entry/InterPro/IPRoo1922/.

Eisen, M.B., Spellman, P.T., Brown, P.O., and Botstein, D. (1998). Cluster analysis and display of genome-wide expression patterns. Proc National Acad Sci 95, 1486314868.

Eshghi, S.T., Au-Yeung, A., Takahashi, C., Bolen, C.R., Nyachienga, M.N., Lear, S.P., Green, C., Mathews, W.R., and O'Gorman, W.E. (2019). Quantitative Comparison of Conventional and t-SNE-guided Gating Analyses. Front Immunol 1O, 1194.

Fan, X., Jin, T., Zhao, S., Liu, C., Han, J., Jiang, X., and Jiang, Y. (2015). Circulating CCR7+ICOS+ Memory T Follicular Helper Cells in Patients with Multiple Sclerosis. Plos One 1O, e0134523.

Faurschou, M., and Borregaard, N. (2003). Neutrophil granules and secretory vesicles in inflammation. Microbes Infect 5, 1317-1327.

Feng, X., Petraglia, A.L., Chen, M., Byskosh, P.V., Boos, M.D., and Reder, A.T. (2002). Low expression of interferon-stimulated genes in active multiple sclerosis is linked to subnormal phosphorylation of STAT1. J Neuroimmunol 129, 205-215.

Ferber, I.A., Brocke, S., Taylor-Edwards, C., Ridgway, W., Dinisco, C., Steinman, L., Dalton, D., and Fathman, C.G. (1996). Mice with a disrupted IFN-gamma gene are susceptible to the induction of experimental autoimmune encephalomyelitis (EAE). $J$ Immunol Baltim Md 1950 156, 5-7. 
Fernandez, E.M., Valenti, V., Rockel, C., Hermann, C., Pot, B., Boneca, I.G., and Grangette, C. (2011). Anti-inflammatory capacity of selected lactobacilli in experimental colitis is driven by NOD2-mediated recognition of a specific peptidoglycan-derived muropeptide. Gut 60, 1050-1059.

Filion, L.G., Graziani-Bowering, G., Matusevicius, D., and Freedman, M.S. (2003). Monocyte-derived cytokines in multiple sclerosis. Clin Exp Immunol 131, 324-334.

Filippini, G., Giovane, C.D., Vacchi, L., D’Amico, R., Pietrantonj, C.D., Beecher, D., and Salanti, G. (2013). Immunomodulators and immunosuppressants for multiple sclerosis: a network meta-analysis. Cochrane Db Syst Rev 6, CDoo8933.

Fillatreau, S., Sweenie, C.H., McGeachy, M.J., Gray, D., and Anderton, S.M. (2002). B cells regulate autoimmunity by provision of IL-10. Nat Immunol 3, 944-950.

Finak, G., Langweiler, M., Jaimes, M., Malek, M., Taghiyar, J., Korin, Y., Raddassi, K., Devine, L., Obermoser, G., Pekalski, M.L., et al. (2016). Standardizing Flow Cytometry Immunophenotyping Analysis from the Human ImmunoPhenotyping Consortium. Sci Rep-UK 6, 20686.

Firestein, G., Budd, R., Gabriel, S.E., McInnes, I.B., and O’Dell, J. (2014). Kelley's Textbook of Rheumatology. Elsevier, UK. $8^{\text {th }}$ Edition. 1-133

Fischer, M.T., Wimmer, I., Höftberger, R., Gerlach, S., Haider, L., Zrzavy, T., Hametner, S., Mahad, D., Binder, C.J., Krumbholz, M., et al. (2013). Disease-specific molecular events in cortical multiple sclerosis lesions. Brain 136, 1799-1815.

Flanagan, R.J., Yusufi, B., and Barnes, T.R.E. (2003). Comparability of whole-blood and plasma clozapine and norclozapine concentrations. Brit J Clin Pharmaco 56, 135-138.

Franchi, L., Warner, N., Viani, K., and Nuñez, G. (2009). Function of Nod-like receptors in microbial recognition and host defense. Immunol Rev 227, 106-128.

Furth, R. van, Cohn, Z.A., Hirsch, J.G., Humphrey, J.H., Spector, W.G., and Langevoort, H.L. (1972). The mononuclear phagocyte system: a new classification of macrophages, monocytes, and their precursor cells. B World Health Organ 46, 845852.

Gale, C.R., and Martyn, C.N. (1995). Migrant studies in multiple sclerosis. Prog Neurobiol 47, 425-448.

Gandhi, R., Laroni, A., and Weiner, H.L. (2010). Role of the innate immune system in the pathogenesis of multiple sclerosis. J Neuroimmunol 221, 7-14.

Gaudillière, B., Fragiadakis, G.K., Bruggner, R.V., Nicolau, M., Finck, R., Tingle, M., Silva, J., Ganio, E.A., Yeh, C.G., Maloney, W.J., et al. (2014). Clinical recovery from surgery correlates with single-cell immune signatures. Sci Transl Med 6, 255ra131.

Gerson, S., and Meltzer, H. (1992). Mechanisms of clozapine-induced agranulocytosis. CNS Drugs 7(2):139-58.

Ghadiri, M., Fitz-Gerald, L., Rezk, A., Li, R., Nyirenda, M., Haegert, D., Giacomini, P.S., Bar-Or, A., and Antel, J. (2017). Reconstitution of the peripheral immune repertoire following withdrawal of fingolimod. Mult Scler J 23, 1225-1232.

Ghosh, M.C., Mondal, A.C., Basu, S., Banerjee, S., Majumder, J., Bhattacharya, D., and Dasgupta, P.S. (2003). Dopamine inhibits cytokine release and expression of 
tyrosine kinases, Lck and Fyn in activated T cells. Int Immunopharmacol 3, 10191026.

Giorelli, M., Livrea, P., and Trojano, M. (2005). Dopamine Fails to Regulate Activation of Peripheral Blood Lymphocytes from Multiple Sclerosis Patients: Effects of IFN- $\beta$. J Interf Cytokine Res 25, 395-406.

Girvan, R.C., Knight, D.A., O’Loughlin, C.J., Hayman, C.M., Hermans, I.F., and Webster, G.A. (2010). MIS416, a non-toxic microparticle adjuvant derived from Propionibacterium acnes comprising immunostimulatory muramyl dipeptide and bacterial DNA promotes cross-priming and Th1 immunity. Vaccine 29, 545-557.

Gjelstrup, M.C., Stilund, M., Petersen, T., Møller, H.J., Petersen, E.L., and Christensen, T. (2017). Subsets of activated monocytes and markers of inflammation in incipient and progressed multiple sclerosis. Immunol Cell Biol 96, 160-174.

Green, L.K., Zareie, P., Templeton, N., Keyzers, R.A., Connor, B., and Flamme, A.C.L. (2017). Enhanced disease reduction using clozapine, an atypical antipsychotic agent, and glatiramer acetate combination therapy in experimental autoimmune encephalomyelitis. Multiple Scler J Exp Transl Clin 3, 205521731769872.

Group, T.L.M.S.S.G. and T.U. of B.C.M.A. (1999). TNF neutralization in MS: Results of a randomized, placebo-controlled multicenter study. Neurology 53, 457-457.

Haas, J., Bekeredjian-Ding, I., Milkova, M., Balint, B., Schwarz, A., Korporal, M., Jarius, S., Fritz, B., Lorenz, H.-M., and Wildemann, B. (2011). B cells undergo unique compartmentalized redistribution in multiple sclerosis. J Autoimmun 37, 289-299.

Hamidi, V., Couto, E., Ringerike, T., and Klemp, M. (2018). A Multiple Treatment Comparison of Eleven Disease-Modifying Drugs Used for Multiple Sclerosis. J Clin Medicine Res 10, 88-105.

Han, S., Lin, Y.C., Wu, T., Salgado, A.D., Mexhitaj, I., Wuest, S.C., Romm, E., Ohayon, J., Goldbach-Mansky, R., Vanderver, A., et al. (2014). Comprehensive Immunophenotyping of Cerebrospinal Fluid Cells in Patients with Neuroimmunological Diseases. J Immunol 192, 2551-2563.

Harrington, L.E., Hatton, R.D., Mangan, P.R., Turner, H., Murphy, T.L., Murphy, K.M., and Weaver, C.T. (2005). Interleukin 17-producing CD4+ effector T cells develop via a lineage distinct from the Thelper type 1 and 2 lineages. Nat Immunol 6, 1123-1132.

Hasan, M., Beitz, B., Rouilly, V., Libri, V., Urrutia, A., Duffy, D., Cassard, L., Santo, J.P.D., Mottez, E., Quintana-Murci, L., et al. (2015). Semi-automated and standardized cytometric procedures for multi-panel and multi-parametric whole blood immunophenotyping. Clin Immunol 157, 261-276.

Hauser, S.L., Waubant, E., Arnold, D.L., Vollmer, T., Antel, J., Fox, R.J., Bar-Or, A., Panzara, M., Sarkar, N., Agarwal, S., et al. (2008). B-Cell Depletion with Rituximab in Relapsing-Remitting Multiple Sclerosis. New Engl J Med 358, 676-688.

Hauser, S.L., Bar-Or, A., Comi, G., Giovannoni, G., Hartung, H.-P., Hemmer, B., Lublin, F., Montalban, X., Rammohan, K.W., Selmaj, K., et al. (2017). Ocrelizumab versus Interferon Beta-1a in Relapsing Multiple Sclerosis. New Engl J Med 376, 221234.

Hawker, K., O’Connor, P., Freedman, M.S., Calabresi, P.A., Antel, J., Simon, J., Hauser, S., Waubant, E., Vollmer, T., Panitch, H., et al. (2009). Rituximab in 
patients with primary progressive multiple sclerosis: Results of a randomized double-blind placebo-controlled multicenter trial. Ann Neurol 66, 460-471.

Hedl, M., Li, J., Cho, J.H., and Abraham, C. (2007). Chronic stimulation of Nod2 mediates tolerance to bacterial products. Proc National Acad Sci 104, 19440-19445.

Herzenberg, L.A., Tung, J., Moore, W.A., Herzenberg, L.A., and Parks, D.R. (2006). Interpreting flow cytometry data: a guide for the perplexed. Nat Immunol 7, 681685 .

Himmerich, H., Schönherr, J., Fulda, S., Sheldrick, A.J., Bauer, K., and Sack, U. (2011). Impact of antipsychotics on cytokine production in-vitro. J Psychiatr Res 45, 1358-1365.

Hinton, G., and Maaten, L. van der (2008). Visualizing Data using t-SNE. Journal of Machine Learning Research 9, 2579-2605.

Hinze-Selch, D., Becker, E.W., Stein, G.M., Berg, P.A., Mullington, J., Holsboer, F., and Pollmächer, T. (1998). Effects of Clozapine on In Vitro Immune Parameters A Longitudinal Study in Clozapine-Treated Schizophrenic Patients.

Neuropsychopharmacol 19, 114-122.

Hla, T., and Brinkmann, V. (2011). Sphingosine 1-phosphate (S1P): Physiology and the effects of $\mathrm{S} 1 \mathrm{P}$ receptor modulation. Neurology $76, \mathrm{~S} 3-\mathrm{S} 8$.

Hulspas, R., O’Gorman, M.R.G., Wood, B.L., Gratama, J.W., and Sutherland, D.R. (2009). Considerations for the control of background fluorescence in clinical flow cytometry. Cytom Part B Clin Cytom 76B, 355-364.

Iezzi, G., Karjalainen, K., and Lanzavecchia, A. (1998). The Duration of Antigenic Stimulation Determines the Fate of Naive and Effector T Cells. Immunity 8, 89-95.

Inshaw, J.R.J., Cutler, A.J., Burren, O.S., Stefana, M.I., and Todd, J.A. (2018). Approaches and advances in the genetic causes of autoimmune disease and their implications. Nat Immunol 19, 674-684.

Ismail, A., Sharrack, B., Saccardi, R., Moore, J.J., and Snowden, J.A. (2019). Autologous haematopoietic stem cell therapy for multiple sclerosis: a review for supportive care clinicians on behalf of the Autoimmune Diseases Working Party of the European Society for Blood and Marrow Transplantation. Curr Opin Support Pa $13,394-401$.

Iwata, Y., Matsushita, T., Horikawa, M., Dilillo, D.J., Yanaba, K., Venturi, G.M., Szabolcs, P.M., Bernstein, S.H., Magro, C.M., Williams, A.D., et al. (2010).

Characterization of a rare IL-10-competent B-cell subset in humans that parallels mouse regulatory B10 cells. Blood 117, 530-541.

Schrader J., Irving C., Octeau J., Christian J., Aballo T. (2019). The differential actions of clozapine and other antipsychotic drugs on the translocation of dopamine D2 receptors to the cell surface. J Biol Chem 294, 5604-5615.

Jager, P.L.D., Rossin, E., Pyne, S., Tamayo, P., Ottoboni, L., Viglietta, V., Weiner, M., Soler, D., Izmailova, E., Faron-Yowe, L., et al. (2008). Cytometric profiling in multiple sclerosis uncovers patient population structure and a reduction of CD8low cells. Brain J Neurology 131, 1701-1711.

Jakimovski, D., Weinstock-Guttman, B., Ramanathan, M., Kolb, C., Hojnacki, D., Minagar, A., and Zivadinov, R. (2017). Ocrelizumab: a B-cell depleting therapy for multiple sclerosis. Expert Opin Biol Th 17, 1163-1172. 
Jensen, P.E. (2007). Recent advances in antigen processing and presentation. Nat Immunol 8, 1041-1048.

Jensen, M.A., Yanowitch, R.N., Reder, A.T., White, D.M., and Arnason, B.G. (2009). Immunoglobulin-like transcript 3 , an inhibitor of $\mathrm{T}$ cell activation, is reduced on blood monocytes during multiple sclerosis relapses and is induced by interferon $\beta$ 1b. Mult Scler J 16, 30-38.

Johnston, L., Harding, S.A., and Flamme, A.C.L. (2015). Comparing methods for ex vivo characterization of human monocyte phenotypes and in vitro responses. Immunobiology 22O, 1305-1310.

Jones, A.P., Kermode, A.G., Lucas, R.M., Carroll, W.M., Nolan, D., and Hart, P.H. (2016). Circulating immune cells in multiple sclerosis. Clin Exp Immunol 187, 193203.

Jong, P.R. de, Schadenberg, A.W.L., Broek, T. van den, Beekman, J.M., Wijk, F. van, Coffer, P.J., Prakken, B.J., and Jansen, N.J.G. (2012). STAT3 Regulates Monocyte TNF-Alpha Production in Systemic Inflammation Caused by Cardiac Surgery with Cardiopulmonary Bypass. Plos One 7, e35070.

Juvet, S.C., and Zhang, L. (2012). Double negative regulatory T cells in transplantation and autoimmunity: recent progress and future directions. J Mol Cell Biol 4, 48-58.

Kang, X., Kim, J., Deng, M., John, S., Chen, H., Wu, G., Phan, H., and Zhang, C.C. (2015). Inhibitory leukocyte immunoglobulin-like receptors: Immune checkpoint proteins and tumor sustaining factors. Cell Cycle 15, 25-40.

Kapoor, R., Davies, M., Blaker, P.A., Hall, S.M., and Smith, K.J. (2003). Blockers of sodium and calcium entry protect axons from nitric oxide-mediated degeneration. Ann Neurol 53, 174-180.

Karni, A., Abraham, M., Monsonego, A., Cai, G., Freeman, G.J., Hafler, D., Khoury, S.J., and Weiner, H.L. (2006). Innate Immunity in Multiple Sclerosis: Myeloid Dendritic Cells in Secondary Progressive Multiple Sclerosis Are Activated and Drive a Proinflammatory Immune Response. J Immunol 177, 4196-4202.

Kastrukoff, L.F., Morgan, N.G., Zecchini, D., White, R., Petkau, A.J., Satoh, J., and Paty, D.W. (1998). A role for natural killer cells in the immunopathogenesis of multiple sclerosis. J Neuroimmunol 86, 123-133.

Kitsos, C.M., Bhamidipati, P., Melnikova, I., Cash, E.P., McNulty, C., Furman, J., Cima, M.J., and Levinson, D. (2007). Combination of automated high throughput platforms, flow cytometry, and hierarchical clustering to detect cell state. Cytom Part A $71 A, 16-27$.

Koenig, J.M., Bliss, J.M., and Mariscalco, M.M. (2017). Fetal and Neonatal Physiology. Sect Xx Dev Immunobiol 1216-1229.e4.

Kouwenhoven, M., Teleshova, N., Özenci, V., Press, R., and Link, H. (2001). Monocytes in multiple sclerosis: phenotype and cytokine profile. $\mathrm{J}$ Neuroimmunol 112, 197-205.

Krause, D., Weidinger, E., Dippel, C., Riedel, M., Schwarz, M.J., Müller, N., and Myint, A.-M. (2013). Impact of different antipsychotics on cytokines and tryptophan metabolites in stimulated cultures from patients with schizophrenia. Psychiat Danub $25,389-397$. 
Krei, K., Fredrikson, S., Fontana, A., and Link, H. (1991). Interleukin-6 is elevated in plasma in multiple sclerosis. J Neuroimmunol 31, 147-153.

Kurtzke, J.F., Beebe, G.W., and Norman, J.E. (1985). Epidemiology of multiple sclerosis in US veterans: III. Migration and the risk of MIS. Neurology 35, 672-672.

Kutzelnigg, A., Lucchinetti, C.F., Stadelmann, C., Brück, W., Rauschka, H., Bergmann, M., Schmidbauer, M., Parisi, J.E., and Lassmann, H. (2005). Cortical demyelination and diffuse white matter injury in multiple sclerosis. Brain 128, 27052712.

Kuuliala, K., Kuuliala, A., Koivuniemi, R., Kautiainen, H., Repo, H., and LeirisaloRepo, M. (2016). STAT6 and STAT1 Pathway Activation in Circulating Lymphocytes and Monocytes as Predictor of Treatment Response in Rheumatoid Arthritis. Plos One 11, e0167975.

Lassmann, H. (2014). Multiple sclerosis: Lessons from molecular neuropathology. Exp Neurol 262, 2-7.

Lassmann, H. (2019). Pathogenic Mechanisms Associated With Different Clinical Courses of Multiple Sclerosis. Front Immunol 9, 3116.

Lassmann, H., Horssen, J. van, and Mahad, D. (2012). Progressive multiple sclerosis: pathology and pathogenesis. Nat Rev Neurology 8, 647-656.

La Flamme, A.C., Abernethy, D., Sim, D., Goode, L., Lockhart, M., Bourke, D., Milner, I., Garrill, T.-M., Joshi, P., Watson, E., et al. (2020). Safety and acceptability of clozapine and risperidone in progressive multiple sclerosis: a phase 1, randomized, blinded, placebo-controlled trial. Medrxiv 2020.03.12.20034983.

Levite, M. (2012). Dopamine in the immune system: dopamine receptors in immune cells, potent effects, endogenous production and involvement in immune and neuropsychiatric diseases. Springer, Wien. 1-359

Li, R., Rezk, A., Ghadiri, M., Luessi, F., Zipp, F., Li, H., Giacomini, P.S., Antel, J., and Bar-Or, A. (2016). Dimethyl Fumarate Treatment Mediates an Anti-Inflammatory Shift in B Cell Subsets of Patients with Multiple Sclerosis. J Immunol Baltim Md $1950198,691-698$.

Lieberman, L.A., Zeng, W., Singh, C., Wang, W., Otipoby, K.L., Loh, C., Plavina, T., Gorelik, L., Ransohoff, R.M., and Cahir-McFarland, E. (2015). CD62L is not a reliable biomarker for predicting PML risk in natalizumab-treated R-MS patients. Neurology 86, 375-381.

Liu, C.-C., Walsh, C.M., and Young, J.D.-E. (1995). Perforin: structure and function. Immunol Today 16, 194-201.

Liu, Z., Pelfrey, C.M., Cotleur, A., Lee, J.-C., and Rudick, R.A. (2001). Immunomodulatory effects of interferon beta-1a in multiple sclerosis. J Neuroimmunol 112, 153-162.

Longbrake, E.E., Ramsbottom, M.J., Cantoni, C., Ghezzi, L., Cross, A.H., and Piccio, L. (2015). Dimethyl fumarate selectively reduces memory T cells in multiple sclerosis patients. Multiple Scler Houndmills Basingstoke Engl 22, 1061-1070.

Lublin, F.D., Reingold, S.C., and Sclerosis*, N.M.S.S. (USA) A.C. on C.T. of N.A. in M. (1996). Defining the clinical course of multiple sclerosis: Results of an international survey. Neurology 46, 907-911. 
Lublin, F.D., Reingold, S.C., Cohen, J.A., Cutter, G.R., Sørensen, P.S., Thompson, A.J., Wolinsky, J.S., Balcer, L.J., Banwell, B., Barkhof, F., et al. (2014). Defining the clinical course of multiple sclerosis: the 2013 revisions. Neurology 83, 278-286.

Luckey, A.M., Anderson, T., Silverman, M.H., and Webster, G. (2015). Safety, tolerability and pharmacodynamics of a novel immunomodulator, MIS416, in patients with chronic progressive multiple sclerosis. Multiple Scler J - Exp Transl Clin 1, 205521731558338.

Lugli, E., Roederer, M., and Cossarizza, A. (2010). Data analysis in flow cytometry: The future just started. Cytom Part A 77A, 705-713.

Ma, W.-T., Gao, F., Gu, K., and Chen, D.-K. (2019). The Role of Monocytes and Macrophages in Autoimmune Diseases: A Comprehensive Review. Front Immunol 10,1140

Maecker, H.T., and Trotter, J. (2006). Flow cytometry controls, instrument setup, and the determination of positivity. Cytom Part A 69A, 1037-1042.

Maecker, H.T., Rinfret, A., D’Souza, P., Darden, J., Roig, E., Landry, C., Hayes, P., Birungi, J., Anzala, O., Garcia, M., et al. (2005). Standardization of cytokine flow cytometry assays. Bmc Immunol 6, 13 .

Maecker, H.T., McCoy, J.P., and Nussenblatt, R. (2012). Standardizing immunophenotyping for the Human Immunology Project. Nat Rev Immunol 12, 191-200.

Mahad, D., Ziabreva, I., Lassmann, H., and Turnbull, D. (2008). Mitochondrial defects in acute multiple sclerosis lesions. Brain 131, 1722-1735.

Maimone, D., Gregory, S., Arnason, B.G.W., and Reder, A.T. (1991). Cytokine levels in the cerebrospinal fluid and serum of patients with multiple sclerosis. $J$

Neuroimmunol 32, 67-74.

Mair, F., Hartmann, F.J., Mrdjen, D., Tosevski, V., Krieg, C., and Becher, B. (2015). The end of gating? An introduction to automated analysis of high dimensional cytometry data. Eur J Immunol 46, 34-43.

Mallucci, G., Peruzzotti-Jametti, L., Bernstock, J.D., and Pluchino, S. (2015). The role of immune cells, glia and neurons in white and gray matter pathology in multiple sclerosis. Prog Neurobiol 127-128, 1-22.

Manel, N., Unutmaz, D., and Littman, D.R. (2008). The differentiation of human $\mathrm{T}(\mathrm{H})-17$ cells requires transforming growth factor-beta and induction of the nuclear receptor RORgammat. Nat Immunol 9, 641-649.

Mantia, L.L., Munari, L.M., and Lovati, R. (2010). Glatiramer acetate for multiple sclerosis. Cochrane Db Syst Rev CDoo4678.

Mantia, L.L., Vacchi, L., Pietrantonj, C.D., Ebers, G., Rovaris, M., Fredrikson, S., and Filippini, G. (2012). Interferon beta for secondary progressive multiple sclerosis. Cochrane Database Syst Rev 1, CDo05181.

Mantia, L.L., Tramacere, I., Firwana, B., Pacchetti, I., Palumbo, R., and Filippini, G. (2016). Fingolimod for relapsing-remitting multiple sclerosis. Cochrane Db Syst Rev 4, CDoo9371.

Marino, F., and Cosentino, M. (2016). Repurposing dopaminergic drugs for MS the evidence mounts. Nat Rev Neurol 12, 191-192. 
Mattoscio, M., Nicholas, R., Sormani, M.P., Malik, O., Lee, J.S., Waldman, A.D., Dazzi, F., and Muraro, P.A. (2015). Hematopoietic mobilization: Potential biomarker of response to natalizumab in multiple sclerosis. Neurology 84, 1473-1482.

McCombe, P., Yan, J., Webster, G., and Greer, J. (2017). Progressive multiple sclerosis patients with NFKBIA mutations are less responsive to the immune changes induced by treatment with MIS416 (P2.349). Neurology 88.

McKenna, F., McLaughlin, P.J., Lewis, B.J., Sibbring, G.C., Cummerson, J.A., Bowen-Jones, D., and Moots, R.J. (2002). Dopamine receptor expression on human T- and B-lymphocytes, monocytes, neutrophils, eosinophils and NK cells: a flow cytometric study. J Neuroimmunol 132, 34-40.

Medscape (2019). Clozapine (Rx).

Https://Reference.Medscape.Com/Drug/Clozaril-Fazaclo-Odt-Clozapine-342972.

Mehling, M., Lindberg, R., Raulf, F., Kuhle, J., Hess, C., Kappos, L., and Brinkmann, V. (2010). Th17 central memory T cells are reduced by FTY720 in patients with multiple sclerosis. Neurology 75, 403-410.

Mei, I. van der, Lucas, R.M., Taylor, B.V., Valery, P.C., Dwyer, T., Kilpatrick, T.J., Pender, M.P., Williams, D., Chapman, C., Otahal, P., et al. (2015). Population attributable fractions and joint effects of key risk factors for multiple sclerosis. Multiple Scler Houndmills Basingstoke Engl 22, 461-469.

Mellergård, J., Edström, M., Jenmalm, M.C., Dahle, C., Vrethem, M., and Ernerudh, J. (2013). Increased B Cell and Cytotoxic NK Cell Proportions and Increased T Cell Responsiveness in Blood of Natalizumab-Treated Multiple Sclerosis Patients. Plos One 8, e81685.

Melnikov, M., Belousova, O., Murugin, V., Pashenkov, M., and Воуко, А. (2016). The role of dopamine in modulation of Th-17 immune response in multiple sclerosis. $\mathrm{J}$ Neuroimmunol 292, 97-101.

Meng, H., Zhao, H., Cao, X., Hao, J., Zhang, H., Liu, Y., Zhu, M.-S., Fan, L., Weng, L., Qian, L., et al. (2019). Double-negative T cells remarkably promote neuroinflammation after ischemic stroke. P Natl Acad Sci Usa 116, 5558-5563.

Meylan, E., Tschopp, J., and Karin, M. (2006). Intracellular pattern recognition receptors in the host response. Nature 442, 39-44.

Michel, L., Chesneau, M., Manceau, P., Genty, A., Garcia, A., Salou, M., Ngono, A.E., Pallier, A., Jacq-Foucher, M., Lefrère, F., et al. (2014). Unaltered regulatory B-cell frequency and function in patients with multiple sclerosis. Clin Immunol Orlando Fla 155, 198-208.

Mikova, O., Yakimova, R., Bosmans, E., Kenis, G., and Maes, M. (2001). Increased serum tumor necrosis factor alpha concentrations in major depression and multiple sclerosis. Eur Neuropsychopharm 11, 203-208.

Mikulak, J., Bozzo, L., Roberto, A., Pontarini, E., Tentorio, P., Hudspeth, K., Lugli, E., and Mavilio, D. (2014). Dopamine Inhibits the Effector Functions of Activated NK Cells via the Upregulation of the D5 Receptor. J Immunol 193, 2792-2800.

Milstein, O., Hagin, D., Lask, A., Reich-Zeliger, S., Shezen, E., Ophir, E., Eidelstein, Y., Afik, R., Antebi, Y.E., Dustin, M.L., et al. (2010). CTLs respond with activation and granule secretion when serving as targets for T-cell recognition. Blood 117, 1042-1052. 
Minagar, A., and Alexander, J.S. (2003). Blood-brain barrier disruption in multiple sclerosis. Mult Scler J 9, 540-549.

Missale, C., Nash, S.R., Robinson, S.W., Jaber, M., and Caron, M.G. (1998).

Dopamine Receptors: From Structure to Function. Physiol Rev 78, 189-225.

Miterski, B., Böhringer, S., Klein, W., Sindern, E., Haupts, M., Schimrigk, S., and Epplen, J.T. (2002). Inhibitors in the NFkB cascade comprise prime candidate genes predisposing to multiple sclerosis, especially in selected combinations. Genes Immun 3, 211-219.

Monici, M. (2005). Biotechnology Annual Review. Biotechnology Annu Rev 11, 227256.

Montalban, X., Hauser, S.L., Kappos, L., Arnold, D.L., Bar-Or, A., Comi, G., Seze, J. de, Giovannoni, G., Hartung, H.-P., Hemmer, B., et al. (2017). Ocrelizumab versus Placebo in Primary Progressive Multiple Sclerosis. New Engl J Med 376, 209-220.

Moretta, A., Marcenaro, E., Parolini, S., Ferlazzo, G., and Moretta, L. (2007). NK cells at the interface between innate and adaptive immunity. Cell Death Differ 15, 226-233.

Mosmann, T.R., Cherwinski, H., Bond, M.W., Giedlin, M.A., and Coffman, R.L. (1986). Two types of murine helper T cell clone. I. Definition according to profiles of lymphokine activities and secreted proteins. J Immunol Baltim Md 1950 136, 23482357.

MSIF (2013). Atlas of MS 2013.

Https://Www.Msif.Org/.

Mukherjee, R., Barman, P.K., Thatoi, P.K., Tripathy, R., Das, B.K., and Ravindran, B. (2015). Non-Classical monocytes display inflammatory features: Validation in Sepsis and Systemic Lupus Erythematous. Sci Rep-Uk 5, 13886.

Murphy, K. (2012). Janeway's Immunobiology. $8^{\text {th }}$ Edition. Garland Science, London. 1-509

Murphy, R.F. (1985). Automated identification of subpopulations in flow cytometric list mode data using cluster analysis. Cytometry 6, 302-309.

Nagata, S., and Suda, T. (1995). Fas and Fas ligand: lpr and gld mutations. Immunol Today $16,39-43$.

Naito, S., Namerow, N., Mickey, M.R., and Terasaki, P.I. (2008). Multiple Sclerosis: Association with HL-A3. Tissue Antigens 2, 1-4.

Nathan, C.F. (1987). Secretory products of macrophages. J Clin Invest 79, 319-326.

Navikas, V., and Link, H. (1996). Cytokines and the pathogenesis of multiple sclerosis. Journal of Neuroscience Research. 45(4) 322-333.

Negrotto, L., Cantó, E., Río, J., Tintoré, M., Montalban, X., and Comabella, M. (2015). Peripheral blood non-MAIT CD8+CD161hi cells are decreased in relapsingremitting multiple sclerosis patients treated with interferon beta. J Neuroimmunol 288, 98-101.

Niemand, C., Nimmesgern, A., Haan, S., Fischer, P., Schaper, F., Rossaint, R., Heinrich, P.C., and Müller-Newen, G. (2003). Activation of STAT3 by IL-6 and IL-10 in Primary Human Macrophages Is Differentially Modulated by Suppressor of Cytokine Signaling 3. J Immunol 170, 3263-3272. 
Niino, M., Hirotani, M., Miyazaki, Y., and Sasaki, H. (2009). Memory and naïve Bcell subsets in patients with multiple sclerosis. Neurosci Lett 464, 74-78.

Niino, M., Fukazawa, T., Minami, N., Amino, I., Tashiro, J., Fujiki, N., Doi, S., and Kikuchi, S. (2012). CD5-positive B cell subsets in secondary progressive multiple sclerosis. Neurosci Lett 523, 56-61.

Nolan, J.P., and Condello, D. (2001). Current Protocols in Cytometry. Curr Protoc Cytom Éditor Board J Paul Robinson Managing Ed Et Al Chapter 1, Unit1.27.

O’Connor, P., Miller, D., Riester, K., Yang, M., Panzara, M., Dalton, C., Miszkiel, K., Khan, O., Rice, G., Sheremata, W., et al. (2005). Relapse rates and enhancing lesions in a phase II trial of natalizumab in multiple sclerosis. Mult Scler J 11, 568-572.

Oh, J., Alikhani, K., Bruno, T., Devonshire, V., Giacomini, P.S., Giuliani, F., Nakhaipour, H.R., Schecter, R., and Larochelle, C. (2019). Diagnosis and management of secondary-progressive multiple sclerosis: time for change.

Neurodegener Dis Management. Vol 9 (6). https://doi.org/10.2217/nmt-2019-0024

Oksenberg, J.R., Barcellos, L.F., Cree, B.A.C., Baranzini, S.E., Bugawan, T.L., Khan, O., Lincoln, R.R., Swerdlin, A., Mignot, E., Lin, L., et al. (2004). Mapping Multiple Sclerosis Susceptibility to the HLA-DR Locus in African Americans. Am J Hum Genetics 74, 160-167.

Olsson, T., Barcellos, L.F., and Alfredsson, L. (2016). Interactions between genetic, lifestyle and environmental risk factors for multiple sclerosis. Nat Rev Neurology 13, $25-36$.

Ontaneda, D., Cohn, S., and Fox, R.J. (2014). Risk stratification and mitigation in multiple sclerosis. Mult Scler Relat Dis 3, 639-649.

Ortiz, G.G., Pacheco-Moisés, F.P., Macías-Islas, M.Á., Flores-Alvarado, L.J., MirelesRamírez, M.A., González-Renovato, E.D., Hernández-Navarro, V.E., Sánchez-López, A.L., and Alatorre-Jiménez, M.A. (2014). Role of the Blood-Brain Barrier in Multiple Sclerosis. Arch Med Res 45, 687-697.

O’Sullivan, D., Green, L., Stone, S., Zareie, P., Kharkrang, M., Fong, D., Connor, B., and Flamme, A.C.L. (2014). Treatment with the antipsychotic agent, risperidone, reduces disease severity in experimental autoimmune encephalomyelitis. Plos One 9, e104430.

Ovchinnikov, D.A. (2008). Macrophages in the embryo and beyond: Much more than just giant phagocytes. Genesis 46, 447-462.

Palanichamy, A., Jahn, S., Nickles, D., Derstine, M., Abounasr, A., Hauser, S.L., Baranzini, S.E., Leppert, D., and Büdingen, H.-C. von (2014). Rituximab efficiently depletes increased CD2O-expressing T cells in multiple sclerosis patients. $\mathrm{J}$ Immunol Baltim Md 1950 193, 580-586.

Palmer, E. (2003). Negative selection - clearing out the bad apples from the T-cell repertoire. Nat Rev Immunol 3, 383-391.

Panitch, H., Haley, A., Hirsch, R., and Johnson, K. (1987). Exacerbations of Multiple Sclerosis in patients treated with gamma interferon. Lancet 329, 893-895.

Parolini, S., Santoro, A., Marcenaro, E., Luini, W., Massardi, L., Facchetti, F., Communi, D., Parmentier, M., Majorana, A., Sironi, M., et al. (2007). The role of 
chemerin in the colocalization of $\mathrm{NK}$ and dendritic cell subsets into inflamed tissues. Blood 109, 3625-3632.

Passweg, J.R., Baldomero, H., Bader, P., Bonini, C., Duarte, R.F., Dufour, C., Gennery, A., Kröger, N., Kuball, J., Lanza, F., et al. (2017). Use of haploidentical stem cell transplantation continues to increase: the 2015 European Society for Blood and Marrow Transplant activity survey report. Bone Marrow Transpl 52, 811-817.

Pender, M.P., Csurhes, P.A., Pfluger, C.M., and Burrows, S.R. (2014). Deficiency of CD8 + effector memory T cells is an early and persistent feature of multiple sclerosis. Mult Scler J 2O, 1825-1832.

Peters, J.H., Ruppert, J., Gieseler, R.K.H., Najar, H.M., and Xu, H. (1991). Differentiation of Human Monocytes into CD14 Negative Accessory Cells: Do Dendritic Cells Derive from the Monocytic Lineage? Pathobiology 59, 122-126.

Peterson, J.W., Bö, L., Mörk, S., Chang, A., and Trapp, B.D. (2001). Transected neurites, apoptotic neurons, and reduced inflammation in cortical multiple sclerosis lesions. Ann Neurol 5o, 389-400.

Plavina, T., Muralidharan, K.K., Kuesters, G., Mikol, D., Evans, K., Subramanyam, M., Nestorov, I., Chen, Y., Dong, Q., Ho, P.-R., et al. (2017). Reversibility of the effects of natalizumab on peripheral immune cell dynamics in MS patients.

Neurology 89, 1584-1593.

Polman, C.H., O’Connor, P.W., Havrdova, E., Hutchinson, M., Kappos, L., Miller, D.H., Phillips, J.T., Lublin, F.D., Giovannoni, G., Wajgt, A., et al. (2006). A Randomized, Placebo-Controlled Trial of Natalizumab for Relapsing Multiple Sclerosis. New Engl J Med 354, 899-910.

Polman, C.H., Reingold, S.C., Banwell, B., Clanet, M., Cohen, J.A., Filippi, M., Fujihara, K., Havrdova, E., Hutchinson, M., Kappos, L., et al. (2011). Diagnostic criteria for multiple sclerosis: 2010 Revisions to the McDonald criteria. Ann Neurol 69, 292-302.

Portevin, D., Pflüger, V., Otieno, P., Brunisholz, R., Vogel, G., and Daubenberger, C. (2015). Quantitative whole-cell MALDI-TOF MS fingerprints distinguishes human monocyte sub-populations activated by distinct microbial ligands. Bmc Biotechnol $15,24$.

Posová, H., Horáková, D., Čapek, V., Uher, T., Hrušková, Z., and Havrdová, E. (2017). Peripheral blood lymphocytes immunophenotyping predicts disease activity in clinically isolated syndrome patients. Bmc Neurol 17, 145 .

Prado, C., Gaiazzi, M., González, H., Ugalde, V., Figueroa, A., Osorio-Barrios, F.J., López, E., Lladser, A., Rasini, E., Marino, F., et al. (2018). Dopaminergic Stimulation of Myeloid Antigen-Presenting Cells Attenuates Signal Transducer and Activator of Transcription 3-Activation Favouring the Development of Experimental Autoimmune Encephalomyelitis. Front Immunol 9, 571.

Praksova, P., Stourac, P., Bednarik, J., Vlckova, E., Mikulkova, Z., and Michalek, J. (2012). Immunoregulatory T cells in multiple sclerosis and the effect of interferon beta and glatiramer acetate treatment on T cell subpopulations. J Neurol Sci 319, 1823.

ProteinAtlas (2019). DRD5 (protein). Https://Www.Proteinatlas.Org/ENSGoooo0169676-DRD5/Tissue. 
Pulliam, L., Gascon, R., Stubblebine, M., McGuire, D., and McGrath, M.S. (1997). Unique monocyte subset in patients with AIDS dementia. Lancet 349, 692-695.

Qiu, P. (2017). Toward deterministic and semiautomated SPADE analysis. Cytom Part A 91, 281-289.

Qiu, P., Simonds, E.F., Bendall, S.C., Gibbs, K.D., Bruggner, R.V., Linderman, M.D., Sachs, K., Nolan, G.P., and Plevritis, S.K. (2011). Extracting a cellular hierarchy from high-dimensional cytometry data with SPADE. Nat Biotechnol 29, 886-891.

Quinn, M.A. (2019). Innate Immunotheraputics 2017 Annual Report. Http://www.innateimmunotherapeutics.com/irm/content/annualreports_RID_319.html

Ransohoff, R.M., Kivisäkk, P., and Kidd, G. (2003). Three or more routes for leukocyte migration into the central nervous system. Nat Rev Immunol 3, 569-581.

Reale, M., Patruno, A., Lutiis, M.A.D., Pesce, M., Felaco, M., Giannantonio, M.D., Nicola, M.D., and Grilli, A. (2011). Dysregulation of chemo-cytokine production in schizophrenic patients versus healthy controls. Bmc Neurosci 12, 13 .

Reder, A.T., and Feng, X. (2014). How type I interferons work in multiple sclerosis and other diseases: some unexpected mechanisms. J Interf Cytokine Res Official $\mathbf{J}$ Int Soc Interf Cytokine Res 34, 589-599.

Rees, A.J. (2010). Monocyte and Macrophage Biology: An Overview. Semin Nephrol $30,216-233$.

Ribeiro, B.M.M., Carmo, M.R.S. do, Freire, R.S., Rocha, N.F.M., Borella, V.C.M., Menezes, A.T. de, Monte, A.S., Gomes, P.X.L., Sousa, F.C.F. de, Vale, M.L., et al. (2013). Evidences for a progressive microglial activation and increase in iNOS expression in rats submitted to a neurodevelopmental model of schizophrenia: Reversal by clozapine. Schizophr Res 151, 12-19.

Robbins, S.H., Walzer, T., Dembélé, D., Thibault, C., Defays, A., Bessou, G., Xu, H., Vivier, E., Sellars, M., Pierre, P., et al. (2008). Novel insights into the relationships between dendritic cell subsets in human and mouse revealed by genome-wide expression profiling. Genome Biol 9, R17.

Robins, H.S., Campregher, P.V., Srivastava, S.K., Wacher, A., Turtle, C.J., Kahsai, O., Riddell, S.R., Warren, E.H., and Carlson, C.S. (2009). Comprehensive assessment of T-cell receptor beta-chain diversity in alphabeta T cells. Blood 114, 4099-4107.

Robinson, A.P., Harp, C.T., Noronha, A., and Miller, S.D. (2014). The experimental autoimmune encephalomyelitis (EAE) model of MS: utility for understanding disease pathophysiology and treatment. Handb Clin Neurology 122, 173-189.

Robinson, S.P., Patterson, S., English, N., Davies, D., Knight, S.C., and Reid, C.D.L. (1999). Human peripheral blood contains two distinct lineages of dendritic cells. Eur J Immunol 29, 2769-2778.

Rodríguez-Martín, E., Picón, C., Costa-Frossard, L., Alenda, R., Maza, S.S. de la, Roldán, E., Espiño, M., Villar, L.M., and Álvarez-Cermeño, J.C. (2015). Natural killer cell subsets in cerebrospinal fluid of patients with multiple sclerosis. Clin Exp Immunol 18o, 243-249.

Rostami, A., and Ciric, B. (2013). Role of Th17 cells in the pathogenesis of CNS inflammatory demyelination. J Neurol Sci 333, 76-87. 
Roth, B., Sheffler, D., and Potkin, S. (2003). Atypical antipsychotic drug actions: unitary or multiple mechanisms for "atypicality"? Clinical Neuroscience Research 3, Issues $1-2,108-117$

Rouvier, E., Luciani, M.F., and Golstein, P. (1993). Fas involvement in $\mathrm{Ca}(2+)-$ independent T cell-mediated cytotoxicity. J Exp Medicine 177, 195-200.

Rudensky, A. (2005). Foxp3 and dominant tolerance. Philosophical Transactions Royal Soc B Biological Sci 360, 1645-1646.

Rudick, R.A., and Ransohoff, R.M. (1992). Cytokine Secretion by Multiple Sclerosis Monocytes. Arch Neurol-Chicago 49, 265.

SAHealth (2019). Clozapine.

Https://Www.Sahealth.Sa.Gov.Au/Wps/Wcm/Connect/Public+content/Sa+health+ internet/Clinical+resources/Clinical+topics/Medicines+and+drugs/Clozapine.

Sakaguchi, S. (2005). Naturally arising Foxp3-expressing CD25+CD4+ regulatory T cells in immunological tolerance to self and non-self. Nat Immunol 6, 345-352.

Sakaguchi, S., Yamaguchi, T., Nomura, T., and Ono, M. (2008). Regulatory T Cells and Immune Tolerance. Cell 133, 775-787.

Sallusto, F., Geginat, J., and Lanzavecchia, A. (2004). C entral M emory and E ffector M emory T C ell S ubsets : Function, Generation, and Maintenance. Annu Rev Immunol 22, 745-763.

Sampath, P., Moideen, K., Ranganathan, U.D., and Bethunaickan, R. (2018). Monocyte Subsets: Phenotypes and Function in Tuberculosis Infection. Front Immunol 9, 1726.

Sarkar, C., Basu, B., Chakroborty, D., Dasgupta, P.S., and Basu, S. (2010). The immunoregulatory role of dopamine: An update. Brain Behav Immun 24, 525-528.

Sato, D.K., Nakashima, I., Bar-Or, A., Misu, T., Suzuki, C., Nishiyama, S., Kuroda, H., Fujihara, K., and Aoki, M. (2014). Changes in Th17 and regulatory T cells after fingolimod initiation to treat multiple sclerosis. J Neuroimmunol 268, 95-98.

Sawcer, S. (2008). The complex genetics of multiple sclerosis: pitfalls and prospects. Brain J Neurology 131, 3118-3131.

Scalfari, A., Knappertz, V., Cutter, G., Goodin, D.S., Ashton, R., and Ebers, G.C. (2013). Mortality in patients with multiple sclerosis. Neurology 81, 184-192.

Schmid, C.L., Streicher, J.M., Meltzer, H.Y., and Bohn, L.M. (2014). Clozapine Acts as an Agonist at Serotonin 2A Receptors to Counter MK-801-Induced Behaviors through a $\beta$ Arrestin2-Independent Activation of Akt. Neuropsychopharmacol 39, 1902-1913.

Schrijver, I.A., Meurs, M. van, Melief, M.-J., Ang, C.W., Buljevac, D., Ravid, R., Hazenberg, M.P., and Laman, J.D. (2001). Bacterial peptidoglycan and immune reactivity in the central nervous system in multiple sclerosis. Brain 124, 1544-1554.

Schubert, R.D., Hu, Y., Kumar, G., Szeto, S., Abraham, P., Winderl, J., Guthridge, J.M., Pardo, G., Dunn, J., Steinman, L., et al. (2015). IFN- $\beta$ treatment requires B cells for efficacy in neuroautoimmunity. J Immunol Baltim Md 1950 194, 2110-2116.

Schulz, K.R., Danna, E.A., Krutzik, P.O., and Nolan, G.P. (2001). Current Protocols in Immunology. Curr Protoc Immunol Ed John E Coligan Et Al Chapter 8, Unit 8.17.120. 
Schwarz, A., Balint, B., Korporal-Kuhnke, M., Jarius, S., Engelhardt, K. von, Fürwentsches, A., Bussmann, C., Ebinger, F., Wildemann, B., and Haas, J. (2016). Bcell populations discriminate between pediatric- and adult-onset multiple sclerosis. Neurology - Neuroimmunol Neuroinflammation 4, e309.

Sedger, L.M., and McDermott, M.F. (2014). TNF and TNF-receptors: From mediators of cell death and inflammation to therapeutic giants - past, present and future. Cytokine Growth F R 25, 453-472.

Seeman, P. (2014). Clozapine, a Fast-Off-D2 Antipsychotic. Acs Chem Neurosci 5, 24-29.

Seidi, O.A., Semra, Y.K., and Sharief, M.K. (2002). Expression of CD5 on B lymphocytes correlates with disease activity in patients with multiple sclerosis. $\mathrm{J}$ Neuroimmunol 133, 205-210.

Sellebjerg, F., Hesse, D., Limborg, S., Lund, H., Sondergaard, H., Krakauer, M., and Sorensen, P. (2012a). Dendritic cell, monocyte and T cell activation and response to glatiramer acetate in multiple sclerosis. Mult Scler J 19, 179-187.

Sellebjerg, F., Krakauer, M., Limborg, S., Hesse, D., Lund, H., Langkilde, A., Søndergaard, H.B., and Sørensen, P.S. (2012b). Endogenous and Recombinant Type I Interferons and Disease Activity in Multiple Sclerosis. Plos One 7, e35927.

Sharief, M.K., and Hentges, R. (1991). Association between Tumor Necrosis Factor- $\alpha$ and Disease Progression in Patients with Multiple Sclerosis. New Engl J Med 325, 467-472.

Sharrack, B., Saccardi, R., Alexander, T., Badoglio, M., Burman, J., Farge, D., Greco, R., Jessop, H., Kazmi, M., Kirgizov, K., et al. (2019). Autologous haematopoietic stem cell transplantation and other cellular therapy in multiple sclerosis and immunemediated neurological diseases: updated guidelines and recommendations from the EBMT Autoimmune Diseases Working Party (ADWP) and the Joint Accreditation Committee of EBMT and ISCT (JACIE). Bone Marrow Transpl 1-24.

Simpson, N., Gatenby, P.A., Wilson, A., Malik, S., Fulcher, D.A., Tangye, S.G., Manku, H., Vyse, T.J., Roncador, G., Huttley, G.A., et al. (2010). Expansion of circulating $\mathrm{T}$ cells resembling follicular helper $\mathrm{T}$ cells is a fixed phenotype that identifies a subset of severe systemic lupus erythematosus. Arthritis Rheumatism 62, 234-244.

Simpson, S., Blizzard, L., Otahal, P., Mei, I.V. der, and Taylor, B. (2011). Latitude is significantly associated with the prevalence of multiple sclerosis: a meta-analysis. $\mathrm{J}$ Neurology Neurosurg Psychiatry 82, 1132-1141.

Skarica, M., Eckstein, C., Whartenby, K.A., and Calabresi, P.A. (2011). Novel mechanisms of immune modulation of natalizumab in multiple sclerosis patients. $\mathrm{J}$ Neuroimmunol 235, 70-76.

Smith, K.J., and McDonald, W.I. (1999). The pathophysiology of multiple sclerosis: the mechanisms underlying the production of symptoms and the natural history of the disease. Philosophical Transactions Royal Soc Lond Ser B Biological Sci 354, 1649-1673.

Smedman, C., Ernemar, T., Gudmundsdotter, L., Gille-Johnson, P., Somell, A., Nihlmark, K., Gårdlund, B., Andersson, J., and Paulie, S. (2012). FluoroSpot Analysis 
of TLR-Activated Monocytes Reveals Several Distinct Cytokine-Secreting Subpopulations. Scand J Immunol 75, 249-258.

Snowden, J.A., Badoglio, M., Labopin, M., Giebel, S., McGrath, E., Marjanovic, Z., Burman, J., Moore, J., Rovira, M., Wulffraat, N.M., et al. (2017). Evolution, trends, outcomes, and economics of hematopoietic stem cell transplantation in severe autoimmune diseases. Blood Adv 1, 2742-2755.

Snowden, J.A., Sharrack, B., Akil, M., Kiely, D.G., Lobo, A., Kazmi, M., Muraro, P.A., and Lindsay, J.O. (2018). Autologous haematopoietic stem cell transplantation (aHSCT) for severe resistant autoimmune and inflammatory diseases - a guide for the generalist. Clin Medicine Lond Engl 18, 329-334.

Spencer, C.M., Crebtree-Hartmann, E.C., Lehmann-Horn, K., Cree, B.A.C., and Zamvil, S.S. (2015). Reduction of CD8 + T lymphocytes in multiple sclerosis patients treated with dimethyl fumarate. Neurology - Neuroimmunol Neuroinflammation 2, e76.

Starr, T.K., Jameson, S.C., and Hogquist, K.A. (2003). Positive and negative selection of T cells. Annu Rev Immunol 21, 139-176.

Staun-Ram, E., and Miller, A. (2017). Effector and regulatory B cells in Multiple Sclerosis. Clin Immunol 184, 11-25.

Stone, K.D., Prussin, C., and Metcalfe, D.D. (2010). IgE, mast cells, basophils, and eosinophils. J Allergy Clin Immunol 125, S73-80.

Streck (2019). Cyto-chex BCT tube. Https://Www.Streck.Com/Collection/Cyto-Chex-Bct/.

Streitz, M., Miloud, T., Kapinsky, M., Reed, M.R., Magari, R., Geissler, E.K., Hutchinson, J.A., Vogt, K., Schlickeiser, S., Kverneland, A., et al. (2013).

Standardization of whole blood immune phenotype monitoring for clinical trials: panels and methods from the ONE study. Transplant Res 2, 17.

Stys, P., Waxman, S., and Ransom, B. (1992). Ionic mechanisms of anoxic injury in mammalian CNS white matter: role of $\mathrm{Na}+$ channels and $\mathrm{Na}(+)-\mathrm{Ca} 2+$ exchanger. $\mathrm{J}$ Neurosci 12, 430-439.

Sun, Z., and Andersson, R. (2002). NF- $\mathrm{B}$ activation and inhibition: a review. SHOCK 18 (2) 99-106

Szabo, S.J., Kim, S.T., Costa, G.L., Zhang, X., Fathman, C.G., and Glimcher, L.H. (2000). A Novel Transcription Factor, T-bet, Directs Th1 Lineage Commitment. Cell $100,655-669$.

Templeton, N., Kivell, B., McCaughey-Chapman, A., Connor, B., and Flamme, A.C.L. (2019). Clozapine administration enhanced functional recovery after cuprizone demyelination. Plos One 14, eo216113.

Teniente-Serra, A., Hervás, J.V., Quirant-Sánchez, B., Mansilla, M.J., Grau-López, L., Ramo-Tello, C., and Martínez-Cáceres, E.M. (2016). Baseline Differences in Minor Lymphocyte Subpopulations may Predict Response to Fingolimod in Relapsing-Remitting Multiple Sclerosis Patients. Cns Neurosci Ther 22, 584-592.

Tesmer, L.A., Lundy, S.K., Sarkar, S., and Fox, D.A. (2008). Th17 cells in human disease. Immunol Rev 223, 87-113. 
Thewissen, K., Nuyts, A.H., Deckx, N., Wijmeersch, B.V., Nagels, G., D’hooghe, M., Willekens, B., Cras, P., Eijnde, B.O., Goossens, H., et al. (2013). Circulating dendritic cells of multiple sclerosis patients are proinflammatory and their frequency is correlated with MS-associated genetic risk factors. Mult Scler J 2O, 548-557.

Thomas, K., Sehr, T., Proschmann, U., Rodriguez-Leal, F.A., Haase, R., and Ziemssen, T. (2017). Fingolimod additionally acts as immunomodulator focused on the innate immune system beyond its prominent effects on lymphocyte recirculation. J Neuroinflamm 14, 41.

Tlaskalová-Hogenová, H., Štěpánková, R., Hudcovic, T., Tučková, L., Cukrowska, B., Lodinová-Žádnı ková, R., Kozáková, H., Rossmann, P., Bártová, J., Sokol, D., et al. (2004). Commensal bacteria (normal microflora), mucosal immunity and chronic inflammatory and autoimmune diseases. Immunol Lett 93, 97-108.

Tonegawa, S. (1983). Somatic generation of antibody diversity. Nature 302, 575-581.

Tramacere, I., Giovane, C.D., Salanti, G., D’Amico, R., and Filippini, G. (2015). Immunomodulators and immunosuppressants for relapsing-remitting multiple sclerosis: a network meta-analysis. Cochrane Db Syst Rev 9, CDo11381.

Trotter, J.L., Collins, K.G., and Veen, R.C. van der (1991). Serum cytokine levels in chronic progressive multiple sclerosis: interleukin-2 levels parallel tumor necrosis factor-a levels. J Neuroimmunol 33, 29-36.

Tsujimura, H., Tamura, T., Kong, H.J., Nishiyama, A., Ishii, K.J., Klinman, D.M., and Ozato, K. (2004). Toll-Like Receptor 9 Signaling Activates NF- $\kappa$ B through IFN Regulatory Factor-8/IFN Consensus Sequence Binding Protein in Dendritic Cells. J Immunol 172, 6820-6827.

Tsukada, N., Miyagi, K., Matsuda, M., Yanagisawa, N., and Yone, K. (1991). Tumor necrosis factor and interleukin-1 in the CSF and sera of patients with multiple sclerosis. J Neurol Sci 104, 230-234.

Venken, K., Hellings, N., Hensen, K., Rummens, J.-L., Medaer, R., D’hooghe, M.B., Dubois, B., Raus, J., and Stinissen, P. (2006). Secondary progressive in contrast to relapsing-remitting multiple sclerosis patients show a normal CD4 + CD25 + regulatory T-cell function and FOXP3 expression. J Neurosci Res 83, 1432-1446.

Voehringer, D. (2009). The role of basophils in helminth infection. Trends Parasitol $25,551-556$.

Vyas, J.M., Veen, A.G.V. der, and Ploegh, H.L. (2008). The known unknowns of antigen processing and presentation. Nat Rev Immunol 8, 607-618.

Walker, L., and Sansom, D. (2011). The emerging role of CTLA4 as a cell-extrinsic regulator of T cell responses. Nat Rev Immunol 11, 852-863.

Wang, D., and Baldwin, A.S. (1998). Activation of Nuclear Factor- $\kappa B-d e p e n d e n t$ Transcription by Tumor Necrosis Factor- $\alpha$ Is Mediated through Phosphorylation of RelA/p65 on Serine 529. J Biol Chem 273, 29411-29416.

Wang, B., Wei, H., Prabhu, L., Zhao, W., Martin, M., Hartley, A.-V., and Lu, T. (2015). Role of Novel Serine 316 Phosphorylation of the p65 Subunit of NF- $\kappa B$ in Differential Gene Regulation. J Biol Chem 290, 20336-20347.

Waschbisch, A., Schröder, S., Schraudner, D., Sammet, L., Weksler, B., Melms, A., Pfeifenbring, S., Stadelmann, C., Schwab, S., and Linker, R.A. (2016). Pivotal Role 
for CD16+ Monocytes in Immune Surveillance of the Central Nervous System. J Immunol Baltim Md 1950 196, 1558-1567.

White, M.P. (2015). Innate immunomodulation with MIS416: mechanism of action in experimental autoimmune encephalomyelitis. Victoria University of Wellington, New Zealand.

White, M., Webster, G., O’Sullivan, D., Stone, S., and La Flamme, A.C. (2014). Targeting innate receptors with MIS416 reshapes Th responses and suppresses CNS disease in a mouse model of multiple sclerosis. Plos One 9, e87712.

White, M.P.J., Webster, G., Leonard, F., and La Flamme, A.C. (2018). Innate IFN- $\gamma$ ameliorates experimental autoimmune encephalomyelitis and promotes myeloid expansion and PDL-1 expression. Sci Rep-Uk 8, 259.

Wiesemann, E., Deb, M., Trebst, C., Hemmer, B., Stangel, M., and Windhagen, A. (2007). Effects of interferon- $\beta$ on co-signaling molecules: upregulation of CD4O, CD86 and PD-L2 on monocytes in relation to clinical response to interferon- $\beta$ treatment in patients with multiple sclerosis. Mult Scler J 14, 166-176.

Wilkins, M.F., Hardy, S.A., Boddy, L., and Morris, C.W. (2001). Comparison of five clustering algorithms to classify phytoplankton from flow cytometry data. Cytometry 44, 210-217.

Wipfler, P., Oppermann, K., Pilz, G., Afazel, S., Haschke-Becher, E., Harrer, A., Huemer, M., Kunz, A., Golaszewski, S., Staffen, W., et al. (2010). Adhesion molecules are promising candidates to establish surrogate markers for natalizumab treatment. Multiple Scler Houndmills Basingstoke Engl 17, 16-23.

Witko-Sarsat, V., Rieu, P., Descamps-Latscha, B., Lesavre, P., and HalbwachsMecarelli, L. (2000). Neutrophils: Molecules, Functions and Pathophysiological Aspects. Lab Invest 8o, 617-653.

Wu, G.F., and Alvarez, E. (2011). The immunopathophysiology of multiple sclerosis. Neurol Clin 29, 257-278.

Xing, L., and Remick, D.G. (2003). Relative cytokine and cytokine inhibitor production by mononuclear cells and neutrophils. Shock 2O, 10-16.

Xu, Z., Zhang, F., Sun, F., Gu, K., Dong, S., and He, D. (2015). Dimethyl fumarate for multiple sclerosis. Cochrane Db Syst Rev 4, CD011076.

Yan, J., Winterford, C.M., Catts, V.S., Pat, B.K., Pender, M.P., McCombe, P.A., and Greer, J.M. (2018). Increased constitutive activation of NF- $\kappa$ B p65 (RelA) in peripheral blood cells of patients with progressive multiple sclerosis. J

Neuroimmunol 320, 111-116.

Zaffaroni, M., Marino, F., Bombelli, R., Rasini, E., Monti, M., Ferrari, M., Ghezzi, A., Comi, G., Lecchini, S., and Cosentino, M. (2008). Therapy with interferon-beta modulates endogenous catecholamines in lymphocytes of patients with multiple sclerosis. Exp Neurol 214, 315-321.

Zareie, P., Connor, B., and La Flamme, A.C. (2017). Amelioration of experimental autoimmune encephalomyelitis by clozapine is not associated with defective CD4 T cell responses. J Neuroinflamm 14, 68.

Zhu, J., Yamane, H., Cote-Sierra, J., Guo, L., and Paul, W.E. (2006). GATA-3 promotes Th2 responses through three different mechanisms: induction of Th2 
cytokine production, selective growth of Th2 cells and inhibition of Th1 cell-specific factors. Cell Res 16, 3-10.

Ziegler-Heitbrock, L. (2006). The CD14+CD16+ blood monocytes: their role in infection and inflammation. J Leukocyte Biol 81, 584-592.

Ziegler-Heitbrock, L., Ancuta, P., Crowe, S., Dalod, M., Grau, V., Hart, D.N., Leenen, P.J.M., Liu, Y.-J., MacPherson, G., Randolph, G.J., et al. (2010). Nomenclature of monocytes and dendritic cells in blood. Blood 116, e74-80.

Zinkernagel, R.M., Bachmann, M.F., Kündig, T.M., Oehen, S., Pirchet, H., and Hengartner, H. (1996). On immunological memory. Annu Rev Immunol 14, 333367.

Zlokovic, B.V. (2008). The Blood-Brain Barrier in Health and Chronic Neurodegenerative Disorders. Neuron 57, 178-201.

Zohren, F., Toutzaris, D., Klärner, V., Hartung, H.-P., Kieseier, B., and Haas, R. (2008). The monoclonal anti-VLA-4 antibody natalizumab mobilizes CD34+ hematopoietic progenitor cells in humans. Blood 111, 3893-3895. 
